University of Tennessee Health Science Center UTHSC Digital Commons

\title{
Application of Different Bioanalytical Workflows for Proteomics of Prostate Cancer
}

\author{
Li Chen \\ University of Tennessee Health Science Center
}

Follow this and additional works at: https://dc.uthsc.edu/dissertations

Part of the Pharmacy and Pharmaceutical Sciences Commons

\section{Recommended Citation}

Chen, Li , "Application of Different Bioanalytical Workflows for Proteomics of Prostate Cancer" (2009). Theses and Dissertations (ETD). Paper 43. http://dx.doi.org/10.21007/etd.cghs.2009.0050. 


\title{
Application of Different Bioanalytical Workflows for Proteomics of Prostate Cancer
}

\begin{abstract}
In the current dissertation, we focused on the development and application of multiple mass spectrometry-based bioanalytical platforms for phosphoproteomic characterization in cell culture and clinical specimens of prostate cancer; and on the application of optimized methods to analysis of differential protein expression to reveal molecular mechanism of drug action in animal model of prostate cancer.
\end{abstract}

Characterization of the phosphoproteome in prostate cancer

Our study in phosphoprotein signatures on a large scale in prostate cancer focused on the LNCaP human prostate cancer cell line, and on human prostate cancer tissue.

For the LNCaP prostate cancer cell line, we applied a combination of analytical platforms: (1) a novel ingel isoelectric focusing (IEF) LC-MS/MS analytical platform; (2) a 2-DE based platform combined with phospho-specific staining.

The in-gel IEF LC-MS/MS analytical methodology used in the study included separation of the LNCaP proteins by in-gel isoelectric focusing; digestion of the proteins with trypsin; enrichment of the digests for phosphopeptides with immobilized metal ion affinity chromatography (IMAC); analysis of the enriched digests by LC-MS/MS; and identification of the phosphorylated peptides/proteins through searches of the Swiss-Prot protein sequence database. With in-gel IEF based analytical platform, we have characterized over 600 different phosphorylation sites in 296 phosphoproteins in the LNCaP prostate cancer cell line. This panel of the LNCaP phosphoproteins was 3-fold larger than the panel obtained in our previous work, and is the largest phosphoprotein panel in prostate cancer reported to date. The phosphoproteins identified in this study belonged to various locations within the cell and were involved in various processes including cell differentiation, transcription regulation, and intercellular signal transduction.

We also developed a 2-DE based platform, in combination with multiplexed staining and LC-MS/MS, for the identification of LNCaP phosphoproteins. In this study, we applied 2-DE as separation technique, Pro$\mathrm{Q}^{\mathrm{TM}}$ Diamond stain as phosphoprotein detection method, LC-MS/MS and database searches for protein identification to investigate the phosphoproteins in the LNCaP prostate cancer cell line. Proteins identified from spots of interest were shown to be highly relevant to prostate cancer. We demonstrated the feasibility of using 2-DE with phospho-specific stain and mass spectrometry to investigate the phosphoproteins in the LNCaP cell line. This methodology complements the in-gel IEF LC-MS/MS platform that we used for phosphoproteomics study; it will be of particular value for future comparative studies of phosphoproteins in various physiological states.

For prostate cancer tissue, a gel-free approach was performed to analyze five prostate cancer tissue specimens to obtain phosphoproteomic signature of prostate cancer for biomarker discovery. Proteins were extracted with Trizol reagent, and then in-solution digested with trypsin. Phosphopeptides were enriched with IMAC, and analyzed the phosphorylated peptides/proteins by LC-MS/MS with identification through searches of the Swiss-Prot protein sequence database. The panels obtained for prostate cancer tissue contain 15-24 phosphoproteins. Some of the characterized phosphoproteins were present in all five specimens; in addition, each specimen also produced a unique set of phosphoproteins. The findings provided a direct glimpse into the phosphoprotein machinery operating within the human prostate cancer tissue. This pilot study focused on a small set of specimens. The phosphoprotein panels that were obtained contained a number of proteins that were unique to a particular specimen. 
Comparative proteomics study of drug effects in prostate cancer

We carried out the first comparative proteomics study for the examination of the effects of bicalutamide/ embelin combination treatment on prostate tumors by characterizing the alterations in protein expression that was induced upon treatment of mice bearing prostate tumors with anticancer combination therapy.

A comparative proteomic strategy based on 2-DE coupled with LC-MS/MS was performed on mouse prostate tumor tissue. Proteins from the mouse prostate tumors were extracted with Trizol, and the protein mixtures were separated by 2-DE. Differences in the protein profiles for the different treatment groups were evaluated by computer-assisted analysis of SYPRO Ruby stained 2-DE gels. LC-MS/MS and database searches were used to identify differentially expressed proteins. Pathway analysis was carried out on the dataset of identified proteins with the Ingenuity bioinformatics tool. Out of the 33 differentially expressed protein spots, 30 protein spots were identified and grouped into various functional classes. The major protein categories were metabolism (52\%), stress response (12\%), protein biosynthesis (13\%) and apoptosis $(11 \%)$, suggesting that alterations in these processes may be involved in the mechanism of drug action. Proteins associated with oxidative stress were up-regulated, which indicated that treatment with bicalutamide/embelin may affect the redox balance within the prostate tumor, and this effect may contribute to tumor suppression.

\section{Document Type}

Dissertation

Degree Name

Doctor of Philosophy (PhD)

Program

Pharmaceutical Sciences

Research Advisor

Sarka Beranova-Giorgianni, Ph.D.

\section{Keywords}

LNCaP; mass spectrometry; phosphoproteomics; prostate cancer; prostate cancer tissue; proteomics

\section{Subject Categories}

Medicine and Health Sciences | Pharmacy and Pharmaceutical Sciences 


\title{
APPLICATION OF DIFFERENT BIOANALYTICAL WORKFLOWS FOR PROTEOMICS OF PROSTATE CANCER
}

\author{
A Dissertation \\ Presented for \\ The Graduate Studies Council \\ The University of Tennessee \\ Health Science Center
}

\author{
In Partial Fulfillment \\ Of the Requirements for the Degree \\ Doctor of Philosophy \\ From The University of Tennessee
}

By

Li Chen

December 2009 
Chapter 2 (C) 2009 by American Chemical Society.

All other material (C) 2009 by Li Chen

All rights reserved 


\section{DEDICATION}

To my husband and son, who remind me daily of the joys of life; and to my parents and my brother for their everlasting love and support. 


\section{ACKNOWLEDGEMENTS}

First and foremost, I would like to express my deepest and most sincere appreciation to my advisor and mentor, Dr. Sarka Beranova-Giorgianni, for the training I received in her lab. Thanks for her supervision, advice and encouragement for the research projects. I also thank her for the time she spent guiding me writing, editing and revising the manuscripts.

I would also like to thank the other members of my committee, Dr. Duane D. Miller, Dr. Ivan C. Gerling, Dr. Dominic Desiderio, and Dr. Wei Li for their invaluable suggestions, guidance, and assistance.

I would like to especially acknowledge Dr. Jeffrey R. Gingrich and Ms. Tina Barrett for generously providing me with the human prostate cancer samples and LNCaP cell lines without which a major portion of this research would not have been possible. I must also thank Dr. Ram I. Mahato and Mr. Michael Danquah for establishing the cooperation research work and providing the mouse prostate tumor samples for comparative proteomic study. I am especially grateful to Dr. Nataliya I. Lenchik in Dr. Gerling's group for her training and assistance in the gel image analysis package and Ingenuity software. I would also like to thank Ms. Margaret M. Jefferson for her help with gel imaging equipment.

I especially thank all former and current members in Dr. Beranova-Giorgianni's Lab, Dr. Francesco Giorgianni, Dr. Amira Wohabrebbi, Dr. Yingxin Zhao, Dr. Bin Fang and Ms. Dina Mohamed Fallog, for technical help, discussions and suggestions.

My thanks are extended to the staff in the Departments of Pharmaceutical Sciences, including Brenda Thornton for her administrative help in committee meeting; and Faith B. Barcroft for her help in reagent purchasing and administrative work.

Tremendous thanks to my husband, my parents, my brother and my son. Without their incredible love and support, I would not accomplish my graduate training. 


\begin{abstract}
In the current dissertation, we focused on the development and application of multiple mass spectrometry-based bioanalytical platforms for phosphoproteomic characterization in cell culture and clinical specimens of prostate cancer; and on the application of optimized methods to analysis of differential protein expression to reveal molecular mechanism of drug action in animal model of prostate cancer.

Characterization of the phosphoproteome in prostate cancer

Our study in phosphoprotein signatures on a large scale in prostate cancer focused on the LNCaP human prostate cancer cell line, and on human prostate cancer tissue.

For the LNCaP prostate cancer cell line, we applied a combination of analytical platforms: (1) a novel in-gel isoelectric focusing (IEF) LC-MS/MS analytical platform; (2) a 2-DE based platform combined with phospho-specific staining.

The in-gel IEF LC-MS/MS analytical methodology used in the study included separation of the $\mathrm{LNCaP}$ proteins by in-gel isoelectric focusing; digestion of the proteins with trypsin; enrichment of the digests for phosphopeptides with immobilized metal ion affinity chromatography (IMAC); analysis of the enriched digests by LC-MS/MS; and identification of the phosphorylated peptides/proteins through searches of the Swiss-Prot protein sequence database. With in-gel IEF based analytical platform, we have characterized over 600 different phosphorylation sites in 296 phosphoproteins in the LNCaP prostate cancer cell line. This panel of the LNCaP phosphoproteins was 3-fold larger than the panel obtained in our previous work, and is the largest phosphoprotein panel in prostate cancer reported to date. The phosphoproteins identified in this study belonged to various locations within the cell and were involved in various processes including cell differentiation, transcription regulation, and intercellular signal transduction.

We also developed a 2-DE based platform, in combination with multiplexed staining and LC-MS/MS, for the identification of LNCaP phosphoproteins. In this study, we applied 2-DE as separation technique, Pro- $\mathrm{Q}^{\mathrm{TM}}$ Diamond stain as phosphoprotein detection method, LC-MS/MS and database searches for protein identification to investigate the phosphoproteins in the $\mathrm{LNCaP}$ prostate cancer cell line. Proteins identified from spots of interest were shown to be highly relevant to prostate cancer. We demonstrated the feasibility of using 2-DE with phospho-specific stain and mass spectrometry to investigate the phosphoproteins in the LNCaP cell line. This methodology complements the in-gel IEF LC-MS/MS platform that we used for phosphoproteomics study; it will be of particular value for future comparative studies of phosphoproteins in various physiological states.
\end{abstract}

For prostate cancer tissue, a gel-free approach was performed to analyze five prostate cancer tissue specimens to obtain phosphoproteomic signature of prostate cancer 
for biomarker discovery. Proteins were extracted with Trizol reagent, and then in-solution digested with trypsin. Phosphopeptides were enriched with IMAC, and analyzed the phosphorylated peptides/proteins by LC-MS/MS with identification through searches of the Swiss-Prot protein sequence database. The panels obtained for prostate cancer tissue contain 15-24 phosphoproteins. Some of the characterized phosphoproteins were present in all five specimens; in addition, each specimen also produced a unique set of phosphoproteins. The findings provided a direct glimpse into the phosphoprotein machinery operating within the human prostate cancer tissue. This pilot study focused on a small set of specimens. The phosphoprotein panels that were obtained contained a number of proteins that were unique to a particular specimen.

\section{Comparative proteomics study of drug effects in prostate cancer}

We carried out the first comparative proteomics study for the examination of the effects of bicalutamide/embelin combination treatment on prostate tumors by characterizing the alterations in protein expression that was induced upon treatment of mice bearing prostate tumors with anticancer combination therapy.

A comparative proteomic strategy based on 2-DE coupled with LC-MS/MS was performed on mouse prostate tumor tissue. Proteins from the mouse prostate tumors were extracted with Trizol, and the protein mixtures were separated by 2-DE. Differences in the protein profiles for the different treatment groups were evaluated by computerassisted analysis of SYPRO Ruby stained 2-DE gels. LC-MS/MS and database searches were used to identify differentially expressed proteins. Pathway analysis was carried out on the dataset of identified proteins with the Ingenuity bioinformatics tool. Out of the 33 differentially expressed protein spots, 30 protein spots were identified and grouped into various functional classes. The major protein categories were metabolism (52\%), stress response $(12 \%)$, protein biosynthesis $(13 \%)$ and apoptosis $(11 \%)$, suggesting that alterations in these processes may be involved in the mechanism of drug action. Proteins associated with oxidative stress were up-regulated, which indicated that treatment with bicalutamide/embelin may affect the redox balance within the prostate tumor, and this effect may contribute to tumor suppression. 


\section{TABLE OF CONTENTS}

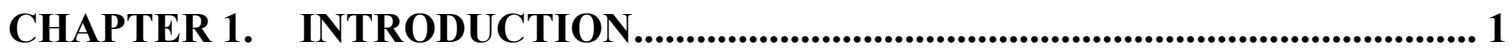

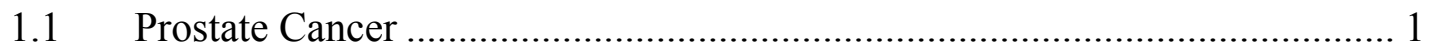

1.2 Why Apply Proteomic Technologies for Biomarker Discovery in Prostate

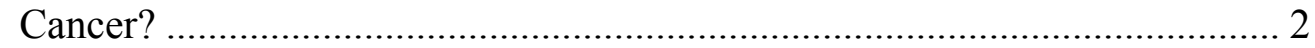

$1.3 \quad$ Elements of Proteomics-based Approach ……………..................................... 3

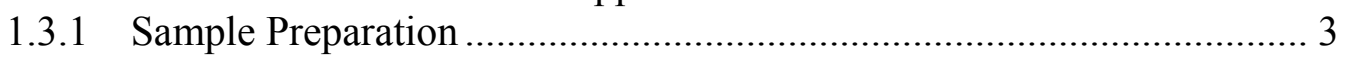

1.3.2 Protein Separation............................................................................ 3

1.3.2.1 Two-dimensional Polyacrylamide Gel Electrophoresis (2-DE) .. 3

1.3.2.2 Other Approaches ................................................................. 7

1.3.3 Mass Spectrometry ............................................................................ 7

1.3.4 Database Searches and Other Bioinformatics Analyses ....................... 10

1.4 Selected Proteomic Technologies Applied in Prostate Cancer Research ..... 12

1.4.1 In-gel-based Platform......................................................................... 12

1.4.2 Gel-free Platform ....................................................................... 16

1.5 Phosphoproteomics Studies of Prostate Cancer............................................ 17

1.5.1 Classical Approaches to Phosphoprotein Analysis................................ 18

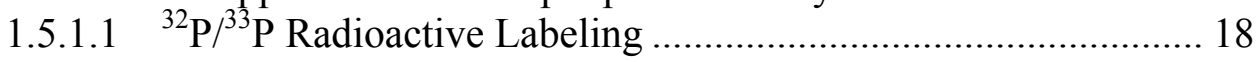

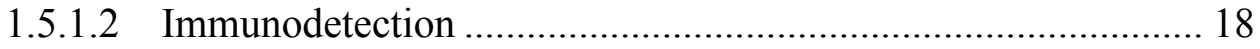

1.5.1.3 Phosphospecific Staining ........................................................ 19

1.5.2 Mass Spectrometry-based Approaches …………………………….... 19

1.5.2.1 Sample Preparation ................................................................ 19

1.5.2.2 Enrichment of Phosphorylated Species .................................... 20

1.5.2.3 Phosphopeptide Sequencing by Tandem Mass Spectrometry ... 22

1.5.2.4 Bioinformatics Resources for Phosphoproteomics .................... 22

$1.6 \quad$ Summary and Research Aims .............................................................. 24

\section{CHAPTER 2. CHARACTERIZATION OF THE PHOSPHOPROTEOME IN LNCAP PROSTATE CANCER CELLS BY IN-GEL ISOELECTRIC FOCUSING AND TANDEM MASS

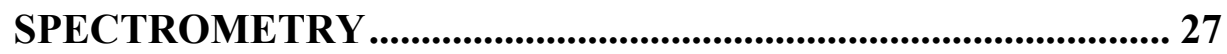

$2.1 \quad$ Introduction.................................................................................... 27

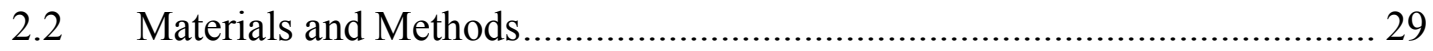

2.2.1 Protein Extraction from LNCaP Cells ................................................ 29

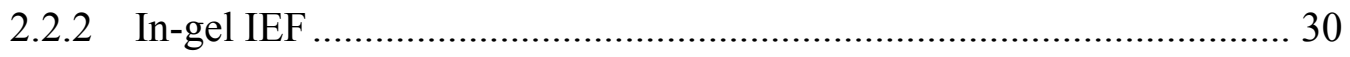

2.2.3 In-gel Digestion ............................................................................ 30

2.2.4 Enrichment of Phosphopeptides with IMAC ……………………....... 30

2.2.5 LC-MS/MS Analysis ....................................................................... 31

2.2.6 Database Searches.......................................................................... 31

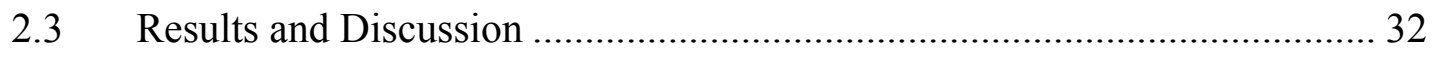

2.3.1 In-gel IEF LC-MS/MS Approach ........................................................ 32

2.3.2 Characteristics of Identified Phosphoproteins/phosphopeptides ........... 34

2.3.3 Classifications of Identified Phosphoproteins ………………………..... 37

2.3.4 Selected Examples of Identified Phosphoproteins.................................. 37 
2.4 Conclusions

\section{CHAPTER 3. INVESTIGATION OF PHOSPHOPROTEIN SIGNATURES IN ARCHIVED HUMAN PROSTATE CANCER TISSUES VIA PROTEOMIC ANALYSIS ........................................................ 41}

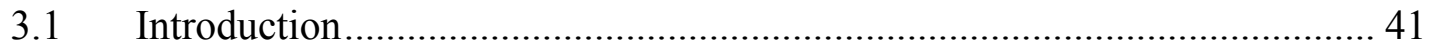

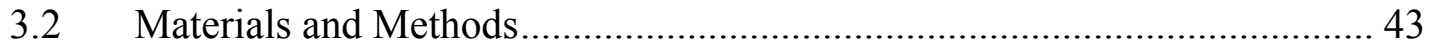

3.2.1 Characteristics of Clinical Samples .................................................. 43

3.2.2 Protein Extraction .................................................................... 43

3.2.3 In-solution Digestion of Proteins ................................................. 43

3.2.4 Enrichment of Phosphopeptides with IMAC ..................................... 44

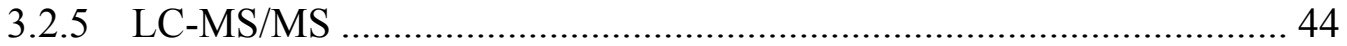

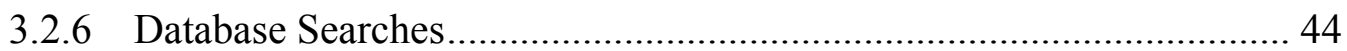

3.2.7 Additional Bioinformatics Analysis ............................................ 45

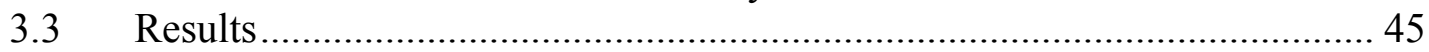

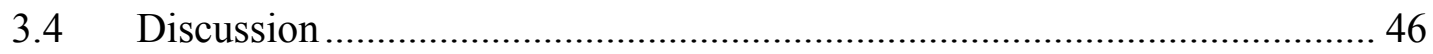

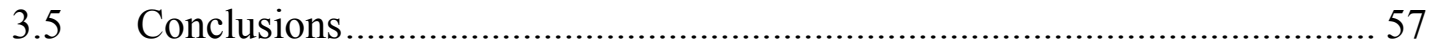

CHAPTER 4. IDENTIFICATION OF PHOSPHOPROTEINS IN THE LNCAP HUMAN PROSTATE CANCER CELL LINE BY A 2-DE AND PHOSPHO-SPECIFIC STAINING-BASED PROTEOMICS PLATFORM ...................................................... 59

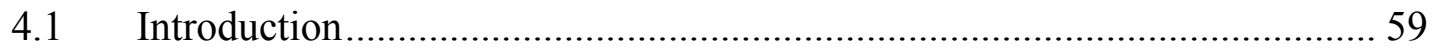

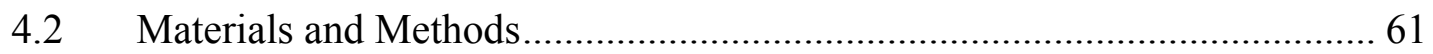

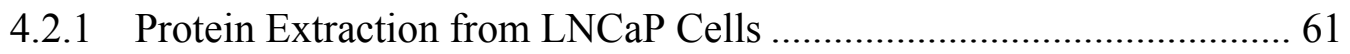

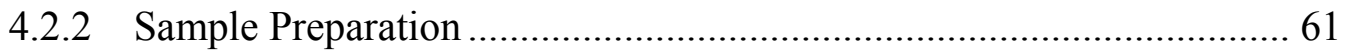

4.2.2.1 Standard Rehydration Buffer ............................................... 61

4.2.2.2 Destreak Rehydration Buffer .............................................. 61

4.2.3 First Dimension: Isoelectric Focusing ......................................... 62

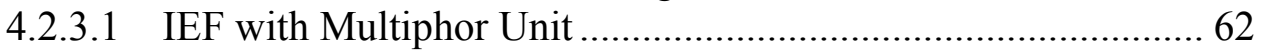

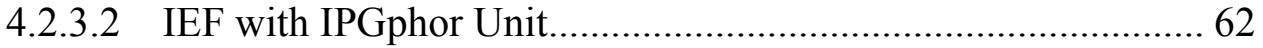

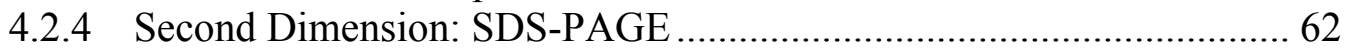

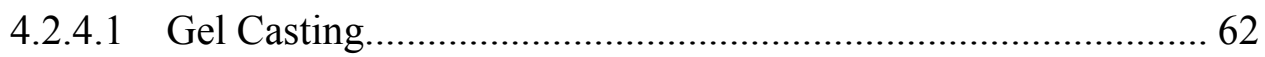

4.2.4.2 SDS-PAGE with Dodeca Cell Unit ....................................... 63

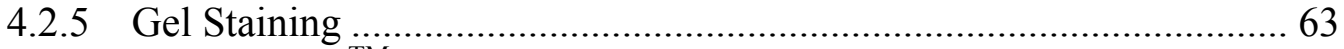

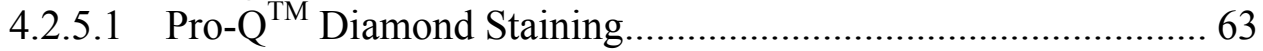

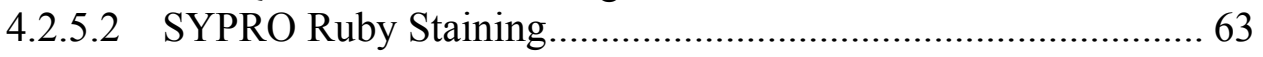

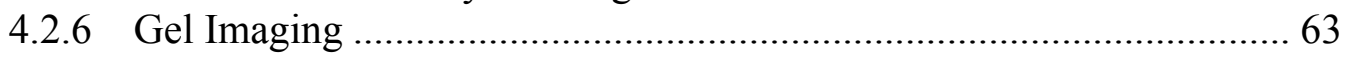

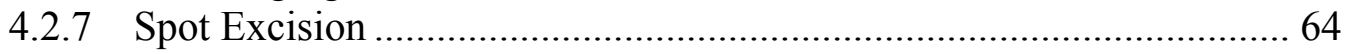

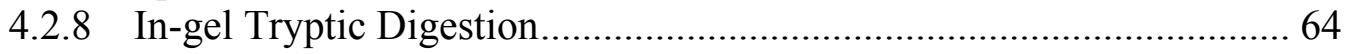

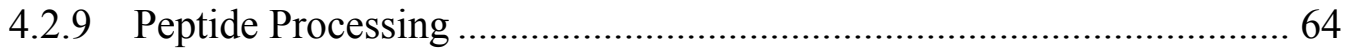

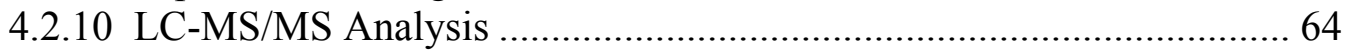

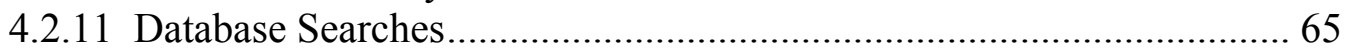

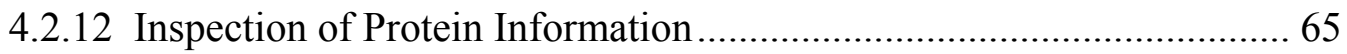

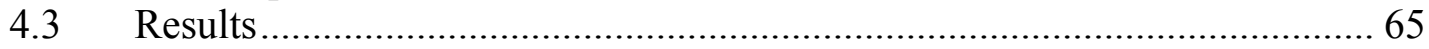


4.3.1 Application of Different Rehydration Buffers: Standard vs.

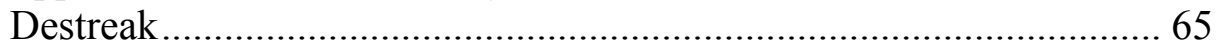

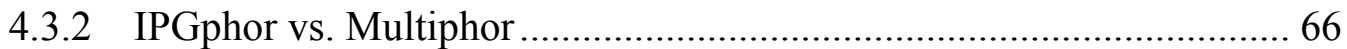

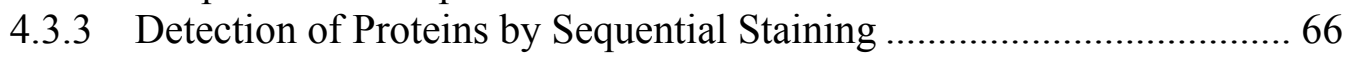

4.3.4 Protein Identification .................................................................... 70

4.3.5. Additional Examination of the Phosphoprotein Panel......................... 70

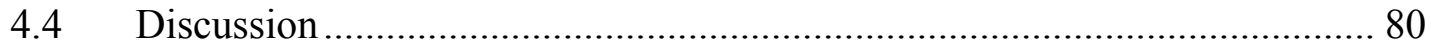

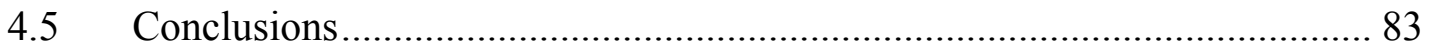

CHAPTER 5. PROTEOMIC ANALYSIS OF PROTEIN ALTERATIONS IN MOUSE PROSTATE CANCER TISSUE INDUCED BY BICALUTAMIDE/EMBELIN COMBINATION

TREATMENT ......................................................................................8 84

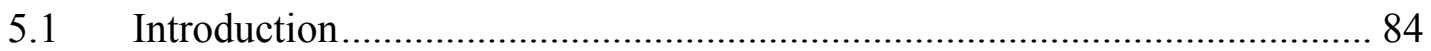

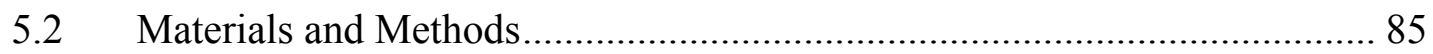

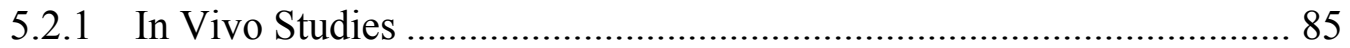

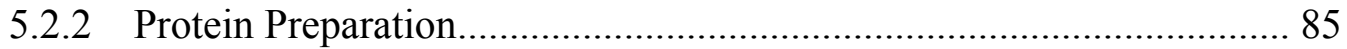

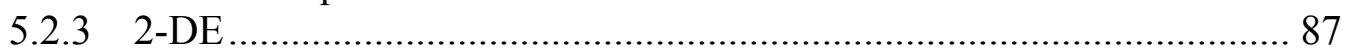

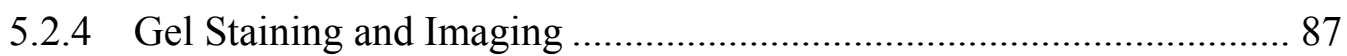

5.2.5 In-gel Tryptic Digestion......................................................... 88

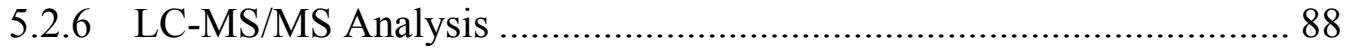

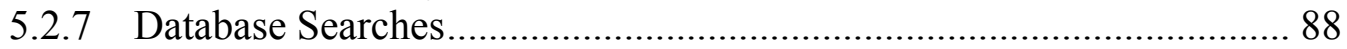

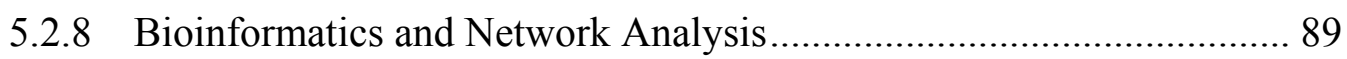

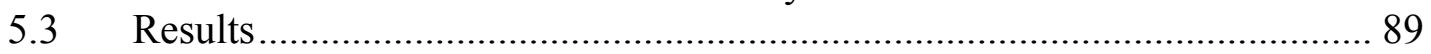

5.3.1 Profile of Mouse Prostate Tumor Specimens ................................... 90

5.3.2 Proteomic Patterns of Bicalutamide/embelin Combination Treatment................................................................................... 90

5.3.3 Identification of Candidate Protein Spots from 2-DE Gels ................. 93

5.3.4 Classification of Identified Proteins................................................. 93

5.3.5 Pathway Analysis of Proteins Modulated in Bicalutamide/embelin Treatment .............................................. 100

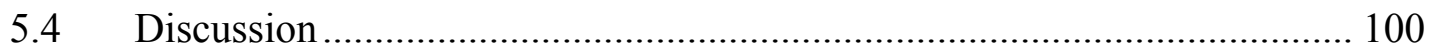

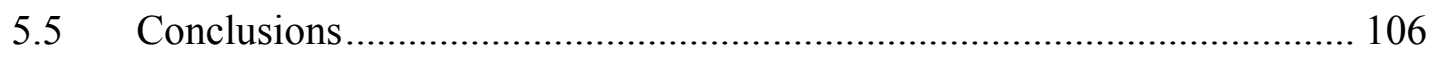

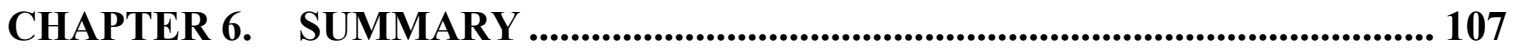

6.1 Discussion of Selected Analytical Aspects .............................................. 107

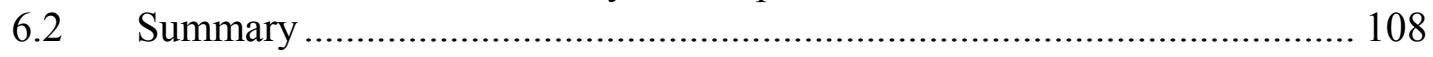

6.2.1 Characterization of the Phosphoproteome in Prostate Cancer........... 108

6.2.2 Comparative Proteomics Study of Drug Effects in Prostate

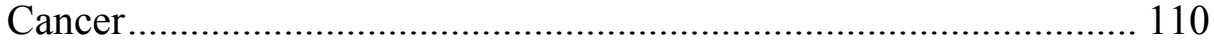

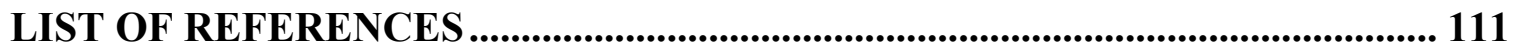

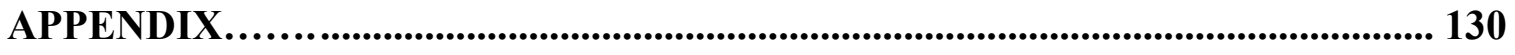

VITA 


\section{LIST OF TABLES}

Table 1-1 Summary of main proteomics methods used in prostate cancer studies ....... 13

Table 1-2 A list of major websites for phosphoproteomics.................................... 25

Table 2-1 Summary of the LNCaP phosphoproteome characterization results: in-gel IEF LC-MS/MS vs. gel-free methodology ............................................... 34

Table 3-1 Characteristics of prostate cancer specimens used in the study .................. 43

Table 3-2 Summary of results for the analyses of the phosphoproteome in human

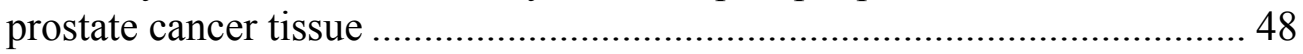

Table 3-3 Phosphoproteins characterized in the prostate cancer specimens ............... 49

Table 4-1 Protein identification results for Pro- $\mathrm{Q}^{\mathrm{TM}}$ Diamond-stained spots from

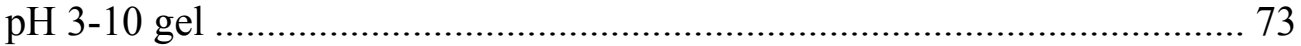

Table 4-2 Protein identification results for Pro- $\mathrm{Q}^{\mathrm{TM}}$ Diamond-stained spots from

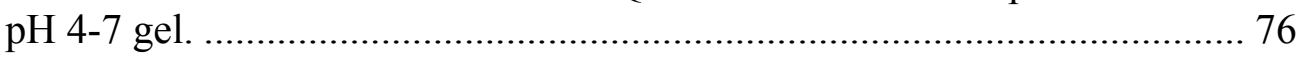

Table 4-3 Example of cancer relevant phosphorproteins identified in LNCaP ............ 82

Table 5-1 Proportion of the matched spots among the different ranges of the CV of

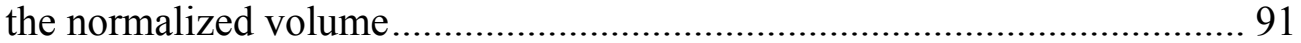

Table 5-2 List of identified protein spots down-regulated by bicalutamide/embelin

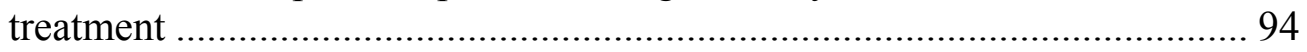

Table 5-3 List of identified protein spots up-regulated by bicalutamide/embelin

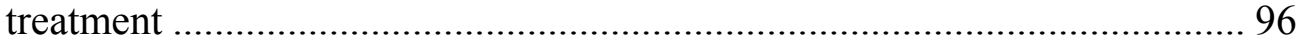

Table 5-4 Protein categories by Gene Ontology ...................................................... 100

Table 5-5 Representative networks associated with identified proteins by IPA software.....

Table A-1 Full list of phosphopeptides and phosphoproteins characterized in LNCaP

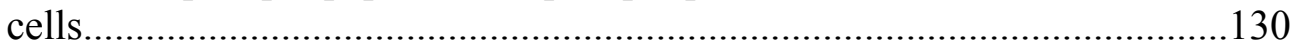

Table A-2 Phosphorylation sites assigned by product ions in MS/MS spectrum........ 167

Table A-3 Subcellular locations and functional categories of the characterized phosphoproteins.

Table A-4 Details of characterized phosphoproteins ............................................... 219

Table A-5 Scansite results for the characterized phosphorylation sites ...................... 224 


\section{LIST OF FIGURES}

Figure 1-1 Elements of proteomics-based study ..................................................... 4

Figure 1-2 Peptide fragmentation series ........................................................... 10

Figure 1-3 An example of tandem MS/MS under data-dependent acquisition ............. 11

Figure 1-4 A representative MS/MS spectrum of a phosphopeptide.............................. 23

Figure 2-1 Outline of the in-gel IEF LC-MS/MS strategy ………………………....... 28

Figure 2-2 A representative MS/MS spectrum obtained in the LC-MS/MS analysis of IMAC-enriched digest from gel section 4 ............................................ 33

Figure 2-3 Distribution of phosphoproteins/phosphopeptides in the IPG strip

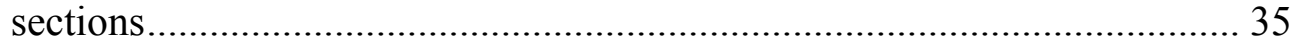

Figure 2-4 Distribution of singly, doubly, and triply phosphorylated peptides............. 36

Figure 2-5 Summary of the sub-cellular locations of the $\mathrm{LNCaP}$ phosphoproteins characterized by in-gel IEF LC-MS/MS ................................................ 38

Figure 2-6 Classification of the characterized LNCaP phosphoproteins based on their involvement in cellular processes ....................................................... 39

Figure 3-1 Outline of the gel-free analytical methodology .......................................... 42

Figure 3-2 An example of MS/MS spectrum of GQLpSDDEKFLFVDK .................... 47

Figure 3-3 Distribution of identified phosphopeptides in five prostate tissues analyzed

Figure 3-4 Classification of identified phosphoproteins by molecular function according to GO annotation at the level 2 .

Figure 4-1 Schematic of 2-DE and phospho-specific staining based proteomics platform.....

Figure 4-2 2-DE gels of LNCaP proteins with $\mathrm{pH}$ 3-10NL using different rehydration buffers (a) Standard rehydration buffer (b) Destreak rehydration solution

Figure 4-3 2-DE gels of LNCaP using different IEF units (a) Multiphor II (b) IPGphor

Figure 4-4 2-DE gels of LNCaP proteins detected with multiplexed staining (a) Pro- $\mathrm{Q}^{\mathrm{TM}}$ Diamond Phosphoprotein staining (b) SYPRO Ruby staining. 69

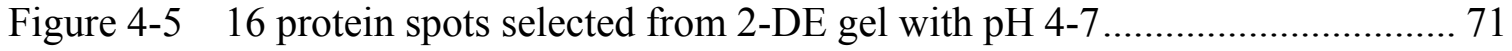

Figure 4-6 14 protein spots selected from 2-DE gel with $\mathrm{pH}$ 3-10 ………………...... 72

Figure 5-1 Outline of 2-DE based comparative proteomics platform .......................... 86 
Figure 5-2 Representative 2-DE gels of mouse prostate cancer proteome

(a) Control (b) Free bicalutamide/embelin treatment.

Figure 5-3 Classification of biological processes of identified proteins according to GO annotations .....

Figure 5-4 Classification of molecular functions of identified proteins according to GO annotations

Figure 5-5 Network of small molecule biochemistry associated with modulated proteins identified in bicalutamide/embelin treatment in mouse prostate tumor tissue. 


\section{LIST OF ABBREVIATIONS}

$\begin{array}{ll}\mu \mathrm{g} & \text { micro gram } \\ \text { AcOH } & \text { acetic acid } \\ \text { ACN } & \text { acetonitrile } \\ \text { ANOVA } & \text { analysis of variance } \\ \text { AR } & \text { androgen receptor } \\ \text { BPH } & \text { benign prostatic hypertrophy } \\ \text { CID } & \text { collision induced dissociation } \\ \text { cm } & \text { centimeter } \\ \text { CV } & \text { coefficient of variation } \\ \text { } \text { C } & \text { degrees Celsius } \\ \text { Da } & \text { dalton } \\ \text { DHB } & \text { 2,5-dihydroxybenzoic acid } \\ \text { DRE } & \text { digital rectal examination } \\ \text { ECD } & \text { electron capture dissociation } \\ \text { ESI } & \text { electrospray ionization } \\ \text { ETD } & \text { electron transfer dissociation } \\ \text { FA } & \text { formic acid } \\ \text { GO } & \text { gene ontology } \\ \text { IDA } & \text { iminodiacetic acid } \\ \text { iTRAQ } & \text { isobaric tags for relative and absolute quantitation } \\ \text { IEF } & \text { isoelectric focusing } \\ \text { IMAC } & \text { immobilized metal ion affinity chromatography } \\ \text { IPA } & \text { ingenuity pathway analysis } \\ \text { IPG } & \text { immobilized pH gradient } \\ \text { HPLC } & \text { high performance liquid chromatography } \\ \text { LC-MS } & \text { liquid chromatography - mass spectrometry } \\ \text { mA } & \text { milliamp } \\ \text { MALDI-TOF } & \text { matrix assisted laser desorption ionization - time of flight } \\ \text { MeOH } & \text { methanol } \\ \text { mL } & \text { milli liter } \\ \text { mm } & \text { milli meter } \\ \text { MS/MS } & \text { tandem mass spectrometry } \\ \text { MudPIT } & \text { multidimensional protein identification technology } \\ \text { MW } & \text { molecular weight } \\ \text { NTA } & \text { nitrilotriacetic acid } \\ \text { pI } & \text { isoelectric point } \\ \text { PMF } & \text { peptide mass fingerprint } \\ \text { ppm } & \text { parts per million } \\ \text { PSA } & \text { prostate specific antigen } \\ \text { PTM } & \text { post translational modification } \\ \text { Rm } & \text { relative migration } \\ \text { RP } & \text { reverse phase } \\ \text { SDS-PAGE } & \text { sodium dodecyl sulfate - polyacrylamide gel electrophoresis } \\ & \end{array}$


SPE

TCA

TFA

2-DE

2D-DIGE solid phase extraction

trichloroacetic acid

trifluoroacetic acid

two dimensional - polyacrylamide gel electrophoresis

two dimensional difference gel electrophoresis 


\section{CHAPTER 1.INTRODUCTION}

\subsection{Prostate Cancer}

Prostate cancer is the most frequently diagnosed cancer and the second leading cause of cancer death among men in the United States (Ann. Intern. Med., 2008). According to Cancer Facts \& Figures 2008 from American Cancer Society, an estimated 186,320 American males will be diagnosed with prostate cancer and 28,660 deaths will result from prostate cancer in the US during 2008. Prostate cancer continues to represent a major health concern.

Clinically, prostate cancer is diagnosed as local or advanced (Miller et al., 2003). During the initial period of prostate cancer, the tumor is dependent on androgen for growth, and therefore responses to therapies of surgical and/or pharmacological depletion of circulating androgens, such as radical local treatment or androgen-deprivation (androgen ablation) treatment. However, the success of the therapies is temporary; most tumors relapse within 2 years as a metastatic and androgen-independent state. Whereas surgery is curative for local prostate cancer, there are not effective treatments for androgen-independent prostate cancer.

Therefore, understanding molecular mechanisms and biological activities of cancer development and progression is the key objective to improve the diagnosis and develop novel prostate cancer therapeutics. Currently, the main tools used for prostate cancer diagnosis include digital rectal examination (DRE), serum prostate specific antigen (PSA) screening and biopsy (Smith et al., 2009).

In the late 1980s, serum PSA was introduced into clinical practice and became the most widely used biomarker for screening and early detection of prostate cancer (Farwell et al., 2007; Rao et al., 2008). The application of serum PSA measurement in clinical practice has dramatically changed detection and treatment of prostate cancer. The incidence of this tumor has increased whereas the impact on mortality rates is less evident. Over diagnosis leading to unnecessary treatment and associated adverse effects have been strongly implicated to occur (Lin et al., 2008).

Using current recommended guidelines, the PSA test suffers from both poor specificity and low sensitivity (Ross et al., 2004). Although higher PSA levels are associated with the risk of cancer, PSA levels can change for many reasons other than cancer, such as enlargement of the prostate (benign prostatic hypertrophy (BPH)) and infection of the prostate (prostatitis). PSA level above $4.0 \mathrm{ng} / \mathrm{ml}$ is the traditional cutoff value for diagnosis of prostate cancer (Welch et al., 2005). However, a single PSA cutoff point lacks specificity and sensitivity needed for an accurate diagnosis of prostate cancer; in men with PSA below $4.0 \mathrm{ng} / \mathrm{ml}$ the risk of cancer is approximately $15 \%$ and $15 \%$ these patients have high grade disease. Conversely for PSA levels above $4.0 \mathrm{ng} / \mathrm{ml}$, cancer is found on biopsy in only $25 \%$ to $30 \%$ of the men evaluated (Parekh et al., 2007; Thompson et al., 2004). 
Several modifications of PSA measurement have been investigated, including PSA velocity, PSA density, PSA glycosylation and PSA doubling time (Carter et al., 1992; Catalona et al., 1994; Fang et al., 2002; Meany et al., 2009). However, none of these modifications are superior to PSA in clinical practice, and since they are more difficult to measure than PSA, they are unlikely to replace PSA for prostate cancer screening and have not been found wide application in clinical practice.

Therefore, new investigation into more accurate diagnostic and prognostic biomarkers to complement PSA is critical to assist in early detection and prognosis, monitor therapeutic response, and to determine the best course of therapy for cancer patients to improve the clinical outcomes. To date, with the advances in biotechnology such as immunohistochemical staining, proteomics, tissue microarray, DNA microarray, many promising biomarkers, such as human kallikrein, hepsin, have been identified and are under investigation and validation.

\subsection{Why Apply Proteomic Technologies for Biomarker Discovery in Prostate Cancer?}

Current biology research efforts often focus on describing the detailed complexity of particular aspects of the total system of cancer development and progression. It is of help to consider systems study, describing multitude of pathways and interactions that regulate biological processes. To capture all interactions and get a complete picture of what is really happening during pathogenesis and disease progression, conventional tools to study single protein or pathway are inadequate and large-scale, high throughput approaches are required to provide complementary information.

Advances in new high throughput technologies, such as genomics, transcriptomics and proteomics, lead to the accelerating growth of information and the improved molecular tools for cancer diagnosis, prognosis and treatment. However, gene expression analysis can not accurately predict protein expression and post-translational modifications (such as phosphorylation) (Blackstock and Weir, 1999). Transcriptome represents a set of messenger RNA (mRNA) produced by a given cell and can reflect the gene expression under a specific condition. But transcriptome neither can completely reflect the underlying biology due to alternative splicing and post-translational modifications in response to changes of external conditions. As the ultimate products for gene expression, proteins are directly responsible for the molecular functions to mediate most changes at the cellular level in cancer. Therefore, protein-directed studies using advanced technologies are of significant interest and significance.

Proteomics is the simultaneous study of multiple proteins on a large scale by high throughput analyses of body fluids, cells and tissues (Anderson and Anderson, 1998). The expansion of the field of proteomics has been enabled by concurrent developments: completion of the human genome project, advances in separation techniques, major improvements in mass spectrometry technologies, and progress in bioinformatics. 
The speed, selectivity and sensitivity of mass spectrometry (MS) make MS-based proteomic methodologies a powerful alternative to traditional biological methods for analyses of proteins in complex biological systems (Feng et al., 2008). The distinct advantage of proteomics and the advances in proteomic technologies have created tremendous opportunities for biomarker discovery and for biological studies of cancer.

Proteomics-based approaches have the potential to provide novel, systems-level insight into the underlying molecular mechanisms of prostate cancer, and also hold great promise for biomarker discovery in prostate cancer.

\subsection{Elements of Proteomics-based Approach}

The bioanalytical strategy of a proteomics-based study involves the incorporation of a number of technologies and knowledge of biochemistry, molecular biology, bioinformatics and bioanalytical chemistry (Patton, 1999). Although a number of options exist within each stage of the proteomic analysis, a typical approach includes the following components: sample preparation, protein separation, protein identification, and data mining (Figure 1-1).

\subsubsection{Sample Preparation}

The first, critically important step of a proteomics-based study is sample preparation. Proteins are isolated from biological samples and then solubilized for separation. The task of sample preparation is to maximally solubilize sample proteins under conditions compatible with subsequent method of analysis, without introducing artefactual modifications. There have been a number of reviews that discussed various approaches to sample preparation and solubilization of proteins for 2-DE (Rabilloud, 1996; Rabilloud, 2009). The use of a protease and/or phosphatase inhibitor cocktail is often included to inhibit protein degradation and/or dephosphorylation. In addition, the application of various combinations of reducing agents, chaotropic agents and detergents is used to disrupt intra- and inter-protein interactions and maximize protein solubilization (Rabilloud et al., 1997).

\subsubsection{Protein Separation}

\subsubsection{Two-dimensional Polyacrylamide Gel Electrophoresis (2-DE)}

2-DE is the most commonly applied technique for the separation of proteins in complex mixtures because of its efficiency and high resolution (Gorg et al., 2004). The technique combines isoelectric focusing (IEF) in the first dimension, and sodium dodecyl sulfate-polyacrylamide gel electrophoresis (SDS-PAGE) in the second dimension. The superior separation power of 2-DE stems from the fact that proteins are separated based on two different physicochemical properties. Proteins are separated based on their 


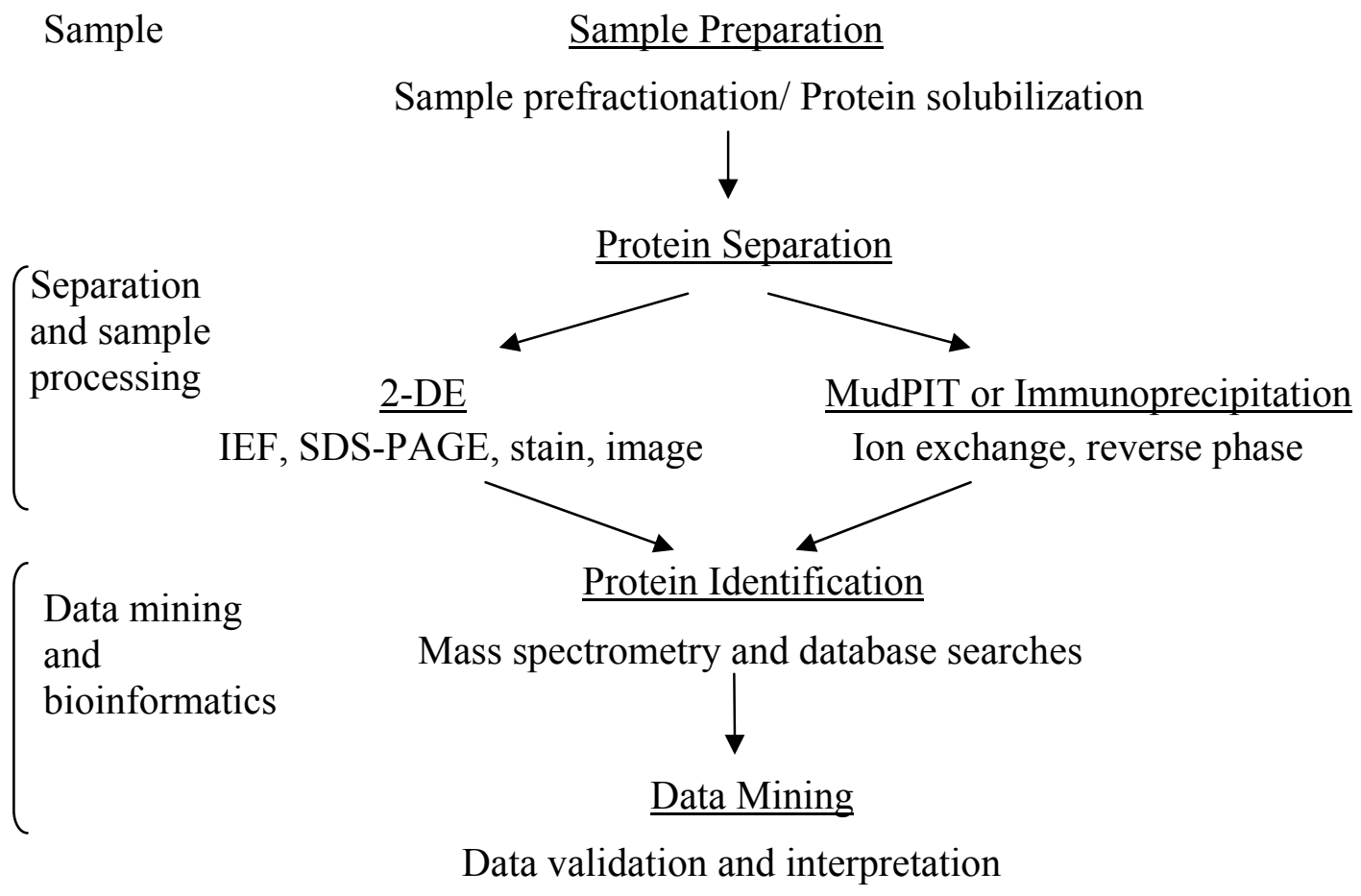

Figure 1-1 Elements of proteomics-based study. 
different isoelectric points in the first dimension, and based on molecular weight in the second dimension. Then proteins are visualized with various staining methods, such as Coomassie Blue, silver staining, or fluorescent stains. Protein profiles can be quantitatively analyzed through 2-DE gel imaging software (Dunn, 1987). Mass spectrometry has been intensively used for identification of proteins of interest in 2-DEbased proteomics studies.

First dimension: Isoelectric focusing (IEF) is a technique for separating proteins by their electric charge differences. Proteins are amphoteric molecules with acidic and basic groups. A protein carries a charge depending on the $\mathrm{pH}$ of its surroundings. This characteristic is used for IEF in $\mathrm{pH}$ gradients. A protein will have positive net charge in a $\mathrm{pH}$ region below its isoelectric point (pI), and in an electric field it will migrate towards the cathode. During protein migration in a $\mathrm{pH}$ gradient, the charge will decrease until the protein reaches the $\mathrm{pH}$ region that corresponds to its $\mathrm{pI}$. At this point it has no net charge and the migration stops. Analogously, negatively charged protein molecules will migrate towards the anode to the protein $\mathrm{pI}$. As a result, the protein is focused into a sharp stationary band at its specific $\mathrm{pI}$ in the $\mathrm{pH}$ gradient.

The great improvement of IEF is the introduction of the immobilized $\mathrm{pH}$ gradient (IPG) strips for the first dimension separation (Bjellqvist et al., 1982). IPG strips are composed of acidic and basic buffering groups covalently linked to large-pore polyacrylamide gel matrix. The polyacrylamide gel is cast onto a plastic backing that allows easy manipulation of the IPG strip. Compared with carrier ampholyte-based IEF, IPG-based IEF has a very high resolution, permits large amount of proteins loading, and presents more reproducible results (Gianazza and Righetti, 2009; Gorg et al., 2000). Moreover, there are pre-cast IPG strips with various lengths and $\mathrm{pH}$ ranges commercially available from several vendors. The development and commercialization of the IPG technology has contributed to the expansion of 2-DE from a handful of highly specialized laboratories to many laboratories in diverse fields of life sciences.

In terms of equipment, several different configurations are used to perform IEF separations. These configurations differ mainly in the procedure for strip rehydration, and in the maximum voltage that can be applied.

Second dimension: Sodium dodecyl sulfate polyacrylamide gel electrophoresis (SDS-PAGE) is used to separate proteins according to their molecular weight. Unlike IEF, SDS-PAGE itself is a widely used, standard analytical technique for protein separation. SDS is used to denature proteins by disrupting hydrogen bonds, blocking hydrophobic interactions and unfolding the proteins to eliminate protein tertiary and secondary structures. Reducing agent (such as DTT) is added to disrupt the disulphide bond between polypeptides and unfold proteins into flexible polypeptides. Then SDS masks the charge of the polypeptides and forms anionic SDS-protein complexes with a constant net negative charge per unit mass. The separation principle of SDS-PAGE involves differential migration of SDS-protein complexes through a restrictive polyacrylamide gel with a define pore size. Under the influence of an electric field, the SDS-protein complexes with a constant charge-to-mass ratio migrate towards the anode. 
Smaller proteins experience less restriction in their movement through gel pores and hence travel the farthest; large proteins are impeded and hence travel the least distance. Thus proteins in a mixture become separated according to their molecular weight (Ibel et al., 1990).

Depending on the specific application, the characteristics of the gel such as size, thickness and percentage of acrylamide should be taken into account. In addition, SDSPAGE systems are available in both horizontal and vertical configurations. Horizontal units are convenient for use with single gels. Vertical units allow simultaneous separations of multiple samples and are often performed for higher throughput analyses minimizing run-to-run variations (Gorg et al., 2004).

Protein detection: After gel electrophoresis, proteins in 2-DE gels are visualized with various staining methods, such as Coomassie Blue, silver staining, or fluorescent stains. Each method has its advantages and disadvantages. Staining with Coomassie Blue dye is simple and provides good linearity; however, the stain is not very sensitive. Silver staining provides much higher detection limits but suffers from reproducibility and linearity issues. Recently, fluorescent stains become an attractive alternative to other staining methods for protein detection. Fluorescent stains (such as SYPRO stains) noncovalently bind to proteins in 2-DE. Compared with Coomassie and silver stains, fluorescent stains offer a number of advantages, including wide dynamic range, high sensitivity, and simplicity of procedure. The SYPRO stains are fully compatible with mass spectrometry analyses, which is an important consideration (Patton, 2000). The shortcomings associated with fluorescent stains are high cost for the requirement of specialized imaging equipment.

Protein imaging: Image analyses are performed with an imaging system that is able to acquire, analyze and store the 2-DE images. In general, image analysis includes spot detection, protein spots matching in different gels, and normalization for protein quantification. A number of computer-based software packages are commercially available for the analysis of 2D gel images, such as PDQuest (Bio-Rad, Hercules, CA), Progenesis (Nonlinear USA Inc, Durham, NC), Z3 2DE analysis system (Compugen, NJ). Although image analysis is a semi-automated procedure, several issues (such as gel distortions, variation in sample loading, staining intensities) require manual intervention.

Recent development in 2-DE: Classical 2-DE, while powerful, suffers from limitations. The main issues include partial proteome coverage, and run-to-run variability. To address some of the issues, improvements of the 2-DE platform have been made. A significant new advancement is the introduction of two-dimensional difference gel electrophoresis (2D-DIGE). 2D-DIGE incorporates differential labeling of different sample populations through the use of fluorescent molecules (CyDyes) (Unlu et al., 1997). Up to three different protein samples can be covalently labeled with fluorescent dyes (Cy3, Cy5, and Cy2), combined and subjected to 2-DE. The differential labeling allows separation of multiple proteomes on a single gel, reducing the gel-to-gel variations. After the gel electrophoresis, images of the gel at different excitation/emission wavelengths (specific for each CyDye) are generated. Images can be overlaid directly by the 
DeCyder $^{\mathrm{TM}}$ software (GE Healthcare) to locate proteins that are differentially expressed between the samples. Advanced data analysis such as hierarchical clustering can be performed (Minden et al., 2009).

\subsubsection{Other Approaches}

In addition to 2-DE based approaches, a number of different separation methodologies have been developed since the advent of proteomics. In general, these methodologies fall within two categories: 1D-gel-based, and gel-free. Furthermore, various methods aimed at reduction of the proteome complexity prior to analysis have been introduced.

One-dimensional gel-based proteomics strategies use a single dimension of electrophoretic separation at the protein level, combined with advanced LC-MS/MS for protein identification. A combination of SDS-PAGE and LC-MS/MS, termed geLC$\mathrm{MS} / \mathrm{MS}$, has been developed and used for numerous applications. Our group has developed a method that combines in-gel IEF at the protein level with LC-MS/MS. This 1D-gel strategy, named in-gel IEF LC-MS/MS, has been applied to analyses of the proteome in the human pituitary (Zhao et al., 2005) and human prostate tissue.

A well-known example of a gel-free proteomics strategy is the Multidimensional protein identification technology (MudPIT). The technique is also known as shotgun proteomics. MudPIT involves multidimensional chromatographic separation of complex mixtures at the peptide level. In brief, a whole protein mixture is digested directly and the resulting peptides are separated by two different types of liquid chromatography (ion exchange and reversed-phase LC), coupled to tandem mass spectrometry. Compared with 2-DE, MudPIT method is unbiased, meaning that proteins that are normally underrepresented in 2D gels (low-abundance proteins, proteins with extremes in $\mathrm{pI}$ and $\mathrm{MW}$, and membrane proteins) can be probed with MudPIT (Washburn et al., 2001).

Immunoprecipitation is an example of a strategy that aims at reducing the complexity of the mixture to be analyzed. Instead of aiming to probe all proteins in a proteome, immunoprecipitation studies focus on targeted analyses of specific proteins or protein groups. Immunoprecipitation methods use an antibody that specifically isolates and concentrates a particular protein or group of proteins from a complex proteome sample. For example, such strategy can be applied to study the composition of protein complexes, or to investigate post-translationally modified proteins.

\subsubsection{Mass Spectrometry}

Mass spectrometry (MS) is a powerful technology for the identification and characterization of peptides, proteins, and other biomolecules. The development of advanced mass spectrometry instrumentation that allowed high-sensitivity, highthroughput protein identification has been one of the enabling events underlying the expansion of proteomics (Yates, III, 2000). New and improved instruments are being 
continuously developed, thus pushing the power of mass spectrometry to higher and higher levels.

MS is an analytical technique that measures the molecular weight of molecules according to their mass-to-charge ratio. A mass spectrometer is composed of three key components: ion source, mass analyzer and detector. Analytes are converted into gasphase ions within an ion source. The ions are then sorted and separated according to their mass-to-charge $(\mathrm{m} / \mathrm{z})$ ratio in a mass analyzer, and the signal is detected by a suitable device such as an electron multiplier. The resulting MS spectra are represented as ion intensity vs. the $\mathrm{m} / \mathrm{z}$ values (Corthals et al., 2000). There are a number of different types of mass spectrometers that include different combination of ion sources and mass analyzers (Patterson and Aebersold, 1995; Yates et al., 2009).

In general, protein identification by mass spectrometry involves cleaving the protein of interest into a set of peptides, which are then subjected to MS analysis. Production of gas-phase ions from peptides is therefore a critical step in mass spectrometry analysis. There are two broadly used ionization methods in proteomics research, namely matrix-assisted laser desorption ionization (MALDI), and electrospray ionization (ESI) (Gevaert and Vandekerckhove 2000). MALDI sources are usually coupled to time-of-flight (TOF) mass analyzers while ESI sources are commonly linked to ion traps or quadrupole mass analyzers (Corthals et al., 2000).

Ionization of analytes by MALDI involves the use of a UV-absorbing matrix. A sample is co-crystallized with the matrix in a well on a samples plate; inside the ion source, the sample-matrix mixture is irradiated with a laser beam. This process results in vaporization of the matrix and analyte molecules are brought into a dense phase (plume) above the sample surface, where they are ionized by proton transfer reactions. Peptides generally ionize through addition of a proton, forming a singly charged molecular ion, $[\mathrm{M}+\mathrm{H}]^{+}$(Henzel et al., 1993).

ESI can directly generate gas-phase ionized molecules from a liquid solution. This feature is of great benefit because it enables on-line coupling of ESI-based mass spectrometers with HPLC. During ESI ionization, the LC eluent is sprayed from a tip of a needle held at a high voltage; through this process, a mist of highly charges droplets is created. Gas and/or heat applied to the droplets causes evaporation of the solvent in the droplets. As the size of droplets decreases, the charge density in the droplets increases. That leads to increase in the surface tension, the droplets dissociate into smaller ones and eventually gas-phase analyte ions are produced. Peptides ionized with ESI usually form multi-protonated molecular ions, $[\mathrm{M}+\mathrm{nH}]^{\mathrm{n}+}, \mathrm{n}=2$ or 3 depending on the number of basic sites in the peptide sequence. The fact that multi-protonated ions are formed upon ESI has important implications for the ability to measure larger peptides, and for gas-phase dissociations of peptide ions (Fenn et al., 1989).

The LTQ two-dimensional linear ion trap mass analyzer is one of commonly used mass analyzers, which is comprised of four parallel hyperbolic shaped rods, segmented in three secions. Ions are trapped radially in a radio frequency (RF) electric field and axially 
in a static electric field using DC voltages. Through the application of appropriate voltages to all three segments, the trapped ions with specific $\mathrm{m} / \mathrm{z}$ ratio are ejected in the radial direction through the two parallel slots in the center section of the linear ion trap. Two highly efficient detectors are placed on either side of the trap to maximize sensitivity. The unique configuration of this device improves the ion storage capacity, trapping efficiency and detection efficiency (Schwartz et al., 2002).

Mass spectrometry measurements can be carried out in two general ways: as single-stage mass spectrometry (MS), or as tandem mass spectrometry (MS/MS). An MS experiment includes ionization of the analytes, and measurement of the $\mathrm{m} / \mathrm{z}$ ratios for the source-generated ions. For peptide ions such as singly-protonated ions produced upon MALDI, information about peptide MWs can be derived easily. This aspect, as it applies to protein identification, is discussed in more detail in the following paragraph. An MS/MS experiment is more complex than MS and includes additional steps. The steps in an MS/MS experiment are: ionization of the analyte molecules and formation of molecular ions; selection of one of the source-generated ions as the so-called precursor ion; activation of the mass selected precursor ion through collisions with a target gas followed by dissociation of the activated precursor ion into a series of structuredetermining product ions; mass analysis of the product ions and recording of the signal. In MS/MS, protonated peptide ions dissociate predominantly via cleavages along the peptide backbone, theoretically forming several different product ion series. Under the conditions used in my research, two major ion series arise from cleavage of the peptide bond: the N-terminal ion series (b-ion series) and the C-terminal ion series (y-ion series) (Figure 1-2). As shown in the figure, the $\mathrm{m} / \mathrm{z}$ of the product ions can be used to derive amino acid sequence information for the peptide under analysis.

Protein identification in proteomics studies may be achieved with MS. The MSbased identification strategy is termed peptide mass fingerprinting (PMF) and it is commonly performed with a MALDI-TOF mass spectrometer. The method includes: cleavage of protein of interest into peptides with a specific enzyme (such as trypsin, which cleaves at the C-terminus of lysine and arginine); measurement of the $\mathrm{m} / \mathrm{z}$ ratios of the peptide ions with MALDI-TOF; comparison of the observed peptide mass fingerprint to the theoretical mass fingerprints of proteins in a protein sequence database; generation of a list of possible candidate proteins based on peptide matching scores. The PMF identification strategy is simple and provides high throughput. It has been applied widely for identification of proteins in $2 \mathrm{D}$ gel spots. It should be noted that PMF is based on measurements of peptide masses, and that it is therefore applicable only to single proteins or simple protein mixtures.

Protein identification may also be accomplished with MS/MS, commonly in conjunction with LC separation (LC-MS/MS). As with PMF, the protein of interest is first cleaved into peptides, usually with trypsin. The tryptic peptides are separated by LC, and the eluting peptides are analyzed by MS/MS. The LC-MS/MS measurements on unknown peptide mixtures are performed using data-dependent acquisition (DDA). During DDA, the instrument cycles through MS and MS/MS (Spahr et al., 2000). This principle is illustrated in Figure 1-3 for a cycle performed for ions eluting with the LC 


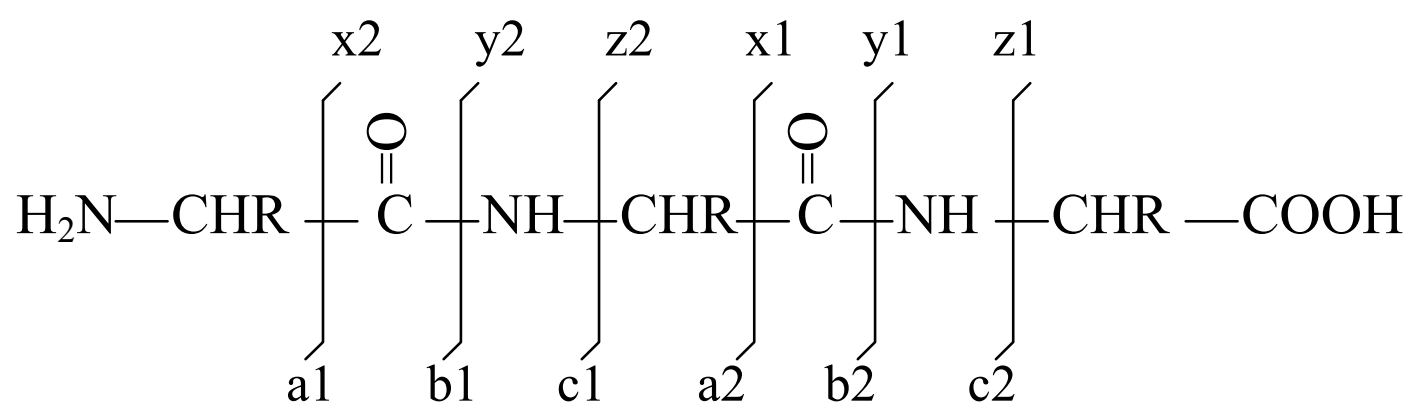

Figure 1-2 Peptide fragmentation series.

retention time around $58.26 \mathrm{~min}$. The instrument acquired an MS spectrum that surveyed the analytes eluting at that particular time. Based on the information from this MS spectrum, subsequent MS/MS measurements were performed on the precursor ions detected in the MS spectrum. One of these MS/MS spectra, acquired for the precursor ion with $\mathrm{m} / \mathrm{z}$ 942. The product ion $\mathrm{m} / \mathrm{z} 893$ is shown in the bottom section of the Figure 1-3. During a single LC-MS/MS analysis, the instrument cycles through many of such MS-MS/MS sequences. The entire MS/MS dataset is used to search a protein sequence database to identify the protein(s) of interest (Yates, III et al., 1995). It is important to point out that, in contrast to PMF that only measures peptide masses, sequence-diagnostic MS/MS data are obtained for each peptide being analyzed. Based on LC-MS/MS data, proteins in 2D gel spots as well as in highly complex protein mixtures can be identified. Therefore, LC-MS/MS is an integral part of 1D-gel and gel-free bioanalytical platforms.

\subsubsection{Database Searches and Other Bioinformatics Analyses}

Identification of proteins of interest based on MS or MS/MS has been enabled by the creation of protein sequence databases that are being continuously updated, and on the development of database search software programs.

In all my projects, sequence information derived from MS/MS was used for database searches with the SEQUEST search engine in the Swiss-Prot protein sequence database. Swiss-Prot is a curated protein sequence database that provides a high level of annotations (such as protein function, protein sequence, post-translational modifications, variants, etc.), minimizes the redundancy and integrates with other databases. Now the Swiss-Prot database has been merged into the UniProt database. SEQUEST is one of the two most widely used searching algorithms (Yates, III et al., 1995). With SEQUEST, proteins in the database are cleaved in silico into peptides, and theoretical product-ion patterns are generated for these peptides. These theoretical patterns are compared to the experimental MS/MS patterns; when a match is retrieved, it is evaluated with a scoring system. Thus, the output of the database search is a list of proteins whose peptide sequences were matched based on MS/MS data. 


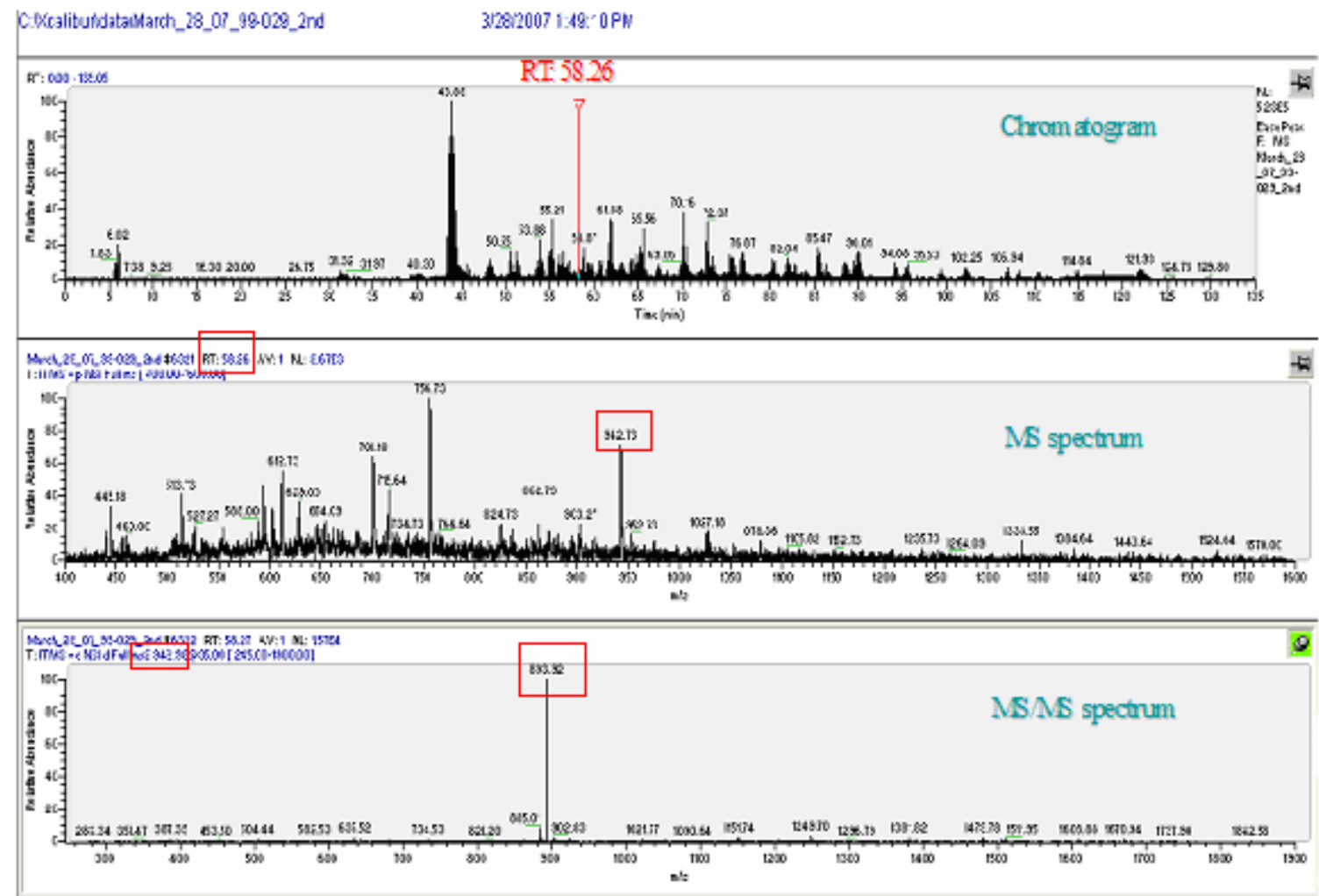

Figure 1-3 An example of tandem MS/MS under data-dependent acquisition.

The top section of the Figure1-3 is chromatogram of LC. The instrument acquired an MS spectrum that surveyed the analytes eluting at the LC retention time around $58.26 \mathrm{~min}$. Based on the information from the MS spectrum, subsequent MS/MS measurements were performed on one of the precursor ions with $\mathrm{m} / \mathrm{z} 942$ detected in the MS spectrum. The product ion $\mathrm{m} / \mathrm{z} 893$ is shown in the MS/MS spectrum in the bottom section of the Figure $1-3$. 
One of the important components of any proteomics research study is to integrate the information about both protein identifications and their corresponding functional characteristics. Gene ontology (GO) is a collection of controlled vocabularies describing the biology of a gene product in any organism (Khatri and Draghici, 2005). GO (http://bioinfo.vanderbilt.edu/webgestalt/) is performed to classify identified proteins according to molecular function, biological process, and molecular component (subcellular location).

Ingenuity Pathway Analysis (IPA) is a powerful curated database and analysis system for understanding how proteins work together to effect cellular changes. It builds hypothetical networks from identified proteins and other proteins, based on published literature; these networks involve interactions between genes, proteins and other biological molecules (Hoorn et al., 2005; Raponi et al., 2004).

\subsection{Selected Proteomic Technologies Applied in Prostate Cancer Research}

Recently, intense efforts have been involved in the application of various proteomics technologies to the study of prostate cancer (Table 1-1). Proteomic studies have generated numerous datasets of potential diagnostic, prognostic, and therapeutic significance for prostate cancer.

\subsubsection{In-gel-based Platform}

Gel-based protein separation is an extensively used proteomics methodology. Generally, proteins are separated with one-dimensional (IEF or SDS-PAGE) or twodimensional (2-DE or 2D-DIGE) gels prior to mass spectrometry identification. The platforms are easily adapted to research projects in various labs and have been widely used.

Malik et al. employed a combination of chemical pre-fractionation, SDS-PAGE, LC-MS/MS and SELDI-based immunoassay to identify discriminatory expression proteins of Dunning rat prostate tumor cell lines of varying metastatic potential (Malik et al., 2007).

Liu et al. identified the alterations in protein expression patterns of LNCaP after incubation with Somatostatin/ Somatostatin derivative using 2-DE in combination with mass spectrometry, and found that $\mathrm{Sms} / \mathrm{smsdx}$ triggered up-regulation of catalytic mitochondrial proteins and seemed to affect apoptosis-related proteins (Liu et al., 2007).

Wu et al. identified key regulatory molecules involved in prostate cancer metastasis in two human androgen-independent Prostate cancer cell lines, highly metastatic 1E8-H and lowly metastatic 2B4-L cells through 2-DE and MS analyses, and demonstrated that up-regulation of vimentin expression positively correlates with the invasion and metastasis of androgen-independent prostate cancer (Wu et al., 2007). 
Table 1-1 Summary of main proteomics methods used in prostate cancer studies.

\begin{tabular}{|c|c|c|c|}
\hline Platform & Method & Sample & Reference \\
\hline $\begin{array}{l}\text { In-gel-based } \\
\text { platform } \\
\text { Sodium dodecyl } \\
\text { sulfate } \\
\text { polyacrylamide gel } \\
\text { electrophoresis } \\
\text { (SDS-PAGE) }\end{array}$ & $\begin{array}{l}\text { SDS-PAGE, LC-MS/MS } \\
\text { and SELDI-based } \\
\text { immunoassay }\end{array}$ & $\begin{array}{l}\text { Dunning rat prostate tumor } \\
\text { cell line }\end{array}$ & $\begin{array}{l}\text { Malik et } \\
\text { al., } 2007\end{array}$ \\
\hline \multirow{6}{*}{$\begin{array}{l}\text { Two-dimensional } \\
\text { polyacrylamide gel } \\
\text { electrophoresis } \\
\text { (2-DE) }\end{array}$} & $\begin{array}{l}\text { 2-DE in combination with } \\
\text { MS }\end{array}$ & $\begin{array}{l}\text { LNCaP after incubation with } \\
\text { Somatostatin/ Somatostatin } \\
\text { derivative }\end{array}$ & $\begin{array}{l}\text { Liu et al., } \\
2007\end{array}$ \\
\hline & $\begin{array}{l}\text { 2-DE in combination with } \\
\text { MS }\end{array}$ & $\begin{array}{l}\text { Two human androgen- } \\
\text { independent prostate cancer } \\
\text { cell lines, highly metastatic } \\
\text { 1E8-H and lowly metastatic } \\
\text { 2B4-L cells }\end{array}$ & $\begin{array}{l}\text { Wu et al. } \\
2007\end{array}$ \\
\hline & 2-DE coupled with MS & $\begin{array}{l}\text { Conditioned medium from } \\
\text { LNCaP, C4-2, and C4-2B } \\
\text { cell }\end{array}$ & $\begin{array}{l}\text { Pang et al., } \\
2009\end{array}$ \\
\hline & $\begin{array}{l}\text { 2-DE followed by matrix- } \\
\text { assisted laser } \\
\text { desorption/time of flight } \\
\text { mass spectrometry } \\
\text { (MALDI-TOF-MS) }\end{array}$ & $\begin{array}{l}\text { The highly metastatic human } \\
\text { prostate epithelial cell line } \\
\text { PC-3M-1E8 (1E8-H) and the } \\
\text { low metastatic line PC-3M- } \\
\text { 2B4 (2B4-L) }\end{array}$ & $\begin{array}{l}\text { Wei et al, } \\
2008\end{array}$ \\
\hline & $\begin{array}{l}\text { 2-DE and MALDI-TOF- } \\
\text { MS/MS }\end{array}$ & $\begin{array}{l}\text { Needle biopsy specimens } \\
\text { from patients with prostate } \\
\text { cancer or benign prostatic } \\
\text { hyperplasia }\end{array}$ & $\begin{array}{l}\text { Lin et al., } \\
2007\end{array}$ \\
\hline & 2-DE coupled with MS & $\begin{array}{l}\text { Biopsy samples from benign } \\
\text { prostate hyperplasia and } \\
\text { prostate cancer }\end{array}$ & $\begin{array}{l}\text { Ummanni et } \\
\text { al., } 2008\end{array}$ \\
\hline \multirow{2}{*}{$\begin{array}{l}\text { Two dimension } \\
\text { difference gel } \\
\text { electrophoresis } \\
\text { (2D-DIGE) }\end{array}$} & $\begin{array}{l}\text { 2D-DIGE technology } \\
\text { coupled with LC-MS/MS }\end{array}$ & $\begin{array}{l}\text { LNCaP human prostate } \\
\text { cancer cell line }\end{array}$ & $\begin{array}{l}\text { Rowland et } \\
\text { al., } 2007\end{array}$ \\
\hline & $\begin{array}{l}\text { 2D-DIGE technology } \\
\text { coupled with MS }\end{array}$ & $\begin{array}{l}\text { Human prostate cancer cells } \\
\text { treated with enzymatically } \\
\text { active free-PSA }\end{array}$ & $\begin{array}{l}\text { Bindukumar } \\
\text { et al., } 2008\end{array}$ \\
\hline
\end{tabular}


Table 1-1 (continued).

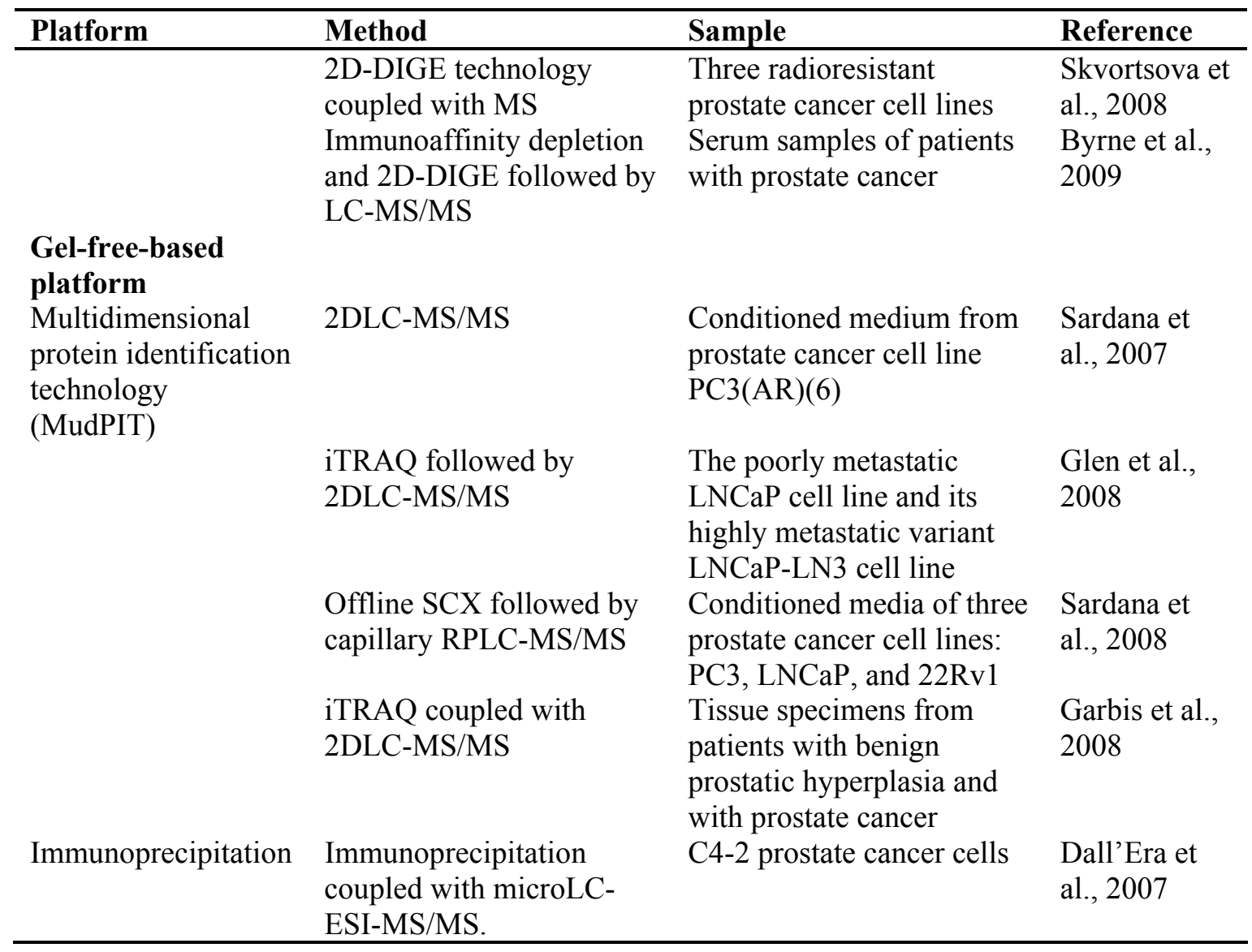


Pang et al. analyzed conditioned medium (CM) from $\mathrm{LNCaP}, \mathrm{C} 4-2$, and $\mathrm{C} 4-2 \mathrm{~B}$ cells using 2-DE coupled with mass spectrometry, and found that higher levels of ubiquitous mitochondrial creatine kinase ( $\mathrm{uMtCK}$ ) in the $\mathrm{CM}$ of androgen independent prostate cancer than that of androgen dependent prostate cancer cells (Pang et al., 2009).

Wei et al. performed comparative proteomic analysis of the highly metastatic human prostate epithelial cell line PC-3M-1E8 (1E8-H) and the low metastatic line PC3M-2B4 (2B4-L) by 2-DE, followed by MALDI-TOF-MS. Highly expressed vimentin was detected in highly metastatic cell line compared to low metastatic cells (Wei et al., 2008).

Lin et al. defined the proteomic features of prostate needle biopsy specimens from patients with prostate cancer or benign prostatic hyperplasia to identify candidate biomarkers for prostate cancer. Using 2-DE, 52 protein spots were exhibited statistically significantly changes among prostate cancer and benign prostatic hyperplasia groups and identified by MALDI-TOF-MS/MS (Lin et al., 2007).

Ummenni et al. investigated biopsy samples from benign prostate hyperplasia and prostate cancer patients by 2-DE and mass spectrometry to identify potential biomarkers which might distinguish the two clinical situations. Eighty eight protein spots were differentially expressed with statistical significance. Prohibitin was significantly upregulated in prostate cancer compared to benign prostatic hyperplasia (Ummanni et al., 2008).

Rowland et al. investigated the effect of androgen (R1881) and anti-androgen (bicalutamide) on the androgen-responsive prostate cancer $\mathrm{LNCaP}$ cell line using 2DDIGE proteomic approach. Out of 197 differentially expressed spots, 165 spots have been successfully identified corresponding to 125 distinct proteins. Identified proteins were involved in diverse processes including the stress response and intracellular signaling (Rowland et al., 2007).

Bindukumar et al. identified proteins modulated in human prostate tumor cells treated with enzymatically active free-PSA by 2D-DIGE technology coupled with LCMS/MS. Several proteins involved in tumor progression had been shown to downregulate by the treatment of PSA (Bindukumar et al., 2008).

Skvortsova et al. determined differences in the proteome profiles in three radioresistant human prostate carcinoma cell lines compared to parental cells using 2DDIGE followed by MS, to define the mechanism involved in the radioresistance development (Skvortsova et al., 2008).

Byrne et al. subjected serum samples to immunoaffinity depletion and analyzed protein expression using 2D-DIGE. Out of 63 differential expression spots between the Gleason score 5 and 7 cohorts $(\mathrm{p}<0.05), 13$ spots were identified using LC-MS/MS. Pigment epithelium-derived factor (PEDF) has been validated in serum and tissue 
samples from the original cohort and also from a larger independent cohort of patients, indicating to be a predictor of early stage prostate cancer (Byrne et al., 2009).

\subsubsection{Gel-free Platform}

Although in-gel-based approaches are the mainstays for prostate cancer analysis, gel-free methodologies including Multidimensional Protein Identification Technology (MudPIT), and immunoprecipitation are emerging as alternative proteomic technologies for prostate cancer studies.

Using strong anion-exchange chromatography, Sardana et al. fractionated the conditioned medium from the prostate cancer cell line PC3(AR)(6), and then further fractionated tryptic fractions by reversed-phase C-18 chromatography before being subjected to ESI-MS/MS, and identified a novel candidate biomarker Mac-2BP (Sardana et al., 2007).

Using the poorly metastatic LNCaP cell line and its highly metastatic variant LNCaP-LN3 cell line as a model, Glen et al. employed a proteomic approach of isobaric tags for relative and absolute quantitation (iTRAQ), followed by strong cation exchange (SCX) chromatography coupled with capillary C-18 reversed-phase liquid chromatography-tandem mass spectrometry (RPLC-MS/MS). Out of the total 280 unique proteins identified, the relative expression data for 176 proteins was obtained. This was the first application of iTRAQ technology for the global proteomic profiling of prostate cancer cells (Glen et al., 2008).

Sardana et al. performed a proteomic analysis of the conditioned media of three prostate cancer cell lines (PC3, LNCaP, and 22Rv1) for discovering novel prostate cancer biomarkers. From the analysis, 2124 proteins were identified by using a bottom-up approach, consisting of offline SCX chromatography followed by RPLC-MS/MS (Sardana et al., 2008b).

Garbis et al. analyzed prostate tissue specimens from patients with benign prostatic hyperplasia and with prostate cancer by isobaric stable isotope labeling (iTRAQ) and two-dimensional liquid chromatography-tandem mass spectrometry (2DLC-MS/MS) approaches (Garbis et al., 2008).

Dall'Era et al. isolated CD10-protein complexes from C4-2 prostate cancer cells by immunoprecipitation using anti-CD10 monoclonal antibodies. Eluted fractions were combined, trypsinized, and the resulting peptides were analyzed by LC-ESI-MS/MS. HSP27 and HSP70 were found to interact with CD10 in C4-2 prostate cancer cells (Dall'Era et al., 2007). 


\subsection{Phosphoproteomics Studies of Prostate Cancer}

Application of DNA microarray and proteomics to profile gene and protein expression shows a big promise in the discovery of prostate cancer markers. However, protein or gene expression does not necessarily reflect protein activity, which is often regulated by post-translational modifications.

Protein phosphorylation is one of the most prominent prost-translational modifications and plays a critical role in the regulation of major cellular processes like proliferation, differentiation or apoptosis through signaling pathways. Phosphorylation of serine, threonine and tyrosine residue is the most common in mammalian cells.

Researchers estimate that as many as $30-50 \%$ of proteins are phosphorylated at any given time (Kalume et al., 2003). Covalent attachment of phosphate group to amino acid changes the charge in the region of a protein and results in a conformation change of the whole protein, which affects both the folding and function of proteins.

Disruptions of phosphorylation-mediated cell signaling are associated with various diseases, including cancer (Benzeno et al., 2006; Iakoucheva et al., 2004; Stephens et al., 2005). Furthermore, distinct phosphotyrosine proteome of the breast and liver tumors were found (Lim et al., 2004; Lim, 2005; Meng et al., 2004). The evidence for the existence of tumor-specific phosphoproteome shows promising to mine the tumor phosphoproteome as potential prostate cancer biomarkers in cancer diagnosis and therapeutics.

The phosphorylation status of proteins is regulated by a complex interplay between protein kinases and protein phosphatases. Protein kinases are the targets of several new cancer drugs and drug candidates. However, the functions of these protein kinases drugs are not fully understood. There is an urgent need to understand and monitor kinase signalling pathways (Ashman and Lopez, 2009).

Thus, the analysis of protein phosphorylation in prostate cancer is of paramount importance. The long-term goal of studying of the phosphorylation status of proteins in prostate cancer with phosphoproteomics methods is to provide large phosphorylation databases for prostate cancer tissues and cell lines, aid in furthering our knowledge of phosphorylation-dependent events associated with prostate cancer, and lead to the identification of new target proteins for clinical research.

Phosphoproteomics is the subdiscipline of proteomics that is focused on the comprehensive study of the extent and dynamics of protein phosphorylation. Its tasks include identification of the phosphoproteins, determination of the phosphorylation sites on the proteins, quantification of the phosphorylation under different conditions, prediction of protein kinases responsible for phosphorylation, and discovery of specific drug targets.

Compared with proteomics, phosphoproteomics faces more technical challenges due to the nature of the biomolecules being studied. Protein phosphorylation is a highly 
dynamic, reversible process, and the ratio of phosphorylated to nonphosphorylated proteins is rather low in vivo. Therefore, phosphoproteomic approaches generally require additional enrichment steps to achieve sufficient sensitivity.

In fact, most phosphoproteomics studies to date have two features in common: protein or peptide fractionation and phosphospecific enrichment. Reduction of the sample complexity is a major step necessary for an effective analysis of low-abundance phosphoproteins; this reduction can be achieved by various fractionation techniques. Furthermore, considerable amount of effort has been devoted to development of methods for phosphospecific enrichment, compatible with mass spectrometry. The objective of this enrichment is isolation of phosphoproteins and/or phosphopeptides.

As the field of phosphoproteomics continues to develop, there have been a number of recent advances in phosphoproteomics technologies, including sample preparation, phospho-specific enrichment, characterization of phosphorylation sites by MS/MS, and bioinformatics analyses of protein phosphorylation. The following subchapters offer a detailed review of protein phosphorylation analysis, emphasizing advancements in MS-based phosphoproteomics platforms.

\subsubsection{Classical Approaches to Phosphoprotein Analysis}

There are several traditional approaches for detecting phosphoproteins. In general, protein samples are separated by one-dimensional or two-dimensional polyacrylamide gel electrophoresis (1-DE or 2-DE) depending on the complexity of the protein sample, and then phosphorylated species are visualized by radiolabeling, immunodetection or phosphospecific staining.

\subsubsection{1 ${ }^{32} P{ }^{33} P$ Radioactive Labeling}

Radioactive labeling is the most sensitive method for phosphoprotein detection, and it is possible to be used both in vivo and in vitro. Phosphoproteins can be radioactively labeled with ${ }^{32} \mathrm{P} /{ }^{33} \mathrm{P}$ and visualized by autoradiography. Several studies have been performed based on radiolabeling (Chen et al., 2004; Czupalla et al., 2003). However, the methodology is not widely used in current proteomics research due to the toxicity of ${ }^{32} \mathrm{P}$ and the possibility of artificially triggered phosphorylation by exposure to ${ }^{32 / 33} \mathrm{P}$-orthophosphate.

\subsubsection{Immunodetection}

Phosphoproteins can be detected by Western blotting using antibodies against phosphoamino acids. This antibody-based approach is widely used in biochemical studies and it is compatible with MS-based phosphoproteome analysis, provided that issues with

protein amounts needed for Western blotting vs. those needed for MS are considered and addressed. However, the efficiency and specificity of the immunodetection method strongly depend on the quality of the applied antibody. While antibodies available for 
phosphotyrosine (pTyr) show high selectivity and therefore can be used to specifically detect phosphotyrosine-containing proteins (Ide et al., 2002), antibodies against phosphoserine (pSer) and phosphothreonine (pThr) lack both selectivity and sensitivity, and are not routinely used (Kaufmann et al., 2001).

\subsubsection{Phosphospecific Staining}

Fluorescence-based protein detection methods have recently shown excellent promise in terms of quantitative accuracy, detection sensitivity, and compatibility with mass spectrometry. Pro- $\mathrm{Q}^{\mathrm{TM}}$ Diamond dye has been developed to detect phosphoproteins directly in polyacrylamide gels or on nitrocellulose membranes after electrophoretic separation. In comparison with radiolabeling and immunodetection approaches, phosphospecific staining offers significant advantages, such as avoidance of radioactivity, no need for expensive antibodies, and direct detection of phosphorylated proteins obtained from cells, tissue, or body fluids. The stain noncovalently binds to phosphoproteins and it is fully compatible with mass spectrometry or Edman sequencing. Commonly, the phosphospecific staining is combined with generic protein staining in a multiplexed fashion - that is, Pro- $\mathrm{Q}^{\mathrm{TM}}$ Diamond stain is applied to a gel to specifically detect phosphorylated proteins, and this staining is followed by detection of all proteins by Sypro Ruby fluorescent stain. Goodman et al detected phosphoproteins on nitrocellulose membranes using the Pro- $\mathrm{Q}^{\mathrm{TM}}$ Diamond staining (Goodman et al., 2004). Stasyk et al. combined a standard 2D-DIGE protocol with subsequent post-staining of gels with phosphospecific fluorescent Pro- $\mathrm{Q}^{\mathrm{TM}}$ Diamond dye for quantitative detection of phosphoproteins in 2-DE gels in mammary epithelial cells (Stasyk et al., 2005). Hopper et al. characterized the phosphoproteome of porcine heart mitochondria, as detected by Pro- $\mathrm{Q}^{\mathrm{TM}}$ Diamond stain using $2-\mathrm{DE}$ and ${ }^{32} \mathrm{P}$ radioisotopic analysis as well as perform an initial screen for mitochondrial kinases and phosphatases associated with these protein phosphorylations (Hopper et al., 2006). Wu et al. devised a sequential protein staining procedure to visualize proteins separated by 2-DE, using Pro- $\mathrm{Q}^{\mathrm{TM}}$ Diamond (phosphoprotein), followed by Pro- $\mathrm{Q}^{\mathrm{TM}}$ Emerald 488 (glycoprotein), followed by SYPRO Ruby stain (general protein stain), and finally silver stain for total protein profile in spleen leukocytes (Wu et al., 2005).

\subsubsection{Mass Spectrometry-based Approaches}

Phosphoproteomics requires proper sample preparation of proteins from biological samples, efficient enrichment of phosphorylated proteins or peptides, and the application of sensitive MS to the identification of phosphorylated proteins and characterization of exact phosphorylation sites.

\subsubsection{Sample Preparation}

In addition to the general concerns discussed previously in the context of protein sample preparation, minimizing phosphatase activities is an important point that needs to be considered for phosphoprotein sample preparation. Performing all sample preparation 
at low temperature will reduce the activities of phosphatases; furthermore, adding phosphatase inhibitors to buffers in the initial step of sample solubilization will inhibit phosphatase action and thus preserve original phosphorylation patterns (Zahedi et al., 2006).

\subsubsection{Enrichment of Phosphorylated Species}

MS approaches are site-specific analyses of protein phosphorylation, requiring that phosphoproteins first be cleaved by site-specific proteases in order to produce phosphopeptides that are amenable to MS analysis. However, digestion of a protein mixture will generate phosphorylated as well as non-phosphorylated peptides. The phosphopeptides will comprise only a small portion of the digest. Furthermore, phosphopeptides are not easy to analyze by MS because of lower ionization efficiency of phosphopeptides in the presence of high-abundance nonphosphorylated peptides. To account for these drawbacks, phosphoproteins and/or phosphopeptides must be specifically enriched prior to MS in order to reduce background and thereby increase sensitivity and improve characterization effectiveness. There are a number of phosphospecific enrichment strategies currently available. Here I will discuss two widely used enrichment methods: immobilized metal ion affinity chromatography (IMAC) and titanium dioxide chromatography $\left(\mathrm{TiO}_{2}\right)$.

Immobilized metal ion affinity chromatography (IMAC): To date, IMAC is an extensively used affinity technique for the isolation of phosphopeptides from peptide mixtures prior to MS analysis (Albuquerque et al., 2008; Gioeli et al., 2002; Hoffmann et al., 2005; Moser and White, 2006; Posewitz and Tempst, 1999). The technique is based on the electrostatic interaction between negatively charged phosphopeptides at low $\mathrm{pH}$ and positively charged metal ions $\left(\mathrm{Fe}^{3+}, \mathrm{Ga}^{3+}, \mathrm{Al}^{3+}, \mathrm{Zr}^{4+}\right)$, which are chelated to nitrilotriacetic acid (NTA) or iminodiacetic acid (IDA) immobilized onto a solid support. Typically, a peptide digest is applied to an IMAC column and the phosphopeptides contained in this digest will bind, while other peptides will wash through. Elution with a suitable buffer will release the bound phosphopeptides. However, IMAC often suffers from limitations, most importantly issues related to specificity, and to recovery of phosphopeptides carrying multiple phosphate groups.

Nonspecific binding of peptides containing acidic amino acid residues to the IMAC columns is one of the main drawbacks associated with the IMAC technique. Peptides containing multiple acidic amino acid residues are often co-purified with the phosphopeptides, thereby reducing the selectivity of the method. To diminish nonspecific binding of acidic peptides, Ficarro and co-workers derivatized the carboxylic groups on acidic amino acid residues in peptides by O-methyl esterification and thereby improved phosphopeptide enrichment (Ficarro et al., 2002). Salomon et al. developed an effective method for the large-scale determination of sites of tyrosine phosphorylation, applying the strategy of phosphotyrosine immunoprecipitation combined with methyl esterification and IMAC of tryptic peptides, and MS identification (Salomon et al., 2003). Kim et al. provided a list of protein phosphorylation sites identified from HT-29 human colon adenocarcinoma cell line by methyl esterification of carboxyl groups on the tryptic 
peptides prior to IMAC enrichment combined with LC-MS/MS analysis (Kim et al., 2005). However, it is argued that reaction conditions of esterification procedure have to be optimized to avoid introducing byproducts and increasing sample complexity.

Furthermore, IMAC is generally biased against multiply phosphorylated peptides due to the strong binding of multiple phosphorylated peptides to IMAC resins, resulting in the reduction of elution efficiency. Optimization of binding, washing and elution conditions of IMAC could improve the efficiency of phosphopeptide enrichment (Dubrovska and Souchelnytskyi, 2005; Hart et al., 2002; Ndassa et al., 2006; Tsai et al., 2008).

Titanium dioxide $\left(\mathrm{TiO}_{2}\right)$ chromatography: Recently, $\mathrm{TiO}_{2}$ chromatography has been introduced as a promising alternative to IMAC. Originally, $\mathrm{TiO}_{2}$ was utilized in bioelectrochemical studies of protein functions using the adsorption of proteins to $\mathrm{TiO}_{2}$ films (Topoglidis et al., 2000) and enrichment of phospholipids (Csucs and Ramsden, 1998), which phosphate groups build self-assembling monolayers on $\mathrm{TiO}_{2}$ surface. The approach is based on the selective interaction of phosphates with porous titanium dioxide microspheres. Phosphopeptides are captured in $\mathrm{TiO}_{2}$ precolumn under acidic conditions and released under basic conditions. Selective interaction with phosphate and the chemical stability make $\mathrm{TiO}_{2}$ a promising enrichment for phosphopeptides. Pinkse et al. presented an online 2D LC-MS/MS strategy for phosphopeptide analysis on the femtomole level, using titanium dioxide precolumn as the first dimension for phosphopeptides enrichment and reverse phase column as the second dimension (Pinkse et al., 2004).

To reduce co-purified acidic peptides, Larsen et al. reported the improvement of the selectivity of $\mathrm{TiO}_{2}$ to phosphopeptide by adding 2,5-dihydroxybenzoic acid (DHB) to the loading buffer. DHB seems to efficiently reduce the binding of nonphosphorylated peptides to $\mathrm{TiO}_{2}$ while keeping its high binding affinity for phosphorylated peptides (Larsen et al., 2005; Thingholm et al., 2006). Yu et al. improved $\mathrm{TiO}_{2}$ enrichment of phosphopeptides from $\mathrm{HeLa}$ cells by the use of $\mathrm{NH}_{4} \mathrm{Glu}$ for a $\mathrm{TiO}_{2}$ column wash, which significantly increased the efficiency of $\mathrm{TiO}_{2}$ phosphopeptide enrichment ( $\mathrm{Yu}$ et al., 2007a).

The utility of zirconium dioxide microtips for phosphopeptide isolation prior to mass spectrometric analysis has been reported. These microtips display similar overall performance as $\mathrm{TiO}_{2}$ microtips. But a slight difference was shown in the selectivity; compared to $\mathrm{ZrO} 2, \mathrm{TiO}_{2}$ preferentially enriched multiply phosphorylated peptides (Kweon and Hakansson, 2006).

Although metal oxides are still not as widespread as IMAC in proteome research, high efficiency for enrichment of phosphopeptides and chemical stability of $\mathrm{TiO}_{2}$ chromatography make the method a potential alternative to IMAC for phosphoproteomic research (Kweon and Hakansson, 2008; Li et al., 2008; Marcantonio et al., 2008). 


\subsubsection{Phosphopeptide Sequencing by Tandem Mass Spectrometry}

Today most phosphopeptides are identified by MS/MS combined with database searches; analysis is performed to obtain MS/MS data diagnostic of the phosphopeptide sequences and to the location of the phosphorylated residues.

Phosphate group-specific dissociation in MS/MS can serve as supporting evidence for the presence of phosphopeptides. When peptide is phosphorylated, the amino acid residue (such as serine, threonine or tyrosine) will have a mass shift owing to the gain of a phosphate group $(80 \mathrm{Da})$. Detection of phosphopeptides by ion trap MS in the positive mode can be achieved on the basis of their tendency to lose the elements of phosphoric acid under low energy dissociation conditions. Under these conditions, MS/MS spectrum of peptides containing phosphoserine or phosphothreonine presents a predominant neutral loss of $98 \mathrm{Da}$ (owing to $\mathrm{H}_{3} \mathrm{PO}_{4}$ loss) during fragmentation by low energy collisions, while peptides bearing phosphotyrosine rarely exhibit a loss of $98 \mathrm{Da}$ in their MS/MS spectra (Figure 1-4) (Tholey et al., 1999). The evidence of the loss of 98 Da can be used as a signature for phosphopeptides and peptide sequence information can be used for determination of phosphorylation site in an ion trap (DeGnore and Qin, 1998).

Low energy CID is widely used to achieve gas-phase dissociation of peptide backbone and to produce ions for sequence analysis and identification of phosphorylation sites. However, the CID process often leads to elimination of phosphoric acid as the major dissociation channel for phospho-serine and phospho-threonine-containing peptides, without sufficient dissociation of the amide bonds along the peptide backbone. This low dissociation extent may result in lack of sequence information in MS/MS spectra. Leitner et al. have recently reported to improve the dissociation efficiency of poorly fragmenting peptides and phosphopeptides during CID by malondialdehyde modification of arginine residues (Leitner et al., 2007). Despite significant technological advancements in peptide sequencing by mass spectrometry, poor fragmentation upon CID remains a challenge for analyzing phosphopeptides.

Electron transfer dissociation (ETD) and Electron capture dissociation (ECD) have been recently introduced as alternatives to CID. These alternative ion activation methods provide a more comprehensive coverage of phosphopeptide sequences (Chi et al., 2007; Molina et al., 2007; Sweet et al., 2006).

\subsubsection{Bioinformatics Resources for Phosphoproteomics}

Identification of phosphopeptides and proteins based on MS/MS data is accomplished using the same principles that were discussed in section 1.3.4. MS/MS datasets are submitted for searches of a protein sequence database; these searches are performed with a suitable search engine such as SEQUEST. For phosphorylation analysis, database search conditions are set to include modifications of Ser, Thr, and/or Tyr residues. Because not all such amino acid residues are modified, phosphorylation is considered as either/or "dynamic" modification. 


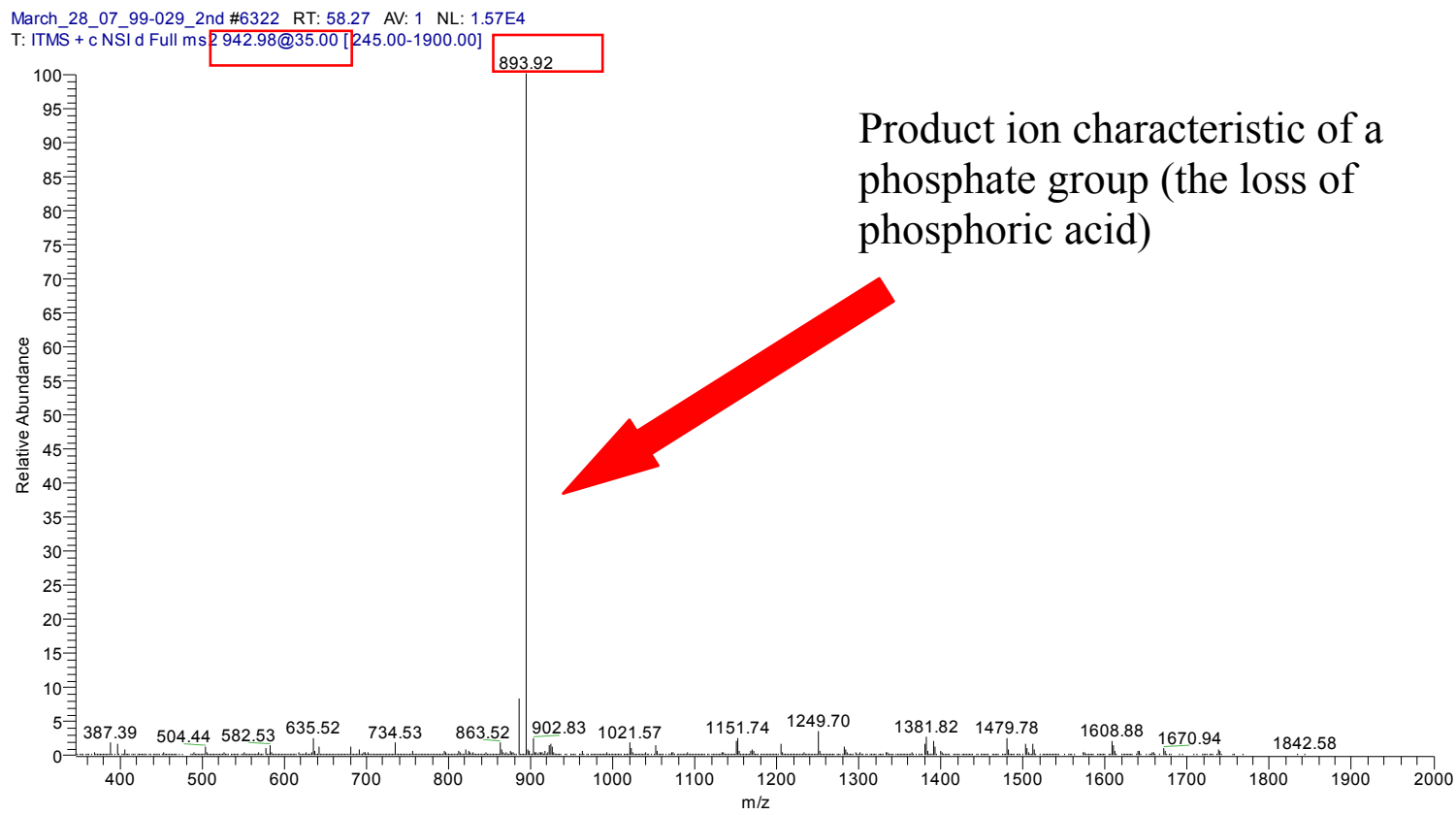

Figure 1-4 A representative MS/MS spectrum of a phosphopeptide.

The MS/MS spectrum is acquired for the precursor ion with $\mathrm{m} / \mathrm{z} 942$. The product ion $\mathrm{m} / \mathrm{z} 893$, a predominant neutral loss of $98 \mathrm{Da}$ (owing to $\mathrm{H}_{3} \mathrm{PO}_{4}$ loss), presents in the MS/MS spectrum. 
Phosphoproteomics research is generating a wealth of novel information. Studies provide important information such as: a list of known phosphoproteins in a particular biological system; novel phosphorylation sites in known phosphoproteins; phosphorylated proteins which have not previously been reported to be phosphorylated; phosphorylation of a novel protein. Therefore, major efforts have been directed towards integration of this information, and towards an effective dissemination to the scientific community.

An important compilation of information on protein phosphorylation (and on other PTMs) is contained in the annotations of the Swiss-Prot protein sequence database. A Swiss-Prot annotation page for a particular protein entry will include information on the location of a phosphorylation site as well as relevant literature references. In addition to Swiss-Prot annotations, new resources related to phosphorylation and other PTMs have been developed. Table 1-2 lists some of the Internet-based resources for phosphoproteomics research.

Several knowledgebases dedicated to protein phosphorylation have been created. One of the most comprehensive knowledgebases is PhosphoSite. PhosphoSite (www.phosphosite.org) is a systems biology resource focused on in vivo protein phosphorylation sites in human, mouse, and other mammalian proteomes. It includes a wealth of information such as the phosphorylated residue, orthologous sites, domains and motifs, additional functionalities etc. Thus, PhosphoSite provides an accurate, comprehensive source of information about mammalian protein phosphorylation sites.

To further the insight derived form large-scale phosphoproteomics studies, bioinformatics tools for additional phosphorylation-related examination have been developed. For example, phosphopeptide sequences identified from phosphoproteomic screens can be analyzed with the Scansite tool to predict protein kinases and/or phosphorylation-dependent binding motifs. Scansite (http://scansite.mit.edu) searches for motifs within proteins that are likely to be phosphorylated by specific protein kinases or bind to domains (Obenauer et al., 2003).

\subsection{Summary and Research Aims}

Rapid development of proteomic technologies for studying prostate cancer shows a great promise for gaining new scientific knowledge that will contribute to the ultimate goal of finding more effective cancer diagnostic methods and therapies. In-gel and gelfree-based methodologies have already been applied in prostate cancer studies of cultured cells and tissue specimens. In the last few years proteomic studies have generated new information of potential diagnostic, prognostic, and therapeutic significance.

Diverse proteomics technologies have adapted for phosphoproteomics. Mass spectrometry-based phosphoproteomic platforms play a major role in current phosphoproteomics research, and advancements in the various components that comprise these platforms have been made, including optimized procedures for sample preparation, 
Table 1-2 A list of major websites for phosphoproteomics.

\begin{tabular}{|c|c|c|}
\hline Name & Comments & URL \\
\hline \multicolumn{3}{|l|}{$\begin{array}{l}\text { Phosphorylation- } \\
\text { site database }\end{array}$} \\
\hline Swiss-Prot & $\begin{array}{l}\text { Protein annotation including } \\
\text { phosphorylation sites and other post } \\
\text { translational modification }\end{array}$ & http://expasy.org \\
\hline PhosphoSite & $\begin{array}{l}\text { Search known phosphorylation sites } \\
\text { in human, mouse or rat }\end{array}$ & http://www.phosphosite.org \\
\hline Phospho.ELM & $\begin{array}{l}\text { Search phosphorylation sites in } \\
\text { proteins }\end{array}$ & http://phospho.elm.eu.org \\
\hline \multicolumn{3}{|l|}{$\begin{array}{l}\text { Phosphorylation- } \\
\text { site prediction }\end{array}$} \\
\hline Scansite & Search for potential kinase motifs & http://scansite.mit.edu \\
\hline Prosite & $\begin{array}{l}\text { Database of protein domains, families } \\
\text { and functional sites }\end{array}$ & http://expasy.org/prosite \\
\hline Ascore & $\begin{array}{l}\text { Phosphorylation site localization } \\
\text { based on the presence and intensity of } \\
\text { site-determining ions in MS/MS } \\
\text { spectra. }\end{array}$ & $\underline{\text { http://ascore.med.harvard.edu }}$ \\
\hline
\end{tabular}

specific and sensitive methods for phosphospecific enrichment, mass spectrometers with lower detection limits and improved mass accuracy, alternative gas-phase dissociation strategies, expanded protein knowledgebases and other bioinformatics resources. These technological advances make it feasible to perform large-scale phosphoproteomic examinations of prostate cancer.

It is an accepted fact that no single bioanalytical platform can be universally applied to proteomics or phosphoproteomics. Due to diverse nature of biological samples, different research objectives, different resources available to investigators, various bioanalytical platforms are being utilized for phosphoproteomics (Neverova and Van Eyk, 2005). Each bioanalytical platform has its unique strengths (and also limitations). Improvements of existing platforms and development of new platforms is a critical aspect of the field of proteomics, since a continued expansion of analytical capabilities will bring more power to penetrate deeper into a proteome or phosphoproteome, to more accurately capture its dynamics, and to integrate information into a systems-level understanding.

In our research group, the long-term focus is on proteomics of human cells, tissues, and biological fluids. Our team has contributed to the efforts directed towards analytical method development. We introduced a new platform that combines IEF and tandem mass spectrometry, and adapted this platform to phosphoproteomics (BeranovaGiorgianni et al., 2006; Giorgianni et al., 2004; Zhao et al., 2005).

Over the past years, our group has pursued research on the prostate cancer proteome and phosphoproteome, focusing on cultured prostate cancer cell lines and on 
human tumor specimens. There were two broad objectives that I set out to achieve in my doctoral research: (1) to build on the successful foundation from previous research in our laboratory to obtain an expanded atlas of the human prostate cancer phosphoproteome; and (2) to complete a first phase of a new collaborative research program aimed at differential profiling of prostate cancer tissue for mechanistic studies of molecular effects of novel anticancer therapies.

The specific aims of my research were:

Specific aim 1 (Chapter 2) was to adapt our newly developed analytical method termed in-gel IEF LC-MS/MS - to mapping of prostate cancer phosphoproteome to characterize on a global scale the phosphoproteome in the $\mathrm{LNCaP}$ human prostate cancer cell line.

Specific aim 2 (Chapter 3) was to apply a gel-free analytical platform to mapping the phosphoprotein signatures in human prostate cancer tissues for biomarkers discovery.

Specific aim 3 (Chapter 4) was to develop a 2-DE based platform combined with phospho-specific staining to characterize the phosphoproteome in the $\mathrm{LNCaP}$ prostate cancer cells.

Specific aim 4 (Chapter 5) was to identify proteins that are differentially expressed in control and bicalutamide/embelin treated mouse prostate tumor specimens, which will aid in the revealing of molecular mechanism of bicalutamide/embelin combination therapy. 


\section{CHAPTER 2. CHARACTERIZATION OF THE PHOSPHOPROTEOME IN LNCAP PROSTATE CANCER CELLS BY IN-GEL ISOELECTRIC FOCUSING AND TANDEM MASS SPECTROMETRY}

\subsection{Introduction}

Prostate cancer is a leading type of cancer in men, and it is the second leading cause of cancer death (Jemal et al., 2008). Despite recent declines in the mortality from prostate cancer, prostate cancer remains a serious public health issue. Therefore, a large number of research efforts aims to increase our understanding of the mechanisms underlying the formation and progression of prostate cancer, in order to improve current diagnostic methods and to discover new drug targets. Analysis of the molecular machinery in prostate cancer cells by proteomics approaches is an integral part of these efforts.

Phosphoproteomics has recently emerged as an approach that focuses on examination of protein phosphorylation on a global-scale. Many fundamental cellular processes are controlled by reversible protein phosphorylation; it is estimated that in mammalian cells, $30 \%$ of proteins are phosphorylated at any given time (Cohen, 2000). Disruptions of phosphorylation-mediated cell signaling are associated with various diseases, including cancer (Benzeno et al., 2006; Iakoucheva et al., 2004; Lim, 2005; Stephens et al., 2005). Therefore, large-scale examination of the prostate cancer phosphoproteome will bring unique mechanistic insight about perturbations of protein networks relevant to cancer. Several recent reviews summarized numerous phosphoproteomic methodologies that have been developed. These methodologies used various combinations of separation strategies, in conjunction with mass spectrometry (Collins et al., 2007; Gafken and Lampe, 2006; Hoffert and Knepper, 2008).

In our research group, the long-term focus is on proteomics of prostate cancer. We have initiated a phosphoproteomic study that aims to map the phosphoproteome in the $\mathrm{LNCaP}$ cell line, including an exact description of the sites of phosphorylation. Towards the aim, we have recently applied a simple gel-free analytical methodology to obtain a first panel of the $\mathrm{LNCaP}$ phosphoproteins, 137 phosphorylation sites in 81 phosphoproteins (Giorgianni et al., 2007). That pilot study underscored the need to enhance the coverage of the LNCaP phosphoproteome through modifications of our analytical strategy.

The aim of this study was to adapt a newly developed analytical methodology, termed in-gel IEF LC-MS/MS (Giorgianni et al., 2003; Zhao et al., 2005), to phosphoproteomics study for prostate cancer, to characterize on a global scale the phosphoproteome in LNCaP human prostate cancer cell line. An outline of the in-gel IEF LC-MS/MS strategy is shown in Figure 2-1. 


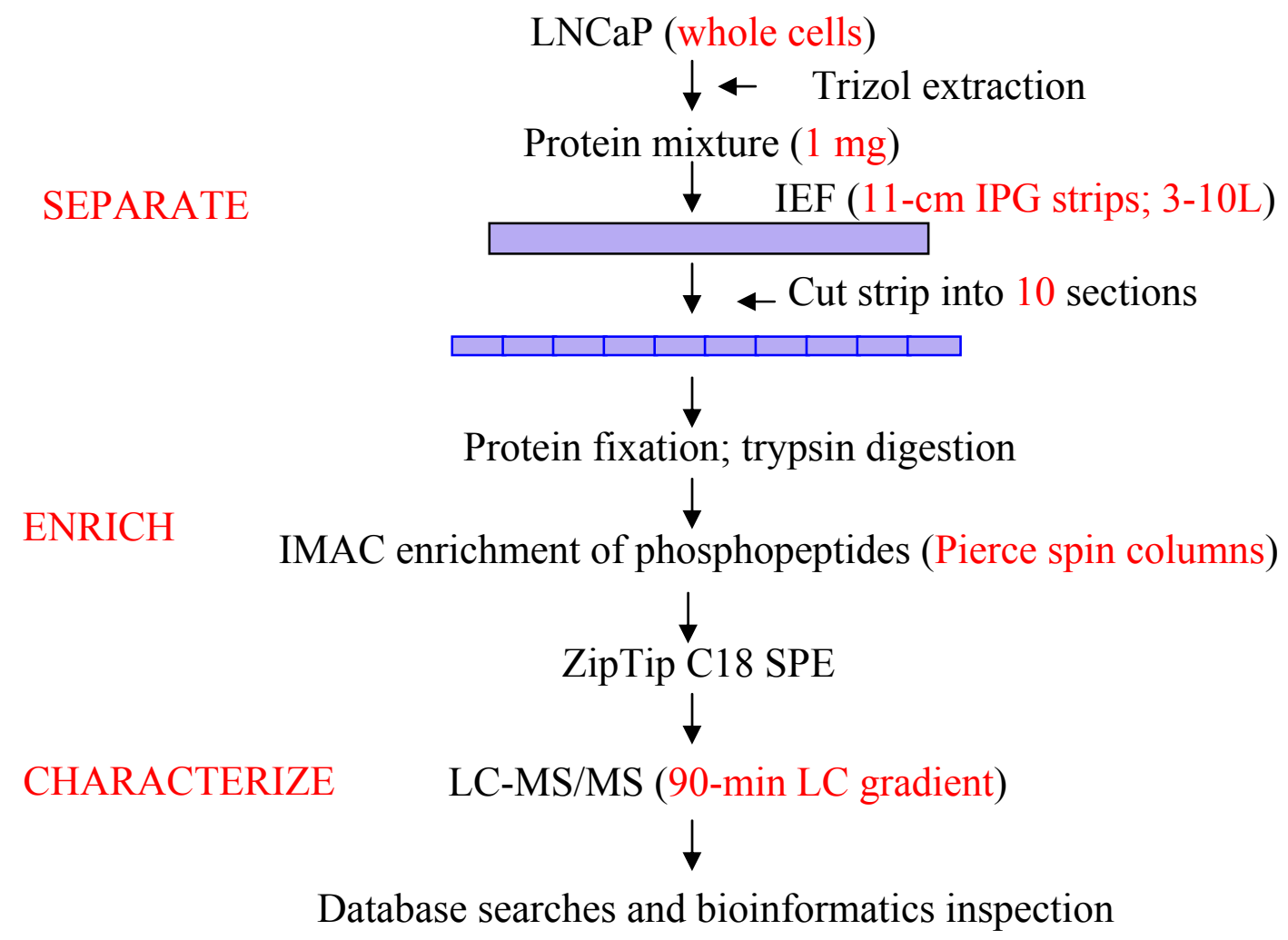

Figure 2-1 Outline of the in-gel IEF LC-MS/MS strategy.

The LNCaP proteins were extracted with Trizol reagent, and separated by IEF in IPG strips. After sectioning of the strips, the proteins in each gel section were fixed, and digested with trypsin. The digests were subjected to IMAC to isolate phosphorylated peptides. The samples were desalted and concentrated by SPE, and analyzed with LCMS/MS. The LC-MS/MS datasets were used to identify the phosphorylated peptides and proteins via searches of a protein sequence database. 


\subsection{Materials and Methods}

\subsubsection{Protein Extraction from LNCaP Cells}

The LNCaP human prostate cancer cells (American Type Culture Collection, Manassas, VA) were cultured under conditions described previously (Giorgianni et al., 2007). Briefly, the LNCaP cells were cultured in RPMI-1640 with $2 \mathrm{mM} \mathrm{L}$-glutamine (American Type Culture Collection, Manassas, VA) supplemented with $10 \%$ fetal bovine serum (Hyclone, Logan, UT) in a $37^{\circ} \mathrm{C}, 5 \% \mathrm{CO} 2$ incubator. The cells were collected while in log-phase growth (approximately $85 \%$ confluency). The media were aspirated from the plates; $0.25 \%$ trypsin- $0.53 \mathrm{mM}$ EDTA (American Type Culture Collection) was added. After a few minutes, complete media were added to the plates to stop the trypsinization, and cells were resuspended and transferred to a conical tube for centrifugation. Cells were pelleted at $150 \times \mathrm{g}$ for $4 \mathrm{~min}$, and resuspended in PBS. The cells were processed for protein extraction in two tubes, each containing $5 \times 10^{6}$ cells. The cells in each tube were resuspended in $1.0 \mathrm{~mL}$ of Trizol reagent (Invitrogen, Carlsbad, CA) containing $5 \mu \mathrm{L}$ of a phosphatase inhibitor cocktail (Sigma, St. Louis, MO), and the cells were lysed by repetitive pipetting. A total of $1 \mathrm{mg}$ of protein was extracted using Trizol reagent following a procedure provided by the manufacturer.

Briefly, cells were resuspended in $1.0 \mathrm{~mL}$ of Trizol reagent containing $5 \mu \mathrm{L}$ of a phosphatase inhibitor cocktail, and the samples were pipetted until full disruption of the cells was achieved. The samples were incubated for 5 minutes at room temperature to permit the complete dissociation of nucleoprotein complexes. Chloroform was added at a proportion of $0.2 \mathrm{~mL}$ per $1 \mathrm{~mL}$ of Trizol reagent. The mixtures were vigorously mixed by hand for 15 seconds. The mixtures were incubated at room temperature for 3 minutes. Centrifugation was performed at $12,000 \mathrm{x}$ g for 15 minutes at $4{ }^{\circ} \mathrm{C}$. Following centrifugation, the mixture separated into a lower red phenol-chloroform phase, an interphase and a colorless upper aqueous phase. The organic phase was retained after carefully removing the aqueous phase (containing RNA). Absolute ethanol was added at a proportion of $0.3 \mathrm{~mL}$ per $1 \mathrm{~mL}$ of Trizol reagent used for the initial homogenization. Samples were mixed by inversion, and were incubated at room temperature for 3 minutes followed by sedimentation of DNA by centrifugation at $2,000 \mathrm{x}$ g for 5 minutes at $4{ }^{\circ} \mathrm{C}$. The protein-containing phenol-ethanol supernatant was retained. Isopropyl alcohol was added to the supernatant at a proportion of $1.5 \mathrm{~mL}$ per $1 \mathrm{~mL}$ of Trizol reagent used for the initial homogenization. The mixture was stored at room temperature for 10 minutes followed by protein sedimentation by centrifugation at $12,000 \mathrm{xg}$ for 10 minutes at $4{ }^{\circ} \mathrm{C}$. Supernatant was removed and protein pellets were washed twice with $0.3 \mathrm{M}$ guanidinium hydrochloride in $95 \%$ ethanol, followed by a single wash with ethanol. The proteins were dried in vacuum centrifuge for 5 minutes. 


\subsubsection{In-gel IEF}

The proteins were solubilized in $600 \mu \mathrm{L}$ of a rehydration buffer containing urea ( 7 M), thiourea (2 M), CHAPS (2\%, w/v), IPG buffer pH 3-10 (2\%, w/v), DTT $(0.3 \%$, w/v), and a trace of Bromophenol Blue dye. The mixture was kept at room temperature for $1 \mathrm{~h}$; during that time, the mixture was sonicated for $20 \mathrm{~s}$ three times. The mixture was divided into three aliquots and centrifuged for $5 \mathrm{~min}$ at $12,000 \times \mathrm{g}$. The aliquots of the sample solution were loaded onto three IPG strips (pH 3-10, $11 \mathrm{~cm}$; GE Healthcare). The strips were allowed to rehydrate overnight in a reswelling tray. The IEF was carried out in a Multiphor II unit (GE Healthcare, Piscataway, NJ) according to manufacturer's instructions. In brief, the Multitemp water circulator was set to $20^{\circ} \mathrm{C}$, and apparatus was leveled, and all cables were connected. The DryStrip tray and the Immobiline strip aligner were placed according to the instructions. The grooves were lined up with the pattern on the cooling plate. The rehydrated IPG strip was taken out of the reswelling tray, and was rinsed slightly with water to remove any residual oil. The strip was placed into a groove in the strip aligner with the gel side up, and anode towards the rear. Two moistened electrode strips were placed on top of the aligned strip, one across the cathode and one across the anode edges. The strip made at least partial contact with the gel surface. Oil was used to completely cover the IPG strip. Focusing was performed at $20^{\circ} \mathrm{C}$ under the following conditions: $100 \mathrm{~V}$ (gradient over $1 \mathrm{~min}$ ); $100 \mathrm{~V}$ (fixed for $120 \mathrm{~min}$ ); $500 \mathrm{~V}$ (gradient over $1 \mathrm{~min}$ ); $3500 \mathrm{~V}$ (gradient over $90 \mathrm{~min}$ ); $3500 \mathrm{~V}$ (fixed for $6 \mathrm{~h}$ ). After IEF, the IPG strips were stored at $-80{ }^{\circ} \mathrm{C}$ until further analysis.

\subsubsection{In-gel Digestion}

After IEF, the IPG strips were rinsed with water, and cut into 10 sections of identical size $(1.10 \mathrm{~cm} \times 0.36 \mathrm{~cm})$. The gel sections were peeled off from the plastic backing with a clean scalpel. The same gel sections from the three strips were combined. Proteins in the IPG strip sections were fixed in $20 \%$ trichloroacetic acid (TCA) for 30min (Zhao et al., 2005). All gel pieces were washed with water and incubated in $200 \mu \mathrm{L}$ of $200 \mathrm{mM}$ ammonium bicarbonate for $10 \mathrm{~min}$, followed by dehydration with $200 \mu \mathrm{L}$ of acetonitrile $(\mathrm{ACN})$. The gel pieces were dried in a vacuum centrifuge. The gel pieces were rehydrated with $100 \mu \mathrm{L}$ of ammonium bicarbonate $(50 \mathrm{mM})$ containing sequencinggrade trypsin (Promega, Madison, WI) at a concentration of $40 \mathrm{ng} / \mu \mathrm{L}$. The samples were incubated overnight at $37^{\circ} \mathrm{C}$.

\subsubsection{Enrichment of Phosphopeptides with IMAC}

The digests from each gel section were dried in a vacuum centrifuge, and redissolved in $50 \mu \mathrm{L}$ of $10 \%$ acetic acid. The phosphopeptides were isolated with an IMAC Gallium (III) spin-column (Phosphopeptide Isolation Kit; Pierce, Rockford, IL). The sample solution was bound to the column by incubation at room temperature for $1 \mathrm{~h}$. The column was washed with the following solutions: two portions of $40 \mu \mathrm{L}$ of $0.1 \%$ $\mathrm{AcOH}$, two portions of $40 \mu \mathrm{L}$ of $0.1 \% \mathrm{AcOH} / 10 \% \mathrm{ACN}$, and two portions of $40 \mu \mathrm{L}$ of 
water. The phosphopeptides were eluted from the IMAC column with two aliquots of $30 \mu \mathrm{L}$ of $200 \mathrm{mM}$ sodium phosphate ( $\mathrm{pH} 8.4$ ), followed by a single elution with $30 \mu \mathrm{L}$ of $100 \mathrm{mM}$ sodium phosphate/ $50 \%$ ACN. The eluates were combined and dried under vacuum. Prior to LC-MS/MS analyses, the IMAC-enriched phosphopeptides were reconstituted with $15 \mu \mathrm{L}$ of $0.1 \%$ trifloroacetic acid (TFA) and desalted with ZipTipC18 (Millipore, Billerica, MA), using the procedure provided by the manufacturer. In brief, the ZipTipC18 column was conditioned with $10 \mu \mathrm{L}$ ACN / 0.1\% TFA (3 times), 50\% ACN / 0.1\% TFA (3 times) and water / 0.1\% TFA (3 times). The peptide mixture was aspirated 10 times in order for the peptides to bind to the cartridge. The cartridge was washed 3 times with $10 \mu \mathrm{L} 0.1 \%$ TFA / water. The phosphopeptides were eluted from the ZipTipC18 column with $3 \mu \mathrm{L}$ of $50 \%$ ACN/0.1\% TFA, and diluted with $3 \mu \mathrm{L}$ of $0.1 \%$ $\mathrm{AcOH}$.

\subsubsection{LC-MS/MS Analysis}

LC-MS/MS experiments were performed on an LTQ linear ion trap mass spectrometer (Thermo Electron, San Jose, CA) coupled to a nanoflow LC system (Dionex, Sunnyvale, CA). Each IMAC-enriched digest obtained from specific IPG gel section was analyzed in triplicate. The peptide mixtures were manually injected onto a PicoTip fused-silica micro-capillary column/spray needle (15 cm length, $75 \mu \mathrm{m}$ ID; New Objective, MA) packed in-house with C18 stationary phase (Michrom Bioresources, Auburn, CA). The peptides were separated using a 90 min-gradient from $0 \%$ to $90 \%$ mobile phase $\mathrm{B}$. The composition of mobile phase $\mathrm{B}$ was $90 \% \mathrm{MeOH} / 10 \%$ water $/ 0.05 \%$ FA; the composition of mobile phase A was $2 \% \mathrm{MeOH} / 98 \%$ water $/ 0.05 \%$ FA. The LTQ mass spectrometer was operated in positive ion mode and data-dependent acquisition was performed. One data-dependent acquisition cycle encompassed a full-range MS scan followed by $7 \mathrm{MS} / \mathrm{MS}$ scans on the most abundant ions from the MS scan.

\subsubsection{Database Searches}

The datasets obtained in the LC-MS/MS analyses of the enriched digests from each gel section were used for database searches with the TurboSEQUEST search engine (Thermo Electron) that is part of Bioworks version 3.2. Using the target-decoy database searching strategy (Ballif et al., 2008; Elias and Gygi, 2007), the data were searched against a composite database containing human protein sequences and their reversed complements. The searches were performed with the following parameters: full-trypsin specificity, dynamic modification of oxidized Met $(+15.9949)$, and dynamic modifications of phosphorylated Ser, Thr, and Tyr $(+79.9663)$. The search results were filtered using the cutoffs of $\Delta \mathrm{Cn}=0.14$, and XCorr of 3.0, 4.0 for doubly and triply charged precursor ions, respectively; this filtering procedure produced a phosphopeptide set with an estimated false-positive rate of $0.8 \%$ (Elias and Gygi, 2007). All entries within this set were manually validated. In addition, lower-scoring phosphopeptides were subjected to manual validation, and those peptides that fulfilled the validation criteria were included in the final phosphopeptide panel. The manual validation involved 
inspection of the corresponding MS/MS data. The criteria used for manual validation included: the presence of a diagnostic product ion corresponding to neutral loss of phosphoric acid (DeGnore and Qin, 1998); and high-quality spectrum in terms of signalto-noise ratio for the sequence-determining product ions. Furthermore, assignments of exact sites of phosphorylation were validated manually through inspection of the corresponding product ions.

\subsection{Results and Discussion}

\subsubsection{In-gel IEF LC-MS/MS Approach}

The analytical methodology, outlined in Figure 2-1, encompassed seven steps: (1) Trizol-based extraction of proteins from the LNCaP cells; (2) separation of the protein mixture by isoelectric focusing in immobilized $\mathrm{pH}$ gradient (IPG) strips; (3) sectioning of the IPG strips; (4) protein fixation followed by digestion of the proteins in each gel section; (5) enrichment of phosphopeptides in the digests by IMAC; (6) analysis of the enriched digests by LC-MS/MS; and (7) identification of the phosphopeptides/proteins through database searches, and assignment of the sites of phosphorylation in these proteins. A representative example of an MS/MS spectrum from the LC-MS/MS datasets is shown in Figure 2-2.

The objective of the study presented here was to obtain an expanded panel of the LNCaP phosphoproteome through application of an improved analytical strategy. In-gel IEF LC-MS/MS, which included separation of the prostate proteins by IEF, was used to provide an additional dimension of separation of the complex mixture. Fractionation of the original protein mixture simplified the samples to be subsequently analyzed by LCMS/MS. The concomitant decrease in throughput (multiple fractions had to be analyzed) was offset by the increase in the amount of information that was gained in the overall output of the analysis.

In combination with IEF separation, phosphopeptide enrichment and a more sensitive mass spectrometer (LTQ vs LCQ Deca XP plus), this bioanalytical strategy yielded a significantly larger phosphoprotein panel, compared to our pilot study of the LNCaP phosphoproteome (Giorgianni et al., 2007). As shown in Table 2-1, a total of 625 phosphorylation sites were identified in 558 phosphopeptides. Together, these phosphopeptides mapped to 296 different proteins. This panel of the LNCaP phosphoproteins was 3.6-fold larger than the panel obtained in our previous work, which attested to the power of the chosen analytical methodology. A full list of the phosphopeptides and phosphoproteins characterized in this study is provided in Table A1. Phosphorylation sites were assigned based on manual inspection of product ion series in the MS/MS spectrum. The full information for each phosphorylation site assignment is provided in Table A-2.

One of tasks we faced in the analysis of tandem mass spectra was to validate 


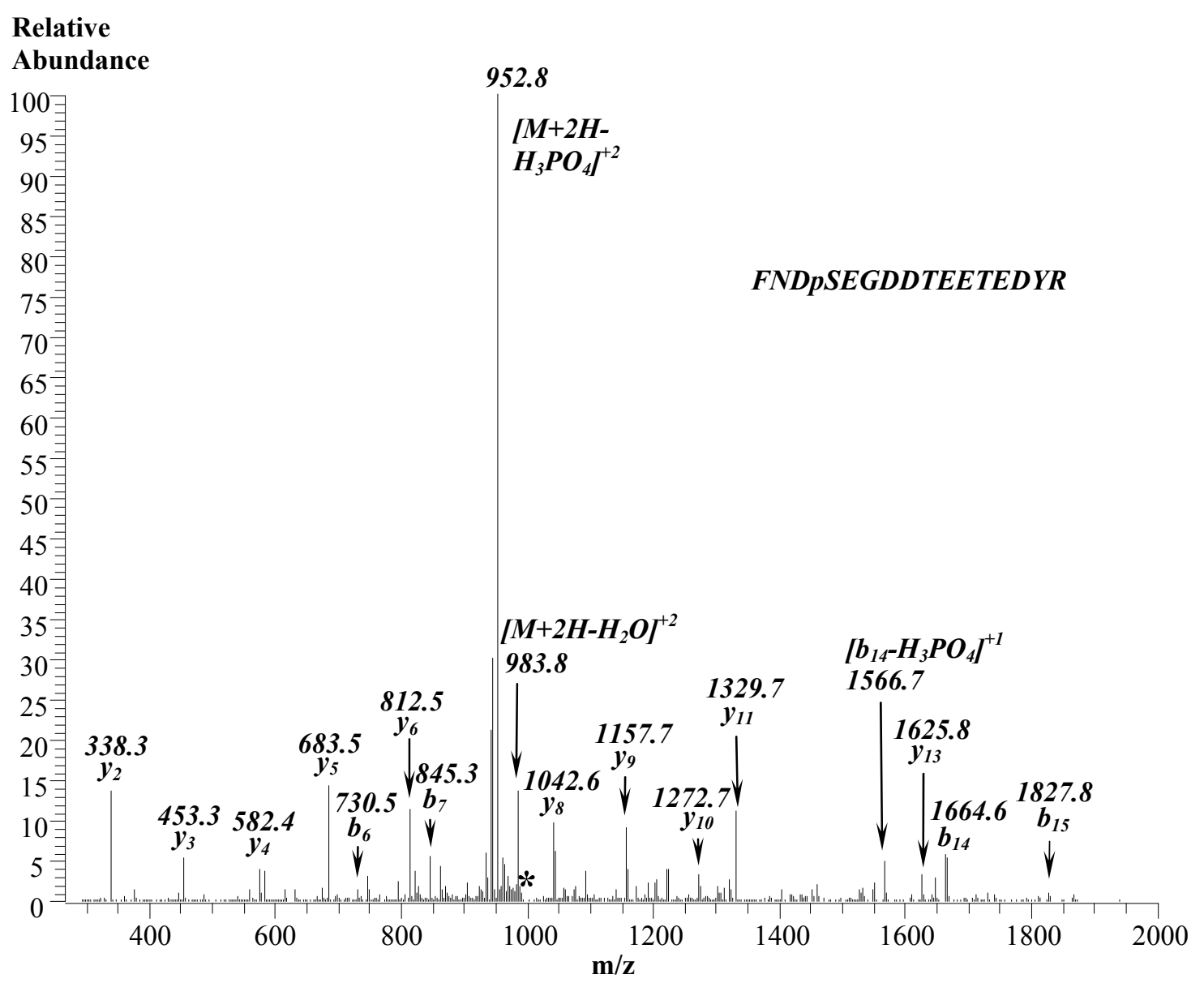

Figure 2-2 A representative MS/MS spectrum obtained in the LC-MS/MS analysis of IMAC-enriched digest from gel section 4.

The spectrum contains a phosphate-diagnostic product ion at $\mathrm{m} / \mathrm{z} 952$, and series of other product ions that determine the sequence of the peptide. Based on these data, the phosphopeptide FNDpSEGDDTEETEDYR $\left(\mathrm{m} / \mathrm{z}\right.$ of the $[\mathrm{M}+2 \mathrm{H}]^{2+}$ molecular ion is 1001.4, marked as * into the spectrum) was identified that belongs to Bcl-2-Associated Transcription Factor 1. 
Table 2-1 Summary of the LNCaP phosphoproteome characterization results: in-gel IEF LC-MS/MS vs. gel-free methodology.

\begin{tabular}{llll}
\hline Method & $\begin{array}{l}\text { Number of } \\
\text { phosphosites }\end{array}$ & $\begin{array}{l}\text { Number of } \\
\text { phosphopeptides }\end{array}$ & $\begin{array}{l}\text { Number of } \\
\text { phosphoproteins }\end{array}$ \\
\hline Gel-free platform & 137 & 115 & 81 \\
& & 558 & 296 \\
\hline In-gel IEF LC-MS/MS & 625 & 58 & \\
\hline
\end{tabular}

peptide-spectrum matched identification. Tandem mass based peptide identifications are dependent on the quality of the match between the observed and predicted sequence spectra. However, in practice matches between observed and expected spectra are not perfect. Therefore, manual inspection of MS/MS spectra of the individual search results is very critical after database search. For the large phosphorylation data set generated by in-gel IEF LC-MS/MS study, we performed a composite target-decoy search strategy (Ballif et al., 2008; Elias and Gygi, 2007), the data were searched against a composite database containing human protein sequences and their reversed complements. The target-decoy strategy helped to estimate how many false positive were associated with the entire data set. We determined the cutoff value for the filters required to achieve a $<1 \%$ false-positive rate. Moreover, we performed manual validation of all hits and included the lower-scoring validated hits in our final panel. Combination the target-decoy strategy and the manual validation strategy allowed us to balance the need to minimize false positive with the need to also reduce false negatives.

\subsubsection{Characteristics of Identified Phosphoproteins/phosphopeptides}

Distribution of the identified phosphoprotein/phosphopeptides across the IPG strip is shown in Figure 2-3. As expected, the largest numbers of phosphoproteins were found in gel sections 4 and 5, corresponding to $\mathrm{pH}$ range 5.1 to 6.5 . In addition, a number of acidic or basic phosphoproteins were found at the extreme positions in the $\mathrm{pH}$ 3-10 IPG strip. For example, a high-pI protein, Cysteine and Glycine-Rich Protein 1 (theoretical pI is 8.90) was identified in gel section 9. These results showed that phosphoproteins with a wide range of physicochemical characteristics were probed by our methodology. It should be noted that the experimental pI information on the intact protein was preserved, unlike in the gel-free approach or other methods that focus on analyses at the peptide level. As shown in Figure 2-4, the majority of the identified phosphopeptides were singly phosphorylated; doubly phosphorylated peptides accounted for $20-30 \%$ of all phosphopeptides, and fewer than $10 \%$ were triply phosphorylated.

The phosphoproteins characterized in the study were evaluated based on their subcellular locations and function, as presented below. The full information for each phosphoprotein is available in Table A-3. 


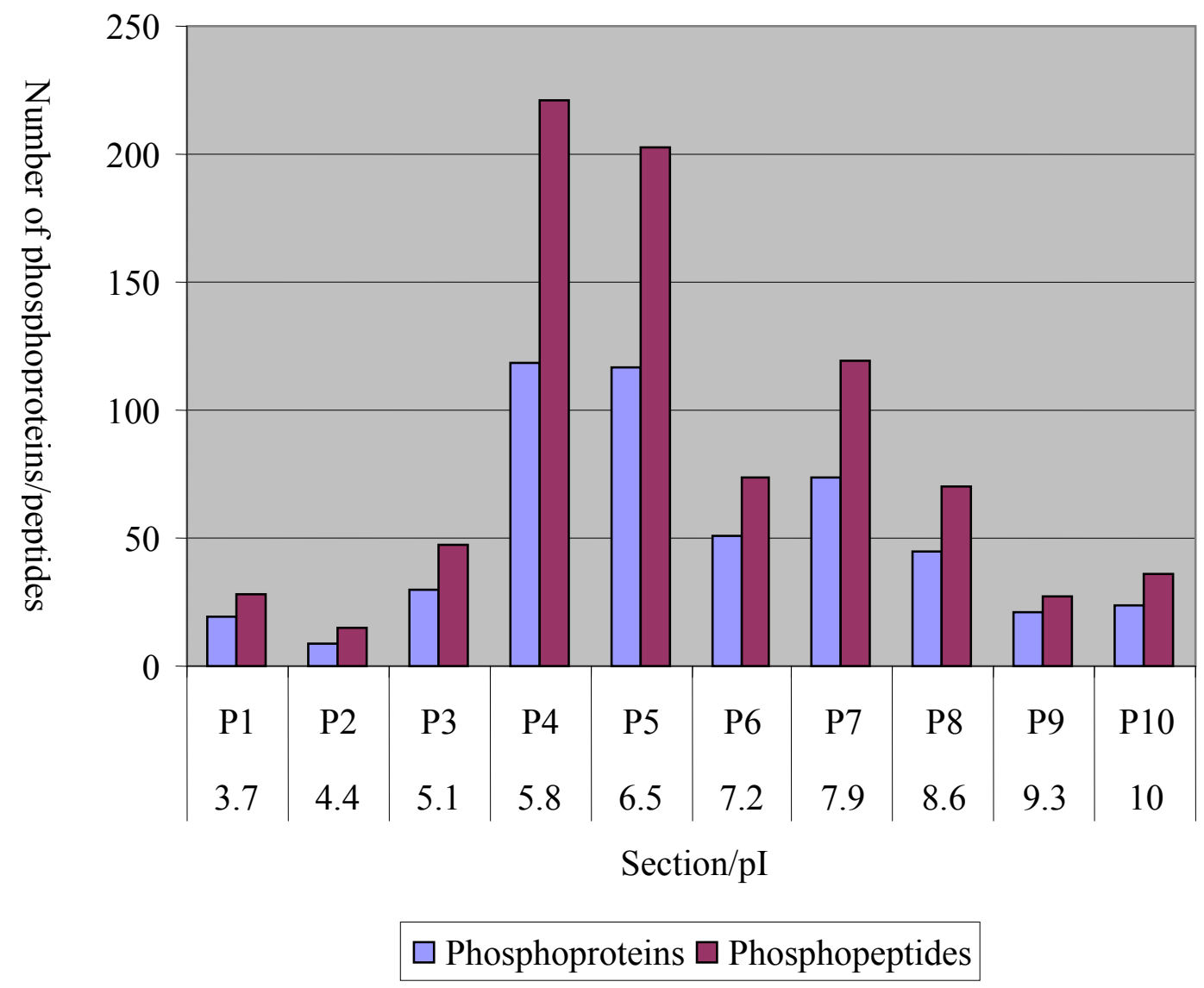

Figure 2-3 Distribution of phosphoproteins/phosphopeptides in the IPG strip sections.

An 11-cm IPG strip (pH range 3-10L) was used and divided into 10 sections of equal size. The labels on the $\mathrm{x}$ axis showed the IPG strip section numbers and corresponding $\mathrm{pI}$ values. 


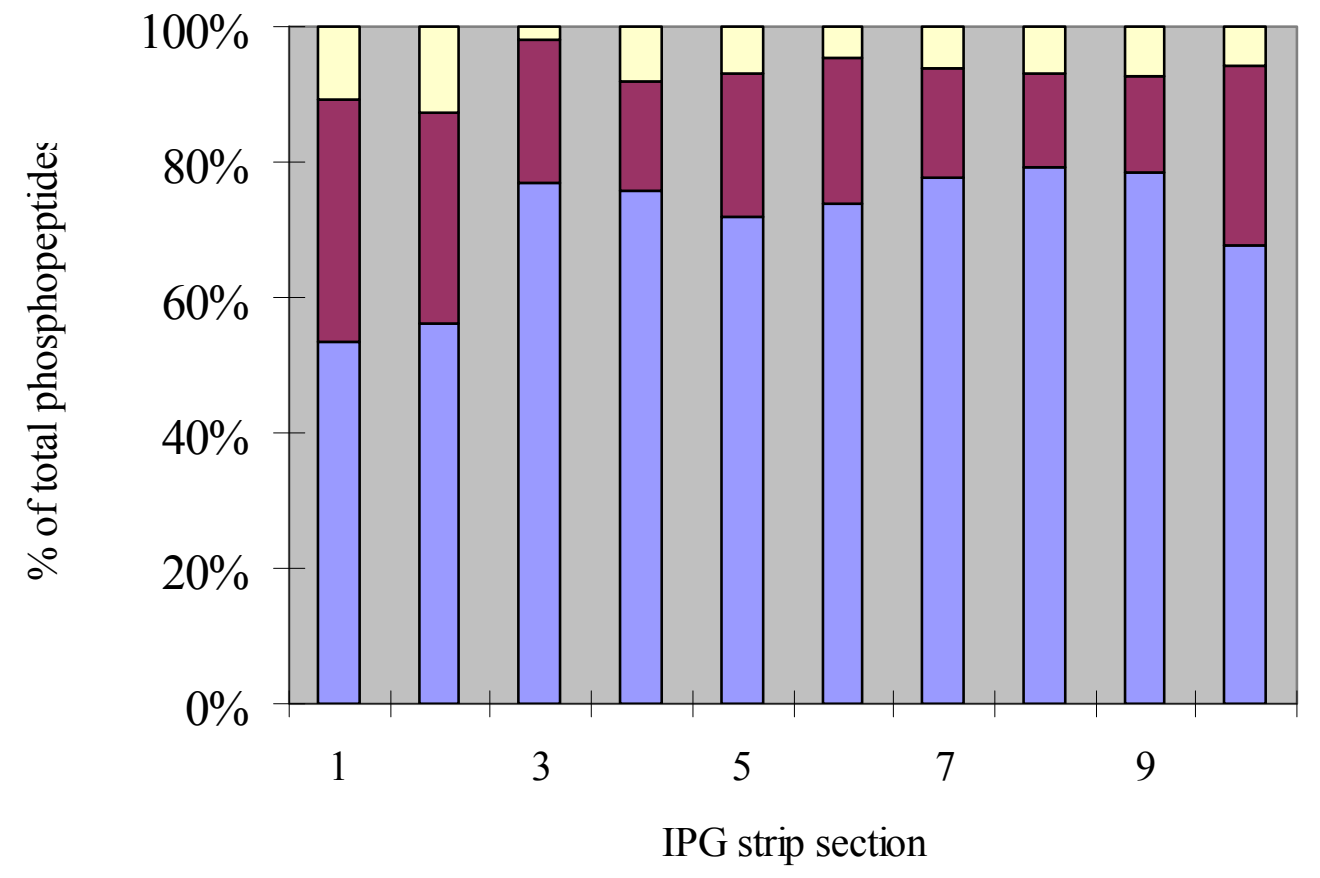

Singly-phosphorylated $\square$ Doubly-phosphorylated $\square$ Triply-phosphorylated

Figure 2-4 Distribution of singly, doubly, and triply phosphorylated peptides. 


\subsubsection{Classifications of Identified Phosphoproteins}

The characterized phosphoproteins belong to various subcellular locations. The subcellular locations of the identified phosphoproteins were assigned based on Swiss-Prot annotations: among the 296 phosphoproteins, 205 proteins were annotated and 91 proteins could not be classified. As depicted in Figure 2-5, proteins from various subcellular compartments were sampled: nucleus, cytoplasm, multiple (that is, proteins that can be found in more than one subcellular location), membrane and mitochondria. It should be noted that the largest group sampled in the study were nuclear proteins and proteins that belong to a cytoplasm/nucleus subgroup within the "multiple" category.

The phosphoproteins characterized in the study are functionally diverse. We grouped the identified phosphoproteins into several categories based on their biological function as annotated in the Swiss-Prot database. The distribution of the phosphoproteins among the various functional categories is shown in Figure 2-6. The largest group was represented by proteins playing a role in transcription, including transcription factors and RNA-processing proteins. Other two large groups were represented by proteins involved in translation and signal transduction.

\subsubsection{Selected Examples of Identified Phosphoproteins}

Selected examples of identified phosphoproteins that are of particular interest in the normal and disease prostate physiology included kinases, steroid-receptor interacting proteins, and other proteins relevant to cancer.

The LNCaP phosphoprotein panel included a number of kinases, for example RAC-alpha Serine/threonine-Protein Kinase, Mitogen-Activated Protein Kinase 2, Serine/threonine-Protein Kinase TAO2, Serine/threonine-protein Kinase D1, and Protein Kinase C. Some of these kinases were known to play a role in prostate cancer. For example, RAC-Alpha Serine/Threonine-Protein Kinase (Protein Kinase B) is part of a central signaling pathway in prostate cancer (Lee et al., 2008); phosphorylation of the site that was found in this study (S473) regulates the activity of this kinase, and phosphorylation at S473 correlates with clinical outcome in prostate cancer (Kreisberg et al., 2004).

A number of other cancer-relevant proteins were identified. RNA-Binding Protein 5 is a tumor suppressor protein that promotes apoptosis and cell cycle arrest. Bcl-2Associated Transcription Factor 1 is a transcriptional repressor that promotes apoptosis. Transforming Acidic Coiled-Coil-Containing Protein 2 is a candidate breast tumor suppressor and biomarker for tumor progression. It may protect nonmalignant cells from tumorigenic conversion by promoting proper cellular organization and tissue morphogenesis (Chen et al., 2000). Programmed Cell Death Protein 4 (Pdcd4) is a novel tumor suppressor. The Pdcd4 gene was first identified as a differentially expressed mRNA when cells were treated with apoptosis inducers. Pdcd4 might suppress tumor progression by regulating the expression of the kinase MAP4K1 (Yang et al., 2006). 


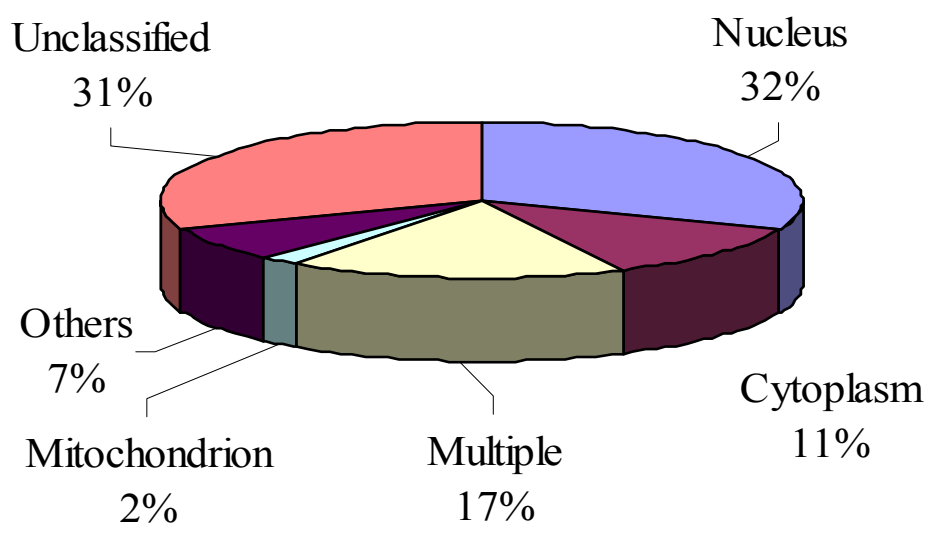

Figure 2-5 Summary of the sub-cellular locations of the LNCaP phosphoproteins characterized by in-gel IEF LC-MS/MS.

Note: The information was compiled from Swiss-Prot annotations. 


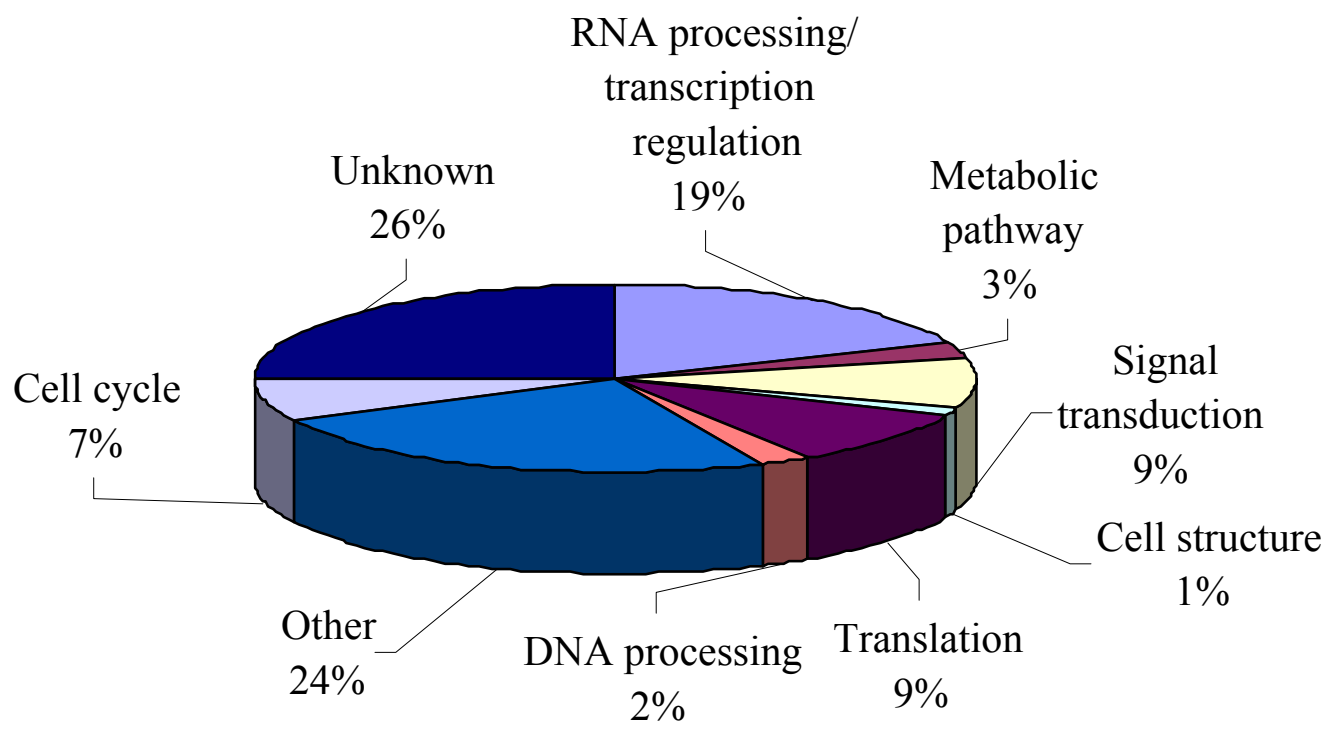

Figure 2-6 Classification of the characterized LNCaP phosphoproteins based on their involvement in cellular processes.

Note: This information was gathered based on Swiss-Prot annotations. 
Several members of the androgen receptor (AR) response pathway were characterized. Several phosphorylated sites in protein from the heat-shock protein (HSP) family were found, such as HSP86, HSPB1 and HSP84. HSPs are bound by the ligandfree AR which is sequestered in the cytoplasm. HSPs function as chaperones to stabilize the AR during folding, protecting it from degradation. When dihydrotestosterone (DHT) binds to the AR, inducing a conformation change, which allows dissociation of HSPs, AR then forms a homo-dimer and is phosphorylated at several sites (Edwards and Bartlett, 2005; Lee and Chang, 2003).

Phosphorylation sites were identified for mitogen-activated protein kinase (MAPK) Ser164, Ser331 and protein kinase C (PKC) Ser354. These kinases help AR sensitize low circulating level of DHT to induce phosphorylation at specific sites of AR and then translocate AR to the nucleus (Rochette-Egly, 2003). PKC is a phospholipiddependent cytoplasmic, serine/threonine kinase, which may be activated by TGF- $\beta$ or intercellular activators such as calcium and diacylglycerol (Lahn et al., 2004). There are PKC phosphorylation consensus sites on the AR at Ser81 and Ser650. This indicates that activating PKC signaling can affect AR phosphorylation.

We also identified proteins involved in Ras signaling: Ras GTPase-activating protein-binding protein 1 (G3BP-1), Ras GTPase-activating protein-binding protein 2 (G3BP-2), and Rab-GTPase. Ras subfamily proteins control signal transduction between the membrane and the nucleus, and activation of Ras-mediated signaling has been implicated in the progression of prostate cancer to androgen independence (Gioeli, 2005).

\subsection{Conclusions}

With in-gel IEF based analytical platform, we have characterized over 600 different phosphorylation sites in 296 phosphoproteins in the LNCaP prostate cancer cell line. This panel of the LNCaP phosphoproteins was 3-fold larger than the panel obtained in our previous work, and was the largest phosphoproteins panel in prostate cancer to date. The phosphoproteins identified in this study belonged to various locations within the cell and were involved in various processes including cell differentiation, transcription regulation, and intercellular signal transduction. The expanded phosphoproteome map obtained in this study was of a satisfactory size to serve as a foundation for future quantitative studies of the effects of pharmaceuticals on the LNCaP prostate cancer cells. 


\section{CHAPTER 3. INVESTIGATION OF PHOSPHOPROTEIN SIGNATURES IN ARCHIVED HUMAN PROSTATE CANCER TISSUES VIA PROTEOMIC ANALYSIS}

\subsection{Introduction}

Early stage detection and diagnosis of cancers decrease the mortality rate of these diseases. The prostate specific antigen (PSA) screen is a commonly used, serum based, biomarker test for the early detection of prostate cancer. It is well known that the PSA screen suffers for low specificity and poor sensitivity (Lin et al., 2007). Therefore, the discovery of new biomarkers of prostate cancer, featuring high specificity and sensitivity, would dramatically improve the survival rate of prostate cancer patients.

Because of its importance, the human prostate tissue has been a prime target of proteomics research efforts. To date, several studies have applied the proteomics approach to study the protein expression patterns in prostate cancer (Sardana et al., 2008b; Semmes et al., 2006; Solassol et al., 2005) as compared to healthy prostate tissues. Most of these studies had a common goal: the discovery of novel biomarkers, and all studies used mass spectrometry (MS) based platforms for their discovery efforts. As a result of these efforts, a large number of molecular markers have been identified; to date none of them has reached clinical use (Sardana et al., 2008a). It is thought that patterns revealed in a panel of proteins (known as a "protein signature") associated with a form of cancer might have better diagnostic and predictive capabilities than the current "one biomarker" approach.

An alternative approach to protein expression analysis for the discovery of disease biomarkers is the comprehensive characterization of protein phosphorylation. Protein phosphorylation is the most widespread signaling mechanism in eukaryotic cells, and it is involved in all fundamental cellular processes. Reversible phosphorylation forms the basis of cell signaling networks and phosphorylation-based signaling networks are key to the cell ability to quickly respond to external and internal environmental changes. The elucidation of the phosphoprotein networks in prostate cancer would therefore deepen our understanding of the mechanisms involved in its formation and progression, and it would improve the accuracy of early detection and it would speed up the discovery rate of targeted and more effective drugs.

We have already applied the gel-free platform to study phosphoproteins in LNCaP cells to obtain the first phosphoprotein networks in a prostate cancer cell line (Giorgianni et al., 2007). Since tissues based proteomic analyses are considered to be potentially more useful for relating protein status directly to diseases, the aim of this study was to apply the gel-free analytical platform to identify phosphoproteins on a global scale in archived prostate cancer tissues for biomarker discovery. An outline of the gel-free approach is shown in Figure 3-1. 


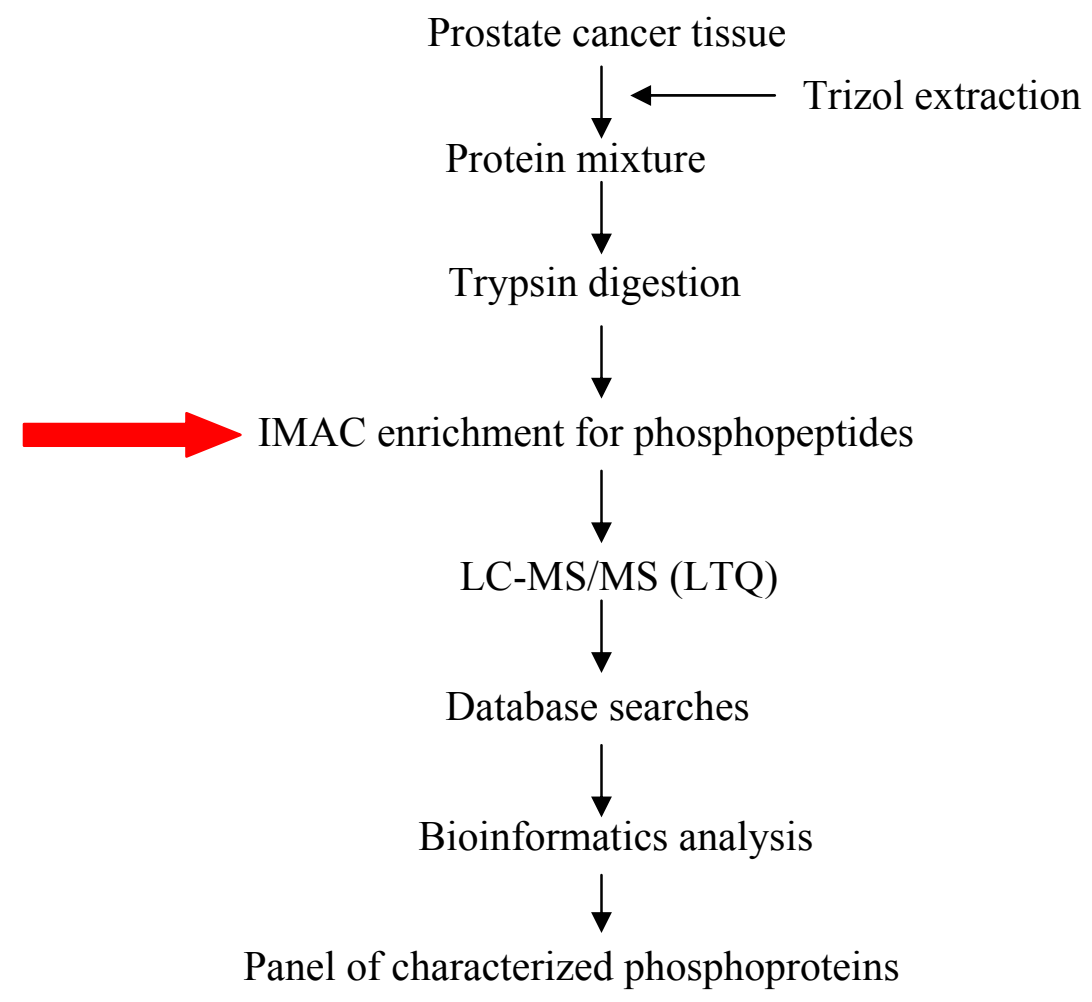

Figure 3-1 Outline of the gel-free analytical methodology.

Proteins from prostate cancer tissues were extracted with Trizol reagent, and then insolution digested with trypsin. The digests were subjected to IMAC to isolate phosphorylated peptides. Enriched peptides were analyzed with LC-MS/MS. The MS/MS datasets were used to identify the phosphorylated peptides and proteins via searches of a protein sequence database. Bioinformatics analyses were performed for the panel of characterized phosphoproteins. 


\subsection{Materials and Methods}

\subsubsection{Characteristics of Clinical Samples}

A total of 5 bulk prostate cancer specimens $(99-010,99-029,98-065,99-045,99-$ 067) have been used in the study. The specimens were obtained from the University of Tennessee Department of Urology Specimen and Tissue Bank. The research project was approved by the UTHSC IRB. The specimens were procured during radical prostatectomy, and were stored in liquid nitrogen until transfer from the Bank; afterwards, they were stored at $-80^{\circ} \mathrm{C}$ until processing. All specimens have been evaluated by a pathologist to confirm the presence of cancer, and to determine the Gleason score. The characteristics of specimens are summarized in Table 3-1.

\subsubsection{Protein Extraction}

Each prostate tumor tissue specimen (50-200 mg wet weight) was quickly minced with a scalpel and the pieces were suspended in $1.0 \mathrm{~mL}$ of Trizol reagent (Invitrogen, Carlsbad, CA) containing $5 \mu \mathrm{L}$ of phosphatase inhibitor cocktail (Sigma, St. Louis, MO). The samples were homogenized until full disruption of the tissue was achieved. The protein, free of nucleic acids, was extracted from each lysate using Trizol reagent according the manufacturer's instruction (details described in Chapter 2). Protein pellets were washed three times with $0.3 \mathrm{M}$ Guanidinium hydrochloride in $95 \%$ ethanol, followed by a single wash with ethanol. Proteins were dried under vacuum for $30 \mathrm{~min}$, and were resuspended in $50 \mathrm{mM}$ ammonium bicarbonate. The protein concentration of the extracts was determined by Bradford assay (GE Healthcare, Piscataway, NJ).

\subsubsection{In-solution Digestion of Proteins}

The extracted proteins (approximately $1 \mathrm{mg}$ ) were re-suspended in $50 \mathrm{mM}$ ammonium bicarbonate buffer $(\mathrm{pH} 8)$ through vigorous vortexing. To produce a homogeneous protein suspension, the samples were sonicated three times for $20 \mathrm{~s}$ each time, with $30 \mathrm{~s}$ intervals between the sonication. Sequencing-grade trypsin (Promega,

Table 3-1 Characteristics of prostate cancer specimens used in the study.

\begin{tabular}{llll}
\hline Specimen code & Donor age & Donor race & $\begin{array}{l}\text { Diagnosis (total } \\
\text { Gleason score) }\end{array}$ \\
\hline $99-010$ & 50 & African American & Cancer (5) \\
$99-029$ & 59 & African American & Cancer (7) \\
$98-065$ & 62 & African American & Cancer (6) \\
$99-045$ & 66 & Caucasian & Cancer (6) \\
$99-067$ & 67 & Caucasian & Cancer (6) \\
\hline
\end{tabular}


Madison, WI, USA) was added to the protein mixture at an enzyme-to-protein ratio of 1:60 (w/w). The digestion proceeded overnight at $37^{\circ} \mathrm{C}$ with gentle agitation. After 16 hours of digestion, undigested protein was visible in some of the samples. Therefore, additional trypsin was added to achieve complete digestion.

\subsubsection{Enrichment of Phosphopeptides with IMAC}

The tryptic digests were dried in a vacuum centrifuge, and re-dissolved in $50 \mu \mathrm{L}$ of $90 \%$ water $/ 10 \% \mathrm{AcOH}$. The resulting solution was applied to an IMAC spin-column (Phosphopeptide Isolation Kit; Pierce, Rockford, IL), and the phosphopeptides were bound by incubation at room temperature for $1 \mathrm{~h}$. The column was washed with the following solutions: $40 \mu \mathrm{L}$ of $0.1 \% \mathrm{AcOH}$ ( 2 washes), $40 \mu \mathrm{L}$ of $0.1 \% \mathrm{AcOH} / 10 \% \mathrm{ACN}$ ( 2 washes), and $40 \mu \mathrm{L}$ of water ( 2 washes). The phosphopeptides were eluted from the

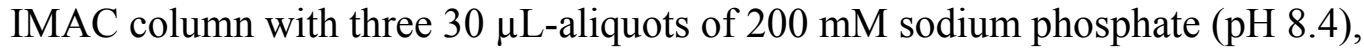
followed by a single elution with $30 \mu \mathrm{L}$ of $100 \mathrm{mM}$ sodium phosphate $/ 50 \% \mathrm{ACN}$. The eluates were combined and dried under vacuum. Prior to LC-MS/MS analysis, the IMAC-enriched phosphopeptides were reconstituted with $15 \mu \mathrm{L}$ of water $/ 2.5 \% \mathrm{FA}$ and desalted with ZipTipC18 (Millipore, Billerica, MA), using the procedure provided by the manufacturer. The phosphopeptides retained on the ZipTipC18 column were eluted with $3 \mu \mathrm{L}$ of $50 \% \mathrm{ACN} / 0.1 \% \mathrm{FA}$ and diluted with $6 \mu \mathrm{L}$ of $0.5 \% \mathrm{FA}$, then analyzed by LCMS/MS.

\subsubsection{LC-MS/MS}

LC-MS/MS experiments were performed on a LTQ linear ion trap mass spectrometer (Thermo Electron, San Jose, CA) coupled to a nanoflow HPLC system (Dionex, Sunnyvale, CA). Each sample was analyzed in triplicate. Peptide mixtures were loaded onto a fused-silica micro-capillary column/spray needle (Picofrit ${ }^{\mathrm{TM}}, 15 \mathrm{~cm}$ length, $75 \mu \mathrm{m}$ ID; New Objective, MA) packed in-house with C18 stationary phase (Michrom Bioresources, Inc., Auburn, CA). The peptides were separated using a 90-min linear gradient from $0 \%$ to $90 \%$ mobile phase B. Mobile phase B was $10 \%$ water $/ 90 \%$ $\mathrm{MeOH} / 0.05 \% \mathrm{FA}$; mobile phase A was $98 \%$ water $/ 2 \% \mathrm{MeOH} / 0.05 \% \mathrm{FA}$. The LTQ mass spectrometer was operated in positive ion mode and data-dependent acquisition was performed. In brief, a scan cycle was initiated with a full MS, which was followed by MS/MS scans on the 7 most abundant precursor ions.

\subsubsection{Database Searches}

The MS/MS data were used to search the Swiss-Prot database (subset of human proteins) with the TurboSEQUEST search engine that was part of Bioworks version 3.2 software suite (Thermo Electron). Searches were performed with the following parameters: full-trypsin specificity, static modifications of oxidized Met (+15.9949), and dynamic modifications of phosphorylated Ser, Thr, and Tyr (+79.9663). All peptide 
matches were filtered to include peptides retrieved with XCorr values $\geq 3.00,2.00$, and 3.50 for singly, doubly and triply charged ions, respectively. The MS/MS spectra of all phosphopeptide candidates that had passed this initial filtering were manually validated through inspection of the MS/MS spectra. This manual validation assessed the following: (1) whether a neutral-loss signal corresponding to $[\mathrm{M}+2 \mathrm{H}-98]^{2+}$ for doubly charged ions or $[\mathrm{M}+3 \mathrm{H}-98]^{3+}$ for triply charged ions was present in the spectrum; and (2) whether a good portion of the peptide sequence was covered by the b- and/or y product-ion series. The exact phosphorylation site assignment was validated by inspecting the $b$ - and/or $y-$ product ions that flanked the phosphorylation site assigned by software.

\subsubsection{Additional Bioinformatics Analysis}

Annotations of the Swiss-Prot database, and the PhosphoSite knowledgebase (www.phosphosite.org) were used to assess whether a particular site has been identified previously in prostate cancer tissue or in other biological systems. Gene Ontology (bioinfor.vanderbilt.edu/webgestalt) was used to classify identified proteins according to molecular function. The bioinformatics tool Scansite (scansite.mit.edu) was used to obtain information about potential kinase and/or phosphorylation-dependent binding motifs.

\subsection{Results}

In this study, a gel-free bioanalytical strategy was used to map the phosphoproteins in archived primary human prostate cancer tissue. One milligram of protein extracted from the tissue specimen was digested in-solution with trypsin. Generally, Trizol-extracted protein mixtures are denatured with $8 \mathrm{M}$ urea and then diluted to 1-2 $\mathrm{M}$ urea prior to trypsin digestion. This strategy is used to digest highly complex protein mixtures, particularly when sample availability is a major concern. However, the urea present in the resulting digest complicates subsequent analysis. A clean, more efficient method for in-solution digestion of protein mixture has been recently reported (Kim et al., 2006), and this method was used in the study reported here. In this method, the extracted proteins were suspended in ammonium bicarbonate and digested with trypsin. The resulting tryptic peptides were dried in a speed vacuum concentrator to remove solvent and volatile buffers. This digestion method eliminated the need for removal of salts, urea or detergents prior to the IMAC step, and thus minimized sample loss.

A group of five archived human prostate tissue specimens was analyzed to obtain initial data on the prostate cancer tissue phosphoproteome. Peptide digests from each specimen were subjected to IMAC to enrich for phosphopeptides, and the enriched digests were analyzed by LC-MS/MS with an LTQ linear ion trap instrument. The phosphopeptides present in each digest were identified through searches of the Swiss-Prot protein sequence database. The identifications were based on MS/MS data that provide information on the peptide sequence and on location of the phosphorylation site(s) in the 
peptide. The database search results were filtered to exclude low-scoring hits, and the phosphopeptides that passed the filters were validated through manual inspection of the corresponding MS/MS data. One of the features that provided supporting evidence for a phosphopeptide was the presence of a phosphate-diagnostic product ion in the MS/MS spectrum. An example of an MS/MS spectrum that illustrated this well-known phenomenon is shown in Figure 3-2. This spectrum displayed a prominent product ion at $\mathrm{m} / \mathrm{z} 812.7$, which corresponded to the loss of the elements of phosphoric acid from the doubly-charged precursor ion.

From each specimen, a number of phosphopeptides were detected; these phosphopeptides mapped to different phosphoproteins. A summary of the results is shown in Table 3-2. These results demonstrated that direct phosphoproteomics of archived tumor specimens was feasible. The size of the phosphoprotein panels ranged from 19 to 34 phosphoproteins encompassing between 25-45 different phosphorylation sites (Table 3-2). The phosphopeptides and sites found for each phosphoprotein are shown in Table 3-3. Additional details on the phosphopeptide/protein identification are provided in Table A-4. Based on an in silico analyses using the Scansite tool, some of the phosphorylation sites were part of known sequence motifs involved in kinase and/or phosphorylation-dependent binding interaction. Information on sites involved in such motifs is provided in Table A-5.

The phosphopeptide signatures varied from specimen to specimen. The overall distribution of the identified phosphopeptides is shown in Figure 3-3. Fifty-three percent of phosphopeptides were uniquely found in each specimen; $17 \%$ were common to all the specimens; $13 \%$ found in 4 out of 5 specimens and $17 \%$ in 2 or 3 out of 5 specimens. The results highlighted the heterogeneity of tissue samples and the need to investigate a larger specimen group. Nevertheless, a number of phosphoproteins were consistenly present in multiple specimens. Among the identified phosphoproteins, nine phosphoproteins were found in most of the specimens. These phosphoproteins included Caldesmone, Desmin, Heat Shock protein beta-6 (HSPB6), Heat Shock protein beta-1 (HSPB1), Synaptopodin2, Filamin-C, DnaJ homolog subfamily C member 5, Tensin-1, and Glyceraldehyde-3phosphate dehydrogenase. Eleven phosphoproteins were found in two or three out of five specimens, such as Myosin-11(MYH11), Septin-2, Desmocollin-1 precursor, Cysteine and glycine-rich protein 1, Transgelin, and Vimentin (Table 3-3).

The characterized phosphoproteins were grouped into functional categories based on GO annotation of the level 2 (Figure 3-4). The characterized phosphoproteins were involved in various cellular functions, such as binding, catalytic activity, enzyme regulator, signal transduction, cell structure, and transcription regulation.

\subsection{Discussion}

Advances in mass spectrometry-based technologies make it feasible to characterize phosphoproteins in complex mixtures, in a high-throughput, large-scale fashion. These technologies have been applied to investigations of cell cultures, tissues 


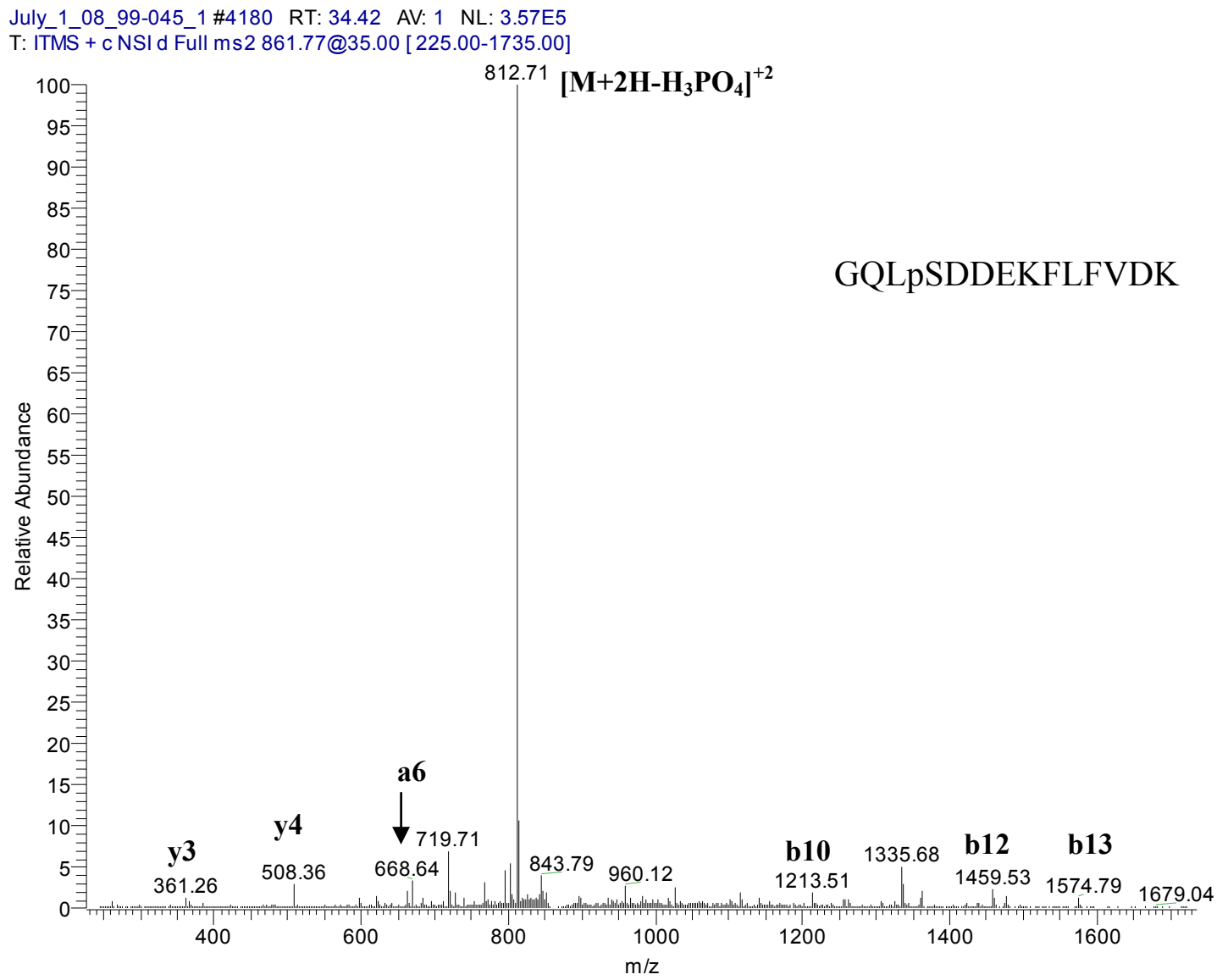

Figure 3-2 An example of MS/MS spectrum of GQLpSDDEKFLFVDK.

The spectrum contains a phosphate diagnostic product ion at $\mathrm{m} / \mathrm{z} 812$, and series of other product ions that determine the sequence of the peptide. Based on these data, the phosphopeptide with the sequence GQLpSDDEKFLFVDK $\left(\mathrm{m} / \mathrm{z}\right.$ of the $[\mathrm{M}+2 \mathrm{H}]^{2+}$ molecular ion is 861.4) was retrieved. The sequence mapped to protein Myosin-11 and included a phosphorylated serine at position 8 . 
Table 3-2 Summary of results for the analyses of the phosphoproteome in human prostate cancer tissue.

\begin{tabular}{llll}
\hline Specimen & $\begin{array}{l}\text { Number of } \\
\text { phosphorylation sites }\end{array}$ & $\begin{array}{l}\text { Number of } \\
\text { phosphopeptides }\end{array}$ & $\begin{array}{l}\text { Number of } \\
\text { phosphoproteins }\end{array}$ \\
\hline $99-010$ & 27 & 24 & 24 \\
$99-029$ & 27 & 24 & 19 \\
$98-065$ & 25 & 26 & 21 \\
$99-045$ & 45 & 40 & 34 \\
$99-067$ & 31 & 34 & 25 \\
\hline
\end{tabular}


Table 3-3 Phosphoproteins characterized in the prostate cancer specimens.

\begin{tabular}{|c|c|c|c|}
\hline $\begin{array}{l}\text { Accession } \\
\text { number }\end{array}$ & Protein & Peptide & Site \\
\hline \multicolumn{4}{|c|}{ Phosphoproteins found in all 5 specimens } \\
\hline \multirow[t]{2}{*}{ Q05682 } & Caldesmon & RGS*IGENQVEVMVEEK & S202 \\
\hline & & RGS*IGENQVEVM\#VEEK & S202 \\
\hline \multirow[t]{3}{*}{ P17661 } & Desmin & TFGGAPGFPLGSPLSS*PVFPR & S32 \\
\hline & & TFGGAPGFPLGS*PLSSPVFPR & S28 \\
\hline & & TFGGAPGFPLGS*PLSS*PVFPR & S32 \\
\hline \multirow[t]{3}{*}{ P04792 } & Heat-shock protein beta-1 & QLS*SGVSEIR & S82 \\
\hline & & GPS*WDPFR & S15 \\
\hline & & GPS*WDPFRDWYPHSR & S15 \\
\hline \multirow[t]{6}{*}{ Q9UMS6 } & Synaptopodin-2 & AQS*PTPSLPASWK & S902 \\
\hline & & AQS*PTPS*LPASWK & S906 \\
\hline & & TAKPFPGSVNQPATPFS*PTR & S604 \\
\hline & & PFPGSVNQPATPFS*PTR & S604 \\
\hline & & $\begin{array}{l}\text { GVSS*PIAGPAQPPPWPQPAPWSQPAF } \\
\text { YDSSER }\end{array}$ & S638 \\
\hline & & SKS*PDPDPNLSHDR & S226 \\
\hline \multicolumn{4}{|c|}{ Phosphoproteins found in 4 out of 5 specimens } \\
\hline O14558 & $\begin{array}{l}\text { Heat-shock protein beta- } 6 \\
\text { (Heat-shock } 20 \mathrm{kDa}-\text { like } \\
\text { protein) }\end{array}$ & RAS*APLPGLSAPGR & S16 \\
\hline Q14315 & Filamin-C & LGS*FGSITR & S2233 \\
\hline \multirow[t]{2}{*}{ Q9H3Z4 } & $\begin{array}{l}\text { DnaJ homolog subfamily } \\
\text { C member } 5\end{array}$ & S*LSTSGESLYHVLGLDK & S8 \\
\hline & & SLSTS*GESLYHVLGLDK & S12 \\
\hline \multirow[t]{6}{*}{ Q9HBL0 } & Tensin-1 & RMS*VGDR & S1393 \\
\hline & & WDS*YDNFSGHR & $\mathrm{S} 338$ \\
\hline & & EAT*SDPSRTPEEEPLNLEGLVAHR & T854 \\
\hline & & T*PTQPLLESGFR & T1105 \\
\hline & & EATSDPSRT*PEEEPLNLEGLVAHR & $\mathrm{T} 860$ \\
\hline & & SQS*FSEAEPQLPPAPVR & S621 \\
\hline P04406 & $\begin{array}{l}\text { Glyceraldehyde-3- } \\
\text { phosphate dehydrogenase }\end{array}$ & VIHDNFGIVEGLMTTVHAITAT*QK & $\mathrm{T} 182$ \\
\hline \multicolumn{4}{|c|}{ Phosphoproteins found in 3 out of 5 specimens } \\
\hline \multirow[t]{3}{*}{ P35749 } & Myosin-11 & GQLS*DDEKFLFVDK & S8 \\
\hline & & VIENADGS*EEETDTR & S1954 \\
\hline & & RVIENADGS*EEETDTR & S1954 \\
\hline
\end{tabular}


Table 3-3 (continued).

\begin{tabular}{|c|c|c|c|}
\hline $\begin{array}{l}\text { Accession } \\
\text { number }\end{array}$ & Protein & Peptide & Site \\
\hline \multicolumn{4}{|c|}{ Phosphoproteins found in 2 out of 5 specimens } \\
\hline Q15019 & Septin-2 & IYHLPDAES*DEDEDFKEQTR & S218 \\
\hline Q08554 & Desmocollin-1 precursor & MKVQDQDLPNT*PHSK & T385 \\
\hline P21291 & $\begin{array}{l}\text { Cysteine and glycine-rich } \\
\text { protein } 1\end{array}$ & GFGFGQGAGALVHS*E & S192 \\
\hline Q8N283 & $\begin{array}{l}\text { Ankyrin repeat domain- } \\
\text { containing protein } 35\end{array}$ & QS*VGLLT*NELAM\#EK & $\begin{array}{l}\text { S672; } \\
\text { T677 }\end{array}$ \\
\hline Q01995 & Transgelin & HVIGLQMGS*NR & S181 \\
\hline P08670 & Vimentin & ISLPLPNFS*SLNLR & S419 \\
\hline Q6P597 & Kinesin light chain 3 & APRTLSAST*QDLS*PH & $\begin{array}{l}\text { T493; } \\
\text { S497 }\end{array}$ \\
\hline O95425 & Supervillin & S*LSDFTGPPQLQALK & S547 \\
\hline Q15746 & Myosin light chain kinase & KSS*TGSPTSPLNAEK & S1773 \\
\hline Q63ZY3 & $\begin{array}{l}\text { Ankyrin repeat domain- } \\
\text { containing protein } 25\end{array}$ & ALAMPGRPES*PPVFR & S375 \\
\hline & & KIS*ITER & S406 \\
\hline \multicolumn{4}{|c|}{ Phosphoproteins found in 1 out of 5 specimens } \\
\hline Q92736 & Ryanodine receptor 2 & SSSENAKVTS*LDS*SSHR & $\begin{array}{l}\text { S4546; } \\
\text { S4549 }\end{array}$ \\
\hline P13796 & Plastin-2 & GS*VSDEEMMELR & S5 \\
\hline Q15283 & $\begin{array}{l}\text { Ras GTPase-activating } \\
\text { protein } 2\end{array}$ & S*SFKETFMCEFFK & S559 \\
\hline P43353 & $\begin{array}{l}\text { Aldehyde dehydrogenase } \\
\text { 3B1 }\end{array}$ & FDY*IFFTGS*PR & $\begin{array}{l}\text { Y183; } \\
\text { S189 }\end{array}$ \\
\hline P24844 & $\begin{array}{l}\text { Myosin regulatory light } \\
\text { chain } 2\end{array}$ & ATS*NVFAMFDQSQIQEFK & S20 \\
\hline P27816 & $\begin{array}{l}\text { Microtubule-associated } \\
\text { protein } 4\end{array}$ & DMES*PTKLDVTLAK & S280 \\
\hline P17252 & $\begin{array}{l}\text { Protein kinase } \mathrm{C} \text { alpha } \\
\text { type }\end{array}$ & STLNPQWNES*FTFK & S226 \\
\hline Q8IYX7 & $\begin{array}{l}\text { Uncharacterized protein } \\
\text { C9orf138 }\end{array}$ & EY*QKGPIPMEGLTTSR & Y59 \\
\hline O75069 & $\begin{array}{l}\text { Transmembrane and } \\
\text { coiled-coil domains } \\
\text { protein } 2\end{array}$ & GAS*LHS*SSGGGSS*GSSSRRTK & $\begin{array}{l}\text { S166; } \\
\text { S169 }\end{array}$ \\
\hline P27216 & Annexin A13 & YQKS*LSDMVRSDTSGDFR & S294 \\
\hline Q6PCE3 & $\begin{array}{l}\text { Phosphoglucomutase-2- } \\
\text { like } 1\end{array}$ & AVAGVMITAS*HNR & S175 \\
\hline P01854 & Ig epsilon chain $\mathrm{C}$ region & VAHTPS*STDWVDNK & S92 \\
\hline
\end{tabular}


Table 3-3 (continued).

\begin{tabular}{|c|c|c|c|}
\hline $\begin{array}{l}\text { Accession } \\
\text { number }\end{array}$ & Protein & Peptide & Site \\
\hline Q8TCU4 & $\begin{array}{l}\text { Alstrom syndrome protein } \\
1\end{array}$ & SPLQEAES*KVSMALEETLR & S2367 \\
\hline P80421 & $\begin{array}{l}\text { Ig heavy chain V-I region } \\
\text { DOT }\end{array}$ & DRLVMSSDTSANTVS*MQLR & S79 \\
\hline Q8TAP8 & $\begin{array}{l}\text { Putative uncharacterized } \\
\text { protein } C 7 \text { orf } 47\end{array}$ & S*SLALGLELR & S108 \\
\hline P36871 & Phosphoglucomutase-1 & AIGGIILTAS*HNPGGPNGDFGIK & $\mathrm{S} 117$ \\
\hline P60174 & Triosephosphate isomerase & KQS*LGELIGTLNAAK & S21 \\
\hline Q9NZN4 & $\begin{array}{l}\text { EH domain-containing } \\
\text { protein } 2\end{array}$ & GPDEAMEDGEEGS*DDEAEWVVTK & S438 \\
\hline P22059 & $\begin{array}{l}\text { Oxysterol-binding protein } \\
1\end{array}$ & $\begin{array}{l}\text { GDMS*DEDDENEFFDAPEIITMPENL } \\
\text { GHK }\end{array}$ & S351 \\
\hline Q32MZ4 & $\begin{array}{l}\text { Leucine-rich repeat } \\
\text { flightless-interacting } \\
\text { protein } 1\end{array}$ & RGS*GDTSISIDTEASIR & S120 \\
\hline Q9UBT6 & DNA polymerase kappa & CDS*YKDDLLLR & S10 \\
\hline Q9Y385 & $\begin{array}{l}\text { Ubiquitin-conjugating } \\
\text { enzyme E2 J1 }\end{array}$ & RLS*TSPDVIQGHQPR & S266 \\
\hline Q86SQ6 & $\begin{array}{l}\text { Probable G-protein } \\
\text { coupled receptor } 123\end{array}$ & QVT*KKAPLCLDTDQPPYPR & T833 \\
\hline Q9Y618 & $\begin{array}{l}\text { Nuclear receptor } \\
\text { corepressor } 2\end{array}$ & REGTPPPPPPS*R & S1390 \\
\hline Q9H2J4 & Phosducin-like protein 3 & LS*ES*GAIMTDLEENPK & $\begin{array}{l}\text { S199; } \\
\text { S201 }\end{array}$ \\
\hline P09496 & Clathrin light chain A & LQS*EPESIR & $\mathrm{S} 105$ \\
\hline O43237 & $\begin{array}{l}\text { Cytoplasmic dynein } 1 \text { light } \\
\text { intermediate chain } 2\end{array}$ & SGQKT*VLSNVQEELDR & $\mathrm{T} 463$ \\
\hline P35558 & $\begin{array}{l}\text { Phosphoenolpyruvate } \\
\text { carboxykinase }\end{array}$ & WM\#S*EEDFEK & S118 \\
\hline P05386 & $\begin{array}{l}\text { 60S acidic ribosomal } \\
\text { protein } \mathrm{P} 1\end{array}$ & KEES*EES*DDDMGFGLFD & $\begin{array}{l}\text { S101; } \\
\text { S104 }\end{array}$ \\
\hline P13861 & $\begin{array}{l}\text { cAMP-dependent protein } \\
\text { kinase type II-alpha } \\
\text { regulatory subunit }\end{array}$ & GDS*ES*EEDEDLEVPVPSR & $\begin{array}{l}\text { S77; } \\
\text { S79 }\end{array}$ \\
\hline Q6P9B9 & $\begin{array}{l}\text { Integrator complex subunit } \\
5\end{array}$ & FQAPSPS*TLLR & S1012 \\
\hline Q14195 & $\begin{array}{l}\text { Dihydropyrimidinase- } \\
\text { related protein } 3\end{array}$ & GSPT*RPNPPVR & T524 \\
\hline Q13618 & Cullin-3 & FLLES*FNNDRLFK & S359 \\
\hline P48681 & Nestin & S*LGEQDQMTLRPPEK & S767 \\
\hline Q9NSE4 & Isoleucyl-tRNA synthetase & GLVYRS*Y*KPVFWSPSSR & $\begin{array}{l}\text { S239; } \\
\text { Y2 } 240\end{array}$ \\
\hline O43432 & $\begin{array}{l}\text { Eukaryotic translation } \\
\text { initiation factor } 4 \text { gamma } 3\end{array}$ & SS*ASSLNR & S1194 \\
\hline P27824 & Calnexin precursor & AEEDEILNRS*PR & S583 \\
\hline
\end{tabular}


Table 3-3 (continued).

\begin{tabular}{|c|c|c|c|}
\hline $\begin{array}{l}\text { Accession } \\
\text { number }\end{array}$ & Protein & Peptide & Site \\
\hline Q96KP6 & $\begin{array}{l}\text { TNFAIP3-interacting } \\
\text { protein } 3\end{array}$ & CS*FSEDCLR & $\mathrm{S} 175$ \\
\hline P20711 & $\begin{array}{l}\text { Aromatic-L-amino-acid } \\
\text { decarboxylase }\end{array}$ & GLQAY*IR & Y377 \\
\hline O76041 & Nebulette & SMQHS*PNLR & S953 \\
\hline P08238 & $\begin{array}{l}\text { Heat shock protein HSP } 90- \\
\text { beta (HSP 90) }\end{array}$ & IEDVGS*DEEDDSGKDKK & S254 \\
\hline O75385 & $\begin{array}{l}\text { Serine/threonine-protein } \\
\text { kinase ULK1 }\end{array}$ & KM\#S*LGGGR & S495 \\
\hline Q9Y5C1 & $\begin{array}{l}\text { Angiopoietin-related } \\
\text { protein } 3 \text { precursor }\end{array}$ & NMSLELNS*K & $\mathrm{S} 122$ \\
\hline Q6KC79 & Nipped-B-like protein & QNEST*IVEPK & T667 \\
\hline P58107 & Epiplakin & QPLQAT*FRGLRKQVS*AR & $\begin{array}{l}\text { T1495, } \\
\text { S1504 }\end{array}$ \\
\hline P02545 & Lamin $\mathrm{A} / \mathrm{C}$ & LRLS*PSPTSQR & S390 \\
\hline Q13242 & $\begin{array}{l}\text { Splicing factor, } \\
\text { arginine/serine-rich } 9\end{array}$ & GS*PHYFSPFRPY & S211 \\
\hline Q13263 & $\begin{array}{l}\text { Transcription intermediary } \\
\text { factor 1-beta }\end{array}$ & SRS*GEGEVSGLMR & S473 \\
\hline P07197 & $\begin{array}{l}\text { Neurofilament triplet } \mathrm{M} \\
\text { protein }\end{array}$ & GKSPVPKS*PVEEK & S645 \\
\hline P15924 & Desmoplakin & GLPSPYNMSSAPGS $*$ R & S2825 \\
\hline O00264 & $\begin{array}{l}\text { Membrane associated } \\
\text { progesterone receptor } \\
\text { component } 1\end{array}$ & LLKEGEEPTVYS*DEEEPKDESAR & S180 \\
\hline
\end{tabular}




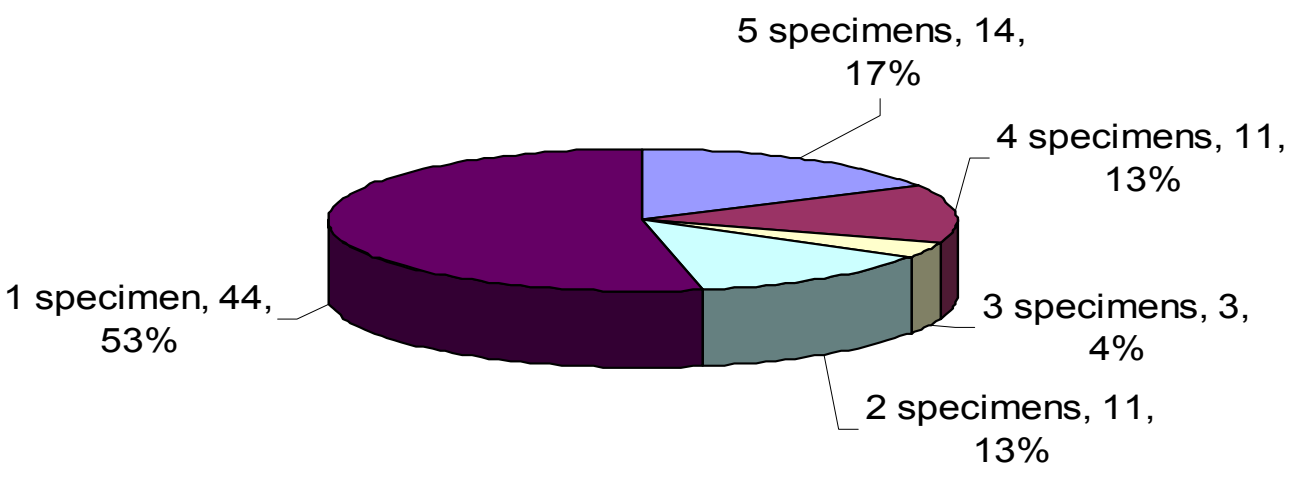

Figure 3-3 Distribution of identified phosphopeptides in five prostate tissues analyzed. 


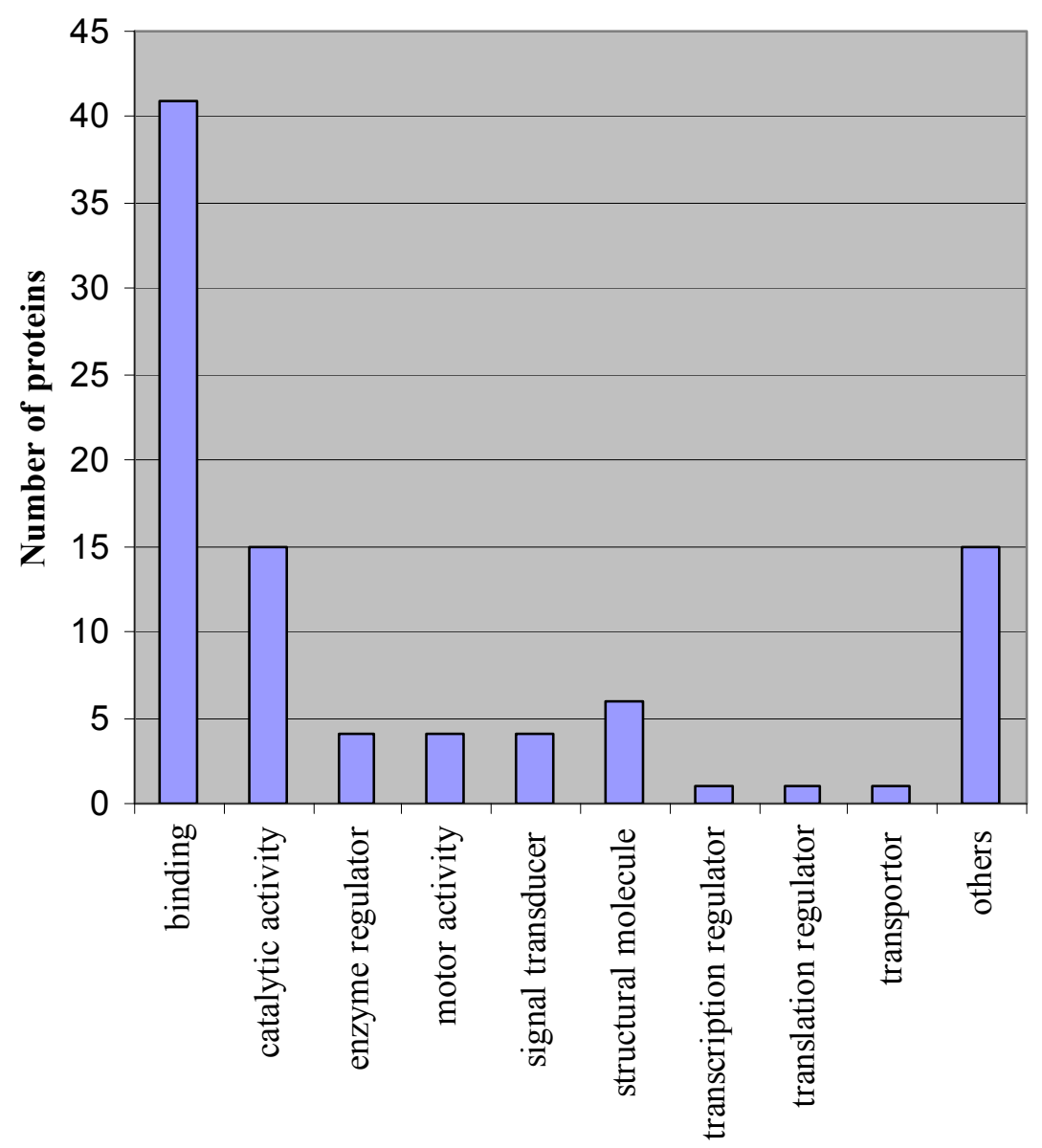

Figure 3-4 Classification of identified phosphoproteins by molecular function according to GO annotation at the level 2. 
and biological fluids to discover cancer biomarkers (Lin et al., 2007; Sardana et al., 2008b; Semmes et al., 2006). Extensive efforts were involved in exploring theapplication of proteomic strategy to study biomarkers in prostate cancer cell lines or tissues, such as 2-DE, 2D-DIGE, multiple dimensional chromatography (Johansson et al., 2006; Lin et al., 2007; Rowland et al., 2007; Sardana et al., 2007; Ummanni et al., 2008; Whitaker et al., 2007). The cell lines PC-3, DU 145, and LNCaP are spontaneously established from human prostate cancer metastases and are frequently used in prostate cancer research. However, these cell lines only partly mimic the biology of prostate cancer. Recently, increased efforts have been directed towards tissue-based proteomic analysis because it can directly reflect the relationship of proteins to diseases. A gel-free bioanalytical methodology has been used in our group to map the phosphoproteome in the LNCaP human prostate cancer cell line (Giorgianni et al., 2007). Recently, we have produced an expanded LNCaP phosphoproteome panel through the application of in-gel IEF LC$\mathrm{MS} / \mathrm{MS}$, which involves one-dimensional protein separation.

In this study, we employed a gel-free methodology to investigate phosphoprotein signatures in archived human prostate cancer tissues. Because of the limited size of a human tissue sample compared to that of cultured cells, it is critical to minimize the loss of sample during the various steps in the analytical workflow. In our previous study of cultured cells (LNCaP) phosphoproteome, we used guanidinium hydrochloride to aid protein solubilization prior to in-solution digestion. With this procedure, the peptide digest had to be subjected to solid phase extraction (SPE) cleanup with a C18 Sep-Pak cartridge before IMAC enrichment. For the present work on human prostate tissue, we sought to avoid this SPE step that may be associated with significant sample loss. Therefore, we adopted a modified protein digestion methodology to our study that was based on recently published report.

Genetic and environmental variability, together with sampling variations due to different cell types, results in the heterogeneity of human prostate cancer tissue specimens. A unique set of phosphoproteins was identified in each tissue specimen. Nevertheless, it was also demonstrated that a subset of the phosphoprotein panel was preserved across multiple specimens; $30 \%$ of the phosphopeptides were identified in $\geq 4$ out of the 5 specimens analyzed.

The characterized phosphoproteins were functionally diverse. Based on published literature, a number of these phosphoproteins were relevant to prostate cancer mechanism. Some of the identified phosphoproteins have previously been reported to be differentially regulated in tumors, including prostate tumors. For example, HSPB1, MYH11, VIME, DESM, TAGL, HSP90A and SEPT2 have been reported as differentially expressed in prostate cancer (Lin et al., 2007; Rowland et al., 2007).

Among the groups of phosphoproteins identified, there were several chaperones, including HSPB1 (HSP27), HSPB6 and HS90B. HSP27, which has been reported upregulated in prostate cancer (Rocchi et al., 2004), is involved in various cellular processes including apoptosis, cell growth and differentiation, actin reorganization and polypeptide renaturation. Cell proliferative activity in prostate cancer specimens was significantly 
associated with HSP27 expression (Kurahashi et al., 2007). HSP 27 is involved in cooperative interaction with androgen receptor (AR), which enhances AR stability, shuttling, and transcriptional activity, thereby increasing prostate cancer cell survival. Androgen-bound AR induces rapid HSP27 phosphorylation on Ser (78) and Ser (82) residues. Phosphorylated HSP27 displaces HSP90 from a complex with AR to chaperone AR into the nucleus and interact with its response elements to enhance its genomic activity. Inhibition of HSP27 phosphorylation, or knockdown using the antisense drug, shifted the association of AR with HSP90 to MDM2, increased AR degradation, decreased AR transcriptional activity, and increased prostate cancer LNCaP cell apoptotic rates (Zoubeidi et al., 2007). Previous study showed increased HSP27 expression was linked with hormone resistance and a poor prostate cancer prognosis (Miyake et al., 2006). HSP27 expression was also highly up-regulated in prostate cancer cells after androgen withdrawal or chemotherapy, to become uniformly highly expressed in androgen-independent prostate cancer. Silencing of HSP27 in prostate cancer cells by siRNA increased apoptotic rates and caused inhibition of cell growth in LNCaP and PC-3 cells (Rocchi et al., 2006). These findings suggested the expression level of HSP27 in prostate cancer tissue could be used as a useful predictor of prostate cancer, and also illustrated the potential utility of HSP27 as future targeted therapy in enhancing the efficacy of chemotherapy in advanced prostate cancer.

Tensin was another important protein found in prostate tissues, which is one of components of phosphatase and tensin homologue deleted on chromosome 10 (PTEN). PTEN has been shown to be inactivated in a wide variety of cancers, and the role of this gene as a tumor suppressor has been well established. PTEN has been found to work as a predictor of prostate cancer in combination with other proteins. Differentiation related gene 1(Drg-1) gene, one of the potential targets of PTEN, has been shown to suppress tumor metastasis in animal models of prostate and colon cancer, and the expression of this gene was significantly reduced with advancement of prostate and breast cancers in clinical setting. PTEN controls Drg-1 by an Akt-dependent pathway, overexpression of PTEN significantly augments the endogenous expression of Drg-1 protein. Combination of the two markers, PTEN and Drg-1, emerged as a significantly better predictor of prostate and breast cancer patient survival than either marker alone (Bandyopadhyay et al., 2004). PTEN, in combination with pAkt, was a better predictor of the risk of biochemical recurrence compared with pAkt alone. Loss of PTEN expression, together with increased Akt phosphorylation and Gleason score, was of significant predictive value for determining, at the time of prostatectomy, the risk of biochemical recurrence (Bedolla et al., 2007). PTEN was found to strongly inhibit the growth of human prostate tumors, especially when combined with radiation therapy, and this effect was mediated by the induction of apoptosis and by the inhibition of angiogenesis and cellular proliferation (Anai et al., 2006). PTEN/phosphatidylinositol 3-kinase (PI3K)/AKT constitute an important pathway regulating the signaling of multiple biological processes such as apoptosis, metabolism, cell proliferation and cell growth. Tissue-specific deletions of PTEN usually provoke cancer. Moreover, an absence of PTEN cooperated with an absence of p53 to promote cancer (Blanco-Aparicio et al., 2007).

A number of filament proteins (desmin, vimentin and caldesmon) were identified 
in our study. Intermediate filaments (IFs) are an important structural feature of eukaryotic cells. They, along with microtubules and actin microfilaments, make up the cytoskeleton. Based on current knowledge, a main function of IFs is to provide protection against various mechanical or other types of stresses. IFs are also involved in maintaining cell shape, and providing flexible scaffold, which undergoes disassembly and reorganization during certain cellular processes including cell division, migration and shape changes in response to their alterations in the microenvironment (Coulombe and Wong, 2004). IFs in smooth muscle are formed by polymerization of the proteins vimentin and desmin and constitute one of the major components of the cytoskeleton (Chang and Goldman, 2004).

Desmin is class-III intermediate filament found in muscle cells. Desmin is important in muscle cell architecture and structure since it connects many components of the cytoplasm. It has been shown that desmin phosphorylation occurred in cells/tissues in response to biological signal/treatment. It is possible that desmin phosphorylation may influence the temporal and spatial array of structural proteins or signal partners in smooth muscle (Tang, 2008).

Vimentin is also a member of class-III intermediate filament family of proteins. Vimentin has been found to be attached to the nucleus, endoplasmic reticulum, and mitochondria, either laterally or terminally. Therefore, vimentin played a significant role in supporting and anchoring the position of the organelles in the cytosol. Vimentin phosphorylation was also found to be associated with its disassembly and spatial reorganization in smooth muscle cells and tissues during agonist activation (Li et al., 2006). Vimentin has been shown to affect motility and invasiveness of prostate cancer cells. A low level of vimentin was expressed in the less invasive cell line, $\mathrm{LNCaP}$, in contrast to the higher level in more aggressive cell lines, DU-145 and PC3 (Mitchell et al., 2000). Downregulation of vimentin expression in PC-3 cells led to a significant decrease in tumor cells motility and invasive activity (Wei et al., 2008; Zhao et al., 2008).

Caldesmon (CaD), a component of microfilaments in all cells and thin filaments in smooth muscle cells, is known to bind to actin, tropomyosin, calmodulin, and myosin and to inhibit actin-activated ATP hydrolysis by smooth muscle myosin. Thus, it is believed to regulate smooth muscle contraction, cell motility and the cytoskeletal structure. $\mathrm{CaD}$ has been found to bind to vimentin and desmin proteins. Assembly of vimentin and desmin into IF was also affected by $\mathrm{CaD}$ silencing, although their expression was not significantly altered when $\mathrm{CaD}$ was silenced. It indicated that $\mathrm{CaD}$ was necessary for the maintenance of actin microfilaments and IF in the cytoskeletal structure. Thus, the alternation of $\mathrm{CaD}$ expression might affect the cytoskeletal structure in smooth muscle, as in smooth muscle de-differentiation and in certain pathological conditions (Deng et al., 2007).

\subsection{Conclusions}

In this study, a gel-free approach was applied to characterize phosphoproteins of archived primary human prostate cancer tissue for biomarker discovery. The panels 
obtained for human prostate cancer tissue contained 15-24 phosphoproteins. Some of the characterized phosphoproteins were present in all specimens; in addition, each specimen also produced a unique set of phosphoproteins. The findings provided a direct glimpse into the phosphoprotein machinery operating within the human prostate cancer tissue and demonstrated that phosphoproteins can be characterized directly from archived tissue specimens. 


\section{CHAPTER 4. IDENTIFICATION OF PHOSPHOPROTEINS IN THE LNCAP HUMAN PROSTATE CANCER CELL LINE BY A 2-DE AND PHOSPHO-SPECIFIC STAINING-BASED PROTEOMICS PLATFORM}

\subsection{Introduction}

Two-dimensional polyacrylamide gel electrophoresis (2-DE) is one of the most commonly used protein separation techniques (Gorg et al., 2000). It can separate thousands of proteins in a specific cell, tissue or biological fluid. It has high resolution and high reproducibility. 2-DE coupled with mass spectrometry (MS) and database searches is a widely used platform in proteomics studies (Gorg et al., 2004). 2-DE combined with MS is able to get a rapid overview of proteomic alterations in different biological systems or physiological states. Combination with multiplexed protein staining makes it possible to investigate various post-translationally modified proteins by 2-DE (Wu et al., 2005).

Pro- $\mathrm{Q}^{\mathrm{TM}}$ Diamond is a fluorescent stain specific for detection of phosphoproteins (Schulenberg et al., 2004; Steinberg et al., 2003). Pro- $\mathrm{Q}^{\mathrm{TM}}$ Diamond can be used to detect phosphorylated serine, tyrosine, or threonine residues. It has been reported that Pro-Q ${ }^{\mathrm{TM}}$ Diamond, combined with SDS-PAGE or 2-DE, was applied to efficiently detect phosphoproteins and to quantitatively measure phosphoprotein expression in various biological systems, such as spleen leukocytes (Wu et al., 2005), eye lenses (Schaefer et al., 2006), dentate gyrus (Chardonnet et al., 2008), heart muscle (Jacques et al., 2008), skeletal muscle (Gannon et al., 2008) et al.

The sub-aims of our study were: (1) to develop a platform for the identification of LNCaP phosphoproteins. This sub-aim included incorporation of multiplexed staining, and evaluation of selected technical aspects. (2) to apply the platform to identify selected phosphoproteins from the LNCaP phosphoproteome. In this study, we applied 2-DE as separation technique, Pro- $\mathrm{Q}^{\mathrm{TM}}$ Diamond as detection method, LC-MS/MS and database searches as protein identification tools to investigate the phosphoproteins in the LNCaP prostate cancer cell line. An outline of the 2-DE with phsopho-specific staining strategy is shown in Figure 4-1.This approach did not require the pre-selection of protein candidates for analysis, rapidly generated a snapshot of the phosphoproteome in the LNCaP cells, and it was particularly useful for an initial screening of the phosphoproteome to evaluate the phosphoproteome coverage for further study of the expression of phosphoproteins in various physiological states. 
LNCaP cells
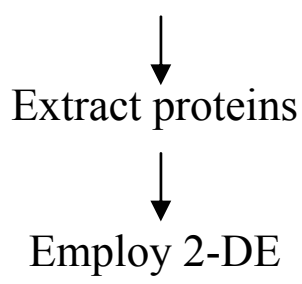

Stain gels with Pro-Q ${ }^{\mathrm{TM}}$ Diamond and SYPRO Ruby

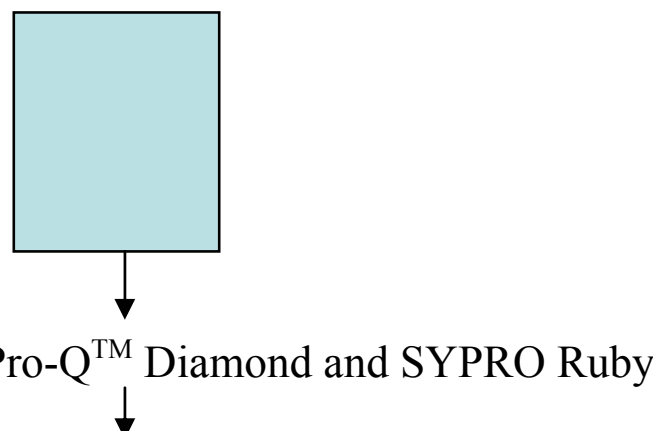

Analyze gel images, select interest spots

Excise spots, digest with trypsin and analyze by LC-MS/MS

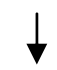

Perform database searches and bioinformatics examination

Figure 4-1 Schematic of 2-DE and phospho-specific staining based proteomics platform.

The LNCaP proteins were extracted with Trizol reagent. Proteins were separated with 2DE. Gels were sequentially stained with Pro- $\mathrm{Q}^{\mathrm{TM}}$ Diamond and SYPRO Ruby staining. Gel images were computer-assisted analyzed. The spots of interest were excised and digested with trypsin. The digests were analyzed with LC-MS/MS. Proteins were identified through database searches and bioinformatics examination of the identified proteins was performed. 


\subsection{Materials and Methods}

\subsubsection{Protein Extraction from LNCaP Cells}

A cell pellet containing $5 \times 10^{6} \mathrm{LNCaP}$ cells was resuspended in $1.0 \mathrm{~mL}$ of Trizol reagent (Invitrogen, Carlsbad, CA) with $5 \mu \mathrm{L}$ of phosphatase inhibitor cocktail (Sigma, St. Louis, MO). The cells were lysed thoroughly by repetitive pipetting. Approximately 1.0 $\mathrm{mg}$ of protein, free of nucleic acids, was extracted from this cell lysate using Trizol reagent according to the manufacturer's instructions (details described in Chapter 2). Protein pellets were washed twice with $0.3 \mathrm{M}$ Guanidinium hydrochloride in $95 \%$ ethanol, followed by a single wash with ethanol. The protein pellet was dried with a vacuum centrifuge for 30 minutes, and was stored at $-80{ }^{\circ} \mathrm{C}$ for further analysis.

\subsubsection{Sample Preparation}

\subsubsection{Standard Rehydration Buffer}

For 2-DE separations, the protein pellet was dissolved with a rehydration buffer that contained urea $(7 \mathrm{M})$, thiourea $(2 \mathrm{M})$, CHAPS detergent $(2 \% \mathrm{w} / \mathrm{v})$, IPG buffer $3-10$ $(2 \% \mathrm{v} / \mathrm{v})$, DTT $(0.3 \%, \mathrm{w} / \mathrm{v})$ and a trace amount of Bromophenol Blue dye. The protein concentration of the extracts was determined by Bradford assay (GE Healthcare, Piscataway, NJ). The final protein concentration in the rehydration buffer was $0.61 \mu \mathrm{g} / \mu \mathrm{L}$. The mixture was vortexed for 1 hour at room temperature. Following solubilization, the sample was centrifuged for 30 minutes at $21,000 \mathrm{x}$ g. Protein sample $(215 \mu \mathrm{g}$ in $350 \mu \mathrm{L}$ of rehydration buffer) was loaded onto a pre-cast immobilized $\mathrm{pH}$ gradient (IPG) strip (pH 3-10NL, $18 \mathrm{~cm}$; GE Healthcare, Piscataway, NJ), and the strip was allowed to rehydrate overnight in a re-swelling tray or a strip holder.

\subsubsection{Destreak Rehydration Buffer}

For 2-DE separations, the protein pellet was dissolved with a DeStreak rehydration solution(Olsson et al., 2002) (GE Healthcare, Piscataway, NJ) with IPG buffer 3-10 (2\% v/v), DTT $(20 \mathrm{mM})$ and a trace amount of Bromophenol Blue dye. Protein concentration was determined by 2-D Quant Kit (GE Healthcare, Piscataway, NJ). The resulting solution $(200 \mu \mathrm{g}$ of proteins in $350 \mu \mathrm{L}$ of the rehydration buffer) was vortexed for 1 hour at room temperature, followed by the centrifugation for 30 minutes at $21,000 \mathrm{x}$. The supernatant was loaded onto a pre-cast IPG strip (pH 3-10NL, $18 \mathrm{~cm}$ ), and the strip was allowed to rehydrate overnight in a re-swelling tray. 


\subsubsection{First Dimension: Isoelectric Focusing}

\subsubsection{IEF with Multiphor Unit}

Isoelectric focusing was performed with a Multiphor II apparatus (GE Healthcare, Piscataway, NJ) according to manufacturer's instructions (details described in Chapter 2). The isoelectric focusing was performed under the following voltage program: $100 \mathrm{~V}$ (gradient over 1 minute); $100 \mathrm{~V}$ (fixed for 2 hours); $500 \mathrm{~V}$ (gradient over 1 minute); 3500 $\mathrm{V}$ (gradient over 90 minutes); $3500 \mathrm{~V}$ (fixed for 8 hours). After the focusing, the strip was wrapped loosely in plastic wrap and was stored at $-80^{\circ} \mathrm{C}$ until the next step.

\subsubsection{IEF with IPGphor Unit}

Isoelectric focusing was performed with the IPGphor apparatus (GE Healthcare, Piscataway, NJ) according to manufacturer's instructions. Briefly, the strip holder was chosen, and $350 \mu \mathrm{L}$ of rehydration solution was applied into the holder. IPG strip was positioned in the holder with the gel side down and without bubbles, making sure that the IPG strip was in contact with the strip holder electrodes at each end. Cover fluid was applied to IPG strip and the cover was placed on the holder. The strip holder was positioned on the IPGphor platform with the two external electrodes of the instrument making contact with the electrodes of the strip holder. The isoelectric focusing was performed under the following voltage program: $30 \mathrm{~V}$ (fixed for 16 hours); $200 \mathrm{~V}$ (fixed for 1 hour); $500 \mathrm{~V}$ (fixed for 1 hour); $1000 \mathrm{~V}$ (fixed for 1 hour); $8000 \mathrm{~V}$ (gradient over 30 minutes); $8000 \mathrm{~V}$ (fixed for 4 hours ). After the focusing, the strip was wrapped loosely in plastic wrap and was stored at $-80{ }^{\circ} \mathrm{C}$ until the next step.

\subsubsection{Second Dimension: SDS-PAGE}

\subsubsection{Gel Casting}

To prepare eight of $12 \%, 20 \times 20 \mathrm{~cm}$ SDS-PAGE gels, gel plates and plastic pads were assembled sequentially into the casting chamber placed on a flat surface. The casting chamber was clamped and the plates were leveled on the bottom. The casting chamber was put on the stand. All reagents were combined, including $120 \mathrm{~mL}$ of $40 \%$ acrylamide/bis, $100 \mathrm{~mL}$ of $1.5 \mathrm{M}$ Tris- $\mathrm{HCl}(\mathrm{pH} 8.8)$ and $180 \mathrm{~mL}$ distilled water and degassed for 15 minutes. When ready to pour the gel, the reagents of $2 \mathrm{~mL}$ of $10 \%$ ammonium persulfate and $100 \mu \mathrm{L}$ TEMED were quickly added and gently mixed. The gel solution was transferred between the glass plates in the casting chamber to about $3 / 4$ inch below the short plate. A small layer of butanol was added on the top of the gel prior to polymerization to straighten the level of the gel and remove unwanted air bubbles that may be present. Once the gel polymerized, the butanol was removed by rinsing the top layer of the gel with distilled water prior to the use. 


\subsubsection{SDS-PAGE with Dodeca Cell Unit}

SDS-PAGE was performed with a ProteanPlus Dodeca Cell apparatus (Bio-Rad, Hercules, CA). The IPG strip was incubated for 15 minutes in an equilibration buffer (50 $\mathrm{mM}$ Tris/ $\mathrm{HCl} \mathrm{pH} 8.8,6 \mathrm{M}$ urea, 30\% glycerol, $2 \% \mathrm{SDS}$ ) with $2.0 \%$ DTT and then in equilibration buffer with $2.5 \%$ iodoacetamide and a trace amount of bromophenol blue dye for an additional 15 minutes. The strip and a sample application piece with the Marker $12 \mathrm{MW}$ maker (Invitrogen, Carisbad, CA) were put on the top of the self-cast $12 \%, 20 \times 20 \mathrm{~cm}$ SDS-PAGE gel and were sealed with 1\% agarose in gel running buffer, which contained $25 \mathrm{mM}$ Tris (pH 8.3), $192 \mathrm{mM}$ glycine and 0.1\% SDS. The SDS-PAGE separation was performed with according to the manufacturer's instructions. Briefly, the temperature setting for the electrophoresis was $15^{\circ} \mathrm{C}$, the voltage applied was $200 \mathrm{~V}$ constant and the electrophoresis time was ca. 8 hours. The SDS-PAGE separation was completed when the Bromophenol Blue dye front migrated to the bottom of the gel.

\subsubsection{Gel Staining}

\subsubsection{Pro- $Q^{T M}$ Diamond Staining}

A series of stains was applied to each 2-DE gel, which was compatible with subsequent mass spectrometric analysis. Briefly, 2-DE gel was fixed in $200 \mathrm{~mL}$ of fixation solution $(50 \% \mathrm{MeOH} / 10 \% \mathrm{AcOH} / 40 \%$ water) for 30 minutes or overnight. The gel was washed three times with distilled water with gentle agitation for 15 minutes each. The gel was incubated in dark with $170 \sim 190 \mathrm{~mL}$ of Pro-Q ${ }^{\mathrm{TM}}$ Diamond phosphoprotein gel stain (Molecular Probes, Eugene, OR) with gentle agitation for 2.5 hours. The gel was incubated with $190 \mathrm{~mL}$ of Pro-Q ${ }^{\mathrm{TM}}$ Diamond phosphoprotein gel destaining solution (Molecular Probes, Eugene, OR) for 2 hours with gentle agitation, followed by two washes with distilled water for 5 minutes each.

\subsubsection{SYPRO Ruby Staining}

After detection of phosphoproteins, the gel was stained in SYPRO Ruby protein stain (Molecular Probes, Eugene, OR). 2-DE gel was rinsed twice with distilled water for 15 minutes each, followed by incubation in $170 \mathrm{~mL}$ of SYPRO Ruby staining solution for 3 hours to overnight in the dark with gentle agitation. After incubation of the staining, the gel was incubated in $200 \mathrm{~mL}$ of $10 \%$ of $\mathrm{MeOH} / 7 \%$ of $\mathrm{AcOH} / 83 \%$ water for 30 minutes. The gel was rinsed twice with distilled water for 5 minutes each.

\subsubsection{Gel Imaging}

The images were acquired with an FX Fluorescence Laser Scanner (Bio-Rad, Hercules, CA). For Pro- ${ }^{\mathrm{TM}}$ Diamond stain, the laser was used with $532 \mathrm{~nm}$ excitation and $555 \mathrm{~nm}$ band pass emission; for SYPRO Ruby stain, proteins were detected using the laser scanner with the excitation $488 \mathrm{~nm}$ and $555 \mathrm{~nm}$ band pass emission. The images 
were displayed with Adobe Photoshop 5.0 LE software, and saved as .TIFF files for future analysis. The Pro- $\mathrm{Q}^{\mathrm{TM}}$ Diamond and SYPRO Ruby images were analyzed using the software PDQuest 7.1.0 (Bio-Rad, Hercules, CA).

\subsubsection{Spot Excision}

Spots in 2-DE gels were excised with cut-to-size $1 \mathrm{~mL}$ pipette tips, and the gel pieces were put into $1.5 \mathrm{~mL}$ siliconized Eppendorf microcentrifuge tubes.

\subsubsection{In-gel Tryptic Digestion}

The gel pieces were incubated in $50 \mathrm{mM}$ ammonium bicarbonate for 10 minutes. After the incubation, the buffer was removed and the gel pieces were washed three times with $50 \%$ ACN $/ 50 \% 50 \mathrm{mM}$ ammonium bicarbonate for 10 minutes each. Gel pieces were dried under vacuum for 30 minutes. The dried gel pieces were rehydrated in a trypsin solution that contained $16.7 \mathrm{ng} / \mu \mathrm{L}$ trypsin (Promega, Madison, WI) in $50 \mathrm{mM}$ ammonium bicarbonate $(\mathrm{pH} 8)$. The digestion solution was incubated at $37^{\circ} \mathrm{C}$ overnight.

\subsubsection{Peptide Processing}

The tryptic digest was centrifuged at $1,000 \mathrm{x}$ g for 1 minute, and the supernatant was transferred to a new $0.6 \mathrm{~mL}$ siliconized microcentrifuge tube. The gel piece was extracted with $60 \%$ ACN $/ 0.01 \%$ TFA three times for 15 minutes each with gentle agitation or sonication. All supernatants were combined and dried with a vacuum centrifuge. Prior to LC-MS/MS analysis, the digest was reconstituted with $15 \mu \mathrm{L}$ of $2.5 \%$ FA/97.5\% water and desalted with ZipTipC18 column (Millipore, Billerica, MA, USA), using the procedure provided by the manufacturer (details described in Chapter 2). The peptides retained on the ZipTipC18 column were eluted with $1.5 \mu \mathrm{L}$ of $50 \% \mathrm{ACN} / 0.1 \%$ FA and diluted with $2.5 \mu \mathrm{L}$ of $0.5 \%$ FA.

\subsubsection{LC-MS/MS Analysis}

LC-MS/MS experiments were performed on a LTQ linear ion trap mass spectrometer (Thermo Electron, San Jose, CA). Peptide mixtures were loaded onto a fused-silica microcapillary column (15 cm length, $75 \mu \mathrm{m}$ ID; New Objective, MA) packed in-house with $\mathrm{C} 18$ resin (Michrom Bioresources, Inc., Auburn, CA) and were separated using a 30-minute linear gradient from $0 \%$ to $90 \%$ solvent $\mathrm{B}(0.05 \% \mathrm{FA} / 90 \%$ $\mathrm{MeOH} / 10 \%$ water). Solvent A was $0.05 \% \mathrm{FA} / 2 \% \mathrm{MeOH} / 98 \%$ water. The LTQ mass spectrometer was operated in positive ion mode and data-dependent acquisition was performed. In brief, a scan cycle was initiated with a full MS scan of wide mass range (m/z 400-2000), which was followed by MS/MS scans on the 7 most abundant precursor ions with dynamic exclusion of previously selected ions. 


\subsubsection{Database Searches}

The MS/MS data were used to search the Swiss-Prot database (subset of human proteins) with the TurboSEQUEST search engine that is part of Bioworks version 3.2 (Thermo Electron, San Jose, CA). Searches were performed with the following parameters: full-trypsin specificity, dynamic modifications of phosphorylated Ser, Thr, and Tyr; dynamic modifications of oxidized methionine, and static modifications of cysteine by carbamidomethylation. All peptide matches were filtered by XCorr larger than 3.00, 2.50, and 3.50 used for singly, doubly and triply charged ions, respectively; peptide probability less than 1.0E-3; different peptides; and only top matches were considered. The peptides retrieved by the searches were validated by manual inspection of the MS/MS data. Criteria used for manual validation included: a high-quality spectrum with product ions clearly above the baseline noise; and sequential members of the band/or $y$-series present in the mass spectra.

\subsubsection{Inspection of Protein Information}

The experimental $\mathrm{pI}$ was estimated according to the $\mathrm{pH}$ gradient chart provided by the manufacturer (GE Healthcare, Piscataway, NJ) based on the relative migration of each protein spot.

A series of protein markers with known molecular weights were used to determine the experimental molecular weight of unknown proteins by comparing the migration distance relative to the markers. The relative migration $(\mathrm{Rm})$ value of protein spots was determined by dividing the total distance they migrated by the distance migrated by the bromphenol blue dye. A straight reference line was plotted, using the $\mathrm{Rm}$ of each MW marker spot and the logarithm of that molecular weight. The relative molecular weight of each protein spot was calculated from that equation.

The theoretical $\mathrm{pI}$ and MW were computed with Swiss-Prot Compute pI/MW tool for each UniProt Knowledgebase entry. The status of protein phosphorylation was inspected in the Swiss-Prot annotations and the PhosphoSite knowledgebase. Information of protein function and subcellualr location was collected from Gene Ontology (http://bioinfo.vanderbilt.edu/webgestalt/) and Swiss-Prot annotation.

\subsection{Results}

\subsubsection{Application of Different Rehydration Buffers: Standard vs. Destreak}

In order to address the issue of streaking in the basic region of the $\mathrm{pH}$ gradient (pH 7-9), we employed the Destreak Rehydration solution in the sample preparation for the first dimension. Destreak Rehydration solution has been reported to transform the 
protein thiol groups into a stable disulfides and hence to protect the disulfide groups from unspecific oxidation (Olsson et al., 2002).

We performed side-by-side separations of LNCaP proteins that were solubilized with standard rehydration buffer or with Destreak Rehydration solution. Comparison of 2-DE gel images of samples using standard rehydration buffer (Figure 4-2a) or Destreak rehydration solution (Figure 4-2b) showed that the protein spot pattern of Destreaksolubilized sample has somewhat improved compared to the patterns obtained using standard rehydration buffer; however, the streaking was not totally eliminated. Therefore, we opted to use IPG strips with $\mathrm{pH}$ range of 4-7 for subsequent experiments.

\subsubsection{IPGphor vs. Multiphor}

As the first dimension of 2-DE, the quality of IEF is critical for the overall success of 2-DE separation. Different configurations of IPG-based IEF equipment are commercially available. The IPGphor and Multiphor II are two widely used systems for IEF.

In the method development phase of our study, we assessed the 2-DE spot patterns of $\mathrm{LNCaP}$ proteins produced by the IPGphor and by the Multiphor units. The same amount of protein was loaded onto two 18-cm IPG strips ( $\mathrm{pH} 4-7$ ), and the strips were focused in Multiphor II and IPGphor, respectively.

Comparison of the 2-DE gel images from the Multiphor II (Figure 4-3a) and the IPGphor (Figure 4-3b) indicated both IEF systems separated proteins with a satisfactory resolution. Multiphor yielded a better separation of proteins in the basic region of the gel.

\subsubsection{Detection of Proteins by Sequential Staining}

In the study, 2-DE gels were first stained with the Pro- $\mathrm{Q}^{\mathrm{TM}}$ Diamond stain; the gels were imaged to capture the phosphoprotein patterns. Subsequently, the gels were stained with SYPRO Ruby total protein stain, and images were obtained to detect the total protein pattern. It has been reported that Pro- $\mathrm{Q}^{\mathrm{TM}}$ Diamond stain did not interfere with the SYPRO Ruby stain, even though there is some overlap in the wavelengths of fluorescence (Wu et al., 2005). The images obtained with Pro- $\mathrm{Q}^{\mathrm{TM}}$ Diamond and with SYPRO Ruby stain are shown in Figure 4-4a and 4-4b, respectively.

Several spots seen with Pro- $\mathrm{Q}^{\mathrm{TM}}$ Diamond stain were not present in the SYPRO Ruby-stained gel. Furthermore, intensities of some spots on Pro- $\mathrm{Q}^{\mathrm{TM}}$ Diamond and SYPRO Ruby stain images are different. 
(a)

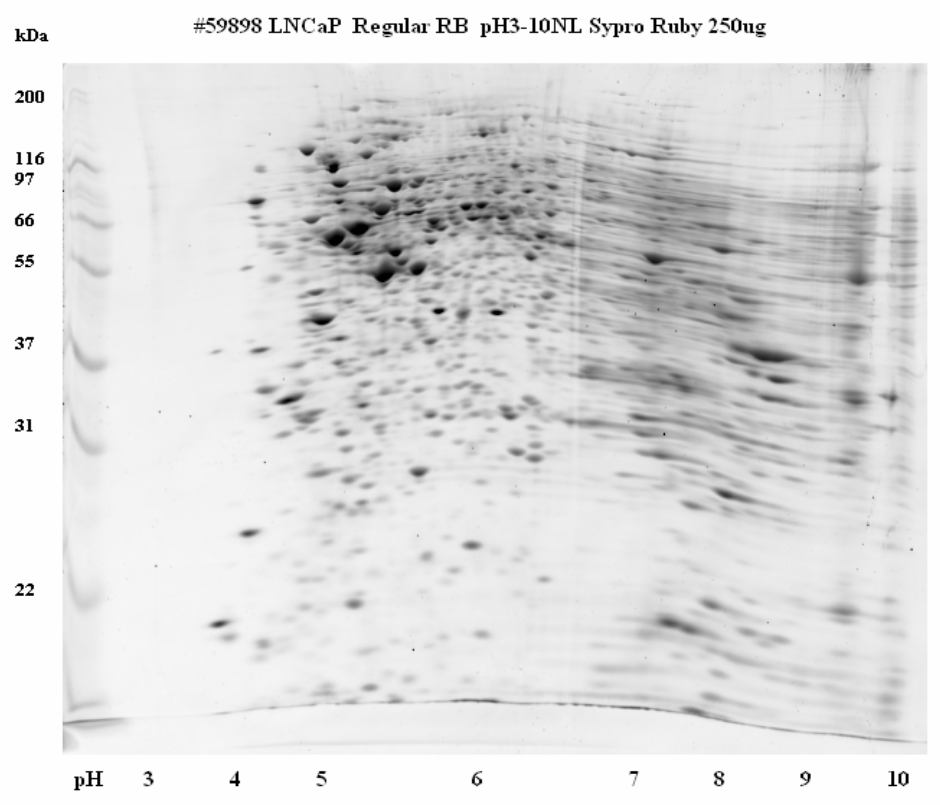

(b)

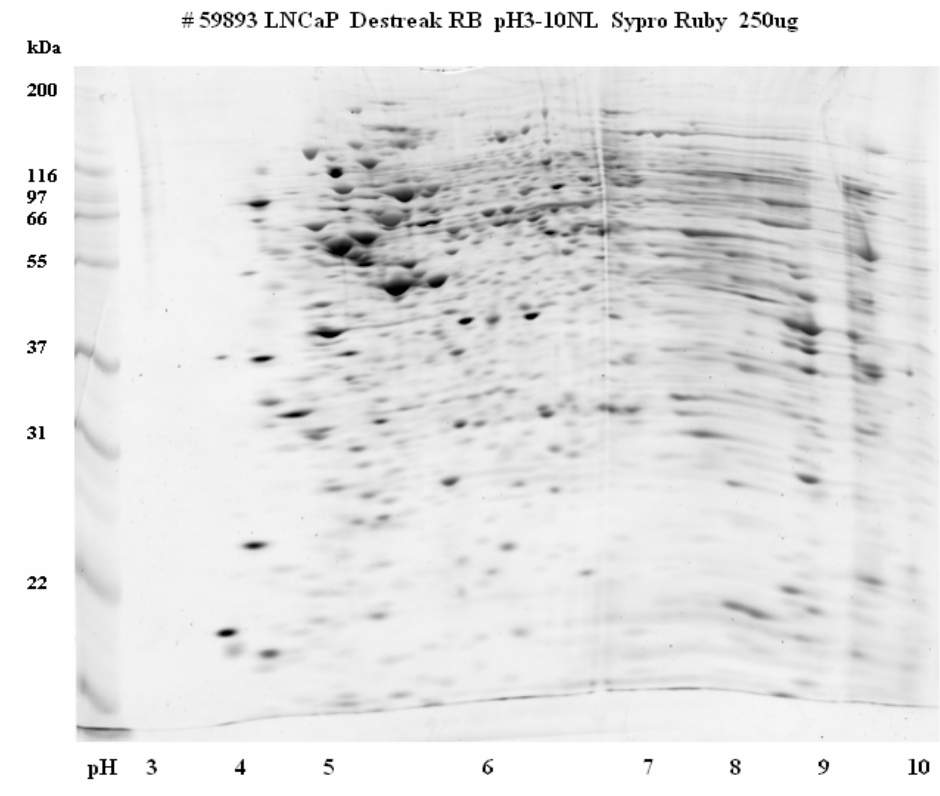

Figure 4-2 2-DE gels of $\mathrm{LNCaP}$ proteins with $\mathrm{pH} 3-10 \mathrm{NL}$ using different rehydration buffers (a) Standard rehydration buffer (b) Destreak rehydration solution. 
(a)

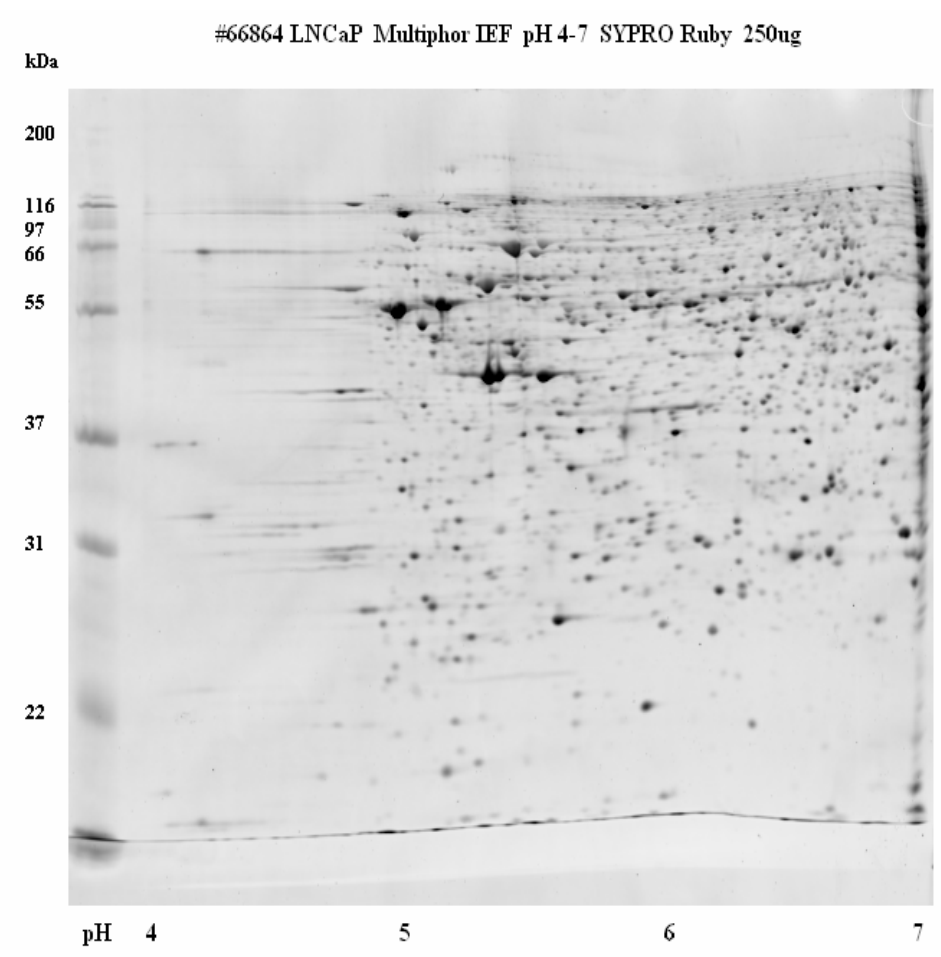

(b)

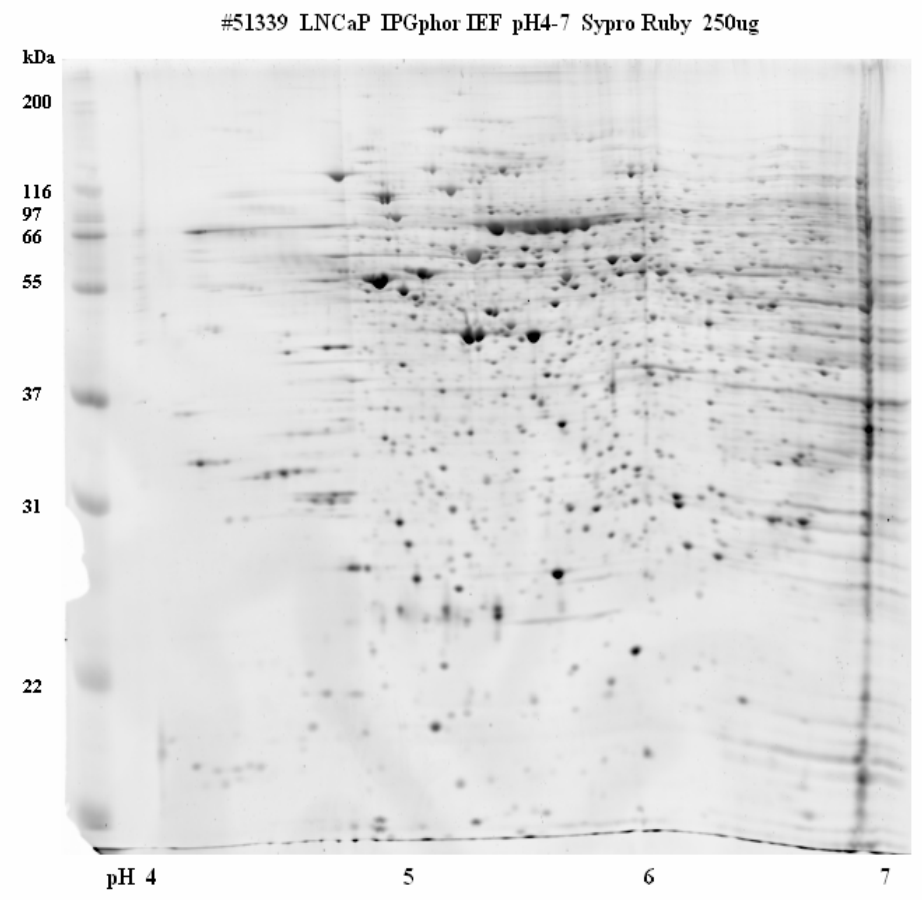

Figure 4-3 2-DE gels of LNCaP using different IEF units (a) Multiphor II (b) IPGphor. 
(a)

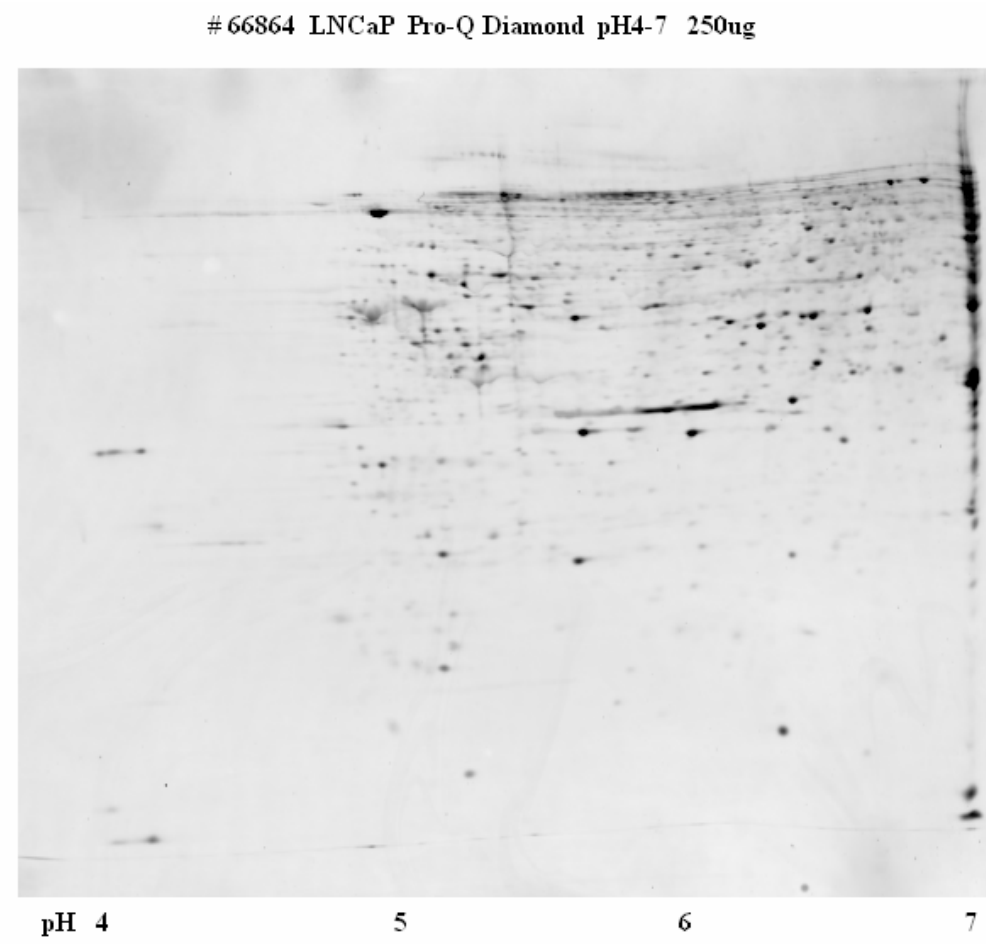

(b)

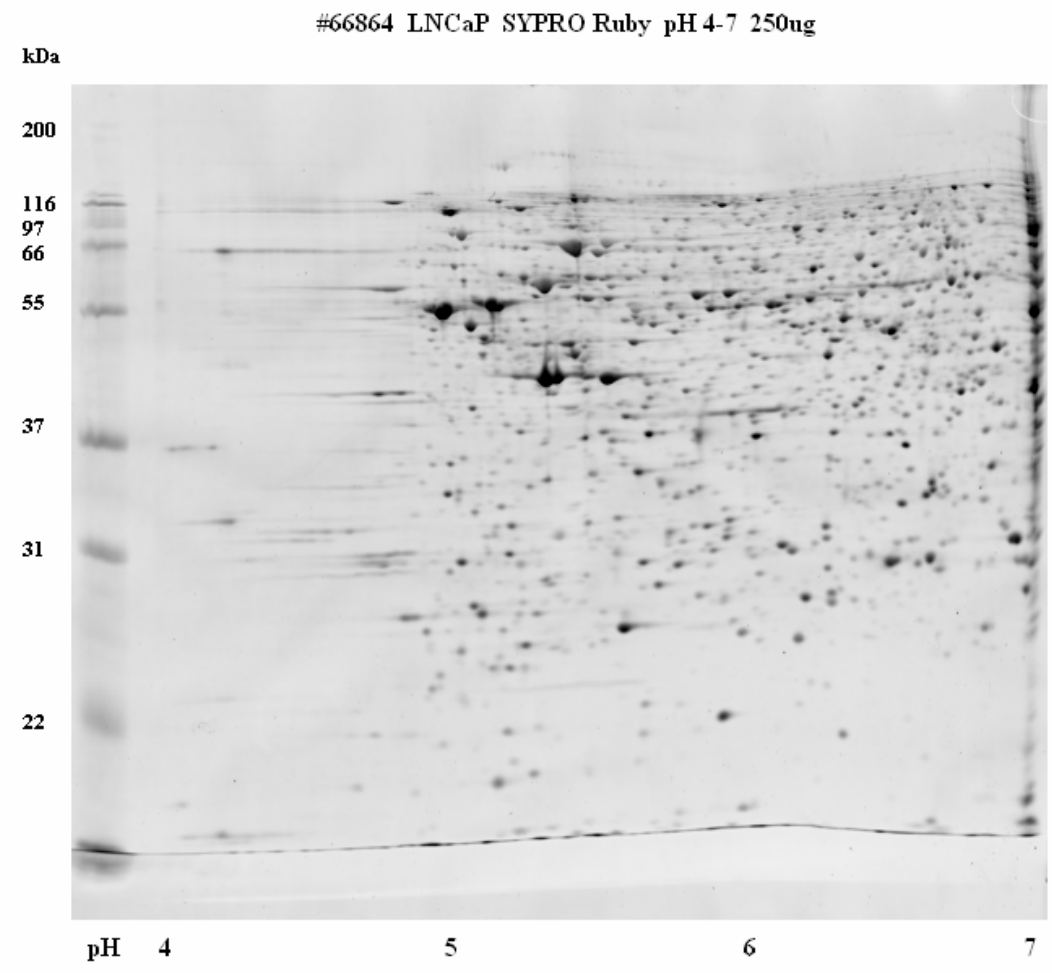

Figure 4-4 2-DE gels of LNCaP proteins detected with multiplexed staining (a) Pro- $\mathrm{Q}^{\mathrm{TM}}$ Diamond Phosphoprotein staining (b) SYPRO Ruby staining. 


\subsubsection{Protein Identification}

We identified a set of proteins that were detected as intense spots in the Pro- $\mathrm{Q}^{\mathrm{TM}}$ Diamond-stained gels. For the identification, we selected 16 spots from the gel with $\mathrm{pH}$ range of 4-7. These spots are labeled in Figure 4-5. In addition, we selected 14 spots from another Pro- $\mathrm{Q}^{\mathrm{TM}}$ Diamond stained gel that included $\mathrm{pH}$ range 3-10 (Figure 4-6). As discussed above, the spot quality in the $\mathrm{pH}$ 3-10 gel was not sufficient for 2-DE-based analysis; however, we used this wide range $\mathrm{pH}$ gradient in our in-gel IEF LC-MS/MS phosphoproteomics platform (see Chapter 2). Therefore, we opted to identify proteins from the $\mathrm{pH}$ 3-10 gel to allow direct comparison of the data.

For identification, the spots were excised and subjected to in-gel tryptic digestion. The extracted peptides were analyzed by LC-MS/MS and the LC-MS/MS data were used for database searches to identify the proteins. Overall, proteins were identified from all thirty protein spots. The identification was based on the presence of $\geq 2$ peptides. Since the spots were taken from a single gel (no pooling), the $100 \%$ success rate attested to the sensitivity of the LC-MS/MS system and to the overall power of our protein identification procedure. Protein identification results for the 14 spots from 2-DE gel with pH 3-10 are shown in Table 4-1; results for the 16 spots from 2-DE gel with $\mathrm{pH}$ 4-7 are shown in Table 4-2. It should be noted that some of the proteins were identified in multiple spots; furthermore, some of the spots contained more than one protein. Both scenarios are frequently encountered in these types of analyses. For example, if several forms of the same protein are present in the mixture, each form will give rise to a different spot. Conversely, different proteins with very similar pI and MW values may co-migrate in 2-DE.

\subsubsection{Additional Examination of the Phosphoprotein Panel}

Inspection of the Swiss-Prot annotations and survey of the PhosphoSite knowledgebase revealed that most of the proteins identified in our study are known to be phosphorylated. This indicated a high specificity of the Pro- $\mathrm{Q}^{\mathrm{TM}}$ Diamond stain.

Gene Ontology and Swiss-Prot annotations were inspected to gather information on subcellular location and on protein function. This information is included in Tables 41 and 4-2.

We compared the phosphoprotein panel obtained in this study to the panel that was produced with in-gel IEF LC-MS/MS (Chapter 2). Out of the 30 proteins, 3 proteins (Lupus la protein, Stress-induced-phosphoprotein1 and Nucleophosmin) were also found with in-gel IEF LC-MS/MS. These results showed that the two platforms provided complementary data on the LNCaP phosphoproteome. 


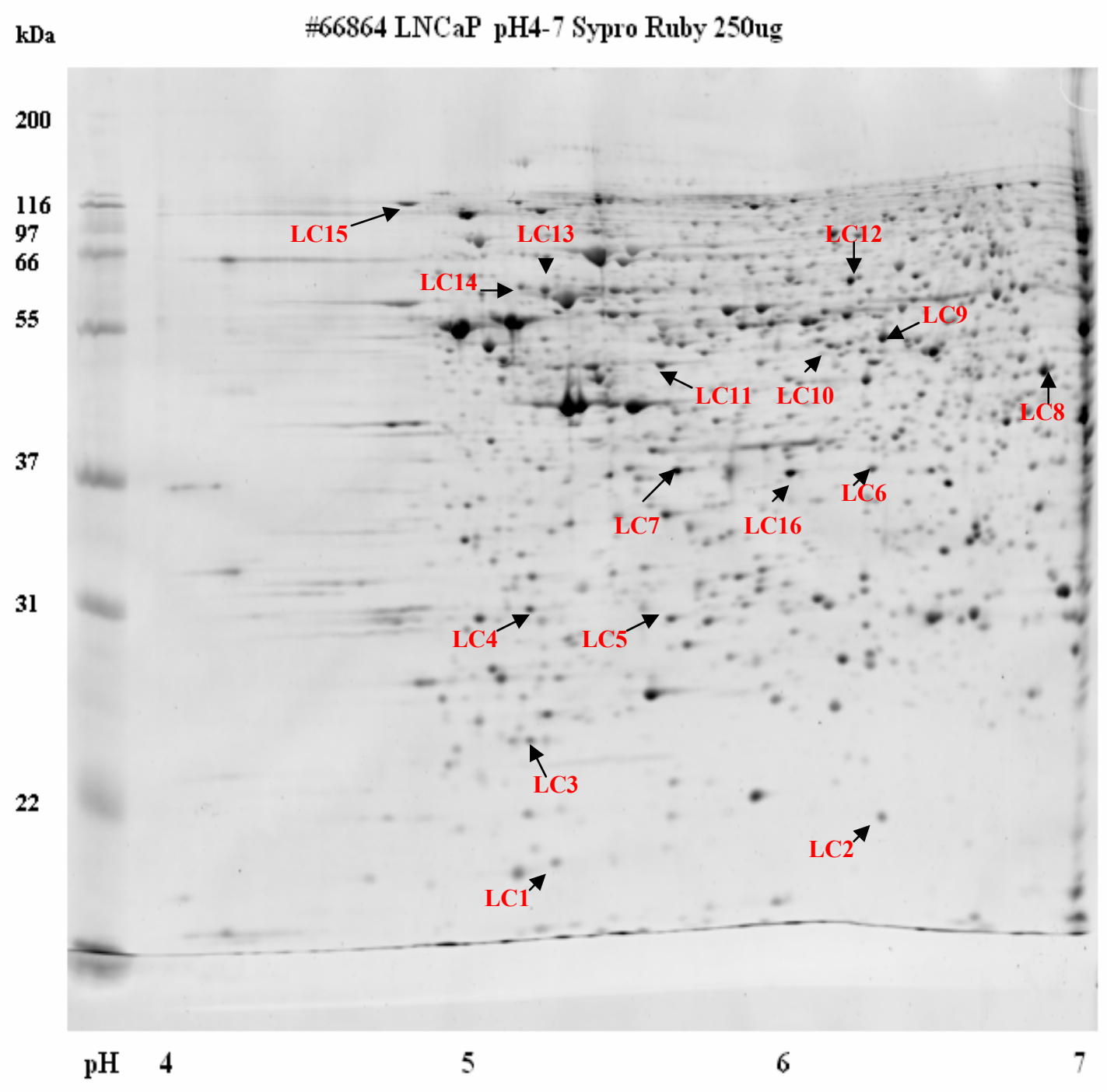

Figure 4-5 16 protein spots selected from 2-DE gel with $\mathrm{pH}$ 4-7. 


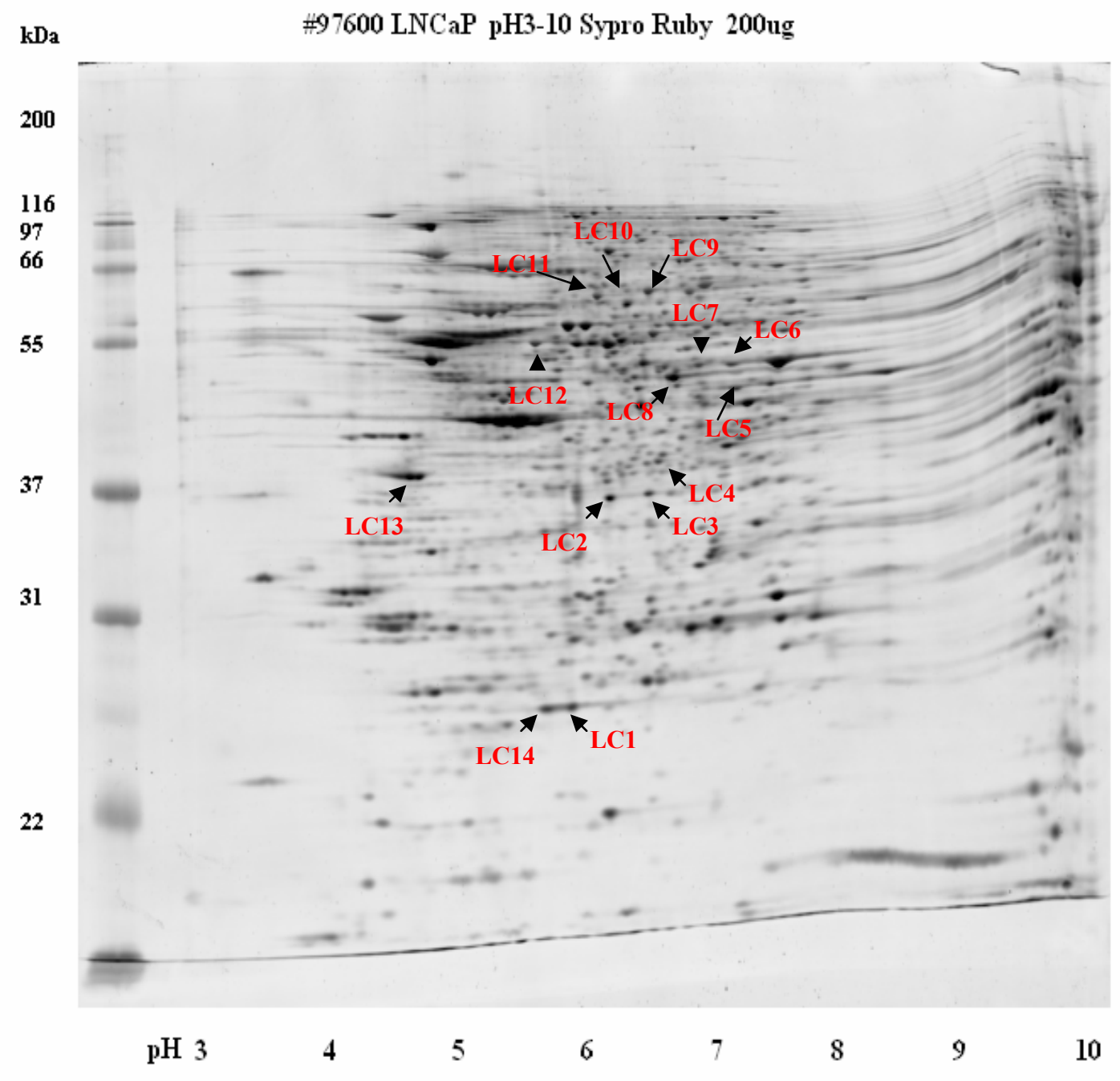

Figure 4-6 14 protein spots selected from 2-DE gel with $\mathrm{pH}$ 3-10. 
Table 4-1 Protein identification results for Pro- $\mathrm{Q}^{\mathrm{TM}}$ Diamond-stained spots from $\mathrm{pH}$ 3-10 gel.

\begin{tabular}{|c|c|c|c|c|c|c|c|}
\hline Spot & $\begin{array}{l}\text { Swiss-Prot } \\
\text { accession number }\end{array}$ & Protein name & $\begin{array}{l}\text { MW } \\
\text { Exp(Theo) }\end{array}$ & $\begin{array}{l}\text { pI } \\
\operatorname{Exp}(\text { Theo) }\end{array}$ & Function $^{\text {a }}$ & Location $^{\text {a }}$ & $\begin{array}{l}\text { Phospho } \\
\text { rylated b }\end{array}$ \\
\hline \multirow[t]{2}{*}{1} & $\begin{array}{l}\text { P32119 } \\
\text { PRDX2_HUMAN }\end{array}$ & Peroxiredoxin-2 & $25.8(21.9)$ & $5.8(5.66)$ & $\begin{array}{l}\text { response to oxidative stress | } \\
\text { regulation of apoptosis | anti- } \\
\text { apoptosis }\end{array}$ & cytoplasm & Yes \\
\hline & $\begin{array}{l}\text { Q06830 } \\
\text { PRDX1_HUMAN }\end{array}$ & Peroxiredoxin-1 & $25.8(22.1)$ & $5.8(8.27)$ & $\begin{array}{l}\text { cell proliferation | skeletal } \\
\text { development }\end{array}$ & $\begin{array}{l}\text { cytoplasm; } \\
\text { melanoso } \\
\text { me }\end{array}$ & Yes \\
\hline 2 & $\begin{array}{l}\text { P04083 } \\
\text { ANXA1_HUMAN }\end{array}$ & Annexin A1 & $42.9(38.7)$ & $6.3(6.57)$ & $\begin{array}{l}\text { cell surface receptor linked signal } \\
\text { transduction | inflammatory } \\
\text { response | keratinocyte } \\
\text { differentiation | lipid metabolism } \\
\text { | peptide cross-linking | anti- } \\
\text { apoptosis | cell motility }\end{array}$ & $\begin{array}{l}\text { nucleus; } \\
\text { cytoplasm }\end{array}$ & Yes \\
\hline \multirow[t]{2}{*}{3} & $\begin{array}{l}\text { P05388 } \\
\text { RLA0_HUMAN }\end{array}$ & $\begin{array}{l}60 \mathrm{~S} \text { acidic } \\
\text { ribosomal protein } \\
\text { P0 }\end{array}$ & $42.9(34.3)$ & $6.5(5.72)$ & $\begin{array}{l}\text { translational elongation | protein } \\
\text { biosynthesis | ribosome } \\
\text { biogenesis and assembly }\end{array}$ & $\begin{array}{l}\text { cytosolic } \\
\text { large } \\
\text { ribosomal } \\
\text { subunit }\end{array}$ & Yes \\
\hline & $\begin{array}{l}\text { P07195 } \\
\text { LDHB_HUMAN }\end{array}$ & $\begin{array}{l}\text { L-lactate } \\
\text { dehydrogenase B } \\
\text { chain }\end{array}$ & $42.9(36.6)$ & $6.5(5.71)$ & $\begin{array}{l}\text { tricarboxylic acid cycle } \\
\text { intermediate metabolism | } \\
\text { anaerobic glycolysis }\end{array}$ & cytoplasm & Yes \\
\hline 4 & $\begin{array}{l}\text { P37837 } \\
\text { TALDO_HUMAN }\end{array}$ & Transaldolase & $46.2(37.5)$ & $6.6(6.36)$ & $\begin{array}{l}\text { metabolism | pentose-phosphate } \\
\text { shunt | carbohydrate metabolism }\end{array}$ & cytoplasm & Yes \\
\hline 5 & $\begin{array}{l}\text { P49411 } \\
\text { EFTU_HUMAN }\end{array}$ & $\begin{array}{l}\text { Elongation factor } \\
\text { Tu, mitochondrial } \\
\text { precursor }\end{array}$ & $55.5(49.5)$ & $7.2(7.26)$ & $\begin{array}{l}\text { translational elongation | protein } \\
\text { biosynthesis }\end{array}$ & $\begin{array}{l}\text { mitochond } \\
\text { rion }\end{array}$ & Yes \\
\hline
\end{tabular}


Table 4-1 (continued).

\begin{tabular}{|c|c|c|c|c|c|c|c|}
\hline Spot & $\begin{array}{l}\text { Swiss-Prot } \\
\text { accession number }\end{array}$ & Protein name & 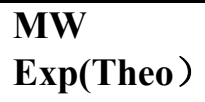 & $\begin{array}{l}\text { pI } \\
\operatorname{Exp}(\text { Theo) }\end{array}$ & Function $^{a}$ & Location $^{\text {a }}$ & $\begin{array}{l}\text { Phospho } \\
\text { rylated }^{b}\end{array}$ \\
\hline 6 & $\begin{array}{l}\text { P06733 } \\
\text { ENOA_HUMAN }\end{array}$ & Alpha-enolase & $58.6(47.2)$ & $7.2(7.01)$ & $\begin{array}{l}\text { glycolysis | growth control | } \\
\text { hypoxia tolerance | allergic } \\
\text { response | transcriptional } \\
\text { repressor }\end{array}$ & $\begin{array}{l}\text { cytoplasm; } \\
\text { cell } \\
\text { membrane } \\
\text { nucleus }\end{array}$ & Yes \\
\hline & $\begin{array}{l}\text { P05455 } \\
\text { LA_HUMAN }\end{array}$ & Lupus La protein & $58.6(46.8)$ & $7.2(6.68)$ & $\begin{array}{l}\text { transcription from RNA } \\
\text { polymerase III promoter | tRNA } \\
\text { modification | RNA processing | } \\
\text { RNA export from nucleus | } \\
\text { histone mRNA metabolism }\end{array}$ & nucleus & Yes \\
\hline 7 & $\begin{array}{l}\text { P06733 } \\
\text { ENOA_HUMAN }\end{array}$ & Alpha-enolase & $58.6(47.2)$ & $6.9(7.01)$ & $\begin{array}{l}\text { glycolysis | growth control } \mid \\
\text { hypoxia tolerance | allergic } \\
\text { response | transcriptional } \\
\text { repressor }\end{array}$ & $\begin{array}{l}\text { cytoplasm; } \\
\text { cell } \\
\text { membrane } \\
\text { nucleus }\end{array}$ & Yes \\
\hline & $\begin{array}{l}\text { P05455 } \\
\text { LA_HUMAN }\end{array}$ & Lupus La protein & $58.6(46.8)$ & $6.9(6.68)$ & $\begin{array}{l}\text { transcription from RNA } \\
\text { polymerase III promoter | tRNA } \\
\text { modification | RNA processing | } \\
\text { RNA export from nucleus | } \\
\text { histone mRNA metabolism }\end{array}$ & nucleus & Yes \\
\hline 8 & $\begin{array}{l}\text { P26641 } \\
\text { EF1G_HUMAN }\end{array}$ & $\begin{array}{l}\text { Elongation factor 1- } \\
\text { gamma }\end{array}$ & $57.6(50.1)$ & $6.6(6.25)$ & $\begin{array}{l}\text { translational elongation | protein } \\
\text { biosynthesis }\end{array}$ & cytosol & Yes \\
\hline 9 & $\begin{array}{l}\text { P31948 } \\
\text { STIP1_HUMAN }\end{array}$ & $\begin{array}{l}\text { Stress-induced- } \\
\text { phosphoprotein } 1\end{array}$ & $70.5(62.6)$ & $6.5(6.40)$ & response to stress & $\begin{array}{l}\text { cytoplasm; } \\
\text { nucleus }\end{array}$ & Yes \\
\hline
\end{tabular}


Table 4-1 (continued).

\begin{tabular}{|c|c|c|c|c|c|c|c|}
\hline Spot & $\begin{array}{l}\text { Swiss-Prot } \\
\text { accession number }\end{array}$ & Protein name & $\begin{array}{l}\text { MW } \\
\text { Exp(Theo) }\end{array}$ & $\begin{array}{l}\text { pI } \\
\operatorname{Exp}(\text { Theo) }\end{array}$ & Function $^{a}$ & Location $^{a}$ & $\begin{array}{l}\text { Phospho } \\
\text { rylated }\end{array}$ \\
\hline 10 & $\begin{array}{l}\text { P40227 } \\
\text { TCPZ_HUMAN }\end{array}$ & $\begin{array}{l}\text { T-complex protein } 1 \\
\text { subunit zeta }\end{array}$ & $67.9(58.0)$ & $6.3(6.24)$ & $\begin{array}{l}\text { nucleotide binding } \mid \text { ATP binding } \\
\mid \text { unfolded protein binding } \mid\end{array}$ & cytoplasm & Yes \\
\hline 11 & $\begin{array}{l}\text { P4936̄ } \\
\text { TCPG_HUMAN }\end{array}$ & $\begin{array}{l}\text { T-complex protein } 1 \\
\text { subunit gamma }\end{array}$ & $69.2(60.5)$ & $6.1(6.10)$ & $\begin{array}{l}\text { nucleotide binding | ATP binding } \\
\mid \text { unfolded protein binding | }\end{array}$ & cytoplasm & Yes \\
\hline \multirow[t]{2}{*}{12} & $\begin{array}{l}\text { P1492 } \overline{3} \\
\text { PLAK_HUMAN }\end{array}$ & $\begin{array}{l}\text { Junction } \\
\text { plakoglobin }\end{array}$ & $61.9(81.6)$ & $5.6(5.95)$ & $\begin{array}{l}\text { structural molecule activity | } \\
\text { cytoskeletal protein binding | }\end{array}$ & $\begin{array}{l}\text { cell } \\
\text { junction; } \\
\text { cytoplasm; } \\
\text { membrane }\end{array}$ & Yes \\
\hline & $\begin{array}{l}\text { P49368 } \\
\text { TCPG_HUMAN }\end{array}$ & $\begin{array}{l}\text { T-complex protein } 1 \\
\text { subunit gamma }\end{array}$ & $61.9(60.5)$ & $5.6(6.10)$ & $\begin{array}{l}\text { nucleotide binding | ATP binding } \\
\mid \text { unfolded protein binding } \mid\end{array}$ & cytoplasm & Yes \\
\hline 13 & $\begin{array}{l}\text { P0674 } \overline{8} \\
\text { NPM_HUMAN }\end{array}$ & Nucleophosmin & $45.3(32.6)$ & $4.6(4.64)$ & $\begin{array}{l}\text { Nucleotide binding | the assembly } \\
\text { and/or transport of ribosome }\end{array}$ & nucleus & Yes \\
\hline 14 & $\begin{array}{l}\text { P32119 } \\
\text { PRDX2_HUMAN }\end{array}$ & Peroxiredoxin-2 & $25.8(21.9)$ & $5.7(5.66)$ & $\begin{array}{l}\text { response to oxidative stress | } \\
\text { regulation of apoptosis | anti- } \\
\text { apoptosis }\end{array}$ & cytoplasm & Yes \\
\hline
\end{tabular}

a. Protein function and sucellular location were obtained based on Gene Ontology and Swiss-Prot annotation.

b. Status of protein phosphorylation was inspected in Swiss-Prot and PhosphoSite. 
Table 4-2 Protein identification results for Pro- $\mathrm{Q}^{\mathrm{TM}}$ Diamond-stained spots from $\mathrm{pH}$ 4-7 gel.

\begin{tabular}{|c|c|c|c|c|c|c|c|}
\hline Spot & $\begin{array}{l}\text { Swiss-Prot } \\
\text { accession number }\end{array}$ & Protein name & $\begin{array}{l}\text { MW } \\
\text { Exp(Theo) }\end{array}$ & $\begin{array}{l}\text { pI } \\
\text { Exp(Theo) }\end{array}$ & Function $^{a}$ & Location $^{a}$ & $\begin{array}{l}\text { Phospho } \\
\text { rylated }\end{array}$ \\
\hline 1 & $\begin{array}{l}\text { P53999 } \\
\text { TCP4_HUMAN }\end{array}$ & $\begin{array}{l}\text { Activated RNA } \\
\text { polymerase II } \\
\text { transcriptional } \\
\text { coactivator p15 }\end{array}$ & $16.7(14.4)$ & $5.3(9.6)$ & $\begin{array}{l}\text { single-stranded DNA binding } \\
\text { | transcription coactivator } \\
\text { activity | DNA binding }\end{array}$ & nucleus & Yes \\
\hline 2 & $\begin{array}{l}\text { P23528 } \\
\text { COF1_HUMAN }\end{array}$ & Cofilin-1 & $18.8(18.5)$ & $6.5(8.22)$ & $\begin{array}{l}\text { actin binding } \mid \text { protein } \\
\text { binding }\end{array}$ & nucleus & Yes \\
\hline \multirow[t]{2}{*}{3} & $\begin{array}{l}\text { Q9Y5S9 } \\
\text { RBM8A_HUMAN }\end{array}$ & $\begin{array}{l}\text { RNA-binding } \\
\text { protein } 8 \mathrm{~A}\end{array}$ & $22.3(19.9)$ & $5.0(5.5)$ & $\begin{array}{l}\text { mRNA binding } \mid \text { protein } \\
\text { binding } \mid \text { nucleotide binding }\end{array}$ & $\begin{array}{l}\text { cytoplasm; } \\
\text { nucleus }\end{array}$ & Yes \\
\hline & $\begin{array}{l}\text { Q13185 } \\
\text { CBX3_HUMAN }\end{array}$ & $\begin{array}{l}\text { Chromobox protein } \\
\text { homolog } 3\end{array}$ & $22.3(20.8)$ & $5.0(5.23)$ & & nucleus & Yes \\
\hline \multirow[t]{2}{*}{4} & $\begin{array}{l}\text { O43399 } \\
\text { TPD54_HUMAN }\end{array}$ & Tumor protein D54 & $31.3(22.2)$ & $5.1(5.26)$ & protein binding & unclassified & Yes \\
\hline & $\begin{array}{l}\text { P25788 } \\
\text { PSA3_HUMAN }\end{array}$ & $\begin{array}{l}\text { Proteasome subunit } \\
\text { alpha type } 3\end{array}$ & $31.3(28.4)$ & $5.1(5.19)$ & $\begin{array}{l}\text { threonine endopeptidase } \\
\text { activity | protein binding }\end{array}$ & $\begin{array}{l}\text { cytoplasm; } \\
\text { nucleus }\end{array}$ & Yes \\
\hline \multirow[t]{2}{*}{5} & $\begin{array}{l}\text { P04792 } \\
\text { HSPB1_HUMAN }\end{array}$ & $\begin{array}{l}\text { Heat-shock protein } \\
\text { beta-1 }\end{array}$ & $30.4(22.8)$ & $5.6(5.98)$ & protein binding & $\begin{array}{l}\text { cytoplasm; } \\
\text { nucleus }\end{array}$ & Yes \\
\hline & $\begin{array}{l}\text { O75489 } \\
\text { NDUS3_HUMAN }\end{array}$ & $\begin{array}{l}\text { NADH } \\
\text { dehydrogenase } \\
\text { [ubiquinone] iron- } \\
\text { sulfur protein } 3\end{array}$ & $30.4(30.2)$ & $5.6(6.98)$ & $\begin{array}{l}\text { NADH dehydrogenase } \\
\text { activity | NADH } \\
\text { dehydrogenase (ubiquinone) } \\
\text { activity }\end{array}$ & mitochondrion & Yes \\
\hline
\end{tabular}


Table 4-2 (continued).

\begin{tabular}{|c|c|c|c|c|c|c|c|}
\hline Spot & $\begin{array}{l}\text { Swiss-Prot } \\
\text { accession number }\end{array}$ & Protein name & $\begin{array}{l}\text { MW } \\
\text { Exp(Theo) }\end{array}$ & $\begin{array}{l}\text { pI } \\
\text { Exp(Theo) }\end{array}$ & Function $^{a}$ & Location $^{a}$ & $\begin{array}{l}\text { Phospho } \\
\text { rylated }\end{array}$ \\
\hline & $\begin{array}{l}\text { Q9BTE7 } \\
\text { DCNL5_HUMAN }\end{array}$ & DCN1-like protein 5 & $30.4(27.5)$ & $5.6(5.44)$ & & unclassified & No \\
\hline 6 & $\begin{array}{l}\text { O00487 } \\
\text { PSDE_HUMAN }\end{array}$ & $\begin{array}{l}\text { 26S proteasome non- } \\
\text { ATPase regulatory } \\
\text { subunit } 14\end{array}$ & $43.9(34.6)$ & $6.2(6.06)$ & $\begin{array}{l}\text { ubiquitin-dependent protein } \\
\text { catabolism }\end{array}$ & unclassified & Yes \\
\hline \multirow{3}{*}{7} & $\begin{array}{l}\text { Q14847 } \\
\text { LASP1_HUMAN }\end{array}$ & $\begin{array}{l}\text { LIM and SH3 domain } \\
\text { protein } 1\end{array}$ & $43.9(29.7)$ & $6.2(6.61)$ & $\begin{array}{l}\text { ion transporter activity | } \\
\text { metal ion binding | actin } \\
\text { binding | SH3/SH2 adaptor } \\
\text { activity | zinc ion binding }\end{array}$ & cytoplasm & Yes \\
\hline & $\begin{array}{l}\text { P05388 } \\
\text { RLA0_HUMAN }\end{array}$ & $\begin{array}{l}60 \mathrm{~S} \text { acidic ribosomal } \\
\text { protein } \mathrm{P} 0\end{array}$ & $43.9(34.3)$ & $5.5(5.72)$ & $\begin{array}{l}\text { structural constituent of } \\
\text { ribosome | RNA binding | } \\
\text { protein binding }\end{array}$ & unclassified & Yes \\
\hline & $\begin{array}{l}\text { P06748 } \\
\text { NPM_HUMAN }\end{array}$ & Nucleophosmin & $43.9(32.6)$ & $5.5(5.0)$ & $\begin{array}{l}\text { Nucleotide binding | the } \\
\text { assembly and/or transport } \\
\text { of ribosome }\end{array}$ & nucleus & Yes \\
\hline 8 & $\begin{array}{l}\text { O75874 } \\
\text { IDHC_HUMAN }\end{array}$ & $\begin{array}{l}\text { Isocitrate } \\
\text { dehydrogenase } \\
\text { [NADP] }\end{array}$ & $56.1(46.7)$ & $6.9(6.53)$ & $\begin{array}{l}\text { oxidoreductase activity | } \\
\text { isocitrate dehydrogenase } \\
(\mathrm{NADP}+) \text { activity | } \\
\text { oxidoreductase activity, } \\
\text { acting on NADH or } \\
\text { NADPH, NAD or NADP as } \\
\text { acceptor }\end{array}$ & cytoplasm & Yes \\
\hline \multirow[t]{2}{*}{9} & $\begin{array}{l}\text { P50395 } \\
\text { GDIB_HUMAN }\end{array}$ & $\begin{array}{l}\text { Rab GDP } \\
\text { dissociation inhibitor } \\
\text { beta }\end{array}$ & $60.5(50.7)$ & $6.3(6.11)$ & $\begin{array}{l}\text { Rab GDP-dissociation } \\
\text { inhibitor activity | GTPase } \\
\text { activator activity }\end{array}$ & $\begin{array}{l}\text { cytoplasm; } \\
\text { membrane }\end{array}$ & Yes \\
\hline & $\begin{array}{l}\text { P31150 } \\
\text { GDIA_HUMAN }\end{array}$ & $\begin{array}{l}\text { Rab GDP } \\
\text { dissociation inhibitor } \\
\text { alpha }\end{array}$ & $60.5(50.6)$ & $6.3(5.0)$ & $\begin{array}{l}\text { Rab GDP-dissociation } \\
\text { inhibitor activity | GTPase } \\
\text { activator activity }\end{array}$ & cytoplasm & Yes \\
\hline
\end{tabular}


Table 4-2 (continued).

\begin{tabular}{|c|c|c|c|c|c|c|c|}
\hline Spot & $\begin{array}{l}\text { Swiss-Prot } \\
\text { accession number }\end{array}$ & Protein name & $\begin{array}{l}\text { MW } \\
\text { Exp(Theo) }\end{array}$ & $\begin{array}{l}\text { pI } \\
\text { Exp(Theo) }\end{array}$ & Function $^{a}$ & Location $^{\text {a }}$ & $\begin{array}{l}\text { Phospho } \\
\text { rylated }\end{array}$ \\
\hline \multirow[t]{3}{*}{10} & $\begin{array}{l}\text { Q99816 } \\
\text { TS101_HUMAN }\end{array}$ & $\begin{array}{l}\text { Tumor } \\
\text { susceptibility gene } \\
101 \text { protein }\end{array}$ & $59.4(43.9)$ & $6.1(6.06)$ & $\begin{array}{l}\text { protein binding | } \\
\text { transcription corepressor } \\
\text { activity | DNA binding | } \\
\text { ubiquitin-protein ligase } \\
\text { activity }\end{array}$ & $\begin{array}{l}\text { cytoplasm; } \\
\text { membrane }\end{array}$ & Yes \\
\hline & $\begin{array}{l}\text { O75439 } \\
\text { MPPB_HUMAN }\end{array}$ & $\begin{array}{l}\text { Mitochondrial- } \\
\text { processing } \\
\text { peptidase subunit } \\
\text { beta }\end{array}$ & $59.4(54.4)$ & $6.1(6.38)$ & $\begin{array}{l}\text { mitochondrial processing } \\
\text { peptidase activity } \mid \text { metal } \\
\text { ion binding | zinc ion } \\
\text { binding }\end{array}$ & mitochondrion & No \\
\hline & $\begin{array}{l}\text { Q9UQ80 } \\
\text { PA2G4_HUMAN }\end{array}$ & $\begin{array}{l}\text { Proliferation- } \\
\text { associated protein } \\
2 \mathrm{G} 4\end{array}$ & $59.4(43.8)$ & $6.1(6.13)$ & $\begin{array}{l}\text { methionyl } \\
\text { aminopeptidase activity } \mid \\
\text { protein binding | } \\
\text { transcription factor } \\
\text { activity | hydrolase } \\
\text { activity | RNA binding | } \\
\text { cobalt ion binding }\end{array}$ & $\begin{array}{l}\text { cytoplasm; } \\
\text { nucleus }\end{array}$ & Yes \\
\hline \multirow[t]{2}{*}{11} & $\begin{array}{l}\text { P31930 } \\
\text { UQCR1_HUMAN }\end{array}$ & $\begin{array}{l}\text { Ubiquinol- } \\
\text { cytochrome-c } \\
\text { reductase complex } \\
\text { core protein } 1\end{array}$ & $56.7(52.6)$ & $5.5(5.94)$ & $\begin{array}{l}\text { oxidoreductase activity | } \\
\text { ubiquinol-cytochrome-c } \\
\text { reductase activity }\end{array}$ & mitochondrion & Yes \\
\hline & $\begin{array}{l}\text { P02647 } \\
\text { APOA1_HUMAN }\end{array}$ & $\begin{array}{l}\text { Apolipoprotein A- } \\
\text { I precursor }\end{array}$ & $56.7(30.8)$ & $5.5(5.56)$ & $\begin{array}{l}\text { lipid binding | high- } \\
\text { density lipoprotein } \\
\text { binding | protein binding | } \\
\text { lipid transporter activity }\end{array}$ & secreted & No \\
\hline 12 & $\begin{array}{l}\text { Q16222 } \\
\text { UAP1_HUMAN }\end{array}$ & $\begin{array}{l}\text { UDP-N- } \\
\text { acetylhexosamine } \\
\text { pyrophosphorylase }\end{array}$ & $70.4(58.8)$ & $6.1(5.92)$ & $\begin{array}{l}\text { UDP-N- } \\
\text { acetylglucosamine } \\
\text { diphosphorylase activity | } \\
\text { nucleotidyltransferase } \\
\text { activity }\end{array}$ & cytoplasm & No \\
\hline
\end{tabular}


Table 4-2 (continued).

\begin{tabular}{|c|c|c|c|c|c|c|c|}
\hline Spot & $\begin{array}{l}\text { Swiss-Prot } \\
\text { accession number }\end{array}$ & Protein name & $\begin{array}{l}\text { MW } \\
\text { Exp(Theo) }\end{array}$ & $\begin{array}{l}\text { pI } \\
\text { Exp(Theo) }\end{array}$ & Function $^{a}$ & Location $^{a}$ & $\begin{array}{l}\text { Phospho } \\
\text { rylated }^{b}\end{array}$ \\
\hline 13 & $\begin{array}{l}\text { P10809 } \\
\text { CH60_HUMAN }\end{array}$ & $\begin{array}{l}60 \mathrm{kDa} \text { heat shock } \\
\text { protein, } \\
\text { mitochondrial } \\
\text { precursor }\end{array}$ & $67.8(61.1)$ & $5.1(5.7)$ & $\begin{array}{l}\text { protein binding | nucleotide } \\
\text { binding | chaperone binding } \mid \\
\text { unfolded protein binding } \mid \\
\text { ATP binding }\end{array}$ & mitochondrion & Yes \\
\hline 14 & $\begin{array}{l}\text { P61978 } \\
\text { HNRPK_HUNMAN }\end{array}$ & $\begin{array}{l}\text { Heterogeneous } \\
\text { nuclear } \\
\text { ribonucleoprotein } \mathrm{K}\end{array}$ & $69.1(51.0)$ & $5.0(5.39)$ & $\begin{array}{l}\text { nucleic acid binding | RNA } \\
\text { binding | DNA binding | } \\
\text { protein binding }\end{array}$ & $\begin{array}{l}\text { cytoplasm/ } \\
\text { nucleus }\end{array}$ & Yes \\
\hline \multirow[t]{3}{*}{15} & $\begin{array}{l}\text { P45974 } \\
\text { UBP5_HUMAN }\end{array}$ & $\begin{array}{l}\text { Ubiquitin carboxyl- } \\
\text { terminal hydrolase } \\
5\end{array}$ & 75.9 (95.8) & $4.9(4.91)$ & $\begin{array}{l}\text { protein binding | zinc ion } \\
\text { binding | metal ion binding | } \\
\text { cysteine-type endopeptidase } \\
\text { activity | ubiquitin-specific } \\
\text { protease } 5 \text { activity }\end{array}$ & unclassified & Yes \\
\hline & $\begin{array}{l}\text { P08238 } \\
\text { HS90B_HUMAN }\end{array}$ & $\begin{array}{l}\text { Heat shock protein } \\
\text { HSP } 90 \text {-beta }\end{array}$ & $75.9(83.3)$ & $4.9(4.97)$ & $\begin{array}{l}\text { nitric-oxide synthase } \\
\text { regulator activity | } \\
\text { nucleotide binding | TPR } \\
\text { domain binding }\end{array}$ & $\begin{array}{l}\text { cytoplasm/ } \\
\text { melanosome }\end{array}$ & Yes \\
\hline & $\begin{array}{l}\text { P07900 } \\
\text { HS90A_HUMAN }\end{array}$ & $\begin{array}{l}\text { Heat shock protein } \\
\text { HSP } 90 \text {-alpha }\end{array}$ & $75.9(84.7)$ & $4.9(4.94)$ & $\begin{array}{l}\text { nitric-oxide synthase } \\
\text { regulator activity | } \\
\text { nucleotide binding | protein } \\
\text { homodimerization activity | } \\
\text { unfolded protein binding | } \\
\text { molecular function unknown } \\
\text { | ATP binding }\end{array}$ & $\begin{array}{l}\text { cytoplasm/ } \\
\text { melanosome }\end{array}$ & Yes \\
\hline 16 & $\begin{array}{l}\text { P05388 } \\
\text { RLA0_HUMAN }\end{array}$ & $\begin{array}{l}60 \mathrm{~S} \text { acidic } \\
\text { ribosomal protein } \\
\mathrm{P} 0\end{array}$ & $43.9(34.3)$ & $6.1(5.72)$ & $\begin{array}{l}\text { structural constituent of } \\
\text { ribosome | RNA binding | } \\
\text { protein binding }\end{array}$ & unclassified & Yes \\
\hline
\end{tabular}

a. Protein function and sucellular location were obtained based on Gene Ontology and Swiss-Prot annotation.

b. Status of protein phosphorylation was inspected in Swiss-Prot and PhosphoSite. 


\subsection{Discussion}

Proteomic technologies enable the study of proteins and their interactions in a systematic manner. Because of the critical role that phosphorylation plays in biological systems, intensive efforts have been centered on the development of improved phosphoproteomic strategies, and many analytical options are available. Before largescale application of a chosen bioanalytical platform to a new biological system, in a new setting, it is necessary to tailor the chosen strategy to the particular task, and to evaluate its performance. In this study, we applied 2-DE with multiplexed Pro- $\mathrm{Q}^{\mathrm{TM}}$ Diamond phsopho-specific stain and SYPRO Ruby stain, followed by protein identification of selected spots using LC-MS/MS, to obtain an overview of phosphoproteins in the LNCaP human prostate cancer cell line.

We found that streaks were present in the $\mathrm{pH}$ range of 7-9 in our 2-DE gels. It is common that horizontal streaking is present at the basic $\mathrm{pH}$ range of 7-9 in a 2-DE gel. The primary reason of streaking is due to the depletion of the reducing agent at the basic end during IEF (Gorg et al., 2000; Olsson et al., 2002). Reducing agent, dithiothreitol (DTT), is a weak acid and therefore charged at a basic $\mathrm{pH}$, which results in the migration of DTT towards the anode during IEF. The depletion of DTT at the cathode leads to oxidation of protein thiol groups and re-formation of inter- and intra- molecular disulfide bridges.

Destreak rehydration solution has been reported to transform the protein thiol groups into a stable disulfide and to protect the disulfide groups from unspecific oxidation (Olsson et al., 2002). We partially reduced streaks and improved the spot patterns on 2-DE gels with $\mathrm{pH}$ range of 3-10 by using the Destreak Rehydration solution. However, the major improvement expected was not reached. Another option to address this issue would be to perform two separations with two different strips: $\mathrm{pH}$ 4-7 and $\mathrm{pH}$ 6-9 (Hoving et al., 2002; Pennington et al., 2004). However, this would mean additional sample and time requirements, plus modification of the experimental procedure.

Therefore, we decided to use IPG strips with $\mathrm{pH}$ range of 4-7 for subsequent experiments; with this option we can achieve an increased resolution in the $\mathrm{pH}$ region where most proteins appear.

Sample application plays an important role in the IPG-based isoelectric focusing. Previous studies showed the best protein recoveries are obtained for all protein loads when samples are applied to IPG strips during rehydration using a single device for both rehydration and IEF. In contrast, the recoveries are poorest when rehydration and IEF are performed in separate devices, and increasing protein loads using separate devices extensively increases sample losses (Zuo and Speicher, 2000).

Compared to Multiphor II that employs separate rehydration and IEF chambers, the IPGphor is an integrated equipment that consists of a high voltage power supply and a solid state cooling system. IPG strips are rehydrated and focused in the same strip holder by automatically programming the initial rehydration step, thus avoiding transfer of the IPG strip from the re-swelling tray to an IEF tray and eliminating further handling of the 
strip. In addition, the advantage of the strip holder over the reswelling tray is that proteins that do not enter the IPG gel during rehydration can enter the gel during the focusing step. Zuo and Speicher reported that the application of low voltage during rehydration did not appear to significantly improve overall protein recovery, but it was beneficial for entry of specific proteins (Zuo and Speicher, 2000). In our study, both IEF systems demonstrated a satisfactory protein separation; either one can be applied in further study.

Both Pro- $\mathrm{Q}^{\mathrm{TM}}$ Diamond stain and SYPRO Ruby stain are fluorescent stains and have similar sensitivity. They can be used subsequently to detect phosphoproteins and total proteins in a single gel. SYPRO Ruby stain has a low background, high resolution and dynamic range, and is easy to manipulate (Lopez et al., 2000). However, Pro- $\mathrm{Q}^{\mathrm{TM}}$ Diamond staining is affected by the expertise that an investigator has with the technique. The stain and destain time points will affect background and resolution. We found that some of the spots that were present on both images sometimes differed in intensity; and there were a few spots only present on Pro- $\mathrm{Q}^{\mathrm{TM}}$ Diamond image but not on SYPRO Ruby. This may be due to the specific proteins that are particularly sensitive to one staining but not to the other (Wu et al., 2005). This sequential staining of proteins in a single gel provided parallel determination of protein expression and preliminary characterization of post-translational modifications of proteins in individual spots on 2-DE gels.

In our determination of the protein identities, we considered the possibility of phosphorylation in our database searches; however, no phosphopeptides were retrieved for the identified proteins. This result was expected. In order to maximize the chance of direct detection of the phosphorylated peptide, an IMAC enrichment step must be included at the peptide level (analogously to the method in Chapter 2). This strategy is possible, and has been used to characterize exact phosphorylation sites, e.g. in the human growth hormone (Zhan et al., 2005). However, the strategy requires a much higher protein amount. In our 2-DE survey of LNCaP phosphoproteins, we chose to focus on maximizing the success rate of determination of protein identity, and we used information from published literature to provide supporting evidence for the phosphorylation status of the identified proteins. To gather this information, we used two bioinformatics resources. First, we inspected the Swiss-Prot annotations for each protein. These annotations summarize information on each Swiss-Prot database entry, including post-translational modifications. Second, we used the PhosphoSite knowledgebase to retrieve information on protein phosphorylation. This strategy takes advantage of the publicly available information sources. Instead of using PubMed, we performed our inspection in Swiss-Prot and PhosphoSite annotation because both of them are populated with information derived from published literatures and as complete and up-to-date as possible.

Another characteristic that we addressed for our phosphoprotein panel concerned relevance of the identified proteins to prostate cancer. Because our study was intended as a prelude to future differential expression studies in the context of prostate cancer, it is important to evaluate whether the phosphoproteins that we probed with the chosen bioanalytical strategy bear potential relevance to cancer. We evaluated this aspect by reviewing published literature. A number of identified proteins have been previously 
reported to be differentially expressed in prostate cancer cell line, such as Peroxiredoxin-2, Annexin A1, Transaldolase, Nucleophosmin et al. The findings indicated that 2-DE strategy is feasible to study the phosphoprotein differential expression in LNCaP. A number of cancer relevant phosphoproteins identified in the present study was shown in Table 4-3 based on their functions.

Peroxiredoxins (PRDXs) are antioxidant enzymes expressed by most free-living organisms, often in multiple isoforms. Shen et al. demonstrated that prostate cancer cells were more sensitive to hydrogen peroxide or an organic hydroperoxide when PRDX1, 2, or 3 was partially suppressed (Shen and Nathan, 2002). Park et al. reported that PRDX1 acted as a key mediator in stimulating the activation of androgen receptor (AR). The high level of PRDX1 was expressed in the aggressive LN3, C4-2, and C4-2B prostate cancer cell lines derived from LNCaP, which displayed PRDX1 is able to sensitize a ligandstimulated AR and serve as a new target in the management of prostate cancer (Park et al., 2007). PRDX2 have been reported to significantly up-regulate in highly metastatic prostate cancer cell line 1E8-H cells (Wu et al., 2007). Nevertheless, PRDX 2 and PRDX1 have been found lacking significant change in prostate needle biopsy speciments (Lin et al., 2007).

Annexin I (ANX I) is a promising prostate cancer biomarker identified by 2-DE and mass spectrometry. Studies have confirmed that ANX I was underexpressed in a majority of early stage prostate cancers (Kang et al., 2002; Ornstein and Tyson, 2006). ANX I expression were also decreased in androgen stimulated prostate cancer, androgenindependent prostate cancer and metastatic prostate cancer cell line (Smitherman et al., 2004; Wu et al., 2007). Although reduced expression levels of ANX I protein is a common finding in all stages of prostate cancer, a causative relationship between ANX I dysregulation and prostate cancer development has not yet been established. ANX I may have tumor suppressor functions in prostate cancer. Hsiang et al. reported that ANX I displayed its pro-apoptotic effect through involving in the activation of $\mathrm{p} 38$ and $\mathrm{JNK}$, which appeared to shift the balance of signal transduction away from proliferation and toward apoptosis (Hsiang et al., 2006).

The nuclear protein B23, nucleophosmin (NPM), is an RNA-associated nucleolus phosphoprotein reported to be more abundant in malignant and growing cells than in

Table 4-3 Example of cancer relevant phosphorproteins identified in LNCaP.

\begin{tabular}{ll}
\hline Functional categories & Protein name \\
\hline Apoptosis modulated protein & Peroxiredoxin-2, Annexin A1 \\
Molecular chaperones & T-complex protein 1 subunit zeta, T- \\
& complex protein 1 subunit gamma, \\
& Nucleophosmin \\
Multifunctional enzyme & Alpha-enolase \\
Carbohydrate metabolism enzyme & Transaldolase \\
Stress response protein & Stress-induced-phosphoprotein 1, \\
& Peroxiredoxin-2 \\
\hline
\end{tabular}


normal nondividing cells (Subong et al., 1999). Leotoing et al. reported the potential of NPM as a tumor marker for human prostate cancer. They found that NPM was overexpressed in prostate cancer compared to normal adjacent tissues, and could modulate AR functions by promoting assembly of AR-containing regulatory complexes. Thus, the high levels of NPM might alter AR functions in prostate cancer (Leotoing et al., 2008).

Two T-complex protein 1 (TCP) isoforms, T-complex protein 1 subunit zeta and T-complex protein 1 subunit gamma, were identified in the study. TCP's function is the binding of nucleotide, ATP and/or unfolded protein (Li et al., 1994). It involves the process of protein folding. Up-regulated TCP was found in highly metastatic prostate cancer cell line 1E8-H (Wu et al., 2007) and the cell line M12 (Liu et al., 2003). The high levels of TCP may reflect the alter AR functions in prostate cancer.

Enolase (ENOA or ENO1) is a multifunctional protein. Besides its role in basic metabolism, Enolase serves as a surface receptor for the binding of plasminogen (Andronicos et al., 1997; Lopez-Alemany et al., 2003). ENO1 has been implicated in numerous diseases, including cancers (Kanemoto et al., 2006; Katayama et al., 2006). Plow et al. have found that ENO1 involved tumor cell invasion and metastasis via initiating the activity of plasminogen activity on the cell surface (Plow et al., 1995). ENO1 has been reported highly expressed in tumor cells (Altenberg et al., 2006; Altenberg and Greulich, 2004). Pancholi demonstrated that the increased expression of ENO1 both at DNA level and at the protein level directly correlated the progression of tumors (Pancholi, 2001).

Transaldolase plays an important role in central metabolism. It is involved in oxidative stress and apoptosis, in multiple sclerosis, and in cancer (Samland and Sprenger, 2009). Transaldolase deficiency has been found to associate with liver cirrhosis in a new inborn (Verhoeven et al., 2001). Recently, Basta et al. demonstrated that genetic polymorphisms in Transaldolase were associated with squamous cell carcinoma of the head and neck (Basta et al., 2008).

\subsection{Conclusions}

In this study, we demonstrated the feasibility of using 2-DE with phsopho-specific staining and tandem mass spectrometry to investigate the phosphoproteins in the LNCaP human prostate cancer cell line. This methodology complements the other platforms that we used for prostate cancer phosphoproteomics research; it will be of particular value for future comparative studies of phosphoproteins in various physiological states. The phosphoproteins probed with the chosen analytical platform are known to be relevant to prostate cancer. 


\section{CHAPTER 5. PROTEOMIC ANALYSIS OF PROTEIN ALTERATIONS IN MOUSE PROSTATE CANCER TISSUE INDUCED BY BICALUTAMIDE/EMBELIN COMBINATION TREATMENT}

\subsection{Introduction}

Prostate cancer is a major health problem in Western countries. It is the most common cancer and the third leading cause of male cancer death (Jemal et al., 2006). During the initial stage of prostate cancer, the tumor is dependent on androgen for growth (Isaacs, 1994; Miller et al., 2003). For those without metastatic disease, androgen withdrawal/androgen ablation is the most effective therapy. Early treatment with the nonsteroidal antiandrogen bicalutamide significantly improves survival in patients with localized disease (Blackledge, 1996). However, the therapeutic success is temporary. Prolonged treatment results in the mutation of androgen receptor, which converts bicalutamide from an antagonist into an agonist, leading to drug resistance. Hence, in the majority of cases, the disease reappears within a few years as an androgen-independent form. There are no effective treatments for androgen-independent prostate cancer. Recently, clinical trials showed that docetaxel, in combination with estramustine could improve the survival of patients with metastatic, androgen-independent prostate cancer, as compared with mitoxantrone and prednisone (Petrylak et al., 2004). The combination of an androgen receptor antagonist (bicalutamide) and XIAP inhibitor (embelin) has been reported to cause regression of prostate tumors (Danquah et al., 2009). That result indicates that a treatment strategy with novel drug combination, delivered in a more effective way, is a promising way to treat androgen-independent prostate cancer.

$\mathrm{X}$-Linked inhibitor of apoptosis protein (XIAP) is a promising molecular target for the design of new anticancer drugs aiming at promoting apoptosis in cancer cells. Embelin is a novel cell-permeable inhibitor of XIAP, which binds to the XIAP BIR3 domain to which Smac and caspase-9 bind. Embelin selectively inhibits cell growth, induces apoptosis, and activates caspase-9 in prostate cancer cells (Chen et al., 2006; Nikolovska-Coleska et al., 2004).

Bicalutamide is a non-steroidal antiandrogen with affinity for androgen receptor. The low solubility/dissolution rate of bicalutamide is still a key factor limiting its oral bioavailability (Le et al., 2009). The use of amphiphilic copolymer micelles as effective carriers for tumor-drug delivery has drawn more attention in the last few years (Blanco et al., 2007; Ishihara et al., 2008; Lee et al., 2006; Yang et al., 2008; Yasugi et al., 1999). The amphiphilic copolymer can form a micelle structure with a hydrophobic inner core and a hydrophilic outer shell in aqueous environments. The core-shell structure makes it easy to incorporate hydrophobic drugs and results in an increased drug concentration in body fluids.

Proteomics is a powerful approach which enables to survey a large number of proteins simultaneously. It has been extensively applied to examine protein expression in various types of human cancers and in the context of resistance to several drugs 
(Cicchillitti et al., 2009; Di Michele et al., 2009; Ou et al., 2008; Rondepierre et al., 2009). Thus, proteomics offers excellent possibilities for determining proteins involved in the complex pharmacological effects of anticancer agents, and to provide an insight into the molecular mechanism of anticancer agents in prostate cancer.

The aim of this study was to identify proteins that are differentially expressed in control and bicalutamide/embelin treated mouse prostate tumor specimens. This study will aid in the revealing of molecular mechanism of bicalutamide/embelin combination therapy. A comparative proteomic approach based on 2-DE coupled with mass spectrometry (LC-MS/MS) was applied to mouse prostate tumors with bicalutamide/embelin combination treatment. An outline of the 2-DE based strategy is shown in Figure 5-1.

\subsection{Materials and Methods}

\subsubsection{In Vivo Studies}

This was a collaboration study with the group of Dr. Ram I. Mahato from the Department of Pharmaceutical Sciences, UTHSC. Dr. Mahato's group performed the in vivo studies. Briefly, all animal experiments were performed in accordance with $\mathrm{NIH}$ animal use guidelines and protocol approved by the Animal Care and Use Committee at the University of Tennessee Health Science Center. Xenograft flank tumors were induced in 8-weeks-old male BALB/C nude mice purchased from The Jackson Laboratory (Bar Harbor, ME) by subcutaneous injection of three million LNCaP cells suspended in 1:1 media and matrigel. When tumors reached approximately $150 \mathrm{~mm}^{3}$, mice were randomized into three groups of five mice, minimizing weight and tumor size difference. Each group was treated with intratumoral injection of saline, sonicated bicalutamide suspension or bicalutamide entrapped in PEG-PLA micelles $(20 \mathrm{mg} / \mathrm{kg})$ three times a week. Mice were treated for three weeks. After three weeks, treatments with bicalutamide were terminated, and treatment with embelin was commenced and continued for two weeks. Mice were sacrificed after bicalutamide /embelin combination treatment. Tumors were surgically removed, frozen in liquid nitrogen and stored at $-80^{\circ} \mathrm{C}$.

\subsubsection{Protein Preparation}

Proteins were extracted from $200 \mathrm{mg}$ of crushed tumor tissues by using $2 \mathrm{~mL}$ of Trizol reagent (Invitrogen, Carlsbad, CA) with $5 \mu \mathrm{L}$ of protease inhibitor cocktail (Sigma, St. Louis, MO) according the manufacturer's instruction (details described in Chapter 2). For 2-DE separations, protein pellet was dissolved with a rehydration buffer that contained urea (7 M), thiourea (2 M), CHAPS detergent (2\% w/v), IPG buffer 3-10 $(2 \% \mathrm{v} / \mathrm{v})$, DTT $(0.3 \%, \mathrm{w} / \mathrm{v})$ and a trace amount of Bromophenol Blue dye. The protein concentration of the extracts was determined by Bradford assay (GE Healthcare, Piscataway, NJ). 


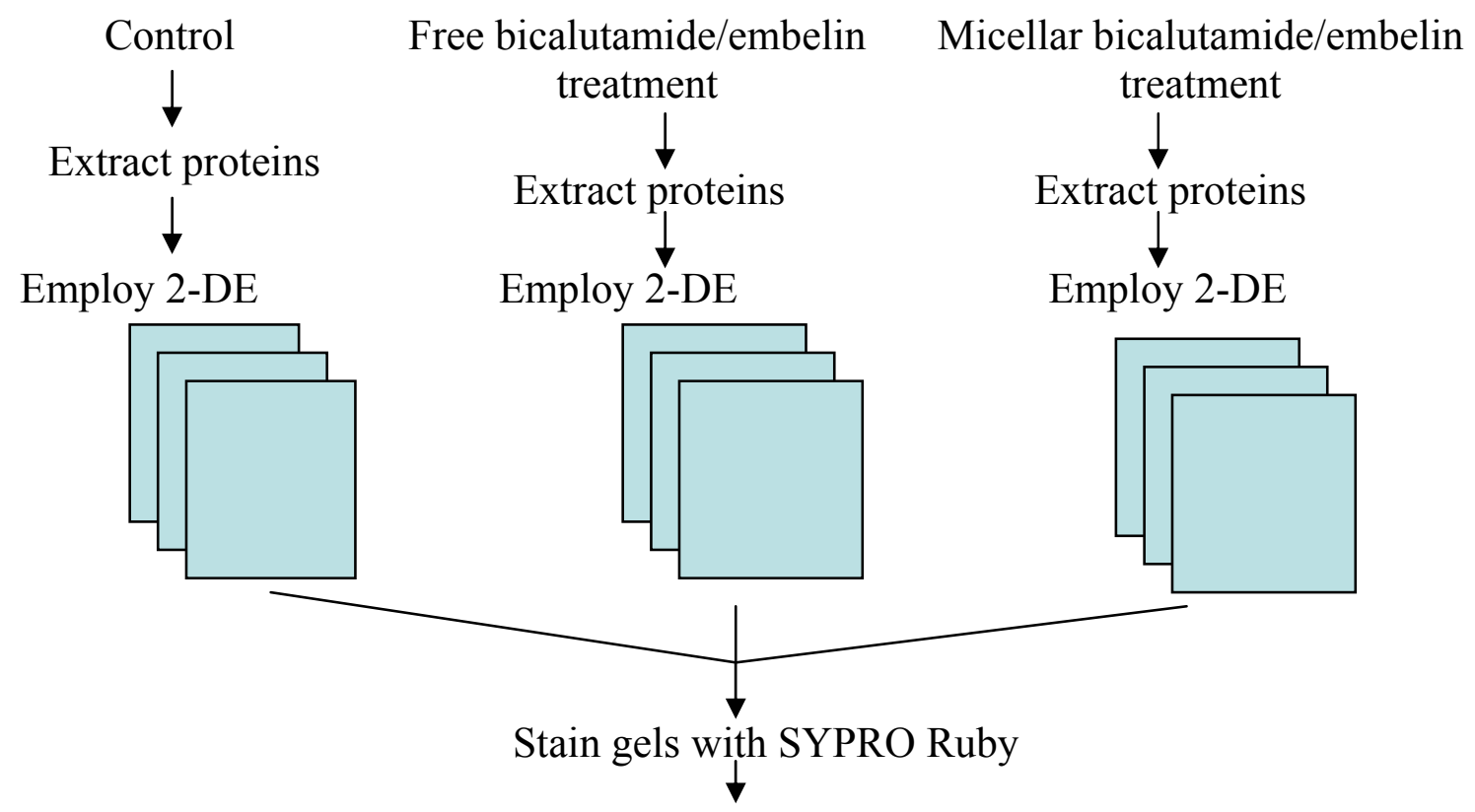

Computer-assisted analyze gel images, select spots with differential expression in three states

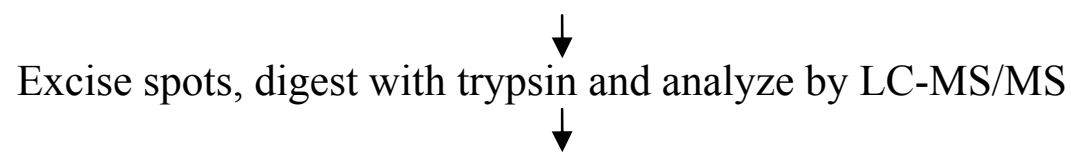

Identify proteins through database searches

Perform bioinformatics analysis for identified protein sets

(Gene Ontology, Ingenuity Pathway Analysis)

Figure 5-1 Outline of 2-DE based comparative proteomics platform.

Proteins were extracted from mouse prostate tumor tissues, and then separated by 2DE. Protein spots on 2-DE gels were visualized by staining with SYPRO Ruby and gel images were analyzed with computer-assisted gel image analysis software. The spots found to be statistically significant $(p<0.05)$ were excised, digested with trypsin, and analyzed by LC-MS/MS. Proteins were identified through database searches, and bioinformatics analyses were performed for final identified proteins. 


\section{$5.2 .3 \quad 2-D E$}

For the first dimension, protein samples $(250 \mu \mathrm{g}$ of protein in $350 \mu \mathrm{L}$ of rehydration buffer) were loaded on pre-cast, $18-\mathrm{cm}$ long immobilized $\mathrm{pH}$ gradient (IPG) stripes ( $\mathrm{pH}$ 4-7) (GE Healthcare, Piscataway, NJ). Isoelectric focusing was performed with the IPGphor apparatus (GE Healthcare, Piscataway, NJ) according to manufacturer's instructions (details described in Chapter 4). The isoelectric focusing was performed under the following voltage program: $30 \mathrm{~V}$ (fixed for 16 hours); $200 \mathrm{~V}$ (fixed for 1 hour); $500 \mathrm{~V}$ (fixed for 1 hour); $1000 \mathrm{~V}$ (fixed for 1 hour); $8000 \mathrm{~V}$ (gradient over 30 minutes); $8000 \mathrm{~V}$ (fixed for 4 hours ).

IPG strips were then incubated for 15 minutes in an equilibration buffer $(50 \mathrm{mM}$ Tris/ $\mathrm{HCl} \mathrm{pH} \mathrm{8.8,} 6 \mathrm{M}$ urea, 30\% glycerol, 2\% SDS) with 2.0\% DTT and then in equilibration buffer with $2.5 \%$ iodoacetamide for an additional 15 minutes. The second dimensional separations were carried out on $12 \%$ SDS- PAGE gels at $15^{\circ} \mathrm{C}$ with $200 \mathrm{~V}$ constant voltage applied for about 8 hours in a Protean Plus Dodeca Cell (Bio-Rad, Hercules, CA), until the bromophenol blue dye front reached the end of the gels.

\subsubsection{Gel Staining and Imaging}

Gels were stained with SYPRO Ruby protein stain (Molecular Probes, Eugene, OR). The image was acquired on an FX Fluorescence Laser Scanner (Bio-Rad, Hercules, CA) with $488 \mathrm{~nm}$ laser excitation and $555 \mathrm{~nm}$ band pass emission filter for SYPRO Ruby stain. The images were displayed with Adobe Photoshop 5.0 LE software, and saved as .TIFF files for future analysis. The gel images were analyzed using two independent and commercially available image analysis software packages, PDQuest version 7.1.0 (Bio-Rad, Hercules, CA) and Progenesis SameSpots version 3.3 (Nonlinear USA Inc, Durham, NC).

Using PDQuest software package, gel images were performed with the "Automated spot detection and matching wizard" tool. After grouping the gels within a specified match set (control, free bicalutamide, or micelle bicalutamide) and spot detection, the software automatically generated a "match set master gel" image, which is a synthetic gel image made up of all the spots that were detected within the match set by PDQuest. Each spots was assigned a unique identified number across all the gels by the software to permit comparison between groups. The density of each spot was normalized to the total density in the gel image and was expressed in parts per million (PPM).

The same 2-DE gel images were also analyzed using Progenesis SameSpot software package. Briefly, gel images were grouped into three categories (control, free bicalutamide and micelle bicalutamide) for comparative analysis. A reference gel was selected from all gel set to serve as a universal index for spot numbering prior to matching analysis. Spot detection, filtering, and background subtraction were automatically performed by the software using its unique nonparametric algorithm and 
applied to all the subgels in the particular group. Spots that did not match were manually warped and updated at the individual and reference gels.

Following confirmation of appropriate spot detection, matching and normalization, spot statistics were reviewed. ANOVA test was performed to quantify differential expression of spots among three groups. The spots found to be statistically significant $(\mathrm{p}<$ $0.05)$ were isolated for further investigation.

\subsubsection{In-gel Tryptic Digestion}

Spots of interest were excised from the gels. The gel pieces were extensively washed with $50 \mathrm{mM}$ ammonium bicarbonate in 50\% ACN for three times, and then dried under vacuum for 30 minutes. The dried gel pieces were rehydrated in a trypsin solution that contained $16.7 \mathrm{ng} / \mu \mathrm{L}$ of trypsin (Promega, Madison, WI) in $50 \mathrm{mM}$ ammonium bicarbonate $(\mathrm{pH} 8)$. The digestion solution was incubated at $37^{\circ} \mathrm{C}$ overnight. The resulting peptides were extracted with $0.01 \%$ TFA/ $60 \%$ ACN three times. All supernatants were combined and dried with a vacuum centrifuge. Prior to LC-MS/MS analysis, the digestions were reconstituted with $15 \mu \mathrm{L}$ of $2.5 \%$ FA and desalted with ZipTipC18 (Millipore, Billerica, MA), using the procedure provided by the manufacturer (details described in Chapter 2). The peptides retained on the ZipTipC18 column were eluted with $1.5 \mu \mathrm{L}$ of $50 \%$ ACN/0.1\% FA and diluted with $2.5 \mu \mathrm{L}$ of $0.5 \%$ FA.

\subsubsection{LC-MS/MS Analysis}

LC-MS/MS experiments were performed on a LTQ linear ion trap mass spectrometer (Thermo Electron, San Jose, CA). Peptide mixtures were loaded onto a fused-silica microcapillary column (15 cm length, $75 \mu \mathrm{m}$ ID; New Objective, MA) packed in-house with $\mathrm{C} 18$ resin (Michrom Bioresources, Inc., Auburn, CA) and were separated using a 30-min gradient from $0 \%$ to $90 \%$ solvent $\mathrm{B}(0.05 \% \mathrm{FA} / 90 \% \mathrm{MeOH})$. Solvent A was $0.05 \% \mathrm{FA} / 2 \% \mathrm{MeOH}$. The LTQ mass spectrometer was operated in positive ion mode and data-dependent acquisition was performed. In brief, a scan cycle was initiated with a full scan of wide mass accuracy ( $\mathrm{m} / \mathrm{z} 400-2000)$ in the ion trap, which was followed by MS/MS scans in the linear ion trap on the 7 most abundant precursor ions with dynamic exclusion of previously selected ions.

\subsubsection{Database Searches}

The MS/MS data were used to search the Swiss-Prot database (subset of mouse proteins) with the TurboSEQUEST search engine (Thermo Electron) that is part of Bioworks version 3.2 (Thermo Electron, San Jose, CA). Searches were performed with the following parameters: full-trypsin specificity, dynamic modifications of oxidized methionine, and static modifications of cystein by carbamidomethylation. All peptide matches were filtered by XCorr larger than 3.00, 2.50, and 3.50 used for singly, doubly 
and triply charged ions, respectively; peptide probability less than 1.0E-3; different peptides; and only the top matches were considered. The peptides retrieved by the searches were validated by manual inspection of the MS/MS data. Criteria used for manual validation included: the spectrum must be of good quality with fragment ion clearly above the baseline noise; sequential members of the b- or y-series were observable in the mass spectra.

\subsubsection{Bioinformatics and Network Analysis}

The proteins identified as being differentially expressed among treatment groups were compared to all annotated proteins using gene ontology (GO). This analysis was carried out using the Web-accessible program (http://bioinfo.vanderbilt.edu/webgestalt/). Proteins were classified with GO annotation at level 4 according to the molecular function that proteins normally carry out, the biological process that proteins are involved in, and the molecular component (subcellular location) of the proteins.

Identified proteins were also analyzed by Ingenuity Pathway Analysis (IPA; Ingenuity System, Mountain View, CA). IPA is a powerful curated database and analysis system for understanding how proteins work together to affect cellular changes. It builds hypothetical networks from identified proteins and other proteins, based on a regularly updated database which consists of millions of individual relationships between genes, proteins and other biological molecules, based on information compiled from published literature. It enables to pinpoint the biological mechanisms, pathways and functions most relevant to the experimental datasets or genes of interest. IPA generates a score for each possible network, which is calculated according to the fit of that network to the input proteins. The higher score indicates a lower possibility that the input proteins occur in a given network by random chance. Networks with scores of 10 or higher have a high confidence of not being generated by random chance alone.

\subsection{Results}

The aim of the study was to identify proteins whose differential expression relates to bicalutamide/embelin treatment in mouse prostate tumors and to reveal the molecular mechanism of prostate tumor regression induced by the combination of anticancer drugs.

We employed a 2-DE based approach coupled with mass spectrometry for protein identification. For gaining insight into the mechanisms of drug action, the ability to reproducibly measure the protein expression levels across the experiments is a key challenge. Hence, it is important to realize the contribution of technical variations in measuring quantitative differences. In the present study, technical variation was determined by employing three gels per sample.

Correlation analysis was used to determine the reproducibility of the spot volume within and between a set of gels (Zhan and Desiderio, 2003). The coefficient of 
correlation of the normalized spot volum across three replicates per sample ranged from 0.89 to 0.98 . The coefficient of variation $(\mathrm{CV})$ is a quantitative index, describing the amount of the variation of volume among the matched-spots. The average CV was calculated from all spots matched on gels within an experiment set. The average CV for replicate 2-DE gels of all three sample types was $20.7 \pm 15.8 \%$, which was comparable with the reported results $35.7 \pm 20.8 \%$ for Dodeca system by Zhan and Desiderio (Zhan and Desiderio, 2003). This result was also consistent with several other published reports on the degree of variation attributed to 2-DE in the hands of the users (Molloy et al., 2003; Saldanha et al., 2008).

In order to describe in the detail of the difference in the amount of variation of the normalized volume of the matched-spots, the proportion of the matched-spots among the different $\mathrm{CV}$ ranges of the normalized volume is described in Table 5-1. The majority of spots was matched within the low $\mathrm{CV}$ range $(70 \%$ of matched spots with $\mathrm{CV}<25 \%, 90 \%$ of matched spots with $\mathrm{CV}<50 \%$ ). Small proportion of spots was matched in high $\mathrm{CV}$ range. The results was also similar to the previous reported results based on the Dodeca system of the study (77\% of matched spots with CV $<50 \%$ ) (Zhan and Desiderio, 2003). The degree of technical variation due to the 2-DE process established an important baseline for uncoupling biological variation from technical variation.

\subsubsection{Profile of Mouse Prostate Tumor Specimens}

To study the molecular mechanisms of bicalutamide/embelin combination therapy in prostate cancer, three groups of mouse prostate tumor specimens were used in this work. Prostate tumors from mice treated with saline were used as controls. The controls were compared to two combination treatments: free-bicalutamide/embelin and PEG-PLA bicalutamide micelles/embelin.

\subsubsection{Proteomic Patterns of Bicalutamide/embelin Combination Treatment}

2-DE was used to separate protein mixture and to locate differentially expressed proteins among control, free-bicalutamide/embelin and PEG-PLA bicalutamide micelles/embelin treated mouse prostate cancer tissues. Patterns from three different mouse specimens were analyzed and three replicate 2-DE gels per specimen were used. Figure 5-2 shows a representative 2-DE gel of proteins for mouse prostate tumor obtained by using an IPG 4-7 strips and a 12\% SDS-PAGE gel. 2-DE patterns from the three treatment groups were obtained and analyzed with two different image analysis software packages (Bio-Rad PDQuest Version 7.1 software and Progenesis Samespots v3.3 software). Overall, more than 600 spots were resolved in the 2-DE gels and the protein expression profiles among the three groups were similar; a set of 33 protein spots exhibiting significantly differential expression by ANOVA test $(\mathrm{p}<0.05)$ are located (Figure 5-2) and analyzed by nano-LC-MS/MS. 
Table 5-1 Proportion of the matched spots among the different ranges of the CV of the normalized volume.

\begin{tabular}{lllllll}
\hline \multirow{2}{*}{$\begin{array}{l}\text { CV of } \\
\text { normalized } \\
\text { volume (\%) }\end{array}$} & $\begin{array}{l}\text { Number of } \\
\text { matched } \\
\text { spots }\end{array}$ & $\begin{array}{l}\text { Propor- } \\
\text { tion (\%) }\end{array}$ & $\begin{array}{l}\text { Number of } \\
\text { matched } \\
\text { spots }\end{array}$ & $\begin{array}{l}\text { Propor- } \\
\text { tion }(\%)\end{array}$ & $\begin{array}{l}\text { Number of } \\
\text { matched } \\
\text { spots }\end{array}$ & $\begin{array}{l}\text { Propor- } \\
\text { tion (\%) }\end{array}$ \\
\hline$<10$ & 93 & 20.9 & 220 & 49.4 & 95 & 21.3 \\
$10-25$ & 175 & 39.4 & 189 & 42.5 & 192 & 43.2 \\
$25-50$ & 123 & 27.6 & 30 & 6.7 & 107 & 24.1 \\
$50-75$ & 41 & 9.2 & 6 & 1.4 & 45 & 10.1 \\
$75-100$ & 10 & 2.2 & 0 & 0 & 5 & 1.1 \\
$>100$ & 3 & 0.7 & 0 & 0 & 1 & 0.2 \\
Total & 445 & 100 & 445 & 100 & 445 & 100 \\
\hline
\end{tabular}


(a)

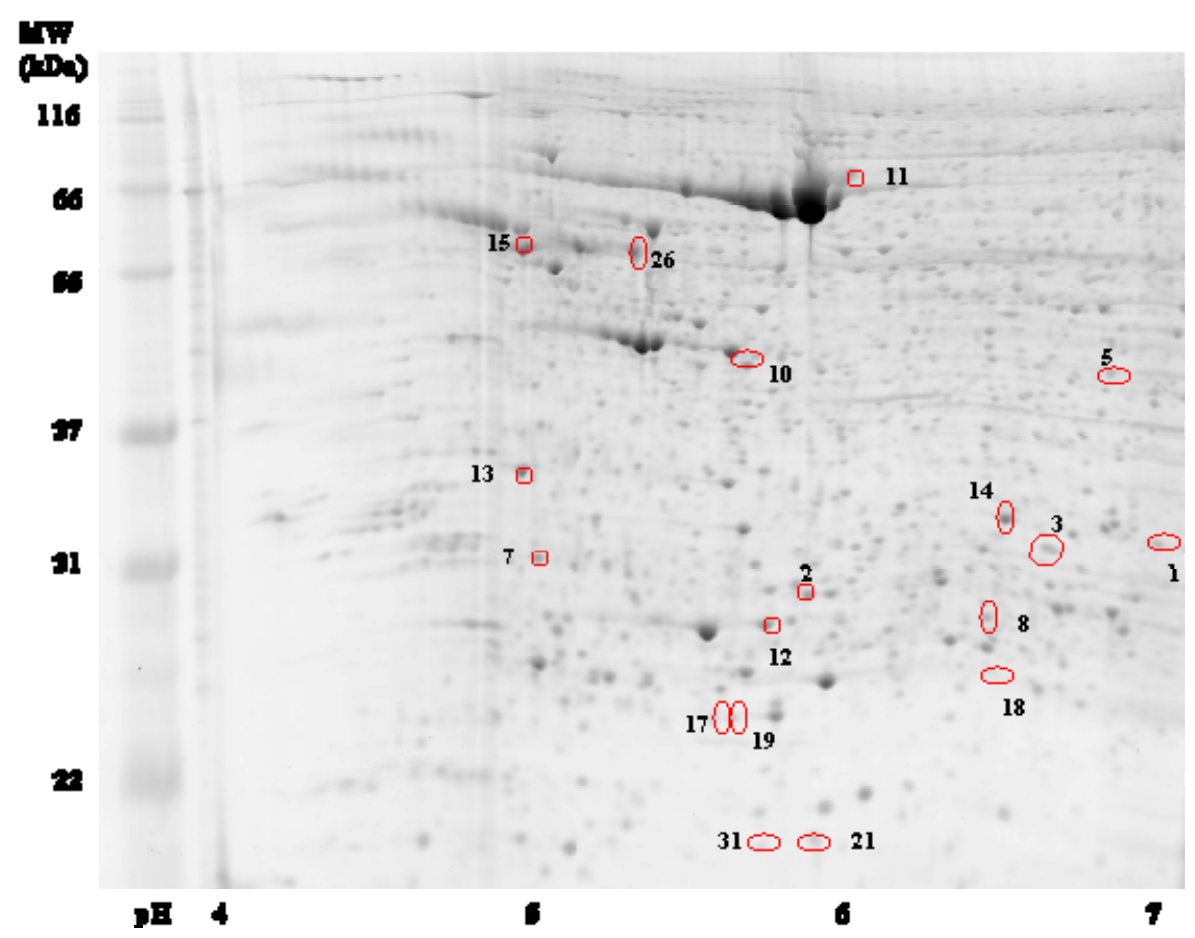

(b)

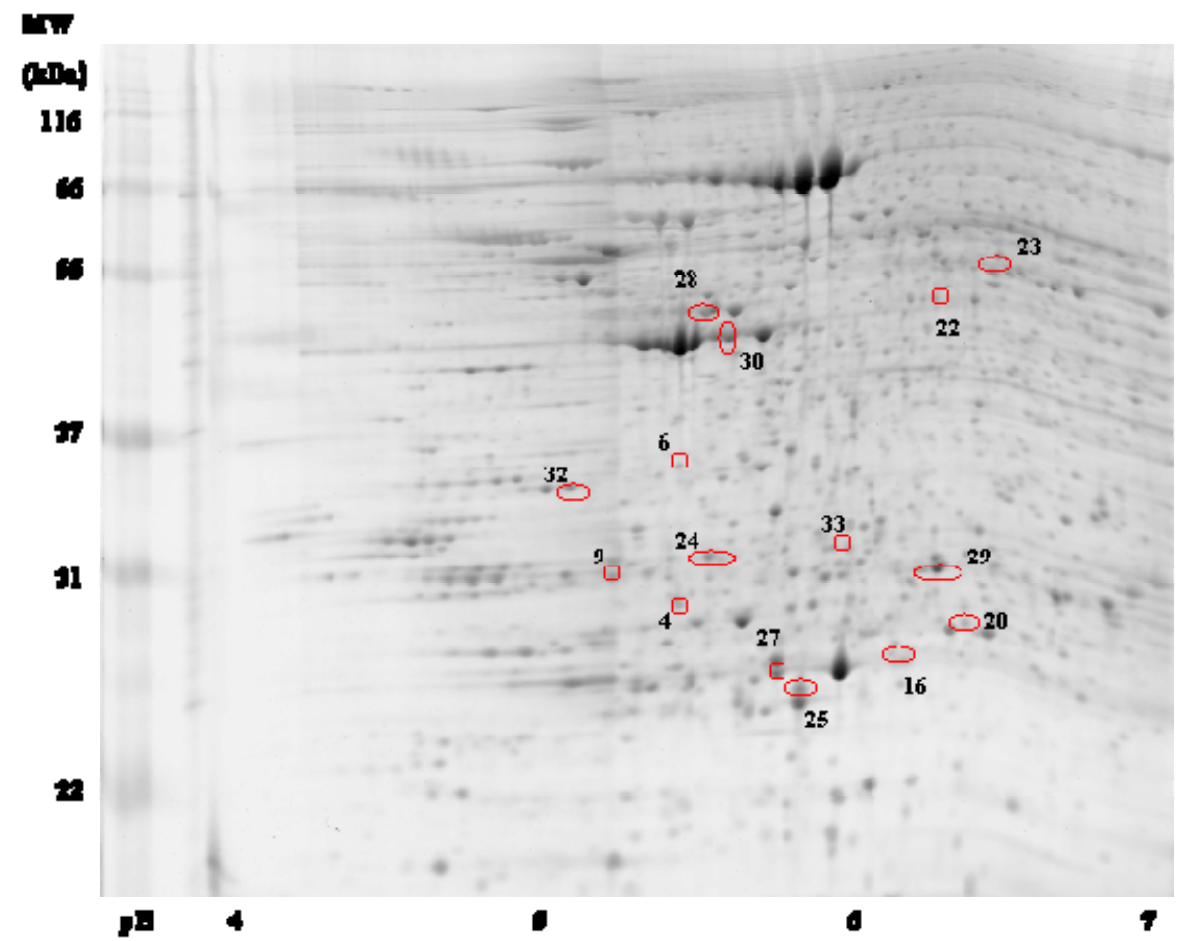

Figure 5-2 Representative 2-DE gels of mouse prostate cancer proteome (a) Control (b) Free bicalutamide/embelin treatment.

33 protein spots marked on the maps were differentially expressed $(p<0.05)$ in the comparisons between control and treated samples; these proteins were identified by MS. 


\subsubsection{Identification of Candidate Protein Spots from 2-DE Gels}

The selected protein spots from SYPRO Ruby stained gels were excised and subjected to in-gel tryptic digestion. The extracted peptides were analyzed by LC-MS/MS to generate sequence-diagnostic MS/MS data. Out of the 33 differentially expressed protein spots, 30 protein spots were successfully identified, corresponding to 38 different proteins (Table 5-2 and Table 5-3). Overall, 19 proteins were identified in protein spots which were significantly up-regulated in bicalutamide/embelin treated specimens compared with control tumor specimens, such as peroxiredoxin-1, Glutathione peroxidase 1, enolase, and peroxiredoxin-2. Moreover, 19 proteins were identified from down-regulated protein spots in bicalutamide/embelin treated specimens compared with control tumor specimens, including plasminogen, annexin 5 and pre-mRNA-splicing factor SPF27. The summary score, protein coverage, and change fold for spots are shown in Table 5-2 and Table 5-3. It should be noted that some of the proteins were identified in multiple spots; furthermore, some of the spots contained more than one protein.

\subsubsection{Classification of Identified Proteins}

To interpret the results systematically, identified proteins were classified with GO annotation of the level 4 according to molecular function that proteins normally carry out, biological process that proteins are involved in, and molecular component (subcellular location).

The majority of the identified proteins were associated with metabolic processes (cellular metabolism $51.5 \%$, primary metabolism $51.5 \%$, and macromolecular metabolism 33.3\%). In addition to metabolic process, the biological processes which the differentially expressed proteins were involved in included localization (51.5\%), transport (33.3\%), response to stress (12.1\%) and apoptosis (12.1\%) (Figure 5-3). Based on GO annotations, identified proteins were involved in various molecular functions (Figure 5-4), such as binding (cation binding 21\%, metal ion binding 24\%, RNA binding $9 \%$ ), oxidoreductase activity ( $9 \%$ ), and peptidase activity (9\%). The classification is redundant since a protein can be classified into more than one category.

To gain further insight in the differentially expressed protein results, separation was made into up-regulated and down-regulated identified proteins (Table 5-4). According to GO annotations, most identified proteins in both groups involved "binding" in molecular function. But proteins in the up-regulated group were associated with "oxidoreductase activity", while very few proteins belonged to that category in the downregulated group. In the category of biological process, both up- and down-regulated proteins were classified into "cellular physiological process" and "metabolism". 
Table 5-2 List of identified protein spots down-regulated by bicalutamide/embelin treatment.

\begin{tabular}{|c|c|c|c|c|c|c|c|c|c|}
\hline Spot & $\begin{array}{l}\text { Accession } \\
\text { number }\end{array}$ & Protein name & $\begin{array}{l}\text { Theor } \\
\text { MW } \\
\text { (kDa)/pI }\end{array}$ & $\begin{array}{l}\text { Peptide } \\
\text { number }\end{array}$ & $\begin{array}{l}\text { Coverage } \\
(\%)\end{array}$ & $\begin{array}{l}\text { Fold change } \\
\text { (Progenesis) }\end{array}$ & $\begin{array}{l}\text { P-value } \\
\text { (Progenesis) }\end{array}$ & $\begin{array}{l}\text { Fold } \\
\text { change } \\
\text { (PDQuest) }\end{array}$ & $\begin{array}{l}\text { P-value } \\
\text { (PDQuest) }\end{array}$ \\
\hline 1 & $\begin{array}{l}\text { P60909 } \\
\text { RS3_MOUSE }\end{array}$ & $\begin{array}{l}40 \mathrm{~S} \text { ribosomal } \\
\text { protein } \mathrm{S} 3\end{array}$ & $26.7 / 9.6$ & 1 & 5.3 & 1.9 & $<0.05$ & 2 & $<0.01$ \\
\hline 2 & $\begin{array}{l}\text { P01843 } \\
\text { LAC1_MOUSE }\end{array}$ & $\begin{array}{l}\text { Ig lambd-1 } \\
\text { chain } \mathrm{C} \text { region }\end{array}$ & $11.6 / 5.8$ & 3 & 46.7 & 1.6 & $<0.05$ & 3.2 & $<0.01$ \\
\hline 3 & $\begin{array}{l}\text { P63159 } \\
\text { HMGB1_MOUS } \\
\text { E }\end{array}$ & $\begin{array}{l}\text { High mobility } \\
\text { group protein } \\
\text { B1 }\end{array}$ & $24.9 / 5.6$ & 4 & 19.1 & 2.6 & $<0.01$ & $\begin{array}{l}\text { Out of } \\
\text { range }\end{array}$ & $<0.01$ \\
\hline 4 & $\begin{array}{l}\text { Q9D287 } \\
\text { BCAS2_MOUSE }\end{array}$ & $\begin{array}{l}\text { Pre-mRNA- } \\
\text { splicing factor } \\
\text { SPF27 }\end{array}$ & $26.1 / 5.4$ & 2 & 11.1 & 1.3 & $>0.05$ & 1.2 & $<0.01$ \\
\hline \multirow[t]{2}{*}{5} & $\begin{array}{l}\text { Q61990 } \\
\text { PCBP2_MOUSE }\end{array}$ & $\begin{array}{l}\text { Poly(rC)- } \\
\text { binding protein } \\
2\end{array}$ & $38.2 / 6.3$ & 9 & 33.1 & 1.4 & $>0.05$ & 1.4 & $<0.01$ \\
\hline & $\begin{array}{l}\text { P61161 } \\
\text { ARP2_MOUSE }\end{array}$ & $\begin{array}{l}\text { Actin-related } \\
\text { protein } 2\end{array}$ & $44.8 / 6.2$ & 6 & 19.8 & & & & \\
\hline 6 & $\begin{array}{l}\text { Q6467̄ } \\
\text { SPEE_MOUSE }\end{array}$ & $\begin{array}{l}\text { Spermidine } \\
\text { synthase }\end{array}$ & $33.9 / 5.3$ & 5 & 29.1 & 1.6 & $<0.05$ & 1.1 & $<0.01$ \\
\hline 7 & $\begin{array}{l}\text { Q99PT̄1 } \\
\text { GDIR_MOUSE }\end{array}$ & $\begin{array}{l}\text { Rho GDP- } \\
\text { dissociation } \\
\text { inhibitor } 1\end{array}$ & $23.4 / 5.1$ & 7 & 38.2 & 1.3 & $<0.05$ & $\begin{array}{l}\text { Out of } \\
\text { range }\end{array}$ & $<0.01$ \\
\hline 8 & $\begin{array}{l}\text { P23506 } \\
\text { PIMT_MOUSE }\end{array}$ & $\begin{array}{l}\text { Protein-L- } \\
\text { isoaspartate(D- } \\
\text { aspartate) O- } \\
\text { methyltransfera } \\
\text { se }\end{array}$ & $24.6 / 7.1$ & 5 & 26.4 & 1.8 & $<0.05$ & 2.6 & $<0.01$ \\
\hline
\end{tabular}


Table 5-2 (continued).

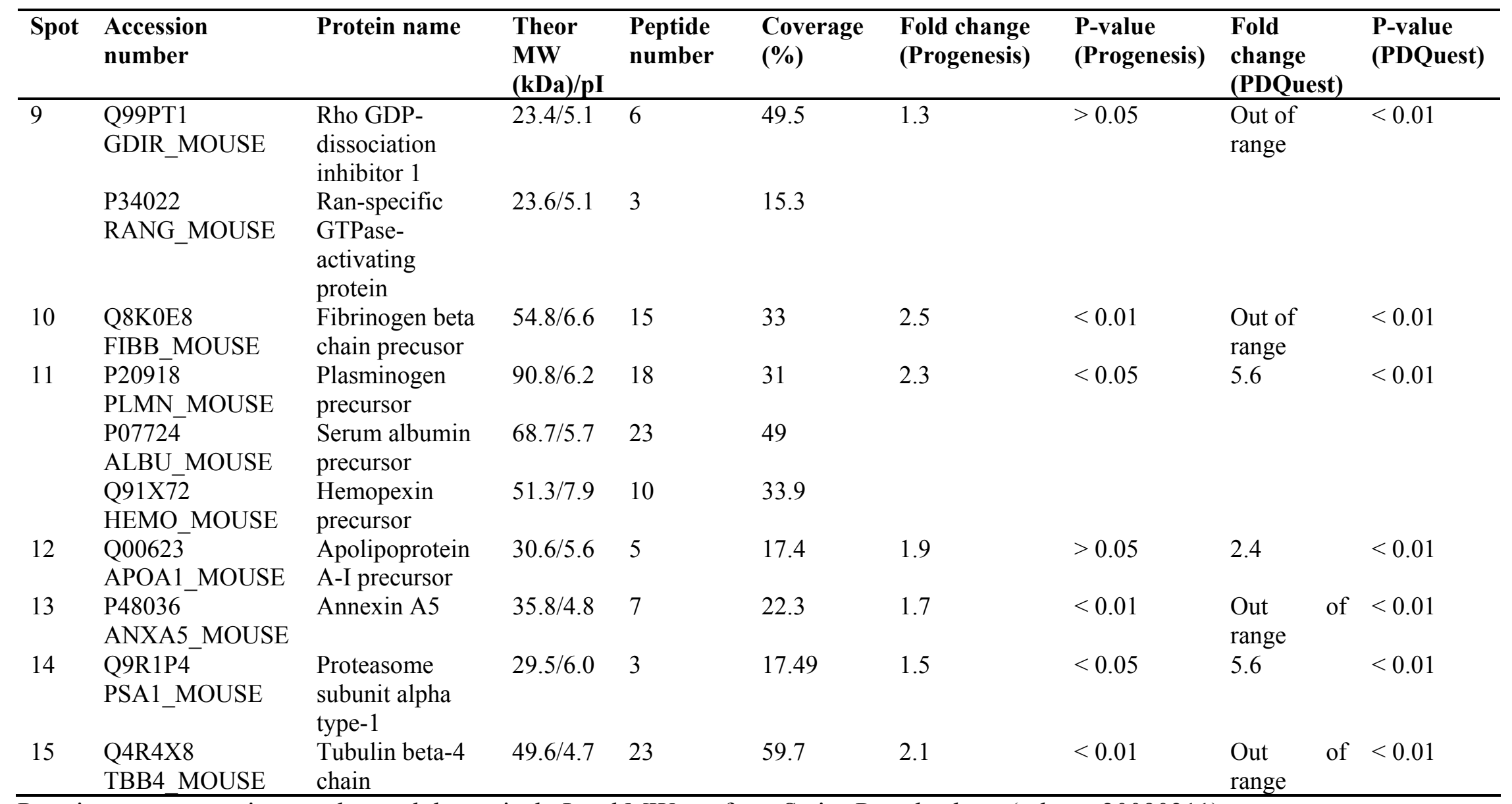

Protein name, accession number and theoretical pI and MW are from Swiss-Prot database (release 20090311)

Out of range indicates spot was absent in replicate gels or was below the detection parameters set for the software package. 
Table 5-3 List of identified protein spots up-regulated by bicalutamide/embelin treatment.

\begin{tabular}{|c|c|c|c|c|c|c|c|c|c|}
\hline Spot & $\begin{array}{l}\text { Accession } \\
\text { number }\end{array}$ & Protein name & $\begin{array}{l}\text { Theor } \\
\text { MW/pI }\end{array}$ & $\begin{array}{l}\text { Peptide } \\
\text { number }\end{array}$ & $\begin{array}{l}\text { Coverage } \\
(\%)\end{array}$ & $\begin{array}{l}\text { Fold } \\
\text { change } \\
\text { (Progenesis) } \\
\end{array}$ & $\begin{array}{l}\text { P-value } \\
\text { (Progenesis) }\end{array}$ & $\begin{array}{l}\text { Fold } \\
\text { change } \\
\text { (PDQuest) }\end{array}$ & $\begin{array}{l}\text { P-value } \\
\text { (PDQuest) }\end{array}$ \\
\hline 16 & $\begin{array}{l}\text { P29391 } \\
\text { FRIL1_MOUSE }\end{array}$ & $\begin{array}{l}\text { Ferritin light } \\
\text { chain1 }\end{array}$ & $20.8 / 5.6$ & 6 & 47.0 & 1.3 & $>0.05$ & $\begin{array}{l}\text { Out of } \\
\text { range }\end{array}$ & $<0.01$ \\
\hline 17 & $\begin{array}{l}\text { P0952 } \overline{8} \\
\text { FRIH_MOUSE }\end{array}$ & $\begin{array}{l}\text { Ferritin heavy } \\
\text { chain }\end{array}$ & $21.1 / 5.5$ & 6 & 40.1 & 1.8 & $<0.01$ & 2.4 & $<0.01$ \\
\hline 18 & $\begin{array}{l}\text { P35700 } \\
\text { PRDX1_MOUS } \\
\text { E }\end{array}$ & Peroxiredoxin-1 & $22.2 / 8.2$ & 5 & 25.1 & 1.3 & $>0.05$ & $\begin{array}{l}\text { Out of } \\
\text { range }\end{array}$ & $<0.01$ \\
\hline 19 & $\begin{array}{l}\text { P46638 } \\
\text { RB11B_MOUS } \\
\text { E }\end{array}$ & $\begin{array}{l}\text { Ras-related } \\
\text { protein Rab-11B }\end{array}$ & $24.5 / 5.6$ & 2 & 10.6 & 2.4 & $<0.01$ & 2.2 & $<0.01$ \\
\hline 20 & $\begin{array}{l}\text { P11352 } \\
\text { GPX1_MOUSE } \\
\text { Q99LX0 } \\
\text { PARK7_MOUS } \\
\text { E }\end{array}$ & $\begin{array}{l}\text { Glutathione } \\
\text { peroxidase } 1 \\
\text { Protein DJ-1 }\end{array}$ & $\begin{array}{l}22.2 / 6.7 \\
20.0 / 6.3\end{array}$ & $\begin{array}{l}8 \\
1\end{array}$ & $\begin{array}{l}55.7 \\
7.90\end{array}$ & 2 & $>0.05$ & $\begin{array}{l}\text { Out of } \\
\text { range }\end{array}$ & $<0.01$ \\
\hline 21 & $\begin{array}{l}\text { P29391 } \\
\text { FRIL1_MOUSE }\end{array}$ & $\begin{array}{l}\text { Ferritin light } \\
\text { chain1 }\end{array}$ & $20.8 / 5.6$ & 5 & 34.4 & 1.4 & $>0.05$ & 1.8 & $<0.01$ \\
\hline 22 & $\begin{array}{l}\text { P50247 } \\
\text { SAHH_MOUS } \\
\text { E }\end{array}$ & $\begin{array}{l}\text { Adenosylhomo- } \\
\text { cysteinase }\end{array}$ & $47.7 / 6.1$ & 10 & 25.7 & 1.6 & $<0.01$ & $\begin{array}{l}\text { Out of } \\
\text { range }\end{array}$ & $<0.01$ \\
\hline 23 & $\begin{array}{l}\text { Q9D8N0 } \\
\text { EF1G_MOUSE } \\
\text { P17182 } \\
\text { ENOA_MOUS } \\
\text { E }\end{array}$ & $\begin{array}{l}\text { Elongation factor } \\
\text { 1-gamma } \\
\text { Alpha-enolase }\end{array}$ & $\begin{array}{l}50.1 / 6.3 \\
47.1 / 6.3\end{array}$ & $\begin{array}{l}10 \\
3\end{array}$ & $\begin{array}{l}30.4 \\
8.80\end{array}$ & 1.9 & $<0.05$ & $\begin{array}{l}\text { Out of } \\
\text { range }\end{array}$ & $<0.01$ \\
\hline 24 & $\begin{array}{l}\text { P01837 } \\
\text { KAC_MOUSE }\end{array}$ & $\begin{array}{l}\text { Ig kappa chain } \mathrm{C} \\
\text { region }\end{array}$ & $11.8 / 5.2$ & 2 & 26.4 & ND & NA & $\begin{array}{l}\text { Out of } \\
\text { range }\end{array}$ & $<0.01$ \\
\hline 25 & 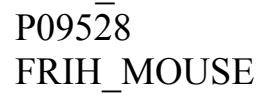 & $\begin{array}{l}\text { Ferritin heavy } \\
\text { chain }\end{array}$ & $21.1 / 5.5$ & 7 & 40.66 & 2.2 & $<0.01$ & 2.1 & $<0.01$ \\
\hline
\end{tabular}


Table 5-3 (continued).

\begin{tabular}{|c|c|c|c|c|c|c|c|c|c|}
\hline Spot & $\begin{array}{l}\text { Accession } \\
\text { number }\end{array}$ & Protein name & $\begin{array}{l}\text { Theor } \\
\text { MW/pI }\end{array}$ & $\begin{array}{l}\text { Peptide } \\
\text { number }\end{array}$ & $\begin{array}{l}\text { Coverage } \\
(\%)\end{array}$ & $\begin{array}{l}\text { Fold } \\
\text { change } \\
\text { (Progenesis) } \\
\end{array}$ & $\begin{array}{l}\text { P-value } \\
\text { (Progenesis) }\end{array}$ & $\begin{array}{l}\text { Fold } \\
\text { change } \\
\text { (PDQuest) }\end{array}$ & $\begin{array}{l}\text { P-value } \\
\text { (PDQuest) }\end{array}$ \\
\hline \multirow[t]{2}{*}{26} & $\begin{array}{l}\text { P21614 } \\
\text { VTDB_MOUSE }\end{array}$ & $\begin{array}{l}\text { Vitamin D- } \\
\text { binding protein } \\
\text { precuosor }\end{array}$ & $53.6 / 5.4$ & 21 & 55.5 & 1.3 & $>0.05$ & 4.1 & $<0.01$ \\
\hline & $\begin{array}{l}\text { Q9D554 } \\
\text { SF3A3_MOUSE }\end{array}$ & $\begin{array}{l}\text { Splicing factor } \\
\text { 3A subunit } 3\end{array}$ & $58.8 / 5.2$ & 5 & 14.2 & & & & \\
\hline 27 & $\begin{array}{l}\text { P29391 } \\
\text { FRIL1_MOUSE } \\
\text { Q61171 } \\
\text { PRDX2_MOUSE }\end{array}$ & $\begin{array}{l}\text { Ferritin light } \\
\text { chain 1 } \\
\text { Peroxiredoxin-2 }\end{array}$ & $\begin{array}{l}20.8 / 5.6 \\
21.8 / 5.2\end{array}$ & 1 & $\begin{array}{l}55.2 \\
5.50\end{array}$ & 2.2 & $<0.01$ & $\begin{array}{l}\text { Out of } \\
\text { range }\end{array}$ & $<0.01$ \\
\hline \multirow[t]{2}{*}{28} & $\begin{array}{l}\text { P60842 } \\
\text { IF2A1_MOUSE }\end{array}$ & $\begin{array}{l}\text { Eukaryotic } \\
\text { initiation factor } \\
\text { 4A-I }\end{array}$ & $46.2 / 5.3$ & 24 & 48.5 & 1.1 & $>0.05$ & $\begin{array}{l}\text { Out of } \\
\text { range }\end{array}$ & $<0.01$ \\
\hline & $\begin{array}{l}\text { P10630 } \\
\text { IF4A2_MOUSE }\end{array}$ & $\begin{array}{l}\text { Eukaryotic } \\
\text { initiation factor } \\
4 \text { A-II }\end{array}$ & $46.4 / 5.3$ & 2 & 8.3 & & & & \\
\hline \multirow[t]{2}{*}{29} & $\begin{array}{l}\text { Q3U0B3 } \\
\text { DHR11_MOUSE }\end{array}$ & $\begin{array}{l}\text { Dehydrogenase/ } \\
\text { reductase SDP } \\
\text { family member } \\
11 \text { precursor }\end{array}$ & $28.3 / 5.9$ & 2 & 11.5 & 1.4 & $>0.05$ & 1.8 & $<0.01$ \\
\hline & $\begin{array}{l}\text { Q9QUM9 } \\
\text { PSA6_MOUSE }\end{array}$ & $\begin{array}{l}\text { Proteasome } \\
\text { subunit alpha } \\
\text { type-6 }\end{array}$ & $27.4 / 6.3$ & 5 & 22.8 & & & & \\
\hline 30 & $\begin{array}{l}\text { Q04447 } \\
\text { KCRB_MOUSE }\end{array}$ & $\begin{array}{l}\text { Creatine kinase } \\
\text { B-type }\end{array}$ & $42.7 / 5.4$ & 11 & 35.7 & 1.7 & $>0.05$ & 2.6 & $<0.01$ \\
\hline
\end{tabular}

Protein name, accession number and theoretical pI and MW are from Swiss-Prot database (release 20090311)

Out of range indicates spot was absent in replicate gels or was below the detection parameters set for the software package. ND means spots not been detected. 


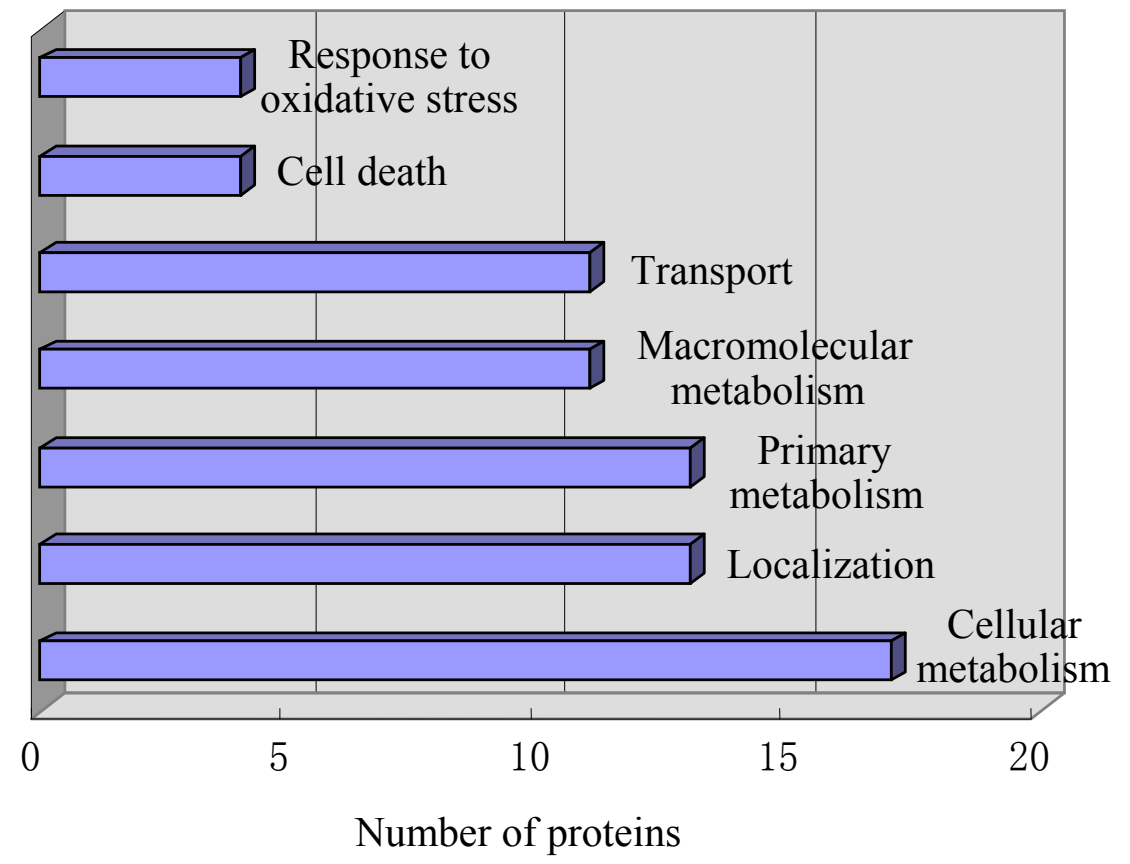

Figure 5-3 Classification of biological processes of identified proteins according to GO annotations. 


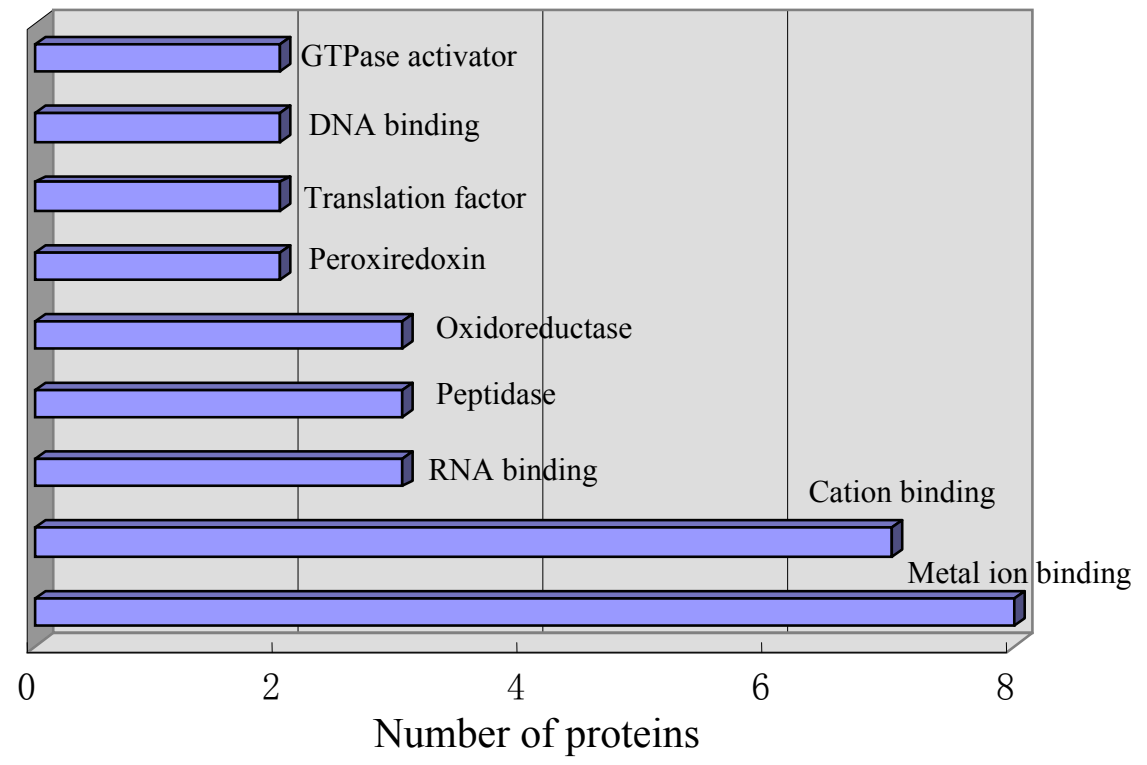

Figure 5-4 Classification of molecular functions of identified proteins according to GO annotations. 
Table 5-4 Protein categories by Gene Ontology.

\begin{tabular}{lcll}
\hline Molecular function & Ratio & Biological process & Ratio \\
\hline Up-regulated proteins & & & \\
Protein binding & $2 / 8$ & Cellular physiological process & $14 / 24$ \\
Ion binding & $3 / 8$ & Metabolism & $10 / 18$ \\
Nucleic binding & $3 / 6$ & Localization & $6 / 13$ \\
Hydrolase activity & $2 / 5$ & Response to stress & $4 / 8$ \\
Oxidoreductase activity & $5 / 5$ & death & $2 / 4$ \\
& & & \\
Down-regulated proteins & & & \\
Protein binding & $6 / 8$ & Cellular physiological process & $10 / 24$ \\
Ion binding & $5 / 8$ & Metabolism & $8 / 18$ \\
Nucleic binding & $3 / 6$ & Localization & $7 / 13$ \\
Hydrolase activity & $3 / 5$ & Response to stress & $4 / 8$ \\
Oxidoreductase activity & $0 / 5$ & death & $2 / 4$ \\
\hline
\end{tabular}

\subsubsection{Pathway Analysis of Proteins Modulated in Bicalutamide/embelin Treatment}

To reveal the potential molecular mechanism of bicalutamide/embelin combination treatment in prostate cancer, pathway analysis was carried out on the dataset of identified proteins through the use of Ingenuity Pathway Analysis software. The most significant interaction networks associated with identified proteins were determined by using published interactions between genes, proteins and other biological molecules. Three high ranking networks were identified: (a) Small molecule biochemistry, tissue morphology; (b) RNA post-transcriptional modification, gene expression, RNA trafficking; (c) Behavior, cancer and carbohydrate metabolism (Table 5-5). Canonical pathways associated with altered proteins included acute phase response signaling, and metabolism. Figure 5-5 is the highest scored cluster of small molecule biochemistry. The score for the depicted network was 32, which indicated the possibility of matching the indicated proteins by a random chance was $10^{-32}$.

\subsection{Discussion}

In the present work, a comparative proteomic study using 2-DE and mass spectrometry was performed and led to the identification of a set of differentially expressed proteins associated with bicalutamide/embelin treatment in mouse prostate tumors. This was the pilot study that examined the effects of bicalutamide/embelin combination treatment on prostate cancer. In this phase, we established a comparative proteomic methodology tailored to mouse tumor tissue, and obtained preliminary results on the effects of this new therapeutic strategy.

There are many proteomic technologies currently available, but 2-DE remains the most accessible platform to separate thousands of proteins simultaneously from multiple 
Table 5-5 Representative networks associated with identified proteins by IPA software.

\begin{tabular}{|c|c|c|c|c|}
\hline ID & Molecules in network & Score & $\begin{array}{l}\text { Focus } \\
\text { molecules }\end{array}$ & Top functions \\
\hline 1 & $\begin{array}{l}\text { ALB, APOA1, CDCP1, CLEC7A, } \\
\text { DHRS11, ENO1, FTH1, FTL, FTMT, } \\
\text { GPX1, HMGB1 (includes EG:25459), } \\
\text { Ho, HPX, IGKC, IL1, IL1F5, IL1F6, } \\
\text { IL1F8, IL1F9, KRT9, KRT16, LSP1, } \\
\text { MIRN198 (includes EG:406975), NFkB } \\
\text { (complex), PCMT1, PELI1, PLG, } \\
\text { PRDX1, PRDX2, PRDX4, SETX, } \\
\text { SRXN1, Tcf 1/3/4, TRPC4AP, UMOD }\end{array}$ & 32 & 14 & $\begin{array}{l}\text { Small molecule } \\
\text { biochemistry, tissue } \\
\text { morphology, hair } \\
\text { and skin } \\
\text { development and } \\
\text { function }\end{array}$ \\
\hline 2 & $\begin{array}{l}\text { ACTR2, AHCY, AHCYL2, ANXA5, } \\
\text { ARHGDIA, CCDC44, CD44, CDC42, } \\
\text { COMMD9, EEF1G, EIF4A1, EIF4A2, } \\
\text { EPB41, ERLIN1, FAM62A, FTSJ1, GC, } \\
\text { GOLIM4, HAL, HNF4A, MRP63, } \\
\text { MRPL49, MRTO4, NUDT11, PABPC1, } \\
\text { PAIP1, PCBP2, PSMA1, PSMA6, } \\
\text { RAB11B, SCYL3, SF3A3, SLC2A4, } \\
\text { TRAF6, TUT1 }\end{array}$ & 29 & 13 & $\begin{array}{l}\text { RNA post- } \\
\text { transcriptional } \\
\text { modification, gene } \\
\text { expression, RNA } \\
\text { trafficking }\end{array}$ \\
\hline 3 & $\begin{array}{l}\text { ALDH7A1, BCAS2, beta-estradiol, } \\
\text { C1QTNF6, C21ORF126, CKB, DSCR3, } \\
\text { ESR1, FAM105B, FGB, GHSR, } \\
\text { GREB1, HCG 401283, HSF2BP, } \\
\text { HSPA13, IL1B, KRT13, KRTAP10-6, } \\
\text { LOC389842, LOC391282, LOC391322, } \\
\text { MED7 (includes EG:66213), MMP2, } \\
\text { NCRNA00162, ODF3B, PARK7, } \\
\text { PNRC2 (includes EG:55629), PRDM2, } \\
\text { SAPS2, SMS, SRM, UCRC, UMODL1, } \\
\text { ZMAT5, ZNF398 }\end{array}$ & 11 & 6 & $\begin{array}{l}\text { Cellular growth and } \\
\text { proliferation, cell- } \\
\text { to-cell signaling and } \\
\text { interaction, cancer }\end{array}$ \\
\hline
\end{tabular}

Genes in 2-DE analysis are shown with bold. 

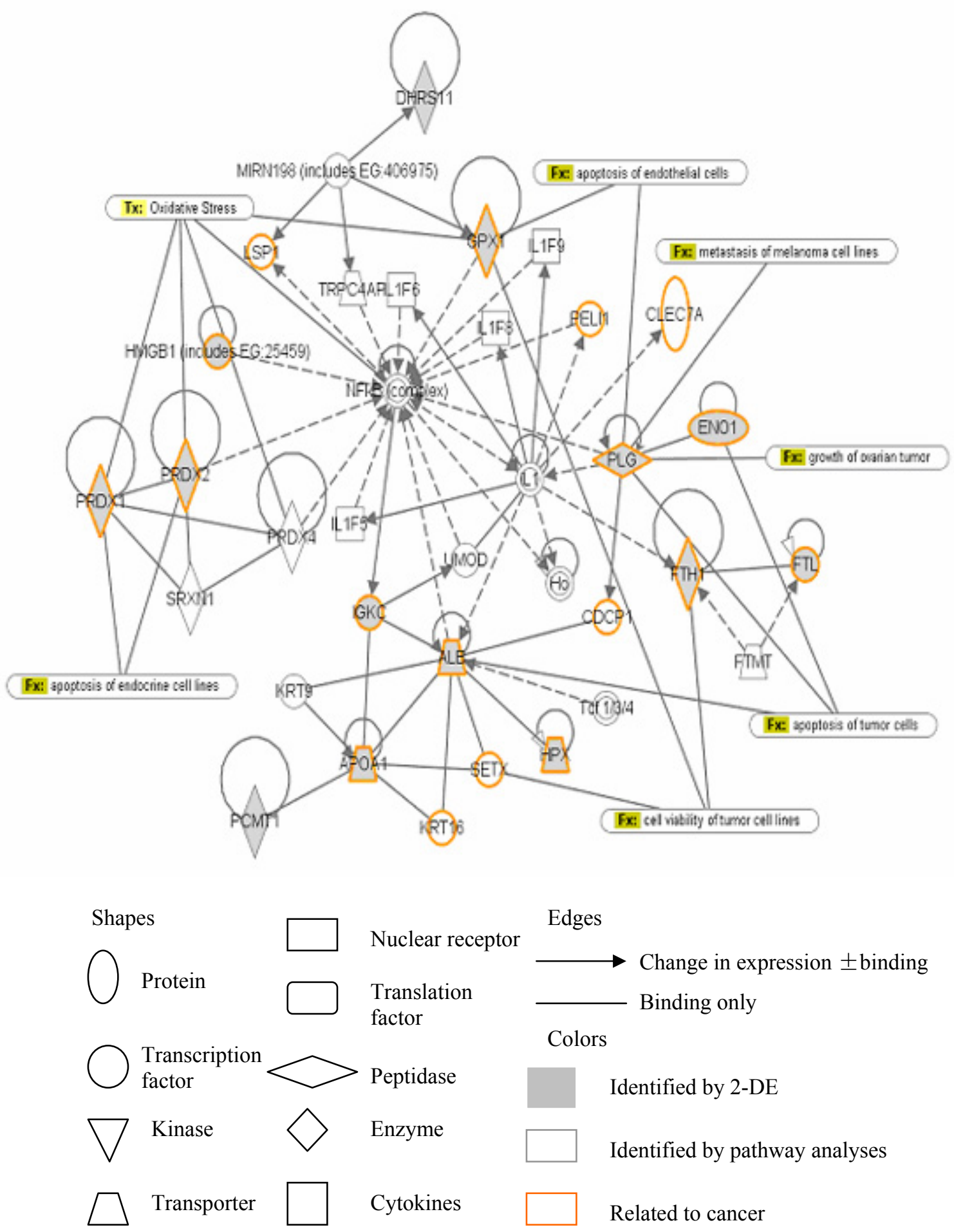

Figure 5-5 Network of small molecule biochemistry associated with modulated proteins identified in bicalutamide/embelin treatment in mouse prostate tumor tissue. 
samples for subsequent protein identification and quantitative comparison (Li et al., 2009; Rondepierre et al., 2009; Tilton et al., 2007; Wu et al., 2007). To ensure high confidence in the identification of differentially expressed protein spots, two independent image analysis software packages, PDQUEST and Progenesis Samespots, were applied to analyze all 2-DE gel images in the study. In total, 33 spots were identified as having statistically significant differential expression, between both software packages. However, only 17 of these were identified using both programs, which is most likely due to the variations in algorithms between the software packages, such as background subtraction or noise correction (Saldanha et al., 2008). The scenarios have been reported by a number of previous publications about the use of independent image analysis packages for the analysis of a gel data set (Byrne et al., 2009; Rosengren et al., 2003; Saldanha et al., 2008).

Analysis of the gel images using PDQuest demanded extensive manual inspection of all gels and subsequent alignment of overlapping gel spots to the master gel image. Parameters were needed to be set up for the examination of the match for the individual gel images to the master gel. Whereas, the Progenesis analysis was entirely automated with minimal manual intervention. Although labor intensive, manual 2-DE image analysis allowed greater accuracy in definition of spot boundaries, especially for low abundance or poorly resolved spots (Saldanha et al., 2008). Apart from confident identification of differentially expressed spots, the application of two independent software programs could increase the coverage of candidate protein spots which might be missed because of different detection cutoff criteria used in the software packages.

In the present study, LC-MS/MS was used for protein spots identification. Protein identification was based on matching of peptide sequence data. Out of the 33 differentially expressed protein spots, 30 spots were identified with adequate coverage. Among the identified protein spots, we found some of the spots contained more than one protein with very similar $\mathrm{pI}$ and MW values. Furthermore, some of the proteins were identified in multiple spots, which may be due to post-translational modifications. Both scenarios are frequently encountered in these types of analyses. Multiple proteins in the same spot would require an independent confirmation through application of biochemical technologies (such as western blotting) to unambiguously pinpoint the exact differentially expressed protein.

Thirty eight differentially expressed proteins induced by bicalutamide/embellin treatment were identified by using 2-DE followed with MS. Both GO analysis and IPA indicated that metabolism and stress response were the two major biological processes associated with differentially expressed proteins in bicalutamide/embellin treatment. Small molecular biochemistry was the pathway in which most of identified proteins were involved. Some of the identified proteins have previously been reported to be modulated in bicalutamide-treated prostate cancer cell lines, supporting the findings of our study (Rowland et al., 2007).

The largest group of identified proteins belonged to the functional class of metabolism. The identified proteins and enzymes, such as Apolipoprotein A-I precursor, 
Vitamin D-binding protein precursor, Dehydrogenase/reductase SDP family member 11, Proteasome subunit alpha type- 6 and Proteasome subunit alpha type-1, were involved in different metabolic pathways. Enolase is a glycolytic enzyme expressed on the surface of eukaryotic cells and also serves as a surface receptor for the binding of plasminogen. Alpha enolase (ENO1) has been implicated in numerous diseases including cancers (Kanemoto et al., 2006; Katayama et al., 2006) and was highly expressed in tumor cells (Altenberg et al., 2006; Altenberg and Greulich, 2004). The role of ENO1 in tumor cell invasion and metastasis may be explained by initiating the activity of plasminogen activity on the cell surface, which implicates various physiological and pathological events such as wound healing, tissue modeling, embryogenesis and the cell spreading (Plow et al., 1995). Up-regulation of alpha-enolase may contribute to an increased metabolic capacity, but not to increased plasminogen activation in our study.

Apart from metabolism, another major group of proteins modulated by the combination treatment were proteins involved in stress response. Proteins in this group are mainly associated with oxidative stress. All proteins identified in this group were upregulated in treated tissue, including peroxiredoxin 1 (PRDX1), peroxiredoxin 2 (PRDX2), glutathione peroxidase 1 (GPX1), protein DJ-1 (PARK7), and Dehydrogenase/reductase SDR family member 11 (DHR11).

Reactive oxygen species (ROS) are oxygen-containing free radicals, including superoxide radical $\left(\mathrm{O}_{2}{ }^{-}\right)$, hydroxyl radical $(\mathrm{OH})$, hydrogen peroxide $\left(\mathrm{H}_{2} \mathrm{O}_{2}\right)$ and reactive nitrogen species nitric oxide (NO) and peroxynitrite radical (ONOO). These species can be generated either through endogenous sources (such as the mitochondrial respiratory chain, the cytochrome P450 metabolic pathway, the inflammatory response etc.), or from external sources (such as chlorinated compounds, metal ions and radiation) (Apel and Hirt, 2004). ROS, at normal physiological concentrations, play important roles in a variety of cellular processes (Muller et al., 2007; Stadtman, 1992).

Oxidative stress occurs through disturbance in the balance between ROS formation and the efficiency of antioxidant processes, which means the net amount of ROS exceeds the antioxidant capacity. Oxidative stress has been linked to a wide range of diseases including Parkinson's disease (Fang et al., 2007), Asthma (Larsen et al., 2006) and cancer (Visconti and Grieco, 2009). A high level of ROS in cancer cells may lead to a variety of biological responses, such as increased proliferation rate, induction of DNA mutations and genetic instability, and resistance to some drugs in anticancer therapy. To date, it is unclear if oxidative stress is the trigger of the disease, or if it is a consequence of the disease and causes the disease symptoms. Recently, Cohen et al.(Cohen et al., 2008) studied the dynamics and variability of the protein response of human cancer cells to a chemotherapy drug, camptothecin. All cells showed rapid translocation of proteins specific to the drug target, and slower nuclear localization changes including the oxidative stress response pathway. This indicated that the anticancer drug treatment led to the modulation of oxidative stress.

It has been reported that patients with prostate cancer or other pathological processes, such as chronic prostatitis or atrophic prostatitis, presented a decreased 
antioxidant power and an increase of lipidic peroxidation (Lopez Laur et al., 2008). GPX is one of primary antioxidant enzymes; it reduces peroxides to their corresponding hydroxylated compounds using glutathione as a hydrogen donor. A recent study showed that the levels of antioxidant enzymes such Superoxide dismutase (SOD) and GPX in prostate cancer subjects were lower than in subjects with benign prostate hyperplasia and control subjects (Aydin et al., 2006). Overexpression of GPX3 in prostate cancer cell lines induced the suppression of colony formation and anchorage-independent growth of PC3, LNCaP, and Du145 cells (Yu et al., 2007b). Peroxiredoxins (PRDXs) are antioxidant enzymes expressed by most free-living organisms, often in multiple isoforms. Prostate cancer cells were shown to be more sensitive to hydrogen peroxide or an organic hydroperoxide when PRDX1, 2, or 3 was partially suppressed (Shen and Nathan, 2002). In the present study, overexpression of GPX, PRDX1 and PRDX2 indicated that administering bicalutamide/embelin perhaps shifts the redox balance within the tumor milieu, ultimately resulting in tumor suppression.

Protein DJ-1, also known as PARK7, is a multifunctional protein related to male fertility, identified as a hydroperoxide-responsive protein, and responsible for Parkinson's disease. Overexpression of DJ-1 has been associated with multiple cancers, including prostate cancer. It is known that DJ-1 is up-regulated by both androgen and antiandrogen treatment and that this increase is due to DJ-1 and androgen receptor protein stability (Pitkanen-Arsiola et al., 2006). It has also been shown that modulation of DJ-1 expression regulated AR transcriptional activity and may be involved in the development of androgen independence (Tillman et al., 2007). In our study, bicalutamide, as an antiandrogen in the combination treatment, resulted in overexpression of DJ-1 in mouse prostate tumors.

Another subgroup of differentially expressed proteins is comprised by proteins that are related to cancer cell metastasis. Annexin A5 is a calcium-dependent phospholipid binding protein, which is commonly used as a probe in Annexin A5 affinity assay to detect cell death. It was reported that down-regulated Annexin A5 reduced migration and invasion capacity of $\mathrm{HaCaT}$ cells which was even stronger in the oral carcinoma (Wehder et al., 2009). High mobility group protein B1 (HMGB1) is known to increase expression in carcinomas and exhibit association with proliferation and metastasis in many tumor types (Suzuki et al., 2009). Breast cancer amplified sequence 2 (BCAS2) was initially identified as a gene that was overexpressed and amplified in some breast cancer cell lines (Qi et al., 2005). In our study, expression levels of these proteins were down-regulated by bicalutamide/embelin combination treatment, indicating the decrease of cancer cell metastasis potential.

Several proteins involved in protein biosynthesis and modification were modulated by the combination treatment. Translation initiation factors (IF2A1, IF4A2) and elongation factor (EF1G) were up-regulated. Furthermore, 40S ribosomal protein (RS3), protein-L-isoaspartate(D-aspartate) O-methyltransferase (PIMT) and spermidine synthase (SPEE) were down-regulated.

In conclusion, bicalutamide/embelin treatment modulated molecular functions of 
metabolism and biosynthesis, and in particular increased the expression levels of antioxidants to reduce oxidative stress.

\subsection{Conclusions}

The present study was the first comparative proteomics examination of the effects of bicalutamide/embelin combination treatment on prostate tumors. Protein alterations of mouse prostate tumors induced by bicalutamide/embelin treatment have been studied using 2-DE and mass spectrometry. This analytical strategy involved total protein separation with 2-DE and protein identification by using nanoLC-MS/MS. Differentially expressed proteins were further investigated by bioinformatics tools (GO and IPA) to reveal the associations with important biological functions and processes. The major functions were related to cancer, inflammatory disease, connective tissue disorders, and skeletal and muscular disorders. The molecular functions of differentially expressed protein were associated with metabolism and stress response. 


\section{CHAPTER 6.SUMMARY}

Advances in technology and understanding of systems biology have provided tremendous strides towards improvement in modern human medicine. Recently, extensive efforts have been involved in the application of various proteomics technologies to the study of prostate cancer to obtain new mechanistic knowledge and to discover novel biomarkers. The desired outcomes of the efforts are markers that will aid in early detection and prognosis of prostate cancer, in monitoring of therapeutic response, and determining best course of therapy for cancer patients to improve clinical outcomes. Despite all these considerable efforts, it remains a challenge to perform a comprehensive proteome analysis.

In the current dissertation, I focused on the development and application of multiple mass spectrometry-based bioanalytical platforms for phosphoproteomic characterization in cell culture and clinical specimens of prostate cancer; and on application of optimized methods to analysis of differential protein expression to reveal molecular mechanism of drug action in mouse model of prostate cancer.

\subsection{Discussion of Selected Analytical Aspects}

In combination with IEF separation and phosphopeptide enrichment, in-gel IEF LC-MS/MS strategy yielded a significantly expanded phosphoprotein panel. This panel of the LNCaP phosphoproteins was 3.6-fold larger than the panel obtained in our previous work, which attests to the power of the chosen analytical methodology. The expanded phosphoproteome map obtained in this study will serve as a foundation for future quantitative studies of the effects of pharmaceuticals on the LNCaP prostate cancer cells; for this purpose, in-gel IEF LC-MS/MS will be combined with stable-isotope labeling such as stable isotope labeling with amino acids in cell culture (SILAC) (Mann, 2006). Furthermore, additional improvements of the in-gel IEF LC-MS/MS methodology will further enhance the coverage of the LNCaP phosphoproteome.

2-DE was one of the main separation methods applied in our studies. We applied 2-DE based platforms to investigation of phosphoproteins in the LNCaP prostate cancer cells, and to obtain protein profiling in mouse prostate tumor tissue treated by anticancer drugs. As an improvement, 2D-DIGE can be integrated into the platform for future continuation of this line of research. 2D-DIGE incorporates labeling of different protein populations with fluorescent molecules (CyDyes) prior to separation by 2-DE. Up to three different protein samples can be covalently labeled with fluorescent dyes and be separated by 2-DE in a single gel. The technique significantly reduces the inter-gel variability encountered with traditional 2-DE platforms (Alban et al., 2003; Minden et al., 2009).

Phosphorylation site assignment is one of challenges for site-specific identification in phosphoproteomics. In our mass spectrometry analyses, CID is the ion 
activation/dissociation method for fragmentation of the peptide backbone and production of ions for sequence analysis and identification of phosphorylation sites. However, CID process often leads to elimination of the molecule of phosphoric acid from peptide ions containing phosphorylated serine and threonine residues; this predominant dissociation pathway results in lack of sequence information in MS/MS spectra. Assignment of phosphorylation sites, especially for peptides containing multiple phosphorylation sites, becomes a challenge. Recently, alternative dissociation methods, such as ETD(Chi et al., 2007; Molina et al., 2007), have been introduced as promising alternatives that provide more comprehensive backbone fragment ion series without loss of water, phosphate groups or phosphoric acid.

In our previous studies, protein function and subcellular location were manually classified based on Swiss-Prot annotations. In large-scale proteome studies, manual annotation strategy is subjective and also labor-intensive. Gene ontology (GO) is a collection of controlled vocabularies describing the biology of a gene product in any organism andit is commonly used to analyze results from high-throughput studies (Khatri and Draghici, 2005; Rhee et al., 2008). GO provides researchers with a broad overview of predominant activities of a specific group of proteins and assists in understanding of large-scale complex biological changes (Dimmer et al., 2008). GO analysis is broadly used in the proteomics community. To interpret our results systematically and to share our results with the research community, GO analysis was adopted in our studies to classify identified proteins according to molecular function, biological process, and molecular component (subcellular location).

Another bioinformatics resource newly applied in our research is the Ingenuity Pathway Analysis. IPA is a powerful curated database and analysis system, which can build hypothetical networks of physical and functional relationships between genes, proteins, and chemicals based on Ingenuity knowledge base. It can help to understand how proteins work together to effect cellular changes (Hoorn et al., 2005; Raponi et al., 2004). Ingenuity helped us to identify the biological mechanisms, pathways and functions most relevant to our experimental datasets of interest.

\subsection{Summary}

\subsubsection{Characterization of the Phosphoproteome in Prostate Cancer}

Our research aimed towards description of the phosphoproteome in prostate cancer focused on the $\mathrm{LNCaP}$ human prostate cancer cell line, and on human prostate cancer tissue.

For the LNCaP prostate cancer cell line, we applied a combination of analytical platforms: (1) a novel in-gel IEF LC-MS/MS analytical platform; (2) a 2-DE based platform combined with phospho-specific staining. 
The in-gel IEF LC-MS/MS analytical methodology used in the study included separation of the LNCaP proteins by in-gel isoelectric focusing; digestion of the proteins with trypsin; enrichment of the digests for phosphopeptides with IMAC; analysis of the enriched digests by LC-MS/MS; and identification of the phosphorylated peptides/proteins through searches of the Swiss-Prot protein sequence database. With ingel IEF based analytical platform, we have characterized over 600 different phosphorylation sites in 296 phosphoproteins in the LNCaP prostate cancer cell line. This panel of the LNCaP phosphoproteins was 3-fold larger than the panel obtained in our previous work (Giorgianni et al., 2007), and is the largest phosphoprotein panel in prostate cancer reported to date. The phosphoproteins identified in this study belonged to various locations within the cell and were involved in various processes including cell differentiation, transcription regulation, and intercellular signal transduction. The expanded phosphoproteome map obtained in this study was of a satisfactory size to serve as a foundation for future quantitative studies of the effects of pharmaceuticals on the LNCaP prostate cancer cells.

We also developed a 2-DE based platform, in combination with multiplexed staining and LC-MS/MS, for the identification of LNCaP phosphoproteins. In this study, we applied 2-DE as separation technique, Pro- $\mathrm{Q}^{\mathrm{TM}}$ Diamond stain as phosphoprotein detection method, LC-MS/MS and database searches for protein identification to investigate the phosphoproteins in the $\mathrm{LNCaP}$ prostate cancer cell line. Proteins identified from spots of interest were shown to be highly relevant to prostate cancer. We demonstrated the feasibility of using 2-DE with phospho-specific stain and mass spectrometry to investigate the phosphoproteins in the LNCaP cell line. This methodology complements the in-gel IEF LC-MS/MS platform that we used for phosphoproteomics study; it will be of particular value for future comparative studies of phosphoproteins in various physiological states.

For prostate cancer tissue, a gel-free approach was applied to analyze five prostate cancer tissue specimens to obtain phosphoproteomic signatures of prostate cancer for biomarker discovery. Proteins were extracted with Trizol reagent, and then in-solution digested with trypsin. Phosphopeptides were enriched with IMAC, and the enriched digests were analzyed by LC-MS/MS with identification through searches of the SwissProt protein sequence database. The panels obtained for prostate cancer tissue contain 1524 phosphoproteins. Some of the characterized phosphoproteins were present in all five specimens; in addition, each specimen also produced a unique set of phosphoproteins. The findings provided a direct glimpse into the phosphoprotein machinery operating within the human prostate cancer tissue. This pilot study focused on a small set of specimens. The phosphoprotein panels that were obtained contained a number of proteins that were unique to a particular specimen. Analyses of a larger group of specimens will provide further knowledge on the phosphoprotein signatures to build the foundation for differential phosphoprotein profiling of cancer vs. control tissue for biomarker discovery. 


\subsubsection{Comparative Proteomics Study of Drug Effects in Prostate Cancer}

We carried out the first comparative proteomics study of the effects of bicalutamide/embelin combination treatment on prostate tumors by characterizing the alterations in protein expression that were induced upon treatment of mice bearing prostate tumors with anticancer combination therapy.

A comparative proteomic strategy based on 2-DE coupled with LC-MS/MS was applied to analysis of mouse prostate tumor tissue. Proteins from the mouse prostate tumors were extracted with Trizol, and the protein mixtures were separated by 2-DE. Differences in the protein profiles for the different treatment groups were evaluated by computer-assisted analysis of SYPRO Ruby stained 2-DE gels. LC-MS/MS and database searches were used to identify differentially expressed proteins. Pathway analysis was carried out on the dataset of identified proteins with the Ingenuity bioinformatics tool. Out of the 33 differentially expressed protein spots, 30 protein spots were identified and grouped into various functional classes. The major protein categories were metabolism $(52 \%)$, stress response (12\%), protein biosynthesis $(13 \%)$ and apoptosis $(11 \%)$, suggesting that alterations in these processes may be involved in the mechanism of drug action. Proteins associated with oxidative stress were up-regulated, which indicated that treatment with bicalutamide/embelin may affect the redox balance within the prostate tumor, and this effect may contribute to tumor suppression.

Future plans for continuation of this study include incorporation of DIGE into the analytical methodology, examination of a larger group so that biological variability can be taken into account, evaluation of the proteomes at different points during the treatment course, etc. Ultimately, this research will bring new insights into the molecular mechanism of anticancer combination therapy.

In conclusion, in the research presented in this dissertation, multiple MS-based bioanalytical platforms were applied successfully to study the phosphoproteome and proteome in prostate cancer. The disease-related proteomic information obtained in my research will contribute to our understanding of the pathogenesis, and it will bring improvements in the diagnosis and treatment of prostate cancer. 


\section{LIST OF REFERENCES}

2008, Screening for prostate cancer: U.S. Preventive Services Task Force recommendation statement: Ann.Intern.Med., v. 149, p. 185-191.

Alban, A, S O David, L Bjorkesten, C Andersson, E Sloge, S Lewis, I Currie, 2003, A novel experimental design for comparative two-dimensional gel analysis: twodimensional difference gel electrophoresis incorporating a pooled internal standard: Proteomics., v. 3, p. 36-44.

Albuquerque, C P, M B Smolka, S H Payne, V Bafna, J Eng, H Zhou, 2008, A multidimensional chromatography technology for in-depth phosphoproteome analysis: Mol.Cell Proteomics., v. 7, p. 1389-1396.

Altenberg, B, C Gemuend, K O Greulich, 2006, Ubiquitous cancer genes: multipurpose molecules for protein micro-arrays: Proteomics., v. 6, p. 67-71.

Altenberg, B, K O Greulich, 2004, Genes of glycolysis are ubiquitously overexpressed in 24 cancer classes: Genomics, v. 84, p. 1014-1020.

Anai, S, S Goodison, K Shiverick, K Iczkowski, M Tanaka, C J Rosser, 2006, Combination of PTEN gene therapy and radiation inhibits the growth of human prostate cancer xenografts: Hum.Gene Ther., v. 17, p. 975-984.

Anderson, N L, N G Anderson, 1998, Proteome and proteomics: new technologies, new concepts, and new words: Electrophoresis, v. 19, p. 1853-1861.

Andronicos, N M, M Ranson, J Bognacki, M S Baker, 1997, The human ENO1 gene product (recombinant human alpha-enolase) displays characteristics required for a plasminogen binding protein: Biochim.Biophys.Acta, v. 1337, p. 27-39.

Apel, K, H Hirt, 2004, Reactive oxygen species: metabolism, oxidative stress, and signal transduction: Annu.Rev.Plant Biol., v. 55, p. 373-399.

Ashman, K, V E Lopez, 2009, Phosphoproteomics and cancer research: Clin.Transl.Oncol., v. 11, p. 356-362.

Aydin, A, Z Arsova-Sarafinovska, A Sayal, A Eken, O Erdem, K Erten, Y Ozgok, A Dimovski, 2006, Oxidative stress and antioxidant status in non-metastatic prostate cancer and benign prostatic hyperplasia: Clin.Biochem., v. 39, p. 176-179.

Ballif, B A, G R Carey, S R Sunyaev, S P Gygi, 2008, Large-scale identification and evolution indexing of tyrosine phosphorylation sites from murine brain: J.Proteome.Res., v. 7, p. 311-318. 
Bandyopadhyay, S, S K Pai, S Hirota, S Hosobe, T Tsukada, K Miura, Y Takano, K Saito, T Commes, D Piquemal, M Watabe, S Gross, Y Wang, J Huggenvik, K Watabe, 2004, PTEN up-regulates the tumor metastasis suppressor gene Drg-1 in prostate and breast cancer: Cancer Res., v. 64, p. 7655-7660.

Basta, P V, J T Bensen, C K Tse, C M Perou, P F Sullivan, A F Olshan, 2008, Genetic variation in Transaldolase 1 and risk of squamous cell carcinoma of the head and neck: Cancer Detect.Prev., v. 32, p. 200-208.

Bedolla, R, T J Prihoda, J I Kreisberg, S N Malik, N K Krishnegowda, D A Troyer, P M Ghosh, 2007, Determining risk of biochemical recurrence in prostate cancer by immunohistochemical detection of PTEN expression and Akt activation: Clin.Cancer Res., v. 13, p. 3860-3867.

Benzeno, S, F Lu, M Guo, O Barbash, F Zhang, J G Herman, P S Klein, A Rustgi, J A Diehl, 2006, Identification of mutations that disrupt phosphorylation-dependent nuclear export of cyclin D1: Oncogene, v. 25, p. 6291-6303.

Beranova-Giorgianni, S, Y Zhao, D M Desiderio, F Giorgianni, 2006, Phosphoproteomic analysis of the human pituitary: Pituitary., v. 9, p. 109-120.

Bindukumar, B, S Schwartz, R Aalinkeel, S Mahajan, A Lieberman, K Chadha, 2008, Proteomic profiling of the effect of prostate-specific antigen on prostate cancer cells: Prostate, v. 68, p. 1531-1545.

Bjellqvist, B, K Ek, P G Righetti, E Gianazza, A Gorg, R Westermeier, W Postel, 1982, Isoelectric focusing in immobilized $\mathrm{pH}$ gradients: principle, methodology and some applications: J.Biochem.Biophys.Methods, v. 6, p. 317-339.

Blackledge, G R, 1996, Clinical progress with a new antiandrogen, Casodex (bicalutamide): Eur.Urol., v. 29 Suppl 2, p. 96-104.

Blackstock, W P, M P Weir, 1999, Proteomics: quantitative and physical mapping of cellular proteins: Trends Biotechnol., v. 17, p. 121-127.

Blanco, E, E A Bey, Y Dong, B D Weinberg, D M Sutton, D A Boothman, J Gao, 2007, Beta-lapachone-containing PEG-PLA polymer micelles as novel nanotherapeutics against NQO1-overexpressing tumor cells: J.Control Release, v. 122, p. 365-374.

Blanco-Aparicio, C, O Renner, J F Leal, A Carnero, 2007, PTEN, more than the AKT pathway: Carcinogenesis, v. 28, p. 1379-1386.

Byrne, J C, M R Downes, N O'Donoghue, C O'Keane, A O'Neill, Y Fan, J M Fitzpatrick, M Dunn, R W Watson, 2009, 2D-DIGE as a strategy to identify serum markers for the progression of prostate cancer: J.Proteome.Res., v. 8, p. 942-957. 
Carter, H B, J D Pearson, E J Metter, L J Brant, D W Chan, R Andres, J L Fozard, P C Walsh, 1992, Longitudinal evaluation of prostate-specific antigen levels in men with and without prostate disease: JAMA, v. 267, p. 2215-2220.

Catalona, W J, J P Richie, J B deKernion, F R Ahmann, T L Ratliff, B L Dalkin, L R Kavoussi, M T MacFarlane, P C Southwick, 1994, Comparison of prostate specific antigen concentration versus prostate specific antigen density in the early detection of prostate cancer: receiver operating characteristic curves: J.Urol., v. 152, p. 2031-2036.

Chang, L, R D Goldman, 2004, Intermediate filaments mediate cytoskeletal crosstalk: Nat.Rev.Mol.Cell Biol., v. 5, p. 601-613.

Chardonnet, S, P Le Marechal, H Cheval, J P Le Caer, P Decottignies, O Laprevote, S Laroche, S Davis, 2008, Large-scale study of phosphoproteins involved in long-term potentiation in the rat dentate gyrus in vivo: Eur.J.Neurosci., v. 27, p. 2985-2998.

Chen, H M, K L Schmeichel, I S Mian, S Lelievre, O W Petersen, M J Bissell, 2000, AZU-1: a candidate breast tumor suppressor and biomarker for tumor progression: Mol.Biol Cell, v. 11, p. 1357-1367.

Chen, J, Z Nikolovska-Coleska, G Wang, S Qiu, S Wang, 2006, Design, synthesis, and characterization of new embelin derivatives as potent inhibitors of X-linked inhibitor of apoptosis protein: Bioorg.Med.Chem.Lett., v. 16, p. 5805-5808.

Chen, Z, K Southwick, C D Thulin, 2004, Initial analysis of the phosphoproteome of Chinese hamster ovary cells using electrophoresis: J.Biomol.Tech., v. 15, p. 249-256.

Chi, A, C Huttenhower, L Y Geer, J J Coon, J E Syka, D L Bai, J Shabanowitz, D J Burke, O G Troyanskaya, D F Hunt, 2007, Analysis of phosphorylation sites on proteins from Saccharomyces cerevisiae by electron transfer dissociation (ETD) mass spectrometry: Proc.Natl.Acad.Sci.U.S.A, v. 104, p. 2193-2198.

Cicchillitti, L, M Di Michele, A Urbani, C Ferlini, M B Donati, G Scambia, D Rotilio, 2009, Comparative proteomic analysis of paclitaxel sensitive A2780 epithelial ovarian cancer and its resistant counterpart A2780TC1 by 2D-DIGE: the role of ERp57:

J.Proteome.Res., v. 8, p. 1902-1912.

Cohen, A A, N Geva-Zatorsky, E Eden, M Frenkel-Morgenstern, I Issaeva, A Sigal, R Milo, C Cohen-Saidon, Y Liron, Z Kam, L Cohen, T Danon, N Perzov, U Alon, 2008, Dynamic proteomics of individual cancer cells in response to a drug: Science, v. 322, p. 1511-1516.

Cohen, P, 2000, The regulation of protein function by multisite phosphorylation--a 25 year update: Trends Biochem.Sci., v. 25, p. 596-601.

Collins, M O, L Yu, J S Choudhary, 2007, Analysis of protein phosphorylation on a proteome-scale: Proteomics., v. 7, p. 2751-2768. 
Corthals, G L, V C Wasinger, D F Hochstrasser, J C Sanchez, 2000, The dynamic range of protein expression: a challenge for proteomic research: Electrophoresis, v. 21, p. 11041115.

Coulombe, P A, P Wong, 2004, Cytoplasmic intermediate filaments revealed as dynamic and multipurpose scaffolds: Nat.Cell Biol., v. 6, p. 699-706.

Csucs, G, J J Ramsden, 1998, Interaction of phospholipid vesicles with smooth metaloxide surfaces: Biochim.Biophys.Acta, v. 1369, p. 61-70.

Czupalla, C, B Nurnberg, E Krause, 2003, Analysis of class I phosphoinositide 3-kinase autophosphorylation sites by mass spectrometry: Rapid Commun.Mass Spectrom., v. 17, p. 690-696.

Dall'Era, M A, A Oudes, D B Martin, A Y Liu, 2007, HSP27 and HSP70 interact with CD10 in C4-2 prostate cancer cells: Prostate, v. 67, p. 714-721.

Danquah, M, F Li, C B Duke, III, D D Miller, R I Mahato, 2009, Micellar delivery of bicalutamide and embelin for treating prostate cancer: Pharm.Res., v. 26, p. 2081-2092.

DeGnore, J P, J Qin, 1998, Fragmentation of phosphopeptides in an ion trap mass spectrometer: J.Am.Soc.Mass Spectrom., v. 9, p. 1175-1188.

Deng, M, S Mohanan, E Polyak, S Chacko, 2007, Caldesmon is necessary for maintaining the actin and intermediate filaments in cultured bladder smooth muscle cells: Cell Motil.Cytoskeleton, v. 64, p. 951-965.

Di Michele, M, C A Della, L Cicchillitti, P Del Boccio, A Urbani, C Ferlini, G Scambia, M B Donati, D Rotilio, 2009, A proteomic approach to paclitaxel chemoresistance in ovarian cancer cell lines: Biochim.Biophys.Acta, v. 1794, p. 225-236.

Dimmer, E C, R P Huntley, D G Barrell, D Binns, S Draghici, E B Camon, M Hubank, P J Talmud, R Apweiler, R C Lovering, 2008, The gene ontology - providing a functional role in proteomic studies: Proteomics., v. 8, p. 2-11.

Dubrovska, A, S Souchelnytskyi, 2005, Efficient enrichment of intact phosphorylated proteins by modified immobilized metal-affinity chromatography: Proteomics., v. 5, p. 4678-4683.

Dunn, M J, 1987, Two-dimensional gel electrophoresis of proteins: J.Chromatogr., v. 418, p. $145-185$.

Edwards, J, J M Bartlett, 2005, The androgen receptor and signal-transduction pathways in hormone-refractory prostate cancer. Part 1: Modifications to the androgen receptor: BJU.Int., v. 95, p. 1320-1326.

Elias, J E, S P Gygi, 2007, Target-decoy search strategy for increased confidence in large-scale protein identifications by mass spectrometry: Nat.Methods, v. 4, p. 207-214. 
Fang, J, E J Metter, P Landis, H B Carter, 2002, PSA velocity for assessing prostate cancer risk in men with PSA levels between 2.0 and $4.0 \mathrm{ng} / \mathrm{ml}$ : Urology, v. 59, p. 889893.

Fang, J, T Nakamura, D H Cho, Z Gu, S A Lipton, 2007, S-nitrosylation of peroxiredoxin 2 promotes oxidative stress-induced neuronal cell death in Parkinson's disease:

Proc.Natl.Acad.Sci.U.S.A, v. 104, p. 18742-18747.

Farwell, W R, J A Linder, A K Jha, 2007, Trends in prostate-specific antigen testing from 1995 through 2004: Arch.Intern.Med., v. 167, p. 2497-2502.

Feng, X, X Liu, Q Luo, B F Liu, 2008, Mass spectrometry in systems biology: an overview: Mass Spectrom.Rev., v. 27, p. 635-660.

Fenn, J B, M Mann, C K Meng, S F Wong, C M Whitehouse, 1989, Electrospray ionization for mass spectrometry of large biomolecules: Science, v. 246, p. 64-71.

Ficarro, S B, M L McCleland, P T Stukenberg, D J Burke, M M Ross, J Shabanowitz, D F Hunt, F M White, 2002, Phosphoproteome analysis by mass spectrometry and its application to Saccharomyces cerevisiae: Nat.Biotechnol., v. 20, p. 301-305.

Gafken, P R, P D Lampe, 2006, Methodologies for characterizing phosphoproteins by mass spectrometry: Cell Commun.Adhes., v. 13, p. 249-262.

Gannon, J, L Staunton, K O'Connell, P Doran, K Ohlendieck, 2008, Phosphoproteomic analysis of aged skeletal muscle: Int.J.Mol.Med., v. 22, p. 33-42.

Garbis, S D, S I Tyritzis, T Roumeliotis, P Zerefos, E G Giannopoulou, A Vlahou, S Kossida, J Diaz, S Vourekas, C Tamvakopoulos, K Pavlakis, D Sanoudou, C A Constantinides, 2008, Search for potential markers for prostate cancer diagnosis, prognosis and treatment in clinical tissue specimens using amine-specific isobaric tagging (iTRAQ) with two-dimensional liquid chromatography and tandem mass spectrometry: J.Proteome.Res., v. 7, p. 3146-3158.

Gianazza, E, P G Righetti, 2009, Immobilized pH gradients: Electrophoresis, v. 30 Suppl 1, p. S112-S121.

Gioeli, D, 2005, Signal transduction in prostate cancer progression: Clin.Sci.(Lond), v. 108, p. 293-308.

Gioeli, D, S B Ficarro, J J Kwiek, D Aaronson, M Hancock, A D Catling, F M White, R E Christian, R E Settlage, J Shabanowitz, D F Hunt, M J Weber, 2002, Androgen receptor phosphorylation. Regulation and identification of the phosphorylation sites: J.Biol.Chem., v. 277, p. 29304-29314.

Giorgianni, F, S Beranova-Giorgianni, D M Desiderio, 2004, Identification and characterization of phosphorylated proteins in the human pituitary: Proteomics., v. 4, p. 587-598. 
Giorgianni, F, D M Desiderio, S Beranova-Giorgianni, 2003, Proteome analysis using isoelectric focusing in immobilized $\mathrm{pH}$ gradient gels followed by mass spectrometry: Electrophoresis, v. 24, p. 253-259.

Giorgianni, F, Y Zhao, D M Desiderio, S Beranova-Giorgianni, 2007, Toward a global characterization of the phosphoproteome in prostate cancer cells: identification of phosphoproteins in the LNCaP cell line: Electrophoresis, v. 28, p. 2027-2034.

Glen, A, C S Gan, F C Hamdy, C L Eaton, S S Cross, J W Catto, P C Wright, I Rehman, 2008, iTRAQ-facilitated proteomic analysis of human prostate cancer cells identifies proteins associated with progression: J.Proteome.Res., v. 7, p. 897-907.

Goodman, T, B Schulenberg, T H Steinberg, W F Patton, 2004, Detection of phosphoproteins on electroblot membranes using a small-molecule organic fluorophore: Electrophoresis, v. 25, p. 2533-2538.

Gorg, A, C Obermaier, G Boguth, A Harder, B Scheibe, R Wildgruber, W Weiss, 2000, The current state of two-dimensional electrophoresis with immobilized $\mathrm{pH}$ gradients: Electrophoresis, v. 21, p. 1037-1053.

Gorg, A, W Weiss, M J Dunn, 2004, Current two-dimensional electrophoresis technology for proteomics: Proteomics., v. 4, p. 3665-3685.

Hart, S R, M D Waterfield, A L Burlingame, R Cramer, 2002, Factors governing the solubilization of phosphopeptides retained on ferric NTA IMAC beads and their analysis by MALDI TOFMS: J.Am.Soc.Mass Spectrom., v. 13, p. 1042-1051.

Henzel, W J, T M Billeci, J T Stults, S C Wong, C Grimley, C Watanabe, 1993, Identifying proteins from two-dimensional gels by molecular mass searching of peptide fragments in protein sequence databases: Proc.Natl.Acad.Sci.U.S.A, v. 90, p. 5011-5015.

Hoffert, J D, M A Knepper, 2008, Taking aim at shotgun phosphoproteomics: Anal.Biochem., v. 375, p. 1-10.

Hoffmann, P, M A Olayioye, R L Moritz, G J Lindeman, J E Visvader, R J Simpson, B E Kemp, 2005, Breast cancer protein StarD10 identified by three-dimensional separation using free-flow electrophoresis, reversed-phase high-performance liquid chromatography, and sodium dodecyl sulfate-polyacrylamide gel electrophoresis: Electrophoresis, v. 26, p. 1029-1037.

Hoorn, E J, J D Hoffert, M A Knepper, 2005, Combined proteomics and pathways analysis of collecting duct reveals a protein regulatory network activated in vasopressin escape: J.Am.Soc.Nephrol., v. 16, p. 2852-2863.

Hopper, R K, S Carroll, A M Aponte, D T Johnson, S French, R F Shen, F A Witzmann, R A Harris, R S Balaban, 2006, Mitochondrial matrix phosphoproteome: effect of extra mitochondrial calcium: Biochemistry, v. 45, p. 2524-2536. 
Hoving, S, B Gerrits, H Voshol, D Muller, R C Roberts, J van Oostrum, 2002, Preparative two-dimensional gel electrophoresis at alkaline $\mathrm{pH}$ using narrow range immobilized pH gradients: Proteomics., v. 2, p. 127-134.

Hsiang, C H, T Tunoda, Y E Whang, D R Tyson, D K Ornstein, 2006, The impact of altered annexin I protein levels on apoptosis and signal transduction pathways in prostate cancer cells: Prostate, v. 66, p. 1413-1424.

Iakoucheva, L M, P Radivojac, C J Brown, T R O'Connor, J G Sikes, Z Obradovic, A K Dunker, 2004, The importance of intrinsic disorder for protein phosphorylation: Nucleic Acids Res., v. 32, p. 1037-1049.

Ibel, K, R P May, K Kirschner, H Szadkowski, E Mascher, P Lundahl, 1990, Proteindecorated micelle structure of sodium-dodecyl-sulfate--protein complexes as determined by neutron scattering: Eur.J.Biochem., v. 190, p. 311-318.

Ide, H, D B Seligson, S Memarzadeh, L Xin, S Horvath, P Dubey, M B Flick, B M Kacinski, A Palotie, O N Witte, 2002, Expression of colony-stimulating factor 1 receptor during prostate development and prostate cancer progression: Proc.Natl.Acad.Sci.U.S.A, v. 99, p. 14404-14409.

Isaacs, J T, 1994, Role of androgens in prostatic cancer: Vitam.Horm., v. 49, p. 433-502.

Ishihara, T, M Goto, H Kanazawa, M Higaki, Y Mizushima, 2008, Efficient entrapment of poorly water-soluble pharmaceuticals in hybrid nanoparticles: J.Pharm.Sci., v. 98, p. 2357-2363.

Jacques, A M, O Copeland, A E Messer, C E Gallon, K King, W J McKenna, V T Tsang, S B Marston, 2008, Myosin binding protein C phosphorylation in normal, hypertrophic and failing human heart muscle: J.Mol.Cell Cardiol., v. 45, p. 209-216.

Jemal, A, R Siegel, E Ward, Y Hao, J Xu, T Murray, M J Thun, 2008, Cancer statistics, 2008: CA Cancer J.Clin., v. 58, p. 71-96.

Jemal, A, R Siegel, E Ward, T Murray, J Xu, C Smigal, M J Thun, 2006, Cancer statistics, 2006: CA Cancer J.Clin., v. 56, p. 106-130.

Johansson, B, M R Pourian, Y C Chuan, I Byman, A Bergh, S T Pang, G Norstedt, T Bergman, A Pousette, 2006, Proteomic comparison of prostate cancer cell lines LNCaPFGC and LNCaP-r reveals heatshock protein 60 as a marker for prostate malignancy: Prostate, v. 66, p. 1235-1244.

Kalume, D E, H Molina, A Pandey, 2003, Tackling the phosphoproteome: tools and strategies: Curr.Opin.Chem.Biol., v. 7, p. 64-69.

Kanemoto, K, H Satoh, H Ishikawa, K Sekizawa, 2006, Neurone-specific enolase and liver metastasis in small cell lung cancer: Clin.Oncol.(R.Coll.Radiol.), v. 18, p. 505. 
Kang, J S, B F Calvo, S J Maygarden, L S Caskey, J L Mohler, D K Ornstein, 2002, Dysregulation of annexin I protein expression in high-grade prostatic intraepithelial neoplasia and prostate cancer: Clin.Cancer Res., v. 8, p. 117-123.

Katayama, M, H Nakano, A Ishiuchi, W Wu, R Oshima, J Sakurai, H Nishikawa, S Yamaguchi, T Otsubo, 2006, Protein pattern difference in the colon cancer cell lines examined by two-dimensional differential in-gel electrophoresis and mass spectrometry: Surg.Today, v. 36, p. 1085-1093.

Kaufmann, H, J E Bailey, M Fussenegger, 2001, Use of antibodies for detection of phosphorylated proteins separated by two-dimensional gel electrophoresis: Proteomics., v. 1, p. 194-199.

Khatri, P, S Draghici, 2005, Ontological analysis of gene expression data: current tools, limitations, and open problems: Bioinformatics., v. 21, p. 3587-3595.

Kim, J E, S R Tannenbaum, F M White, 2005, Global phosphoproteome of HT-29 human colon adenocarcinoma cells: J.Proteome.Res., v. 4, p. 1339-1346.

Kim, S C, Y Chen, S Mirza, Y Xu, J Lee, P Liu, Y Zhao, 2006, A clean, more efficient method for in-solution digestion of protein mixtures without detergent or urea:

J.Proteome.Res., v. 5, p. 3446-3452.

Kreisberg, J I, S N Malik, T J Prihoda, R G Bedolla, D A Troyer, S Kreisberg, P M Ghosh, 2004, Phosphorylation of Akt (Ser473) is an excellent predictor of poor clinical outcome in prostate cancer: Cancer Res., v. 64, p. 5232-5236.

Kurahashi, T, H Miyake, I Hara, M Fujisawa, 2007, Expression of major heat shock proteins in prostate cancer: correlation with clinicopathological outcomes in patients undergoing radical prostatectomy: J.Urol., v. 177, p. 757-761.

Kweon, H K, K Hakansson, 2006, Selective zirconium dioxide-based enrichment of phosphorylated peptides for mass spectrometric analysis: Anal.Chem., v. 78, p. 17431749.

Kweon, H K, K Hakansson, 2008, Metal oxide-based enrichment combined with gasphase ion-electron reactions for improved mass spectrometric characterization of protein phosphorylation: J.Proteome.Res., v. 7, p. 749-755.

Lahn, M, K Sundell, M Gleave, F Ladan, C Su, S Li, D Ma, B M Paterson, T F Bumol, 2004, Protein kinase C-alpha in prostate cancer: BJU.Int., v. 93, p. 1076-1081.

Larsen, K, J Malmstrom, M Wildt, C Dahlqvist, L Hansson, G Marko-Varga, L Bjermer, A Scheja, G Westergren-Thorsson, 2006, Functional and phenotypical comparison of myofibroblasts derived from biopsies and bronchoalveolar lavage in mild asthma and scleroderma: Respir.Res., v. 7, p. 11. 
Larsen, M R, T E Thingholm, O N Jensen, P Roepstorff, T J Jorgensen, 2005, Highly selective enrichment of phosphorylated peptides from peptide mixtures using titanium dioxide microcolumns: Mol.Cell Proteomics., v. 4, p. 873-886.

Le, Y, H Ji, J F Chen, Z Shen, J Yun, M Pu, 2009, Nanosized bicalutamide and its molecular structure in solvents: Int.J.Pharm., v. 370, p. 175-180.

Lee, H J, C Chang, 2003, Recent advances in androgen receptor action: Cell Mol.Life Sci., v. 60, p. 1613-1622.

Lee, J T, B D Lehmann, D M Terrian, W H Chappell, F Stivala, M Libra, A M Martelli, L S Steelman, J A McCubrey, 2008, Targeting prostate cancer based on signal transduction and cell cycle pathways: Cell Cycle, v. 7, p. 1745-1762.

Lee, W C, Y C Li, I M Chu, 2006, Amphiphilic poly(D,L-lactic acid)/poly(ethylene glycol)/poly(D,L-lactic acid) nanogels for controlled release of hydrophobic drugs: Macromol.Biosci., v. 6, p. 846-854.

Leitner, A, A Foettinger, W Lindner, 2007, Improving fragmentation of poorly fragmenting peptides and phosphopeptides during collision-induced dissociation by malondialdehyde modification of arginine residues: J.Mass Spectrom., v. 42, p. 950-959.

Leotoing, L, L Meunier, M Manin, C Mauduit, M Decaussin, G Verrijdt, F Claessens, M Benahmed, G Veyssiere, L Morel, C Beaudoin, 2008, Influence of nucleophosmin/B23 on DNA binding and transcriptional activity of the androgen receptor in prostate cancer cell: Oncogene, v. 27, p. 2858-2867.

Li, Q F, A M Spinelli, R Wang, Y Anfinogenova, H A Singer, D D Tang, 2006, Critical role of vimentin phosphorylation at Ser-56 by $\mathrm{p} 21$-activated kinase in vimentin cytoskeleton signaling: J.Biol.Chem., v. 281, p. 34716-34724.

Li, W H, X H Miao, Z T Qi, W Ni, S Y Zhu, F Fang, 2009, Proteomic analysis of differently expressed proteins in human hepatocellular carcinoma cell lines HepG2 with transfecting hepatitis B virus X gene: Chin Med.J.(Engl.), v. 122, p. 15-23.

Li, W Z, P Lin, J Frydman, T R Boal, T S Cardillo, L M Richard, D Toth, M A Lichtman, F U Hartl, F Sherman, 1994, Tcp20, a subunit of the eukaryotic TRiC chaperonin from humans and yeast: J.Biol.Chem., v. 269, p. 18616-18622.

Li, Y, X Xu, D Qi, C Deng, P Yang, X Zhang, 2008, Novel Fe3O4@TiO2 core-shell microspheres for selective enrichment of phosphopeptides in phosphoproteome analysis: J.Proteome.Res., v. 7, p. 2526-2538.

Lim, Y P, 2005, Mining the tumor phosphoproteome for cancer markers: Clin.Cancer Res., v. 11, p. 3163-3169. 
Lim, Y P, C Y Wong, L L Ooi, B J Druker, R J Epstein, 2004, Selective tyrosine hyperphosphorylation of cytoskeletal and stress proteins in primary human breast cancers: implications for adjuvant use of kinase-inhibitory drugs: Clin.Cancer Res., v. 10, p. 39803987.

Lin, J F, J Xu, H Y Tian, X Gao, Q X Chen, Q Gu, G J Xu, J D Song, F K Zhao, 2007, Identification of candidate prostate cancer biomarkers in prostate needle biopsy specimens using proteomic analysis: Int.J.Cancer, v. 121, p. 2596-2605.

Lin, K, R Lipsitz, T Miller, S Janakiraman, 2008, Benefits and harms of prostate-specific antigen screening for prostate cancer: an evidence update for the U.S. Preventive Services Task Force: Ann.Intern.Med., v. 149, p. 192-199.

Liu, X, Y Wu, Z E Zehner, C Jackson-Cook, J L Ware, 2003, Proteomic analysis of the tumorigenic human prostate cell line M12 after microcell-mediated transfer of chromosome 19 demonstrates reduction of vimentin: Electrophoresis, v. 24, p. 3445-3453.

Liu, Z, S Bengtsson, M Krogh, M Marquez, S Nilsson, P James, A Aliaya, A R Holmberg, 2007, Somatostatin effects on the proteome of the LNCaP cell-line: Int.J.Oncol., v. 30, p. 1173-1179.

Lopez Laur, J D, M Abud, F C Lopez, J Silva, Y Cisella, E R Perez, A Ortiz, 2008, [Antioxidant power and cellular damage in prostate cancer]: Arch.Esp.Urol., v. 61, p. 563-569.

Lopez, M F, K Berggren, E Chernokalskaya, A Lazarev, M Robinson, W F Patton, 2000, A comparison of silver stain and SYPRO Ruby Protein Gel Stain with respect to protein detection in two-dimensional gels and identification by peptide mass profiling: Electrophoresis, v. 21, p. 3673-3683.

Lopez-Alemany, R, C Longstaff, S Hawley, M Mirshahi, P Fabregas, M Jardi, E Merton, L A Miles, J Felez, 2003, Inhibition of cell surface mediated plasminogen activation by a monoclonal antibody against alpha-Enolase: Am.J.Hematol., v. 72, p. 234-242.

Malik, G, E Rojahn, M D Ward, M B Gretzer, A W Partin, O J Semmes, R W Veltri, 2007, SELDI protein profiling of dunning R-3327 derived cell lines: identification of molecular markers of prostate cancer progression: Prostate, v. 67, p. 1565-1575.

Mann, M, 2006, Functional and quantitative proteomics using SILAC: Nat.Rev.Mol.Cell Biol., v. 7, p. 952-958.

Marcantonio, M, M Trost, M Courcelles, M Desjardins, P Thibault, 2008, Combined enzymatic and data mining approaches for comprehensive phosphoproteome analyses: application to cell signaling events of interferon-gamma-stimulated macrophages:

Mol.Cell Proteomics., v. 7, p. 645-660. 
Meany, D L, Z Zhang, L J Sokoll, H Zhang, D W Chan, 2009, Glycoproteomics for prostate cancer detection: changes in serum PSA glycosylation patterns: J.Proteome.Res., v. 8, p. 613-619.

Meng, S, D Tripathy, S Shete, R Ashfaq, B Haley, S Perkins, P Beitsch, A Khan, D Euhus, C Osborne, E Frenkel, S Hoover, M Leitch, E Clifford, E Vitetta, L Morrison, D Herlyn, L W Terstappen, T Fleming, T Fehm, T Tucker, N Lane, J Wang, J Uhr, 2004, HER-2 gene amplification can be acquired as breast cancer progresses:

Proc.Natl.Acad.Sci.U.S.A, v. 101, p. 9393-9398.

Miller, D C, K S Hafez, A Stewart, J E Montie, J T Wei, 2003, Prostate carcinoma presentation, diagnosis, and staging: an update form the National Cancer Data Base: Cancer, v. 98, p. 1169-1178.

Minden, J S, S R Dowd, H E Meyer, K Stuhler, 2009, Difference gel electrophoresis: Electrophoresis, v. 30 Suppl 1, p. S156-S161.

Mitchell, S, P Abel, M Ware, G Stamp, E Lalani, 2000, Phenotypic and genotypic characterization of commonly used human prostatic cell lines: BJU.Int., v. 85, p. 932-944.

Miyake, H, M Muramaki, T Kurahashi, K Yamanaka, I Hara, M Fujisawa, 2006, Enhanced expression of heat shock protein 27 following neoadjuvant hormonal therapy is associated with poor clinical outcome in patients undergoing radical prostatectomy for prostate cancer: Anticancer Res., v. 26, p. 1583-1587.

Molina, H, D M Horn, N Tang, S Mathivanan, A Pandey, 2007, Global proteomic profiling of phosphopeptides using electron transfer dissociation tandem mass spectrometry: Proc.Natl.Acad.Sci.U.S.A, v. 104, p. $2199-2204$.

Molloy, M P, E E Brzezinski, J Hang, M T McDowell, R A VanBogelen, 2003, Overcoming technical variation and biological variation in quantitative proteomics: Proteomics., v. 3, p. 1912-1919.

Moser, K, F M White, 2006, Phosphoproteomic analysis of rat liver by high capacity IMAC and LC-MS/MS: J.Proteome.Res., v. 5, p. 98-104.

Muller, F L, M S Lustgarten, Y Jang, A Richardson, H Van Remmen, 2007, Trends in oxidative aging theories: Free Radic.Biol.Med., v. 43, p. 477-503.

Ndassa, Y M, C Orsi, J A Marto, S Chen, M M Ross, 2006, Improved immobilized metal affinity chromatography for large-scale phosphoproteomics applications: J.Proteome.Res., v. 5, p. 2789-2799.

Neverova, I, J E Van Eyk, 2005, Role of chromatographic techniques in proteomic analysis: J.Chromatogr.B Analyt.Technol.Biomed.Life Sci., v. 815, p. 51-63. 
Nikolovska-Coleska, Z, L Xu, Z Hu, Y Tomita, P Li, P P Roller, R Wang, X Fang, R Guo, M Zhang, M E Lippman, D Yang, S Wang, 2004, Discovery of embelin as a cellpermeable, small-molecular weight inhibitor of XIAP through structure-based computational screening of a traditional herbal medicine three-dimensional structure database: J.Med.Chem., v. 47, p. 2430-2440.

Obenauer, J C, L C Cantley, M B Yaffe, 2003, Scansite 2.0: Proteome-wide prediction of cell signaling interactions using short sequence motifs: Nucleic Acids Res., v. 31, p. 3635-3641.

Olsson, I, K Larsson, R Palmgren, B Bjellqvist, 2002, Organic disulfides as a means to generate streak-free two-dimensional maps with narrow range basic immobilized $\mathrm{pH}$ gradient strips as first dimension: Proteomics., v. 2, p. 1630-1632.

Ornstein, D K, D R Tyson, 2006, Proteomics for the identification of new prostate cancer biomarkers: Urol.Oncol., v. 24, p. 231-236.

Ou, K, K Yu, D Kesuma, M Hooi, N Huang, W Chen, S Y Lee, X P Goh, L K Tan, J Liu, S Y Soon, R S Bin Abdul, T C Putti, H Jikuya, T Ichikawa, O Nishimura, M Salto-Tellez, $\mathrm{P}$ Tan, 2008, Novel breast cancer biomarkers identified by integrative proteomic and gene expression mapping: J.Proteome.Res., v. 7, p. 1518-1528.

Pancholi, V, 2001, Multifunctional alpha-enolase: its role in diseases: Cell Mol.Life Sci., v. 58, p. 902-920.

Pang, B, H Zhang, J Wang, W Z Chen, S H Li, Q G Shi, R X Liang, B X Xie, R Q Wu, X L Qian, L Yu, Q M Li, C F Huang, J G Zhou, 2009, Ubiquitous mitochondrial creatine kinase is overexpressed in the conditioned medium and the extract of LNCaP lineaged androgen independent cell lines and facilitates prostate cancer progression: Prostate., v. 69, p. 1176-1187.

Parekh, D J, D P Ankerst, D Troyer, S Srivastava, I M Thompson, 2007, Biomarkers for prostate cancer detection: J.Urol., v. 178, p. 2252-2259.

Park, S Y, X Yu, C Ip, J L Mohler, P N Bogner, Y M Park, 2007, Peroxiredoxin 1 interacts with androgen receptor and enhances its transactivation: Cancer Res., v. 67, p. 9294-9303.

Patterson, S D, R Aebersold, 1995, Mass spectrometric approaches for the identification of gel-separated proteins: Electrophoresis, v. 16, p. 1791-1814.

Patton, W F, 1999, Proteome analysis. II. Protein subcellular redistribution: linking physiology to genomics via the proteome and separation technologies involved: J.Chromatogr.B Biomed.Sci.Appl., v. 722, p. 203-223.

Patton, W F, 2000, A thousand points of light: the application of fluorescence detection technologies to two-dimensional gel electrophoresis and proteomics: Electrophoresis, v. 21, p. 1123-1144. 
Pennington, K, E McGregor, C L Beasley, I Everall, D Cotter, M J Dunn, 2004, Optimization of the first dimension for separation by two-dimensional gel electrophoresis of basic proteins from human brain tissue: Proteomics., v. 4, p. 27-30.

Petrylak, D P, C M Tangen, M H Hussain, P N Lara, Jr., J A Jones, M E Taplin, P A Burch, D Berry, C Moinpour, M Kohli, M C Benson, E J Small, D Raghavan, E D Crawford, 2004, Docetaxel and estramustine compared with mitoxantrone and prednisone for advanced refractory prostate cancer: N.Engl.J.Med., v. 351, p. 1513-1520.

Pinkse, M W, P M Uitto, M J Hilhorst, B Ooms, A J Heck, 2004, Selective isolation at the femtomole level of phosphopeptides from proteolytic digests using 2D-NanoLC-ESIMS/MS and titanium oxide precolumns: Anal.Chem., v. 76, p. 3935-3943.

Pitkanen-Arsiola, T, J E Tillman, G Gu, J Yuan, R L Roberts, M Wantroba, G A Coetzee, M S Cookson, S Kasper, 2006, Androgen and anti-androgen treatment modulates androgen receptor activity and DJ-1 stability: Prostate, v. 66, p. 1177-1193.

Plow, E F, T Herren, A Redlitz, L A Miles, J L Hoover-Plow, 1995, The cell biology of the plasminogen system: FASEB J., v. 9, p. 939-945.

Posewitz, M C, P Tempst, 1999, Immobilized gallium(III) affinity chromatography of phosphopeptides: Anal.Chem., v. 71, p. 2883-2892.

Qi, C, Y T Zhu, J Chang, A V Yeldandi, M S Rao, Y J Zhu, 2005, Potentiation of estrogen receptor transcriptional activity by breast cancer amplified sequence 2 : Biochem.Biophys.Res.Commun., v. 328, p. 393-398.

Rabilloud, T, 1996, Solubilization of proteins for electrophoretic analyses:

Electrophoresis, v. 17, p. 813-829.

Rabilloud, T, 2009, Solubilization of proteins in 2DE: an outline: Methods Mol.Biol., v. 519, p. 19-30.

Rabilloud, T, C Adessi, A Giraudel, J Lunardi, 1997, Improvement of the solubilization of proteins in two-dimensional electrophoresis with immobilized $\mathrm{pH}$ gradients:

Electrophoresis, v. 18, p. 307-316.

Rao, A R, H G Motiwala, O M Karim, 2008, The discovery of prostate-specific antigen: BJU.Int., v. 101, p. 5-10.

Raponi, M, R T Belly, J E Karp, J E Lancet, D Atkins, Y Wang, 2004, Microarray analysis reveals genetic pathways modulated by tipifarnib in acute myeloid leukemia: BMC.Cancer, v. 4, p. 56.

Rhee, S Y, V Wood, K Dolinski, S Draghici, 2008, Use and misuse of the gene ontology annotations: Nat.Rev.Genet., v. 9, p. 509-515. 
Rocchi, P, P Jugpal, A So, S Sinneman, S Ettinger, L Fazli, C Nelson, M Gleave, 2006, Small interference RNA targeting heat-shock protein 27 inhibits the growth of prostatic cell lines and induces apoptosis via caspase-3 activation in vitro: BJU.Int., v. 98, p. 10821089.

Rocchi, P, A So, S Kojima, M Signaevsky, E Beraldi, L Fazli, A Hurtado-Coll, K Yamanaka, M Gleave, 2004, Heat shock protein 27 increases after androgen ablation and plays a cytoprotective role in hormone-refractory prostate cancer: Cancer Res., v. 64, p. 6595-6602.

Rochette-Egly, C, 2003, Nuclear receptors: integration of multiple signalling pathways through phosphorylation: Cell Signal., v. 15, p. 355-366.

Rondepierre, F, B Bouchon, J Papon, M Bonnet-Duquennoy, R Kintossou, N Moins, J Maublant, J C Madelmont, M D'Incan, F Degoul, 2009, Proteomic studies of B16 lines: involvement of annexin A1 in melanoma dissemination: Biochim.Biophys.Acta, v. 1794, p. 61-69.

Rosengren, A T, J M Salmi, T Aittokallio, J Westerholm, R Lahesmaa, T A Nyman, O S Nevalainen, 2003, Comparison of PDQuest and Progenesis software packages in the analysis of two-dimensional electrophoresis gels: Proteomics., v. 3, p. 1936-1946.

Ross, L E, R J Coates, N Breen, R J Uhler, A L Potosky, D Blackman, 2004, Prostatespecific antigen test use reported in the 2000 National Health Interview Survey: Prev.Med., v. 38, p. 732-744.

Rowland, J G, J L Robson, W J Simon, H Y Leung, A R Slabas, 2007, Evaluation of an in vitro model of androgen ablation and identification of the androgen responsive proteome in LNCaP cells: Proteomics., v. 7, p. 47-63.

Saldanha, R G, N Xu, M P Molloy, D A Veal, M S Baker, 2008, Differential proteome expression associated with urokinase plasminogen activator receptor (uPAR) suppression in malignant epithelial cancer: J.Proteome.Res., v. 7, p. 4792-4806.

Salomon, A R, S B Ficarro, L M Brill, A Brinker, Q T Phung, C Ericson, K Sauer, A Brock, D M Horn, P G Schultz, E C Peters, 2003, Profiling of tyrosine phosphorylation pathways in human cells using mass spectrometry: Proc.Natl.Acad.Sci.U.S.A, v. 100, p. 443-448.

Samland, A K, G A Sprenger, 2009, Transaldolase: from biochemistry to human disease: Int.J.Biochem.Cell Biol., v. 41, p. 1482-1494.

Sardana, G, B Dowell, E P Diamandis, 2008a, Emerging biomarkers for the diagnosis and prognosis of prostate cancer: Clin.Chem., v. 54, p. 1951-1960. 
Sardana, G, K Jung, C Stephan, E P Diamandis, 2008b, Proteomic analysis of conditioned media from the PC3, LNCaP, and 22Rv1 prostate cancer cell lines: discovery and validation of candidate prostate cancer biomarkers: J.Proteome.Res., v. 7, p. 33293338 .

Sardana, G, J Marshall, E P Diamandis, 2007, Discovery of candidate tumor markers for prostate cancer via proteomic analysis of cell culture-conditioned medium: Clin.Chem., v. 53, p. 429-437.

Schaefer, H, D C Chamrad, M Herrmann, J Stuwe, G Becker, J Klose, M Blueggel, H E Meyer, K Marcus, 2006, Study of posttranslational modifications in lenticular alphaACrystallin of mice using proteomic analysis techniques: Biochim.Biophys.Acta, v. 1764, p. 1948-1962.

Schulenberg, B, T N Goodman, R Aggeler, R A Capaldi, W F Patton, 2004, Characterization of dynamic and steady-state protein phosphorylation using a fluorescent phosphoprotein gel stain and mass spectrometry: Electrophoresis, v. 25, p. 2526-2532.

Schwartz, J C, M W Senko, J E Syka, 2002, A two-dimensional quadrupole ion trap mass spectrometer: J.Am.Soc.Mass Spectrom., v. 13, p. 659-669.

Semmes, O J, G Malik, M Ward, 2006, Application of mass spectrometry to the discovery of biomarkers for detection of prostate cancer: J.Cell Biochem., v. 98, p. 496503.

Shen, C, C Nathan, 2002, Nonredundant antioxidant defense by multiple two-cysteine peroxiredoxins in human prostate cancer cells: Mol.Med., v. 8, p. 95-102.

Skvortsova, I, S Skvortsov, T Stasyk, U Raju, B A Popper, B Schiestl, E von Guggenberg, A Neher, G K Bonn, L A Huber, P Lukas, 2008, Intracellular signaling pathways regulating radioresistance of human prostate carcinoma cells: Proteomics., v. 8, p. 45214533.

Smith, R A, V Cokkinides, O W Brawley, 2009, Cancer screening in the United States, 2009: a review of current American Cancer Society guidelines and issues in cancer screening: CA Cancer J.Clin., v. 59, p. 27-41.

Smitherman, A B, J L Mohler, S J Maygarden, D K Ornstein, 2004, Expression of annexin I, II and VII proteins in androgen stimulated and recurrent prostate cancer: J.Urol., v. 171, p. 916-920.

Solassol, J, P Marin, E Demettre, P Rouanet, J Bockaert, T Maudelonde, A Mange, 2005, Proteomic detection of prostate-specific antigen using a serum fractionation procedure: potential implication for new low-abundance cancer biomarkers detection: Anal.Biochem., v. 338, p. 26-31. 
Spahr, C S, S A Susin, E J Bures, J H Robinson, M T Davis, M D McGinley, G Kroemer, S D Patterson, 2000, Simplification of complex peptide mixtures for proteomic analysis: reversible biotinylation of cysteinyl peptides: Electrophoresis, v. 21, p. 1635-1650.

Stadtman, E R, 1992, Protein oxidation and aging: Science, v. 257, p. 1220-1224.

Stasyk, T, S Morandell, R Bakry, I Feuerstein, C W Huck, G Stecher, G K Bonn, L A Huber, 2005, Quantitative detection of phosphoproteins by combination of twodimensional difference gel electrophoresis and phosphospecific fluorescent staining: Electrophoresis, v. 26, p. 2850-2854.

Steinberg, T H, B J Agnew, K R Gee, W Y Leung, T Goodman, B Schulenberg, J Hendrickson, J M Beechem, R P Haugland, W F Patton, 2003, Global quantitative phosphoprotein analysis using Multiplexed Proteomics technology: Proteomics., v. 3, p. 1128-1144.

Stephens, B J, H Han, V Gokhale, D D Von Hoff, 2005, PRL phosphatases as potential molecular targets in cancer: Mol.Cancer Ther., v. 4, p. 1653-1661.

Subong, E N, M J Shue, J I Epstein, J V Briggman, P K Chan, A W Partin, 1999, Monoclonal antibody to prostate cancer nuclear matrix protein (PRO:4-216) recognizes nucleophosmin/B23: Prostate, v. 39, p. 298-304.

Suzuki, S, K Takeshita, M Asamoto, S Takahashi, H Kandori, K Tsujimura, F Saito, K Masuko, T Shirai, 2009, High mobility group box associated with cell proliferation appears to play an important role in hepatocellular carcinogenesis in rats and humans: Toxicology, v. 255, p. 160-170.

Sweet, S M, A J Creese, H J Cooper, 2006, Strategy for the identification of sites of phosphorylation in proteins: neutral loss triggered electron capture dissociation: Anal.Chem., v. 78, p. 7563-7569.

Tang, D D, 2008, Intermediate filaments in smooth muscle: Am.J.Physiol Cell Physiol, v. 294, p. C869-C878.

Thingholm, T E, T J Jorgensen, O N Jensen, M R Larsen, 2006, Highly selective enrichment of phosphorylated peptides using titanium dioxide: Nat.Protoc., v. 1, p. 19291935.

Tholey, A, J Reed, W D Lehmann, 1999, Electrospray tandem mass spectrometric studies of phosphopeptides and phosphopeptide analogues: J.Mass Spectrom., v. 34, p. 117-123.

Thompson, I M, D K Pauler, P J Goodman, C M Tangen, M S Lucia, H L Parnes, L M Minasian, L G Ford, S M Lippman, E D Crawford, J J Crowley, C A Coltman, Jr., 2004, Prevalence of prostate cancer among men with a prostate-specific antigen level $<$ or $=4.0$ ng per milliliter: N.Engl.J.Med., v. 350, p. 2239-2246. 
Tillman, J E, J Yuan, G Gu, L Fazli, R Ghosh, A S Flynt, M Gleave, P S Rennie, S Kasper, 2007, DJ-1 binds androgen receptor directly and mediates its activity in hormonally treated prostate cancer cells: Cancer Res., v. 67, p. 4630-4637.

Tilton, R G, S J Haidacher, W S Lejeune, X Zhang, Y Zhao, A Kurosky, A R Brasier, L Denner, 2007, Diabetes-induced changes in the renal cortical proteome assessed with two-dimensional gel electrophoresis and mass spectrometry: Proteomics., v. 7, p. 17291742.

Topoglidis, E, T Lutz, R L Willis, C J Barnett, A E Cass, J R Durrant, 2000, Protein adsorption on nanoporous TiO2 films: a novel approach to studying photoinduced protein/electrode transfer reactions: Faraday Discuss., p. 35-46.

Tsai, C F, Y T Wang, Y R Chen, C Y Lai, P Y Lin, K T Pan, J Y Chen, K H Khoo, Y J Chen, 2008, Immobilized metal affinity chromatography revisited: $\mathrm{pH} /$ acid control toward high selectivity in phosphoproteomics: J.Proteome.Res., v. 7, p. 4058-4069.

Ummanni, R, H Junker, U Zimmermann, S Venz, S Teller, J Giebel, C Scharf, C Woenckhaus, F Dombrowski, R Walther, 2008, Prohibitin identified by proteomic analysis of prostate biopsies distinguishes hyperplasia and cancer: Cancer Lett., v. 266, p. 171-185.

Unlu, M, M E Morgan, J S Minden, 1997, Difference gel electrophoresis: a single gel method for detecting changes in protein extracts: Electrophoresis, v. 18, p. 2071-2077.

Verhoeven, N M, J H Huck, B Roos, E A Struys, G S Salomons, A C Douwes, M S van der Knaap, C Jakobs, 2001, Transaldolase deficiency: liver cirrhosis associated with a new inborn error in the pentose phosphate pathway: Am.J.Hum.Genet., v. 68, p. 10861092.

Visconti, R, D Grieco, 2009, New insights on oxidative stress in cancer: Curr.Opin.Drug Discov.Devel., v. 12, p. 240-245.

Washburn, M P, D Wolters, J R Yates, III, 2001, Large-scale analysis of the yeast proteome by multidimensional protein identification technology: Nat.Biotechnol., v. 19, p. 242-247.

Wehder, L, S Arndt, U Murzik, A K Bosserhoff, R Kob, F von Eggeling, C Melle, 2009, Annexin A5 is involved in migration and invasion of oral carcinoma: Cell Cycle, v. 8, p. 1552-1558.

Wei, J, G Xu, M Wu, Y Zhang, Q Li, P Liu, T Zhu, A Song, L Zhao, Z Han, G Chen, S Wang, L Meng, J Zhou, Y Lu, S Wang, D Ma, 2008, Overexpression of vimentin contributes to prostate cancer invasion and metastasis via src regulation: Anticancer Res., v. 28, p. 327-334. 
Welch, H G, L M Schwartz, S Woloshin, 2005, Prostate-specific antigen levels in the United States: implications of various definitions for abnormal: J.Natl.Cancer Inst., v. 97, p. 1132-1137.

Whitaker, H C, D P Stanbury, C Brinham, J Girling, S Hanrahan, N Totty, D E Neal, 2007, Labeling and identification of $\mathrm{LNCaP}$ cell surface proteins: a pilot study: Prostate, v. 67, p. 943-954.

Wu, J, N J Lenchik, M J Pabst, S S Solomon, J Shull, I C Gerling, 2005, Functional characterization of two-dimensional gel-separated proteins using sequential staining: Electrophoresis, v. 26, p. 225-237.

Wu, M, X Bai, G Xu, J Wei, T Zhu, Y Zhang, Q Li, P Liu, A Song, L Zhao, C Gang, Z Han, S Wang, J Zhou, Y Lu, D Ma, 2007, Proteome analysis of human androgenindependent prostate cancer cell lines: variable metastatic potentials correlated with vimentin expression: Proteomics., v. 7, p. 1973-1983.

Yang, H S, C P Matthews, T Clair, Q Wang, A R Baker, C C Li, T H Tan, N H Colburn, 2006, Tumorigenesis suppressor Pdcd4 down-regulates mitogen-activated protein kinase kinase kinase kinase 1 expression to suppress colon carcinoma cell invasion: Mol.Cell Biol, v. 26, p. 1297-1306.

Yang, Z L, X R Li, K W Yang, Y Liu, 2008, Amphotericin B-loaded poly(ethylene glycol)-poly(lactide) micelles: preparation, freeze-drying, and in vitro release:

J.Biomed.Mater.Res.A, v. 85, p. 539-546.

Yasugi, K, Y Nagasaki, M Kato, K Kataoka, 1999, Preparation and characterization of polymer micelles from poly(ethylene glycol)-poly(D,L-lactide) block copolymers as potential drug carrier: J.Control Release, v. 62, p. 89-100.

Yates, J R, III, 2000, Mass spectrometry. From genomics to proteomics: Trends Genet., v. 16 , p. 5-8.

Yates, J R, III, J K Eng, A L McCormack, D Schieltz, 1995, Method to correlate tandem mass spectra of modified peptides to amino acid sequences in the protein database:

Anal.Chem., v. 67, p. 1426-1436.

Yates, J R, C I Ruse, A Nakorchevsky, 2009, Proteomics by mass spectrometry: approaches, advances, and applications: Annu.Rev.Biomed.Eng, v. 11, p. 49-79.

Yu, L R, Z Zhu, K C Chan, H J Issaq, D S Dimitrov, T D Veenstra, 2007a, Improved titanium dioxide enrichment of phosphopeptides from HeLa cells and high confident phosphopeptide identification by cross-validation of MS/MS and MS/MS/MS spectra: J.Proteome.Res., v. 6, p. 4150-4162. 
Yu, Y P, G Yu, G Tseng, K Cieply, J Nelson, M Defrances, R Zarnegar, G Michalopoulos, J H Luo, 2007b, Glutathione peroxidase 3, deleted or methylated in prostate cancer, suppresses prostate cancer growth and metastasis: Cancer Res., v. 67, p. 8043-8050.

Zahedi, R P, A J Begonja, S Gambaryan, A Sickmann, 2006, Phosphoproteomics of human platelets: A quest for novel activation pathways: Biochim.Biophys.Acta, v. 1764, p. 1963-1976.

Zhan, X, D M Desiderio, 2003, Differences in the spatial and quantitative reproducibility between two second-dimensional gel electrophoresis systems: Electrophoresis, v. 24, p. 1834-1846.

Zhan, X, F Giorgianni, D M Desiderio, 2005, Proteomics analysis of growth hormone isoforms in the human pituitary: Proteomics., v. 5, p. 1228-1241.

Zhao, Y, F Giorgianni, D M Desiderio, B Fang, S Beranova-Giorgianni, 2005, Toward a global analysis of the human pituitary proteome by multiple gel-based technology: Anal.Chem., v. 77, p. 5324-5331.

Zhao, Y, Q Yan, X Long, X Chen, Y Wang, 2008, Vimentin affects the mobility and invasiveness of prostate cancer cells: Cell Biochem.Funct., v. 26, p. 571-577.

Zoubeidi, A, A Zardan, E Beraldi, L Fazli, R Sowery, P Rennie, C Nelson, M Gleave, 2007, Cooperative interactions between androgen receptor (AR) and heat-shock protein 27 facilitate AR transcriptional activity: Cancer Res., v. 67, p. 10455-10465.

Zuo, X, D W Speicher, 2000, Quantitative evaluation of protein recoveries in twodimensional electrophoresis with immobilized $\mathrm{pH}$ gradients: Electrophoresis, v. 21, p. 3035-3047. 


\section{APPENDIX}

Table A-1 Full list of phosphopeptides and phosphoproteins characterized in LNCaP cells.

\begin{tabular}{|c|c|c|c|c|c|c|}
\hline $\begin{array}{l}\text { Accession } \\
\text { number }\end{array}$ & Peptide & МH+ & $\mathbf{z}$ & $\mathbf{X C}$ & $\begin{array}{l}\text { Site \# in Swiss/Phosphosite } \\
(06 / 17 / 08)\end{array}$ & $\begin{array}{l}\text { Present in Phosphosite } \\
(06 / 17 / 08)\end{array}$ \\
\hline \multirow[t]{4}{*}{ P07900 } & HS90A_HUMAN Heat shock protein HSP 90-alpha (HSP 86) & & & 20.21 & & \\
\hline & K.ESEDKPEIEDVGS*DEEEEK.K & 2272.88640 & 2 & 4.15 & 263 Swiss/ 263 phos & Yes \\
\hline & K.ESEDKPEIEDVGS*DEEEEKK.D & 2400.98137 & 3 & 3.93 & see line 4 & \\
\hline & R.DKEVS*DDEAEEK.E & 1473.56282 & 2 & 4.89 & 231 Swiss/ 231 phos & Yes \\
\hline \multirow[t]{4}{*}{ Q15019 } & SEPT2_HUMAN Septin-2 (Protein NEDD5) & & & 10.27 & & \\
\hline & K.IYHLPDAES*DEDEDFKEQTR.L & 2517.04530 & 3 & 4.28 & see line 10 & \\
\hline & R.YLHDES*GLNR.R & 1283.54158 & 2 & 2.41 & 134 NEW & No \\
\hline & K.IYHLPDAES*DEDEDFK.E & 2002.79534 & 2 & 3.37 & 218 Swiss/ 218 phos & Yes \\
\hline \multirow[t]{3}{*}{ Q15185 } & TEBP_HUMAN Prostaglandin E synthase 3 (Cytosolic prostagland & synthase) & & 10.35 & & \\
\hline & K.DWEDDS*DEDMSNFDR.F & 1955.62729 & 2 & 5.85 & 113 Swiss/ 113 phos & Yes \\
\hline & K.DWEDDS*DEDM\#SNFDR.F & 1971.62219 & 2 & 2.92 & see line 12 & \\
\hline \multirow[t]{11}{*}{ O60716 } & CTND1_HUMAN Catenin delta-1 (p120 catenin) & & & 70.35 & & \\
\hline & R.LRSY*EDMIGEEVPSDQYYWAPLAQHER.G & 3362.48235 & 3 & 6.95 & 321 Swiss/ 321 phos & Yes \\
\hline & R.HYEDGYPGGSDNYGS*LSR.V & 2053.79232 & 2 & 4.83 & 230 Swiss/ 230 phos & Yes \\
\hline & R.SQSSHSYDDST*LPLIDR.N & 2000.85968 & 3 & 4.73 & 869 Swiss & No \\
\hline & R.S*YEDMIGEEVPSDQYYWAPLAQHER.G & 3093.29718 & 3 & 3.65 & 320 Swiss/ 320 phos & Yes \\
\hline & R.VGGS*SVDLHR.F & 1106.49898 & 2 & 3.19 & 268 Swiss/ 268 phos & Yes \\
\hline & R.S*GDLGDMEPLK.G & 1241.51192 & 2 & 3.14 & $920 \mathrm{NEW}$ & No \\
\hline & R.TLDRS*GDLGDMEPLK.G & 1726.77171 & 2 & 2.77 & $920 \mathrm{NEW}$ & No \\
\hline & R.S*MGYDDLDYGMMSDYGTAR.R & 2227.80177 & 2 & 5.45 & 288 Swiss/ 288 phos & Yes \\
\hline & R.T*LDRSGDLGDMEPLK.G & 1726.77171 & 2 & 2.65 & 916 Swiss/ 916 phos & Yes \\
\hline & R.HYEDGYPGGS*DNY*GSLSRVT*RIEER.Y & 3097.21249 & 3 & 3.04 & 228 Swiss/ 228 phos & Yes \\
\hline
\end{tabular}


Table A-1 (continued).

\begin{tabular}{|c|c|c|c|c|c|c|}
\hline $\begin{array}{l}\text { Accession } \\
\text { number }\end{array}$ & Peptide & MH+ & $\mathbf{z}$ & $\mathbf{X C}$ & $\begin{array}{l}\text { Site \# in Swiss/Phosphosite } \\
(06 / 17 / 08)\end{array}$ & $\begin{array}{l}\text { Present in Phosphosite } \\
(06 / 17 / 08)\end{array}$ \\
\hline & R.S*MGYDDLDYGMMSDYGTAR.R & 2227.80177 & 2 & 3.41 & see line 22 & \\
\hline & R.VGGS*SVDLHR.F & 1106.49898 & 2 & 2.44 & see line 19 & \\
\hline & K.WAHDKFS*GEEGEIEDDES*GTENR.E & 2797.02977 & 3 & 6.50 & 939 Swiss/ 939 phos & Yes \\
\hline & K.WAHDKFS*GEEGEIEDDESGTENR.E & 2717.06347 & 3 & 5.99 & 928 Swiss/ 928 phos & Yes \\
\hline & K.WAHDKFS*GEEGEIEDDESGT*ENREEK.D & 3183.20992 & 3 & 3.70 & 941 Swiss/ 941 phos & Yes \\
\hline & R.ERS*PALKS*PLQSVVVR.R & 1925.96089 & 3 & 3.42 & $\begin{array}{l}248 \text { Swiss } / 248 \text { phos } \& 253 \\
\text { Swiss/ } 253 \text { phos }\end{array}$ & Yes \\
\hline & R.IDIS*PSTFR.K & 1115.51324 & 2 & 3.28 & 682 Swiss/ 682 phos & Yes \\
\hline & R.ASAVSELS*PR.E & 1096.50340 & 2 & 3.01 & 243 Swiss/ 243 phos & Yes \\
\hline & K.S*PPSTGSTYGSSQK.E & 1463.60496 & 2 & 2.82 & 320 Swiss/ 320 phos & Yes \\
\hline & R.DSS*HS*RERS*AEK.T & 1628.55019 & 2 & 2.35 & $\begin{array}{l}747 \text { NEW \& } 749 \text { NEW \& } 753 \\
\text { NEW }\end{array}$ & No \\
\hline & R.RIDIS*PSTFR.K & 1271.61435 & 2 & 2.15 & see line 32 & \\
\hline & K.SPPSTGST*Y*GSS*QK.E & 1623.53756 & 2 & 2.09 & $\begin{array}{l}324 \text { Swiss/ } 324 \text { phos \& } 325 \\
\text { NEW \& } 328 \text { NEW }\end{array}$ & Yes \& No \& No \\
\hline \multirow[t]{3}{*}{ P08651 } & NFIC_HUMAN Nuclear factor 1 C-type (Nuclear factor 1/C) & & & 20.25 & & \\
\hline & R.NWTEDMEGGISS*PVK.K & 1729.71386 & 2 & 4.96 & 323 Swiss/ 323 phos & Yes \\
\hline & R.NWTEDMEGGISS*PVKK.T & 1857.80883 & 2 & 4.41 & see line 39 & \\
\hline \multirow[t]{3}{*}{ Q13283 } & \multicolumn{3}{|c|}{ G3BP1_HUMAN Ras GTPase-activating protein-binding protein 1 (G3BP-1) } & 20.26 & & \\
\hline & K.SSS*PAPADIAQTVQEDLR.T & 1964.89606 & 2 & 4.55 & 232 Swiss/ 232 phos & Yes \\
\hline & R.YQDEVFGGFVTEPQEES*EEEVEEPEER.Q & 3296.33143 & 3 & 3.25 & 149 Swiss/ 149 phos & Yes \\
\hline \multirow[t]{4}{*}{ Q9Y608 } & LRRF2_HUMAN Leucine-rich repeat flightless-interacting protein 2 & & & 20.25 & & \\
\hline & R.RGS*GDTSSLIDPDTSLSELR.D & 2185.99723 & 2 & 4.94 & 328 Swiss/ 328 phos & Yes \\
\hline & R.IS*S*ARSS*PGFTNDDTASIVSSDRASR.G & 2924.18594 & 3 & 3.04 & $\begin{array}{l}231 \text { NEW \& } 232 \text { NEW \& } 236 \\
\text { NEW }\end{array}$ & No \\
\hline & R.PSSRNS*ASATT*PLSGNSS*R.R & 2116.80966 & 2 & 2.34 & $\begin{array}{l}312 \text { NEW \& } 317 \text { NEW \& } 324 \\
\text { NEW }\end{array}$ & Yes \\
\hline \multirow[t]{2}{*}{ Q5VTR2 } & BRE1A_HUMAN Ubiquitin-protein ligase BRE1A (BRE1-A) & & & 20.18 & & \\
\hline & K.ALVVPEPEPDSDS*NQER.K & 1961.84878 & 2 & 3.70 & 138 Swiss/ 138 phos & Yes \\
\hline
\end{tabular}


Table A-1 (continued).

\begin{tabular}{|c|c|c|c|c|c|c|}
\hline $\begin{array}{l}\text { Accession } \\
\text { number }\end{array}$ & Peptide & MH+ & $\mathbf{z}$ & $\mathbf{X C}$ & $\begin{array}{l}\text { Site \# in Swiss/Phosphosite } \\
(06 / 17 / 08)\end{array}$ & $\begin{array}{l}\text { Present in Phosphosite } \\
(06 / 17 / 08)\end{array}$ \\
\hline & K.PDSEDLSSQSS*AS*KASQEDANEIK.S & 2683.06549 & 3 & 3.42 & 548 NEW \& 550 NEW & No \\
\hline \multirow[t]{5}{*}{ Q8N6T3 } & ARFG1_HUMAN ADP-ribosylation factor GTPase-activating protein 1 & & & 20.21 & & \\
\hline & R.RSS*DSWEVWGSASTNR.N & 1904.79226 & 2 & 3.98 & see line 54 & \\
\hline & R.EFLESQEDY*DPCWS*LQEK.Y & 2405.89201 & 2 & 2.15 & 93 New \& 98 New & No \\
\hline & R.SS*DSWEVWGSASTNR.N & 1748.69115 & 2 & 4.03 & 361 Swiss/ New in phos & No \\
\hline & K.ASELGHS*LNENVLKPAQEK.V & 2144.03831 & 3 & 3.39 & 246 Swiss/ 246 phos & Yes \\
\hline \multirow[t]{4}{*}{ Q9Y6G9 } & DC1L1_HUMAN Cytoplasmic dynein 1 light intermediate chain 1 & & & 30.31 & & \\
\hline & R.DFQEYVEPGEDFPAS*PQR.R & 2190.90154 & 2 & 5.94 & 207 Swiss/ 207 phos & Yes \\
\hline & K.KTGSPGGPGVSGGS*PAGGAGGGSS*GLPPSTKK.S & 2811.27095 & 3 & 3.77 & 468 NEW \& 478 NEW & No \\
\hline & K.PVT*VS*PTTPTSPTEGEAS* & 1997.74287 & 2 & 2.22 & $\begin{array}{l}508 \text { NEW \& } 510 \text { Swiss/510 } \\
\text { phos \& } 523 \text { NEW }\end{array}$ & No \& Yes \& No \\
\hline \multirow[t]{5}{*}{ Q9Y2U5 } & M3K2_HUMAN Mitogen-activated protein kinase kinase kinase 2 & & & 40.21 & & \\
\hline & R.DRSS*PPPGYIPDELHQVAR.N & 2214.03389 & 3 & 4.27 & 164 Swiss/ 164 phos & Yes \\
\hline & R.AQS*YPDNHQEFSDYDNPIFEK.F & 2624.06129 & 3 & 3.87 & $239 \mathrm{NEW}$ & No \\
\hline & K.RLS*IIGPTSR.D & 1179.62452 & 2 & 2.62 & $153 \mathrm{NEW}$ & No \\
\hline & R.GS*DIDNPT*LTVM\#DISPPSR.S & 2190.90250 & 2 & 2.38 & $\begin{array}{l}331 \text { Swiss/ } 331 \text { phos \& } 337 \\
\text { NEW }\end{array}$ & Yes \& No \\
\hline \multirow[t]{2}{*}{ O00193 } & SMAP_HUMAN Small acidic protein & & & 10.34 & & \\
\hline & R.S*ASPDDDLGSSNWEAADLGNEER.K & 2514.98925 & 2 & 6.76 & 15 Swiss/ 15 phos & Yes \\
\hline \multirow[t]{6}{*}{ O95218 } & ZRAB2_HUMAN Zinc finger Ran-binding domain-containing protein 2 & & & 20.20 & & \\
\hline & R.ENVEYIEREES*DGEYDEFGR.K & 2545.00383 & 3 & 3.45 & see line 69 & \\
\hline & R.EES*DGEYDEFGR.K & 1512.51621 & 2 & 3.21 & 120 Swiss/ 120 phos & Yes \\
\hline & K.YNLDAS*EEEDSNK.K & 1593.59519 & 2 & 5.17 & 188 Swiss/ 188 phos & Yes \\
\hline & K.EVEDKES*EGEEEDEDEDLSK.Y & 2419.90318 & 3 & 3.62 & 153 Swiss/ 153 phos & Yes \\
\hline & R.S*SSRS*S*SPSSSRSR.S & 1694.59312 & 2 & 2.06 & $\begin{array}{l}213 \text { NEW \& } 217 \text { NEW \& } \\
218 \text { NEW }\end{array}$ & No \\
\hline
\end{tabular}


Table A-1 (continued).

\begin{tabular}{|c|c|c|c|c|c|c|}
\hline $\begin{array}{l}\text { Accession } \\
\text { number }\end{array}$ & Peptide & MH+ & $\mathbf{z}$ & $\mathbf{X C}$ & $\begin{array}{l}\text { Site \# in Swiss/Phosphosite } \\
(06 / 17 / 08)\end{array}$ & $\begin{array}{l}\text { Present in Phosphosite } \\
(06 / 17 / 08)\end{array}$ \\
\hline \multirow[t]{3}{*}{ Q8N5F7 } & NKAP_HUMAN NF-kappa-B-activating protein & & & 10.23 & & \\
\hline & R.IGELGAPEVWGLS*PK.N & 1632.80327 & 2 & 4.59 & 149 Swiss/ 149 phos & Yes \\
\hline & K.Y*S*EDS*DSDSDSETDSSDEDNK.R & 2566.70622 & 3 & 3.41 & $\begin{array}{l}209 \text { NEW \& } 210 \text { NEW \& } \\
213 \text { NEW }\end{array}$ & No \\
\hline \multirow[t]{2}{*}{ Q9NSK0 } & KLC4_HUMAN Kinesin light chain 4 (KLC 4) (Kinesin-like protein 8) & & & 10.25 & & \\
\hline & R.AAS*LNYLNQPSAAPLQVSR.G & 2080.02226 & 2 & 5.05 & 590 Swiss/ 590 phos & Yes \\
\hline \multirow[t]{2}{*}{ Q9H1E3 } & NUCKS_HUMAN Nuclear ubiquitous casein and cyclin-dependent kinas & s substrate & & 10.28 & & \\
\hline & K.VVDYSQFQES*DDADEDYGR.D & 2317.87684 & 2 & 5.65 & 19 Swiss/ 19 phos & Yes \\
\hline \multirow[t]{2}{*}{ Q92625 } & ANKS1_HUMAN Ankyrin repeat and SAM domain-containing protein 1 & A (Odin) & & 10.21 & & \\
\hline & K.SPS*FASEWDEIEK.I & 1604.65158 & 2 & 4.30 & 663 Swiss/ 662 phos & Yes (same AA, diff \#) \\
\hline \multirow[t]{3}{*}{ O00192 } & ARVC_HUMAN Armadillo repeat protein deleted in velo-cardio-facial s & ndrome & & 20.28 & & \\
\hline & R.S*LAADDEGGPELEPDYGTATR.R & 2243.93396 & 2 & 5.66 & 267 Swiss/ 267 phos & Yes \\
\hline & R.NFDT*LDLPK.R & 1142.51290 & 2 & 2.61 & 642 Swiss/ 642 phos & Yes \\
\hline \multirow[t]{2}{*}{ O15027 } & K0310_HUMAN Uncharacterized protein KIAA0310 & & & 10.23 & & \\
\hline & R.FTGS*FDDDPDPHRDPYGEEVDR.R & 2646.04162 & 3 & 4.15 & 1149 Swiss/ New in phos & No \\
\hline \multirow[t]{7}{*}{ Q14247 } & SRC8_HUMAN Src substrate cortactin (Amplaxin) (Oncogene EMS1) & & & 30.24 & & \\
\hline & R.LPSS*PVYEDAASFK.A & 1590.70870 & 2 & 4.83 & 418 Swiss/ 418 phos & Yes \\
\hline & K.TQT*PPVS*PAPQPTEER.L & 1894.79830 & 2 & 4.19 & $\begin{array}{l}401 \text { Swiss/ } 401 \text { phos \& } 405 \\
\text { Swiss/ } 405 \text { phos }\end{array}$ & Yes \& Yes \\
\hline & R.VDKSAVGFDY*QGKT*EK.H & 1931.81871 & 2 & 2.19 & $\begin{array}{l}178 \text { NEW in Swiss/ } 178 \\
\text { phos \& } 182 \text { NEW }\end{array}$ & Yes \& No \\
\hline & K.GRY*GLFPANY*VELR.Q & 1814.80260 & 3 & 3.27 & 538 NEW \& 545 NEW & No \\
\hline & K.LS*KHCS*QVDSVRGFGGK.F & 1964.84488 & 2 & 2.12 & $\begin{array}{l}109 \text { NEW \& } 113 \text { Swiss/ } 113 \\
\text { phos }\end{array}$ & No \& Yes \\
\hline & R.LPSS*PVY*EDAASFK.A & 1670.67500 & 2 & 2.47 & $\begin{array}{l}418 \text { Swiss/ } 418 \text { phos \& } 412 \\
\text { Swiss/ } 412 \text { phos }\end{array}$ & Yes \& Yes \\
\hline \multirow[t]{3}{*}{ Q14498 } & RBM39_HUMAN RNA-binding protein 39 (RNA-binding motif protein & & & 20.26 & & \\
\hline & K.DKS*PVREPIDNLTPEER.D & 2074.98046 & 3 & 5.11 & 136 Swiss/ 136 phos & \\
\hline & R.YRS*PYSGPK.F & 1134.49792 & 2 & 2.90 & 97 Swiss/ 97 phos & \\
\hline
\end{tabular}


Table A-1 (continued).

\begin{tabular}{|c|c|c|c|c|c|c|}
\hline $\begin{array}{l}\text { Accession } \\
\text { number }\end{array}$ & Peptide & MH+ & $\mathbf{z}$ & $\mathbf{X C}$ & $\begin{array}{l}\text { Site \# in Swiss/Phosphosite } \\
(06 / 17 / 08)\end{array}$ & $\begin{array}{l}\text { Present in Phosphosite } \\
(06 / 17 / 08)\end{array}$ \\
\hline \multirow[t]{2}{*}{ Q6ICG6 } & CV009_HUMAN Uncharacterized protein C22orf9 & & & 10.20 & & \\
\hline & K.SHS*ANDSEEFFR.E & 1505.56925 & 2 & 3.98 & $324 \mathrm{NEW}$ & No \\
\hline \multirow[t]{13}{*}{ Q9NYF8 } & BCLF1_HUMAN Bcl-2-associated transcription factor 1 (Btf) & & & 80.23 & & \\
\hline & K.FNDS*EGDDTEETEDYR.Q & 2001.68692 & 2 & 4.61 & 397 Swiss/ 397 phos & Yes \\
\hline & R.AEGEWEDQEALDYFS*DKESGK.Q & 2513.00277 & 3 & 4.42 & 385 Swiss/ 385 phos & Yes \\
\hline & K.KAEGEPQEES*PLK.S & 1521.68321 & 2 & 4.20 & 177 Swiss/ 177 phos & Yes \\
\hline & K.DLFDYS*PPLHK.N & 1411.62933 & 2 & 3.84 & 512 Swiss/ 512 phos & Yes \\
\hline & K.LKDLFDYS*PPLHK.N & 1652.80836 & 3 & 3.04 & see line 103 & \\
\hline & R.IDIS*PSTLR.K & 1081.52889 & 2 & 2.83 & 658 Swiss/ 658 phos & Yes \\
\hline & R.YSPS*QNS*PIHHIPSR.R & 1879.78874 & 2 & 2.81 & $\begin{array}{l}287 \text { Swiss/ } 287 \text { phos \& } 290 \\
\text { Swiss/ } 290 \text { phos }\end{array}$ & Yes \& Yes \\
\hline & R.AEGEWEDQEALDYFS*DK.E & 2111.81172 & 2 & 2.64 & 385 Swiss/ 385 phos & Yes \\
\hline & R.RIDIS*PSTLR.K & 1237.63000 & 2 & 2.02 & see line 105 & \\
\hline & R.SSSSS*AS*PSSPSS*REEK.E & 1936.66093 & 2 & 2.10 & $\begin{array}{l}755 \text { NEW \& } 757 \text { NEW \& } \\
763 \text { NEW }\end{array}$ & No \& No \& No \\
\hline & R.S*SSSRSSSPYSKSPVS*K.R & 1917.79903 & 2 & 2.01 & 141 NEW \& 156 NEW & No \& No \\
\hline & R.YSPS*QNS*PIHHIPSR.R & 1879.78874 & 2 & 2.34 & see line 106 & \\
\hline \multirow[t]{5}{*}{ P42167 } & LAP2B_HUMAN Lamina-associated polypeptide 2, isoforms beta/gamma & & & 20.23 & & \\
\hline & K.GPPDFS*SDEEREPTPVLGSGAAAAGR.S & 2650.17805 & 3 & 4.54 & 66 Swiss/ 66 phos & Yes \\
\hline & R.SST*PLPTISSSAENTR.Q & 1727.78472 & 2 & 2.99 & $\begin{array}{l}160 \text { Swiss/ } 160 \text { NEW in } \\
\text { phos }\end{array}$ & No \\
\hline & K.GPPDFSSDEEREPT*PVLGSGAAAAGR.S & 2650.17805 & 3 & 4.12 & 174 Swiss/ 173 phos & Yes (same AA, diff \#) \\
\hline & R.EQGTES*RSST*PLPTISSSAENTR.Q & 2595.09706 & 3 & 3.03 & $\begin{array}{l}156 \text { Swiss/ } 155 \text { phos } \& 160 \\
\text { Swiss/ } 159 \text { phos }\end{array}$ & Yes (same AA, diff \#) \\
\hline \multirow[t]{4}{*}{ P54578 } & UBP14_HUMAN Ubiquitin carboxyl-terminal hydrolase 14 & & & 10.30 & & \\
\hline & R.AS*GEMASAQYITAALR.D & 1719.77713 & 2 & 5.99 & 143 Swiss/ 142 phos & Yes (same AA, diff \#) \\
\hline & R.AS*GEM\#ASAQYITAALR.D & 1735.77203 & 2 & 2.67 & see line 118 & \\
\hline & K.S*S*KISRLPAYLTIQMVR.F & 2123.04833 & 2 & 2.06 & 314 NEW \& 315 NEW & No \& No \\
\hline
\end{tabular}


Table A-1 (continued).

\begin{tabular}{|c|c|c|c|c|c|c|}
\hline $\begin{array}{l}\text { Accession } \\
\text { number }\end{array}$ & Peptide & MH+ & $\mathbf{z}$ & $\mathbf{X C}$ & $\begin{array}{l}\text { Site \# in Swiss/Phosphosite } \\
(06 / 17 / 08)\end{array}$ & $\begin{array}{l}\text { Present in Phosphosite } \\
(06 / 17 / 08)\end{array}$ \\
\hline \multirow[t]{2}{*}{ Q8NDX5 } & \multicolumn{3}{|l|}{ PHC3_HUMAN Polyhomeotic-like protein 3 (hPH3) (Homolog of polyhomeotic 3) } & 10.33 & & \\
\hline & R.MDRT*PPPPTLS*PAAITVGR.G & 2136.99121 & 3 & 5.84 & $\begin{array}{l}609 \text { Swiss/ } 609 \text { phos \& } 616 \\
\text { Swiss/ } 616 \text { phos }\end{array}$ & Yes \& Yes \\
\hline \multirow[t]{9}{*}{ Q8IYB3 } & SRRM1_HUMAN Serine/arginine repetitive matrix protein 1 & & & 50.35 & & \\
\hline & K.KETES*EAEDNLDDLEK.H & 1944.79574 & 2 & 6.65 & 874 Swiss/ 874 phos & Yes \\
\hline & K.KPPAPPS*PVQSQS*PSTNWSPAVPVK.K & 2743.28916 & 3 & 5.37 & $\begin{array}{l}769 \text { Swiss/ } 769 \text { phos } \& 775 \\
\text { Swiss/ } 775 \text { phos }\end{array}$ & Yes \& Yes \\
\hline & K.KPPAPPS*PVQSQS*PSTNWS*PAVPVK.K & 2823.25546 & 3 & 4.63 & $\begin{array}{l}\text { see line } 125 \text { also } 781 \text { Swiss/ } \\
781 \text { phos }\end{array}$ & Yes \\
\hline & K.EKT*PELPEPSVK.V & 1433.69232 & 2 & 2.41 & 220 Swiss/ 220 phos & Yes \\
\hline & K.SRVS*VS*PGR.T & 1104.45980 & 2 & 2.06 & $\begin{array}{l}429 \text { Swiss/ } 429 \text { phos \& } 431 \\
\text { Swiss/ } 431 \text { phos }\end{array}$ & Yes \& Yes \\
\hline & R.RYS*PPIQR.R & 1096.52989 & 2 & 2.20 & 597 Swiss/ 597 phos & Yes \\
\hline & R.RLS*PSAS*PPR.R & 1227.52822 & 2 & 2.09 & $\begin{array}{l}389 \text { Swiss/ } 389 \text { phos \& } 393 \\
\text { Swiss/ } 393 \text { phos }\end{array}$ & Yes \& Yes \\
\hline & R.HRPS*PPAT*PPPK.T & 1441.63883 & 2 & 2.56 & $\begin{array}{l}402 \text { Swiss/ } 402 \text { phos \& } 406 \\
\text { Swiss/ } 406 \text { phos }\end{array}$ & Yes \& Yes \\
\hline P10644 & \multicolumn{3}{|l|}{ KAP0_HUMAN cAMP-dependent protein kinase type I-alpha regulatory subunit } & 10.20 & & \\
\hline & R.EDEIS*PPPPNPVVK.G & 1597.75090 & 2 & 3.90 & 83 Swiss/ 83 phos & Yes \\
\hline & R.TDSREDEIS*PPPPNPVVK.G & 2056.95866 & 2 & 2.86 & see line 133 & \\
\hline \multirow[t]{3}{*}{ P06748 } & \multicolumn{3}{|l|}{ NPM_HUMAN Nucleophosmin (NPM) (Nucleolar phosphoprotein B23) } & 20.30 & & \\
\hline & K.DELHIVEAEAMNYEGS*PIK.V & 2224.98316 & 2 & 5.47 & 70 Swiss/ 70 phos & Yes \\
\hline & K.DELHIVEAEAMNYEGS*PIKVTLATLK.M & 2951.44714 & 3 & 3.33 & see line 136 & \\
\hline \multirow[t]{2}{*}{ P31943 } & \multicolumn{3}{|l|}{ HNRH1_HUMAN Heterogeneous nuclear ribonucleoprotein H (hnRNP H) } & 10.30 & & \\
\hline & R.PSGEAFVELES*EDEVK.L & 1844.78372 & 2 & 3.17 & 63 Swiss/ New in phos & No \\
\hline \multirow[t]{3}{*}{ P16949 } & STMN1_HUMAN Stathmin (Phosphoprotein p19) (pp19) & & & 10.15 & & \\
\hline & K.ESVPEFPLS*PPK.K & 1406.66029 & 2 & 2.42 & 38 Swiss/ 37 in phos & Yes (same AA, diff \#) \\
\hline & R.ASGQAFELILS*PR.S & 1468.71954 & 2 & 4.00 & 25 Swiss/ 24 in phos & Yes (same AA, diff \#) \\
\hline \multirow[t]{2}{*}{ Q14694 } & UBP10_HUMAN Ubiquitin carboxyl-terminal hydrolase 10 & & & 10.27 & & \\
\hline & K.NHSVNEEEQEEQGEGS*EDEWEQVGPR.N & 3107.21338 & 3 & 5.31 & 576 Swiss/ 576 phos & Yes \\
\hline
\end{tabular}


Table A-1 (continued).

\begin{tabular}{|c|c|c|c|c|c|c|}
\hline $\begin{array}{l}\text { Accession } \\
\text { number }\end{array}$ & Peptide & MH+ & $\overline{\mathbf{z}}$ & $\mathbf{X C}$ & $\begin{array}{l}\text { Site \# in Swiss/Phosphosite } \\
(06 / 17 / 08)\end{array}$ & $\begin{array}{l}\text { Present in Phosphosite } \\
(06 / 17 / 08)\end{array}$ \\
\hline \multirow[t]{3}{*}{ Q9UK76 } & HN1_HUMAN Hematological and neurological expressed 1 protein & & & 10.29 & & \\
\hline & R.RNS*SEASSGDFLDLK.G & 1705.74285 & 2 & 5.40 & 87 Swiss/ 87 phos & Yes \\
\hline & R.NS*SEASSGDFLDLK.G & 1549.64174 & 2 & 5.22 & see line 146 & \\
\hline \multirow[t]{4}{*}{ Q13263 } & TIF1B_HUMAN Transcription intermediary factor 1-beta (TIF1-beta) & & & 20.24 & & \\
\hline & R.STAPSAAASASASAAASS*PAGGGAEALELLEHCGVCR.E & 3408.51954 & 3 & 4.90 & 50 Swiss/ New in phos & No \\
\hline & R.SRS*GEGEVSGLMR.K & 1444.62499 & 2 & 2.88 & 473 Swiss/ 473 phos & Yes \\
\hline & R.S*GEGEVSGLMR.K & 1201.49185 & 2 & 4.01 & see line 150 & \\
\hline \multirow[t]{3}{*}{ Q53SF7 } & CBLL1_HUMAN Cordon-bleu protein-like 1 & & & 10.24 & & \\
\hline & R.AGS*LQLSSMSAGNSSLR.R & 1745.78876 & 2 & 4.78 & 382 NEW & No \\
\hline & R.QS*SLTFQSSDPEQMR.Q & 1820.75204 & 2 & 2.48 & 1146 Swiss/ New in phos & No \\
\hline \multirow[t]{2}{*}{ O75821 } & IF34_HUMAN Eukaryotic translation initiation factor 3 subunit 4 (eIF-3 delta) & & & 10.19 & & \\
\hline & K.GIPLATGDT*SPEPELLPGAPLPPPK.E & 2544.29967 & 3 & 3.15 & 41 Swiss/ 41 phos & Yes \\
\hline \multirow[t]{2}{*}{ Q9UKL0 } & RCOR1_HUMAN REST corepressor 1 (Protein CoREST) & & & 10.17 & & \\
\hline & R.EREES*EDELEEANGNNPIDIEVDQNK.E & 3095.29605 & 3 & 3.44 & 257 Swiss/ 257 phos & Yes \\
\hline \multirow[t]{7}{*}{ Q6PKG0 } & LARP1_HUMAN La-related protein 1 & & & 30.23 & & \\
\hline & K.GLSAS*LPDLDSENWIEVK.K & 2052.95251 & 2 & 4.67 & 548 Swiss/ 548 phos & Yes \\
\hline & R.S*LPTTVPESPNY*R.N & 1620.67058 & 2 & 2.21 & 766 Swiss/ 766 phos & Yes \\
\hline & R.SLPTTVPES*PNYR.N & 1540.70428 & 2 & 2.17 & 774 Swiss/ 774 phos & Yes \\
\hline & K.ILIVTQT*PHYMR.R & 1551.77528 & 2 & 3.86 & 649 Swiss/ 649 phos & Yes \\
\hline & R.EHRPRTASISSS*PSEGTPT*VGSYGCTPQSLPK.F & 3474.53959 & 3 & 3.77 & $\begin{array}{l}851 \text { Swiss/ } 851 \text { phos \& } 858 \\
\text { New in Swiss/ } 858 \text { phos }\end{array}$ & Yes \& Yes \\
\hline & K.QEVENFKKVNM\#IS*R.E & 1817.86152 & 2 & 2.70 & $716 \mathrm{NEW}$ & No \\
\hline \multirow[t]{2}{*}{ O43741 } & AAKB2_HUMAN 5'-AMP-activated protein kinase subunit beta-2 (AMPK beta & -2 chain) & & 10.18 & & \\
\hline & R.DLSSS*PPGPYGQEMYAFR.S & 2081.86740 & 2 & 3.58 & 184 New in Swiss/ 184 phos & Yes \\
\hline
\end{tabular}


Table A-1 (continued).

\begin{tabular}{|c|c|c|c|c|c|c|}
\hline $\begin{array}{l}\text { Accession } \\
\text { number }\end{array}$ & Peptide & MH+ & $\mathbf{z}$ & $\mathbf{X C}$ & $\begin{array}{l}\text { Site \# in Swiss/Phosphosite } \\
(06 / 17 / 08)\end{array}$ & $\begin{array}{l}\text { Present in Phosphosite } \\
(06 / 17 / 08)\end{array}$ \\
\hline \multirow[t]{2}{*}{ Q9Y2V2 } & CHSP1_HUMAN Calcium-regulated heat stable protein 1 & & & 10.12 & & \\
\hline & R.GNVVPS*PLPTR.R & 1216.60853 & 2 & 2.47 & 41 Swiss/ 41 phos & Yes \\
\hline \multirow[t]{3}{*}{ P12694 } & ODBA_HUMAN 2-oxoisovalerate dehydrogenase subunit alph & precursor & & 20.23 & & \\
\hline & R.S*VDEVNYWDK.Q & 1334.53001 & 2 & 4.36 & 347 Swiss/ 347 phos & Yes \\
\hline & R.IGHHS*TSDDSSAYR.S & 1612.63872 & 3 & 3.68 & 337 Swiss/ 337 phos & Yes \\
\hline \multirow[t]{2}{*}{ Q8NEY8 } & PPHLN_HUMAN Periphilin-1 (Gastric cancer antigen Ga50) & & & 10.16 & & \\
\hline & R.DNTFFRES*PVGR.K & 1504.65800 & 2 & 3.12 & 133 Swiss/ 133 phos & Yes \\
\hline \multirow[t]{10}{*}{ Q96JM3 } & K1802_HUMAN Zinc finger protein KIAA1802 & & & 70.24 & & \\
\hline & R.KPSPSES*PEPWKPFPAVS*PEPR.R & 2606.17274 & 3 & 3.89 & $\begin{array}{l}286 \text { Swiss/ New in phos \& } \\
297 \text { NEW }\end{array}$ & No \& No \\
\hline & R.KPSGS*PDLWK.L & 1194.55544 & 2 & 3.88 & 445 Swiss/ New in phos & No \\
\hline & R.KPS*PS*ESPEPWKPFPAVSPEPR.R & 2606.17274 & 3 & 3.41 & $\begin{array}{l}282 \text { Swiss/ New in phos \& } \\
284 \text { NEW }\end{array}$ & No \& No \\
\hline & K.TAPTLS*PEHWK.A & 1346.61401 & 2 & 3.05 & 405 Swiss/ New in phos & No \\
\hline & R.RPAPAVS*PGSWK.P & 1332.64598 & 2 & 2.96 & 308 Swiss/ 308 phos & Yes \\
\hline & R.GGS*PDLWK.S & 939.39714 & 2 & 2.23 & 476 Swiss/ 476 phos & Yes \\
\hline & R.KPGPPLS*PEIR.S & 1270.65548 & 2 & 2.17 & 427 Swiss/ 427 phos & Yes \\
\hline & R.KTS*PASLDFPESQK.S & 1614.74106 & 2 & 4.31 & 459 Swiss/ New in phos & No \\
\hline & R.KT*SPASLDFPES*QKS*SR.G & 2104.83883 & 2 & 2.27 & $\begin{array}{l}458 \text { NEW \& } 468 \text { NEW \& } \\
471 \text { NEW }\end{array}$ & No \& No \& No \\
\hline \multirow[t]{2}{*}{ Q15459 } & SF3A1_HUMAN Splicing factor 3 subunit 1 (Spliceosome-ass & 14) & & 10.25 & & \\
\hline & K.FGESEEVEMEVES*DEEDDKQEK.A & 2697.02806 & 3 & 3.62 & 329 Swiss/ 329 phos & Yes \\
\hline \multirow[t]{3}{*}{ Q9UL54 } & TAOK2_HUMAN Serine/threonine-protein kinase TAO2 & & & 20.19 & & \\
\hline & K.MLLARHS*LDQDLLR.E & 1760.88769 & 3 & 3.51 & $656 \mathrm{NEW}$ & No \\
\hline & R.HS*LDQDLLR.E & 1176.54085 & 2 & 2.14 & see line 188 & \\
\hline
\end{tabular}


Table A-1 (continued).

\begin{tabular}{|c|c|c|c|c|c|c|}
\hline $\begin{array}{l}\text { Accession } \\
\text { number }\end{array}$ & Peptide & MH+ & $\mathbf{z}$ & $\mathbf{X C}$ & $\begin{array}{l}\text { Site \# in Swiss/Phosphosite } \\
(06 / 17 / 08)\end{array}$ & $\begin{array}{l}\text { Present in Phosphosite } \\
(06 / 17 / 08)\end{array}$ \\
\hline \multirow[t]{4}{*}{ Q15139 } & KPCD1_HUMAN Serine/threonine-protein kinase D1 (nPKC-D1) & & & 20.21 & & \\
\hline & R.RLS*NVS*LTGVSTIR.T & 1662.79752 & 2 & 4.19 & 205 Swiss/ 205 phos \& 208 & Yes \& Yes \\
\hline & R.RLS*NVSLTGVSTIR.T & 1582.83122 & 2 & 3.50 & $\begin{array}{l}\text { New in Swiss/ } 208 \text { phos } \\
\text { see line } 191\end{array}$ & \\
\hline & R.T*SS*AELST*SAPDEPLLQKSPSESFIGREK.R & 3331.44188 & 3 & 3.04 & $\begin{array}{l}217 \text { NEW \& } 219 \text { New in } \\
\text { Swiss/ } 219 \text { phos \& } 224 \\
\text { NEW }\end{array}$ & No \& Yes \& No \\
\hline \multirow[t]{3}{*}{ Q9UEW8 } & STK39_HUMAN STE20/SPS1-related proline-alanine-rich protein kinase & & & 20.27 & & \\
\hline & K.TEDGDWEWS*DDEMDEK.S & 2066.68447 & 2 & 4.93 & 387 New in Swiss/ 387 phos & Yes \\
\hline & K.ADMWSFGITAIELATGAAPY*HKY*PPMK.V & 3126.39053 & 3 & 3.26 & 275 NEW \& 278 NEW & No \& No \\
\hline \multirow[t]{4}{*}{ Q9H0B6 } & KLC2_HUMAN Kinesin light chain 2 (KLC 2) & & & 30.21 & & \\
\hline & R.RDS*APYGEYGSWYK.A & 1758.71591 & 2 & 4.27 & $428 \mathrm{NEW}$ & No \\
\hline & R.ASS*LNFLNK.S & 1073.50267 & 2 & 3.81 & 528 Swiss/ 528 phos & Yes \\
\hline & R.TLSSS*SMDLSR.R & 1263.52863 & 2 & 3.67 & 610 New in Swiss/ 610 phos & Yes \\
\hline \multirow[t]{2}{*}{ Q32MZ4 } & LRRF1_HUMAN Leucine-rich repeat flightless-interacting protein 1 & & & 10.20 & & \\
\hline & R.RGS*GDTSISIDTEASIR.E & 1844.83855 & 2 & 3.99 & 120 New in Swiss/ 120 phos & Yes \\
\hline \multirow[t]{2}{*}{ Q15052 } & ARHG6_HUMAN Rho guanine nucleotide exchange factor 6 & & & 10.16 & & \\
\hline & R.MS*GFIYQGK.I & 1110.46893 & 2 & 2.94 & 488 Swiss/ 488 phos & Yes \\
\hline \multirow[t]{2}{*}{ P21127 } & CD2L1_HUMAN PITSLRE serine/threonine-protein kinase CDC2L1 & & & 10.13 & & \\
\hline & K.AYT*PVVVTLWYR.A & 1547.76576 & 2 & 2.68 & 595 Swiss/ 595 phos & Yes \\
\hline \multirow[t]{3}{*}{ P17812 } & PYRG1_HUMAN CTP synthase 1 (UTP--ammonia ligase 1) & & & 20.29 & & \\
\hline & R.SGSSS*PDSEITELK.F & 1516.64141 & 2 & 4.44 & 575 Swiss/ 575 phos & Yes \\
\hline & K.YIDSADLEPITS*QEEPVR.Y & 2141.96381 & 2 & 2.29 & $347 \mathrm{NEW}$ & No \\
\hline \multirow[t]{3}{*}{ P25490 } & TYY1_HUMAN Transcriptional repressor protein YY1 (Yin and yang 1) (Y & & & 20.22 & & \\
\hline & K.DIDHETVVEEQIIGENS*PPDYSEYMTGK.K & 3275.39735 & 3 & 4.41 & 247 Swiss/ 247 phos & Yes \\
\hline & R.EEVVGGDDS*DGLR.A & 1427.56858 & 2 & 2.26 & 118 Swiss/ 118 phos & Yes \\
\hline
\end{tabular}


Table A-1 (continued).

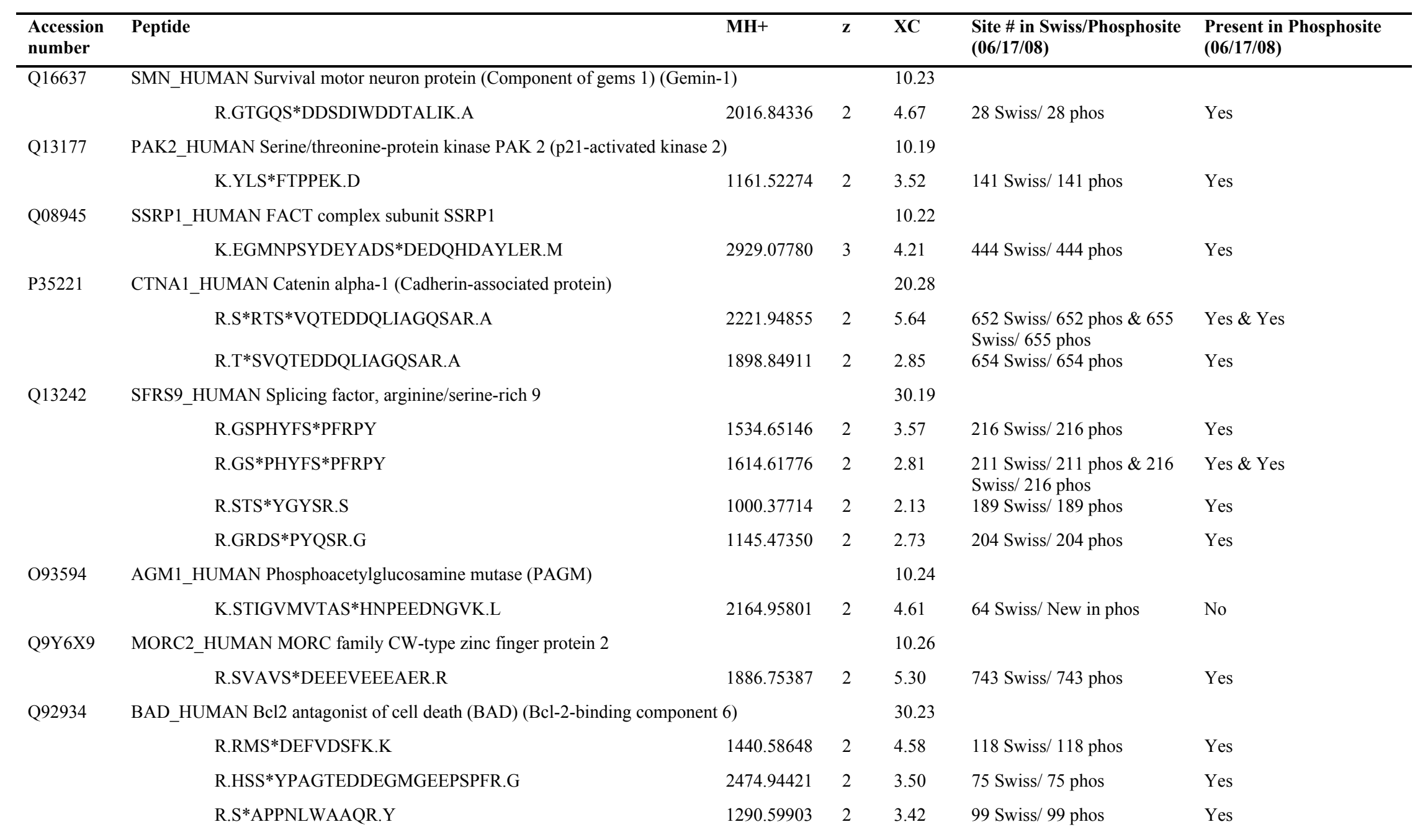


Table A-1 (continued).

\begin{tabular}{|c|c|c|c|c|c|c|}
\hline $\begin{array}{l}\text { Accession } \\
\text { number }\end{array}$ & Peptide & MH+ & $\mathbf{z}$ & $\mathbf{X C}$ & $\begin{array}{l}\text { Site \# in Swiss/Phosphosite } \\
(06 / 17 / 08)\end{array}$ & $\begin{array}{l}\text { Present in Phosphosite } \\
(06 / 17 / 08)\end{array}$ \\
\hline \multirow[t]{4}{*}{$\overline{Q 9 C 0 C 2}$} & TB182_HUMAN $182 \mathrm{kDa}$ tankyrase 1-binding protein & & & 10.20 & & \\
\hline & R.RFS*EGVLQSPSQDQEK.L & 1914.85928 & 2 & 3.98 & $\begin{array}{l}429 \text { New in Swiss/ } 429 \\
\text { phosq }\end{array}$ & Yes \\
\hline & R.S*QEADVQDWEFR.K & 1589.62676 & 2 & 3.95 & 836 Swiss/ 836 phos & Yes \\
\hline & K.VST*LRES*SAMAS*PLPR.E & 1941.79413 & 2 & 2.04 & $\begin{array}{l}5 \text { NEW \& } 9 \text { NEW \& } 14 \\
\text { NEW }\end{array}$ & No \& No \& No \\
\hline \multirow[t]{5}{*}{ Q96B36 } & AKTS1_HUMAN Proline-rich AKT1 substrate 1 (40 kDa proline-rich AK & ubstrate) & & 10.22 & & \\
\hline & R.AATAARPPAPPPAPQPPS*PTPS*PPRPTLAR.E & 3124.54923 & 3 & 4.30 & $\begin{array}{l}88 \text { Swiss/ } 88 \text { phos \& } 92 \\
\text { Swiss/ } 92 \text { phos }\end{array}$ & Yes \& Yes \\
\hline & K.S*LPVSVPVWGFK.E & 1395.70718 & 2 & 3.70 & 183 Swiss/ 183 phos & Yes \\
\hline & R.LNT*SDFQK.L & 1032.43974 & 2 & 2.56 & 246 Swiss/ 246 phos & Yes \\
\hline & R.AAT*AARPPAPPPAPQPPS*PT*PSPPR.P & 2666.19280 & 2 & 2.55 & $\begin{array}{l}73 \text { NEW \& } 88 \text { Swiss/ } 88 \\
\text { phos \& } 90 \text { NEW }\end{array}$ & No \& Yes \& No \\
\hline \multirow[t]{3}{*}{ Q14671 } & PUM1_HUMAN Pumilio homolog 1 (Pumilio-1) (HsPUM) & & & 10.20 & & \\
\hline & R.RDS*LTGSSDLYK.R & 1421.63079 & 2 & 4.05 & 709 New in Swiss/ 709 phos & Yes \\
\hline & R.S*ASSASSLFSPSSTLFSS*SR.L & 2152.88349 & 2 & 2.23 & 797 NEW & No \\
\hline \multirow[t]{4}{*}{ P08559 } & ODPA_HUMAN Pyruvate dehydrogenase E1 component alpha subunit & & & 20.25 & & \\
\hline & R.YHGHS*MSDPGVS*YR.T & 1752.62365 & 2 & 4.94 & $\begin{array}{l}293 \text { Swiss/ } 293 \text { phos } \& 300 \\
\text { Swiss/ } 300 \text { phos }\end{array}$ & Yes \& Yes \\
\hline & R.YGMGTS*VER.A & 1079.42271 & 2 & 3.43 & 232 Swiss/ 232 phos & Yes \\
\hline & R.YHGHSMS*DPGVSYR.T & 1672.65735 & 3 & 3.83 & 295 Swiss/ 295 phos & Yes \\
\hline \multirow[t]{7}{*}{ Q9UQ35 } & SRRM2_HUMAN Serine/arginine repetitive matrix protein 2 & & & 30.31 & & \\
\hline & R.HGGS*PQPLATTPLSQEPVNPPSEAS*PTR.D & 3012.34993 & 3 & 6.13 & $\begin{array}{l}377 \text { Swiss/ } 377 \text { phos \& } 398 \\
\text { Swiss/ } 398 \text { phos }\end{array}$ & Yes \& Yes \\
\hline & K.SST*PPGESYFGVSSLQLK.G & 1963.90483 & 2 & 3.36 & 1043 Swiss/ 1043 phos & Yes \\
\hline & R.S*RT*PLLPR.K & 1099.50603 & 2 & 2.13 & $\begin{array}{l}2032 \text { Swiss/ } 2032 \text { phos } \& \\
2034 \text { Swiss/ } 2034 \text { phos }\end{array}$ & Yes \& Yes \\
\hline & R.GPS*PEGSS*ST*ESSPEHPPK.S & 2133.74499 & 2 & 2.18 & $\begin{array}{l}1648 \text { NEW \& } 1653 \text { NEW \& } \\
1655 \text { NEW }\end{array}$ & No \& No \& No \\
\hline & R.AHRSTSADS*ASSS*DTS*R.S & 1962.66266 & 2 & 2.07 & $\begin{array}{l}268 \text { NEW \& } 272 \text { NEW \& } \\
275 \text { NEW }\end{array}$ & No \& No \& No \\
\hline & R.QS*PSRS*SSPQPK.V & 1445.58210 & 2 & 2.01 & $\begin{array}{l}908 \text { Swiss/ } 908 \text { phos \& } 912 \\
\text { NEW }\end{array}$ & Yes \& No \\
\hline
\end{tabular}


Table A-1 (continued).

\begin{tabular}{|c|c|c|c|c|c|c|}
\hline $\begin{array}{l}\text { Accession } \\
\text { number }\end{array}$ & Peptide & MH+ & $\mathbf{z}$ & $\mathbf{X C}$ & $\begin{array}{l}\text { Site \# in Swiss/Phosphosite } \\
(06 / 17 / 08)\end{array}$ & $\begin{array}{l}\text { Present in Phosphosite } \\
(06 / 17 / 08)\end{array}$ \\
\hline \multirow[t]{3}{*}{ P98175 } & RBM10_HUMAN RNA-binding protein 10 (RNA-binding motif protein 10) & & & 20.16 & & \\
\hline & R.HRHS*PTGPPGFPR.D & 1522.70629 & 3 & 3.06 & $189 \mathrm{NEW}$ & No \\
\hline & R.Y*GATDRSQDDGGENRS*R.D & 2043.75527 & 2 & 2.07 & $16 \mathrm{NEW}$ & No \\
\hline \multirow[t]{2}{*}{ Q9BW71 } & HIRP3_HUMAN HIRA-interacting protein 3 & & & 10.24 & & \\
\hline & K.SLKES*EQES*EEEILAQK.K & 2136.89847 & 2 & 4.73 & $\begin{array}{l}223 \text { Swiss/ } 223 \text { phos } \& 227 \\
\text { Swiss/ } 227 \text { phos }\end{array}$ & Yes \\
\hline \multirow[t]{2}{*}{ Q9BSJ8 } & FA62A_HUMAN Protein FAM62A (Membrane-bound C2 domain-containin & g protein) & & 10.12 & & \\
\hline & R.KLDVSVKSNS*SFMS*R.E & 1844.80128 & 2 & 2.43 & 1063 NEW \& 1067 NEW & No \& No \\
\hline \multirow[t]{2}{*}{ Q9UKS6 } & PACN3_HUMAN Protein kinase $\mathrm{C}$ and casein kinase substrate in neurons pr & otein 3 & & 10.23 & & \\
\hline & R.DGTAPPPQSPGSPGTGQDEEWS*DEESPR.K & 2990.19594 & 3 & 4.58 & 354 Swiss/ 354 phos & Yes \\
\hline \multirow[t]{2}{*}{ P24844 } & MLRN_HUMAN Myosin regulatory light chain 2, smooth muscle isoform & & & 10.19 & & \\
\hline & R.ATS*NVFAMFDQSQIQEFK.E & 2170.95147 & 2 & 3.81 & 20 Swiss/ 20 phos & Yes \\
\hline \multirow[t]{3}{*}{ P04792 } & HSPB1_HUMAN Heat-shock protein beta-1 (HspB1) (Heat shock $27 \mathrm{kDa}$ pr & otein) & & 20.26 & & \\
\hline & R.QLS*SGVSEIR.H & 1155.54051 & 2 & 2.96 & 82 Swiss/ 82 phos & Yes \\
\hline & R.GPS*WDPFR.D & 1041.41894 & 2 & 2.81 & 15 Swiss/ 15 phos & Yes \\
\hline \multirow[t]{3}{*}{ P05455 } & LA_HUMAN Lupus La protein (Sjoegren syndrome type B antigen) & & & 20.26 & & \\
\hline & K.FAS*DDEHDEHDENGATGPVK.R & 2249.86186 & 3 & 3.37 & 366 Swiss/ 366 phos & Yes \\
\hline & R.SPS*KPLPEVTDEYK.N & 1669.77203 & 2 & 3.12 & 94 NEW & No \\
\hline \multirow[t]{2}{*}{ O60841 } & IF2P_HUMAN Eukaryotic translation initiation factor 5B (eIF-5B) & & & 10.20 & & \\
\hline & K.NKPGPNIES*GNEDDDASFK.I & 2113.87097 & 2 & 4.07 & 214 Swiss/ 214 phos & Yes \\
\hline \multirow[t]{3}{*}{ P51114 } & FXR1_HUMAN Fragile $\mathrm{X}$ mental retardation syndrome-related protein $1(\mathrm{hF}$ & XR1p) & & 20.27 & & \\
\hline & R.RGPNYTSGYGTNSELSNPS*ETESER.K & 2812.16934 & 3 & 5.34 & 409 Swiss/ 409 phos & Yes \\
\hline & R.RGPNYTSGYGTNSELS*NPS*ETESER.K & 2892.13564 & 3 & 3.84 & $\begin{array}{l}406 \text { Swiss/ } 406 \text { phos \& } 409 \\
\text { Swiss/ } 409 \text { phos }\end{array}$ & Yes \& Yes \\
\hline
\end{tabular}


Table A-1 (continued).

\begin{tabular}{|c|c|c|c|c|c|c|}
\hline $\begin{array}{l}\text { Accession } \\
\text { number }\end{array}$ & Peptide & MH+ & $\overline{\mathbf{z}}$ & $\mathbf{X C}$ & $\begin{array}{l}\text { Site \# in Swiss/Phosphosite } \\
(06 / 17 / 08)\end{array}$ & $\begin{array}{l}\text { Present in Phosphosite } \\
(06 / 17 / 08)\end{array}$ \\
\hline \multirow[t]{5}{*}{$\mathrm{P} 08238$} & HS90B_HUMAN Heat shock protein HSP 90-beta (HSP 84) (HSP 90) & & & 30.23 & & \\
\hline & K.IEDVGS*DEEDDSGKDK.K & 1817.69602 & 2 & 4.59 & 255 Swiss/ 254 phos & Yes (same AA, diff \#) \\
\hline & K.IEDVGS*DEEDDSGK.D & 1574.57412 & 2 & 3.78 & see line 281 & \\
\hline & K.IEDVGS*DEEDDSGKDKK.K & 1945.79099 & 3 & 3.00 & see line 281 & \\
\hline & R.EKEIS*DDEAEEEK.G & 1630.63672 & 2 & 4.07 & 226 Swiss/ 225 phos & Yes (same AA, diff \#) \\
\hline \multirow[t]{2}{*}{ Q9UEE9 } & CFDP1_HUMAN Craniofacial development protein 1 (Bucentaur) & & & 10.18 & & \\
\hline & K.LDWES*FKEEEGIGEELAIHNR.G & 2581.16061 & 3 & 3.66 & 250 New in Swiss/ 250 phos & Yes \\
\hline \multirow[t]{2}{*}{ Q9Y383 } & LC7L2_HUMAN Putative RNA-binding protein Luc7-like 2 & & & 10.18 & & \\
\hline & R.AMLDQLMGTS*R.D & 1302.55815 & 2 & 3.69 & 18 New in Swiss/ 18 phos & Yes \\
\hline \multirow[t]{2}{*}{ Q99613 } & IF38_HUMAN Eukaryotic translation initiation factor 3 subunit 8 (eIF3 p110) & & & 10.13 & & \\
\hline & K.QPLLLS*EDEEDTKR.V & 1752.80512 & 2 & 2.05 & 39 Swiss 39 phos & Yes \\
\hline \multirow[t]{5}{*}{ O14974 } & MYPT1_HUMAN Protein phosphatase 1 regulatory subunit 12A & & & 20.22 & & \\
\hline & K.TGS*YGALAEITASK.E & 1448.66684 & 2 & 4.43 & 445 Swiss/ 445 phos & Yes \\
\hline & K.SPLIES*TANMDNNQSQKTFKNK.E & 2575.18578 & 2 & 2.49 & 304 NEW & No \\
\hline & R.RST*QGVTLTDLQEAEK.T & 1855.87968 & 2 & 5.08 & 696 Swiss/ 696 phos & Yes \\
\hline & R.KTGS*YGALAEITASK.E & 1576.76180 & 2 & 4.22 & see line 292 & \\
\hline \multirow[t]{2}{*}{ Q9Y3S2 } & ZN330_HUMAN Zinc finger protein 330 (Nucleolar cysteine-rich protein) & & & 10.20 & & \\
\hline & R.KDS*DTESSDLFTNLNLGR.T & 2091.92300 & 2 & 4.00 & $291 \mathrm{NEW}$ & No \\
\hline \multirow[t]{5}{*}{ P61978 } & HNRPK_HUMAN Heterogeneous nuclear ribonucleoprotein K & & & 20.27 & & \\
\hline & R.GSY*GDLGGPIITTQVTIPK.D & 1996.99907 & 2 & 4.79 & 380 New in Swiss/ 380 phos & Yes \\
\hline & R.RDYDDMS*PR.R & 1234.45580 & 2 & 2.26 & 284 Swiss/ 284 phos & Yes \\
\hline & R.GSY*GDLGGPIITT*QVTIPK.D & 2076.96537 & 2 & 3.32 & see line 299 & \\
\hline & R.DYDDMS*PR.R & 1078.35469 & 2 & 2.55 & see line 300 & \\
\hline
\end{tabular}


Table A-1 (continued).

\begin{tabular}{|c|c|c|c|c|c|c|}
\hline $\begin{array}{l}\text { Accession } \\
\text { number }\end{array}$ & Peptide & MH+ & $\mathbf{z}$ & $\mathbf{X C}$ & $\begin{array}{l}\text { Site \# in Swiss/Phosphosite } \\
(06 / 17 / 08)\end{array}$ & $\begin{array}{l}\text { Present in Phosphosite } \\
(06 / 17 / 08)\end{array}$ \\
\hline \multirow[t]{7}{*}{ P51858 } & HDGF_HUMAN Hepatoma-derived growth factor (HDGF) & & & 10.13 & & \\
\hline & R.AGDLLEDS*PK.R & 1124.48708 & 2 & 2.54 & 165 Swiss/ 165 phos & Yes \\
\hline & R.RAGDLLEDS*PK.R & 1280.58819 & 2 & 3.54 & see line 304 & \\
\hline & R.AGDLLEDS*PKRPK.E & 1505.73592 & 2 & 2.64 & see line 304 & \\
\hline & K.GNAEGS*S*DEEGKLVIDEPAK.E & 2204.89953 & 2 & 5.87 & $\begin{array}{l}132 \text { Swiss/ } 132 \text { phos \& } 133 \\
\text { Swiss/ } 133 \text { phos }\end{array}$ & Yes \& Yes \\
\hline & K.KGNAEGS*S*DEEGKLVIDEPAK.E & 2332.99450 & 3 & 3.80 & see line 307 & \\
\hline & K.GNAEGS*SDEEGKLVIDEPAK.E & 2124.93323 & 2 & 4.76 & 132 Swiss/ 132 phos & Yes \\
\hline \multirow[t]{3}{*}{ P22059 } & OSBP1_HUMAN Oxysterol-binding protein 1 & & & 20.20 & & \\
\hline & R.TGS*NISGASSDISLDEQYK.H & 2051.88047 & 2 & 4.00 & 379 Swiss/ 379 phos & Yes \\
\hline & K.GDMS*DEDDENEFFDAPEIITMPENLGHK.R & 3275.30682 & 3 & 3.34 & 351 Swiss/ 351 phos & Yes \\
\hline \multirow[t]{3}{*}{ Q8NE71 } & ABCF1_HUMAN ATP-binding cassette sub-family F member 1 & & & 20.17 & & \\
\hline & K.LSVPTS*DEEDEVPAPK.P & 1792.78880 & 2 & 3.36 & 109 Swiss/ 109 phos & Yes \\
\hline & K.LSVPTS*DEEDEVPAPKPR.G & 2045.94268 & 2 & 3.26 & see line 314 & \\
\hline \multirow[t]{2}{*}{ P55795 } & HNRH2_HUMAN Heterogeneous nuclear ribonucleoprotein $\mathrm{H}^{\prime}\left(\mathrm{hnRNP} \mathrm{H}^{\prime}\right)\left(\mathrm{F}^{\prime}\right.$ & FTP-3) & & 10.28 & & \\
\hline & K.HTGPNS*PDTANDGFVR.L & 1764.73369 & 2 & 4.12 & 104 Swiss/ 104 phos & Yes \\
\hline \multirow[t]{2}{*}{ Q9BYG3 } & MK67I_HUMAN MKI67 FHA domain-interacting nucleolar phosphoprotein & & & 10.19 & & \\
\hline & K.S*QVAELNDDDKDDEIVFK.Q & 2159.93799 & 2 & 3.71 & 247 Swiss/ 247 phos & Yes \\
\hline \multirow[t]{6}{*}{ P02545 } & LMNA_HUMAN Lamin-A/C (70 kDa lamin) (Renal carcinoma antigen NY-R & (EN-32) & & 30.20 & & \\
\hline & R.NKS*NEDQSMGNWQIK.R & 1858.77892 & 2 & 3.00 & $458 \mathrm{NEW}$ & No \\
\hline & R.LRLS*PSPTSQR.S & 1321.66236 & 2 & 2.65 & 390 Swiss/ 390 phos & Yes \\
\hline & R.KLEST*ES*RSSFSQHAR.T & 2009.84771 & 2 & 2.05 & 424 Swiss/ 424 phos & Yes \\
\hline & R.LRLS*PS*PTSQR.S & 1401.62866 & 2 & 3.69 & $\begin{array}{l}390 \text { Swiss/ } 390 \text { phos } \& 392 \\
\text { Swiss/ } 392 \text { phos }\end{array}$ & Yes \& Yes \\
\hline & R.SVGGS*GGGSFGDNLVTR.S & 1646.71697 & 2 & 2.11 & 632 Swiss/ 632 phos & Yes \\
\hline
\end{tabular}


Table A-1 (continued).

\begin{tabular}{|c|c|c|c|c|c|c|}
\hline $\begin{array}{l}\text { Accession } \\
\text { number }\end{array}$ & Peptide & MH+ & $\overline{\mathbf{z}}$ & $\mathbf{X C}$ & $\begin{array}{l}\text { Site \# in Swiss/Phosphosite } \\
(06 / 17 / 08)\end{array}$ & $\begin{array}{l}\text { Present in Phosphosite } \\
(06 / 17 / 08)\end{array}$ \\
\hline \multirow[t]{2}{*}{ P06493 } & CDC2_HUMAN Cell division control protein 2 homolog (p34 protein kinase) & & & 10.16 & & \\
\hline & R.VYT*HEVVTLWYR.S & 1645.77739 & 2 & 3.27 & 161 Swiss/ 161 phos & Yes \\
\hline \multirow[t]{3}{*}{ P49585 } & PCY1A_HUMAN Choline-phosphate cytidylyltransferase A & & & 10.23 & & \\
\hline & R.MLQAIS*PK.Q & 967.46820 & 2 & 2.61 & 315 Swiss/ 315 phos & Yes \\
\hline & K.T*SPPCS*PANLSR.H & 1389.52690 & 2 & 3.20 & $\begin{array}{l}342 \text { New in Swiss/ } 324 \text { phos } \\
\& 347 \text { Swiss/ } 347 \text { phos }\end{array}$ & Yes \& Yes \\
\hline \multirow[t]{6}{*}{ Q16204 } & CCDC6_HUMAN Coiled-coil domain-containing protein 6 (H4 protein) & & & 40.26 & & \\
\hline & R.PIS*PGLSYASHTVGFTPPTSLTR.A & 2466.20644 & 3 & 5.14 & 367 Swiss/ New in phos & No \\
\hline & R.T*VSSPIPYTPSPSSSR.P & 1742.79964 & 2 & 4.29 & 349 NEW & No \\
\hline & R.PIS*PGLSYASHTVGFT*PPTSLTR.A & 2546.17274 & 3 & 3.02 & $\begin{array}{l}367 \text { Swiss/ New in phos \& } \\
380 \text { NEW }\end{array}$ & No \& No \\
\hline & R.QLSESES*SLEMDDER.Y & 1834.70481 & 2 & 2.68 & $327 \mathrm{NEW}$ & No \\
\hline & K.LDQPVS*APPS*PR.D & 1423.60178 & 2 & 3.09 & $\begin{array}{l}240 \text { Swiss/ } 240 \text { phos } \& 244 \\
\text { Swiss/ } 244 \text { phos }\end{array}$ & Yes \& Yes \\
\hline \multirow[t]{3}{*}{ Q9NTJ3 } & SMC4_HUMAN Structural maintenance of chromosomes protein 4 & & & 10.16 & & \\
\hline & R.T*ESPATAAETASEELDNR.S & 1971.81787 & 2 & 3.25 & $39 \mathrm{NEW}$ & No \\
\hline & K.S*S*LAMNRS*R.G & 1261.41964 & 2 & 2.25 & $\begin{array}{l}588 \text { NEW \& } 589 \text { NEW \& } 595 \\
\text { NEW }\end{array}$ & No \\
\hline \multirow[t]{3}{*}{ Q8TAQ2 } & $\begin{array}{l}\text { SMRC2_HUMAN SWI/SNF-related matrix-associated actin-dependent regulato } \\
\text { subfamily C member } 2\end{array}$ & tor of chromatin & & 10.15 & & \\
\hline & R.KRS*PSPSPT*PEAK.K & 1541.67600 & 2 & 2.81 & 302 Swiss/ 302 phos & Yes \\
\hline & K.DMDEPS*PVPNVEEVTLPK.T & 2075.92425 & 2 & 3.05 & 347 Swiss/ 347 phos & Yes \\
\hline \multirow[t]{2}{*}{ Q14676 } & MDC1_HUMAN Mediator of DNA damage checkpoint protein 1 & & & 10.14 & & \\
\hline & R.LLLAEDS*EEEVDFLSER.R & 2073.92636 & 2 & 2.88 & 168 Swiss/ 168 phos & Yes \\
\hline \multirow[t]{3}{*}{ Q8WUA2 } & PPIL4_HUMAN Peptidyl-prolyl cis-trans isomerase-like 4 (PPIase) & & & 10.16 & & \\
\hline & R.INHTVILDDPFDDPPDLLIPDRSPEPT*R.E & 3277.57763 & 3 & 3.15 & $182 \mathrm{NEW}$ & No \\
\hline & R.INHTVILDDPFDDPPDLLIPDRS*PEPTR.E & 3277.57763 & 3 & 5.89 & 178 Swiss/ 178 phos & Yes \\
\hline \multirow[t]{2}{*}{ P18887 } & XRCC1_HUMAN DNA-repair protein XRCC1 & & & 10.20 & & \\
\hline & K.TKPTQAAGPSS*PQKPPT*PEETK.A & 2437.10472 & 3 & 4.07 & $\begin{array}{l}447 \text { Swiss/ } 447 \text { phos \& } 453 \\
\text { Swiss/ } 453 \text { phos }\end{array}$ & Yes \& Yes \\
\hline
\end{tabular}


Table A-1 (continued).

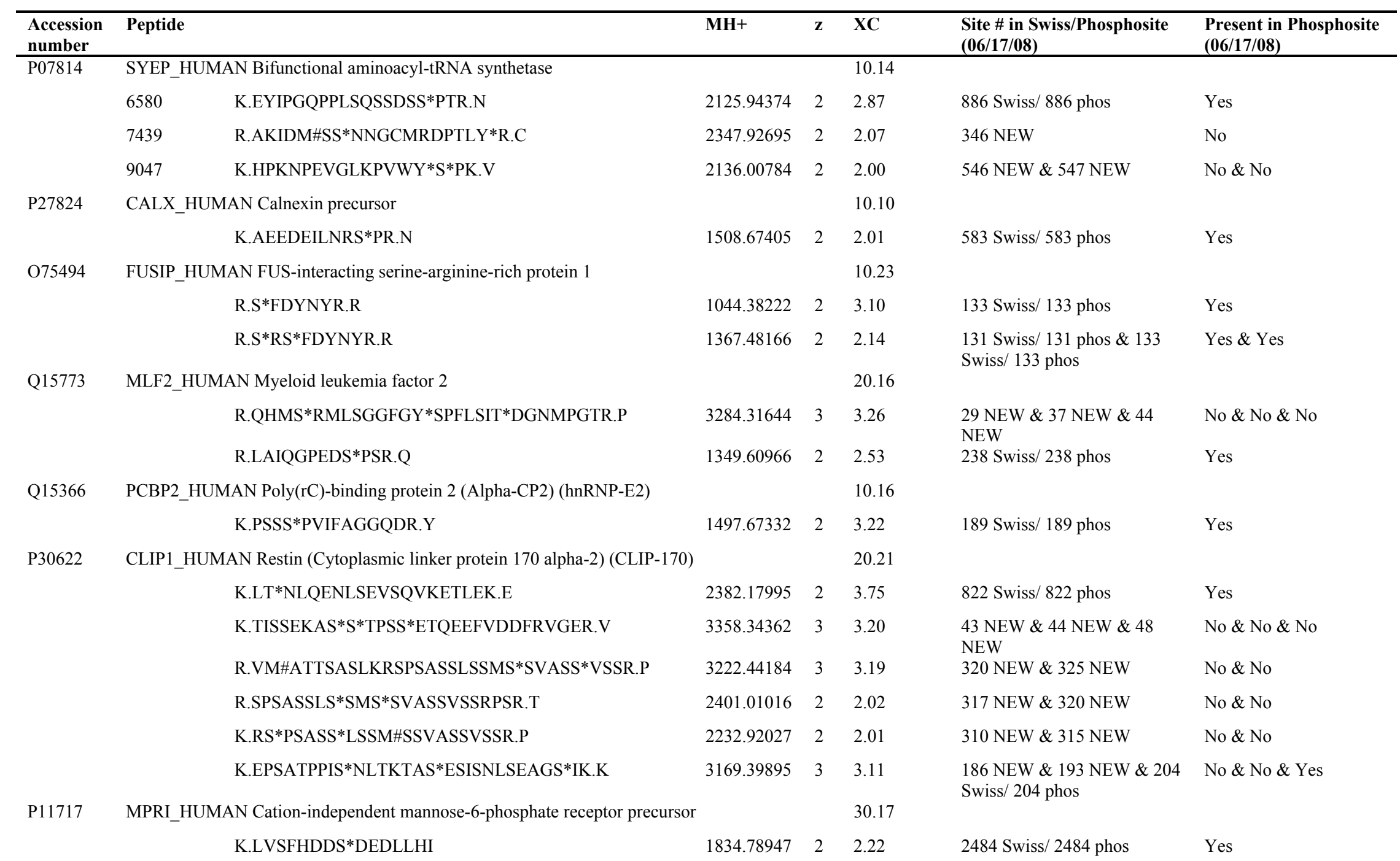


Table A-1 (continued).

\begin{tabular}{|c|c|c|c|c|c|c|}
\hline $\begin{array}{l}\text { Accession } \\
\text { number }\end{array}$ & Peptide & MH+ & $\overline{\mathbf{z}}$ & $\mathbf{X C}$ & $\begin{array}{l}\text { Site \# in Swiss/Phosphosite } \\
(06 / 17 / 08)\end{array}$ & $\begin{array}{l}\text { Present in Phosphosite } \\
(06 / 17 / 08)\end{array}$ \\
\hline \multirow[t]{2}{*}{$\mathrm{O} 00567$} & NOP56_HUMAN Nucleolar protein Nop56 (Nucleolar protein 5A) & & & 10.15 & & \\
\hline & K.EELMSS*DLEETAGSTSIPK.R & 2103.90391 & 2 & 3.07 & 520 Swiss/ 520 phos & Yes \\
\hline \multirow[t]{2}{*}{ P52756 } & RBM5_HUMAN RNA-binding protein 5 (RNA-binding motif protein 5) & & & 10.18 & & \\
\hline & R.GLVAAYSGDS*DNEEELVER.L & 2132.90193 & 2 & 3.53 & 624 Swiss/ 624 phos & Yes \\
\hline \multirow[t]{2}{*}{ Q8ND30 } & LIPB2_HUMAN Liprin-beta-2 & & & 10.16 & & \\
\hline & R.T*QSGNFYTDTLGMAEFR.R & 2017.83610 & 2 & 3.11 & $510 \mathrm{NEW}$ & No \\
\hline \multirow[t]{2}{*}{ P40222 } & TXLNA_HUMAN Alpha-taxilin & & & 10.13 & & \\
\hline & R.RPEGPGAQAPSS*PR.V & 1486.67980 & 2 & 2.58 & 515 Swiss/ 515 phos & Yes \\
\hline \multirow[t]{2}{*}{ Q8IWS0 } & PHF6_HUMAN PHD finger protein 6 (PHD-like zinc finger protein) & & & 10.17 & & \\
\hline & K.TAHNSEADLEESFNEHELEPSS*PK.S & 2777.15737 & 3 & 3.42 & 155 Swiss/ 155 phos & Yes \\
\hline \multirow[t]{7}{*}{ Q13595 } & TRA2A_HUMAN Transformer-2 protein homolog (TRA-2 alpha) & & & 30.15 & & \\
\hline & R.PTHS*GGGGGGGGGGGGGGGGRRR.D & 1901.82228 & 3 & 3.05 & $215 \mathrm{NEW}$ & No \\
\hline & R.AHT*PTPGIYMGR.P & 1380.61297 & 2 & 2.77 & $202 \mathrm{NEW}$ & No \\
\hline & R.RS*PS*PYYSR.Y & 1272.48093 & 2 & 2.09 & $\begin{array}{l}260 \text { Swiss/ } 260 \text { phos } \& 262 \\
\text { Swiss/ } 262 \text { phos }\end{array}$ & Yes \& Yes \\
\hline & R.S*PS*PYYSR.Y & 1116.37982 & 2 & 2.99 & see line 386 & \\
\hline & R.RRDSY*YDR.G & 1210.50005 & 2 & 2.27 & $237 \mathrm{NEW}$ & No \\
\hline & R.RRS*PSPYY*SR.Y & 1428.58204 & 2 & 2.02 & $\begin{array}{l}260 \text { Swiss/ } 260 \text { phos \& } 265 \\
\text { New in Swiss/ } 265 \text { in phos }\end{array}$ & Yes \& Yes \\
\hline \multirow[t]{2}{*}{ Q13489 } & BIRC3_HUMAN Baculoviral IAP repeat-containing protein 3 & & & 10.19 & & \\
\hline & -.M\#NIVENS*IFLS*NLMK.S & 1928.82979 & 2 & 3.74 & 7 NEW \& 11 NEW & No \& No \\
\hline \multirow[t]{3}{*}{ P49736 } & MCM2_HUMAN DNA replication licensing factor MCM2 & & & 10.15 & & \\
\hline & R.GLLYDS*DEEDEERPAR.K & 1973.81239 & 2 & 2.73 & 139 Swiss/ 139 phos & Yes \\
\hline & R.VM\#LES*FIDTQK.F & 1406.62726 & 2 & 2.69 & $801 \mathrm{NEW}$ & No \\
\hline
\end{tabular}


Table A-1 (continued).

\begin{tabular}{|c|c|c|c|c|c|c|}
\hline $\begin{array}{l}\text { Accession } \\
\text { number }\end{array}$ & Peptide & MH+ & $\mathbf{z}$ & $\mathbf{X C}$ & $\begin{array}{l}\text { Site \# in Swiss/Phosphosite } \\
(06 / 17 / 08)\end{array}$ & $\begin{array}{l}\text { Present in Phosphosite } \\
(06 / 17 / 08)\end{array}$ \\
\hline \multirow[t]{2}{*}{ Q12968 } & NFAC3_HUMAN Nuclear factor of activated T-cells, cytoplasmic 3 (NF-ATc? & & & 10.13 & & \\
\hline & R.PS*S*DSGCSHDSVLS*GQR.S & 1958.63875 & 2 & 2.66 & $\begin{array}{l}730 \text { NEW \& } 731 \text { NEW \& } 742 \\
\text { NEW }\end{array}$ & No \& No \& No \\
\hline \multirow[t]{2}{*}{ Q9H410 } & CT172_HUMAN Uncharacterized protein C20orf172 & & & 10.20 & & \\
\hline & K.SLHLS*PQEQSASYQDR.R & 1925.83888 & 2 & 3.94 & $81 \mathrm{NEW}$ & No \\
\hline \multirow[t]{3}{*}{ Q9ULU4 } & PKCB1_HUMAN Protein kinase C-binding protein 1 (Rack7) & & & 10.15 & & \\
\hline & R.RIS*LSDMPR.S & 1154.53874 & 2 & 2.48 & 425 New in Swiss/ 425 phos & Yes \\
\hline & K.S*DSSDSEYISDDEQK.S & 1784.63817 & 2 & 2.95 & $595 \mathrm{NEW}$ & No \\
\hline \multirow[t]{2}{*}{ P31321 } & KAP1_HUMAN cAMP-dependent protein kinase type I-beta regulatory subuni & & & 10.14 & & \\
\hline & -.M\#ASPPACPS*EEDESLK.G & 1786.69106 & 2 & 2.77 & $9 \mathrm{NEW}$ & No \\
\hline \multirow[t]{2}{*}{ Q7L7X3 } & TAOK1_HUMAN Serine/threonine-protein kinase TAO1 & & & 10.14 & & \\
\hline & R.AS*DPQSPPQVS*R.H & 1428.55555 & 2 & 2.22 & 417 New in Swiss/ 417 phos & Yes \\
\hline \multirow[t]{2}{*}{ P51587 } & BRCA2_HUMAN Breast cancer type 2 susceptibility protein & & & 10.15 & & \\
\hline & K.VFADIQS*EEILQHNQNMSGLEKVS*K.I & 3004.35223 & 3 & 3.01 & $1926 \mathrm{NEW}$ & No \\
\hline \multirow[t]{2}{*}{ Q8TF66 } & LRC15_HUMAN Leucine-rich repeat-containing protein 15 precursor (hLib) & & & 10.13 & & \\
\hline & R.M\#LANLQNIS*LQNNRLR.Q & 1994.00007 & 2 & 2.64 & $371 \mathrm{NEW}$ & No \\
\hline \multirow[t]{3}{*}{ Q5VT25 } & MRCKA_HUMAN Serine/threonine-protein kinase MRCK alpha & & & 20.12 & & \\
\hline & R.HSTAS*NSS*NLSSPPS*PASPR.K & 2220.83587 & 2 & 2.16 & $\begin{array}{l}1700 \text { NEW \& } 1703 \text { NEW \& } \\
1710 \text { NEW }\end{array}$ & No \& No \& No \\
\hline & R.TVFSGS*VSIPS*ITK.S & 1582.71647 & 2 & 2.05 & 1611 NEW \& 1616 NEW & No \& No \\
\hline \multirow[t]{2}{*}{ Q9UMY1 } & NOL7_HUMAN Nucleolar protein 7 (Nucleolar protein of $27 \mathrm{kDa}$ ) & & & 10.15 & & \\
\hline & K.VQSVS*QNKSY*LAVRLK.D & 1979.97146 & 2 & 2.94 & 167 NEW \& 172 NEW & No \& No \\
\hline \multirow[t]{2}{*}{ P28161 } & GSTM2_HUMAN Glutathione S-transferase Mu 2 (GSTM2-2) (GST class-mu & & & 10.16 & & \\
\hline & K.S*S*RFLPRPVFTK.M & 1594.75419 & 2 & 3.02 & 200 NEW \& 201 NEW & No \& No \\
\hline
\end{tabular}


Table A-1 (continued).

\begin{tabular}{|c|c|c|c|c|c|c|}
\hline $\begin{array}{l}\text { Accession } \\
\text { number }\end{array}$ & Peptide & MH+ & $\overline{\mathbf{z}}$ & $\mathbf{X C}$ & $\begin{array}{l}\text { Site \# in Swiss/Phosphosite } \\
(06 / 17 / 08)\end{array}$ & $\begin{array}{l}\text { Present in Phosphosite } \\
(06 / 17 / 08)\end{array}$ \\
\hline \multirow[t]{2}{*}{ Q9UKX3 } & MYH13_HUMAN Myosin-13 (Myosin heavy chain 13) & & & 10.14 & & \\
\hline & R.VEEKES*LISQLT*K.S & 1663.75906 & 2 & 2.79 & 1300 NEW \& 1306 NEW & No \& No \\
\hline \multirow[t]{2}{*}{ P55199 } & ELL_HUMAN RNA polymerase II elongation factor ELL & & & 10.14 & & \\
\hline & R.KSGAS*AVSGGS*GVS*QR.P & 1674.62844 & 2 & 2.82 & $\begin{array}{l}194 \text { NEW \& } 200 \text { NEW \& } 203 \\
\text { NEW }\end{array}$ & No \& No \& No \\
\hline \multirow[t]{3}{*}{ Q9BXT6 } & M10L1_HUMAN Putative helicase Mov1011 & & & 20.13 & & \\
\hline & K.WEDDSRNHGSPS*DCGPR.V & 1994.74466 & 2 & 2.63 & $113 \mathrm{NEW}$ & No \\
\hline & K.SSQALT*S*AKTTVVVTAQK.R & 1979.94497 & 2 & 2.59 & 472 NEW \& 473 NEW & No \& No \\
\hline \multirow[t]{2}{*}{ Q14181 } & DPOA2_HUMAN DNA polymerase subunit alpha B & & & 10.17 & & \\
\hline & R.GGAGNIS*LKVLGCPEALTGSYKSMFQK.L & 2836.37728 & 3 & 3.42 & $192 \mathrm{NEW}$ & No \\
\hline \multirow[t]{2}{*}{ Q12824 } & $\begin{array}{l}\text { SNF5_HUMAN SWI/SNF-related matrix-associated actin-dependent regulator } \\
\text { subfamily B member } 1\end{array}$ & of chromatin & & 10.14 & & \\
\hline & R.YPS*LWRRLATVEER.K & 1855.92143 & 2 & 2.26 & $49 \mathrm{NEW}$ & No \\
\hline \multirow[t]{3}{*}{ Q8IWV8 } & UBR2_HUMAN E3 ubiquitin-protein ligase UBR2 (N-recognin-2) & & & 20.16 & & \\
\hline & K.M\#RES*SPTSPVAET*EGTIMEESSRDK.D & 2930.18316 & 3 & 3.28 & 1005 NEW \& 1014 NEW & No \& No \\
\hline & K.MRESS*PT*SPVAETEGTIMEES*SRDK.D & 2994.15456 & 3 & 3.01 & $\begin{array}{l}1006 \text { NEW \& } 1008 \text { NEW \& } \\
1022 \text { NEW }\end{array}$ & No \& No \& No \\
\hline \multirow[t]{4}{*}{ P49006 } & MRP_HUMAN MARCKS-related protein (MARCKS-like protein 1) & & & 30.22 & & \\
\hline & R.GDVTAEEAAGAS*PAK.A & 1453.62061 & 2 & 4.03 & 22 Swiss/ 21 phos & Yes (same AA, diff \#) \\
\hline & K.LSGLS*FK.R & 831.40117 & 2 & 2.35 & 104 Swiss/ 103 phos & Yes (same AA, diff \#) \\
\hline & K.LSGLS*FKR.N & 987.50228 & 2 & 2.21 & see line 433 & \\
\hline \multirow[t]{2}{*}{ Q99733 } & NP1L4_HUMAN Nucleosome assembly protein 1-like 4 & & & 10.24 & & \\
\hline & R.REFITGDVEPTDAESEWHS*ENEEEEK.L & 3172.29024 & 3 & 3.40 & 125 Swiss/ 125 phos & Yes \\
\hline \multirow[t]{3}{*}{ Q13541 } & 4EBP1_HUMAN Eukaryotic translation initiation factor 4E-binding protein 1 & & & 20.24 & & \\
\hline & R.VVLGDGVQLPPGDYSTT*PGGTLFSTT*PGGTR.I & 3207.46462 & 3 & 4.48 & \multirow{2}{*}{$\begin{array}{l}37 \text { Swiss/ } 36 \text { phos \& } 46 \\
\text { Swiss/ } 45 \text { phos } \\
\text { see line } 438\end{array}$} & Yes (same AA, diff \#) \\
\hline & R.RVVLGDGVQLPPGDYSTT*PGGTLFSTT*PGGTR.I & 3363.56573 & 3 & 4.23 & & \\
\hline
\end{tabular}


Table A-1 (continued).

\begin{tabular}{|c|c|c|c|c|c|c|}
\hline $\begin{array}{l}\text { Accession } \\
\text { number }\end{array}$ & Peptide & MH+ & $\mathbf{z}$ & $\mathbf{X C}$ & $\begin{array}{l}\text { Site \# in Swiss/Phosphosite } \\
(06 / 17 / 08)\end{array}$ & $\begin{array}{l}\text { Present in Phosphosite } \\
(06 / 17 / 08)\end{array}$ \\
\hline \multirow[t]{2}{*}{ Q9H6Z4 } & RANB3_HUMAN Ran-binding protein 3 (RanBP3) & & & 10.18 & & \\
\hline & R.TSS*LTQFPPSQSEER.S & 1773.76907 & 2 & 3.57 & 126 Swiss/ 126 phos & Yes \\
\hline \multirow[t]{2}{*}{ P42566 } & EP15_HUMAN Epidermal growth factor receptor substrate 15 (Protein E & ps15) & & 10.17 & & \\
\hline & R.S*SPELLPSGVTDENEVTTAVTEK.V & 2483.14362 & 2 & 3.43 & 562 Swiss/ New in phos & No \\
\hline \multirow[t]{2}{*}{ P62258 } & 1433E_HUMAN 14-3-3 protein epsilon (14-3-3E) & & & 10.32 & & \\
\hline & K.AAFDDAIAELDTLS*EESYK.D & 2167.93184 & 2 & 3.58 & 210 Swiss/ New in phos & No \\
\hline \multirow[t]{2}{*}{ O46021 } & RL1D1_HUMAN Ribosomal L1 domain-containing protein 1 & & & 10.12 & & \\
\hline & K.ATNES*EDEIPQLVPIGK.K & 1919.89975 & 2 & 2.09 & 361 Swiss/ 361 phos & Yes \\
\hline \multirow[t]{3}{*}{ P29966 } & MARCS_HUMAN Myristoylated alanine-rich C-kinase substrate (MARC & (KS) & & 20.11 & & \\
\hline & K.LSGFS*FK.K & 865.38552 & 2 & 2.27 & 170 Swiss/ 170 phos & Yes \\
\hline & K.LSGFS*FKK.N & 993.48048 & 2 & 2.25 & see line 449 & \\
\hline \multirow[t]{2}{*}{ O95400 } & CD2B2_HUMAN CD2 antigen cytoplasmic tail-binding protein 2 & & & 10.19 & & \\
\hline & K.HSLDS*DEEEDDDDGGSSK.Y & 2016.68256 & 2 & 3.71 & 49 Swiss/ 49 phos & Yes \\
\hline \multirow[t]{6}{*}{ P05783 } & K1C18_HUMAN Keratin, type I cytoskeletal 18 (Cytokeratin-18) & & & 50.33 & & \\
\hline & R.PVSSAAS*VYAGAGGSGSR.I & 1660.73262 & 2 & 6.03 & 34 Swiss/ 33 phos & Yes (Same AA, diff \#) \\
\hline & R.LLEDGEDFNLGDALDSSNS*MQTIQK.T & 2820.22810 & 3 & 4.90 & 401 Swiss/ 400 phos & Yes (Same AA, diff \#) \\
\hline & R.STS*FRGGM\#GSGGLATGIAGGLAGMGGIQNEK.E & 2935.34374 & 3 & 3.62 & 53 Swiss/ 52 phos & Yes (Same AA, diff \#) \\
\hline & R.ST*FSTNYR.S & 1055.41934 & 2 & 2.57 & 8 Swiss/ 7 phos & Yes (Same AA, diff \#) \\
\hline & R.S*LGSVQAPSYGAR.P & 1372.62564 & 2 & 2.47 & 15 Swiss/ New in phos & No \\
\hline \multirow[t]{4}{*}{ Q96QR8 } & PURB_HUMAN Transcriptional activator protein Pur-beta & & & 30.26 & & \\
\hline & R.DSLGDFIEHYAQLGPSS*PEQLAAGAEEGGGPR.R & 3335.48519 & 3 & 5.18 & 101 Swiss/ 101 phos & Yes \\
\hline & R.RGGGS*GGGEES*EGEEVDED & 2011.64371 & 2 & 2.62 & $\begin{array}{l}298 \text { Swiss/ } 298 \text { phos \& } 304 \text { Swiss/ } \\
304 \text { phos }\end{array}$ & Yes \& Yes \\
\hline & R.RGGGSGGGEES*EGEEVDED & 1931.67741 & 2 & 2.41 & see line 461 & \\
\hline
\end{tabular}


Table A-1 (continued).

\begin{tabular}{|c|c|c|c|c|c|c|}
\hline $\begin{array}{l}\text { Accession } \\
\text { number }\end{array}$ & Peptide & MH+ & $\overline{\mathbf{z}}$ & $\mathbf{X C}$ & $\begin{array}{l}\text { Site \# in Swiss/Phosphosite } \\
(06 / 17 / 08)\end{array}$ & $\begin{array}{l}\text { Present in Phosphosite } \\
(06 / 17 / 08)\end{array}$ \\
\hline \multirow[t]{2}{*}{ Q5BKZ1 } & ZN326_HUMAN Zinc finger protein 326 & & & 10.21 & & \\
\hline & R.SMDSYLNQS*YGMDNHSGGGGGSR.F & 2456.92310 & 3 & 4.21 & $56 \mathrm{NEW}$ & No \\
\hline \multirow[t]{2}{*}{ P29692 } & EF1D_HUMAN Elongation factor 1-delta (EF-1-delta) & & & 10.20 & & \\
\hline & R.ATAPQTQHVS*PMR.Q & 1503.67736 & 2 & 3.75 & 133 Swiss/ New in phos & No \\
\hline \multirow[t]{2}{*}{ Q9UGV2 } & NDRG3_HUMAN Protein NDRG3 & & & 10.25 & & \\
\hline & R.THS*TSSSLGSGESPFSR.S & 1803.75448 & 2 & 5.00 & 331 Swiss/ 331 phos & Yes \\
\hline \multirow[t]{3}{*}{ Q05519 } & SFR11_HUMAN Splicing factor arginine/serine-rich 11 & & & 20.21 & & \\
\hline & K.LNHVAAGLVS*PSLK.S & 1485.78248 & 2 & 4.28 & $207 \mathrm{Swiss} / 207 \mathrm{New}$ in pho & No \\
\hline & R.DYDEEEQGYDS*EKEK.K & 1943.70659 & 2 & 3.00 & 434 Swiss/ 434 phso & Yes \\
\hline \multirow[t]{2}{*}{ P25788 } & PSA3_HUMAN Proteasome subunit alpha type 3 (Proteasome componen & at C8) & & 10.28 & & \\
\hline & K.ESLKEEDES*DDDNM & 1735.58878 & 2 & 5.58 & 250 Swiss/ 249 phos & Yes (Same AA, diff \#) \\
\hline \multirow[t]{2}{*}{ Q6UN15 } & FIP1_HUMAN Pre-mRNA 3'-end-processing factor FIP1 (FIP1-like 1) & & & 10.20 & & \\
\hline & R.DHS*PTPSVFNSDEER.Y & 1796.71228 & 2 & 3.83 & 492 Swiss/ 492 pho & Yes \\
\hline \multirow[t]{2}{*}{ Q9NYB0 } & TE2IP_HUMAN Telomeric repeat-binding factor 2-interacting protein 1 & & & 10.21 & & \\
\hline & K.YLLGDAPVS*PSSQK.L & 1541.72469 & 2 & 4.30 & 203 Swiss/ 203 phos & Yes \\
\hline \multirow[t]{6}{*}{ P27816 } & MAP4_HUMAN Microtubule-associated protein 4 (MAP 4) & & & 40.27 & & \\
\hline & K.DMES*PTKLDVTLAK.D & 1627.76484 & 2 & 4.73 & 280 Swiss/ 280 phos & Yes \\
\hline & K.VGS*LDNVGHLPAGGAVK.T & 1670.82613 & 2 & 3.62 & 1073 Swiss/ 1073 phos & Yes \\
\hline & K.DMS*PLSETEMALGK.D & 1588.66341 & 2 & 3.06 & 507 Swiss/ 507 phos & Yes \\
\hline & K.T*STSKAKTQPTSLPK.Q & 1654.84111 & 2 & 2.19 & $712 \mathrm{NEW}$ & No \\
\hline & K.VGS*TENIK.H & 927.41827 & 2 & 2.02 & $941 \mathrm{NEW}$ & No \\
\hline \multirow[t]{3}{*}{ P08729 } & K2C7_HUMAN Keratin, type II cytoskeletal 7 (Cytokeratin-7) & & & 20.32 & & \\
\hline & R.T*LNETELTELQS*QISDTSVVLSMDNSR.S & 3170.38472 & 3 & 6.39 & 227 NEW \& 238 NEW & No \& No \\
\hline & R.T*LNETELT*ELQSQISDTSVVLSM\#DNSR.S & 3186.37962 & 3 & 4.13 & 227 NEW \& 234 NEW & No \& No \\
\hline
\end{tabular}


Table A-1 (continued).

\begin{tabular}{|c|c|c|c|c|c|c|}
\hline $\begin{array}{l}\text { Accession } \\
\text { number }\end{array}$ & Peptide & MH+ & $\overline{\mathbf{z}}$ & $\mathbf{X C}$ & $\begin{array}{l}\text { Site \# in Swiss/Phosphosite } \\
(06 / 17 / 08)\end{array}$ & $\begin{array}{l}\text { Present in Phosphosite } \\
(06 / 17 / 08)\end{array}$ \\
\hline \multirow[t]{2}{*}{ Q9H307 } & PININ_HUMAN Pinin (140 kDa nuclear and cell adhesion-related phosp & phoprotein) & & 10.20 & & \\
\hline & K.S*LSPGKENVSALDMEK.E & 1784.81358 & 2 & 3.95 & 441 Swiss/ 440 phos & Yes (Same AA, diff \#) \\
\hline \multirow[t]{2}{*}{ Q9BQE3 } & TBA6_HUMAN Tubulin alpha-6 chain (Alpha-tubulin 6) & & & 10.31 & & \\
\hline & K.TIGGGDDS*FNTFFSETGAGK.H & 2087.85934 & 2 & 5.65 & 48 Swiss/ 48 phos & Yes \\
\hline \multirow[t]{2}{*}{ Q93009 } & UBP7_HUMAN Ubiquitin carboxyl-terminal hydrolase 7 (Ubiquitin thio & oesterase 7) & & 10.21 & & \\
\hline & K.AGEQQLS*EPEDMEMEAGDTDDPPR.I & 2727.04333 & 3 & 4.29 & 18 Swiss/ 18 phos & Yes \\
\hline \multirow[t]{2}{*}{ P52597 } & HNRPF_HUMAN Heterogeneous nuclear ribonucleoprotein F (hnRNP F & & & 10.32 & & \\
\hline & K.ATENDIYNFFS*PLNPVR.V & 2076.94262 & 2 & 3.97 & 310 Swiss/ New in pho & No \\
\hline \multirow[t]{2}{*}{ Q13185 } & CBX3_HUMAN Chromobox protein homolog 3 & & & 10.27 & & \\
\hline & K.SLS*DSESDDSK.S & 1249.44673 & 2 & 3.53 & 95 Swiss/ 95 phos & Yes \\
\hline \multirow[t]{3}{*}{ Q8TE77 } & SSH3_HUMAN Protein phosphatase Slingshot homolog 3 (SSH-3L) (hS & SSH-3L) & & 20.26 & & \\
\hline & R.S*PPGSGASTPVGPWDQAVQR.R & 2073.93893 & 2 & 4.31 & 4 NEW & No \\
\hline & R.RQS*FAVLR.G & 1056.53497 & 2 & 3.05 & 37 New in Swiss/ 37 phos & Yes \\
\hline \multirow[t]{2}{*}{ Q9NR30 } & DDX21_HUMAN Nucleolar RNA helicase 2 (Nucleolar RNA helicase II & & & 10.20 & & \\
\hline & K.NEEPS*EEEIDAPKPK.K & 1791.76840 & 2 & 4.09 & 121 Swiss/ 121 pho & Yes \\
\hline \multirow[t]{2}{*}{ O95359 } & TACC2_HUMAN Transforming acidic coiled-coil-containing protein 2 & & & 10.18 & & \\
\hline & K.LDNTPAS*PPRS*PAEPNDIPIAK.G & 2460.12070 & 3 & 3.68 & $\begin{array}{l}2317 \text { Swiss/ New in phos \& } 2321 \\
\text { NEW }\end{array}$ & No \& No \\
\hline \multirow[t]{2}{*}{ Q86W92 } & LIPB1_HUMAN Liprin-beta-1 & & & 10.26 & & \\
\hline & R.SQS*TTFNPDDMSEPEFK.R & 2039.79396 & 2 & 5.12 & 601 Swiss/ New in phos & No \\
\hline
\end{tabular}


Table A-1 (continued).

\begin{tabular}{|c|c|c|c|c|c|c|}
\hline $\begin{array}{l}\text { Accession } \\
\text { number }\end{array}$ & Peptide & MH+ & $\mathbf{z}$ & $\mathbf{X C}$ & $\begin{array}{l}\text { Site \# in Swiss/Phosphosite } \\
(06 / 17 / 08)\end{array}$ & $\begin{array}{l}\text { Present in Phosphosite } \\
(06 / 17 / 08)\end{array}$ \\
\hline \multirow[t]{7}{*}{$\mathrm{P} 23588$} & IF4B_HUMAN Eukaryotic translation initiation factor 4B (eIF-4B) & & & 50.27 & & \\
\hline & K.SPPY*TAFLGNLPYDVTEESIK.E & 2421.12612 & 2 & 4.84 & $96 \mathrm{NEW}$ & No \\
\hline & R.RES*EKS*LENETLNK.E & 1836.77757 & 2 & 3.92 & $\begin{array}{l}442 \text { NEW \& } 445 \text { Swiss/ New in } \\
\text { phos }\end{array}$ & No \& No \\
\hline & R.ARPATDS*FDDYPPR.R & 1687.71116 & 2 & 3.20 & $207 \mathrm{NEW}$ & No \\
\hline & R.HPS*WRS*EETQER.E & 1701.64174 & 2 & 2.11 & 406 Swiss/ 406 phos \& 409 NEW & Yes \& No \\
\hline & K.PRST*PEEDDSS*AS*TSQSTR.A & 2277.79446 & 2 & 2.07 & 341 NEW \& 348 NEW \& 350 NEW & No \& No \& No \\
\hline & K.YAALS*VDGEDENEGEDYAE & 2155.78630 & 2 & 4.50 & 597 Swiss/ New in phos & No \\
\hline \multirow[t]{3}{*}{ O15164 } & TIF1A_HUMAN Transcription intermediary factor 1-alpha (TIF1-alpha) & & & 20.16 & & \\
\hline & K.SEWLDPSQKS*PLHVGETR.K & 2145.99644 & 3 & 3.26 & 811 Swiss/ New in phos & No \\
\hline & R.SILTSLLLNSS*QSS*T*SEETVLR.S & 2605.14458 & 2 & 2.00 & $\begin{array}{l}768 \text { Swiss/ } 768 \text { phos \& } 771 \text { NEW \& } \\
772 \text { NEW }\end{array}$ & Yes \& No \& No \\
\hline \multirow[t]{3}{*}{ P46821 } & MAP1B_HUMAN Microtubule-associated protein 1B (MAP 1B) & & & 20.18 & & \\
\hline & K.LGDVS*PTQIDVSQFGSFK.E & 2004.93138 & 2 & 3.59 & 1501 Swiss/ 1501 phos & Yes \\
\hline & K.TTSPPEVS*GYS*Y*EK.T & 1784.61039 & 2 & 2.12 & $\begin{array}{l}1970 \text { NEW \& } 1973 \text { NEW \& } 1974 \\
\text { Swiss/ } 1974 \text { phos }\end{array}$ & No \& No \& Yes \\
\hline \multirow[t]{2}{*}{ Q06265 } & EXOS9_HUMAN Exosome complex exonuclease RRP45 (Exosome comp & onent 9) & & 20.20 & & \\
\hline & K.APIDTS*DVEEK.A & 1283.54024 & 2 & 3.89 & $306 \mathrm{NEW}$ & No \\
\hline \multirow[t]{3}{*}{ Q8WUZ0 } & BCL7C_HUMAN B-cell CLL/lymphoma 7 protein family member C & & & 20.28 & & \\
\hline & K.GTEPS*PGGTPQPSRPVS*PAGPPEGVPEEAQPPR.L & 3419.53041 & 3 & 5.64 & $\begin{array}{l}114 \text { Swiss/ } 114 \text { phos \& } 126 \text { Swiss/ } \\
126 \text { phos }\end{array}$ & Yes \& Yes \\
\hline & K.GTEPS*PGGT*PQPSRPVSPAGPPEGVPEEAQPPR.L & 3419.53041 & 3 & 3.07 & 118 Swiss/ 118 phos & Yes \\
\hline \multirow[t]{6}{*}{ O43290 } & SNUT1_HUMAN U4/U6.U5 tri-snRNP-associated protein 1 & & & 10.26 & & \\
\hline & R.RVS*EVEEEKEPVPQPLPSDDTR.V & 2616.21885 & 3 & 5.18 & 448 Swiss/ 448 phos & Yes \\
\hline & R.DLQGLT*VEHAIDSFREGETMILT*LKDK.G & 3219.50438 & 3 & 3.16 & $258 \mathrm{NEW}$ & No \\
\hline & K.KMSSS*DT*PLGT*VALLQEK.Q & 2144.89865 & 2 & 2.08 & 762 NEW \& 764 NEW \& 768 NEW & No \& No \& No \\
\hline & K.KMS*S*S*DTPLGTVALLQEKQK.A & 2401.05220 & 2 & 2.36 & 760 NEW \& 761 NEW \& 762 NEW & No \& No \& No \\
\hline & K.M\#SS*SDT*PLGTVALLQEK.Q & 1952.83229 & 2 & 2.13 & 761 NEW \& 764 NEW & No \& No \\
\hline
\end{tabular}


Table A-1 (continued).

\begin{tabular}{|c|c|c|c|c|c|c|}
\hline $\begin{array}{l}\text { Accession } \\
\text { number }\end{array}$ & Peptide & MH+ & $\overline{\mathbf{z}}$ & $\mathbf{X C}$ & $\begin{array}{l}\text { Site \# in Swiss/Phosphosite } \\
(06 / 17 / 08)\end{array}$ & $\begin{array}{l}\text { Present in Phosphosite } \\
(06 / 17 / 08)\end{array}$ \\
\hline \multirow[t]{3}{*}{ P30291 } & WEE1_HUMAN Wee1-like protein kinase (Wee1A kinase) (WEE1hu) & & & 20.15 & & \\
\hline & K.S*PAAPY*FLGSSFS*PVRCGGPGDASPR.G & 2820.12888 & 3 & 3.04 & $\begin{array}{l}127 \text { Swiss/ New in phos \& } 132 \\
\text { NEW \& } 139 \text { Swiss/ } 139 \text { phos }\end{array}$ & No \& No \& Yes \\
\hline & K.S*RYTTEFHELEKIGS*GEFGSVFK.C & 2808.23171 & 3 & 3.03 & 293 NEW \& 307 NEW & No \& No \\
\hline \multirow[t]{2}{*}{ P16333 } & NCK1_HUMAN Cytoplasmic protein NCK1 (NCK adaptor protein 1) & & & 10.17 & & \\
\hline & K.RKPS*VPDSASPADDSFVDPGER.L & 2409.07179 & 3 & 3.42 & 85 Swiss/ 85 phos & Yes \\
\hline \multirow[t]{2}{*}{ Q9UNE7 } & STUB1_HUMAN STIP1 homology and U box-containing protein 1 & & & 10.20 & & \\
\hline & R.LGAGGGS*PEKS*PSAQELK.E & 1872.81395 & 2 & 3.92 & $\begin{array}{l}19 \text { Swiss/ } 19 \text { phos \& } 23 \text { Swiss/ } 23 \\
\text { phos }\end{array}$ & Yes \& Yes \\
\hline \multirow[t]{2}{*}{ Q9BTC0 } & DIDO1_HUMAN Death-inducer obliterator 1 (DIO-1) & & & 10.18 & & \\
\hline & R.RNS*VERPAEPVAGAATPSLVEQQK.M & 2614.29844 & 3 & 3.66 & 1456 Swiss/ New in phos & No \\
\hline \multirow[t]{3}{*}{ Q96I25 } & SPF45_HUMAN Splicing factor 45 (45 kDa-splicing factor) & & & 20.20 & & \\
\hline & R.S*MGGAAIAPPTSLVEK.D & 1608.77026 & 2 & 4.06 & $169 \mathrm{NEW}$ & No \\
\hline & R.SPT*GPSNSFLANMGGTVAHK.I & 2052.92084 & 2 & 3.75 & 224 NEW & No \\
\hline \multirow[t]{4}{*}{ Q53EL6 } & \multicolumn{3}{|l|}{ PDCD4_HUMAN Programmed cell death protein 4 (Nuclear antigen H731-like) } & 30.20 & & \\
\hline & R.KDS*VWGSGGGQQSVNHLVK.E & 2062.97056 & 3 & 3.98 & 313 NEW & No \\
\hline & R.SGLTVPTS*PK.G & 1066.51799 & 2 & 2.62 & 94 Swiss/ 94 phos & Yes \\
\hline & K.NSSRDS*GRGDS*VS*DSGSDALR.S & 2364.84896 & 2 & 2.17 & 71 NEW \& 76 Swiss/ New in phos & No \& No \\
\hline \multirow[t]{3}{*}{ Q9H2U2 } & \multicolumn{3}{|l|}{ IPYR2_HUMAN Inorganic pyrophosphatase 2, mitochondrial precursor (PPase 2) } & 20.28 & & \\
\hline & R.SLVESVSSS*PNKESNEEEQVWHFLGK & 3026.37787 & 3 & 5.60 & 317 Swiss/ 317 phos & Yes \\
\hline & K.FKPGYLEATLNWFRLY*K.V & 2226.11471 & 3 & 3.71 & 241 New in Swiss/ 241 phos & Yes \\
\hline \multirow[t]{3}{*}{ Q8ND56 } & LS14A_HUMAN LSM14 protein homolog A (Protein SCD6 homolog) & & & 20.17 & & \\
\hline & K.S*PTMEQAVQTASAHLPAPAAVGR.R & 2370.12714 & 3 & 3.50 & 192 Swiss/ 192 phos & Yes \\
\hline & R.SS*PQLDPLR.K & 1092.50849 & 2 & 2.38 & 183 Swiss/ 183 phos & Yes \\
\hline \multirow[t]{3}{*}{ Q13247 } & SFRS6_HUMAN Splicing factor, arginine/serine-rich 6 & & & 20.18 & & \\
\hline & R.SNS*PLPVPPSK.A & 1202.58165 & 2 & 3.19 & 303 Swiss/ 303 phos & Yes \\
\hline & R.LIVENLSS*R.C & 1110.55544 & 2 & 3.14 & 119 NEW & No \\
\hline
\end{tabular}


Table A-1 (continued).

\begin{tabular}{|c|c|c|c|c|c|c|}
\hline $\begin{array}{l}\text { Accession } \\
\text { number }\end{array}$ & Peptide & MH+ & $\mathbf{z}$ & $\mathbf{X C}$ & $\begin{array}{l}\text { Site \# in Swiss/Phosphosite } \\
(06 / 17 / 08)\end{array}$ & $\begin{array}{l}\text { Present in Phosphosite } \\
(06 / 17 / 08)\end{array}$ \\
\hline \multirow[t]{2}{*}{ Q9H7L9 } & \multicolumn{3}{|c|}{ SDS3_HUMAN Sin3 histone deacetylase corepressor complex component SDS3 } & 10.24 & & \\
\hline & K.RPAS*PSS*PEHLPATPAESPAQR.F & 2443.08023 & 3 & 4.74 & 234 Swiss/ 234 phos & Yes \\
\hline \multirow[t]{3}{*}{ Q9BXP5 } & ARS2_HUMAN Arsenite-resistance protein 2 & & & 20.17 & & \\
\hline & R.TQLWASEPGT*PPLPTSLPSQNPILK.N & 2752.39569 & 3 & 3.35 & 544 Swiss/ 544 phos & Yes \\
\hline & R.HELS*PPQK.R & 1015.46081 & 2 & 2.44 & 74 Swiss/ 74 phos & Yes \\
\hline \multirow[t]{2}{*}{ P68366 } & TBA1_HUMAN Tubulin alpha-1 chain (Alpha-tubulin 1) & & & 10.16 & & \\
\hline & R.EDM\#AALEKDY*EEVGIDSYEDEDEGEE & 3105.14492 & 3 & 3.15 & $432 \mathrm{NEW}$ & No \\
\hline \multirow[t]{4}{*}{ Q96D71 } & REPS1_HUMAN RalBP1-associated Eps domain-containing protein 1 & & & 30.20 & & \\
\hline & R.TSADAQEPASPVVSPQQS*PPTS*PHTWR.K & 3018.30298 & 3 & 4.01 & $\begin{array}{l}118 \text { Swiss/ New in phos \& } 122 \\
\text { Swiss/ New in phos }\end{array}$ & No \& No \\
\hline & R.RQS*SSYDDPWK.I & 1448.58417 & 2 & 3.75 & 220 Swiss/ New in phos & No \\
\hline & R.HAAS*YS*SDSENQGSY*SGVIPPPPGRGQVKK.G & 3340.40717 & 3 & 3.14 & $\begin{array}{l}63 \text { NEW \& } 65 \text { NEW \& } 74 \text { New in } \\
\text { Swis/s } 74 \text { phos }\end{array}$ & No \& No \& Yes \\
\hline \multirow[t]{4}{*}{ O94888 } & UBXD7_HUMAN UBX domain-containing protein 7 & & & 10.17 & & \\
\hline & R.SESLIDASEDS*QLEAAIR.A & 2013.90121 & 2 & 3.34 & 288 Swiss/ 288 phos & Yes \\
\hline & R.SES*LIDASEDSQLEAAIR.A & 2013.90121 & 2 & 5.19 & 280 Swiss/ 280 phos & Yes \\
\hline & R.DFQT*ET*IRQEQELR.N & 1952.81502 & 2 & 2.66 & 116 NEW \& 118 NEW & No \& No \\
\hline \multirow[t]{3}{*}{ P67809 } & YBOX1_HUMAN Nuclease sensitive element-binding protein 1 & & & 20.24 & & \\
\hline & R.NYQQNYQNS*ESGEKNEGSESAPEGQAQQR.R & 3337.36251 & 3 & 4.15 & 165 Swiss/ 165 phos & Yes \\
\hline & K.NEGSES*APEGQAQQR.R & 1667.66567 & 2 & 2.98 & 176 Swiss/ 176 phso & Yes \\
\hline \multirow[t]{2}{*}{ Q9UN36 } & NDRG2_HUMAN Protein NDRG2 (Protein Syld709613) & & & 10.24 & & \\
\hline & R.SRT*AS*LTSAASVDGNR.S & 1752.73129 & 2 & 4.75 & $\begin{array}{l}330 \text { Swiss/ } 330 \text { phos } \& 332 \text { Swiss/ } \\
332 \text { phos }\end{array}$ & Yes \& Yes \\
\hline \multirow[t]{2}{*}{ O00264 } & PGRC1_HUMAN Membrane-associated progesterone receptor compone & $\mathrm{t} 1$ (mPR) & & 10.17 & & \\
\hline & K.EGEEPTVYS*DEEEPKDESAR.K & 2375.93984 & 3 & 3.38 & 181 Swiss/ 180 phos & Yes (Same AA, diff \#) \\
\hline
\end{tabular}


Table A-1 (continued).

\begin{tabular}{|c|c|c|c|c|c|c|}
\hline $\begin{array}{l}\text { Accession } \\
\text { number }\end{array}$ & Peptide & MH+ & $\mathbf{z}$ & $\mathbf{X C}$ & $\begin{array}{l}\text { Site \# in Swiss/Phosphosite } \\
(06 / 17 / 08)\end{array}$ & $\begin{array}{l}\text { Present in Phosphosite } \\
(06 / 17 / 08)\end{array}$ \\
\hline \multirow[t]{3}{*}{ Q9UBC2 } & EP15R_HUMAN Epidermal growth factor receptor substrate 15-like 1 & & & 20.23 & & \\
\hline & K.FHDTS*SPLMVTPPSAEAHWAVR.V & 2516.14279 & 3 & 4.60 & $107 \mathrm{NEW}$ & No \\
\hline & K.DS*LRSTPS*HGSVSSLNSTGSLSPKHS*LK.Q & 3106.36424 & 3 & 3.07 & 235 NEW \& 241 Swiss/ 241 phos & No \& Yes \\
\hline \multirow[t]{3}{*}{ O00499 } & \multicolumn{3}{|l|}{ BIN1_HUMAN Myc box-dependent-interacting protein 1 (Bridging integrator 1) } & 20.22 & & \\
\hline & K.S*PSPPDGS*PAATPEIR.V & 1738.70843 & 2 & 4.46 & $\begin{array}{l}296 \text { Swiss/ } 296 \text { phos \& } 303 \text { Swiss/ } \\
303 \text { phos }\end{array}$ & Yes \& Yes \\
\hline & K.S*PSPPDGSPAATPEIR.V & 1658.74213 & 2 & 3.86 & see line 582 & \\
\hline \multirow[t]{2}{*}{ Q9BQI5 } & SGIP1_HUMAN SH3-containing GRB2-like protein 3-interacting proteir & & & 10.12 & & \\
\hline & K.WVHFSDTSPEHVTPELT*PR.E & 2315.04921 & 2 & 2.12 & $328 \mathrm{NEW}$ & No \\
\hline \multirow[t]{2}{*}{ O95460 } & MATN4_HUMAN Matrilin-4 precursor & & & 10.16 & & \\
\hline & R.VGVIQYS*SQVQSVFPLR.A & 1987.00482 & 2 & 3.13 & 79 NEW & No \\
\hline \multirow[t]{2}{*}{ Q9ULM3 } & YETS2_HUMAN YEATS domain-containing protein 2 & & & 10.14 & & \\
\hline & K.T*EES*S*ELGNYVIK.I & 1708.61548 & 2 & 2.85 & $\begin{array}{l}1131 \text { NEW \& } 1134 \text { NEW \& } 1135 \\
\text { NEW }\end{array}$ & No \& No \& No \\
\hline \multirow[t]{2}{*}{ Q9Y233 } & PDE10_HUMAN cAMP and cAMP-inhibited cGMP 3',5'-cyclic phospho & liesterase $10 \mathrm{~A}$ & & 10.15 & & \\
\hline & K.S*QHLTGLTDEKVK.A & 1535.74648 & 2 & 2.90 & $8 \mathrm{NEW}$ & No \\
\hline \multirow[t]{4}{*}{ Q15054 } & DPOD3_HUMAN DNA polymerase subunit delta 3 (DNA polymerase st & ounit delta p66) & & 20.14 & & \\
\hline & K.TEPEPPSVKS*S*SGENK.R & 1832.73504 & 2 & 2.76 & 374 NEW \& 375 NEW \& 376 NEW & No \& No \& No \\
\hline & K.S*KLAVT*ASIHVY*SIQK.A & 1984.89450 & 2 & 2.16 & 90 NEW \& 95 NEW \& 101 NEW & No \& No \& No \\
\hline & R.VALS*DDETKETENMR.K & 1817.76227 & 2 & 2.38 & 307 Swiss/ New in phos & No \\
\hline \multirow[t]{2}{*}{ Q9UNZ2 } & NSF1C_HUMAN NSFL1 cofactor p47 (p97 cofactor p47) & & & 10.17 & & \\
\hline & R.KKS*PNELVDDLFK.G & 1612.79818 & 2 & 3.44 & 114 Swiss/ 114 phos & Yes \\
\hline \multirow[t]{3}{*}{ Q9Y6W5 } & WASF2_HUMAN Wiskott-Aldrich syndrome protein family member 2 & & & 10.23 & & \\
\hline & K.RS*SVVSPSHPPPAPPLGSPPGPK.P & 2325.17508 & 3 & 4.64 & $292 \mathrm{NEW}$ & No \\
\hline & R.S*S*VVS*PSHPPPAPPLGSPPGPK.P & 2329.00657 & 3 & 3.68 & 292 NEW \& 293 NEW \& 296 NEW & No \& No \& No \\
\hline
\end{tabular}


Table A-1 (continued).

\begin{tabular}{|c|c|c|c|c|c|c|}
\hline $\begin{array}{l}\text { Accession } \\
\text { number }\end{array}$ & Peptide & MH+ & $\mathbf{z}$ & $\mathbf{X C}$ & $\begin{array}{l}\text { Site \# in Swiss/Phosphosite } \\
(06 / 17 / 08)\end{array}$ & $\begin{array}{l}\text { Present in Phosphosite } \\
(06 / 17 / 08)\end{array}$ \\
\hline \multirow[t]{2}{*}{ Q9BQA1 } & MEP50_HUMAN Methylosome protein 50 (MEP50 protein) (WD repeat $\mathrm{p}$ & protein 77) & & 10.16 & & \\
\hline & R.KET*PPPLVPPAAR.E & 1452.76101 & 2 & 3.22 & 5 Swiss/ 5 phos & Yes \\
\hline \multirow[t]{2}{*}{ P49407 } & ARRB1_HUMAN Beta-arrestin-1 (Arrestin beta 1) & & & 10.16 & & \\
\hline & R.KDLFVANVQS*FPPAPEDK.K & 2081.99432 & 2 & 3.22 & $86 \mathrm{NEW}$ & No \\
\hline \multirow[t]{2}{*}{ Q9P2B4 } & CT2NL_HUMAN CTTNBP2 N-terminal-like protein & & & 10.12 & & \\
\hline & K.EQKKLS*SQLEEER.S & 1683.79489 & 2 & 2.45 & $165 \mathrm{NEW}$ & No \\
\hline \multirow[t]{2}{*}{ Q5JTD0 } & TJAP1_HUMAN Tight junction-associated protein 1 (Tight junction protei & in 4) & & 10.16 & & \\
\hline & R.KDS*LTQAQEQGNLLN & 1738.80070 & 2 & 3.19 & 545 Swiss/ 545 phos & Yes \\
\hline \multirow[t]{2}{*}{ P24043 } & LAMA2_HUMAN Laminin subunit alpha-2 precursor (Laminin M chain) & & & 10.16 & & \\
\hline & R.NS*HIAIAFDDT*KVKNR.L & 1988.89902 & 2 & 3.24 & 2772 NEW \& 2781 NEW & No \& No \\
\hline \multirow[t]{2}{*}{ Q6Y7W6 } & PERQ2_HUMAN PERQ amino acid-rich with GYF domain-containing pro & otein 2 & & 10.14 & & \\
\hline & R.PGT*PSDHQSQEASQFER.K & 1980.80831 & 2 & 2.51 & 382 Swiss/ New in phos & No \\
\hline \multirow[t]{3}{*}{ Q86WB0 } & NIPA_HUMAN Nuclear-interacting partner of ALK & & & 20.16 & & \\
\hline & K.QSSQPAETDS*MSLSEKS*RK.V & 2255.92504 & 3 & 3.15 & 479 NEW \& 486 NEW & No \& No \\
\hline & R.SMGTGDT*PGLEVPSS*PLR.K & 1960.81224 & 2 & 2.26 & 387 NEW \& 395 Swiss/ 395 phos & No \& Yes \\
\hline \multirow[t]{2}{*}{ Q12955 } & ANK3_HUMAN Ankyrin-3 (ANK-3) (Ankyrin-G) & & & 10.15 & & \\
\hline & R.RQS*FASLALR.K & 1228.61977 & 2 & 2.97 & 1459 Swiss/ 1459 phos & Yes \\
\hline \multirow[t]{2}{*}{ Q15642 } & CIP4_HUMAN Cdc42-interacting protein 4 & & & 10.15 & & \\
\hline & R.APSDS*SLGTPSDGRPELR.G & 1921.86510 & 2 & 3.04 & 298 New in Swiss/ 298 phos & Yes \\
\hline \multirow[t]{2}{*}{$\mathrm{O} 15173$} & PGRC2_HUMAN Membrane-associated progesterone receptor component & & & 10.18 & & \\
\hline & R.LLKPGEEPSEY*TDEEDTK.D & 2159.92675 & 3 & 3.57 & 210 New in Swiss/ 210 phos & Yes \\
\hline \multirow[t]{3}{*}{ Q8N5S9 } & KKCC1_HUMAN Calcium/calmodulin-dependent protein kinase kinase 1 & & & 20.12 & & \\
\hline & R.KLS*LQER.P & 953.48154 & 2 & 2.35 & 74 Swiss/ 74 phos & Yes \\
\hline & R.LIPS*WTT*VILVKS*MLRK.R & 2225.09690 & 2 & 2.14 & 443 NEW \& 446 NEW \& 452 NEW & No \& No \& No \\
\hline
\end{tabular}


Table A-1 (continued).

\begin{tabular}{|c|c|c|c|c|c|c|}
\hline $\begin{array}{l}\text { Accession } \\
\text { number }\end{array}$ & Peptide & MH+ & $\mathbf{z}$ & $\mathbf{X C}$ & $\begin{array}{l}\text { Site \# in Swiss/Phosphosite } \\
(06 / 17 / 08)\end{array}$ & $\begin{array}{l}\text { Present in Phosphosite } \\
(06 / 17 / 08)\end{array}$ \\
\hline \multirow[t]{2}{*}{$\mathrm{P} 30086$} & PEBP1_HUMAN Phosphatidylethanolamine-binding protein 1 (PEBP-1) & & & 10.23 & & \\
\hline & K.NRPTS*ISWDGLDSGK.L & 1712.76392 & 2 & 4.63 & 52 Swiss/ New in phos & No \\
\hline \multirow[t]{2}{*}{ Q9UQB8 } & BAIP2_HUMAN Brain-specific angiogenesis inhibitor 1-associated prote & & & 10.22 & & \\
\hline & K.LSDSYSNT*LPVR.K & 1431.65152 & 2 & 3.93 & 340 New in Swiss/ 340 phos & Yes \\
\hline \multirow[t]{2}{*}{$\mathrm{O} 15530$} & PDPK1_HUMAN 3-phosphoinositide-dependent protein kinase 1 (hPDK1 & & & 10.31 & & \\
\hline & R.ANS*FVGTAQYVSPELLTEK.S & 2134.01036 & 2 & 6.10 & 241 Swiss/ 241 phos & Yes \\
\hline \multirow[t]{2}{*}{ Q9H788 } & SH24A_HUMAN SH2 domain-containing protein 4A (Protein SH(2)A) & & & 10.21 & & \\
\hline & R.TLS*SSAQEDIIR.W & 1399.64644 & 2 & 4.13 & 315 Swiss/ 315 phos & Yes \\
\hline \multirow[t]{4}{*}{ P18615 } & NELFE_HUMAN Negative elongation factor E (NELF-E) (RD protein) & & & 10.18 & & \\
\hline & R.SIS*ADDDLQESSR.R & 1502.60061 & 2 & 3.56 & 115 Swiss/ 115 phos & Yes \\
\hline & K.QPMLDAAT*GKSVWGS*LAVQNS*PKGCHR.D & 3078.31268 & 3 & 3.44 & $\begin{array}{l}340 \text { NEW \& } 347 \text { NEW \& } 353 \\
\text { Swiss/ } 353 \text { phos }\end{array}$ & No \& No \& Yes \\
\hline & R.S*LSEQPVMDTATATEQAK.Q & 1986.87255 & 2 & 3.56 & 49 NEW & No \\
\hline \multirow[t]{4}{*}{ Q9H1B7 } & CN004_HUMAN Protein C14orf4 & & & 20.18 & & \\
\hline & R.KAS*PEPPDSAEGALK.L & 1576.72541 & 2 & 3.57 & 547 Swiss/ 547 phos & Yes \\
\hline & R.RNS*SS*PVSPASVPGQR.R & 1785.76801 & 2 & 3.32 & $\begin{array}{l}657 \text { Swiss/ } 657 \text { phos \& } 659 \text { Swiss/ } \\
659 \text { phos }\end{array}$ & Yes \& Yes \\
\hline & R.QSPNSSSAAAS*VAS*RR.G & 1735.71597 & 2 & 2.23 & $\begin{array}{l}224 \text { NEW \& } 227 \text { Swiss/ New in } \\
\text { phos }\end{array}$ & No \& No \\
\hline \multirow[t]{3}{*}{ Q12857 } & NFIA_HUMAN Nuclear factor 1 A-type (Nuclear factor 1/A) & & & 20.24 & & \\
\hline & R.S*PGSGSQSSGWHEVEPGMPSPTTLK.K & 2620.13849 & 3 & 4.79 & 300 Swiss/ 300 phos & Yes \\
\hline & R.RSLPSTS*ST*SSTKR.L & 1654.71966 & 2 & 2.49 & 273 NEW \& 275 NEW & No \& No \\
\hline \multirow[t]{2}{*}{ Q5UE93 } & PI3R6_HUMAN Phosphoinositide 3-kinase regulatory subunit 6 & & & 10.16 & & \\
\hline & R.PS*GDGEM\#LPGVSRLHTAR.V & 1975.90551 & 2 & 3.21 & $401 \mathrm{NEW}$ & No \\
\hline
\end{tabular}


Table A-1 (continued).

\begin{tabular}{|c|c|c|c|c|c|c|}
\hline $\begin{array}{l}\text { Accession } \\
\text { number }\end{array}$ & Peptide & MH+ & $\overline{\mathbf{z}}$ & $\mathbf{X C}$ & $\begin{array}{l}\text { Site \# in Swiss/Phosphosite } \\
(06 / 17 / 08)\end{array}$ & $\begin{array}{l}\text { Present in Phosphosite } \\
(06 / 17 / 08)\end{array}$ \\
\hline \multirow[t]{5}{*}{$\mathrm{P} 21675$} & TAF1_HUMAN Transcription initiation factor TFIID subunit 1 & & & 30.15 & & \\
\hline & R.M\#LLAAGS*AASGNNHRDDDTASVTSLNS*SATGR.C & 3323.39946 & 3 & 3.04 & 1138 NEW \& 1158 NEW & No \& No \\
\hline & R.TDPMVTLSSILES*IINDM\#R.D & 2231.03347 & 2 & 2.21 & $1391 \mathrm{NEW}$ & No \\
\hline & R.VGT*TVHCDYLNR.P & 1457.62426 & 2 & 2.17 & & \\
\hline & R.VGTTVHCDY*LNRPHK.S & 1819.83090 & 2 & 2.89 & $1366 \mathrm{NEW}$ & No \\
\hline \multirow[t]{2}{*}{ Q8N1N0 } & CLC4F_HUMAN C-type lectin domain family 4 member F & & & 10.13 & & \\
\hline & K.SSFDNT*S*AEIQFLR.G & 1774.70843 & 2 & 2.70 & 313 NEW \& 314 NEW & No \& No \\
\hline \multirow[t]{5}{*}{ Q04726 } & TLE3_HUMAN Transducin-like enhancer protein 3 (ESG3) & & & 20.18 & & \\
\hline & R.ESSANNSVS*PSESLR.A & 1643.69082 & 2 & 3.65 & 203 Swiss/ 203 phos & Yes \\
\hline & K.DLGHNDKS*STPGLKSNTPT*PR.N & 2382.04860 & 2 & 2.32 & $310 \mathrm{NEW}$ & No \\
\hline & K.SDDLVVDVSNEDPAT*PRVSPAHS*PPENGLDK.A & 3417.48827 & 3 & 3.78 & $\begin{array}{l}259 \text { Swiss/ } 259 \text { New in phos \& } \\
267 \text { Swiss/ } 267 \text { phos }\end{array}$ & No \& Yes \\
\hline & R.VS*PAHS*PPENGLDK.A & 1607.65018 & 2 & 2.57 & $\begin{array}{l}263 \text { Swiss/ } 263 \text { phos \& } 267 \\
\text { Swiss/ } 267 \text { phos }\end{array}$ & Yes \& Yes \\
\hline \multirow[t]{2}{*}{ Q5VV41 } & ARHGG_HUMAN Rho guanine nucleotide exchange factor 16 & & & 10.17 & & \\
\hline & R.HQS*FGAAVLSR.E & 1252.58338 & 2 & 3.25 & $107 \mathrm{NEW}$ & No \\
\hline \multirow[t]{2}{*}{ Q12906 } & ILF3_HUMAN Interleukin enhancer-binding factor 3 & & & 10.16 & & \\
\hline & K.RPMEEDGEEKS*PSK.K & 1698.70403 & 3 & 3.13 & 382 Swiss/ 382 phos & Yes \\
\hline \multirow[t]{2}{*}{ Q96IT1 } & ZN496_HUMAN Zinc finger protein 496 & & & 10.16 & & \\
\hline & R.PPS*QLS*GDPVLQDAFLLQEENVR.D & 2712.23171 & 2 & 2.93 & $\begin{array}{l}185 \text { Swiss/ New in phos \& } 188 \\
\text { NEW }\end{array}$ & No \& No \\
\hline \multirow[t]{2}{*}{ Q9UN86 } & G3BP2_HUMAN Ras GTPase-activating protein-binding protein 2 (G3BP-2) & & & 10.17 & & \\
\hline & K.STT*PPPAEPVSLPQEPPK.A & 1951.94122 & 2 & 3.33 & 227 Swiss/ 227 phos & Yes \\
\hline \multirow[t]{2}{*}{ P55317 } & HNF3A_HUMAN Hepatocyte nuclear factor 3-alpha (HNF-3A) & & & 10.17 & & \\
\hline & K.TGQLEGAPAPGPAAS*PQTLDHSGATATGGASELK.T & 3225.50593 & 3 & 3.46 & $332 \mathrm{NEW}$ & No \\
\hline \multirow[t]{2}{*}{ P35579 } & MYH9_HUMAN Myosin-9 (Myosin heavy chain 9) & & & 10.17 & & \\
\hline & K.KMEDS*VGCLET*AEEVK.R & 1927.74653 & 2 & 3.31 & 1376 NEW \& 1382 NEW & No \& No \\
\hline
\end{tabular}


Table A-1 (continued).

\begin{tabular}{|c|c|c|c|c|c|c|}
\hline $\begin{array}{l}\text { Accession } \\
\text { number }\end{array}$ & Peptide & MH+ & $\mathbf{z}$ & $\mathbf{X C}$ & $\begin{array}{l}\text { Site \# in Swiss/Phosphosite } \\
(06 / 17 / 08)\end{array}$ & $\begin{array}{l}\text { Present in Phosphosite } \\
(06 / 17 / 08)\end{array}$ \\
\hline \multirow[t]{3}{*}{$\mathrm{P} 08621$} & \multicolumn{3}{|l|}{ RU17_HUMAN U1 small nuclear ribonucleoprotein $70 \mathrm{kDa}$ (U1 snRNP $70 \mathrm{kDa})$} & 10.18 & & \\
\hline & R.YDERPGPS*PLPHR.D & 1600.72675 & 3 & 3.68 & 226 Swiss/ 226 phos & Yes \\
\hline & R.GGGGGQDNGLEGLGNDS*R.D & 1739.69803 & 2 & 3.35 & 410 Swiss/ 410 phos & Yes \\
\hline \multirow[t]{3}{*}{ Q13315 } & \multicolumn{3}{|l|}{ ATM_HUMAN Serine-protein kinase ATM (Ataxia telangiectasia mutated) } & 20.16 & & \\
\hline & K.RSLES*VYS*LYPTLSR.L & 1930.87107 & 2 & 3.21 & 2165 NEW \& 2168 NEW & No \& No \\
\hline & K.M\#LQPIT*R.L & 954.44778 & 2 & 2.01 & $2031 \mathrm{NEW}$ & No \\
\hline \multirow[t]{3}{*}{ Q9UKM9 } & \multicolumn{3}{|l|}{ RALY_HUMAN RNA-binding protein Raly } & \multicolumn{2}{|l|}{10.13} & \\
\hline & R.GRLS*PVPVPR.A & 1157.61904 & 2 & 2.68 & 135 Swiss/ 135 phos & Yes \\
\hline & R.AAS*AIYSGYIFDYDYYR.D & 2117.88918 & 2 & 5.64 & $206 \mathrm{NEW}$ & No \\
\hline \multirow[t]{2}{*}{ Q9BSG1 } & \multicolumn{3}{|l|}{ ZNF2_HUMAN Zinc finger protein 2 (Zinc finger 2.2) (Zinc finger protein 661) } & \multicolumn{2}{|l|}{10.15} & \\
\hline & K.VFSSKS*SVIQHQR.R & 1582.77370 & 2 & 2.67 & $410 \mathrm{NEW}$ & No \\
\hline \multirow[t]{2}{*}{ Q6ZMT1 } & \multicolumn{3}{|l|}{ STAC2_HUMAN SH3 and cysteine-rich domain-containing protein 2} & \multicolumn{2}{|l|}{10.19} & \\
\hline & R.SSFSS $* T * S E S P T R S L S E R . D$ & 2004.79468 & 2 & 3.71 & 224 NEW \& 225 NEW & No \& No \\
\hline \multirow[t]{3}{*}{ P85037 } & \multicolumn{3}{|l|}{ FOXK1_HUMAN Forkhead box protein K1 (Myocyte nuclear factor) } & \multicolumn{2}{|l|}{20.14} & \\
\hline & R.S*APAS*PTHPGLMSPR.S & 1665.68552 & 2 & 2.77 & $\begin{array}{l}416 \text { Swiss/ } 416 \text { phos } \& 420 \\
\text { Swiss/ } 420 \text { phos }\end{array}$ & Yes \& Yes \\
\hline & R.EGS*PIPHDPEFGSK.L & 1576.66790 & 2 & 2.13 & 445 Swiss/ 445 phos & \\
\hline \multirow[t]{2}{*}{ O15062 } & \multicolumn{3}{|l|}{ ZBTB5_HUMAN Zinc finger and BTB domain-containing protein 5} & \multicolumn{2}{|l|}{10.13} & \\
\hline & K.HYLTTRTLPMS*PPSER.V & 1965.92519 & 2 & 2.59 & $127 \mathrm{NEW}$ & $\mathrm{No} \backslash$ \\
\hline \multirow[t]{2}{*}{ Q9H2M9 } & \multicolumn{3}{|l|}{ RBGPR_HUMAN Rab3 GTPase-activating protein non-catalytic subunit } & \multicolumn{2}{|l|}{10.16} & \\
\hline & K.QDFS*PEVLKLANEER.D & 1854.86330 & 2 & 3.28 & $952 \mathrm{NEW}$ & No \\
\hline \multirow[t]{5}{*}{ P22626 } & \multicolumn{3}{|c|}{ ROA2_HUMAN Heterogeneous nuclear ribonucleoproteins A2/B1 (hnRNP A2 / hnRNP B1) } & 30.33 & & \\
\hline & R.GFGDGYNGYGGGPGGGNFGGS*PGYGGGR.G & 2575.00584 & 3 & 6.53 & 259 Swiss/ 259 pho & Yes \\
\hline & R.NMGGPYGGGNYGPGGSGGS*GGYGGR.S & 2269.87165 & 2 & 6.11 & see line 693 & \\
\hline & R.GGNFGFGDS*R.G & 1093.40983 & 2 & 3.41 & 212 Swiss/ 212 phos & Yes \\
\hline & R.NMGGPYGGGNYGPGGS*GGSGGYGGR.S & 2269.87165 & 2 & 3.97 & 341 Swiss/ 341 phos & Yes \\
\hline
\end{tabular}


Table A-1 (continued).

\begin{tabular}{|c|c|c|c|c|c|c|}
\hline $\begin{array}{l}\text { Accession } \\
\text { number }\end{array}$ & Peptide & MH+ & $\overline{\mathbf{z}}$ & $\mathbf{X C}$ & $\begin{array}{l}\text { Site \# in Swiss/Phosphosite } \\
(06 / 17 / 08)\end{array}$ & $\begin{array}{l}\text { Present in Phosphosite } \\
(06 / 17 / 08)\end{array}$ \\
\hline \multirow[t]{4}{*}{ Q9UHD8 } & SEPT9_HUMAN Septin-9 (MLL septin-like fusion protein) & & & 10.29 & & \\
\hline & R.S*FEVEEVETPNSTPPR.R & 1897.82150 & 2 & 5.71 & 30 Swiss/ 30 phos & Yes \\
\hline & K.RS*FEVEEVETPNSTPPR.R & 2053.92261 & 2 & 3.64 & see line 698 & \\
\hline & R.S*FEVEEVET*PNSTPPR.R & 1977.78780 & 2 & 2.43 & 38 NEW \& 30 Swiss/ 30 phos & No \& Yes \\
\hline \multirow[t]{4}{*}{ Q8TEA8 } & DTD1_HUMAN Probable D-tyrosyl-tRNA(Tyr) deacylase 1 & & & 30.18 & & \\
\hline & R.S*ASSGAEGDVSSEREP & 1644.63845 & 2 & 3.65 & 194 NEW & No \\
\hline & K.EDRS*ASSGAEGDVS*S*EREP & 2204.74170 & 2 & 2.43 & $\begin{array}{l}194 \text { NEW \& } 204 \text { Swiss/ } 204 \\
\text { phos \& } 205 \text { Swiss/ } 205 \text { phos }\end{array}$ & No \& Yes \& Yes \\
\hline & K.EDRSAS*SGAEGDVS*S*ER.E & 1978.64634 & 2 & 2.37 & $\begin{array}{l}196 \text { Swiss/ } 196 \text { phos \& see line } \\
703 \text { for } 204 \& 205\end{array}$ & Yes \& Yes \& Yes \\
\hline \multirow[t]{2}{*}{ Q5JSZ5 } & K0515_HUMAN Uncharacterized protein KIAA0515 & & & 10.22 & & \\
\hline & K.LKFS*DDEEEEEVVK.D & 1775.76225 & 2 & 4.37 & 388 Swiss/ 388 phos & Yes \\
\hline \multirow[t]{2}{*}{ P25205 } & MCM3_HUMAN DNA replication licensing factor MCM3 & & & 10.18 & & \\
\hline & K.DGDSYDPYDFS*DTEEEMPQVHTPK.T & 2882.10223 & 3 & 3.58 & 711 Swiss/ 711 phos & Yes \\
\hline \multirow[t]{2}{*}{ O95248 } & MTMR5_HUMAN SET-binding factor 1 (Sbf1) (Myotubular & n 5) & & 10.16 & & \\
\hline & R.RTTVPSGPPMT*AILER.C & 1805.89792 & 2 & 3.18 & $546 \mathrm{NEW}$ & No \\
\hline \multirow[t]{5}{*}{ Q6P6C2 } & ALKB5_HUMAN Alkylated repair protein alkB homolog 5 & & & 10.14 & & \\
\hline & R.RGS*FSSENYWR.K & 1468.60049 & 2 & 2.75 & 361 Swiss/ 361 phos & Yes \\
\hline & K.YQEDS*DPERSDYEEQQLQK.E & 2466.99327 & 3 & 3.45 & 64 New in Swiss/ 64 phos & Yes \\
\hline & R.GS*FSSENYWR.K & 1312.49938 & 2 & 2.31 & see line 712 & \\
\hline & R.LET*KS*LSSSVLPPSYASDRLSGNNR.D & 2838.30700 & 3 & 3.15 & 294 NEW \& 296 NEW & Yes \& Yes \\
\hline \multirow[t]{2}{*}{ Q9NX63 } & CHCH3_HUMAN Coiled-coil-helix-coiled-coil-helix domain & tein 3 & & 10.26 & & \\
\hline & R.YS*GAYGASVSDEELK.R & 1655.68361 & 2 & 5.21 & 50 Swiss/ 50 phos & Yes \\
\hline \multirow[t]{2}{*}{ P21796 } & VDAC1_HUMAN Voltage-dependent anion-selective channe & $\mathrm{AC}-1)$ & & 10.20 & & \\
\hline & K.LTFDSSFS*PNTGK.K & 1480.63554 & 2 & 3.97 & 104 Swiss/ 103 pho & Yes (Same AA, diff \#) \\
\hline \multirow[t]{2}{*}{ Q76KP1 } & B4GN4_HUMAN N-acetyl-beta-glucosaminyl-glycoprotein 4 & alactosam & & 10.14 & & \\
\hline & R.TLGPAAPTVDS*NLS*SEAR.P & 1945.83033 & 2 & 2.81 & 616 NEW \& 619 NEW & No \& No \\
\hline
\end{tabular}


Table A-1 (continued).

\begin{tabular}{|c|c|c|c|c|c|c|}
\hline $\begin{array}{l}\text { Accession } \\
\text { number }\end{array}$ & Peptide & MH+ & $\overline{\mathbf{z}}$ & $\mathbf{X C}$ & $\begin{array}{l}\text { Site \# in Swiss/Phosphosite } \\
(06 / 17 / 08)\end{array}$ & $\begin{array}{l}\text { Present in Phosphosite } \\
(06 / 17 / 08)\end{array}$ \\
\hline \multirow[t]{2}{*}{ Q13573 } & SNW1_HUMAN SNW domain-containing protein 1 (Nuclear protein SkiP) & & & 10.20 & & \\
\hline & R.GPPS*PPAPVMHS*PSR.K & 1673.69061 & 2 & 3.62 & $\begin{array}{l}224 \text { Swiss/ } 224 \text { phos } \& 232 \\
\text { Swiss/ } 232 \text { phos }\end{array}$ & Yes \& Yes \\
\hline \multirow[t]{2}{*}{ Q9NPH0 } & PPA6_HUMAN Lysophosphatidic acid phosphatase type 6 precursor & & & 10.13 & & \\
\hline & R.GRRQTAS*LQPGIS*EDLK.K & 2015.93105 & 2 & 2.42 & 223 NEW \& 229 NEW & No \& No \\
\hline \multirow[t]{2}{*}{ Q6WKZ4 } & RFIP1_HUMAN Rab11 family-interacting protein 1 (Rab11-FIP1) & & & 10.27 & & \\
\hline & K.HLFSS*TENLAAGSWK.E & 1727.77885 & 2 & 5.33 & 357 Swiss/ New in phos & No \\
\hline \multirow[t]{3}{*}{ P23193 } & TCEA1_HUMAN Transcription elongation factor A protein 1 & & & 10.27 & & \\
\hline & K.EPAITSQNS*PEAR.E & 1479.64750 & 2 & 2.81 & 100 Swiss/ 100 phos & Yes \\
\hline & K.KKEPAITSQNS*PEAR.E & 1735.83742 & 3 & 3.12 & see line 729 & \\
\hline \multirow[t]{2}{*}{ O75446 } & SAP30_HUMAN Histone deacetylase complex subunit SAP30 & & & 10.15 & & \\
\hline & K.DTLTYFIY*SVKNDK.N & 1786.82988 & 2 & 3.06 & 202 NEW & No \\
\hline \multirow[t]{2}{*}{ Q07666 } & SAM68_HUMAN KH domain-containing, RNA-binding, signal transduction & ciated protein 1 & & 10.16 & & \\
\hline & R.SGS*MDPSGAHPSVR.Q & 1464.59369 & 2 & 3.21 & 20 Swiss/ 20 phos & Yes \\
\hline \multirow[t]{2}{*}{ Q9UHR4 } & BI2L1_HUMAN Brain-specific angiogenesis inhibitor 1-associated protein 2 & protein 1 & & 10.21 & & \\
\hline & K.TPASTPVSGT*PQAS*PMIER.S & 2086.89155 & 2 & 4.27 & $\begin{array}{l}257 \text { Swis/ } 257 \text { phos \& } 261 \text { Swiss/ } \\
261 \text { phos }\end{array}$ & Yes \& Yes \\
\hline \multirow[t]{3}{*}{ P09651 } & ROA1_HUMAN Heterogeneous nuclear ribonucleoprotein A1 & & & 10.32 & & \\
\hline & K.S*ESPKEPEQLR.K & 1379.62022 & 2 & 2.54 & 4 Swiss/ 4 phos & Yes \\
\hline & R.SS*GPYGGGGQYFAK.P & 1455.59401 & 2 & 2.23 & 338 Swiss/ 338 phos & Yes \\
\hline \multirow[t]{3}{*}{ Q9Y446 } & PKP3_HUMAN Plakophilin-3 & & & 10.21 & & \\
\hline & R.LS*SGFDDIDLPSAVK.Y & 1643.75638 & 2 & 4.14 & 313 Swiss/ New in phos & No \\
\hline & R.ADYDTLS*LR.S & 1133.48742 & 2 & 2.91 & 180 New in Swiss/ 180 phos & Yes \\
\hline \multirow[t]{2}{*}{ Q96T21 } & SEBP2_HUMAN SECIS-binding protein 2 & & & 10.15 & & \\
\hline & K.S*ARGSHHLSIYAENSLK.S & 1949.92288 & 2 & 2.43 & 174 NEW & No \\
\hline \multirow[t]{2}{*}{ P52948 } & NUP98_HUMAN Nuclear pore complex protein Nup98-Nup96 precursor & & & 10.17 & & \\
\hline & R.DSENLAS*PSEYPENGER.F & 1973.77601 & 2 & 3.38 & 623 Swiss/ 623 phos & Yes \\
\hline
\end{tabular}


Table A-1 (continued).

\begin{tabular}{|c|c|c|c|c|c|c|}
\hline $\begin{array}{l}\text { Accession } \\
\text { number }\end{array}$ & Peptide & MH+ & $\overline{\mathbf{z}}$ & $\mathbf{X C}$ & $\begin{array}{l}\text { Site \# in Swiss/Phosphosite } \\
(06 / 17 / 08)\end{array}$ & $\begin{array}{l}\text { Present in Phosphosite } \\
(06 / 17 / 08)\end{array}$ \\
\hline \multirow[t]{3}{*}{ P35527 } & K1C9_HUMAN Keratin, type I cytoskeletal 9 (Cytokeratin-9) & & & 20.18 & & \\
\hline & R.GSRGGSGGSY*GGGGS*GGGYGGGSGS*R.G & 2331.78121 & 2 & 3.63 & 497 NEW \& 502 NEW \& 512 NEW & No \& No \& No \\
\hline & R.GSRGGSGGS*Y*GGGGSGGGYGGGS*GSR.G & 2331.78121 & 2 & 2.82 & 496 NEW \& 497 NEW \& 510 NEW & No \& No \& No \\
\hline \multirow[t]{4}{*}{ P51991 } & ROA3_HUMAN Heterogeneous nuclear ribonucleoprotein A3 ( $\mathrm{hnRN}$ & A3) & & 20.29 & & \\
\hline & R.SSGS*PYGGGYGSGGGSGGYGSR.R & 1990.75627 & 2 & 5.90 & 358 Swiss/ 358 phos & Yes \\
\hline & R.SSGSPY*GGGYGS*GGGS*GGYGSR.R & 2150.68887 & 2 & 2.04 & $\begin{array}{l}360 \text { Swiss/ } 360 \text { phos \& } 366 \text { NEW \& } \\
370 \text { Swiss/ } 370 \text { phos }\end{array}$ & Yes \& No \& Yes \\
\hline & R.SS*GSPY*GGGYGSGGGS*GGYGSRRF & 2453.85840 & 3 & 3.00 & $\begin{array}{l}356 \text { Swiss } / 356 \text { phos \& see line } 758 \\
\text { for } 360 \text { and } 370\end{array}$ & Yes \& Yes \& Yes \\
\hline \multirow[t]{2}{*}{ Q6P5R6 } & RL22L_HUMAN Ribosomal protein L22-like 1 & & & 10.19 & & \\
\hline & R.YFQISQDEDES*ESED & 1900.66439 & 2 & 3.83 & 118 Swiss/ 118 phos & Yes \\
\hline \multirow[t]{4}{*}{ Q96T37 } & RBM15_HUMAN Putative RNA-binding protein 15 (RNA-binding m & tif protein 15$)$ & & 20.21 & & \\
\hline & R.SLS*PGGAALGYR.D & 1228.57215 & 2 & 2.74 & 294 Swiss/ 294 phos & Yes \\
\hline & K.NS*S*GGGESRSSSRGGGGES*R.S & 2107.72263 & 2 & 2.34 & 134 NEW \& 135 NEW \& 151 NEW & No \& No \& No \\
\hline & R.SRS*PLDKDTY*PPSASVVGASVGGHR.H & 2700.21779 & 3 & 4.27 & $\begin{array}{l}259 \text { New in Swiss/ } 259 \text { phos \& } 266 \\
\text { NEW }\end{array}$ & Yes \& No \\
\hline \multirow[t]{2}{*}{ Q9UQF2 } & JIP1_HUMAN C-jun-amino-terminal kinase-interacting protein 1 & & & 10.14 & & \\
\hline & K.QFVEYT*CPTEDIYLE & 1929.78636 & 2 & 2.89 & $702 \mathrm{NEW}$ & No \\
\hline \multirow[t]{2}{*}{ O96013 } & PAK4_HUMAN Serine/threonine-protein kinase PAK 4 (p21-activate & kinase 4) & & 10.14 & & \\
\hline & R.PFNT*YPR.A & 974.41313 & 2 & 2.17 & 207 Swiss/ New in phos & No \\
\hline \multirow[t]{2}{*}{ Q15233 } & NONO_HUMAN Non-POU domain-containing octamer-binding prot & n (NonO prote & & 10.24 & & \\
\hline & R.FGQAATMEGIGAIGGT*PPAFNR.A & 2243.03145 & 3 & 3.66 & 450 Swiss/ 450 phos & Yes \\
\hline \multirow[t]{2}{*}{ Q96PK6 } & RBM14_HUMAN RNA-binding protein 14 (RNA-binding motif prote & n 14) & & 10.13 & & \\
\hline & R.RLS*ESQLSFR.R & 1302.62016 & 2 & 2.27 & 618 Swiss/ 618 phos & Yes \\
\hline \multirow[t]{2}{*}{ P21291 } & CSRP1_HUMAN Cysteine and glycine-rich protein 1 (Cysteine-rich $\mathrm{p}$ & otein 1) & & 10.16 & & \\
\hline & K.GFGFGQGAGALVHS*E & 1513.64710 & 2 & 2.84 & 192 Swiss/ 191 phos & Yes (Same AA, diff \#) \\
\hline \multirow[t]{2}{*}{ Q9P2K5 } & MYEF2_HUMAN Myelin expression factor 2 (MyEF-2) (MST156) & & & 10.18 & & \\
\hline & K.AEVPGATGGDS*PHLQPAEPPGEPR.R & 2446.10343 & 3 & 3.66 & 17 Swiss/ 17 phos & Yes \\
\hline
\end{tabular}


Table A-1 (continued).

\begin{tabular}{|c|c|c|c|c|c|c|}
\hline $\begin{array}{l}\text { Accession } \\
\text { number }\end{array}$ & Peptide & MH+ & $\overline{\mathbf{z}}$ & $\mathbf{X C}$ & $\begin{array}{l}\text { Site \# in Swiss/Phosphosite } \\
(06 / 17 / 08)\end{array}$ & $\begin{array}{l}\text { Present in Phosphosite } \\
(06 / 17 / 08)\end{array}$ \\
\hline \multirow[t]{2}{*}{ Q96J84 } & KIRR1_HUMAN Kin of IRRE-like protein 1 precursor & & & 10.15 & & \\
\hline & R.FSQEPADQT*VVAGQR.A & 1712.76392 & 2 & 2.98 & $31 \mathrm{NEW}$ & No \\
\hline \multirow[t]{2}{*}{ Q9UHL0 } & DDX25_HUMAN ATP-dependent RNA helicase DDX25 (DEAD box & $\mathrm{x}$ protein 25$)$ & & 10.18 & & \\
\hline & K.WLTVEMIQDGHQVS*LLS*GELTVEQR.A & 3028.38862 & 3 & 3.50 & 366 NEW \& 369 NEW & No \& No \\
\hline \multirow[t]{2}{*}{ Q5T8P6 } & RBM26_HUMAN RNA-binding protein 26 (RNA-binding motif prote & ein 26) & & 10.13 & & \\
\hline & K.T*QMQKELLDT*ELDLYKK.M & 2256.02698 & 2 & 2.67 & 808 NEW \& 817 NEW & No \& No \\
\hline \multirow[t]{2}{*}{ Q9Y3Q4 } & $\begin{array}{l}\text { HCN4_HUMAN Potassium/sodium hyperpolarization-activated cyclic } \\
\text { channel } 4\end{array}$ & c nucleotide-g & & 10.12 & & \\
\hline & R.RGTPPLT*PGR.L & 1131.56700 & 2 & 2.47 & $1075 \mathrm{NEW}$ & No \\
\hline \multirow[t]{2}{*}{ Q8TEW8 } & PAR3L_HUMAN Partitioning-defective 3 homolog B (PAR3-beta) & & & 10.20 & & \\
\hline & R.T*QEELVAMLRST*K.Q & 1665.73180 & 2 & 4.02 & $445 \mathrm{NEW}$ & No \\
\hline \multirow[t]{2}{*}{ O60825 } & F262_HUMAN 6-phosphofructo-2-kinase/fructose-2,6-biphosphatase & & & 10.16 & & \\
\hline & R.RNS*FTPLSSSNTIR.R & 1659.78499 & 2 & 3.16 & 446 Swiss/ 446 phos & Yes \\
\hline \multirow[t]{2}{*}{ Q96JC9 } & EAF1_HUMAN ELL-associated factor 1 & & & 10.16 & & \\
\hline & K.T*SPLKDNPS*PEPQLDDIKR.E & 2310.04139 & 2 & 3.15 & $\begin{array}{l}158 \text { Swiss/ } 158 \text { phos \& } 165 \text { Swiss/ } \\
165 \text { phos }\end{array}$ & Yes \& Yes \\
\hline \multirow[t]{2}{*}{ O43583 } & DENR_HUMAN Density-regulated protein (DRP) (Protein DRP1) & & & 20.30 & & \\
\hline & K.LTVENS*PKQEAGISEGQGTAGEEEEK.K & 2797.24110 & 3 & 5.95 & 73 Swiss/ 73 phos & Yes \\
\hline \multirow[t]{2}{*}{ Q9C0C2 } & TB182_HUMAN $182 \mathrm{kDa}$ tankyrase 1-binding protein & & & 40.21 & & \\
\hline & R.SPS*QDFSFIEDTEILDSAMYR.S & 2531.06835 & 2 & 4.20 & 1554 Swiss/ 1554 phos & Yes \\
\hline \multirow[t]{4}{*}{ O14745 } & NHERF_HUMAN Ezrin-radixin-moesin-binding phosphoprotein 50( & (EBP50) & & 30.33 & & \\
\hline & R.S*ASSDTSEELNSQDSPPK.Q & 1958.78624 & 2 & 6.59 & 288 Swiss/ 287 phos & Yes (Same AA, diff \#) \\
\hline & R.SAS*SDTSEELNSQDSPPK.Q & 1958.78624 & 2 & 6.11 & 290 Swiss/ 289 phos & Yes (Same AA, diff \#) \\
\hline & R.EALAEAALES*PRPALVR.S & 1872.95787 & 2 & 2.72 & 280 Swiss/ 279 phos & Yes (Same AA, diff \#) \\
\hline \multirow[t]{2}{*}{ P42858 } & HD_HUMAN Huntingtin (Huntington disease protein) (HD protein) & & & 10.16 & & \\
\hline & R.VNHCLTICENIVAQS*VR.N & 1978.92381 & 2 & 3.27 & $116 \mathrm{NEW}$ & No \\
\hline
\end{tabular}


Table A-1 (continued).

\begin{tabular}{|c|c|c|c|c|c|c|}
\hline $\begin{array}{l}\text { Accession } \\
\text { number }\end{array}$ & Peptide & MH+ & $\overline{\mathbf{z}}$ & $\mathbf{X C}$ & $\begin{array}{l}\text { Site \# in Swiss/Phosphosite } \\
(06 / 17 / 08)\end{array}$ & $\begin{array}{l}\text { Present in Phosphosite } \\
(06 / 17 / 08)\end{array}$ \\
\hline \multirow[t]{2}{*}{ Q9Y266 } & NUDC_HUMAN Nuclear migration protein nudC & & & 20.23 & & \\
\hline & K.NGSLDS*PGKQDTEEDEEEDEKDK.G & 2674.05230 & 3 & 4.56 & 139 Swiss/ 139 phos & Yes \\
\hline \multirow[t]{2}{*}{ O00264 } & PGRC1_HUMAN Membrane-associated progesterone receptor comp & onent 1 (mPR) & & 40.17 & & \\
\hline & K.EGEEPTVYS*DEEEPK.D & 1817.70005 & 2 & 2.75 & 181 Swiss/ 180 phos & Yes (Same AA, diff \#) \\
\hline \multirow[t]{2}{*}{ Q9BVG4 } & CX026_HUMAN UPF0368 protein Cxorf26 & & & 10.16 & & \\
\hline & K.GADS*GEEKEEGINR.E & 1570.63806 & 2 & 3.20 & 197 NEW & No \\
\hline \multirow[t]{2}{*}{ Q13286 } & CLN3_HUMAN Battenin (Protein CLN3) (Batten disease protein) & & & 10.14 & & \\
\hline & R.RFS*DS*EGEET*VPEPR.L & 1974.69183 & 2 & 2.88 & $\begin{array}{l}12 \text { Swiss/ } 12 \text { phos \& } 14 \text { Swiss/ } 14 \\
\text { phos \& } 19 \text { NEW }\end{array}$ & Yes \& Yes \& No \\
\hline \multirow[t]{3}{*}{ Q5JTV8 } & TOIP1_HUMAN Torsin-1A-interacting protein 1 & & & 30.23 & & \\
\hline & R.LQQQHSEQPPLQPS*PVMTR.R & 2281.07946 & 3 & 3.90 & 143 Swiss/ 143 New in phos & No \\
\hline & K.VNFSEEGET*EEDDQDSSHSSVTTVK.A & 2836.13161 & 3 & 3.60 & 220 Swiss/ 220 New in phos & No \\
\hline \multirow[t]{2}{*}{ Q13501 } & SQSTM_HUMAN Sequestosome-1 & & & 10.15 & & \\
\hline & R.LTPVS*PESSSTEEK.S & 1570.68836 & 2 & 2.56 & 272 Swiss/ 272 phos & Yes \\
\hline \multirow[t]{3}{*}{ P20700 } & LMNB1_HUMAN Lamin-B1 & & & 30.30 & & \\
\hline & R.LKLS*PS*PSSR.V & 1231.54828 & 2 & 3.11 & $\begin{array}{l}391 \text { Swiss/ } 390 \text { phos \& } 393 \text { Swiss/ } \\
392 \text { phos }\end{array}$ & Yes (Same AA, diff \#) \\
\hline & R.LKLS*PSPSSR.V & 1151.58198 & 2 & 2.63 & see line 810 & \\
\hline \multirow[t]{2}{*}{ Q8IXT5 } & RB12B_HUMAN RNA-binding protein 12B (RNA-binding motif pr & otein 12B) & & 20.18 & & \\
\hline & R.FPPEDFRHS*PEDFR.R & 1855.77991 & 3 & 3.55 & 575 Swiss/ 575 phos & Yes \\
\hline \multirow[t]{2}{*}{ O14578 } & CTRO_HUMAN Citron Rho-interacting kinase (CRIK) & & & 10.16 & & \\
\hline & R.TPLSQVNKVWDQS*S*V & 1847.79758 & 2 & 2.53 & 2025 NEW \& 2026 NEW & No \& No \\
\hline \multirow[t]{2}{*}{ Q8WWM7 } & ATX2L_HUMAN Ataxin-2-like protein (Ataxin-2 domain protein) & & & 10.17 & & \\
\hline & K.EVDGLLTSEPMGS*PVSSK.T & 1912.86092 & 2 & 3.27 & 594 Swiss/ 594 phos & Yes \\
\hline \multirow[t]{2}{*}{ P62995 } & TRA2B_HUMAN Arginine/serine-rich-splicing factor 10 (Transform & er-2-beta) & & 10.26 & & \\
\hline & K.RPHT*PTPGIYMGR.P & 1562.72973 & 3 & 3.52 & 201 Swiss/ 201 phos & Yes \\
\hline
\end{tabular}


Table A-1 (continued).

\begin{tabular}{|c|c|c|c|c|c|c|}
\hline $\begin{array}{l}\text { Accession } \\
\text { number }\end{array}$ & Peptide & MH+ & $\overline{\mathbf{z}}$ & $\mathbf{X C}$ & $\begin{array}{l}\text { Site \# in Swiss/Phosphosite } \\
(06 / 17 / 08)\end{array}$ & $\begin{array}{l}\text { Present in Phosphosite } \\
(06 / 17 / 08)\end{array}$ \\
\hline \multirow[t]{3}{*}{ Q14157 } & UBP2L_HUMAN Ubiquitin-associated protein 2-like (Protein NICE-4) & & & 30.22 & & \\
\hline & R.RYPSSISSS*PQK.D & 1416.65185 & 2 & 2.58 & 609 Swiss/ 609 phos & Yes \\
\hline & K.NPS*DSAVHSPFTK.R & 1466.63112 & 2 & 2.57 & $410 \mathrm{NEW}$ & No \\
\hline \multirow[t]{2}{*}{ P31749 } & AKT1_HUMAN RAC-alpha serine/threonine-protein kinase (RAC-PK-a & lpha) & & 20.18 & & \\
\hline & R.RPHFPQFS*YSASGTA & 1732.74788 & 2 & 2.83 & 473 Swiss/ 473 phos & Yes \\
\hline \multirow[t]{3}{*}{ O14646 } & CHD1_HUMAN Chromodomain-helicase-DNA-binding protein 1 & & & 20.17 & & \\
\hline & K.S*DSSPLPSEKSDEDDDK.L & 1930.74370 & 2 & 3.06 & 1353 Swiss/ 1353 phos & Yes \\
\hline & K.SDS*SPLPSEKSDEDDDK.L & 1930.74370 & 2 & 2.75 & 1355 Swiss/ 1355 phos & Yes \\
\hline \multirow[t]{2}{*}{ O00257 } & CBX4_HUMAN E3 SUMO-protein ligase CBX4 (Chromobox protein $\mathrm{h}$ & molog 4) & & 20.16 & & \\
\hline & R.EEEVSGVS*DPQPQDAGSR.K & 1966.80255 & 2 & 3.20 & 334 NEW & No \\
\hline \multirow[t]{2}{*}{ O14874 } & BCKD_HUMAN [3-methyl-2-oxobutanoate dehydrogenase [lipoamide] & kinase & & 20.15 & & \\
\hline & R.ARS*TSAT*DTHHVEMAR.E & 1929.76736 & 2 & 3.10 & $\begin{array}{l}31 \text { Swiss/ } 31 \text { phos } \& 35 \text { Swiss/ } 35 \\
\text { phos }\end{array}$ & Yes \& Yes \\
\hline \multirow[t]{2}{*}{ Q15056 } & IF4H_HUMAN Eukaryotic translation initiation factor 4H (eIF-4H) & & & 10.26 & & \\
\hline & R.AY*SS*FGGGR.G & 1061.34886 & 2 & 2.54 & 12 Swiss/ 11 phos \& 14 NEW & $\begin{array}{l}\text { Yes (Same AA, diff \#) \& } \\
\text { No }\end{array}$ \\
\hline \multirow[t]{2}{*}{ Q8NC51 } & PAIRB_HUMAN Plasminogen activator inhibitor 1 RNA-binding protei & & & 10.25 & & \\
\hline & K.SKS*EEAHAEDSVMDHHFR.K & 2191.88624 & 3 & 3.62 & 330 Swiss/ 330 phos & Yes \\
\hline \multirow[t]{2}{*}{ Q9Y478 } & AAKB1_HUMAN 5'-AMP-activated protein kinase subunit beta-1 & & & 10.24 & & \\
\hline & R.S*HNNFVAILDLPEGEHQYK.F & 2291.04921 & 3 & 4.80 & 108 Swiss/ 107 phos & Yes (Same AA, diff \#) \\
\hline \multirow[t]{2}{*}{ P43243 } & MATR3_HUMAN Matrin-3 & & & 10.23 & & \\
\hline & R.RDS*FDDR.G & 990.36763 & 2 & 2.57 & 188 Swiss/ 188 phos & Yes \\
\hline \multirow[t]{2}{*}{ P18669 } & PGAM1_HUMAN Phosphoglycerate mutase 1 (Phosphoglycerate mutas & isozyme B) & & 10.21 & & \\
\hline & R.HGES*AWNLENR.F & 1392.56919 & 2 & 3.91 & 11 Swiss/ 13 phos & Yes (Same AA, diff \#) \\
\hline \multirow[t]{2}{*}{ Q96AT1 } & K1143_HUMAN Uncharacterized protein KIAA1143 & & & 10.20 & & \\
\hline & R.IQPQPPDEDGDHS*DKEDEQPQVVVLK.K & 3022.36770 & 3 & 3.96 & 50 Swiss/ 50 phos & Yes \\
\hline
\end{tabular}


Table A-1 (continued).

\begin{tabular}{|c|c|c|c|c|c|c|}
\hline $\begin{array}{l}\text { Accession } \\
\text { number }\end{array}$ & Peptide & MH+ & $\mathbf{z}$ & $\mathbf{X C}$ & $\begin{array}{l}\text { Site \# in Swiss/Phosphosite } \\
(06 / 17 / 08)\end{array}$ & $\begin{array}{l}\text { Present in Phosphosite } \\
(06 / 17 / 08)\end{array}$ \\
\hline \multirow[t]{2}{*}{ P47736 } & RGP2_HUMAN Rap1 GTPase-activating protein 1 (Rap1GAP) & & & 10.18 & & \\
\hline & R.RSS*AIGIENIQEVQEK.R & 1880.91132 & 2 & 3.68 & $499 \mathrm{NEW}$ & No \\
\hline \multirow[t]{2}{*}{ Q9BUZ4 } & TRAF4_HUMAN TNF receptor-associated factor 4 & & & 10.17 & & \\
\hline & R.GS*LDESSLGFGYPK.F & 1536.66175 & 2 & 3.48 & $426 \mathrm{NEW}$ & No \\
\hline \multirow[t]{2}{*}{ Q96T58 } & MINT_HUMAN Msx2-interacting protein (SPEN homolog) & & & 10.17 & & \\
\hline & K.FGKVTSVQIHGTS*EER.Y & 1854.87454 & 2 & 2.66 & 374 NEW & No \\
\hline \multirow[t]{2}{*}{ Q5HYJ3 } & FA76B_HUMAN Protein FAM76B & & & 10.17 & & \\
\hline & K.ISNLS*PEEEQGLWK.Q & 1709.77818 & 2 & 2.64 & 193 Swiss/ 193 phos & Yes \\
\hline \multirow[t]{2}{*}{ Q13427 } & PPIG_HUMAN Peptidyl-prolyl cis-trans isomerase G & & & 10.17 & & \\
\hline & R.RS*ET*PPHWR.Q & 1325.51872 & 2 & 2.81 & $\begin{array}{l}356 \text { Swiss/ } 356 \text { phos \& } 358 \text { Swiss/ } \\
358 \text { phos }\end{array}$ & Yes \& Yes \\
\hline \multirow[t]{2}{*}{ Q9UDY2 } & ZO2_HUMAN Tight junction protein $\mathrm{ZO}-2$ (Zonula occludens 2 protein) & & & 10.16 & & \\
\hline & R.DNS*PPPAFKPEPPK.A & 1600.74067 & 2 & 3.20 & 986 Swiss/ 986 phos & Yes \\
\hline \multirow[t]{2}{*}{ Q9HC52 } & CBX8_HUMAN Chromobox protein homolog 8 (Polycomb 3 homolog) & & & 10.14 & & \\
\hline & R.HGS*GPPSSGGGLYR.D & 1408.60049 & 2 & 2.62 & 311 New in Swiss/ 311 phos & Yes \\
\hline
\end{tabular}


Table A-2 Phosphorylation sites assigned by product ions in MS/MS spectrum.

\begin{tabular}{|c|c|c|c|}
\hline $\begin{array}{l}\text { Accession } \\
\text { number }\end{array}$ & $\operatorname{Scan}(\mathbf{s})$ & Peptide & Phosphorylation sites \\
\hline \multirow[t]{5}{*}{ P07900 } & $\begin{array}{l}\text { HS90A_HUMAN Heat shock } \\
\text { protein HSP 90-alpha (HSP } \\
\text { 86) }\end{array}$ & & \\
\hline & & K.ESEDKPEIEDVGS*DEEEEK.K & $\begin{array}{l}\text { S263 confirmed by y } 6, \text { y } 7 \\
\text { S } 252 \text { not phosphorylated by b4, b5 }\end{array}$ \\
\hline & & K.ESEDKPEIEDVGS*DEEEEKK. & S263 confirmed by y 7, y9 \\
\hline & & & S252 not phosphorylated by b4, b6 \\
\hline & & R.DKEVS*DDEAEEK.E & S231 confirmed by y7, y8 \\
\hline \multirow[t]{6}{*}{ Q15019 } & $\begin{array}{l}\text { SEPT2_HUMAN Septin-2 } \\
\text { (Protein NEDD5) }\end{array}$ & & \\
\hline & & K.IYHLPDAES*DEDEDFKEQTR. & S 218 confirmed by y 11 and y 12 \\
\hline & & & Y211 not phosphorylated by b2, b3 \\
\hline & & R.YLHDES*GLNR.R & S 134 confirmed by y $3, y 5$ \\
\hline & & & Y129 not phosphorylated by b2, b4 \\
\hline & & K.IYHLPDAES*DEDEDFK.E & S218 confirmed by y6, y10 \\
\hline \multirow[t]{5}{*}{ Q15185 } & TEBP_HUMAN & & \\
\hline & Prostaglandin E synthase 3 & & \\
\hline & $\begin{array}{l}\text { (Cytosolic prostaglandin E2 } \\
\text { synthase) }\end{array}$ & & \\
\hline & & K.DWEDDS*DEDMSNFDR.F & S113 confirmed by y 9, y 10 \\
\hline & & K.DWEDDS*DEDM\#SNFDR.F & \\
\hline \multirow[t]{23}{*}{ O60716 } & $\begin{array}{l}\text { CTND1_HUMAN Catenin } \\
\text { delta-1 (p120 catenin) }\end{array}$ & & \\
\hline & & R.LRSY*EDMIGEEVPSDQYYW & S320 not confirmed \\
\hline & & APLAQHER.G & Y321 not confirmed \\
\hline & & & $\begin{array}{l}\text { S331 not phosphorylated by y13, } \\
\text { y14 }\end{array}$ \\
\hline & & & $\begin{array}{l}\text { Y334 not phosphorylated by y10, } \\
\text { y11 }\end{array}$ \\
\hline & & & Y335 not phosphorylated by y9, y10 \\
\hline & & R.HYEDGYPGGSDNYGS*LSR.V & S230 confirmed by y3, y5 \\
\hline & & & Y217 not phosphorylated by b2, b3 \\
\hline & & & Y221 not phosphorylated by b5, b6 \\
\hline & & & S225 not phosphorylated by y8, y9 \\
\hline & & & $\begin{array}{l}\text { Y228 not phosphorylated by b12, } \\
\text { b13 }\end{array}$ \\
\hline & & & S232 not phosphorylated by y3 \\
\hline & & R.SQSSHSYDDST*LPLIDR.N & T869 confirmed by y6, y7 \\
\hline & & $\begin{array}{l}\text { R.S*YEDMIGEEVPSDQYYWAP } \\
\text { LAQHER.G }\end{array}$ & S320 not confirmed \\
\hline & & R.VGGS*SVDLHR.F & S268 confirmed by y6, y7 \\
\hline & & R.S*GDLGDMEPLK.G & S 920 confirmed by b3 and b4 \\
\hline & & R.TLDRS*GDLGDMEPLK.G & S920 confirmed by y10, y11 \\
\hline & & R.S*MGYDDLDYGMMSDYGTA & S288 confirmed by b3 \\
\hline & & R.R & S300 not phosphorylated by y 7 \\
\hline & & R.T*LDRSGDLGDMEPLK.G & T916 confirmed by b3 \\
\hline & & $\begin{array}{l}\text { R.HYEDGYPGGS*DNY*GSLSR } \\
\text { VT*RIEER.Y }\end{array}$ & Y228 not confirmed \\
\hline & & $\begin{array}{l}\text { R.S*MGYDDLDYGMMSDYGTA } \\
\text { R R }\end{array}$ & S288 \\
\hline & & R.VGGS*SVDLHR.F & S268 \\
\hline \multirow[t]{5}{*}{ Q9Y2W1 } & $\begin{array}{l}\text { TR150_HUMAN Thyroid } \\
\text { hormone receptor-associated } \\
\text { protein } 3\end{array}$ & & \\
\hline & & K.WAHDKFS*GEEGEIEDDES*G & S928 confirmed by b6, b9 \\
\hline & & TENR.E & S 939 confirmed by y 5, y 6 \\
\hline & & K.WAHDKFS*GEEGEIEDDESGT & S928 confirmed by b6, b9 \\
\hline & & ENR.E & S939 not phosphorylated by y5, y6 \\
\hline
\end{tabular}


Table A-2 (continued).

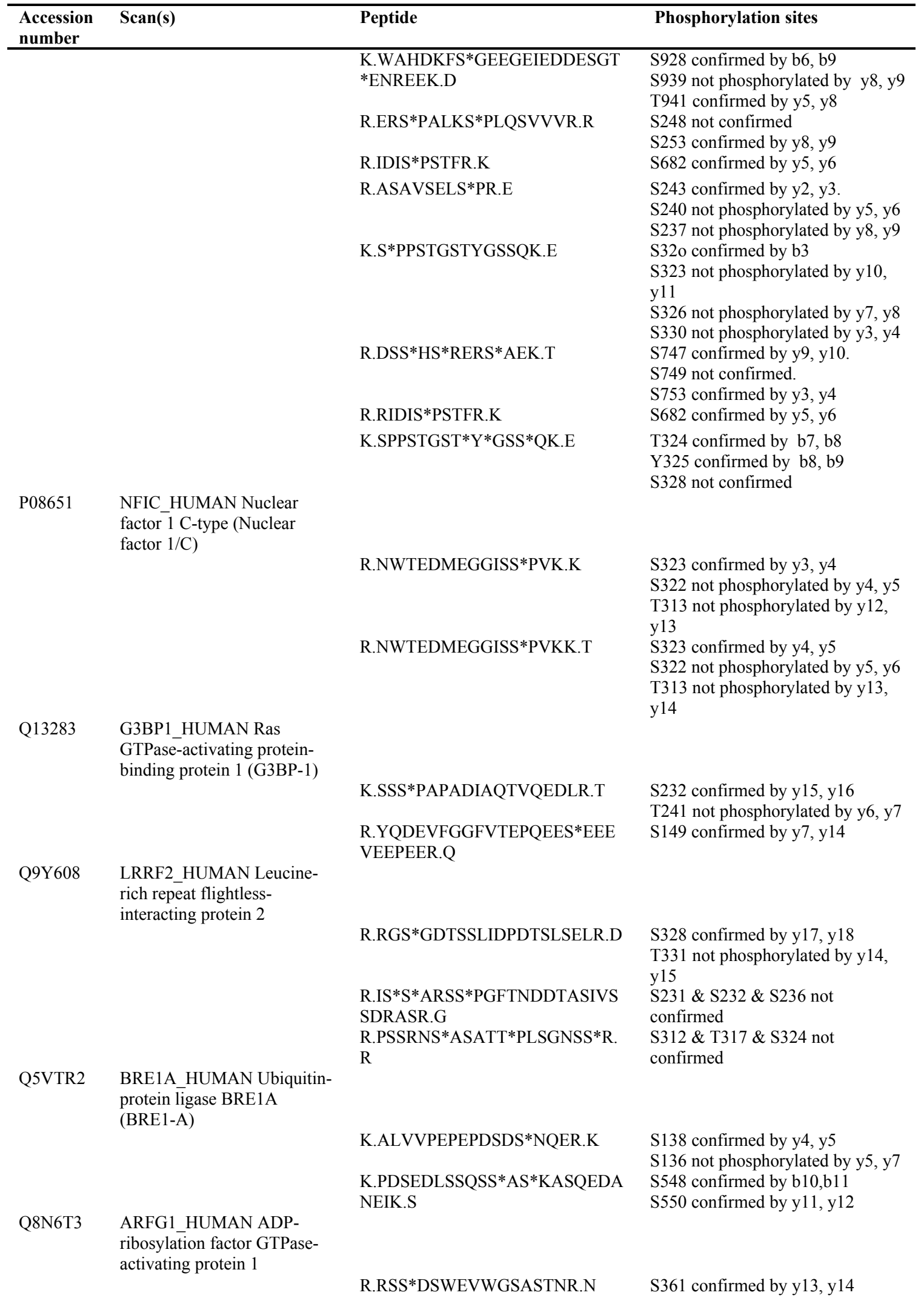


Table A-2 (continued).

\begin{tabular}{|c|c|c|c|}
\hline $\begin{array}{l}\text { Accession } \\
\text { number }\end{array}$ & Scan(s) & Peptide & Phosphorylation sites \\
\hline & & R.EFLESQEDY*DPCWS*LQEK.Y & $\begin{array}{l}\text { Y93 not confirmed } \\
\text { S98 confirmed by b13, b14 }\end{array}$ \\
\hline & & R.SS*DSWEVWGSASTNR.N & S361 \\
\hline & & K.ASELGHS*LNENVLKPAQEK.V & S246 not confirmed \\
\hline \multirow[t]{4}{*}{ Q9Y6G9 } & $\begin{array}{l}\text { DC1L1_HUMAN } \\
\text { Cytoplasmic dynein } 1 \text { light } \\
\text { intermediate chain } 1\end{array}$ & & \\
\hline & & R.DFQEYVEPGEDFPAS*PQR.R & S207 confirmed by y3, y5 \\
\hline & & $\begin{array}{l}\text { K.KTGSPGGPGVSGGS*PAGGAGGG } \\
\text { SS*GLPPSTKK.S }\end{array}$ & S468 confirmed by b11, b15 \\
\hline & & K.PVT*VS*PTTPTSPTEGEAS* & $\begin{array}{l}\text { T508 confirmed by b3 } \\
\text { S510 confirmed by y13, y14 }\end{array}$ \\
\hline \multirow[t]{5}{*}{ Q9Y2U5 } & $\begin{array}{l}\text { M3K2_HUMAN Mitogen- } \\
\text { activated protein kinase } \\
\text { kinase kinase } 2\end{array}$ & & \\
\hline & & R.DRSS*PPPGYIPDELHQVAR.N & S164 confirmed by b3, b4 \\
\hline & & R.AQS*YPDNHQEFSDYDNPIFEK.F & S239 confirmed by b3, b4 \\
\hline & & K.RLS*IIGPTSR.D & S153 confirmed by y7, y8 \\
\hline & & R.GS*DIDNPT*LTVM\#DISPPSR.S & $\begin{array}{l}\text { S331 confirmed by b3 } \\
\text { T337 not confirmed }\end{array}$ \\
\hline \multirow[t]{2}{*}{ O00193 } & $\begin{array}{l}\text { SMAP_HUMAN Small } \\
\text { acidic protein }\end{array}$ & & \\
\hline & & $\begin{array}{l}\text { R.S*ASPDDDLGSSNWEAADLGNEE } \\
\text { R.K }\end{array}$ & S15 not confirmed \\
\hline \multirow[t]{6}{*}{ O95218 } & $\begin{array}{l}\text { ZRAB2_HUMAN Zinc } \\
\text { finger Ran-binding domain- } \\
\text { containing protein } 2\end{array}$ & & \\
\hline & & R.ENVEYIEREES*DGEYDEFGR.K & S120 \\
\hline & & R.EES*DGEYDEFGR.K & S120 confirmed by y9, y10 \\
\hline & & K.YNLDAS*EEEDSNK.K & S188 confirmed by y7, y8 \\
\hline & & K.EVEDKES*EGEEEDEDEDLSK.Y & S153 confirmed by b7 \\
\hline & & R.S*SSRS*S*SPSSSRSR.S & $\begin{array}{l}\text { S213 \& S217 \& S218 not } \\
\text { confirmed }\end{array}$ \\
\hline \multirow[t]{3}{*}{ Q8N5F7 } & $\begin{array}{l}\text { NKAP_HUMAN NF- } \\
\text { kappa-B-activating protein }\end{array}$ & & \\
\hline & & R.IGELGAPEVWGLS*PK.N & S149 confirmed by b12, b13 \\
\hline & & $\begin{array}{l}\text { K.Y*S*EDS*DSDSDSETDSSDEDNK. } \\
\text { R }\end{array}$ & $\begin{array}{l}\text { Y209 \&S } 210 \& \text { S213 not } \\
\text { confirmed }\end{array}$ \\
\hline \multirow[t]{2}{*}{ Q9NSK0 } & $\begin{array}{l}\text { KLC4_HUMAN Kinesin } \\
\text { light chain } 4\end{array}$ & & \\
\hline & & R.AAS*LNYLNQPSAAPLQVSR.G & $\begin{array}{l}\text { S590 confirmed by b3 } \\
\text { Y593 not phosphorylated by y } 12 \text {, } \\
\text { y14 }\end{array}$ \\
\hline \multirow[t]{2}{*}{ Q9H1E3 } & $\begin{array}{l}\text { NUCKS_HUMAN Nuclear } \\
\text { ubiquitous casein and } \\
\text { cyclin-dependent kinases } \\
\text { substrate }\end{array}$ & & \\
\hline & & K.VVDYSQFQES*DDADEDYGR.D & S19 confirmed by y8, y10 \\
\hline \multirow[t]{2}{*}{ Q92625 } & $\begin{array}{l}\text { ANKS1_HUMAN Ankyrin } \\
\text { repeat and SAM domain- } \\
\text { containing protein 1A } \\
\text { (Odin) }\end{array}$ & & \\
\hline & & K.SPS*FASEWDEIEK.I & S663 confirmed by y10, y11 \\
\hline \multirow[t]{2}{*}{ O00192 } & $\begin{array}{l}\text { ARVC_HUMAN Armadillo } \\
\text { repeat protein deleted in } \\
\text { velo-cardio-facial syndrome }\end{array}$ & & \\
\hline & & R.S*LAADDEGGPELEPDYGTATR.R & S267 confirmed by b3 \\
\hline
\end{tabular}


Table A-2 (continued).

\begin{tabular}{|c|c|c|c|}
\hline $\begin{array}{l}\text { Accession } \\
\text { number }\end{array}$ & Scan(s) & Peptide & Phosphorylation sites \\
\hline & & R.NFDT*LDLPK.R & T642 confirmed by y5, y6 \\
\hline \multirow[t]{3}{*}{ O15027 } & $\begin{array}{l}\text { K0310_HUMAN } \\
\text { Uncharacterized protein } \\
\text { KIAA0310 }\end{array}$ & & \\
\hline & & R.FTGS*FDDDPDPHRDPYGEE & S1149 confirmed by b2, b5 \\
\hline & & VDR.R & Y1161 not phosphorylated by y4, y7 \\
\hline \multirow[t]{7}{*}{ Q14247 } & $\begin{array}{l}\text { SRC8_HUMAN Src } \\
\text { substrate cortactin }\end{array}$ & & \\
\hline & & R.LPSS*PVYEDAASFK.A & S418 confirmed by y 10, y11 \\
\hline & & K.TQT*PPVS*PAPQPTEER.L & $\begin{array}{l}\text { T401 confirmed by y } 13, \text { y } 14 \\
\text { S } 405 \text { confirmed by y } 9, \text { y10 }\end{array}$ \\
\hline & & R.VDKSAVGFDY*QGKT*EK.H & $\begin{array}{l}\text { Y178 confirmed by b6, b10 } \\
\text { T182 confirmed by b13, b14 }\end{array}$ \\
\hline & & K.GRY*GLFPANY*VELR.Q & $\begin{array}{l}\text { Y538 confirmed by b3 } \\
\text { Y545 confirmed by y } 2, \text { y } 5\end{array}$ \\
\hline & & K.LS*KHCS*QVDSVRGFGGK.F & $\begin{array}{l}\text { S109 confirmed by b3 } \\
\text { S113 confirmed by y11, y13 }\end{array}$ \\
\hline & & R.LPSS*PVY*EDAASFK.A & $\begin{array}{l}\text { S418 confirmed by y } 10, \text { y } 11 \\
\text { Y421 not confirmed }\end{array}$ \\
\hline \multirow[t]{3}{*}{ Q14498 } & $\begin{array}{l}\text { RBM39_HUMAN RNA- } \\
\text { binding protein } 39\end{array}$ & & \\
\hline & & K.DKS*PVREPIDNLTPEER.D & S136 confirmed by b3 \\
\hline & & R.YRS*PYSGPK.F & 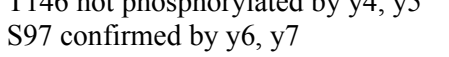 \\
\hline \multirow[t]{2}{*}{ Q6ICG6 } & $\begin{array}{l}\text { CV009_HUMAN } \\
\text { Uncharacterized protein } \\
\text { C22orf9 }\end{array}$ & & \\
\hline & & K.SHS*ANDSEEFFR.E & S324 confirmed by y9, y10 \\
\hline \multirow[t]{13}{*}{ Q9NYF8 } & $\begin{array}{l}\text { BCLF1_HUMAN Bcl-2- } \\
\text { associated transcription } \\
\text { factor } 1\end{array}$ & & \\
\hline & & K.FNDS*EGDDTEETEDYR.Q & S397 confirmed by y11, y13 \\
\hline & & $\begin{array}{l}\text { R.AEGEWEDQEALDYFS*DKES } \\
\text { GK.Q }\end{array}$ & S385 not confirmed \\
\hline & & K.KAEGEPQEES*PLK.S & S177 confirmed by b9, b10 \\
\hline & & K.DLFDYS*PPLHK.N & S512 confirmed by y5, y6 \\
\hline & & K.LKDLFDYS*PPLHK.N & \\
\hline & & R.IDIS*PSTLR.K & S658 confirmed by y5, y6 \\
\hline & & R.YSPS*QNS*PIHHIPSR.R & $\begin{array}{l}\text { S } 287 \text { not confirmed } \\
\text { S } 290 \text { confirmed by y } 8, \text { y } 9\end{array}$ \\
\hline & & R.AEGEWEDQEALDYFS*DK.E & S385 confirmed by b14, b15 \\
\hline & & R.RIDIS*PSTLR.K & S658 \\
\hline & & R.SSSSS*AS*PSSPSS*REEK.E & $\begin{array}{l}\text { S755 confirmed by b4, b5 } \\
\text { S757 confirmed by b6, b7 S763 not } \\
\text { confirmed }\end{array}$ \\
\hline & & R.S*SSSRSSSPYSKSPVS*K.R & $\begin{array}{l}\text { S141 not confirmed } \\
\text { S156 confirmed by y } 3\end{array}$ \\
\hline & & R.YSPS*QNS*PIHHIPSR.R & \\
\hline \multirow[t]{5}{*}{ P42167 } & $\begin{array}{l}\text { LAP2B_HUMAN Lamina- } \\
\text { associated polypeptide } 2 \text {, } \\
\text { isoforms beta/gamma }\end{array}$ & & \\
\hline & & $\begin{array}{l}\text { K.GPPDFS*SDEEREPTPVLGSG } \\
\text { AAAAGR.S }\end{array}$ & S66 not confirmed \\
\hline & & R.SST*PLPTISSSAENTR.Q & $\mathrm{T} 160$ confirmed by y $13, \mathrm{y} 14$ \\
\hline & & $\begin{array}{l}\text { K.GPPDFSSDEEREPT*PVLGSG } \\
\text { AAAAGR.S }\end{array}$ & T174 confirmed by b12, b14 \\
\hline & & $\begin{array}{l}\text { R.EQGTES*RSST*PLPTISSSAE } \\
\text { NTR.Q }\end{array}$ & $\begin{array}{l}\text { S156 not confirmed } \\
\text { T160 confirmed by y } 13, \text { y } 14\end{array}$ \\
\hline
\end{tabular}


Table A-2 (continued).

\begin{tabular}{|c|c|c|c|}
\hline $\begin{array}{l}\text { Accession } \\
\text { number }\end{array}$ & $\operatorname{Scan}(\mathbf{s})$ & Peptide & Phosphorylation sites \\
\hline \multirow[t]{4}{*}{ P54578 } & $\begin{array}{l}\text { UBP14_HUMAN Ubiquitin } \\
\text { carboxyl-terminal hydrolase } \\
14\end{array}$ & & \\
\hline & & R.AS*GEMASAQYITAALR.D & S143 confirmed by b5, b6 \\
\hline & & R.AS*GEM\#ASAQYITAALR.D & see line 118 \\
\hline & & K.S*S*KISRLPAYLTIQMVR.F & S314 \& S315 not confirmed \\
\hline \multirow[t]{3}{*}{ Q8NDX5 } & PHC3_HUMAN & & \\
\hline & Polyhomeotic-like protein 3 & & \\
\hline & & R.MDRT*PPPPTLS*PAAITVGR.G & $\begin{array}{l}\text { T } 609 \text { confirmed by b4, b5 } \\
\text { S } 616 \text { confirmed by y } 8, \text { y } 9\end{array}$ \\
\hline \multirow[t]{8}{*}{ Q8IYB3 } & $\begin{array}{l}\text { SRRM1_HUMAN } \\
\text { Serine/arginine repetitive } \\
\text { matrix protein } 1\end{array}$ & & \\
\hline & & K.KETES*EAEDNLDDLEK.H & S874 confirmed by y 11, y 12 \\
\hline & & $\begin{array}{l}\text { K.KPPAPPS*PVQSQS*PSTNWSP } \\
\text { AVPVK.K } \\
\text { K.KPPAPPS*PVQSQS*PSTNWS* } \\
\text { PAVPVK.K }\end{array}$ & $\begin{array}{l}\text { S769 confirmed by b5, b7 } \\
\text { S775 confirmed by y12, y13 } \\
\text { S } 781 \text { confirmed by y6, y7 }\end{array}$ \\
\hline & & K.EKT*PELPEPSVK.V & T220 confirmed by y9, y10 \\
\hline & & K.SRVS*VS*PGR.T & $\begin{array}{l}\text { S429 confirmed by y } 5, \text { y } 6 \\
\text { S431 confirmed by y3, y } 4\end{array}$ \\
\hline & & R.RYS*PPIQR.R & S597 confirmed y5, y6 \\
\hline & & R.RLS*PSAS*PPR.R & $\begin{array}{l}\text { S389 confirmed by b2, b3 } \\
\text { S393 confirmed by b6, b7 }\end{array}$ \\
\hline & & R.HRPS*PPAT*PPPK.T & $\begin{array}{l}\text { S } 402 \text { confirmed by b5 } \\
\text { T406 confirmed by y } 5\end{array}$ \\
\hline \multirow[t]{3}{*}{ P10644 } & $\begin{array}{l}\text { KAP0_HUMAN cAMP- } \\
\text { dependent protein kinase type } \\
\text { I-alpha regulatory subunit }\end{array}$ & & \\
\hline & & R.EDEIS*PPPPNPVVK.G & S83 confirmed by y 9, y10 \\
\hline & & R.TDSREDEIS*PPPPNPVVK.G & \\
\hline \multirow[t]{3}{*}{ P06748 } & NPM_HUMAN & & \\
\hline & Nucleophosmin & K.DELHIVEAEAMNYEGS*PIK.V & S70 confirmed by y3, y4 \\
\hline & & $\begin{array}{l}\text { K.DELHIVEAEAMNYEGS*PIKV } \\
\text { TLATLK.M }\end{array}$ & \\
\hline \multirow[t]{2}{*}{ P31943 } & $\begin{array}{l}\text { HNRH1_HUMAN } \\
\text { Heterogeneous nuclear } \\
\text { ribonucleoprotein } \mathrm{H}\end{array}$ & & \\
\hline & & R.PSGEAFVELES*EDEVK.L & S63 confirmed by y 5 , y6 \\
\hline \multirow[t]{3}{*}{ P16949 } & STMN1_HUMAN Stathmin & & \\
\hline & & K.ESVPEFPLS*PPK.K & $\begin{array}{l}\text { S38 confirmed by } y 3, y 4 \\
\text { S31 not phosphorylated by b2, b3 }\end{array}$ \\
\hline & & R.ASGQAFELILS*PR.S & S 25 confirmed by $\mathrm{y} 3, \mathrm{y} 4$ \\
\hline \multirow[t]{2}{*}{ Q14694 } & $\begin{array}{l}\text { UBP10_HUMAN Ubiquitin } \\
\text { carboxyl-terminal hydrolase } \\
10\end{array}$ & & \\
\hline & & $\begin{array}{l}\text { K.NHSVNEEEQEEQGEGS*EDEW } \\
\text { EQVGPR.N }\end{array}$ & S576 confirmed by y8, y11 \\
\hline \multirow[t]{2}{*}{ Q9UK76 } & $\begin{array}{l}\text { HN1_HUMAN } \\
\text { Hematological and } \\
\text { neurological expressed } 1 \\
\text { protein }\end{array}$ & & \\
\hline & & R.RNS*SEASSGDFLDLK.G & $\begin{array}{l}\text { S87 confirmed by y12, y13 } \\
\text { S88 not phosphorylated by y } 11, \\
\text { y12 } \\
\text { S91 not phosphorylated by y } 8, \text { y }\end{array}$ \\
\hline
\end{tabular}


Table A-2 (continued).

\begin{tabular}{|c|c|c|c|}
\hline $\begin{array}{l}\text { Accession } \\
\text { number }\end{array}$ & $\operatorname{Scan}(\mathbf{s})$ & Peptide & Phosphorylation sites \\
\hline \multirow[t]{4}{*}{ Q13263 } & $\begin{array}{l}\text { TIF1B_HUMAN } \\
\text { Transcription intermediary } \\
\text { factor 1-beta }\end{array}$ & & \\
\hline & & $\begin{array}{l}\text { R.STAPSAAASASASAAASS*PAGGGAE } \\
\text { ALELLEHCGVCR.E }\end{array}$ & S50 confirmed by b17, b18 \\
\hline & & R.SRS*GEGEVSGLMR.K & S473 confirmed by b2, b3 \\
\hline & & R.S*GEGEVSGLMR.K & \\
\hline \multirow[t]{3}{*}{ Q53SF7 } & $\begin{array}{l}\text { CBLL1_HUMAN Cordon- } \\
\text { bleu protein-like } 1\end{array}$ & & \\
\hline & & R.AGS*LQLSSMSAGNSSLR.R & S382 confirmed by y 14, y 15 \\
\hline & & R.QS*SLTFQSSDPEQMR.Q & S1146 confirmed by y14 \\
\hline \multirow[t]{2}{*}{ O75821 } & $\begin{array}{l}\text { IF34_HUMAN Eukaryotic } \\
\text { translation initiation factor } 3 \\
\text { subunit } 4 \text { (eIF-3 delta) }\end{array}$ & & \\
\hline & & K.GIPLATGDT*SPEPELLPGAPLPPPK.E & T41 not confirmed \\
\hline \multirow[t]{2}{*}{ Q9UKL0 } & $\begin{array}{l}\text { RCOR1_HUMAN REST } \\
\text { corepressor } 1\end{array}$ & & \\
\hline & & $\begin{array}{l}\text { R.EREES*EDELEEANGNNPIDIEVDQN } \\
\text { K.E }\end{array}$ & S257 confirmed by b4, b6 \\
\hline \multirow[t]{6}{*}{ Q6PKG0 } & $\begin{array}{l}\text { LARP1_HUMAN La-related } \\
\text { protein } 1\end{array}$ & & \\
\hline & & K.GLSAS*LPDLDSENWIEVK.K & S548 confirmed by y13, y14 \\
\hline & & R.S*LPTTVPESPNY*R.N & $\begin{array}{l}\text { S766 confirmed by b2 } \\
\text { Y777 confirmed by b1 } 1, \mathrm{~b} 12\end{array}$ \\
\hline & & R.SLPTTVPES*PNYR.N & S774 confirmed by y 4, y6 \\
\hline & & K.ILIVTQT*PHYMR.R & T649 not confirmed \\
\hline & & $\begin{array}{l}\text { R.EHRPRTASISSS*PSEGTPT*VGSYGC } \\
\text { TPQSLPK.F } \\
\text { K.QEVENFKKVNM\#IS*R.E }\end{array}$ & $\begin{array}{l}\text { S851 not confirmed } \\
\text { S858 not confirmed } \\
\text { S716 not confirmed }\end{array}$ \\
\hline \multirow[t]{2}{*}{ O43741 } & $\begin{array}{l}\text { AAKB2_HUMAN 5'-AMP- } \\
\text { activated protein kinase } \\
\text { subunit beta-2 }\end{array}$ & & \\
\hline & & R.DLSSS*PPGPYGQEMYAFR.S & S184 confirmed by y13, y14 \\
\hline \multirow[t]{2}{*}{ Q9Y2V2 } & $\begin{array}{l}\text { CHSP1_HUMAN Calcium- } \\
\text { regulated heat stable protein } 1\end{array}$ & & \\
\hline & & R.GNVVPS*PLPTR.R & S41 confirmed by y 5, y 6 \\
\hline \multirow[t]{3}{*}{ P12694 } & $\begin{array}{l}\text { ODBA_HUMAN 2- } \\
\text { oxoisovalerate dehydrogenase } \\
\text { subunit alpha, mitochondrial } \\
\text { precursor }\end{array}$ & & \\
\hline & & R.S*VDEVNYWDK.Q & S347 confirmed by b2, b3 \\
\hline & & R.IGHHS*TSDDSSAYR.S & S337 not confirmed \\
\hline \multirow[t]{2}{*}{ Q8NEY8 } & $\begin{array}{l}\text { PPHLN_HUMAN Periphilin- } \\
1\end{array}$ & & \\
\hline & & R.DNTFFRES*PVGR.K & S133 confirmed by y4, y 5 \\
\hline \multirow[t]{8}{*}{ Q96JM3 } & $\begin{array}{l}\text { K1802_HUMAN Zinc finger } \\
\text { protein KIAA1802 }\end{array}$ & & \\
\hline & & R.KPSPSES*PEPWKPFPAVS*PEPR.R & $\begin{array}{l}\text { S286 confirmed by b6, b9 } \\
\text { S } 297 \text { confirmed by y } 4, \text { y } 7\end{array}$ \\
\hline & & R.KPSGS*PDLWK.L & S445 confirmed by y 5, y 7 \\
\hline & & R.KPS*PS*ESPEPWKPFPAVSPEPR.R & $\begin{array}{l}\text { S282 not confirmed } \\
\text { S284 not confirmed }\end{array}$ \\
\hline & & K.TAPTLS*PEHWK.A & S405 confirmed by y 5 , y6 \\
\hline & & R.RPAPAVS*PGSWK.P & S308 confirmed by y 5 , y6 \\
\hline & & R.GGS*PDLWK.S & S476 confirmed by y 5, y 6 \\
\hline & & R.KPGPPLS*PEIR.S & S427 confirmed by y4, y5 \\
\hline
\end{tabular}


Table A-2 (continued).

\begin{tabular}{|c|c|c|c|}
\hline $\begin{array}{l}\text { Accession } \\
\text { number }\end{array}$ & Scan(s) & Peptide & Phosphorylation sites \\
\hline & & R.KTS*PASLDFPESQK.S & S459 not confirmed \\
\hline & & R.KT*SPASLDFPES*QKS*SR.G & $\begin{array}{l}\text { S458 \& S468 \& S471 not } \\
\text { confirmed }\end{array}$ \\
\hline \multirow[t]{2}{*}{ Q15459 } & $\begin{array}{l}\text { SF3A1_HUMAN Splicing } \\
\text { factor } 3 \text { subunit } 1\end{array}$ & & \\
\hline & & K.FGESEEVEMEVES*DEEDDKQEK.A & S329 confirmed by y9, y10 \\
\hline \multirow[t]{3}{*}{ Q9UL54 } & $\begin{array}{l}\text { TAOK2_HUMAN } \\
\text { Serine/threonine-protein } \\
\text { kinase TAO2 }\end{array}$ & & \\
\hline & & K.MLLARHS*LDQDLLR.E & S656 confirmed by y7, y9 \\
\hline & & R.HS*LDQDLLR.E & S656 confirmed by b5, b6 \\
\hline \multirow[t]{4}{*}{ Q15139 } & $\begin{array}{l}\text { KPCD1_HUMAN } \\
\text { Serine/threonine-protein } \\
\text { kinase D1 }\end{array}$ & & \\
\hline & & R.RLS*NVS*LTGVSTIR.T & $\begin{array}{l}\text { S205 confirmed by y11. y12 } \\
\text { S208 confirmed by y } 7, \text { y } 9\end{array}$ \\
\hline & & R.RLS*NVSLTGVSTIR.T & S205 confirmed by y 11, y 12 \\
\hline & & $\begin{array}{l}\text { R.T*SS*AELST*SAPDEPLLQKSPSESFI } \\
\text { GREK.R }\end{array}$ & $\begin{array}{l}\text { T217 \& S219 \& T224 not } \\
\text { confirmed }\end{array}$ \\
\hline \multirow[t]{3}{*}{ Q9UEW8 } & $\begin{array}{l}\text { STK39_HUMAN } \\
\text { STE20/SPS1-related proline- } \\
\text { alanine-rich protein kinase }\end{array}$ & & \\
\hline & & K.TEDGDWEWS*DDEMDEK.S & S387 confirmed by y6, y9 \\
\hline & & $\begin{array}{l}\text { K.ADMWSFGITAIELATGAAPY*HKY* } \\
\text { PPMK.V }\end{array}$ & $\begin{array}{l}\text { Y275 \& Y278 not } \\
\text { confirmed }\end{array}$ \\
\hline \multirow[t]{4}{*}{ Q9H0B6 } & $\begin{array}{l}\text { KLC2_HUMAN Kinesin } \\
\text { light chain } 2 \text { (KLC 2) }\end{array}$ & & \\
\hline & & R.RDS*APYGEYGSWYK.A & S428 confirmed by y 11, y 12 \\
\hline & & R.ASS*LNFLNK.S & S528 confirmed by y6, y7 \\
\hline & & R.TLSSS*SMDLSR.R & S610 confirmed by y6, y7 \\
\hline \multirow[t]{2}{*}{ Q32MZ4 } & $\begin{array}{l}\text { LRRF1_HUMAN Leucine- } \\
\text { rich repeat flightless- } \\
\text { interacting protein } 1\end{array}$ & & \\
\hline & & R.RGS*GDTSISIDTEASIR.E & S120 confirmed by y 14, y 15 \\
\hline \multirow[t]{2}{*}{ Q15052 } & $\begin{array}{l}\text { ARHG6_HUMAN Rho } \\
\text { guanine nucleotide exchange } \\
\text { factor } 6\end{array}$ & & \\
\hline & & R.MS*GFIYQGK.I & S488 confirmed by y $7, y 8$ \\
\hline \multirow[t]{2}{*}{ P21127 } & $\begin{array}{l}\text { CD2L1_HUMAN PITSLRE } \\
\text { serine/threonine-protein } \\
\text { kinase CDC2L1 }\end{array}$ & & \\
\hline & & K.AYT*PVVVTLWYR.A & T595 confirmed by y9, y10 \\
\hline \multirow[t]{3}{*}{ P17812 } & $\begin{array}{l}\text { PYRG1_HUMAN CTP } \\
\text { synthase } 1\end{array}$ & & \\
\hline & & R.SGSSS*PDSEITELK.F & S575 confirmed by y9, y10 \\
\hline & & K.YIDSADLEPITS*QEEPVR.Y & S347 confirmed by y5, y7 \\
\hline \multirow[t]{3}{*}{ P25490 } & $\begin{array}{l}\text { TYY1_HUMAN } \\
\text { Transcriptional repressor } \\
\text { protein YY1 }\end{array}$ & & \\
\hline & & $\begin{array}{l}\text { K.DIDHETVVEEQIIGENS*PPDYSEYM } \\
\text { TGK.K }\end{array}$ & S247 confirmed by y11, y12 \\
\hline & & R.EEVVGGDDS*DGLR.A & S118 confirmed by y4, y7 \\
\hline Q16637 & $\begin{array}{l}\text { SMN_HUMAN Survival } \\
\text { motor neuron protein }\end{array}$ & & \\
\hline
\end{tabular}


Table A-2 (continued).

\begin{tabular}{|c|c|c|c|}
\hline $\begin{array}{l}\text { Accession } \\
\text { number }\end{array}$ & Scan(s) & Peptide & Phosphorylation sites \\
\hline & & R.GTGQS*DDSDIWDDTALIK.A & S28 confirmed by y13, y14 \\
\hline \multirow[t]{3}{*}{ Q13177 } & PAK2_HUMAN & & \\
\hline & $\begin{array}{l}\text { Serine/threonine-protein } \\
\text { kinase PAK } 2\end{array}$ & & \\
\hline & & K.YLS*FTPPEK.D & S141 confirmed by y 5, y 7 \\
\hline \multirow[t]{2}{*}{ Q08945 } & $\begin{array}{l}\text { SSRP1_HUMAN FACT } \\
\text { complex subunit SSRP1 }\end{array}$ & & \\
\hline & & $\begin{array}{l}\text { K.EGMNPSYDEYADS*DEDQHDAYL } \\
\text { ER.M }\end{array}$ & S444 confirmed by b12, b14 \\
\hline \multirow[t]{3}{*}{ P35221 } & $\begin{array}{l}\text { CTNA1_HUMAN Catenin } \\
\text { alpha-1 }\end{array}$ & & \\
\hline & & R.S*RTS*VQTEDDQLIAGQSAR.A & $\begin{array}{l}\text { S652 no confirmed } \\
\text { S } 655 \text { confirmed by y } 14 \text { y } 16\end{array}$ \\
\hline & & R.T*SVQTEDDQLIAGQSAR.A & T654 no confirmed \\
\hline \multirow[t]{5}{*}{ Q13242 } & $\begin{array}{l}\text { SFRS9_HUMAN Splicing } \\
\text { factor, arginine/serine-rich } 9\end{array}$ & & \\
\hline & & R.GSPHYFS*PFRPY & S216 confirmed by y 5 , y 6 \\
\hline & & R.GS*PHYFS*PFRPY & $\begin{array}{l}\text { S211 confirmed by b2 } \\
\text { S216 confirmed by y } 5 \text {, y } 6\end{array}$ \\
\hline & & R.STS*YGYSR.S & S189 confirmed by y5, y6 \\
\hline & & R.GRDS*PYQSR.G & S204 not confirmed \\
\hline \multirow[t]{3}{*}{ O93594 } & AGM1_HUMAN & & \\
\hline & $\begin{array}{l}\text { Phosphoacetylglucosamine } \\
\text { mutase (PAGM) }\end{array}$ & & \\
\hline & & K.STIGVMVTAS*HNPEEDNGVK.L & S64 confirmed by y10, y12 \\
\hline \multirow[t]{2}{*}{ Q9Y6X9 } & $\begin{array}{l}\text { MORC2 HUMAN MORC } \\
\text { family } \mathrm{CW} \text {-type zinc finger } \\
\text { protein } 2\end{array}$ & & \\
\hline & & R.SVAVS*DEEEVEEEAER.R & S743 confirmed by y9, y12 \\
\hline \multirow[t]{4}{*}{ Q92934 } & $\begin{array}{l}\text { BAD_HUMAN Bcl2 } \\
\text { antagonist of cell death }\end{array}$ & & \\
\hline & & R.RMS*DEFVDSFK.K & S118 confirmed by b2, b4 \\
\hline & & R.HSS*YPAGTEDDEGMGEEPSPFR.G & S75 no confirmed \\
\hline & & R.S*APPNLWAAQR.Y & S99 confirmed by b2, b7 \\
\hline \multirow[t]{4}{*}{ Q9C0C2 } & $\begin{array}{l}\text { TB182_HUMAN } 182 \mathrm{kDa} \\
\text { tankyrase 1-binding protein }\end{array}$ & & \\
\hline & & R.RFS*EGVLQSPSQDQEK.L & $\begin{array}{l}\text { S429 confirmed by b2, b4 } \\
\text { S435 not phosphorylated by } \\
\text { y } 7, \text { y8 } \\
\text { S } 437 \text { not phosphorylated by } \\
\text { y4, y7 }\end{array}$ \\
\hline & & R.S*QEADVQDWEFR.K & S836 not confirmed \\
\hline & & K.VST*LRES*SAMAS*PLPR.E & $\begin{array}{l}\text { T5 \& S } 9 \& \text { S14 not } \\
\text { confirmed }\end{array}$ \\
\hline \multirow[t]{6}{*}{ Q96B36 } & $\begin{array}{l}\text { AKTS1_HUMAN Proline- } \\
\text { rich AKT1 substrate }\end{array}$ & & \\
\hline & & R.AATAARPPAPPPAPQPPS*PTPS*PP & S 88 confirmed by y12, y13 \\
\hline & & RPTLAR.E & S92 confirmed by y8, y9 \\
\hline & & K.S*LPVSVPVWGFK.E & S183 not confirmed \\
\hline & & R.LNT*SDFQK.L & T246 not confirmed \\
\hline & & $\begin{array}{l}\text { R.AAT*AARPPAPPPAPQPPS*PT*PSP } \\
\text { PR.P }\end{array}$ & $\begin{array}{l}\text { T73 \& S88 \& T } 90 \text { not } \\
\text { confirmed }\end{array}$ \\
\hline \multirow[t]{3}{*}{ Q14671 } & $\begin{array}{l}\text { PUM1_HUMAN Pumilio } \\
\text { homolog } 1 \text { (Pumilio-1) }\end{array}$ & & \\
\hline & & R.RDS*LTGSSDLYK.R & S709 confirmed by y9, y10 \\
\hline & & R.S*ASSASSLFSPSSTLFSS*SR.L & S797 not confirmed \\
\hline
\end{tabular}


Table A-2 (continued).

\begin{tabular}{|c|c|c|c|}
\hline $\begin{array}{l}\text { Accession } \\
\text { number }\end{array}$ & $\operatorname{Scan}(\mathbf{s})$ & Peptide & Phosphorylation sites \\
\hline \multirow[t]{4}{*}{$\mathrm{P} 08559$} & $\begin{array}{l}\text { ODPA_HUMAN Pyruvate } \\
\text { dehydrogenase E1 } \\
\text { component alpha subunit }\end{array}$ & & \\
\hline & & R.YHGHS*MSDPGVS*YR.T & $\begin{array}{l}\text { S } 293 \text { confirmed by y } 9, \text { y } 10 \\
\text { S } 300 \text { confirmed by b11,b12 }\end{array}$ \\
\hline & & R.YGMGTS*VER.A & S232 confirmed by y3, y4 \\
\hline & & R.YHGHSMS*DPGVSYR.T & S295 not confirmed \\
\hline \multirow[t]{8}{*}{ Q9UQ35 } & $\begin{array}{l}\text { SRRM2_HUMAN } \\
\text { Serine/arginine repetitive } \\
\text { matrix protein } 2\end{array}$ & & \\
\hline & & R.HGGS*PQPLATTPLSQEPVNPPSEA & S377 no confirmed \\
\hline & & S*PTR.D & S398 confirmed by y $3, \mathrm{y} 4$ \\
\hline & & K.SST*PPGESYFGVSSLQLK.G & T1043 no confirmed \\
\hline & & R.S*RT*PLLPR.K & $\begin{array}{l}\text { S2032 no confirmed } \\
\text { T2034 confirmed by y } 5, \text { y } 6\end{array}$ \\
\hline & & R.GPS*PEGSS*ST*ESSPEHPPK.S & $\begin{array}{l}\text { S1648 confirmed by b4 } \\
\text { S1653 \& T } 1655 \text { not confirmed }\end{array}$ \\
\hline & & R.AHRSTSADS*ASSS*DTS*R.S & $\begin{array}{l}\text { S268 \&S } 272 \& \text { T } 275 \text { not } \\
\text { confirmed }\end{array}$ \\
\hline & & R.QS*PSRS*SSPQPK.V & S908 \& S912 not confirmed \\
\hline \multirow[t]{3}{*}{ P98175 } & $\begin{array}{l}\text { RBM10_HUMAN RNA- } \\
\text { binding protein } 10\end{array}$ & & \\
\hline & & R.HRHS*PTGPPGFPR.D & S189 confirmed by b2, b4 \\
\hline & & R.Y*GATDRSQDDGGENRS*R.D & Y16 confirmed by b3 \\
\hline \multirow[t]{2}{*}{ Q9BW71 } & $\begin{array}{l}\text { HIRP3_HUMAN HIRA- } \\
\text { interacting protein } 3\end{array}$ & & \\
\hline & & K.SLKES*EQES*EEEILAQK.K & $\begin{array}{l}\text { S } 223 \text { confirmed by y } 12, \text { y } 14 \\
\text { S227 confirmed by b8, b9 }\end{array}$ \\
\hline \multirow[t]{2}{*}{ Q9BSJ8 } & $\begin{array}{l}\text { FA62A_HUMAN Protein } \\
\text { FAM62A }\end{array}$ & & \\
\hline & & R.KLDVSVKSNS*SFMS*R.E & $\begin{array}{l}\text { S1063 no confirmed } \\
\text { S1067 confirmed by y } 2, \text { y } 4\end{array}$ \\
\hline \multirow[t]{2}{*}{ Q9UKS6 } & $\begin{array}{l}\text { PACN3_HUMAN Protein } \\
\text { kinase } \mathrm{C} \text { and casein kinase } \\
\text { substrate in neurons protein } 3\end{array}$ & & \\
\hline & & $\begin{array}{l}\text { R.DGTAPPPQSPGSPGTGQDEEWS*D } \\
\text { EESPR.K }\end{array}$ & S354 confirmed by y6, y7 \\
\hline \multirow[t]{2}{*}{ P24844 } & $\begin{array}{l}\text { MLRN_HUMAN Myosin } \\
\text { regulatory light chain } 2 \text {, } \\
\text { smooth muscle isoform }\end{array}$ & & \\
\hline & & R.ATS*NVFAMFDQSQIQEFK.E & S20 no confirmed \\
\hline \multirow[t]{3}{*}{ P04792 } & $\begin{array}{l}\text { HSPB1_HUMAN Heat- } \\
\text { shock protein beta-1 (HspB1) } \\
\text { (Heat shock } 27 \mathrm{kDa} \text { protein) }\end{array}$ & & \\
\hline & & R.QLS*SGVSEIR.H & $\begin{array}{l}\text { S82 confirmed by y } 7, \text { y } 8 \\
\text { S83 not phosphorylated by y6, } \\
\text { y7 }\end{array}$ \\
\hline & & R.GPS*WDPFR.D & $\mathrm{S} 15$ confirmed by $\mathrm{b} 2, \mathrm{~b} 3$ \\
\hline \multirow[t]{3}{*}{ P05455 } & $\begin{array}{l}\text { LA_HUMAN Lupus La } \\
\text { protein (Sjoegren syndrome } \\
\text { type B antigen) }\end{array}$ & & \\
\hline & & K.FAS*DDEHDEHDENGATGPVK.R & S366 confirmed by b2, b3 \\
\hline & & R.SPS*KPLPEVTDEYK.N & S94 confirmed by y11, y13 \\
\hline \multirow[t]{2}{*}{ O60841 } & $\begin{array}{l}\text { IF2P_HUMAN Eukaryotic } \\
\text { translation initiation factor } \\
5 \mathrm{~B}\end{array}$ & & \\
\hline & & K.NKPGPNIES*GNEDDDASFK.I & S214 confirmed by y10, y11 \\
\hline
\end{tabular}


Table A-2 (continued).

\begin{tabular}{|c|c|c|c|}
\hline $\begin{array}{l}\begin{array}{l}\text { Accession } \\
\text { number }\end{array} \\
\end{array}$ & Scan(s) & Peptide & Phosphorylation sites \\
\hline \multirow[t]{3}{*}{$\begin{array}{l}\mathrm{P} 51114 \\
\end{array}$} & $\begin{array}{l}\text { FXR1_HUMAN Fragile X } \\
\text { mental retardation syndrome- } \\
\text { related protein } 1 \text { (hFXR1p) }\end{array}$ & & \\
\hline & & $\begin{array}{l}\text { R.RGPNYTSGYGTNSELSNPS*ETES } \\
\text { ER.K }\end{array}$ & S409 confirmed by y5, y7 \\
\hline & & $\begin{array}{l}\text { R.RGPNYTSGYGTNSELS*NPS*ETE } \\
\text { SER.K }\end{array}$ & S406 confirmed by y9, y10 \\
\hline \multirow[t]{5}{*}{ P08238 } & $\begin{array}{l}\text { HS90B_HUMAN Heat shock } \\
\text { protein HSP 90-beta (HSP 84) } \\
\text { (HSP 90) }\end{array}$ & & \\
\hline & & K.IEDVGS*DEEDDSGKDK.K & $\begin{array}{l}\text { S255 confirmed by b4, b6 } \\
\text { S261 not phosphorylated by } \\
\text { b11, b12 }\end{array}$ \\
\hline & & K.IEDVGS*DEEDDSGK.D & S255 confirmed by y 7, y 10 \\
\hline & & K.IEDVGS*DEEDDSGKDKK.K & \\
\hline & & R.EKEIS*DDEAEEEK.G & S226 confirmed by y8, y9 \\
\hline \multirow[t]{2}{*}{ Q9UEE9 } & $\begin{array}{l}\text { CFDP1_HUMAN Craniofacial } \\
\text { development protein } 1\end{array}$ & & \\
\hline & & K.LDWES*FKEEEGIGEELAIHNR.G & S250 confirmed by b4, b9 \\
\hline \multirow[t]{2}{*}{ Q9Y383 } & $\begin{array}{l}\text { LC7L2_HUMAN Putative } \\
\text { RNA-binding protein Luc7-like }\end{array}$ & & \\
\hline & & R.AMLDQLMGTS*R.D & $\begin{array}{l}\text { S18 confirmed by y2, y3 } \\
\text { T17 not phosphorylated by } \\
\text { y3, y4 }\end{array}$ \\
\hline \multirow[t]{2}{*}{ Q99613 } & $\begin{array}{l}\text { IF38_HUMAN Eukaryotic } \\
\text { translation initiation factor } 3 \\
\text { subunit } 8 \text { (eIF3 p110) }\end{array}$ & & \\
\hline & & K.QPLLLS*EDEEDTKR.V & S39 confirmed by y8, y9 \\
\hline \multirow[t]{5}{*}{ O14974 } & $\begin{array}{l}\text { MYPT1_HUMAN Protein } \\
\text { phosphatase } 1 \text { regulatory } \\
\text { subunit } 12 \mathrm{~A}\end{array}$ & & \\
\hline & & K.TGS*YGALAEITASK.E & S445 confirmed by y11,y12 \\
\hline & & $\begin{array}{l}\text { K.SPLIES*TANMDNNQSQKTFKNK. } \\
\text { E }\end{array}$ & S304 confirmed by b5, b6 \\
\hline & & R.RST*QGVTLTDLQEAEK.T & T696 not confirmed \\
\hline & & R.KTGS*YGALAEITASK.E & S445 \\
\hline \multirow[t]{2}{*}{ Q9Y3S2 } & $\begin{array}{l}\text { ZN330_HUMAN Zinc finger } \\
\text { protein } 330\end{array}$ & & \\
\hline & & R.KDS*DTESSDLFTNLNLGR.T & S291 confirmed by y15, y16 \\
\hline \multirow[t]{5}{*}{ P61978 } & $\begin{array}{l}\text { HNRPK_HUMAN } \\
\text { Heterogeneous nuclear } \\
\text { ribonucleoprotein K }\end{array}$ & & \\
\hline & & R.GSY*GDLGGPIITTQVTIPK.D & Y380 confirmed by y14, y17 \\
\hline & & R.RDYDDMS*PR.R & S284 confirmed by y2, y3 \\
\hline & & R.GSY*GDLGGPIITT*QVTIPK.D & \\
\hline & & R.DYDDMS*PR.R & S284 \\
\hline \multirow[t]{7}{*}{ P51858 } & $\begin{array}{l}\text { HDGF_HUMAN Hepatoma- } \\
\text { derived growth factor (HDGF) }\end{array}$ & & \\
\hline & & R.AGDLLEDS*PK.R & S165 confirmed by y3, y4 \\
\hline & & R.RAGDLLEDS*PK.R & \\
\hline & & R.AGDLLEDS*PKRPK.E & \\
\hline & & K.GNAEGS*S*DEEGKLVIDEPAK.E & $\begin{array}{l}\text { S132 not confirmed } \\
\text { S133 not confirmed }\end{array}$ \\
\hline & & $\begin{array}{l}\text { K.KGNAEGS*S*DEEGKLVIDEPAK. } \\
\text { E }\end{array}$ & \\
\hline & & K.GNAEGS*SDEEGKLVIDEPAK.E & S132 confirmed by b5, b6 \\
\hline
\end{tabular}


Table A-2 (continued).

\begin{tabular}{|c|c|c|c|}
\hline $\begin{array}{l}\text { Accession } \\
\text { number }\end{array}$ & Scan(s) & Peptide & Phosphorylation sites \\
\hline \multirow[t]{3}{*}{ P22059 } & $\begin{array}{l}\text { OSBP1_HUMAN Oxysterol- } \\
\text { binding protein } 1\end{array}$ & & \\
\hline & & R.TGS*NISGASSDISLDEQYK.H & S379 confirmed by y 16, y 17 \\
\hline & & $\begin{array}{l}\text { K.GDMS*DEDDENEFFDAPEIITMPE } \\
\text { NLGHK.R }\end{array}$ & S351 not confirmed \\
\hline \multirow[t]{3}{*}{ Q8NE71 } & $\begin{array}{l}\text { ABCF1_HUMAN ATP-binding } \\
\text { cassette sub-family F member } 1\end{array}$ & & \\
\hline & & K.LSVPTS*DEEDEVPAPK.P & S109 confirmed by y9, y11 \\
\hline & & K.LSVPTS*DEEDEVPAPKPR.G & S109 confirmed by y 12, y 13 \\
\hline \multirow[t]{3}{*}{ P55795 } & HNRH2_HUMAN & & \\
\hline & $\begin{array}{l}\text { Heterogeneous nuclear } \\
\text { ribonucleoprotein } \mathrm{H}^{\prime}\end{array}$ & & \\
\hline & & K.HTGPNS*PDTANDGFVR.L & S104 confirmed by y10, y11 \\
\hline \multirow[t]{2}{*}{ Q9BYG3 } & $\begin{array}{l}\text { MK67I_HUMAN MKI67 FHA } \\
\text { domain-interacting nucleolar } \\
\text { phosphoprotein }\end{array}$ & & \\
\hline & & K.S*QVAELNDDDKDDEIVFK.Q & S247 confirmed by b3 \\
\hline \multirow[t]{6}{*}{ P02545 } & $\begin{array}{l}\text { LMNA_HUMAN Lamin-A/C } \\
\text { (70 kDa lamin) }\end{array}$ & & \\
\hline & & R.NKS*NEDQSMGNWQIK.R & S458 confirmed by b4 \\
\hline & & R.LRLS*PSPTSQR.S & S390 confirmed by y7, y8 \\
\hline & & R.KLEST*ES*RSSFSQHAR.T & $\begin{array}{l}\text { T424 confirmed by y } 11, \text { y } 12 \\
\text { S } 426 \text { no confirmed }\end{array}$ \\
\hline & & R.LRLS*PS*PTSQR.S & $\begin{array}{l}\text { S390 confirmed by y } 7, \text { y } 8 \\
\text { S392 confirmed by y5, y6 }\end{array}$ \\
\hline & & R.SVGGS*GGGSFGDNLVTR.S & S632 not confirmed \\
\hline \multirow[t]{2}{*}{ P06493 } & $\begin{array}{l}\text { CDC2_HUMAN Cell division } \\
\text { control protein } 2 \text { homolog (p34 } \\
\text { protein kinase) }\end{array}$ & & \\
\hline & & R.VYT*HEVVTLWYR.S & T161 confirmed by y9, y10 \\
\hline \multirow[t]{3}{*}{ P49585 } & $\begin{array}{l}\text { PCY1A_HUMAN Choline- } \\
\text { phosphate cytidylyltransferase A }\end{array}$ & & \\
\hline & & R.MLQAIS*PK.Q & S315 confirmed by b5,b6 \\
\hline & & K.T*SPPCS*PANLSR.H & $\begin{array}{l}\text { T342 not confirmed } \\
\text { S347 confirmed by y6, y7 }\end{array}$ \\
\hline \multirow[t]{5}{*}{ Q16204 } & $\begin{array}{l}\text { CCDC6_HUMAN Coiled-coil } \\
\text { domain-containing protein } 6 \text { (H4 } \\
\text { protein) }\end{array}$ & & \\
\hline & & $\begin{array}{l}\text { R.PIS*PGLSYASHTVGFTPPTSLTR. } \\
\text { A }\end{array}$ & S367 confirmed by b3 \\
\hline & & R.T*VSSPIPYTPSPSSSR.P & T349 no confirmed \\
\hline & & $\begin{array}{l}\text { R.PIS*PGLSYASHTVGFT*PPTSLTR. } \\
\text { A } \\
\text { R.QLSESES*SLEMDDER.Y }\end{array}$ & $\begin{array}{l}\text { S367 confirmed by b3 T380 } \\
\text { not confirmed } \\
\text { S327 no confirmed }\end{array}$ \\
\hline & & K.LDQPVS*APPS*PR.D & $\begin{array}{l}\text { S240 confirmed by b5, b7 } \\
\text { S244 confirmed by y2, y3 }\end{array}$ \\
\hline \multirow[t]{3}{*}{ Q9NTJ3 } & $\begin{array}{l}\text { SMC4_HUMAN Structural } \\
\text { maintenance of chromosomes } \\
\text { protein } 4\end{array}$ & & \\
\hline & & R.T*ESPATAAETASEELDNR.S & T39 no confirmed \\
\hline & & K.S*S*LAMNRS*R.G & $\begin{array}{l}\text { S588 \& S589 not confirmed } \\
\text { S595 confirmed by y3 }\end{array}$ \\
\hline
\end{tabular}


Table A-2 (continued).

\begin{tabular}{|c|c|c|c|}
\hline $\begin{array}{l}\text { Accession } \\
\text { number }\end{array}$ & $\operatorname{Scan}(\mathbf{s})$ & Peptide & Phosphorylation sites \\
\hline \multirow[t]{3}{*}{$\overline{\text { Q8TAQ2 }}$} & $\begin{array}{l}\text { SMRC2_HUMAN SWI/SNF- } \\
\text { related matrix-associated actin- } \\
\text { dependent regulator of } \\
\text { chromatin subfamily C member } \\
2\end{array}$ & & \\
\hline & & R.KRS*PSPSPT*PEAK.K & S302 confirmed by b2, b3 \\
\hline & & K.DMDEPS*PVPNVEEVTLPK.T & $\begin{array}{l}\text { T308 confirmed by y } 4, y 6 \\
\text { S347 confirmed by y12, y14 }\end{array}$ \\
\hline \multirow[t]{2}{*}{ Q14676 } & $\begin{array}{l}\text { MDC1_HUMAN Mediator of } \\
\text { DNA damage checkpoint } \\
\text { protein } 1\end{array}$ & & \\
\hline & & R.LLLAEDS*EEEVDFLSER.R & S168 confirmed by y9, y11 \\
\hline \multirow[t]{3}{*}{ Q8WUA2 } & $\begin{array}{l}\text { PPIL4_HUMAN Peptidyl- } \\
\text { prolyl cis-trans isomerase-like } \\
4 \text { (PPIase) }\end{array}$ & & \\
\hline & & $\begin{array}{l}\text { R.INHTVILDDPFDDPPDLLIPDRSPE } \\
\text { PT*R.E }\end{array}$ & T182 not confirmed \\
\hline & & $\begin{array}{l}\text { R.INHTVILDDPFDDPPDLLIPDRS*P } \\
\text { EPTR.E }\end{array}$ & S178 confirmed by y 5, y \\
\hline \multirow[t]{2}{*}{ P18887 } & $\begin{array}{l}\text { XRCC1_HUMAN DNA-repair } \\
\text { protein XRCC1 }\end{array}$ & & \\
\hline & & $\begin{array}{l}\text { K.TKPTQAAGPSS*PQKPPT*PEETK. } \\
\text { A }\end{array}$ & $\begin{array}{l}\text { S447 no confirmed } \\
\text { T453 confirmed by y5, y7 }\end{array}$ \\
\hline \multirow[t]{4}{*}{ P07814 } & $\begin{array}{l}\text { SYEP_HUMAN Bifunctional } \\
\text { aminoacyl-tRNA synthetase }\end{array}$ & & \\
\hline & & K.EYIPGQPPLSQSSDSS*PTR.N & S886 confirmed by y3, y4 \\
\hline & & R.AKIDM\#SS*NNGCMRDPTLY*R.C & S346 not confirmed \\
\hline & & K.HPKNPEVGLKPVWY*S*PK.V & Y546 \& S547 not confirmed \\
\hline \multirow[t]{2}{*}{ P27824 } & $\begin{array}{l}\text { CALX_HUMAN Calnexin } \\
\text { precursor }\end{array}$ & & \\
\hline & & K.AEEDEILNRS*PR.N & S583 confirmed by b9, b10 \\
\hline \multirow[t]{3}{*}{ O75494 } & $\begin{array}{l}\text { FUSIP_HUMAN FUS- } \\
\text { interacting serine-arginine-rich } \\
\text { protein } 1\end{array}$ & & \\
\hline & & R.S*FDYNYR.R & S133 confirmed by b2 \\
\hline & & R.S*RS*FDYNYR.R & $\begin{array}{l}\text { S131 confirmed by b2 } \\
\text { S133 confirmed by b2, b4 }\end{array}$ \\
\hline \multirow[t]{3}{*}{ Q15773 } & $\begin{array}{l}\text { MLF2_HUMAN Myeloid } \\
\text { leukemia factor } 2\end{array}$ & & \\
\hline & & $\begin{array}{l}\text { R.QHMS*RMLSGGFGY*SPFLSIT*D } \\
\text { GNMPGTR.P }\end{array}$ & $\begin{array}{l}\text { S29 \& Y37 \& T44 not } \\
\text { confirmed }\end{array}$ \\
\hline & & R.LAIQGPEDS*PSR.Q & S238 confirmed by y3, y4 \\
\hline \multirow[t]{2}{*}{ Q15366 } & $\begin{array}{l}\text { PCBP2_HUMAN Poly(rC)- } \\
\text { binding protein } 2 \text { (Alpha-CP2) }\end{array}$ & & \\
\hline & & K.PSSS*PVIFAGGQDR.Y & S189 confirmed by b3, b4 \\
\hline \multirow[t]{6}{*}{ P30622 } & CLIP1_HUMAN Restin & & \\
\hline & & K.LT*NLQENLSEVSQVKETLEK.E & T822 confirmed by b4 \\
\hline & & $\begin{array}{l}\text { K.TISSEKAS*S*TPSS*ETQEEFVDD } \\
\text { FRVGER.V } \\
\text { R.VM\#ATTSASLKRSPSASSLSSMS* } \\
\text { SVASS*VSSR.P }\end{array}$ & $\begin{array}{l}\text { S43 \& S44 \& S48 not } \\
\text { confirmed } \\
\text { S320 \& S325 not confirmed }\end{array}$ \\
\hline & & R.SPSASSLS*SMS*SVASSVSSRPSR. & S317 confirmed by y 15, y16 \\
\hline & & K.RS*PSASS*LSSM\#SSVASSVSSR.P & $\begin{array}{l}\text { S320 not confirmed } \\
\text { S310 confirmed by b2 }\end{array}$ \\
\hline & & $\begin{array}{l}\text { K.EPSATPPIS*NLTKTAS*ESISNLSE } \\
\text { AGS*IK.K }\end{array}$ & $\begin{array}{l}\text { S315 not confirmed } \\
\text { S186 \& S193 not confirmed } \\
\text { S204 confirmed by y } 3, \text { y }\end{array}$ \\
\hline
\end{tabular}


Table A-2 (continued).

\begin{tabular}{|c|c|c|c|}
\hline $\begin{array}{l}\text { Accession } \\
\text { number }\end{array}$ & Scan(s) & Peptide & Phosphorylation sites \\
\hline \multirow[t]{2}{*}{ P11717 } & $\begin{array}{l}\text { MPRI_HUMAN Cation- } \\
\text { independent mannose-6- } \\
\text { phosphate receptor precursor }\end{array}$ & & \\
\hline & & K.LVSFHDDS*DEDLLHI & S2484 confirmed by y6, y8 \\
\hline \multirow[t]{2}{*}{ O00567 } & $\begin{array}{l}\text { NOP56_HUMAN Nucleolar } \\
\text { protein Nop56 }\end{array}$ & & \\
\hline & & K.EELMSS*DLEETAGSTSIPK.R & S520 confirmed by y 13, y 14 \\
\hline \multirow[t]{2}{*}{ P52756 } & $\begin{array}{l}\text { RBM5_HUMAN RNA-binding } \\
\text { protein } 5\end{array}$ & & \\
\hline & & R.GLVAAYSGDS*DNEEELVER.L & S624 confirmed by y9, y12 \\
\hline \multirow[t]{2}{*}{ Q8ND30 } & LIPB2_HUMAN Liprin-beta-2 & & \\
\hline & & R.T*QSGNFYTDTLGMAEFR.R & T510 not confirmed \\
\hline \multirow[t]{2}{*}{ P40222 } & $\begin{array}{l}\text { TXLNA_HUMAN Alpha- } \\
\text { taxilin }\end{array}$ & & \\
\hline & & R.RPEGPGAQAPSS*PR.V & S515 confirmed by y $2, \mathrm{y} 3$ \\
\hline \multirow[t]{2}{*}{ Q8IWS0 } & $\begin{array}{l}\text { PHF6_HUMAN PHD finger } \\
\text { protein } 6 \text { (PHD-like zinc finger } \\
\text { protein) }\end{array}$ & & \\
\hline & & $\begin{array}{l}\text { K.TAHNSEADLEESFNEHELEPSS*P } \\
\text { K.S }\end{array}$ & S155 confirmed by y3 \\
\hline \multirow[t]{7}{*}{ Q13595 } & $\begin{array}{l}\text { TRA2A_HUMAN } \\
\text { Transformer-2 protein homolog } \\
\text { (TRA-2 alpha) }\end{array}$ & & \\
\hline & & $\begin{array}{l}\text { R.PTHS*GGGGGGGGGGGGGGGG } \\
\text { RRR.D }\end{array}$ & S215 not confirmed \\
\hline & & R.AHT*PTPGIYMGR.P & T202 confirmed by y9, y10 \\
\hline & & R.RS*PS*PYYSR.Y & $\begin{array}{l}\text { S260 confirmed by b2 } \\
\text { S262 confirmed by b3, b4 }\end{array}$ \\
\hline & & R.S*PS*PYYSR.Y & \\
\hline & & R.RRDSY*YDR.G & Y237 not confirmed \\
\hline & & R.RRS*PSPYY*SR.Y & $\begin{array}{l}\text { S260 confirmed by b2, b3 } \\
\text { Y } 265 \text { confirmed by y2, y3 }\end{array}$ \\
\hline \multirow[t]{2}{*}{ Q13489 } & $\begin{array}{l}\text { BIRC3_HUMAN Baculoviral } \\
\text { IAP repeat-containing protein } 3\end{array}$ & & \\
\hline & & -.M\#NIVENS*IFLS*NLMK.S & $\begin{array}{l}\text { S7 confirmed by y7, y10 } \\
\text { S11 confirmed by y4, y7 }\end{array}$ \\
\hline \multirow[t]{3}{*}{ P49736 } & $\begin{array}{l}\text { MCM2_HUMAN DNA } \\
\text { replication licensing factor } \\
\text { MCM2 }\end{array}$ & & \\
\hline & & R.GLLYDS*DEEDEERPAR.K & S139 confirmed by y9, y11 \\
\hline & & R.VM\#LES*FIDTQK.F & S801 confirmed by b3, b5 \\
\hline \multirow[t]{2}{*}{ Q12968 } & $\begin{array}{l}\text { NFAC3 HUMAN Nuclear } \\
\text { factor of activated T-cells, } \\
\text { cytoplasmic } 3 \text { (NF-ATc3) }\end{array}$ & & \\
\hline & & R.PS*S*DSGCSHDSVLS*GQR.S & $\begin{array}{l}\text { S730 not confirmed } \\
\text { S731 not confirmed } \\
\text { S742 confirmed by y } 3 \text {, y } 5\end{array}$ \\
\hline \multirow[t]{2}{*}{ Q9H410 } & $\begin{array}{l}\text { CT172_HUMAN } \\
\text { Uncharacterized protein } \\
\text { C20orf172 }\end{array}$ & & \\
\hline & & K.SLHLS*PQEQSASYQDR.R & S81 confirmed by y11, y12 \\
\hline \multirow[t]{2}{*}{ Q9ULU4 } & $\begin{array}{l}\text { PKCB1_HUMAN Protein } \\
\text { kinase C-binding protein } 1\end{array}$ & & \\
\hline & & K.S*DSSDSEYISDDEQK.S & $\begin{array}{l}\text { S425 confirmed by y6, y8 } \\
\text { S427 not phosphorylated by } \\
\text { y3, y5 } \\
\text { S595 not confirmed }\end{array}$ \\
\hline
\end{tabular}


Table A-2 (continued).

\begin{tabular}{|c|c|c|c|}
\hline $\begin{array}{l}\text { Accession } \\
\text { number }\end{array}$ & $\operatorname{Scan}(\mathbf{s})$ & Peptide & Phosphorylation sites \\
\hline \multirow[t]{2}{*}{$\mathrm{P} 31321$} & $\begin{array}{l}\text { KAP1_HUMAN cAMP- } \\
\text { dependent protein kinase type I- } \\
\text { beta regulatory subunit }\end{array}$ & & \\
\hline & & -.M\#ASPPACPS*EEDESLK.G & S9 confirmed by b8, b11 \\
\hline \multirow[t]{3}{*}{ Q7L7X3 } & TAOK1_HUMAN & & \\
\hline & $\begin{array}{l}\text { Serine/threonine-protein kinase } \\
\text { TAO1 }\end{array}$ & & \\
\hline & & R.AS*DPQSPPQVS*R.H & $\begin{array}{l}\text { S417 confirmed by b3 } \\
\text { S426 confirmed by b9, b11 }\end{array}$ \\
\hline \multirow[t]{2}{*}{ P51587 } & $\begin{array}{l}\text { BRCA2_HUMAN Breast cancer } \\
\text { type } 2 \text { susceptibility protein }\end{array}$ & & \\
\hline & & $\begin{array}{l}\text { K.VFADIQS*EEILQHNQNMSGLE } \\
\text { KVS*K.I }\end{array}$ & S1926 not confirmed \\
\hline \multirow[t]{2}{*}{ Q8TF66 } & $\begin{array}{l}\text { LRC15_HUMAN Leucine-rich } \\
\text { repeat-containing protein } 15 \\
\text { precursor (hLib) }\end{array}$ & & \\
\hline & & R.M\#LANLQNIS*LQNNRLR.Q & S371 confirmed by y6, y9 \\
\hline \multirow[t]{4}{*}{ Q5VT25 } & MRCKA_HUMAN & & \\
\hline & $\begin{array}{l}\text { Serine/threonine-protein kinase } \\
\text { MRCK alpha }\end{array}$ & & \\
\hline & & $\begin{array}{l}\text { R.HSTAS*NSS*NLSSPPS*PASPR. } \\
\text { K }\end{array}$ & $\begin{array}{l}\text { S1700 \& S1703 \& S1710 not } \\
\text { confirmed }\end{array}$ \\
\hline & & R.TVFSGS*VSIPS*ITK.S & 1611 NEW \& 1616 NEW \\
\hline \multirow[t]{2}{*}{ Q9UMY1 } & $\begin{array}{l}\text { NOL7_HUMAN Nucleolar } \\
\text { protein } 7\end{array}$ & & \\
\hline & & K.VQSVS*QNKSY*LAVRLK.D & $\begin{array}{l}\text { S167 confirmed by y } 11, \text { y12 } \\
\text { Y172 confirmed by y } 4, y 7\end{array}$ \\
\hline \multirow[t]{2}{*}{ P28161 } & $\begin{array}{l}\text { GSTM2_HUMAN Glutathione } \\
\text { S-transferase Mu } 2 \text { (GSTM2-2) }\end{array}$ & & \\
\hline & & K.S*S*RFLPRPVFTK.M & S200 \& S201 not confirmed \\
\hline \multirow[t]{2}{*}{ Q9UKX3 } & $\begin{array}{l}\text { MYH13_HUMAN Myosin-13 } \\
\text { (Myosin heavy chain 13) }\end{array}$ & & \\
\hline & & R.VEEKES*LISQLT*K.S & $\begin{array}{l}\text { S1300 confirmed by b4, b6 } \\
\text { T1306 confirmed by b11, b12 }\end{array}$ \\
\hline \multirow[t]{2}{*}{ P55199 } & $\begin{array}{l}\text { ELL_HUMAN RNA polymerase } \\
\text { II elongation factor ELL }\end{array}$ & & \\
\hline & & R.KSGAS*AVSGGS*GVS*QR.P & $\begin{array}{l}\text { S194 not confirmed } \\
\text { S200 confirmed by b10, b12 } \\
\text { S203 confirmed by y2, y3 }\end{array}$ \\
\hline \multirow[t]{3}{*}{ Q9BXT6 } & $\begin{array}{l}\text { M10L1_HUMAN Putative } \\
\text { helicase Mov1011 }\end{array}$ & & \\
\hline & & K.WEDDSRNHGSPS*DCGPR.V & S113 confirmed by b11, b12 \\
\hline & & K.SSQALT*S*AKTTVVVTAQK.R & T472 \& S473 not confirmed \\
\hline \multirow[t]{2}{*}{ Q14181 } & $\begin{array}{l}\text { DPOA2_HUMAN DNA } \\
\text { polymerase subunit alpha B }\end{array}$ & & \\
\hline & & $\begin{array}{l}\text { R.GGAGNIS*LKVLGCPEALTGSY } \\
\text { KSMFQK.L }\end{array}$ & S192 confirmed by b5, b7 \\
\hline \multirow[t]{2}{*}{ Q12824 } & $\begin{array}{l}\text { SNF5_HUMAN SWI/SNF- } \\
\text { related matrix-associated actin- } \\
\text { dependent regulator of chromatin } \\
\text { subfamily B member } 1\end{array}$ & & \\
\hline & & R.YPS*LWRRLATVEER.K & S49 confirmed by b2,b6 \\
\hline \multirow[t]{2}{*}{ Q8IWV8 } & $\begin{array}{l}\text { UBR2_HUMAN E3 ubiquitin- } \\
\text { protein ligase UBR2 }\end{array}$ & & \\
\hline & & $\begin{array}{l}\text { K.M\#RES*SPTSPVAET*EGTIMEE } \\
\text { SSRDK.D }\end{array}$ & $\begin{array}{l}\text { S1005 not confirmed } \\
\text { T1014 confirmed by y12, y13 }\end{array}$ \\
\hline
\end{tabular}


Table A-2 (continued).

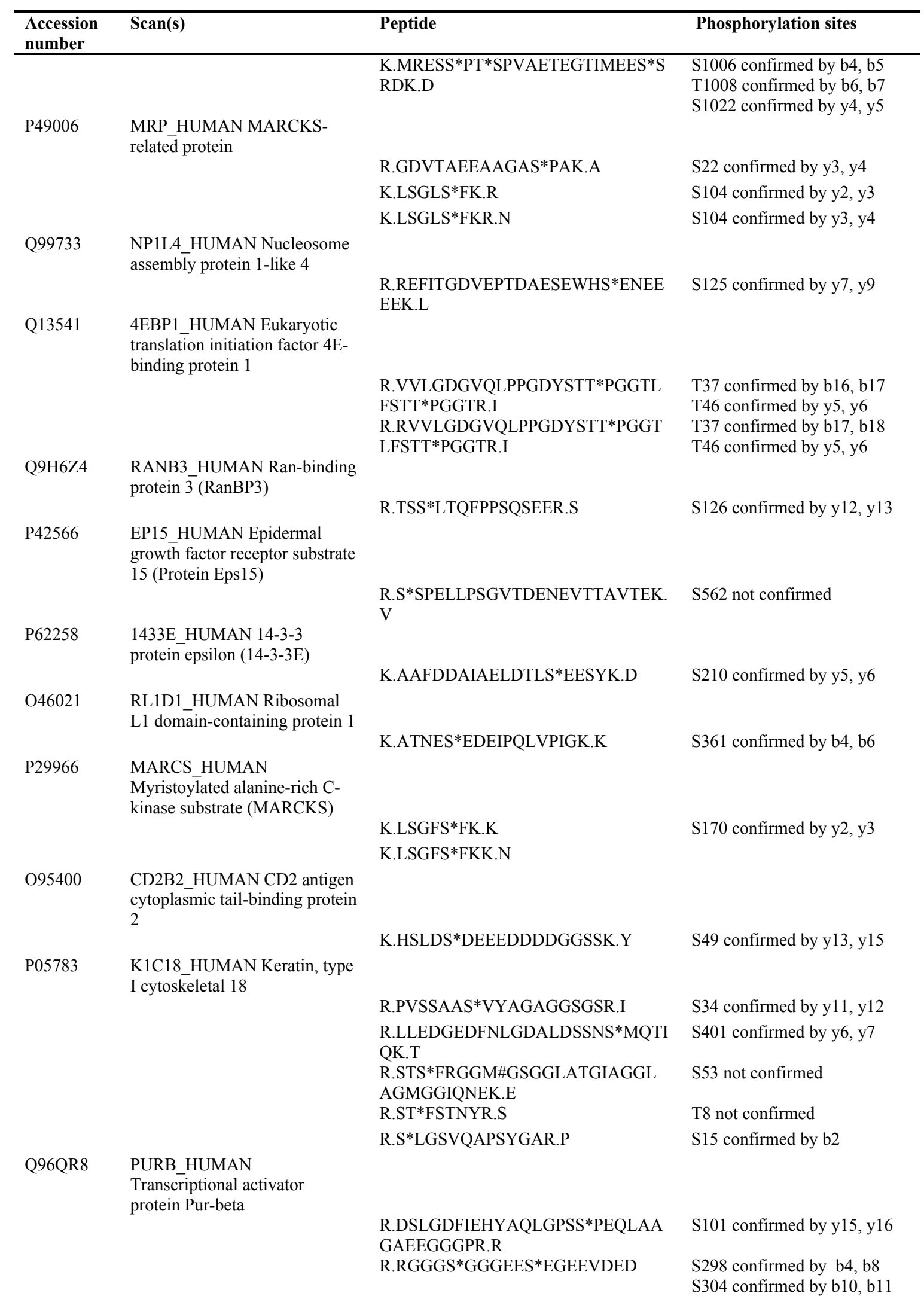


Table A-2 (continued).

\begin{tabular}{|c|c|c|c|}
\hline $\begin{array}{l}\text { Accession } \\
\text { number }\end{array}$ & Scan(s) & Peptide & Phosphorylation sites \\
\hline & & R.RGGGSGGGEES*EGEEVDED & S304 confirmed by b10, b12 \\
\hline \multirow[t]{2}{*}{ Q5BKZ1 } & $\begin{array}{l}\text { ZN326_HUMAN Zinc finger } \\
\text { protein } 326\end{array}$ & & \\
\hline & & $\begin{array}{l}\text { R.SMDSYLNQS*YGMDNHSGGGGG } \\
\text { SR.F }\end{array}$ & S56 not confirmed \\
\hline \multirow[t]{2}{*}{ P29692 } & $\begin{array}{l}\text { EF1D_HUMAN Elongation } \\
\text { factor 1-delta (EF-1-delta) }\end{array}$ & & \\
\hline & & R.ATAPQTQHVS*PMR.Q & S133 confirmed by b9, b10 \\
\hline \multirow[t]{2}{*}{ Q9UGV2 } & $\begin{array}{l}\text { NDRG3_HUMAN Protein } \\
\text { NDRG3 }\end{array}$ & & \\
\hline & & R.THS*TSSSLGSGESPFSR.S & S331 confirmed by y 14, y 15 \\
\hline \multirow[t]{3}{*}{ Q05519 } & $\begin{array}{l}\text { SFR11_HUMAN Splicing } \\
\text { factor arginine/serine-rich } 11\end{array}$ & & \\
\hline & & K.LNHVAAGLVS*PSLK.S & S207 confirmed by y4, y 5 \\
\hline & & R.DYDEEEQGYDS*EKEK.K & S434 confirmed by y4, y5 \\
\hline \multirow[t]{2}{*}{ P25788 } & $\begin{array}{l}\text { PSA3_HUMAN Proteasome } \\
\text { subunit alpha type } 3\end{array}$ & & \\
\hline & & K.ESLKEEDES*DDDNM & S250 confirmed by y 5 , y6 \\
\hline \multirow[t]{2}{*}{ Q6UN15 } & $\begin{array}{l}\text { FIP1_HUMAN Pre-mRNA 3'- } \\
\text { end-processing factor FIP1 }\end{array}$ & & \\
\hline & & R.DHS*PTPSVFNSDEER.Y & S492 confirmed by b2, b3 \\
\hline \multirow[t]{2}{*}{ Q9NYB0 } & $\begin{array}{l}\text { TE2IP_HUMAN Telomeric } \\
\text { repeat-binding factor } 2- \\
\text { interacting protein } 1\end{array}$ & & \\
\hline & & K.YLLGDAPVS*PSSQK.L & S203 confirmed by y 5 , y6 \\
\hline \multirow[t]{6}{*}{ P27816 } & $\begin{array}{l}\text { MAP4_HUMAN Microtubule- } \\
\text { associated protein } 4 \text { (MAP 4) }\end{array}$ & & \\
\hline & & K.DMES*PTKLDVTLAK.D & S280 confirmed by b2, b4 \\
\hline & & K.VGS*LDNVGHLPAGGAVK.T & S1073 not confirmed \\
\hline & & K.DMS*PLSETEMALGK.D & S507 confirmed by b2, b3 \\
\hline & & K.T*STSKAKTQPTSLPK.Q & T712 not confirmed \\
\hline & & K.VGS*TENIK.H & S941 not confirmed \\
\hline \multirow[t]{4}{*}{ P08729 } & $\begin{array}{l}\text { K2C7_HUMAN Keratin, type } \\
\text { II cytoskeletal } 7 \text { (Cytokeratin- } \\
\text { 7) }\end{array}$ & & \\
\hline & & R.T*LNETELTELQS*QISDTSVVLSM & T227 not confirmed \\
\hline & & DNSR.S & S238 confirmed by y 15, y 17 \\
\hline & & $\begin{array}{l}\text { R.T*LNETELT*ELQSQISDTSVVLSM } \\
\text { \#DNSR.S }\end{array}$ & $\begin{array}{l}\text { T227 not confirmed } \\
\text { T234 confirmed by b7, b11 }\end{array}$ \\
\hline \multirow[t]{2}{*}{ Q9H307 } & $\begin{array}{l}\text { PININ_HUMAN Pinin ( } 140 \\
\text { kDa nuclear and cell adhesion- } \\
\text { related phosphoprotein) }\end{array}$ & & \\
\hline & & K.S*LSPGKENVSALDMEK.E & S441 confirmed by b2 \\
\hline \multirow[t]{2}{*}{ Q9BQE3 } & $\begin{array}{l}\text { TBA6_HUMAN Tubulin } \\
\text { alpha-6 chain (Alpha-tubulin 6) }\end{array}$ & & \\
\hline & & K.TIGGGDDS*FNTFFSETGAGK.H & S48 confirmed by y12, y13 \\
\hline \multirow[t]{2}{*}{ Q93009 } & $\begin{array}{l}\text { UBP7_HUMAN Ubiquitin } \\
\text { carboxyl-terminal hydrolase } 7\end{array}$ & & \\
\hline & & $\begin{array}{l}\text { K.AGEQQLS*EPEDMEMEAGDTDDP } \\
\text { PR.I }\end{array}$ & S18 confirmed by b6, b8 \\
\hline \multirow[t]{2}{*}{ P52597 } & $\begin{array}{l}\text { HNRPF_HUMAN } \\
\text { Heterogeneous nuclear } \\
\text { ribonucleoprotein F (hnRNP F) }\end{array}$ & & \\
\hline & & K.ATENDIYNFFS*PLNPVR.V & S310 confirmed by y6, y7 \\
\hline
\end{tabular}


Table A-2 (continued).

\begin{tabular}{|c|c|c|c|}
\hline $\begin{array}{l}\text { Accession } \\
\text { number }\end{array}$ & $\operatorname{Scan}(\mathbf{s})$ & Peptide & Phosphorylation sites \\
\hline \multirow[t]{2}{*}{ Q13185 } & $\begin{array}{l}\text { CBX3_HUMAN Chromobox } \\
\text { protein homolog } 3\end{array}$ & & \\
\hline & & K.SLS*DSESDDSK.S & S95 confirmed by y8, y9 \\
\hline \multirow[t]{3}{*}{ Q8TE77 } & $\begin{array}{l}\text { SSH3_HUMAN Protein } \\
\text { phosphatase Slingshot homolog } \\
3 \text { (SSH-3L) (hSSH-3L) }\end{array}$ & & \\
\hline & & R.S*PPGSGASTPVGPWDQAVQR.R & S4 not confirmed \\
\hline & & R.RQS*FAVLR.G & S37 confirmed by b2, b5 \\
\hline \multirow[t]{2}{*}{ Q9NR30 } & $\begin{array}{l}\text { DDX21_HUMAN Nucleolar } \\
\text { RNA helicase } 2\end{array}$ & & \\
\hline & & K.NEEPS*EEEIDAPKPK.K & S121 confirmed by b4, b6 \\
\hline \multirow[t]{3}{*}{ O95359 } & TACC2_HUMAN & & \\
\hline & $\begin{array}{l}\text { Transforming acidic coiled- } \\
\text { coil-containing protein } 2\end{array}$ & & \\
\hline & & K.LDNTPAS*PPRS*PAEPNDIPIAK. & S2317 not confirmed \\
\hline \multirow[t]{2}{*}{ Q86W92 } & LIPB1_HUMAN Liprin-beta-1 & & \\
\hline & & R.SQS*TTFNPDDMSEPEFK.R & S601 confirmed by y14, y15 \\
\hline \multirow[t]{7}{*}{ P23588 } & $\begin{array}{l}\text { IF4B_HUMAN Eukaryotic } \\
\text { translation initiation factor 4B }\end{array}$ & & \\
\hline & & K.SPPY*TAFLGNLPYDVTEESIK.E & Y96 not confirmed \\
\hline & & R.RES*EKS*LENETLNK.E & $\begin{array}{l}\text { S } 442 \text { confirmed by b2, b4 } \\
\text { S } 445 \text { confirmed by y } 8, \text { y } 9\end{array}$ \\
\hline & & R.ARPATDS*FDDYPPR.R & S207 confirmed by y7, y8 \\
\hline & & R.HPS*WRS*EETQER.E & $\begin{array}{l}\text { S406 confirmed by y9, y } 10 \\
\text { S409 confirmed by b5, b7 }\end{array}$ \\
\hline & & K.PRST*PEEDDSS*AS*TSQSTR.A & $\begin{array}{l}\text { T341 confirmed by b3, b5 } \\
\text { S348 \& S350 not confirmed }\end{array}$ \\
\hline & & K.YAALS*VDGEDENEGEDYAE & S597 confirmed by y14, y15 \\
\hline \multirow[t]{3}{*}{ O15164 } & $\begin{array}{l}\text { TIF1A_HUMAN Transcription } \\
\text { intermediary factor } 1 \text {-alpha }\end{array}$ & & \\
\hline & & K.SEWLDPSQKS*PLHVGETR.K & S811 confirmed by b9, b10 \\
\hline & & $\begin{array}{l}\text { R.SILTSLLLNSS*QSS*T*SEETVLR. } \\
\mathrm{S}\end{array}$ & $\begin{array}{l}\text { S768 confirmed by y } 11, \text { y12 } \\
\text { S771 \& T772 not confirmed }\end{array}$ \\
\hline \multirow[t]{3}{*}{ P46821 } & $\begin{array}{l}\text { MAP1B_HUMAN } \\
\text { Microtubule-associated protein } \\
\text { 1B (MAP 1B) }\end{array}$ & & \\
\hline & & K.LGDVS*PTQIDVSQFGSFK.E & S1501 confirmed by y 13, y 14 \\
\hline & & K.TTSPPEVS*GYS*Y*EK.T & $\begin{array}{l}\text { S1970 \& S1973 } \\
\& \text { Y1974 not confirmed }\end{array}$ \\
\hline \multirow[t]{2}{*}{ Q06265 } & $\begin{array}{l}\text { EXOS9_HUMAN Exosome } \\
\text { complex exonuclease RRP45 } \\
\text { (Exosome component 9) }\end{array}$ & & \\
\hline & & K.APIDTS*DVEEK.A & S306 confirmed by y4, y6 \\
\hline \multirow[t]{4}{*}{ Q8WUZ0 } & $\begin{array}{l}\text { BCL7C_HUMAN B-cell } \\
\text { CLL/lymphoma } 7 \text { protein } \\
\text { family member C }\end{array}$ & & \\
\hline & & K.GTEPS*PGGTPQPSRPVS*PAGPP & S114 not confirmed \\
\hline & & EGVPEEAQPPR.L & S126 confirmed by b16, b19 \\
\hline & & $\begin{array}{l}\text { K.GTEPS*PGGT*PQPSRPVSPAGPP } \\
\text { EGVPEEAQPPR.L }\end{array}$ & T118 not confirmed \\
\hline
\end{tabular}


Table A-2 (continued).

\begin{tabular}{|c|c|c|c|}
\hline $\begin{array}{l}\text { Accession } \\
\text { number }\end{array}$ & Scan(s) & Peptide & Phosphorylation sites \\
\hline \multirow[t]{6}{*}{ O43290 } & $\begin{array}{l}\text { SNUT1_HUMAN U4/U6.U5 } \\
\text { tri-snRNP-associated protein } 1\end{array}$ & & \\
\hline & & $\begin{array}{l}\text { R.RVS*EVEEEKEPVPQPLPSDDTR. } \\
\text { V }\end{array}$ & S448 confirmed by b2, b4 \\
\hline & & $\begin{array}{l}\text { R.DLQGLT*VEHAIDSFREGETMILT } \\
\text { *LKDK.G }\end{array}$ & T258 not confirmed \\
\hline & & K.KMSSS*DT*PLGT*VALLQEK.Q & $\begin{array}{l}\text { S762 \& T764 \& T768 not } \\
\text { confirmed }\end{array}$ \\
\hline & & $\begin{array}{l}\text { K.KMS*S*S*DTPLGTVALLQEKQK. } \\
\text { A }\end{array}$ & $\begin{array}{l}\text { S760 \& S761 \& S762 not } \\
\text { confirmed }\end{array}$ \\
\hline & & K.M\#SS*SDT*PLGTVALLQEK.Q & S761\&T764 not confirmed \\
\hline \multirow[t]{5}{*}{ P30291 } & $\begin{array}{l}\text { WEE1_HUMAN Wee1-like } \\
\text { protein kinase (Wee1A kinase) }\end{array}$ & & \\
\hline & & K.S*PAAPY*FLGSSFS*PVRCGGPG & S127 \& Y132 not confirmed \\
\hline & & DASPR.G & S139 confirmed by y13, y14 \\
\hline & & K.S*RYTTEFHELEKIGS*GEFGSVFK & S293 not confirmed \\
\hline & &. $\mathrm{C}$ & S307 confirmed by y 5 , y9 \\
\hline \multirow[t]{2}{*}{ P16333 } & $\begin{array}{l}\text { NCK1_HUMAN Cytoplasmic } \\
\text { protein NCK1 }\end{array}$ & & \\
\hline & & $\begin{array}{l}\text { K.RKPS*VPDSASPADDSFVDPGER. } \\
\text { L }\end{array}$ & S 85 confirmed by b3, b4 \\
\hline \multirow[t]{2}{*}{ Q9UNE7 } & $\begin{array}{l}\text { STUB1_HUMAN STIP1 } \\
\text { homology and U box- } \\
\text { containing protein } 1\end{array}$ & & \\
\hline & & R.LGAGGGS*PEKS*PSAQELK.E & $\begin{array}{l}\text { S19 confirmed by b5, b7 } \\
\text { S23 confirmed by y } 7, \text { y } 9\end{array}$ \\
\hline \multirow[t]{2}{*}{ Q9BTC0 } & $\begin{array}{l}\text { DIDO1_HUMAN Death- } \\
\text { inducer obliterator } 1 \text { (DIO-1) }\end{array}$ & & \\
\hline & & $\begin{array}{l}\text { R.RNS*VERPAEPVAGAATPSLVEQ } \\
\text { QK.M }\end{array}$ & S1456 confirmed by b2, b5 \\
\hline \multirow[t]{3}{*}{ Q96I25 } & $\begin{array}{l}\text { SPF45_HUMAN Splicing } \\
\text { factor } 45\end{array}$ & & \\
\hline & & R.S*MGGAAIAPPTSLVEK.D & S169 confirmed by b2 \\
\hline & & R.SPT*GPSNSFLANMGGTVAHK.I & T224 not confirmed \\
\hline \multirow[t]{5}{*}{ Q53EL6 } & PDCD4_HUMAN & & \\
\hline & $\begin{array}{l}\text { Programmed cell death protein } \\
4 \text { (Nuclear antigen H731-like) }\end{array}$ & & \\
\hline & & R.KDS*VWGSGGGQQSVNHLVK.E & S313 confirmed by b2,b3 \\
\hline & & R.SGLTVPTS*PK.G & S94 confirmed by y 2, y 3 \\
\hline & & $\begin{array}{l}\text { K.NSSRDS*GRGDS*VS*DSGSDALR } \\
\text {.S }\end{array}$ & S71 \& S76 not confirmed \\
\hline \multirow[t]{3}{*}{ Q9H2U2 } & $\begin{array}{l}\text { IPYR2_HUMAN Inorganic } \\
\text { pyrophosphatase } 2 \text {, } \\
\text { mitochondrial precursor }\end{array}$ & & \\
\hline & & $\begin{array}{l}\text { R.SLVESVSSS*PNKESNEEEQVWHF } \\
\text { LGK }\end{array}$ & S317 not confirmed \\
\hline & & K.FKPGYLEATLNWFRLY*K.V & Y241 not confirmed \\
\hline \multirow[t]{3}{*}{ Q8ND56 } & $\begin{array}{l}\text { LS14A_HUMAN LSM14 } \\
\text { protein homolog A }\end{array}$ & & \\
\hline & & $\begin{array}{l}\text { K.S*PTMEQAVQTASAHLPAPAAVG } \\
\text { R.R }\end{array}$ & S192 confirmed by b2 \\
\hline & & R.SS*PQLDPLR.K & S183 not confirmed \\
\hline \multirow[t]{3}{*}{ Q13247 } & $\begin{array}{l}\text { SFRS6_HUMAN Splicing } \\
\text { factor, arginine/serine-rich } 6\end{array}$ & & \\
\hline & & R.SNS*PLPVPPSK.A & S303 confirmed by b2, b3 \\
\hline & & R.LIVENLSS*R.C & $\begin{array}{l}\text { S119 confirmed by y1, y2 } \\
\text { S118 not phosphorylated by } \\
\text { y2, y3 }\end{array}$ \\
\hline
\end{tabular}


Table A-2 (continued).

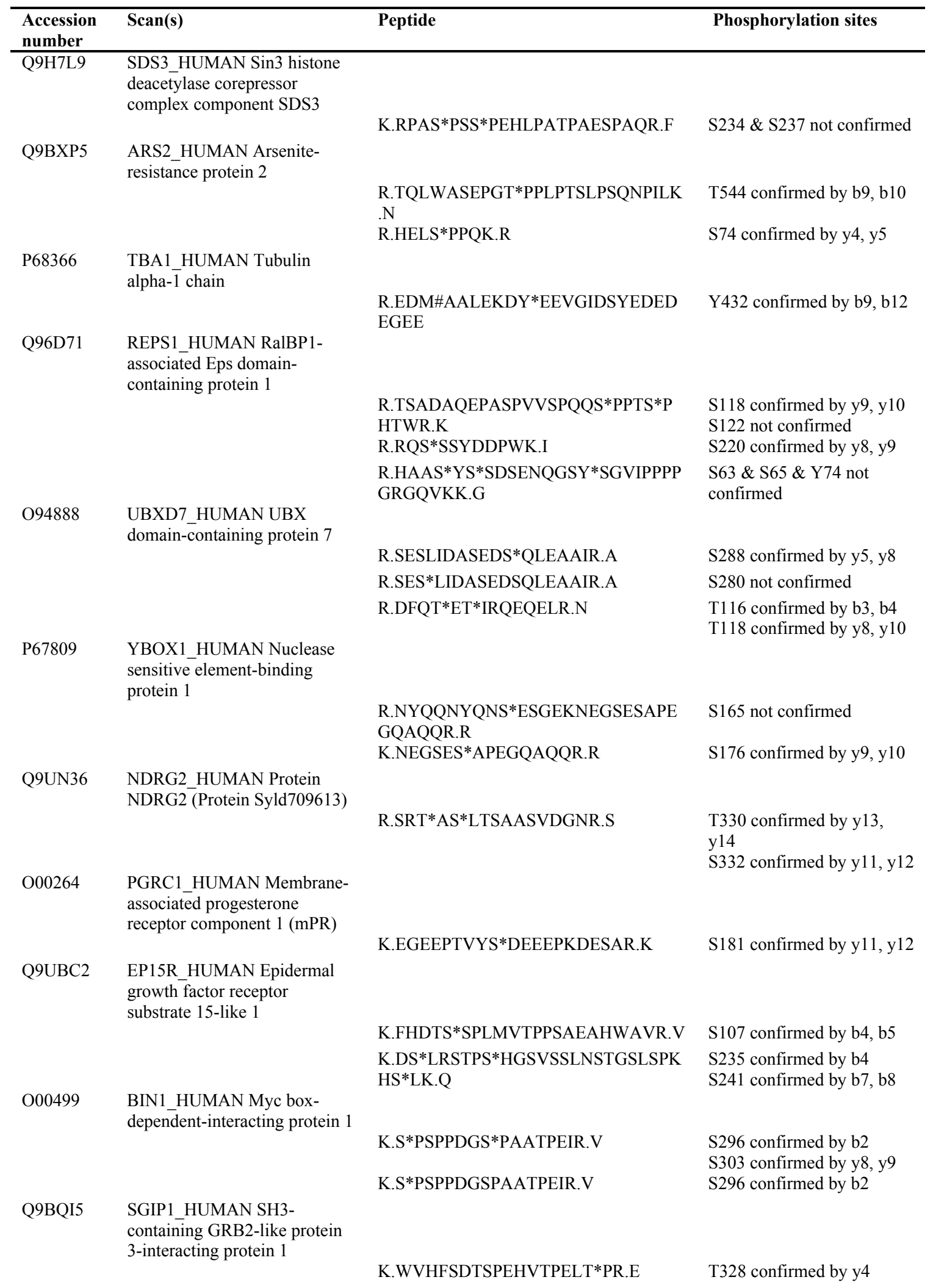


Table A-2 (continued).

\begin{tabular}{|c|c|c|c|}
\hline $\begin{array}{l}\text { Accession } \\
\text { number }\end{array}$ & Scan(s) & Peptide & Phosphorylation sites \\
\hline \multirow[t]{2}{*}{ Q9ULM3 } & $\begin{array}{l}\text { YETS2_HUMAN YEATS } \\
\text { domain-containing protein } 2\end{array}$ & & \\
\hline & & K.T*EES*S*ELGNYVIK.I & $\begin{array}{l}\text { T1131 confirmed by b3 } \\
\text { S1134 \& S1135 not } \\
\text { confirmed }\end{array}$ \\
\hline \multirow[t]{2}{*}{ Q9Y233 } & $\begin{array}{l}\text { PDE10_HUMAN cAMP and } \\
\text { cAMP-inhibited cGMP } 3 ', 5^{\prime}- \\
\text { cyclic phosphodiesterase } 10 \mathrm{~A}\end{array}$ & & \\
\hline & & K.S*QHLTGLTDEKVK.A & S8 confirmed by b3 \\
\hline \multirow[t]{4}{*}{ Q15054 } & $\begin{array}{l}\text { DPOD3_HUMAN DNA } \\
\text { polymerase subunit delta } 3 \\
\text { (DNA polymerase subunit delta } \\
\text { p66) }\end{array}$ & & \\
\hline & & K.TEPEPPSVKS*S*SGENK.R & S374 \& S375 not confirmed \\
\hline & & K.S*KLAVT*ASIHVY*SIQK.A & $\begin{array}{l}\text { S90 confirmed by b3 } \\
\text { T95 confirmed by b3, b6 } \\
\text { Y101 not confirmed }\end{array}$ \\
\hline & & R.VALS*DDETKETENMR.K & S307 confirmed by b3, b5 \\
\hline \multirow[t]{2}{*}{ Q9UNZ2 } & $\begin{array}{l}\text { NSF1C_HUMAN NSFL1 } \\
\text { cofactor p47 (p97 cofactor p47) }\end{array}$ & & \\
\hline & & R.KKS*PNELVDDLFK.G & S114 confirmed by y10, y11 \\
\hline \multirow[t]{3}{*}{ Q9Y6W5 } & $\begin{array}{l}\text { WASF2_HUMAN Wiskott- } \\
\text { Aldrich syndrome protein } \\
\text { family member } 2\end{array}$ & & \\
\hline & & K.RS*SVVSPSHPPPAPPLGSPPGPK.P & S292 not confirmed \\
\hline & & R.S*S*VVS*PSHPPPAPPLGSPPGPK.P & $\begin{array}{l}\text { S292 \& S293 \& S296 not } \\
\text { confirmed }\end{array}$ \\
\hline \multirow[t]{2}{*}{ Q9BQA1 } & $\begin{array}{l}\text { MEP50_HUMAN } \\
\text { Methylosome protein } 50 \\
\text { (MEP50 protein) (WD repeat } \\
\text { protein 77) }\end{array}$ & & \\
\hline & & R.KET*PPPLVPPAAR.E & T5 confirmed by y10, y11 \\
\hline \multirow[t]{2}{*}{ P49407 } & $\begin{array}{l}\text { ARRB1_HUMAN Beta- } \\
\text { arrestin-1 (Arrestin beta 1) }\end{array}$ & & \\
\hline & & R.KDLFVANVQS*FPPAPEDK.K & S86 confirmed by y8, y10 \\
\hline Q9P2B4 & $\begin{array}{l}\text { CT2NL_HUMAN CTTNBP2 } \\
\text { N-terminal-like protein } \\
7470\end{array}$ & K.EQKKLS*SQLEEER.S & S165 confirmed by b4, b6 \\
\hline \multirow[t]{2}{*}{ Q5JTD0 } & $\begin{array}{l}\text { TJAP1_HUMAN Tight } \\
\text { junction-associated protein } 1 \\
\text { (Tight junction protein } 4 \text { ) }\end{array}$ & & \\
\hline & & R.KDS*LTQAQEQGNLLN & S545 confirmed by b3 \\
\hline \multirow[t]{2}{*}{ P24043 } & $\begin{array}{l}\text { LAMA2_HUMAN Laminin } \\
\text { subunit alpha-2 precursor } \\
\text { (Laminin M chain) }\end{array}$ & & \\
\hline & & R.NS*HIAIAFDDT*KVKNR.L & $\begin{array}{l}\text { S2772 confirmed by b4 } \\
\text { T2781 confirmed by y } 5 \text {, y } 6\end{array}$ \\
\hline \multirow[t]{2}{*}{ Q6Y7W6 } & $\begin{array}{l}\text { PERQ2_HUMAN PERQ } \\
\text { amino acid-rich with GYF } \\
\text { domain-containing protein } 2\end{array}$ & & \\
\hline & & R.PGT*PSDHQSQEASQFER.K & T382 confirmed by b3 \\
\hline \multirow[t]{3}{*}{ Q86WB0 } & $\begin{array}{l}\text { NIPA_HUMAN Nuclear- } \\
\text { interacting partner of ALK }\end{array}$ & & \\
\hline & & K.QSSQPAETDS*MSLSEKS*RK.V & S479 \& S486 not confirmed \\
\hline & & R.SMGTGDT*PGLEVPSS*PLR.K & T387 \& S395 not confirmed \\
\hline
\end{tabular}


Table A-2 (continued).

\begin{tabular}{|c|c|c|c|}
\hline $\begin{array}{l}\text { Accession } \\
\text { number }\end{array}$ & Scan(s) & Peptide & Phosphorylation sites \\
\hline \multirow[t]{2}{*}{ Q12955 } & $\begin{array}{l}\text { ANK3_HUMAN Ankyrin-3 } \\
\text { (ANK-3) (Ankyrin-G) }\end{array}$ & & \\
\hline & & R.RQS*FASLALR.K & S1459 confirmed by y7, y8 \\
\hline \multirow[t]{2}{*}{ Q15642 } & $\begin{array}{ll}\text { CIP4_HUMAN } \\
\text { interacting protein } 4\end{array}$ & & \\
\hline & & R.APSDS*SLGTPSDGRPELR.G & S298 not confirmed \\
\hline \multirow[t]{2}{*}{ O15173 } & $\begin{array}{l}\text { PGRC2_HUMAN Membrane- } \\
\text { associated progesterone } \\
\text { receptor component } 2\end{array}$ & & \\
\hline & & R.LLKPGEEPSEY*TDEEDTK.D & Y210 not confirmed \\
\hline \multirow[t]{3}{*}{ Q8N5S9 } & $\begin{array}{l}\text { KKCC1_HUMAN } \\
\text { Calcium/calmodulin-dependent } \\
\text { protein kinase kinase } 1\end{array}$ & & \\
\hline & & R.KLS*LQER.P & S74 confirmed by y4, y 5 \\
\hline & & R.LIPS*WTT*VILVKS*MLRK.R & $\begin{array}{l}\text { S443 not confirmed } \\
\text { T446 confirmed by b6, b7 } \\
\text { S452 confirmed by b10, b13 }\end{array}$ \\
\hline \multirow[t]{2}{*}{ P30086 } & $\begin{array}{l}\text { PEBP1_HUMAN } \\
\text { Phosphatidylethanolamine- } \\
\text { binding protein } 1 \text { (PEBP-1) }\end{array}$ & & \\
\hline & & K.NRPTS*ISWDGLDSGK.L & S52 confirmed by y10, y11 \\
\hline \multirow[t]{2}{*}{ Q9UQB8 } & $\begin{array}{l}\text { BAIP2_HUMAN Brain- } \\
\text { specific angiogenesis inhibitor } \\
\text { 1-associated protein 2 }\end{array}$ & & \\
\hline & & K.LSDSYSNT*LPVR.K & T340 confirmed by y4, y6 \\
\hline \multirow[t]{2}{*}{$\mathrm{O} 15530$} & $\begin{array}{l}\text { PDPK1_HUMAN } \\
\text { phosphoinositide-dependent } \\
\text { protein kinase 1 (hPDK1) }\end{array}$ & & \\
\hline & & R.ANS*FVGTAQYVSPELLTEK.S & S241 confirmed by b4, b5 \\
\hline \multirow[t]{2}{*}{ Q9H788 } & 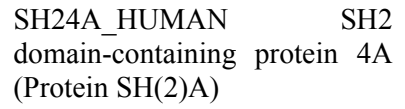 & & \\
\hline & & R.TLS*SSAQEDIIR.W & S315 confirmed by y9, y10 \\
\hline \multirow[t]{3}{*}{ P18615 } & $\begin{array}{l}\text { NELFE_HUMAN } \\
\text { elongation factor E (NELF-E) } \\
\text { (RD protein) }\end{array}$ & & \\
\hline & & R.SIS*ADDDLQESSR.R & S115 confirmed by y9, y11 \\
\hline & & $\begin{array}{l}\text { K.QPMLDAAT*GKSVWGS*LAVQNS* } \\
\text { PKGCHR.D } \\
\text { R.S*LSEQPVMDTATATEQAK.Q }\end{array}$ & $\begin{array}{l}\text { T340 \& S347 \& S353 not } \\
\text { confirmed } \\
\text { S49 not confirmed }\end{array}$ \\
\hline \multirow[t]{4}{*}{ Q9H1B7 } & $\begin{array}{l}\text { CN004_HUMAN } \\
\text { C14orf4 }\end{array}$ & & \\
\hline & & R.KAS*PEPPDSAEGALK.L & S547 confirmed by b3 \\
\hline & & R.RNS*SS*PVSPASVPGQR.R & $\begin{array}{l}\text { S657 confirmed by b2, b3 } \\
\text { S659 confirmed by y11, y12 }\end{array}$ \\
\hline & & R.QSPNSSSAAAS*VAS*RR.G & $\begin{array}{l}\text { S224 confirmed by y5, y } 7 \\
\text { S227 confirmed by b13, b15 }\end{array}$ \\
\hline \multirow[t]{3}{*}{ Q12857 } & $\begin{array}{l}\text { NFIA_HUMAN Nuclear factor } \\
1 \text { A-type (Nuclear factor } 1 / A \text { ) }\end{array}$ & & \\
\hline & & $\begin{array}{l}\text { R.S*PGSGSQSSGWHEVEPGMPSPTTL } \\
\text { K.K }\end{array}$ & S300 confirmed by b3 \\
\hline & & R.RSLPSTS*ST*SSTKR.L & S273 \& T275 not confirmed \\
\hline \multirow[t]{2}{*}{ UE93 } & $\begin{array}{l}\text { PI3R6_HUMAN } \\
\text { Phosphoinositide } \\
\text { regulatory subunit } 6\end{array}$ & & \\
\hline & & R.PS*GDGEM\#LPGVSRLHTAR.V & S401 confirmed by b3, y17 \\
\hline
\end{tabular}


Table A-2 (continued).

\begin{tabular}{|c|c|c|c|}
\hline $\begin{array}{l}\text { Accession } \\
\text { number }\end{array}$ & Scan(s) & Peptide & Phosphorylation sites \\
\hline \multirow[t]{6}{*}{$\mathrm{P} 21675$} & $\begin{array}{l}\text { TAF1_HUMAN Transcription } \\
\text { initiation factor TFIID subunit } \\
1\end{array}$ & & \\
\hline & & R.M\#LLAAGS*AASGNNHRDDDTAS & S1138 confirmed by b5, b9 \\
\hline & & VTSLNS*SATGR.C & S1158 not confirmed \\
\hline & & R.TDPMVTLSSILES*IINDM\#R.D & $\begin{array}{l}\text { S1391 confirmed by b12, } \\
\text { b14 }\end{array}$ \\
\hline & & R.VGT*TVHCDYLNR.P & T1360 not confirmed \\
\hline & & R.VGTTVHCDY*LNRPHK.S & Y1366 not confirmed \\
\hline \multirow[t]{2}{*}{ Q8N1N0 } & $\begin{array}{l}\text { CLC4F_HUMAN C-type lectin } \\
\text { domain family } 4 \text { member } F\end{array}$ & & \\
\hline & & K.SSFDNT*S*AEIQFLR.G & T313 \& S314 not confirmed \\
\hline \multirow[t]{7}{*}{ Q04726 } & $\begin{array}{l}\text { TLE3_HUMAN Transducin- } \\
\text { like enhancer protein } 3 \text { (ESG3) }\end{array}$ & & \\
\hline & & R.ESSANNSVS*PSESLR.A & S203 confirmed by y6, y7 \\
\hline & & K.DLGHNDKS*STPGLKSNTPT*PR.N & S310 confirmed by b7, b8 \\
\hline & & $\begin{array}{l}\text { K.SDDLVVDVSNEDPAT*PRVSPAHS* } \\
\text { PPENGLDK.A }\end{array}$ & $\begin{array}{l}\text { T259 confirmed by y15, } \\
\text { y17 }\end{array}$ \\
\hline & & & S267 not confirmed \\
\hline & & R.VS*PAHS*PPENGLDK.A & S263 not confirmed \\
\hline & & & S267 not confirmed \\
\hline \multirow[t]{2}{*}{ Q5VV41 } & $\begin{array}{l}\text { ARHGG_HUMAN Rho } \\
\text { guanine nucleotide exchange } \\
\text { factor } 16\end{array}$ & & \\
\hline & & R.HQS*FGAAVLSR.E & S107 confirmed by y8, y9 \\
\hline \multirow[t]{2}{*}{ Q12906 } & $\begin{array}{l}\text { ILF3_HUMAN Interleukin } \\
\text { enhancer-binding factor } 3\end{array}$ & & \\
\hline & & K.RPMEEDGEEKS*PSK.K & S382 confirmed by y3, y 5 \\
\hline \multirow[t]{2}{*}{ Q96IT1 } & $\begin{array}{l}\text { ZN496_HUMAN Zinc finger } \\
\text { protein } 496\end{array}$ & & \\
\hline & & $\begin{array}{l}\text { R.PPS*QLS*GDPVLQDAFLLQEENVR } \\
\text {.D }\end{array}$ & $\begin{array}{l}\text { S185 confirmed by b4 } \\
\text { S188 not confirmed }\end{array}$ \\
\hline \multirow[t]{2}{*}{ Q9UN86 } & $\begin{array}{l}\text { G3BP2_HUMAN Ras GTPase- } \\
\text { activating protein-binding } \\
\text { protein } 2 \text { (G3BP-2) }\end{array}$ & & \\
\hline & & K.STT*PPPAEPVSLPQEPPK.A & T227 not confirmed \\
\hline \multirow[t]{2}{*}{ P55317 } & $\begin{array}{l}\text { HNF3A_HUMAN Hepatocyte } \\
\text { nuclear factor 3-alpha }\end{array}$ & & \\
\hline & & $\begin{array}{l}\text { K.TGQLEGAPAPGPAAS*PQTLDHSG } \\
\text { ATATGGASELK.T }\end{array}$ & S332 confirmed by b10, b16 \\
\hline \multirow[t]{2}{*}{ P35579 } & $\begin{array}{l}\text { MYH9_HUMAN Myosin-9 } \\
\text { (Myosin heavy chain 9) }\end{array}$ & & \\
\hline & & K.KMEDS*VGCLET*AEEVK.R & $\begin{array}{l}\text { S1376 confirmed by y11, } \\
\text { y14 } \\
\text { T1382 confirmed by b9, } \\
\text { b12 }\end{array}$ \\
\hline \multirow[t]{3}{*}{ P08621 } & $\begin{array}{l}\text { RU17_HUMAN U1 small } \\
\text { nuclear ribonucleoprotein } 70 \\
\mathrm{kDa}(\mathrm{U} 1 \text { snRNP } 70 \mathrm{kDa})\end{array}$ & & \\
\hline & & R.YDERPGPS*PLPHR.D & S226 confirmed by y5, y7 \\
\hline & & R.GGGGGQDNGLEGLGNDS*R.D & S410 confirmed by y2, y4 \\
\hline \multirow[t]{3}{*}{ Q13315 } & $\begin{array}{l}\text { ATM_HUMAN Serine-protein } \\
\text { kinase ATM (Ataxia } \\
\text { telangiectasia mutated) }\end{array}$ & & \\
\hline & & K.RSLES*VYS*LYPTLSR.L & $\begin{array}{l}\text { S2 } 165 \text { confirmed by b3, b5 } \\
\text { S2168 confirmed by b7, b8 }\end{array}$ \\
\hline & & K.M\#LQPIT*R.L & T2031 confirmed by b5, b6 \\
\hline
\end{tabular}


Table A-2 (continued).

\begin{tabular}{|c|c|c|c|}
\hline $\begin{array}{l}\text { Accession } \\
\text { number }\end{array}$ & Scan(s) & Peptide & Phosphorylation sites \\
\hline \multirow[t]{3}{*}{ Q9UKM9 } & $\begin{array}{l}\text { RALY_HUMAN RNA-binding } \\
\text { protein Raly }\end{array}$ & & \\
\hline & & R.GRLS*PVPVPR.A & S135 confirmed by y6, y7 \\
\hline & & R.AAS*AIYSGYIFDYDYYR.D & S206 confirmed by b4 \\
\hline \multirow[t]{2}{*}{ Q9BSG1 } & $\begin{array}{l}\text { ZNF2_HUMAN Zinc finger } \\
\text { protein } 2 \text { (Zinc finger 2.2) } \\
\text { (Zinc finger protein 661) }\end{array}$ & & \\
\hline & & K.VFSSKS*SVIQHQR.R & S410 not confirmed \\
\hline \multirow[t]{2}{*}{ Q6ZMT1 } & $\begin{array}{l}\text { STAC2_HUMAN SH3 and } \\
\text { cysteine-rich domain- } \\
\text { containing protein } 2\end{array}$ & & \\
\hline & & R.SSFSS*T*SESPTRSLSER.D & S224 \& T225 not confirmed \\
\hline \multirow[t]{3}{*}{ P85037 } & $\begin{array}{l}\text { FOXK1_HUMAN Forkhead } \\
\text { box protein K1 (Myocyte } \\
\text { nuclear factor) }\end{array}$ & & \\
\hline & & R.S*APAS*PTHPGLMSPR.S & $\begin{array}{l}\text { S416 confirmed by b4 } \\
\text { S420 confirmed by y } 10 \text {, y11 }\end{array}$ \\
\hline & & R.EGS*PIPHDPEFGSK.L & S445 confirmed by b3 \\
\hline \multirow[t]{2}{*}{ O15062 } & $\begin{array}{l}\text { ZBTB5 HUMAN Zinc finger } \\
\text { and BTB domain-containing } \\
\text { protein } 5\end{array}$ & & \\
\hline & & K.HYLTTRTLPMS*PPSER.V & S127 confirmed by b9, b11 \\
\hline \multirow[t]{2}{*}{ Q9H2M9 } & $\begin{array}{l}\text { RBGPR_HUMAN Rab3 } \\
\text { GTPase-activating protein non- } \\
\text { catalytic subunit }\end{array}$ & & \\
\hline & & K.QDFS*PEVLKLANEER.D & S952 confirmed by b3, b6 \\
\hline \multirow[t]{5}{*}{ P22626 } & $\begin{array}{l}\text { ROA2_HUMAN } \\
\text { Heterogeneous nuclear } \\
\text { ribonucleoproteins A2/B1 }\end{array}$ & & \\
\hline & & $\begin{array}{l}\text { R.GFGDGYNGYGGGPGGGNFGGS*P } \\
\text { GYGGGR.G }\end{array}$ & S259 confirmed by y7, y8 \\
\hline & & & S259 confirmed by y6, y7 \\
\hline & & R.GGNFGFGDS*R.G & S212 confirmed by b8, b9 \\
\hline & & $\begin{array}{l}\text { R.NMGGPYGGGNYGPGGS*GGSGGY } \\
\text { GGR.S }\end{array}$ & S341 confirmed by y9, y10 \\
\hline \multirow[t]{4}{*}{ Q9UHD8 } & $\begin{array}{l}\text { SEPT9_HUMAN Septin-9 } \\
\text { (MLL septin-like fusion } \\
\text { protein) }\end{array}$ & & \\
\hline & & R.S*FEVEEVETPNSTPPR.R & S30 confirmed by b3 \\
\hline & & K.RS*FEVEEVETPNSTPPR.R & \\
\hline & & R.S*FEVEEVET*PNSTPPR.R & $\begin{array}{l}\text { S38 confirmed by b6 } \\
\text { T30 confirmed by y } 7 \text {, y8 }\end{array}$ \\
\hline \multirow[t]{3}{*}{ Q8TEA8 } & $\begin{array}{l}\text { DTD1_HUMAN Probable D- } \\
\text { tyrosyl-tRNA(Tyr) deacylase } 1\end{array}$ & & \\
\hline & & R.S*ASSGAEGDVSSEREP & S194 confirmed by b2 \\
\hline & & $\begin{array}{l}\text { K.EDRS*ASSGAEGDVS*S*EREP } \\
\text { K.EDRSAS*SGAEGDVS*S*ER.E }\end{array}$ & $\begin{array}{l}\text { S } 194 \text { confirmed by b3, b4 } \\
\text { S204 \& S205 not confirmed } \\
\text { S196 not confirmed } \\
\text { S204 confirmed by b13, b14 } \\
\text { S205 confirmed by b14, b16 }\end{array}$ \\
\hline \multirow[t]{2}{*}{ Q5JSZ5 } & $\begin{array}{l}\text { K0515_HUMAN } \\
\text { Uncharacterized protein } \\
\text { KIAA0515 }\end{array}$ & & \\
\hline & & K.LKFS*DDEEEEEVVK.D & S388 confirmed by y $10, \mathrm{y} 11$ \\
\hline O95460 & $\begin{array}{l}\text { MATN4_HUMAN Matrilin-4 } \\
\text { precursor }\end{array}$ & & S70 nt onfirmed \\
\hline
\end{tabular}


Table A-2 (continued).

\begin{tabular}{|c|c|c|c|}
\hline $\begin{array}{l}\text { Accession } \\
\text { number }\end{array}$ & Scan(s) & Peptide & Phosphorylation sites \\
\hline \multirow[t]{2}{*}{$\mathrm{P} 25205$} & $\begin{array}{l}\text { MCM3_HUMAN DNA } \\
\text { replication licensing factor } \\
\text { MCM3 }\end{array}$ & & \\
\hline & & $\begin{array}{l}\text { K.DGDSYDPYDFS*DTEEEMPQVHTP } \\
\text { K.T }\end{array}$ & S711 confirmed by y 13, y14 \\
\hline \multirow[t]{2}{*}{ O95248 } & $\begin{array}{l}\text { MTMR5_HUMAN SET- } \\
\text { binding factor } 1 \text { (Sbf1) } \\
\text { (Myotubularin-related protein } \\
\text { 5) }\end{array}$ & & \\
\hline & & R.RTTVPSGPPMT*AILER.C & T546 confirmed by y 5 , y 6 \\
\hline \multirow[t]{5}{*}{ Q6P6C2 } & $\begin{array}{l}\text { ALKB5_HUMAN Alkylated } \\
\text { repair protein alkB homolog } 5\end{array}$ & & \\
\hline & & R.RGS*FSSENYWR.K & S361 confirmed by y8, y9 \\
\hline & & K.YQEDS*DPERSDYEEQQLQK.E & S64 not confirmed \\
\hline & & R.GS*FSSENYWR.K & $\mathrm{S} 361$ \\
\hline & & $\begin{array}{l}\text { R.LET*KS*LSSSVLPPSYASDRLSGN } \\
\text { NR.D }\end{array}$ & T294 \& S296 not confirmed \\
\hline \multirow[t]{2}{*}{ Q9NX63 } & $\begin{array}{l}\text { CHCH3_HUMAN Coiled-coil- } \\
\text { helix-coiled-coil-helix domain- } \\
\text { containing protein } 3\end{array}$ & & \\
\hline & & R.YS*GAYGASVSDEELK.R & S50 confirmed by y 14, b2 \\
\hline \multirow[t]{2}{*}{ P21796 } & $\begin{array}{l}\text { VDAC1_HUMAN Voltage- } \\
\text { dependent anion-selective } \\
\text { channel protein } 1 \text { (VDAC-1) }\end{array}$ & & \\
\hline & & K.LTFDSSFS*PNTGK.K & S104 confirmed by y 5 , y 6 \\
\hline \multirow[t]{2}{*}{ Q76KP1 } & $\begin{array}{l}\text { B4GN4_HUMAN N-acetyl- } \\
\text { beta-glucosaminyl-glycoprotein } \\
\text { 4-beta-N-acetylgalactosam }\end{array}$ & & \\
\hline & & R.TLGPAAPTVDS*NLS*SEAR.P & $\begin{array}{l}\text { S616 confirmed by b10, b12 } \\
\text { S619 not confirmed }\end{array}$ \\
\hline \multirow[t]{3}{*}{ Q13573 } & $\begin{array}{l}\text { SNW1_HUMAN SNW } \\
\text { domain-containing protein } 1 \\
\text { (Nuclear protein SkiP) }\end{array}$ & & \\
\hline & & R.GPPS*PPAPVMHS*PSR.K & $\begin{array}{l}\text { S224 confirmed by y11, } \\
\text { y12 }\end{array}$ \\
\hline & & & S232 confirmed by y $3, y 4$ \\
\hline \multirow[t]{2}{*}{ Q9NPH0 } & $\begin{array}{l}\text { PPA6_HUMAN } \\
\text { Lysophosphatidic acid } \\
\text { phosphatase type } 6 \text { precursor }\end{array}$ & & \\
\hline & & R.GRRQTAS*LQPGIS*EDLK.K & $\begin{array}{l}\text { S223 confirmed by y10, y11 } \\
\text { S229 confirmed by b12, b14 }\end{array}$ \\
\hline \multirow[t]{2}{*}{ Q6WKZ4 } & $\begin{array}{l}\text { RFIP1_HUMAN Rab11 } \\
\text { family-interacting protein } 1 \\
\text { (Rab11-FIP1) }\end{array}$ & & \\
\hline & & K.HLFSS*TENLAAGSWK.E & S357 confirmed by y10, y11 \\
\hline \multirow[t]{3}{*}{ P23193 } & $\begin{array}{l}\text { TCEA1_HUMAN } \\
\text { Transcription elongation factor } \\
\text { A protein } 1\end{array}$ & & \\
\hline & & K.EPAITSQNS*PEAR.E & S100 confirmed by y 4, y5 \\
\hline & & K.KKEPAITSQNS*PEAR.E & \\
\hline \multirow[t]{2}{*}{ O75446 } & $\begin{array}{l}\text { SAP30_HUMAN Histone } \\
\text { deacetylase complex subunit } \\
\text { SAP30 }\end{array}$ & & \\
\hline & & K.DTLTYFIY*SVKNDK.N & Y202 confirmed by b7, b8 \\
\hline \multirow[t]{2}{*}{ Q07666 } & $\begin{array}{l}\text { SAM68_HUMAN KH domain-c } \\
\text { transduction-associated protein } 1\end{array}$ & ntaining, RNA-binding, signal & \\
\hline & & R.SGS*MDPSGAHPSVR.Q & S20 not confirmed \\
\hline
\end{tabular}


Table A-2 (continued).

\begin{tabular}{|c|c|c|c|}
\hline $\begin{array}{l}\text { Accession } \\
\text { number }\end{array}$ & Scan(s) & Peptide & Phosphorylation sites \\
\hline \multirow[t]{2}{*}{ Q9UHR4 } & $\begin{array}{l}\text { BI2L1_HUMAN Brain-specific } \\
\text { angiogenesis inhibitor 1- } \\
\text { associated protein 2-like } \\
\text { protein } 1\end{array}$ & & \\
\hline & & K.TPASTPVSGT*PQAS*PMIER.S & $\begin{array}{l}\text { T257 confirmed by y9, y11 } \\
\text { S261 confirmed by y5, y } 6\end{array}$ \\
\hline \multirow[t]{3}{*}{ P09651 } & $\begin{array}{l}\text { ROA1_HUMAN } \\
\text { Heterogeneous nuclear } \\
\text { ribonucleoprotein A1 }\end{array}$ & & \\
\hline & & K.S*ESPKEPEQLR.K & S4 confirmed by b2 \\
\hline & & R.SS*GPYGGGGQYFAK.P & S338 not confirmed \\
\hline \multirow[t]{3}{*}{ Q9Y446 } & PKP3 HUMAN Plakophilin-3 & & \\
\hline & & R.LS*SGFDDIDLPSAVK.Y & S313 confirmed by b2, y14 \\
\hline & & R.ADYDTLS*LR.S & S180 confirmed by y2, y3 \\
\hline \multirow[t]{2}{*}{ Q96T21 } & $\begin{array}{l}\text { SEBP2_HUMAN SECIS- } \\
\text { binding protein } 2\end{array}$ & & \\
\hline & & K.S*ARGSHHLSIYAENSLK.S & S174 confirmed by b4 \\
\hline \multirow[t]{2}{*}{ P52948 } & $\begin{array}{l}\text { NUP98_HUMAN Nuclear pore } \\
\text { complex protein Nup98-Nup } 96 \\
\text { precursor }\end{array}$ & & \\
\hline & & R.DSENLAS*PSEYPENGER.F & S623 confirmed by y 10, y 11 \\
\hline \multirow[t]{3}{*}{ P35527 } & $\begin{array}{l}\text { K1C9_HUMAN Keratin, type I } \\
\text { cytoskeletal } 9 \text { (Cytokeratin-9) }\end{array}$ & & \\
\hline & & $\begin{array}{l}\text { R.GSRGGSGGSY*GGGGS*GGGYGG } \\
\text { GSGS*R.G }\end{array}$ & $\begin{array}{l}\text { Y } 497 \text { \& S502 \& S512 not } \\
\text { confirmed }\end{array}$ \\
\hline & & $\begin{array}{l}\text { R.GSRGGSGGS*Y*GGGGSGGGYGG } \\
\text { GS*GSR.G }\end{array}$ & $\begin{array}{l}\text { S496 \& Y497 \& S510 not } \\
\text { confirmed }\end{array}$ \\
\hline \multirow[t]{5}{*}{ P51991 } & ROA3_HUMAN & & \\
\hline & $\begin{array}{l}\text { Heterogeneous nuclear } \\
\text { ribonucleoprotein A3 }\end{array}$ & & \\
\hline & & R.SSGS*PYGGGYGSGGGSGGYGSR.R & S358 confirmed by y 18, y 20 \\
\hline & & $\begin{array}{l}\text { R.SSGSPY*GGGYGS*GGGS*GGYGS } \\
\text { R.R }\end{array}$ & $\begin{array}{l}\text { Y360 \& S366 \& S370 not } \\
\text { confirmed }\end{array}$ \\
\hline & & $\begin{array}{l}\text { R.SS*GSPY*GGGYGSGGGS*GGYGS } \\
\text { RRF }\end{array}$ & $\begin{array}{l}\text { S356 \& Y360 not } \\
\text { confirmed }\end{array}$ \\
\hline \multirow[t]{2}{*}{ Q6P5R6 } & $\begin{array}{l}\text { RL22L_HUMAN Ribosomal } \\
\text { protein L22-like } 1\end{array}$ & & \\
\hline & & R.YFQISQDEDES*ESED & S1 18 confirmed by b10, b12 \\
\hline \multirow[t]{4}{*}{ Q96T37 } & RBM15_HUMAN Putative & & \\
\hline & & R.SLS*PGGAALGYR.D & S294 confirmed by y 9 , y10 \\
\hline & & K.NS*S*GGGESRSSSRGGGGES*R.S & $\begin{array}{l}\text { S134 \& S135 not confirmed } \\
\text { S151 confirmed by b18, b19 }\end{array}$ \\
\hline & & $\begin{array}{l}\text { R.SRS*PLDKDTY*PPSASVVGASVGG } \\
\text { HR.H }\end{array}$ & $\begin{array}{l}\text { S259 \& Y266 not } \\
\text { confirmed }\end{array}$ \\
\hline \multirow[t]{2}{*}{ Q9UQF2 } & $\begin{array}{l}\text { JIP1_HUMAN C-jun-amino- } \\
\text { terminal kinase-interacting } \\
\text { protein } 1\end{array}$ & & \\
\hline & & K.QFVEYT*CPTEDIYLE & T702 confirmed by y9, y10 \\
\hline \multirow[t]{2}{*}{ O96013 } & $\begin{array}{l}\text { PAK4_HUMAN } \\
\text { Serine/threonine-protein kinase } \\
\text { PAK 4(2)1-activated kinase 4) }\end{array}$ & & \\
\hline & & R.PFNT*YPR.A & T207 confirmed by y3, y5 \\
\hline \multirow[t]{2}{*}{ Q15233 } & $\begin{array}{l}\text { NONO_HUMAN Non-POU } \\
\text { domain-containing octamer- } \\
\text { binding protein }\end{array}$ & & \\
\hline & & R.FGQAATMEGIGAIGGT*PPAFNR.A & T450 confirmed by y6, y8 \\
\hline
\end{tabular}


Table A-2 (continued).

\begin{tabular}{|c|c|c|c|}
\hline $\begin{array}{l}\text { Accession } \\
\text { number }\end{array}$ & Scan(s) & Peptide & Phosphorylation sites \\
\hline \multirow[t]{2}{*}{ Q96PK6 } & $\begin{array}{l}\text { RBM14_HUMAN RNA- } \\
\text { binding protein } 14\end{array}$ & & \\
\hline & & R.RLS*ESQLSFR.R & S618 confirmed by y $7, y 8$ \\
\hline \multirow[t]{2}{*}{ P21291 } & $\begin{array}{l}\text { CSRP1_HUMAN Cysteine and } \\
\text { glycine-rich protein } 1\end{array}$ & & \\
\hline & & K.GFGFGQGAGALVHS*E & S192 confirmed by y3 \\
\hline \multirow[t]{2}{*}{ Q9P2K5 } & $\begin{array}{l}\text { MYEF2_HUMAN Myelin } \\
\text { expression factor } 2 \text { (MyEF-2) } \\
\text { (MST156) }\end{array}$ & & \\
\hline & & $\begin{array}{l}\text { K.AEVPGATGGDS*PHLQPAEPPGEP } \\
\text { R.R }\end{array}$ & S17 confirmed by y13, y14 \\
\hline \multirow[t]{2}{*}{ Q96J84 } & $\begin{array}{l}\text { KIRR1_HUMAN Kin of } \\
\text { IRRE-like protein } 1 \text { precursor }\end{array}$ & & \\
\hline & & R.FSQEPADQT*VVAGQR.A & T31 confirmed by b7, b11 \\
\hline \multirow[t]{2}{*}{ Q9UHL0 } & $\begin{array}{l}\text { DDX25_HUMAN ATP- } \\
\text { dependent RNA helicase } \\
\text { DDX25 (DEAD box protein } \\
\text { 25) }\end{array}$ & & \\
\hline & & $\begin{array}{l}\text { K.WLTVEMIQDGHQVS*LLS*GELTV } \\
\text { EQR.A }\end{array}$ & S366 \& S369 not confirmed \\
\hline \multirow[t]{2}{*}{ Q5T8P6 } & $\begin{array}{l}\text { RBM26_HUMAN RNA- } \\
\text { binding protein } 26\end{array}$ & & \\
\hline & & K.T*QMQKELLDT*ELDLYKK.M & T808 \& T817 not confirmed \\
\hline \multirow[t]{2}{*}{ Q9Y3Q4 } & $\begin{array}{l}\text { HCN4_HUMAN } \\
\text { Potassium/sodium } \\
\text { hyperpolarization-activated } \\
\text { cyclic nucleotide-gated channel } \\
4\end{array}$ & & \\
\hline & & R.RGTPPLT*PGR.L & T1075 confirmed by y2, y6 \\
\hline \multirow[t]{2}{*}{ Q8TEW8 } & $\begin{array}{l}\text { PAR3L_HUMAN Partitioning- } \\
\text { defective } 3 \text { homolog B (PAR3- } \\
\text { beta) }\end{array}$ & & \\
\hline & & R.T*QEELVAMLRST*K.Q & $\begin{array}{l}\text { T445 confirmed by b3 } \\
\text { TT456 not confirmed }\end{array}$ \\
\hline \multirow[t]{2}{*}{ O60825 } & $\begin{array}{l}\text { F262_HUMAN 6- } \\
\text { phosphofructo-2- } \\
\text { kinase/fructose-2,6- } \\
\text { biphosphatase } 2\end{array}$ & & \\
\hline & & R.RNS*FTPLSSSNTIR.R & S446 confirmed by b2, b3 \\
\hline \multirow[t]{2}{*}{ Q96JC9 } & $\begin{array}{l}\text { EAF1_HUMAN ELL- } \\
\text { associated factor } 1\end{array}$ & & \\
\hline & & K.T*SPLKDNPS*PEPQLDDIKR.E & $\begin{array}{l}\text { T158 not confirmed } \\
\text { S165 confirmed by y } 8, \text { y11 }\end{array}$ \\
\hline \multirow[t]{2}{*}{ O43583 } & $\begin{array}{l}\text { DENR_HUMAN Density- } \\
\text { regulated protein (DRP) }\end{array}$ & & \\
\hline & & $\begin{array}{l}\text { K.LTVENS*PKQEAGISEGQGTAGEE } \\
\text { EEK.K }\end{array}$ & S73 confirmed by b5, b6 \\
\hline \multirow[t]{2}{*}{ Q9C0C2 } & $\begin{array}{l}\text { TB182_HUMAN } 182 \mathrm{kDa} \\
\text { tankyrase 1-binding protein }\end{array}$ & & \\
\hline & & R.SPS*QDFSFIEDTEILDSAMYR.S & S1554 confirmed by b3 \\
\hline \multirow[t]{4}{*}{ O14745 } & $\begin{array}{l}\text { NHERF_HUMAN Ezrin- } \\
\text { radixin-moesin-binding } \\
\text { phosphoprotein } 50 \text { (EBP50) }\end{array}$ & & \\
\hline & & R.S*ASSDTSEELNSQDSPPK.Q & S288 not confirmed \\
\hline & & R.SAS*SDTSEELNSQDSPPK.Q & S290 not confirmed \\
\hline & & R.EALAEAALES*PRPALVR.S & S280 confirmed by y $7, y 8$ \\
\hline
\end{tabular}


Table A-2 (continued).

\begin{tabular}{|c|c|c|c|}
\hline $\begin{array}{l}\text { Accession } \\
\text { number }\end{array}$ & Scan(s) & Peptide & Phosphorylation sites \\
\hline \multirow[t]{2}{*}{$\mathrm{P} 42858$} & $\begin{array}{l}\text { HD_HUMAN Huntingtin } \\
\text { (Huntington disease protein) (HD } \\
\text { protein) }\end{array}$ & & \\
\hline & & R.VNHCLTICENIVAQS*VR.N & S116 confirmed by b13, b15 \\
\hline \multirow[t]{2}{*}{ Q9Y266 } & $\begin{array}{l}\text { NUDC_HUMAN Nuclear } \\
\text { migration protein nudC }\end{array}$ & & \\
\hline & & $\begin{array}{l}\text { K.NGSLDS*PGKQDTEEDEEEDEKD } \\
\text { K.G }\end{array}$ & S139 confirmed by b4, b9 \\
\hline \multirow[t]{2}{*}{ O00264 } & $\begin{array}{l}\text { PGRC1_HUMAN Membrane- } \\
\text { associated progesterone receptor } \\
\text { component } 1 \text { (mPR) }\end{array}$ & & \\
\hline & & K.EGEEPTVYS*DEEEPK.D & S181 not confirmed \\
\hline \multirow[t]{2}{*}{ Q9BVG4 } & $\begin{array}{l}\text { CX026_HUMAN UPF0368 } \\
\text { protein Cxorf26 }\end{array}$ & & \\
\hline & & K.GADS*GEEKEEGINR.E & S197 confirmed by b3, b5 \\
\hline \multirow[t]{2}{*}{ Q13286 } & $\begin{array}{l}\text { CLN3_HUMAN Battenin } \\
\text { (Protein CLN3) }\end{array}$ & & \\
\hline & & R.RFS*DS*EGEET*VPEPR.L & $\begin{array}{l}\text { S12 confirmed by y13 } \\
\text { S14 confirmed by y10, y11 } \\
\text { T19 confirmed by y5, y9 }\end{array}$ \\
\hline \multirow[t]{3}{*}{ Q5JTV8 } & $\begin{array}{l}\text { TOIP1_HUMAN Torsin-1A- } \\
\text { interacting protein } 1\end{array}$ & & \\
\hline & & R.LQQQHSEQPPLQPS*PVMTR.R & S143 not confirmed \\
\hline & & $\begin{array}{l}\text { K.VNFSEEGET*EEDDQDSSHSSVTT } \\
\text { VK.A }\end{array}$ & T220 confirmed by b7, b9 \\
\hline \multirow[t]{2}{*}{ Q13501 } & $\begin{array}{l}\text { SQSTM_HUMAN } \\
\text { Sequestosome-1 }\end{array}$ & & \\
\hline & & R.LTPVS*PESSSTEEK.S & S272 confirmed by y9, y10 \\
\hline \multirow[t]{3}{*}{ P20700 } & LMNB1_HUMAN Lamin-B1 & & \\
\hline & & R.LKLS*PS*PSSR.V & $\begin{array}{l}\text { S391 confirmed by b3, b4 } \\
\text { S393 confirmed by y4, y6 }\end{array}$ \\
\hline & & R.LKLS*PSPSSR.V & S391 confirmed by y6, y7 \\
\hline \multirow[t]{2}{*}{ Q8IXT5 } & $\begin{array}{l}\text { RB12B_HUMAN RNA-binding } \\
\text { protein } 12 \mathrm{~B}\end{array}$ & & \\
\hline & & R.FPPEDFRHS*PEDFR.R & S575 confirmed by y5, y6 \\
\hline \multirow[t]{2}{*}{ O14578 } & $\begin{array}{l}\text { CTRO_HUMAN Citron Rho- } \\
\text { interacting kinase (CRIK) }\end{array}$ & & \\
\hline & & R.TPLSQVNKVWDQS*S*V & $\begin{array}{l}\text { S2025 not confirmed } \\
\text { S2026 confirmed by y2 }\end{array}$ \\
\hline \multirow[t]{2}{*}{$\begin{array}{l}\text { Q8WWM } \\
7\end{array}$} & $\begin{array}{l}\text { ATX2L_HUMAN Ataxin-2-like } \\
\text { protein (Ataxin-2 domain } \\
\text { protein) }\end{array}$ & & \\
\hline & & K.EVDGLLTSEPMGS*PVSSK.T & S594 confirmed by y5, y7 \\
\hline \multirow[t]{2}{*}{ P62995 } & $\begin{array}{l}\text { TRA2B_HUMAN } \\
\text { Arginine/serine-rich-splicing } \\
\text { factor } 10 \text { (Transformer-2-beta) }\end{array}$ & & \\
\hline & & K.RPHT*PTPGIYMGR.P & T201 confirmed by b2, b4 \\
\hline \multirow[t]{3}{*}{ Q14157 } & $\begin{array}{l}\text { UBP2L_HUMAN Ubiquitin- } \\
\text { associated protein 2-like (Protein } \\
\text { NICE-4) }\end{array}$ & & \\
\hline & & R.RYPSSISSS*PQK.D & S609 confirmed by y3, y4 \\
\hline & & K.NPS*DSAVHSPFTK.R & S410 confirmed by b3 \\
\hline \multirow[t]{2}{*}{ P31749 } & $\begin{array}{l}\text { AKT1_HUMAN RAC-alpha } \\
\text { serine/threonine-protein kinase }\end{array}$ & & \\
\hline & & R.RPHFPQFS*YSASGTA & S473 confirmed by b7, b8 \\
\hline
\end{tabular}


Table A-2 (continued).

\begin{tabular}{|c|c|c|c|}
\hline $\begin{array}{l}\text { Accession } \\
\text { number }\end{array}$ & Scan(s) & Peptide & Phosphorylation sites \\
\hline \multirow{4}{*}{$\mathrm{O} 14646$} & CHD1_HUMAN & & \\
\hline & $\begin{array}{l}\text { Chromodomain-helicase-DNA- } \\
\text { binding protein } 1\end{array}$ & & \\
\hline & & K.S*DSSPLPSEKSDEDDDK.L & S1353 not confirmed \\
\hline & & K.SDS*SPLPSEKSDEDDDK.L & S1355 confirmed by b3 \\
\hline \multirow[t]{3}{*}{ O00257 } & $\begin{array}{l}\text { CBX4_HUMAN E3 SUMO- } \\
\text { protein ligase CBX4 }\end{array}$ & & \\
\hline & (Chromobox protein homolog 4) & & \\
\hline & & R.EEEVSGVS*DPQPQDAGSR.K & S334 not confirmed \\
\hline \multirow[t]{2}{*}{ O14874 } & $\begin{array}{l}\text { BCKD_HUMAN [3-methyl-2- } \\
\text { oxobutanoate dehydrogenase } \\
\text { [lipoamide]] kinase }\end{array}$ & & \\
\hline & & R.ARS*TSAT*DTHHVEMAR.E & $\begin{array}{l}\text { S31 not confirmed } \\
\text { T35 confirmed by y } 9 \text {, y } 10\end{array}$ \\
\hline \multirow[t]{3}{*}{ Q15056 } & $\begin{array}{l}\text { IF4H_HUMAN Eukaryotic } \\
\text { translation initiation factor } 4 \mathrm{H} \\
(\mathrm{eIF}-4 \mathrm{H})\end{array}$ & & \\
\hline & & R.AY*SS*FGGGR.G & Y12 confirmed by b2 \\
\hline & & & S14 not confirmed \\
\hline \multirow[t]{2}{*}{ Q8NC51 } & $\begin{array}{l}\text { PAIRB_HUMAN Plasminogen } \\
\text { activator inhibitor } 1 \text { RNA- } \\
\text { binding protein }\end{array}$ & & \\
\hline & & K.SKS*EEAHAEDSVMDHHFR.K & S330 confirmed by b2, b3 \\
\hline \multirow[t]{2}{*}{ Q9Y478 } & $\begin{array}{l}\text { AAKB1_HUMAN 5'-AMP- } \\
\text { activated protein kinase subunit } \\
\text { beta-1 }\end{array}$ & & \\
\hline & & R.S*HNNFVAILDLPEGEHQYK.F & S108 confirmed by b2 \\
\hline \multirow[t]{2}{*}{ P43243 } & MATR3_HUMAN Matrin-3 & & \\
\hline & & R.RDS*FDDR.G & S188 confirmed by b2, b3 \\
\hline \multirow[t]{3}{*}{ P18669 } & PGAM1_HUMAN & & \\
\hline & Phosphoglycerate mutase 1 & & \\
\hline & & R.HGES*AWNLENR.F & S11 confirmed by y/, y \\
\hline \multirow[t]{3}{*}{ Q96AT1 } & & & \\
\hline & $\begin{array}{l}\text { Uncharacterized protein } \\
\text { KIAA } 1143\end{array}$ & & \\
\hline & & $\begin{array}{l}\text { R.IQPQPPDEDGDHS*DKEDEQPQV } \\
\text { VVLK.K }\end{array}$ & S50 confirmed by y 13, y 14 \\
\hline \multirow[t]{2}{*}{ P47736 } & $\begin{array}{l}\text { RGP2_HUMAN Rap1 GTPase- } \\
\text { activating protein } 1 \text { (Rap1GAP) }\end{array}$ & & \\
\hline & & R.RSS*AIGIENIQEVQEK.R & $\begin{array}{l}\text { S499 confirmed by y13, } \\
\text { y14 }\end{array}$ \\
\hline \multirow[t]{2}{*}{ Q9BUZ4 } & $\begin{array}{l}\text { TRAF4_HUMAN TNF receptor- } \\
\text { associated factor } 4\end{array}$ & & \\
\hline & & R.GS*LDESSLGFGYPK.F & S426 confirmed by b2 \\
\hline \multirow[t]{2}{*}{ Q96T58 } & $\begin{array}{l}\text { MINT_HUMAN Msx2- } \\
\text { interacting }\end{array}$ & & \\
\hline & & K.FGKVTSVQIHGTS*EER.Y & $\begin{array}{l}\text { S374 confirmed by b12, } \\
\text { b13 }\end{array}$ \\
\hline \multirow[t]{2}{*}{ Q5HYJ3 } & $\begin{array}{l}\text { FA76B_HUMAN Protein } \\
\text { FAM76B }\end{array}$ & & \\
\hline & & K.ISNLS*PEEEQGLWK.Q & S193 confirmed by y9, y10 \\
\hline \multirow[t]{2}{*}{ Q13427 } & $\begin{array}{l}\text { PPIG_HUMAN Peptidyl-prolyl } \\
\text { cis-trans isomerase G }\end{array}$ & & \\
\hline & & R.RS*ET*PPHWR.Q & $\begin{array}{l}\text { S356 confirmed by b2 } \\
\text { T358 confirmed by y } 5, \text { y } 6\end{array}$ \\
\hline
\end{tabular}


Table A-2 (continued).

\begin{tabular}{llll}
\hline $\begin{array}{l}\text { Accession } \\
\text { number }\end{array}$ & Scan(s) & Peptide & Phosphorylation sites \\
\hline Q9UDY2 & $\begin{array}{l}\text { ZO2_HUMAN Tight junction } \\
\text { protein ZO-2 (Zonula occludens } \\
\text { 2 protein) }\end{array}$ & R.DNS*PPPAFKPEPPK.A & S986 confirmed by b3, b4 \\
Q9HC52 & $\begin{array}{l}\text { CBX8_HUMAN Chromobox } \\
\text { protein homolog 8 }\end{array}$ & R.HGS*GPPSSGGGLYR.D & S311 confirmed by b3, b5 \\
\hline
\end{tabular}


Table A-3 Subcellular locations and functional categories of the characterized phosphoproteins.

\begin{tabular}{|c|c|c|c|}
\hline $\begin{array}{l}\text { Swissprot } \\
\text { code }\end{array}$ & Protein name & $\begin{array}{l}\text { Subcellular } \\
\text { localization }\end{array}$ & Functional category \\
\hline HS90A & $\begin{array}{l}\text { HUMAN Heat shock protein HSP } \\
\text { 90-alpha (HSP 86) }\end{array}$ & Cytoplasm & Molecular chaperone \\
\hline SEPT2 & $\begin{array}{l}\text { HUMAN Septin-2 (Protein } \\
\text { NEDD5) }\end{array}$ & unknown & Involved in cytokinesis \\
\hline TEBP & $\begin{array}{l}\text { HUMAN Prostaglandin E } \\
\text { synthase } 3 \text { (Cytosolic } \\
\text { prostaglandin E2 synthase) }\end{array}$ & Cytoplasm & $\begin{array}{l}\text { RNA processing and Transcription } \\
\text { regulation }\end{array}$ \\
\hline CTND1 & $\begin{array}{l}\text { HUMAN Catenin delta-1 (p120 } \\
\text { catenin) }\end{array}$ & $\begin{array}{l}\text { Cytoplasm. } \\
\text { Nucleus }\end{array}$ & Transcription regulation \\
\hline TR150 & $\begin{array}{l}\text { HUMAN Thyroid hormone } \\
\text { receptor-associated protein } 3\end{array}$ & Nucleus & $\begin{array}{l}\text { RNA processing and Transcription } \\
\text { regulation }\end{array}$ \\
\hline NFIC & $\begin{array}{l}\text { HUMAN Nuclear factor 1 C-type } \\
\text { (Nuclear factor } 1 / \mathrm{C} \text { ) (NF1-C) }\end{array}$ & Unknown & $\begin{array}{l}\text { RNA processing and Transcription } \\
\text { regulation }\end{array}$ \\
\hline G3BP1 & $\begin{array}{l}\text { HUMAN Ras GTPase-activating } \\
\text { protein-binding protein } 1 \text { (G3BP- } \\
\text { 1) }\end{array}$ & $\begin{array}{l}\text { Cytoplasm. } \\
\text { Cytoplasm; } \\
\text { cytosol. Cell } \\
\text { membrane. } \\
\text { Nucleus. }\end{array}$ & DNA processing \\
\hline LRRF2 & $\begin{array}{l}\text { HUMAN Leucine-rich repeat } \\
\text { flightless-interacting protein } 2\end{array}$ & Unknown & Signal Transduction \\
\hline BRE1A & $\begin{array}{l}\text { HUMAN Ubiquitin-protein ligase } \\
\text { BRE1A (BRE1-A) (hBRE1) }\end{array}$ & Nucleus & $\begin{array}{l}\text { RNA processing and Transcription } \\
\text { regulation }\end{array}$ \\
\hline ARFG1 & $\begin{array}{l}\text { HUMAN ADP-ribosylation } \\
\text { factor GTPase-activating protein } \\
1\end{array}$ & Cytoplasm & Unknown \\
\hline DC1L1 & $\begin{array}{l}\text { HUMAN Cytoplasmic dynein } 1 \\
\text { light intermediate chain } 1\end{array}$ & Unknown & $\begin{array}{l}\text { May play a role in binding dynein to } \\
\text { membranous organelles or } \\
\text { chromosomes. May associate with } \\
\text { the heavy chains in the dynein head } \\
\text { where they might regulate enzyme } \\
\text { activity }\end{array}$ \\
\hline
\end{tabular}


Table A-3 (continued).

\begin{tabular}{|c|c|c|c|}
\hline $\begin{array}{l}\text { Swissprot } \\
\text { code }\end{array}$ & Protein name & $\begin{array}{l}\text { Subcellular } \\
\text { localization }\end{array}$ & Functional category \\
\hline M3K2 & $\begin{array}{l}\text { HUMAN Mitogen-activated } \\
\text { protein kinase kinase kinase } 2\end{array}$ & Cytoplasm & Signal transduction \\
\hline SMAP & HUMAN Small acidic protein & Unknown & Unknown \\
\hline ZRAB2 & $\begin{array}{l}\text { HUMAN Zinc finger Ran- } \\
\text { binding domain-containing } \\
\text { protein } 2\end{array}$ & Nucleus & Translation \\
\hline NKAP & $\begin{array}{l}\text { HUMAN NF-kappa-B-activating } \\
\text { protein }\end{array}$ & Nucleus & $\begin{array}{l}\text { Regulator of TNF and IL-1 induced } \\
\text { NF-kappa-B activation }\end{array}$ \\
\hline KLC4 & $\begin{array}{l}\text { HUMAN Kinesin light chain } 4 \\
\text { (KLC 4) (Kinesin-like protein } 8 \text { ) }\end{array}$ & Unknown & $\begin{array}{l}\text { Kinesin is a microtubule-associated } \\
\text { force-producing protein that may } \\
\text { play a role in organelle transport. The } \\
\text { light chain may function in coupling } \\
\text { of cargo to the heavy chain or in the } \\
\text { modulation of its ATPase activity }\end{array}$ \\
\hline NUCKS & $\begin{array}{l}\text { HUMAN Nuclear ubiquitous } \\
\text { casein and cyclin-dependent } \\
\text { kinases substrate }\end{array}$ & Nucleus & Unknown \\
\hline ANKS1 & $\begin{array}{l}\text { HUMAN Ankyrin repeat and } \\
\text { SAM domain-containing protein } \\
\text { 1A (Odin) }\end{array}$ & Cytoplasm & Signal transduction \\
\hline ARVC & $\begin{array}{l}\text { HUMAN Armadillo repeat } \\
\text { protein deleted in velo-cardio- } \\
\text { facial syndrome }\end{array}$ & Unknown & $\begin{array}{l}\text { Involved in protein-protein } \\
\text { interactions at adherent junctions }\end{array}$ \\
\hline K0310 & $\begin{array}{l}\text { HUMAN Uncharacterized } \\
\text { protein KIAA0310 }\end{array}$ & Unknown & Unknown \\
\hline SRC8 & $\begin{array}{l}\text { HUMAN Src substrate cortactin } \\
\text { (Amplaxin) (Oncogene EMS1) }\end{array}$ & Cytoplasm & Cell cycle \\
\hline RBM39 & $\begin{array}{l}\text { HUMAN RNA-binding protein } \\
39 \text { (RNA-binding motif protein } \\
\text { 39) }\end{array}$ & Nucleus & $\begin{array}{l}\text { RNA processing and Transcription } \\
\text { regulation }\end{array}$ \\
\hline CV009 & $\begin{array}{l}\text { HUMAN Uncharacterized } \\
\text { protein C22orf9 }\end{array}$ & Unknown & Unknown \\
\hline BCLF1 & $\begin{array}{l}\text { HUMAN Bcl-2-associated } \\
\text { transcription factor } 1 \text { (Btf) }\end{array}$ & $\begin{array}{l}\text { Cytoplasm. } \\
\text { Nucleus }\end{array}$ & $\begin{array}{l}\text { Death-promoting transcriptional } \\
\text { repressor }\end{array}$ \\
\hline
\end{tabular}


Table A-3 (continued).

\begin{tabular}{|c|c|c|c|}
\hline $\begin{array}{l}\text { Swissprot } \\
\text { code }\end{array}$ & Protein name & $\begin{array}{l}\text { Subcellular } \\
\text { localization }\end{array}$ & Functional category \\
\hline LAP2B & $\begin{array}{l}\text { HUMAN Lamina-associated } \\
\text { polypeptide } 2 \text {, isoforms } \\
\text { beta/gamma }\end{array}$ & Nucleus & DNA processing \\
\hline UBP14 & $\begin{array}{l}\text { HUMAN Ubiquitin carboxyl- } \\
\text { terminal hydrolase } 14\end{array}$ & Unknown & Unknown \\
\hline PHC3 & $\begin{array}{l}\text { HUMAN Polyhomeotic-like } \\
\text { protein } 3(\mathrm{hPH} 3) \text { (Homolog of } \\
\text { polyhomeotic } 3)\end{array}$ & Nucleus & $\begin{array}{l}\text { RNA processing and Transcription } \\
\text { regulation }\end{array}$ \\
\hline SRRM1 & $\begin{array}{l}\text { HUMAN Serine/arginine } \\
\text { repetitive matrix protein } 1\end{array}$ & Nucleus & Translation \\
\hline KAP0 & $\begin{array}{l}\text { HUMAN cAMP-dependent } \\
\text { protein kinase type I-alpha } \\
\text { regulatory subunit }\end{array}$ & Unknown & Unknown \\
\hline NPM & $\begin{array}{l}\text { HUMAN Nucleophosmin (NPM) } \\
\text { (Nucleolar phosphoprotein B23) }\end{array}$ & Nucleus & $\begin{array}{l}\text { Associated with nucleolar } \\
\text { ribonucleoprotein structures and bind } \\
\text { single-stranded nucleic acids. It may } \\
\text { function in the assembly and/or } \\
\text { transport of ribosome }\end{array}$ \\
\hline HNRH1 & $\begin{array}{l}\text { HUMAN Heterogeneous nuclear } \\
\text { ribonucleoprotein H (hnRNP H) }\end{array}$ & $\begin{array}{l}\text { Nucleus; } \\
\text { nucleoplasm. }\end{array}$ & $\begin{array}{l}\text { RNA processing and Transcription } \\
\text { regulation }\end{array}$ \\
\hline STMN1 & $\begin{array}{l}\text { HUMAN Stathmin } \\
\text { (Phosphoprotein p19) (pp19) } \\
\text { (Oncoprotein 18) }\end{array}$ & Cytoplasm & $\begin{array}{l}\text { Involved in the regulation of the } \\
\text { microtubule (MT) }\end{array}$ \\
\hline UBP10 & $\begin{array}{l}\text { HUMAN Ubiquitin carboxyl- } \\
\text { terminal hydrolase } 10\end{array}$ & Unknown & Ubiquitin specific protease \\
\hline HN1 & $\begin{array}{l}\text { HUMAN Hematological and } \\
\text { neurological expressed } 1 \text { protein }\end{array}$ & Nucleus & Unknown \\
\hline TIF1B & $\begin{array}{l}\text { HUMAN Transcription } \\
\text { intermediary factor 1-beta (TIF1- } \\
\text { beta) }\end{array}$ & Nucleus & Transcription factor \\
\hline CBLL1 & $\begin{array}{l}\text { HUMAN Cordon-bleu protein- } \\
\text { like } 1\end{array}$ & Unknown & Unknown \\
\hline IF34 & $\begin{array}{l}\text { HUMAN Eukaryotic translation } \\
\text { initiation factor } 3 \text { subunit } 4 \text { (eIF- } \\
3 \text { delta) }\end{array}$ & Unknown & Translation \\
\hline RCOR1 & HUMAN REST corepressor 1 & Nucleus & $\begin{array}{l}\text { RNA processing and Transcription } \\
\text { regulation }\end{array}$ \\
\hline
\end{tabular}


Table A-3 (continued).

\begin{tabular}{|c|c|c|c|}
\hline $\begin{array}{l}\text { Swissprot } \\
\text { code }\end{array}$ & Protein name & $\begin{array}{l}\text { Subcellular } \\
\text { localization }\end{array}$ & Functional category \\
\hline LARP1 & HUMAN La-related protein 1 & Unknown & Unknown \\
\hline AAKB2 & $\begin{array}{l}\text { HUMAN 5'-AMP-activated } \\
\text { protein kinase subunit beta-2 } \\
\text { (AMPK beta- } 2 \text { chain) }\end{array}$ & Unknown & $\begin{array}{l}\text { AMPK is responsible for the } \\
\text { regulation of fatty acid synthesis by } \\
\text { phosphorylation of acetyl-CoA } \\
\text { carboxylase }\end{array}$ \\
\hline CHSP1 & $\begin{array}{l}\text { HUMAN Calcium-regulated heat } \\
\text { stable protein } 1\end{array}$ & Cytoplasm & $\begin{array}{l}\text { May play a role in acinar cell } \\
\text { metabolism }\end{array}$ \\
\hline ODBA & $\begin{array}{l}\text { HUMAN 2-oxoisovalerate } \\
\text { dehydrogenase subunit alpha, } \\
\text { mitochondrial precursor }\end{array}$ & $\begin{array}{l}\text { Mitochondrio } \\
\mathrm{n} \text {; } \\
\text { mitochondria } \\
1 \text { matrix }\end{array}$ & $\begin{array}{l}\text { The branched-chain alpha-keto } \\
\text { dehydrogenase complex }\end{array}$ \\
\hline PPHLN & $\begin{array}{l}\text { HUMAN Periphilin-1 (Gastric } \\
\text { cancer antigen Ga50) }\end{array}$ & $\begin{array}{l}\text { Nucleus. } \\
\text { Cytoplasm }\end{array}$ & $\begin{array}{l}\text { Involved in epithelial differentiation } \\
\text { and contributes }\end{array}$ \\
\hline K1802 & $\begin{array}{l}\text { HUMAN Zinc finger protein } \\
\text { KIAA } 1802\end{array}$ & Unknown & Unknown \\
\hline SF3A1 & $\begin{array}{l}\text { HUMAN Splicing factor } 3 \\
\text { subunit } 1 \text { (Spliceosome- } \\
\text { associated protein 114) }\end{array}$ & Nucleus & Unknown \\
\hline TAOK2 & $\begin{array}{l}\text { HUMAN Serine/threonine- } \\
\text { protein kinase TAO2 }\end{array}$ & Cytoplasm & $\begin{array}{l}\text { Activates the JNK MAP kinase } \\
\text { pathway through the specific } \\
\text { activation of the upstream MKK3 } \\
\text { and MKK6 kinases. }\end{array}$ \\
\hline KPCD1 & $\begin{array}{l}\text { HUMAN Serine/threonine- } \\
\text { protein kinase D1 (nPKC-D1) } \\
\text { (Protein kinase D) }\end{array}$ & Unknown & Unknown \\
\hline STK39 & $\begin{array}{l}\text { HUMAN STE20/SPS1-related } \\
\text { proline-alanine-rich protein } \\
\text { kinase }\end{array}$ & $\begin{array}{l}\text { Cytoplasm } \\
\text { (Probable). } \\
\text { Nucleus } \\
\text { (Probable). }\end{array}$ & Signal transduction \\
\hline KLC2 & $\begin{array}{l}\text { HUMAN Kinesin light chain } 2 \\
\text { (KLC 2) }\end{array}$ & Unknown & $\begin{array}{l}\text { Kinesin is a microtubule-associated } \\
\text { force-producing protein that may } \\
\text { play a role in organelle transport. The } \\
\text { light chain may function in coupling } \\
\text { of cargo to the heavy chain or in the } \\
\text { modulation of its ATPase activity }\end{array}$ \\
\hline LRRF1 & $\begin{array}{l}\text { HUMAN Leucine-rich repeat } \\
\text { flightless-interacting protein } 1\end{array}$ & $\begin{array}{l}\text { Nucleus. } \\
\text { Cytoplasm. }\end{array}$ & $\begin{array}{l}\text { RNA processing and Transcription } \\
\text { regulation }\end{array}$ \\
\hline
\end{tabular}


Table A-3 (continued).

\begin{tabular}{|c|c|c|c|}
\hline $\begin{array}{l}\text { Swissprot } \\
\text { code }\end{array}$ & Protein name & $\begin{array}{l}\text { Subcellular } \\
\text { localization }\end{array}$ & Functional category \\
\hline ARHG6 & $\begin{array}{l}\text { HUMAN Rho guanine nucleotide } \\
\text { exchange factor } 6\end{array}$ & Unknown & $\begin{array}{l}\text { Acts as a RAC1 guanine nucleotide } \\
\text { exchange factor }\end{array}$ \\
\hline CD2L1 & $\begin{array}{l}\text { HUMAN PITSLRE } \\
\text { serine/threonine-protein kinase } \\
\text { CDC2L1 }\end{array}$ & $\begin{array}{l}\text { Cytoplasm. } \\
\text { Nucleus }\end{array}$ & $\begin{array}{l}\text { Appears to play multiple roles in cell } \\
\text { cycle progression, cytokinesis and } \\
\text { apoptosis }\end{array}$ \\
\hline PYRG1 & $\begin{array}{l}\text { HUMAN CTP synthase } 1 \text { (UTP-- } \\
\text { ammonia ligase 1) }\end{array}$ & Unknown & $\begin{array}{l}\text { Catalyzes the ATP-dependent } \\
\text { amination of UTP to CTP with either } \\
\text { L-glutamine or ammonia as the } \\
\text { source of nitrogen. }\end{array}$ \\
\hline TYY1_ & $\begin{array}{l}\text { HUMAN Transcriptional } \\
\text { repressor protein YY1 (Yin and } \\
\text { yang 1) (YY-1) }\end{array}$ & Nucleus & Transcription factor \\
\hline SMN & $\begin{array}{l}\text { HUMAN Survival motor neuron } \\
\text { protein (Component of gems 1) } \\
\text { (Gemin-1) }\end{array}$ & $\begin{array}{l}\text { Cytoplasm. } \\
\text { Nucleus }\end{array}$ & $\begin{array}{l}\text { The SMN complex plays an essential } \\
\text { role in spliceosomal snRNP assembly } \\
\text { in the cytoplasm and is required for } \\
\text { pre-mRNA splicing in the nucleus. It } \\
\text { may also play a role in the } \\
\text { metabolism of snoRNPs. }\end{array}$ \\
\hline PAK2 & $\begin{array}{l}\text { HUMAN Serine/threonine- } \\
\text { protein kinase PAK } 2 \text { (p21- } \\
\text { activated kinase 2) }\end{array}$ & Unknown & $\begin{array}{l}\text { The activated kinase acts on a variety } \\
\text { of targets. Phosphorylates ribosomal } \\
\text { protein S6, histone } \mathrm{H} 4 \text { and myelin } \\
\text { basic protein. }\end{array}$ \\
\hline SSRP1 & $\begin{array}{l}\text { HUMAN FACT complex subunit } \\
\text { SSRP1 }\end{array}$ & Nucleus & $\begin{array}{l}\text { Component of the FACT complex, a } \\
\text { general chromatin factor that acts to } \\
\text { reorganize nucleosomes. }\end{array}$ \\
\hline CTNA1 & $\begin{array}{l}\text { HUMAN Catenin alpha-1 } \\
\text { (Cadherin-associated protein) }\end{array}$ & Cell junction & $\begin{array}{l}\text { May play a crucial role in cell } \\
\text { differentiation }\end{array}$ \\
\hline SFRS9 & $\begin{array}{l}\text { HUMAN Splicing factor, } \\
\text { arginine/serine-rich } 9\end{array}$ & Nucleus & Translation \\
\hline AGM1 & $\begin{array}{l}\text { HUMAN } \\
\text { Phosphoacetylglucosamine } \\
\text { mutase (PAGM) }\end{array}$ & Unknown & $\begin{array}{l}\text { Interconvert GlcNAc-6-P and } \\
\text { GlcNAc-1-P }\end{array}$ \\
\hline MORC2 & $\begin{array}{l}\text { HUMAN MORC family CW-type } \\
\text { zinc finger protein } 2\end{array}$ & Unknown & Unknown \\
\hline BAD & $\begin{array}{l}\text { HUMAN Bcl2 antagonist of cell } \\
\text { death (BAD) }\end{array}$ & $\begin{array}{l}\text { Mitochondrio } \\
\mathrm{n} \text {; } \\
\text { mitochondria } \\
\text { l outer } \\
\text { membrane }\end{array}$ & Cell cycle \\
\hline TB182 & $\begin{array}{l}\text { HUMAN } 182 \mathrm{kDa} \text { tankyrase } 1- \\
\text { binding protein }\end{array}$ & $\begin{array}{l}\text { Nucleus. } \\
\text { Cytoplasm }\end{array}$ & $\begin{array}{l}\text { Binds to the ANK repeat domain of } \\
\text { TNKS1 and TNKS2 }\end{array}$ \\
\hline AKTS1 & $\begin{array}{l}\text { HUMAN Proline-rich AKT1 } \\
\text { substrate } 1 \text { ( } 40 \mathrm{kDa} \text { proline-rich } \\
\text { AKT substrate) }\end{array}$ & $\begin{array}{l}\text { Cytoplasm; } \\
\text { cytosol }\end{array}$ & Signal transduction \\
\hline
\end{tabular}


Table A-3 (continued).

\begin{tabular}{|c|c|c|c|}
\hline $\begin{array}{l}\text { Swissprot } \\
\text { code }\end{array}$ & Protein name & $\begin{array}{l}\text { Subcellular } \\
\text { localization }\end{array}$ & Functional category \\
\hline PUM1 & $\begin{array}{l}\text { HUMAN Pumilio homolog } 1 \\
\text { (Pumilio-1) (HsPUM) }\end{array}$ & Cytoplasm & $\begin{array}{l}\text { RNA processing and Transcription } \\
\text { regulation }\end{array}$ \\
\hline ODPA & $\begin{array}{l}\text { HUMAN Pyruvate dehydrogenase } \\
\text { E1 component alpha subunit }\end{array}$ & $\begin{array}{l}\text { Mitochondrio } \\
\mathrm{n} \text {; } \\
\text { mitochondria } \\
1 \text { matrix. }\end{array}$ & Metabolic pathway \\
\hline SRRM2 & $\begin{array}{l}\text { HUMAN Serine/arginine } \\
\text { repetitive matrix protein } 2\end{array}$ & Nucleus & Translation \\
\hline RBM10 & $\begin{array}{l}\text { HUMAN RNA-binding protein } 10 \\
\text { (RNA-binding motif protein 10) }\end{array}$ & Nucleus & Unknown \\
\hline HIRP3 & $\begin{array}{l}\text { HUMAN HIRA-interacting } \\
\text { protein } 3\end{array}$ & Nucleus & $\begin{array}{l}\text { May play a role in chromatin } \\
\text { function and histone metabolism via } \\
\text { its interaction with HIRA and } \\
\text { histones. }\end{array}$ \\
\hline FA62A & $\begin{array}{l}\text { HUMAN Protein FAM62A } \\
\text { (Membrane-bound C2 domain- } \\
\text { containing protein) }\end{array}$ & Membrane & Unknown \\
\hline PACN3 & $\begin{array}{l}\text { HUMAN Protein kinase } \mathrm{C} \text { and } \\
\text { casein kinase substrate in neurons } \\
\text { protein } 3\end{array}$ & $\begin{array}{l}\text { Cytoplasm. } \\
\text { Cell } \\
\text { membrane; } \\
\text { peripheral } \\
\text { membrane } \\
\text { protein; } \\
\text { cytoplasmic } \\
\text { side. }\end{array}$ & $\begin{array}{l}\text { May play a role in vesicle formation } \\
\text { and transport. }\end{array}$ \\
\hline MLRN & $\begin{array}{l}\text { HUMAN Myosin regulatory light } \\
\text { chain } 2 \text {, smooth muscle isoform }\end{array}$ & Unknown & $\begin{array}{l}\text { Plays an important role in regulation } \\
\text { of both smooth muscle and } \\
\text { nonmuscle cell contractile activity }\end{array}$ \\
\hline HSPB1 & $\begin{array}{l}\text { HUMAN Heat-shock protein beta- } \\
1 \text { (HspB1) (Heat shock } 27 \mathrm{kDa} \\
\text { protein) }\end{array}$ & $\begin{array}{l}\text { Cytoplasm, } \\
\text { Nucleus }\end{array}$ & $\begin{array}{l}\text { Involved in stress resistance and } \\
\text { actin organization. }\end{array}$ \\
\hline LA & $\begin{array}{l}\text { HUMAN Lupus La protein } \\
\text { (Sjoegren syndrome type B } \\
\text { antigen) }\end{array}$ & Nucleus & $\begin{array}{l}\text { RNA processing and Transcription } \\
\text { regulation }\end{array}$ \\
\hline IF2P & $\begin{array}{l}\text { HUMAN Eukaryotic translation } \\
\text { initiation factor } 5 \mathrm{~B} \text { (eIF-5B) }\end{array}$ & Unknown & Translation \\
\hline FXR1 & $\begin{array}{l}\text { HUMAN Fragile X mental } \\
\text { retardation syndrome-related } \\
\text { protein } 1 \text { (hFXR1p) }\end{array}$ & Cytoplasm. & $\begin{array}{l}\text { RNA processing and Transcription } \\
\text { regulation }\end{array}$ \\
\hline HS90B & $\begin{array}{l}\text { HUMAN Heat shock protein HSP } \\
\text { 90-beta (HSP 84) }\end{array}$ & Cytoplasm & Molecular chaperone \\
\hline
\end{tabular}


Table A-3 (continued).

\begin{tabular}{|c|c|c|c|}
\hline $\begin{array}{l}\text { Swissprot } \\
\text { code }\end{array}$ & Protein name & $\begin{array}{l}\text { Subcellular } \\
\text { localization }\end{array}$ & Functional category \\
\hline CFDP1 & $\begin{array}{l}\text { HUMAN Craniofacial } \\
\text { development protein } 1 \\
\text { (Bucentaur) }\end{array}$ & Unknown & $\begin{array}{l}\text { May play a role during } \\
\text { embryogenesis (By similarity). }\end{array}$ \\
\hline LC7L2 & $\begin{array}{l}\text { HUMAN Putative RNA-binding } \\
\text { protein Luc7-like } 2\end{array}$ & Unknown & $\begin{array}{l}\text { May bind to RNA via its Arg/Ser- } \\
\text { rich domain }\end{array}$ \\
\hline IF38 & $\begin{array}{l}\text { HUMAN Eukaryotic translation } \\
\text { initiation factor } 3 \text { subunit } 8 \text { (eIF3 } \\
\text { p110) }\end{array}$ & Unknown & Translation \\
\hline MYPT1 & $\begin{array}{l}\text { HUMAN Protein phosphatase } 1 \\
\text { regulatory subunit } 12 \mathrm{~A}\end{array}$ & Cytoplasm & $\begin{array}{l}\text { Regulates myosin phosphatase } \\
\text { activity }\end{array}$ \\
\hline ZN330 & $\begin{array}{l}\text { HUMAN Zinc finger protein } 330 \\
\text { (Nucleolar cysteine-rich protein) }\end{array}$ & Nucleus & Unknown \\
\hline HNRPK & $\begin{array}{l}\text { HUMAN Heterogeneous nuclear } \\
\text { ribonucleoprotein K }\end{array}$ & $\begin{array}{l}\text { Cytoplasm. } \\
\text { Nucleus; } \\
\text { nucleoplasm. }\end{array}$ & $\begin{array}{l}\text { One of the major pre-mRNA-binding } \\
\text { proteins. }\end{array}$ \\
\hline HDGF & $\begin{array}{l}\text { HUMAN Hepatoma-derived } \\
\text { growth factor (HDGF) }\end{array}$ & Cytoplasm & $\begin{array}{l}\text { Heparin-binding protein, with } \\
\text { mitogenic activity for fibroblasts. }\end{array}$ \\
\hline OSBP1 & $\begin{array}{l}\text { HUMAN Oxysterol-binding } \\
\text { protein } 1\end{array}$ & Cytoplasm & $\begin{array}{l}\text { May play a role in the regulation of } \\
\text { sterol metabolism }\end{array}$ \\
\hline $\mathrm{ABCF} 1$ & $\begin{array}{l}\text { HUMAN ATP-binding cassette } \\
\text { sub-family F member } 1\end{array}$ & Unknown & Translation \\
\hline HNRH2 & $\begin{array}{l}\text { HUMAN Heterogeneous nuclear } \\
\text { ribonucleoprotein H' (hnRNP H') } \\
\text { (FTP-3) }\end{array}$ & $\begin{array}{l}\text { Nucleus; } \\
\text { nucleoplasm }\end{array}$ & Translation \\
\hline MK67I & $\begin{array}{l}\text { HUMAN MKI67 FHA domain- } \\
\text { interacting nucleolar } \\
\text { phosphoprotein }\end{array}$ & $\begin{array}{l}\text { Nucleus; } \\
\text { nucleolus }\end{array}$ & Unknown \\
\hline LMNA & $\begin{array}{l}\text { HUMAN Lamin-A/C (70 kDa } \\
\text { lamin) }\end{array}$ & Nucleus. & Unknown \\
\hline $\mathrm{CDC} 2$ & $\begin{array}{l}\text { HUMAN Cell division control } \\
\text { protein } 2 \text { homolog (p34 protein } \\
\text { kinase) }\end{array}$ & Nucleus & Cell cycle \\
\hline PCY1A & $\begin{array}{l}\text { HUMAN Choline-phosphate } \\
\text { cytidylyltransferase A }\end{array}$ & $\begin{array}{l}\text { Cytoplasm; } \\
\text { cytosol. } \\
\text { Membrane; } \\
\text { peripheral } \\
\text { membrane } \\
\text { protein. }\end{array}$ & $\begin{array}{l}\text { Controls phosphatidylcholine } \\
\text { synthesis }\end{array}$ \\
\hline
\end{tabular}


Table A-3 (continued).

\begin{tabular}{|c|c|c|c|}
\hline $\begin{array}{l}\text { Swissprot } \\
\text { code }\end{array}$ & Protein name & $\begin{array}{l}\text { Subcellular } \\
\text { localization }\end{array}$ & Functional category \\
\hline CCDC6 & $\begin{array}{l}\text { HUMAN Coiled-coil domain- } \\
\text { containing protein } 6 \text { (H4 protein) }\end{array}$ & $\begin{array}{l}\text { Cytoplasm. } \\
\text { Cytoplasm; } \\
\text { cytoskeleto } \\
\text { n }\end{array}$ & Unknown \\
\hline SMC4 & $\begin{array}{l}\text { HUMAN Structural maintenance } \\
\text { of chromosomes protein } 4\end{array}$ & $\begin{array}{l}\text { Nucleus. } \\
\text { Cytoplasm }\end{array}$ & DNA processing \\
\hline SMRC2 & $\begin{array}{l}\text { HUMAN SWI/SNF-related } \\
\text { matrix-associated actin- } \\
\text { dependent regulator of chromatin } \\
\text { subfamily C member } 2\end{array}$ & Nucleus & $\begin{array}{l}\text { transcriptional activation and } \\
\text { repression }\end{array}$ \\
\hline MDC1 & $\begin{array}{l}\text { HUMAN Mediator of DNA } \\
\text { damage checkpoint protein } 1\end{array}$ & nucleus & Cell cycle \\
\hline PPIL4 & $\begin{array}{l}\text { HUMAN Peptidyl-prolyl cis- } \\
\text { trans isomerase-like } 4\end{array}$ & Nucleus & $\begin{array}{l}\text { PPIases accelerate the folding of } \\
\text { proteins }\end{array}$ \\
\hline $\mathrm{XRCC1}$ & $\begin{array}{l}\text { HUMAN DNA-repair protein } \\
\text { XRCC1 }\end{array}$ & Nucleus & Unknown \\
\hline SYEP & $\begin{array}{l}\text { HUMAN Bifunctional } \\
\text { aminoacyl-tRNA synthetase }\end{array}$ & Unknown & Unknown \\
\hline CALX & HUMAN Calnexin precursor & $\begin{array}{l}\text { Endoplasmi } \\
\text { c reticulum }\end{array}$ & Translation \\
\hline FUSIP & $\begin{array}{l}\text { HUMAN FUS-interacting serine- } \\
\text { arginine-rich protein } 1\end{array}$ & $\begin{array}{l}\text { Nucleus; } \\
\text { cytoplasm }\end{array}$ & Translation \\
\hline MLF2 & $\begin{array}{l}\text { HUMAN Myeloid leukemia } \\
\text { factor } 2\end{array}$ & $\begin{array}{l}\text { Cytoplasm; } \\
\text { nucleus }\end{array}$ & Unknown \\
\hline PCBP2 & $\begin{array}{l}\text { HUMAN Poly(rC)-binding } \\
\text { protein } 2 \text { (Alpha-CP2) }\end{array}$ & $\begin{array}{l}\text { Nucleus; } \\
\text { cytoplasm }\end{array}$ & $\begin{array}{l}\text { Major cellular poly(rC)-binding } \\
\text { protein }\end{array}$ \\
\hline CLIP1 & HUMAN Restin & cytoplasm & Unknown \\
\hline MPRI & $\begin{array}{l}\text { HUMAN Cation-independent } \\
\text { mannose-6-phosphate receptor } \\
\text { precursor }\end{array}$ & $\begin{array}{l}\text { Lysosome; } \\
\text { lysosomal } \\
\text { membrane }\end{array}$ & Signal transduction \\
\hline NOP56 & $\begin{array}{l}\text { HUMAN Nucleolar protein } \\
\text { Nop56 }\end{array}$ & Nucleus & Translation \\
\hline RBM5 & HUMAN RNA-binding protein 5 & Nucleus & $\begin{array}{l}\text { Tumor suppressor protein that reduces } \\
\text { cell proliferation and promotes } \\
\text { apoptosis and cell cycle arrest }\end{array}$ \\
\hline LIPB2 & HUMAN Liprin-beta-2 & Unknown & $\begin{array}{l}\text { May regulate the disassembly of focal } \\
\text { adhesions. Did not bind receptor-like } \\
\text { tyrosine phosphatases type } 2 \mathrm{~A}\end{array}$ \\
\hline
\end{tabular}


Table A-3 (continued).

\begin{tabular}{|c|c|c|c|}
\hline $\begin{array}{l}\text { Swissprot } \\
\text { code }\end{array}$ & Protein name & $\begin{array}{l}\text { Subcellular } \\
\text { localization }\end{array}$ & Functional category \\
\hline TXLNA & HUMAN Alpha-taxilin & Unknown & Unknown \\
\hline PHF6 & $\begin{array}{l}\text { HUMAN PHD finger protein } 6 \\
\text { (PHD-like zinc finger protein) }\end{array}$ & Nucleus & Transcription regulation \\
\hline TRA2A & $\begin{array}{l}\text { HUMAN Transformer-2 protein } \\
\text { homolog }\end{array}$ & Nucleus & Translation \\
\hline BIRC3 & $\begin{array}{l}\text { HUMAN Baculoviral IAP } \\
\text { repeat-containing protein } 3\end{array}$ & Unknown & Unknown \\
\hline MCM2 & $\begin{array}{l}\text { HUMAN DNA replication } \\
\text { licensing factor MCM2 }\end{array}$ & Nucleus & Cell cycle \\
\hline NFAC3 & $\begin{array}{l}\text { HUMAN Nuclear factor of } \\
\text { activated T-cells, cytoplasmic } 3 \\
\text { (NF-ATc3) }\end{array}$ & $\begin{array}{l}\text { Cytoplasm, } \\
\text { nucleus }\end{array}$ & $\begin{array}{l}\text { Plays a role in the inducible expression } \\
\text { of cytokine genes in T-cells, especially } \\
\text { in the induction of the IL-2. }\end{array}$ \\
\hline CT172 & $\begin{array}{l}\text { HUMAN Uncharacterized } \\
\text { protein C20orf172 }\end{array}$ & Nucleus & Cell cycle \\
\hline PKCB1 & $\begin{array}{l}\text { HUMAN Protein kinase C- } \\
\text { binding protein } 1 \text { (Rack7) }\end{array}$ & Unknown & Unknown \\
\hline KAP1 & $\begin{array}{l}\text { HUMAN cAMP-dependent } \\
\text { protein kinase type I-beta } \\
\text { regulatory subunit }\end{array}$ & Unknown & Unknown \\
\hline TAOK1 & $\begin{array}{l}\text { HUMAN Serine/threonine- } \\
\text { protein kinase TAO1 }\end{array}$ & Cytoplasm & Signal transduction \\
\hline BRCA2 & $\begin{array}{l}\text { HUMAN Breast cancer type } 2 \\
\text { susceptibility protein }\end{array}$ & Unknown & Cell cycle \\
\hline LRC15 & $\begin{array}{l}\text { HUMAN Leucine-rich repeat- } \\
\text { containing protein } 15 \text { precursor } \\
\text { (hLib) }\end{array}$ & Membrane & Unknown \\
\hline MRCKA & $\begin{array}{l}\text { HUMAN Serine/threonine- } \\
\text { protein kinase MRCK alpha }\end{array}$ & Cytoplasm & Cell cycle \\
\hline NOL7 & HUMAN Nucleolar protein 7 & Nucleus & Unknown \\
\hline GSTM2 & $\begin{array}{l}\text { HUMAN Glutathione S- } \\
\text { transferase } \mathrm{Mu} 2\end{array}$ & Cytoplasm & Unknown \\
\hline MYH13 & $\begin{array}{l}\text { HUMAN Myosin-13 (Myosin } \\
\text { heavy chain 13) }\end{array}$ & Unknown & Muscle contraction \\
\hline
\end{tabular}


Table A-3 (continued).

\begin{tabular}{|c|c|c|c|}
\hline $\begin{array}{l}\text { Swissprot } \\
\text { code }\end{array}$ & Protein name & $\begin{array}{l}\text { Subcellular } \\
\text { localization }\end{array}$ & Functional category \\
\hline ELL & $\begin{array}{l}\text { HUMAN RNA polymerase II } \\
\text { elongation factor ELL }\end{array}$ & Nucleus & Elongation factor \\
\hline M10L1 & $\begin{array}{l}\text { HUMAN Putative helicase } \\
\text { Mov1011 }\end{array}$ & Unknown & $\begin{array}{l}\text { Putative RNA helicase. Isoform } 1 \text { may } \\
\text { play a role in male germ cell } \\
\text { development. }\end{array}$ \\
\hline DPOA2 & $\begin{array}{l}\text { HUMAN DNA polymerase } \\
\text { subunit alpha B }\end{array}$ & Nucleus. & $\begin{array}{l}\text { RNA processing and transcription } \\
\text { regulation }\end{array}$ \\
\hline SNF5 & $\begin{array}{l}\text { HUMAN SWI/SNF-related } \\
\text { matrix-associated actin-dependent } \\
\text { regulator of chromatin subfamily } \\
\text { B member } 1\end{array}$ & Nucleus & Cell cycle \\
\hline UBR2 & $\begin{array}{l}\text { HUMAN E3 ubiquitin-protein } \\
\text { ligase UBR2 (N-recognin-2) }\end{array}$ & nucleus & Signal transduction \\
\hline MRP & $\begin{array}{l}\text { HUMAN MARCKS-related } \\
\text { protein (MARCKS-like protein 1) }\end{array}$ & Unknown & Signal transduction \\
\hline NP1L4 & $\begin{array}{l}\text { HUMAN Nucleosome assembly } \\
\text { protein 1-like } 4\end{array}$ & Nucleus & Unknown \\
\hline 4EBP1 & $\begin{array}{l}\text { HUMAN Eukaryotic translation } \\
\text { initiation factor 4E-binding } \\
\text { protein } 1\end{array}$ & Unknown & Translation \\
\hline RANB3 & $\begin{array}{l}\text { HUMAN Ran-binding protein } 3 \\
\text { (RanBP3) }\end{array}$ & $\begin{array}{l}\text { Cytoplasm. } \\
\text { Nucleus. }\end{array}$ & $\begin{array}{l}\text { Acts as a cofactor for XPO1/CRM1- } \\
\text { mediated nuclear export, perhaps as } \\
\text { export complex scaffolding protein. }\end{array}$ \\
\hline EP15 & $\begin{array}{l}\text { HUMAN Epidermal growth } \\
\text { factor receptor substrate } 15 \\
\text { (Protein Eps15) }\end{array}$ & $\begin{array}{l}\text { Cytoplasm, } \\
\text { cell } \\
\text { membrane }\end{array}$ & Cell growth \\
\hline $1433 \mathrm{E}$ & $\begin{array}{l}\text { HUMAN 14-3-3 protein epsilon } \\
(14-3-3 E)\end{array}$ & Cytoplasm & Signal transduction \\
\hline RL1D1 & $\begin{array}{l}\text { HUMAN Ribosomal L1 domain- } \\
\text { containing protein } 1\end{array}$ & Nucleus & Unknown \\
\hline MARCS & $\begin{array}{l}\text { HUMAN Myristoylated alanine- } \\
\text { rich C-kinase substrate } \\
\text { (MARCKS) }\end{array}$ & Unknown & $\begin{array}{l}\text { MARCKS is the most prominent } \\
\text { cellular substrate for protein kinase } \mathrm{C}\end{array}$ \\
\hline CD2B2 & $\begin{array}{l}\text { HUMAN CD2 antigen } \\
\text { cytoplasmic tail-binding protein } 2\end{array}$ & Cytoplasm & Unknown \\
\hline $\mathrm{K} 1 \mathrm{C} 18$ & $\begin{array}{l}\text { HUMAN Keratin, type I } \\
\text { cytoskeletal } 18 \text { (Cytokeratin-18) } \\
\text { (CK-18) }\end{array}$ & Unknown & Unknown \\
\hline PURB & $\begin{array}{l}\text { HUMAN Transcriptional } \\
\text { activator protein Pur-beta }\end{array}$ & Nucleus & $\begin{array}{l}\text { Has capacity to bind repeated elements } \\
\text { in single-stranded DNA such as the } \\
\text { purine-rich single strand of the PUR } \\
\text { element located upstream of the MYC } \\
\text { gene. }\end{array}$ \\
\hline
\end{tabular}


Table A-3 (continued).

\begin{tabular}{|c|c|c|c|}
\hline $\begin{array}{l}\text { Swissprot } \\
\text { code }\end{array}$ & Protein name & $\begin{array}{l}\text { Subcellular } \\
\text { localization }\end{array}$ & Functional category \\
\hline ZN326 & HUMAN Zinc finger protein 326 & Nucleus & transcriptional activator \\
\hline EF1D & $\begin{array}{l}\text { HUMAN Elongation factor 1- } \\
\text { delta (EF-1-delta) (Antigen NY- } \\
\text { CO-4) }\end{array}$ & Unknown & Translation \\
\hline NDRG3 & HUMAN Protein NDRG3 & Unknown & Unknown \\
\hline SFR11 & $\begin{array}{l}\text { HUMAN Splicing factor } \\
\text { arginine/serine-rich } 11\end{array}$ & Nucleus & $\begin{array}{l}\text { RNA processing and Transcription } \\
\text { regulation }\end{array}$ \\
\hline PSA3 & $\begin{array}{l}\text { HUMAN Proteasome subunit } \\
\text { alpha type } 3 \text { (Proteasome } \\
\text { component C } 8 \text { ) }\end{array}$ & $\begin{array}{l}\text { Cytoplasm, } \\
\text { nucleus }\end{array}$ & proteasome \\
\hline FIP1 & $\begin{array}{l}\text { HUMAN Pre-mRNA 3'-end- } \\
\text { processing factor FIP1 (FIP1-like } \\
\text { 1) }\end{array}$ & Unknown & $\begin{array}{l}\text { RNA processing and Transcription } \\
\text { regulation }\end{array}$ \\
\hline TE2IP & $\begin{array}{l}\text { HUMAN Telomeric repeat- } \\
\text { binding factor 2-interacting } \\
\text { protein } 1\end{array}$ & Nucleus & $\begin{array}{l}\text { May play a role in telomere length } \\
\text { regulation. }\end{array}$ \\
\hline MAP4 & $\begin{array}{l}\text { HUMAN Microtubule-associated } \\
\text { protein } 4 \text { (MAP 4) }\end{array}$ & Unknown & $\begin{array}{l}\text { Non-neuronal microtubule-associated } \\
\text { protein. Promotes }\end{array}$ \\
\hline $\mathrm{K} 2 \mathrm{C} 7$ & $\begin{array}{l}\text { HUMAN Keratin, type II } \\
\text { cytoskeletal } 7\end{array}$ & Unknown & Unknown \\
\hline PININ & $\begin{array}{l}\text { HUMAN Pinin }(140 \mathrm{kDa} \text { nuclear } \\
\text { and cell adhesion-related } \\
\text { phosphoprotein) }\end{array}$ & $\begin{array}{l}\text { Nucleus; } \\
\text { nuclear } \\
\text { speckle. } \\
\text { Cell } \\
\text { junction; }\end{array}$ & $\begin{array}{l}\text { RNA processing and Transcription } \\
\text { regulation }\end{array}$ \\
\hline TBA6 & $\begin{array}{l}\text { HUMAN Tubulin alpha-6 chain } \\
\text { (Alpha-tubulin 6) }\end{array}$ & Unknown & $\begin{array}{l}\text { Tubulin is the major constituent of } \\
\text { microtubules. It binds two moles of } \\
\text { GTP, one at an exchangeable site on } \\
\text { the beta chain and one at a non- } \\
\text { exchangeable site on the alpha-chain. }\end{array}$ \\
\hline UBP7 & $\begin{array}{l}\text { HUMAN Ubiquitin carboxyl- } \\
\text { terminal hydrolase } 7 \text { (Ubiquitin } \\
\text { thioesterase } 7 \text { ) }\end{array}$ & Nucleus & Cell cycle \\
\hline HNRPF & $\begin{array}{l}\text { HUMAN Heterogeneous nuclear } \\
\text { ribonucleoprotein F (hnRNP F) }\end{array}$ & $\begin{array}{l}\text { Nucleus; } \\
\text { nucleoplasm }\end{array}$ & $\begin{array}{l}\text { Component of the heterogeneous } \\
\text { nuclear ribonucleoprotein (hnRNP) } \\
\text { complexes which provide the substrate } \\
\text { for the processing events that pre- } \\
\text { mRNAs undergo before becoming } \\
\text { functional, translatable mRNAs in the } \\
\text { cytoplasm. Probably binds G-rich } \\
\text { sequences in pre-mRNAs }\end{array}$ \\
\hline
\end{tabular}


Table A-3 (continued).

\begin{tabular}{|c|c|c|c|}
\hline $\begin{array}{l}\text { Swissprot } \\
\text { code }\end{array}$ & Protein name & $\begin{array}{l}\text { Subcellular } \\
\text { localization }\end{array}$ & Functional category \\
\hline CBX3 & $\begin{array}{l}\text { HUMAN Chromobox protein } \\
\text { homolog } 3\end{array}$ & $\begin{array}{l}\text { Nucleus } \\
\text { (Probable). }\end{array}$ & $\begin{array}{l}\text { RNA processing and Transcription } \\
\text { regulation }\end{array}$ \\
\hline SSH3 & $\begin{array}{l}\text { HUMAN Protein phosphatase } \\
\text { Slingshot homolog } 3 \text { (SSH-3L) }\end{array}$ & $\begin{array}{l}\text { Cytoplasm, } \\
\text { nucleus }\end{array}$ & $\begin{array}{l}\text { Protein phosphatase which may play a } \\
\text { role in the regulation of actin filament } \\
\text { dynamics }\end{array}$ \\
\hline DDX21 & $\begin{array}{l}\text { HUMAN Nucleolar RNA } \\
\text { helicase } 2 \text { (Nucleolar RNA } \\
\text { helicase II) }\end{array}$ & $\begin{array}{l}\text { Nucleus; } \\
\text { nucleolus. }\end{array}$ & $\begin{array}{l}\text { RNA processing and Transcription } \\
\text { regulation }\end{array}$ \\
\hline TACC2 & $\begin{array}{l}\text { HUMAN Transforming acidic } \\
\text { coiled-coil-containing protein } 2\end{array}$ & $\begin{array}{l}\text { Cytoplasm. } \\
\text { Nucleus. } \\
\text { Centrosome. }\end{array}$ & $\begin{array}{l}\text { May play a role in organizing } \\
\text { centrosomal microtubules. May act as } \\
\text { a tumor suppressor protein. May } \\
\text { represent a tumor progression marker }\end{array}$ \\
\hline LIPB1 & HUMAN Liprin-beta-1 & Unknown & $\begin{array}{l}\text { May regulate the disassembly of focal } \\
\text { adhesions. Did not bind receptor-like } \\
\text { tyrosine phosphatases type } 2 \text { A. }\end{array}$ \\
\hline IF4B & $\begin{array}{l}\text { HUMAN Eukaryotic translation } \\
\text { initiation factor 4B (eIF-4B) }\end{array}$ & Unknown & $\begin{array}{l}\text { RNA processing and Transcription } \\
\text { regulation }\end{array}$ \\
\hline TIF1A & $\begin{array}{l}\text { HUMAN Transcription } \\
\text { intermediary factor 1-alpha } \\
\text { (TIF1-alpha) }\end{array}$ & Nucleus & $\begin{array}{l}\text { Interacts selectively in vitro with the } \\
\text { AF2-activating domain of the estrogen } \\
\text { receptors. Association with DNA- } \\
\text { bound estrogen receptors requires the } \\
\text { presence of estradiol. }\end{array}$ \\
\hline MAP1B & $\begin{array}{l}\text { HUMAN Microtubule-associated } \\
\text { protein 1B (MAP 1B) }\end{array}$ & Unknown & $\begin{array}{l}\text { The function of brain MAPS is } \\
\text { essentially unknown. Phosphorylated } \\
\text { MAP1B may play a role in the } \\
\text { cytoskeletal changes that accompany } \\
\text { neurite extension }\end{array}$ \\
\hline EXOS9 & $\begin{array}{l}\text { HUMAN Exosome complex } \\
\text { exonuclease RRP45 (Exosome } \\
\text { component 9) }\end{array}$ & $\begin{array}{l}\text { Cytoplasm. } \\
\text { Nucleus; } \\
\text { nucleolus. }\end{array}$ & $\begin{array}{l}\text { RNA processing and Transcription } \\
\text { regulation }\end{array}$ \\
\hline BCL7C & $\begin{array}{l}\text { HUMAN B-cell CLL/lymphoma } \\
7 \text { protein family member C }\end{array}$ & Unknown & May play an anti-apoptotic role \\
\hline SNUT1 & $\begin{array}{l}\text { HUMAN U4/U6.U5 tri-snRNP- } \\
\text { associated protein } 1\end{array}$ & Nucleus & Translation \\
\hline WEE1 & $\begin{array}{l}\text { HUMAN Wee1-like protein } \\
\text { kinase (Wee1A kinase) } \\
\text { (WEE1hu) }\end{array}$ & Nucleus & Cell cycle \\
\hline
\end{tabular}


Table A-3 (continued).

\begin{tabular}{|c|c|c|c|}
\hline $\begin{array}{l}\text { Swissprot } \\
\text { code }\end{array}$ & Protein name & $\begin{array}{l}\text { Subcellular } \\
\text { localization }\end{array}$ & Functional category \\
\hline NCK1 & $\begin{array}{l}\text { HUMAN Cytoplasmic protein } \\
\text { NCK1 (NCK adaptor protein 1) }\end{array}$ & Cytoplasm & $\begin{array}{l}\text { Adapter protein which associates with } \\
\text { tyrosine-phosphorylated growth factor } \\
\text { receptors or their cellular substrates. }\end{array}$ \\
\hline STUB1 & $\begin{array}{l}\text { HUMAN STIP1 homology and U } \\
\text { box-containing protein } 1\end{array}$ & Cytoplasm & Unknown \\
\hline DIDO1 & $\begin{array}{l}\text { HUMAN Death-inducer } \\
\text { obliterator } 1 \text { (DIO-1) }\end{array}$ & $\begin{array}{l}\text { Cytoplasm. } \\
\text { Nucleus }\end{array}$ & $\begin{array}{l}\text { RNA processing and Transcription } \\
\text { regulation }\end{array}$ \\
\hline SPF45 & $\begin{array}{l}\text { HUMAN Splicing factor } 45 \text { ( } 45 \\
\text { kDa-splicing factor) }\end{array}$ & Nucleus. & RNA processing \\
\hline PDCD4 & $\begin{array}{l}\text { HUMAN Programmed cell death } \\
\text { protein } 4 \text { (Nuclear antigen H731- } \\
\text { like) }\end{array}$ & $\begin{array}{l}\text { Nucleus. } \\
\text { Cytoplasm }\end{array}$ & $\begin{array}{l}\text { Tumor suppressor. Inhibits tumor } \\
\text { promoter-induced }\end{array}$ \\
\hline IPYR2 & $\begin{array}{l}\text { HUMAN Inorganic } \\
\text { pyrophosphatase 2, mitochondrial } \\
\text { precursor (PPase 2) }\end{array}$ & $\begin{array}{l}\text { Mitochondr } \\
\text { ion }\end{array}$ & Unknown \\
\hline LS14A & $\begin{array}{l}\text { HUMAN LSM14 protein } \\
\text { homolog A (Protein SCD6 } \\
\text { homolog) }\end{array}$ & Unknown & Unknown \\
\hline SFRS6 & $\begin{array}{l}\text { HUMAN Splicing factor, } \\
\text { arginine/serine-rich } 6\end{array}$ & Nucleus & $\begin{array}{l}\text { Plays a role in constitutive splicing and } \\
\text { can modulate the selection of } \\
\text { alternative splice sites }\end{array}$ \\
\hline SDS3 & $\begin{array}{l}\text { HUMAN Sin3 histone } \\
\text { deacetylase corepressor complex } \\
\text { component SDS3 }\end{array}$ & Unknown & $\begin{array}{l}\text { Regulatory protein which represses } \\
\text { transcription and augments histone } \\
\text { deacetylase activity of HDAC1. May } \\
\text { have a potential role in tumor } \\
\text { suppressor pathways. May function in } \\
\text { the assembly and/or enzymatic activity } \\
\text { of the mSin3A corepressor complex or } \\
\text { in mediating interactions between the } \\
\text { complex and other regulatory } \\
\text { complexes }\end{array}$ \\
\hline ARS2 & $\begin{array}{l}\text { HUMAN Arsenite-resistance } \\
\text { protein } 2\end{array}$ & Unknown & Confers arsenite resistance \\
\hline TBA1 & $\begin{array}{l}\text { HUMAN Tubulin alpha-1 chain } \\
\text { (Alpha-tubulin 1) }\end{array}$ & Unknown & $\begin{array}{l}\text { Tubulin is the major constituent of } \\
\text { microtubules. It binds two moles of } \\
\text { GTP, one at an exchangeable site on } \\
\text { the beta chain and one at a non- } \\
\text { exchangeable site on the alpha-chain }\end{array}$ \\
\hline REPS1 & $\begin{array}{l}\text { HUMAN RalBP1-associated Eps } \\
\text { domain-containing protein } 1\end{array}$ & Unknown & Unknown \\
\hline
\end{tabular}


Table A-3 (continued).

\begin{tabular}{|c|c|c|c|}
\hline $\begin{array}{l}\text { Swissprot } \\
\text { code }\end{array}$ & Protein name & $\begin{array}{l}\text { Subcellular } \\
\text { localization }\end{array}$ & Functional category \\
\hline UBXD7 & $\begin{array}{l}\text { HUMAN UBX domain- } \\
\text { containing protein } 7\end{array}$ & Unknown & Unknown \\
\hline YBOX1 & $\begin{array}{l}\text { HUMAN Nuclease sensitive } \\
\text { element-binding protein } 1\end{array}$ & $\begin{array}{l}\text { Cytoplasm. } \\
\text { Nucleus }\end{array}$ & Translation \\
\hline NDRG2 & $\begin{array}{l}\text { HUMAN Protein NDRG2 } \\
\text { (Protein Syld709613) }\end{array}$ & $\begin{array}{l}\text { Cytoplasm. } \\
\text { Cytoplasm; } \\
\text { perinuclear } \\
\text { region }\end{array}$ & $\begin{array}{l}\text { May be involved in dendritic cell and } \\
\text { neuron differentiation. May have anti- } \\
\text { tumor activity. }\end{array}$ \\
\hline PGRC1 & $\begin{array}{l}\text { HUMAN Membrane-associated } \\
\text { progesterone receptor component } \\
1 \text { (mPR) }\end{array}$ & $\begin{array}{l}\text { Microsome; } \\
\text { microsomal } \\
\text { membrane; }\end{array}$ & Receptor for progesterone \\
\hline EP15R & $\begin{array}{l}\text { HUMAN Epidermal growth } \\
\text { factor receptor substrate } 15 \text {-like } 1\end{array}$ & $\begin{array}{l}\text { Cell } \\
\text { membrane }\end{array}$ & $\begin{array}{l}\text { Seems to be a constitutive component } \\
\text { of clathrin-coated pits that is required } \\
\text { for receptor-mediated endocytosis }\end{array}$ \\
\hline BIN1 & $\begin{array}{l}\text { HUMAN Myc box-dependent- } \\
\text { interacting protein } 1 \text { (Bridging } \\
\text { integrator 1) }\end{array}$ & $\begin{array}{l}\text { Nucleus } \\
\text { cytoplasm }\end{array}$ & Unknown \\
\hline SGIP1 & $\begin{array}{l}\text { HUMAN SH3-containing GRB2- } \\
\text { like protein 3-interacting protein } \\
1\end{array}$ & Unknown & $\begin{array}{l}\text { Possible role in the regulation of } \\
\text { energy homeostasis }\end{array}$ \\
\hline MATN4 & HUMAN Matrilin-4 precursor & $\begin{array}{l}\text { Secreted } \\
\text { protein }\end{array}$ & Unknown \\
\hline YETS2 & $\begin{array}{l}\text { HUMAN YEATS domain- } \\
\text { containing protein } 2\end{array}$ & Nucleus & Unknown \\
\hline PDE10 & $\begin{array}{l}\text { HUMAN cAMP and cAMP- } \\
\text { inhibited cGMP 3',5'-cyclic } \\
\text { phosphodiesterase } 10 \mathrm{~A}\end{array}$ & $\begin{array}{l}\text { Soluble } \\
\text { cellular } \\
\text { fractions }\end{array}$ & Signal transduction \\
\hline DPOD3 & $\begin{array}{l}\text { HUMAN DNA polymerase } \\
\text { subunit delta } 3 \text { (DNA polymerase } \\
\text { subunit delta p66) }\end{array}$ & Nucleus. & Unknown \\
\hline NSF1C & $\begin{array}{l}\text { HUMAN NSFL1 cofactor } \mathrm{p} 47 \\
\text { (p97 cofactor } \mathrm{p} 47 \text { ) }\end{array}$ & $\begin{array}{l}\text { Nucleus. } \\
\text { Golgi } \\
\text { apparatus; }\end{array}$ & Translation \\
\hline WASF2 & $\begin{array}{l}\text { HUMAN Wiskott-Aldrich } \\
\text { syndrome protein family member } \\
2\end{array}$ & Cytoplasm & Signal transduction \\
\hline MEP50 & $\begin{array}{l}\text { HUMAN Methylosome protein } \\
50 \text { (MEP50 protein) (WD repeat } \\
\text { protein } 77 \text { ) }\end{array}$ & Unknown & $\begin{array}{l}\text { The methylosome may regulate an } \\
\text { early step in the assembly of U } \\
\text { snRNPs, possibly the transfer of Sm } \\
\text { proteins to the SMN-complex }\end{array}$ \\
\hline
\end{tabular}


Table A-3 (continued).

\begin{tabular}{|c|c|c|c|}
\hline $\begin{array}{l}\text { Swissprot } \\
\text { code }\end{array}$ & Protein name & $\begin{array}{l}\text { Subcellular } \\
\text { localization }\end{array}$ & Functional category \\
\hline ARRB1 & $\begin{array}{l}\text { HUMAN Beta-arrestin-1 } \\
\text { (Arrestin beta 1) }\end{array}$ & Unknown & $\begin{array}{l}\text { Regulates beta-adrenergic receptor } \\
\text { function. Beta-arrestins seem to bind } \\
\text { phosphorylated beta-adrenergic } \\
\text { receptors, thereby causing a significant } \\
\text { impairment of their capacity to } \\
\text { activate } G(S) \text { protein }\end{array}$ \\
\hline CT2NL & $\begin{array}{l}\text { HUMAN CTTNBP2 N-terminal- } \\
\text { like protein }\end{array}$ & Unknown & Unknown \\
\hline TJAP1 & $\begin{array}{l}\text { HUMAN Tight junction- } \\
\text { associated protein } 1 \text { (Tight } \\
\text { junction protein } 4 \text { ) }\end{array}$ & $\begin{array}{l}\text { Golgi } \\
\text { apparatus }\end{array}$ & Unknown \\
\hline LAMA2 & $\begin{array}{l}\text { HUMAN Laminin subunit alpha- } \\
2 \text { precursor (Laminin M chain) }\end{array}$ & $\begin{array}{l}\text { Secreted } \\
\text { protein; } \\
\text { extracellular } \\
\text { space; } \\
\text { extracellular }\end{array}$ & $\begin{array}{l}\text { Binding to cells via a high affinity } \\
\text { receptor, laminin is thought to mediate } \\
\text { the attachment, migration and } \\
\text { organization of cells into tissues } \\
\text { during embryonic development by } \\
\text { interacting with other extracellular } \\
\text { matrix components }\end{array}$ \\
\hline PERQ2 & $\begin{array}{l}\text { HUMAN PERQ amino acid-rich } \\
\text { with GYF domain-containing } \\
\text { protein } 2\end{array}$ & Unknown & Signal transduction \\
\hline NIPA & $\begin{array}{l}\text { HUMAN Nuclear-interacting } \\
\text { partner of ALK }\end{array}$ & Nucleus & Signal transduction \\
\hline ANK3 & $\begin{array}{l}\text { HUMAN Ankyrin-3 (ANK-3) } \\
\text { (Ankyrin-G) }\end{array}$ & Unknown & Unknown \\
\hline CIP4 & $\begin{array}{l}\text { HUMAN Cdc42-interacting } \\
\text { protein } 4\end{array}$ & $\begin{array}{l}\text { Cytoplasm; } \\
\text { lysosome; } \\
\text { Golgi } \\
\text { apparatus. } \\
\text { Cell } \\
\text { membrane }\end{array}$ & Signal transduction \\
\hline PGRC2 & $\begin{array}{l}\text { HUMAN Membrane-associated } \\
\text { progesterone receptor component } \\
2\end{array}$ & Unknown & Receptor for steroids \\
\hline $\mathrm{KKCC1}$ & $\begin{array}{l}\text { HUMAN Calcium/calmodulin- } \\
\text { dependent protein kinase kinase } 1\end{array}$ & $\begin{array}{l}\text { Cytoplasm, } \\
\text { nucleus }\end{array}$ & $\begin{array}{l}\text { Signal transduction } \\
\text { Calcium/calmodulin-dependent } \\
\text { protein kinase that belongs to a } \\
\text { proposed calcium-triggered signaling } \\
\text { cascade involved in a number of } \\
\text { cellular processes }\end{array}$ \\
\hline PEBP1 & $\begin{array}{l}\text { HUMAN } \\
\text { Phosphatidylethanolamine- } \\
\text { binding protein } 1 \text { (PEBP-1) }\end{array}$ & Cytoplasm & Unknown \\
\hline
\end{tabular}


Table A-3 (continued).

\begin{tabular}{|c|c|c|c|}
\hline $\begin{array}{l}\text { Swissprot } \\
\text { code }\end{array}$ & Protein name & $\begin{array}{l}\text { Subcellular } \\
\text { localization }\end{array}$ & Functional category \\
\hline BAIP2 & $\begin{array}{l}\text { HUMAN Brain-specific } \\
\text { angiogenesis inhibitor 1- } \\
\text { associated protein } 2\end{array}$ & $\begin{array}{l}\text { Cytoplasm, } \\
\text { membrane }\end{array}$ & $\begin{array}{l}\text { Adapter protein that links membrane- } \\
\text { bound small G-proteins to cytoplasmic } \\
\text { effector proteins. Necessary for } \\
\text { CDC42-mediated reorganization of the } \\
\text { actin cytoskeleton and for RAC1- } \\
\text { mediated membrane ruffling. }\end{array}$ \\
\hline PDPK1 & $\begin{array}{l}\text { HUMAN 3-phosphoinositide- } \\
\text { dependent protein kinase } 1 \\
\text { (hPDK1) }\end{array}$ & $\begin{array}{l}\text { Cytoplasm. } \\
\text { membrane; } \\
\text { peripheral } \\
\text { membrane }\end{array}$ & Signal transduction \\
\hline SH24A & $\begin{array}{l}\text { HUMAN SH2 domain-containing } \\
\text { protein } 4 \mathrm{~A}\end{array}$ & Unknown & Unknown \\
\hline NELFE & $\begin{array}{l}\text { HUMAN Negative elongation } \\
\text { factor E (NELF-E) }\end{array}$ & Nucleus & Transcription regulation \\
\hline CN004 & HUMAN Protein C14orf4 & Nucleus & Unknown \\
\hline NFIA & $\begin{array}{l}\text { HUMAN Nuclear factor } 1 \text { A-type } \\
\text { (Nuclear factor } 1 / \mathrm{A} \text { ) }\end{array}$ & Nucleus & $\begin{array}{l}\text { RNA processing and Transcription } \\
\text { regulation }\end{array}$ \\
\hline PI3R6 & $\begin{array}{l}\text { HUMAN Phosphoinositide } 3 \text { - } \\
\text { kinase regulatory subunit } 6\end{array}$ & Cytoplasm & Unknown \\
\hline TAF1 & $\begin{array}{l}\text { HUMAN Transcription initiation } \\
\text { factor TFIID subunit } 1\end{array}$ & Nucleus & Transcription regulation \\
\hline CLC4F & $\begin{array}{l}\text { HUMAN C-type lectin domain } \\
\text { family } 4 \text { member } F\end{array}$ & Membrane & $\begin{array}{l}\text { Receptor with an affinity for galactose } \\
\text { and fucose. Could be involved in } \\
\text { endocytosis }\end{array}$ \\
\hline TLE3 & $\begin{array}{l}\text { HUMAN Transducin-like } \\
\text { enhancer protein } 3 \text { (ESG3) }\end{array}$ & Unknown & Transcription regulation \\
\hline ARHGG & $\begin{array}{l}\text { HUMAN Rho guanine nucleotide } \\
\text { exchange factor } 16\end{array}$ & Unknown & Unknown \\
\hline ILF3 & $\begin{array}{l}\text { HUMAN Interleukin enhancer- } \\
\text { binding factor } 3\end{array}$ & Nucleus & DNA processing \\
\hline ZN496 & HUMAN Zinc finger protein 496 & Nucleus & Transcription regulation \\
\hline G3BP2 & $\begin{array}{l}\text { HUMAN Ras GTPase-activating } \\
\text { protein-binding protein } 2 \text { (G3BP- } \\
\text { 2) }\end{array}$ & Unknown & Scaffold protein \\
\hline
\end{tabular}


Table A-3 (continued).

\begin{tabular}{|c|c|c|c|}
\hline $\begin{array}{l}\text { Swissprot } \\
\text { code }\end{array}$ & Protein name & $\begin{array}{l}\text { Subcellular } \\
\text { localization }\end{array}$ & Functional category \\
\hline HNF3A & $\begin{array}{l}\text { HUMAN Hepatocyte nuclear } \\
\text { factor 3-alpha }\end{array}$ & Nucleus & Transcription activator \\
\hline MYH9 & $\begin{array}{l}\text { HUMAN Myosin-9 (Myosin } \\
\text { heavy chain 9) }\end{array}$ & Unknown & Cell cycle \\
\hline RU17 & $\begin{array}{l}\text { HUMAN U1 small nuclear } \\
\text { ribonucleoprotein } 70 \mathrm{kDa} \text { (U1 } \\
\text { snRNP } 70 \mathrm{kDa})\end{array}$ & Unknown & Unknown \\
\hline ATM & $\begin{array}{l}\text { HUMAN Serine-protein kinase } \\
\text { ATM (Ataxia telangiectasia } \\
\text { mutated) }\end{array}$ & $\begin{array}{l}\text { Nucleus. } \\
\text { Cytoplasmic } \\
\text { vesicle }\end{array}$ & Signal transduction \\
\hline RALY & $\begin{array}{l}\text { HUMAN RNA-binding protein } \\
\text { Raly }\end{array}$ & Nucleus & Translation \\
\hline ZNF2 & $\begin{array}{l}\text { HUMAN Zinc finger protein } 2 \\
\text { (Zinc finger 2.2) (Zinc finger } \\
\text { protein 661) }\end{array}$ & Nucleus & Transcription regulation \\
\hline STAC2 & $\begin{array}{l}\text { HUMAN SH3 and cysteine-rich } \\
\text { domain-containing protein } 2\end{array}$ & Unknown & Unknown \\
\hline FOXK1 & $\begin{array}{l}\text { HUMAN Forkhead box protein } \\
\text { K1 (Myocyte nuclear factor) } \\
\text { (MNF) }\end{array}$ & Unknown & Transcription regulation \\
\hline ZBTB5 & $\begin{array}{l}\text { HUMAN Zinc finger and BTB } \\
\text { domain-containing protein } 5\end{array}$ & Nucleus & Transcription regulation \\
\hline RBGPR & $\begin{array}{l}\text { HUMAN Rab3 GTPase-activating } \\
\text { protein non-catalytic subunit }\end{array}$ & Cytoplasm & $\begin{array}{l}\text { Regulatory subunit of a GTPase } \\
\text { activating protein that has specificity } \\
\text { for Rab3 subfamily (RAB3A, } \\
\text { RAB3B, RAB3C and RAB3D). Rab3 } \\
\text { proteins are involved in regulated } \\
\text { exocytosis of neurotransmitters and } \\
\text { hormones. Rab3 GTPase-activating } \\
\text { complex specifically converts active } \\
\text { Rab3-GTP to the inactive form Rab3- } \\
\text { GDP. Required for normal eye and } \\
\text { brain development. }\end{array}$ \\
\hline ROA2 & $\begin{array}{l}\text { HUMAN Heterogeneous nuclear } \\
\text { ribonucleoproteins A2/B1 } \\
\text { (hnRNP A2/hnRNP B1) }\end{array}$ & Nucleus & $\begin{array}{l}\text { RNA processing and Transcription } \\
\text { regulation }\end{array}$ \\
\hline
\end{tabular}


Table A-3 (continued).

\begin{tabular}{|c|c|c|c|}
\hline $\begin{array}{l}\text { Swissprot } \\
\text { code }\end{array}$ & Protein name & $\begin{array}{l}\text { Subcellular } \\
\text { localization }\end{array}$ & Functional category \\
\hline SEPT9 & $\begin{array}{l}\text { HUMAN Septin-9 (MLL septin- } \\
\text { like fusion protein) }\end{array}$ & $\begin{array}{l}\text { Cell } \\
\text { membrane }\end{array}$ & $\begin{array}{l}\text { Involved in cytokinesis. May } \\
\text { function as an ovarian tumor } \\
\text { suppressor }\end{array}$ \\
\hline DTD1 & $\begin{array}{l}\text { HUMAN Probable D-tyrosyl- } \\
\text { tRNA(Tyr) deacylase } 1\end{array}$ & Cytoplasm & $\begin{array}{l}\text { Hydrolyzes D-tyrosyl-tRNA(Tyr) } \\
\text { into D-tyrosine and free } \\
\text { tRNA(Tyr). Could be a defense } \\
\text { mechanism against a harmful effect } \\
\text { of D-tyrosine }\end{array}$ \\
\hline K0515 & $\begin{array}{l}\text { HUMAN Uncharacterized protein } \\
\text { KIAA0515 }\end{array}$ & Unknown & Unknown \\
\hline MCM3 & $\begin{array}{l}\text { HUMAN DNA replication } \\
\text { licensing factor MCM3 }\end{array}$ & Nucleus & DNA processing \\
\hline MTMR5 & $\begin{array}{l}\text { HUMAN SET-binding factor } 1 \\
\text { (Sbf1) (Myotubularin-related } \\
\text { protein 5) }\end{array}$ & Nucleus & $\begin{array}{l}\text { Probable pseudophosphatase. Lacks } \\
\text { several amino acids in the catalytic } \\
\text { pocket which renders it catalytically } \\
\text { inactive as a phosphatase. The } \\
\text { pocket is however sufficiently } \\
\text { preserved to bind phosphorylated } \\
\text { substrates, and maybe protect them } \\
\text { from phosphatases. Inhibits } \\
\text { myoblast differentiation in vitro and } \\
\text { induces oncogenic transformation } \\
\text { in fibroblasts }\end{array}$ \\
\hline ALKB5 & $\begin{array}{l}\text { HUMAN Alkylated repair protein } \\
\text { alkB homolog } 5\end{array}$ & Unknown & Unknown \\
\hline $\mathrm{CHCH} 3$ & $\begin{array}{l}\text { HUMAN Coiled-coil-helix-coiled- } \\
\text { coil-helix domain-containing } \\
\text { protein } 3\end{array}$ & Unknown & Unknown \\
\hline VDAC1 & $\begin{array}{l}\text { HUMAN Voltage-dependent } \\
\text { anion-selective channel protein } 1 \\
\text { (VDAC-1) }\end{array}$ & $\begin{array}{l}\text { Mitochondrio } \\
\mathrm{n} \text {; } \\
\text { mitochondrial } \\
\text { outer } \\
\text { membrane. }\end{array}$ & Unknown \\
\hline B4GN4 & $\begin{array}{l}\text { HUMAN N-acetyl-beta- } \\
\text { glucosaminyl-glycoprotein 4-beta- } \\
\text { N-acetylgalactosaminyltransferase } \\
1\end{array}$ & $\begin{array}{l}\text { Golgi } \\
\text { apparatus }\end{array}$ & $\begin{array}{l}\text { Transfers N-acetylgalactosamine } \\
\text { (GalNAc) from UDP-GalNAc to N- } \\
\text { acetylglucosamine-beta-benzyl with } \\
\text { a beta-1,4-linkage to form N,N'- } \\
\text { diacetyllactosediamine, GalNAc- } \\
\text { beta-1,4-GlcNAc structures in N- } \\
\text { linked glycans and probably O- } \\
\text { linked glycans. }\end{array}$ \\
\hline SNW1 & $\begin{array}{l}\text { HUMAN SNW domain-containing } \\
\text { protein } 1 \text { (Nuclear protein SkiP) }\end{array}$ & Nucleus & $\begin{array}{l}\text { RNA processing and Transcription } \\
\text { regulation }\end{array}$ \\
\hline PPA6 & $\begin{array}{l}\text { HUMAN Lysophosphatidic acid } \\
\text { phosphatase type } 6 \text { precursor }\end{array}$ & $\begin{array}{l}\text { Secreted } \\
\text { protein }\end{array}$ & $\begin{array}{l}\text { Hydrolyzes lysophosphatidic acid } \\
\text { to monoacylglycerol }\end{array}$ \\
\hline
\end{tabular}


Table A-3 (continued).

\begin{tabular}{|c|c|c|c|}
\hline $\begin{array}{l}\text { Swissprot } \\
\text { code }\end{array}$ & Protein name & $\begin{array}{l}\text { Subcellular } \\
\text { localization }\end{array}$ & Functional category \\
\hline RFIP1 & $\begin{array}{l}\text { HUMAN Rab11 family- } \\
\text { interacting protein } 1 \text { (Rab11- } \\
\text { FIP1) }\end{array}$ & $\begin{array}{l}\text { Endosome; } \\
\text { recycling } \\
\text { endosome. } \\
\text { Isoform 2: }\end{array}$ & $\begin{array}{l}\text { A Rab11 effector protein involved in } \\
\text { the endosomal recycling process. Also } \\
\text { involved in controlling membrane } \\
\text { trafficking along the phagocytic } \\
\text { pathway and in phagocytosis }\end{array}$ \\
\hline TCEA1 & $\begin{array}{l}\text { HUMAN Transcription } \\
\text { elongation factor A protein } 1\end{array}$ & Nucleus & Transcription regulation \\
\hline SAP30 & $\begin{array}{l}\text { HUMAN Histone deacetylase } \\
\text { complex subunit SAP30 }\end{array}$ & Nucleus & Transcription regulation \\
\hline SAM68 & $\begin{array}{l}\text { HUMAN KH domain-containing, } \\
\text { RNA-binding, signal } \\
\text { transduction-associated protein1 }\end{array}$ & $\begin{array}{l}\text { Nucleus. } \\
\text { Membrane. }\end{array}$ & $\begin{array}{l}\text { Signal transduction, transcription } \\
\text { regulation and cell cycle }\end{array}$ \\
\hline $\mathrm{BI} 2 \mathrm{~L} 1$ & $\begin{array}{l}\text { HUMAN Brain-specific } \\
\text { angiogenesis inhibitor 1- } \\
\text { associated protein 2-like protein1 }\end{array}$ & Unknown & May function as adapter protein \\
\hline ROA1 & $\begin{array}{l}\text { HUMAN Heterogeneous nuclear } \\
\text { ribonucleoprotein A1 }\end{array}$ & $\begin{array}{l}\text { Nucleus, } \\
\text { cytoplasm }\end{array}$ & $\begin{array}{l}\text { Involved in the packaging of pre- } \\
\text { mRNA into hnRNP particles, transport } \\
\text { of poly(A) mRNA from the nucleus to } \\
\text { the cytoplasm and may modulate } \\
\text { splice site selection }\end{array}$ \\
\hline PKP3 & HUMAN Plakophilin-3 & $\begin{array}{l}\text { Nucleus. } \\
\text { Cell } \\
\text { junction; } \\
\text { desmosome. }\end{array}$ & May play a role in junctional plaques. \\
\hline SEBP2 & $\begin{array}{l}\text { HUMAN SECIS-binding protein } \\
2\end{array}$ & Nucleus & $\begin{array}{l}\text { Binds to the SECIS element in the } 3 \text { ' } \\
\text { UTR of some mRNAs encoding } \\
\text { selenoproteins. Binding is stimulated } \\
\text { by SELB. }\end{array}$ \\
\hline NUP98 & $\begin{array}{l}\text { HUMAN Nuclear pore complex } \\
\text { protein Nup98-Nup96 precursor }\end{array}$ & Nucleus & $\begin{array}{l}\text { Nup98 and Nup96 play a role in the } \\
\text { bidirectional transport across the } \\
\text { nucleoporin complex (NPC). The } \\
\text { repeat domain in Nup } 98 \text { has a direct } \\
\text { role in the transport }\end{array}$ \\
\hline K1C9 & $\begin{array}{l}\text { HUMAN Keratin, type I } \\
\text { cytoskeletal } 9 \text { (Cytokeratin-9) }\end{array}$ & Unknown & $\begin{array}{l}\text { May serve an important special } \\
\text { function either in the mature palmar } \\
\text { and plantar skin tissue or in the } \\
\text { morphogenic program of the formation } \\
\text { of these tissues }\end{array}$ \\
\hline ROA3 & $\begin{array}{l}\text { HUMAN Heterogeneous nuclear } \\
\text { ribonucleoprotein A3 (hnRNP } \\
\text { A3) }\end{array}$ & Nucleus & $\begin{array}{l}\text { RNA processing and Transcription } \\
\text { regulation }\end{array}$ \\
\hline RL22L & $\begin{array}{l}\text { HUMAN Ribosomal protein L22- } \\
\text { like } 1\end{array}$ & Unknown & Unknown \\
\hline
\end{tabular}


Table A-3 (continued).

\begin{tabular}{|c|c|c|c|}
\hline $\begin{array}{l}\text { Swissprot } \\
\text { code }\end{array}$ & Protein name & $\begin{array}{l}\text { Subcellular } \\
\text { localization }\end{array}$ & Functional category \\
\hline RBM15 & $\begin{array}{l}\text { HUMAN Putative RNA-binding } \\
\text { protein } 15 \text { (RNA-binding motif } \\
\text { protein 15) }\end{array}$ & Nucleus & $\begin{array}{l}\text { May be implicated in HOX gene } \\
\text { regulation }\end{array}$ \\
\hline JIP1 & $\begin{array}{l}\text { HUMAN C-jun-amino-terminal } \\
\text { kinase-interacting protein } 1\end{array}$ & $\begin{array}{l}\text { Cytoplasm; } \\
\text { nucleus }\end{array}$ & Signal transduction \\
\hline PAK4 & $\begin{array}{l}\text { HUMAN Serine/threonine- } \\
\text { protein kinase PAK } 4 \text { (p21- } \\
\text { activated kinase 4) }\end{array}$ & Unknown & Signal transduction \\
\hline NONO & $\begin{array}{l}\text { HUMAN Non-POU domain- } \\
\text { containing octamer-binding } \\
\text { protein (NonO protein) }\end{array}$ & Nucleus & $\begin{array}{l}\text { RNA processing and Transcription } \\
\text { regulation }\end{array}$ \\
\hline RBM14 & $\begin{array}{l}\text { HUMAN RNA-binding protein } \\
14 \text { (RNA-binding motif protein } \\
14 \text { ) }\end{array}$ & Unknown & Transcription regulation \\
\hline CSRP1 & $\begin{array}{l}\text { HUMAN Cysteine and glycine- } \\
\text { rich protein } 1 \text { (Cysteine-rich } \\
\text { protein 1) }\end{array}$ & Nucleus & $\begin{array}{l}\text { Could play a role in neuronal } \\
\text { development }\end{array}$ \\
\hline MYEF2 & $\begin{array}{l}\text { HUMAN Myelin expression } \\
\text { factor } 2 \text { (MyEF-2) (MST156) }\end{array}$ & Nucleus & Transcription repressor \\
\hline KIRR1 & $\begin{array}{l}\text { HUMAN Kin of IRRE-like } \\
\text { protein } 1 \text { precursor }\end{array}$ & Membrane & Signal transduction \\
\hline DDX25 & $\begin{array}{l}\text { HUMAN ATP-dependent RNA } \\
\text { helicase DDX25 (DEAD box } \\
\text { protein 25) }\end{array}$ & Cytoplasm & ATP-dependent RNA helicase \\
\hline RBM26 & $\begin{array}{l}\text { HUMAN RNA-binding protein } \\
26 \text { (RNA-binding motif protein } \\
\text { 26) }\end{array}$ & Unknown & Unknown \\
\hline HCN4 & $\begin{array}{l}\text { HUMAN Potassium/sodium } \\
\text { hyperpolarization-activated cyclic } \\
\text { nucleotide-gated channel } 4\end{array}$ & Membrane & $\begin{array}{l}\text { Hyperpolarization-activated ion } \\
\text { channel with very slow activation and } \\
\text { inactivation exhibiting weak } \\
\text { selectivity for potassium over sodium } \\
\text { ions. May contribute to the native } \\
\text { pacemaker currents in heart (If) and in } \\
\text { neurons (Ih). Activated by cAMP. } \\
\text { May mediate responses to sour stimuli. }\end{array}$ \\
\hline
\end{tabular}


Table A-3 (continued).

\begin{tabular}{|c|c|c|c|}
\hline $\begin{array}{l}\text { Swissprot } \\
\text { code }\end{array}$ & Protein name & $\begin{array}{l}\text { Subcellular } \\
\text { localization }\end{array}$ & Functional category \\
\hline PAR3L & $\begin{array}{l}\text { HUMAN Partitioning-defective } 3 \\
\text { homolog B (PAR3-beta) }\end{array}$ & $\begin{array}{l}\text { Intracytopla } \\
\text { smic } \\
\text { membrane. } \\
\text { Cell } \\
\text { junction }\end{array}$ & $\begin{array}{l}\text { Putative adapter protein involved in } \\
\text { asymmetrical cell division and cell } \\
\text { polarization processes. May play a role } \\
\text { in the formation of epithelial tight } \\
\text { junctions }\end{array}$ \\
\hline F262 & $\begin{array}{l}\text { HUMAN 6-phosphofructo-2- } \\
\text { kinase/fructose-2,6-biphosphatase } \\
2\end{array}$ & Unknown & $\begin{array}{l}\text { Synthesis and degradation of fructose } \\
\text { 2,6-bisphosphate }\end{array}$ \\
\hline EAF1 & HUMAN ELL-associated factor 1 & Nucleus & Transcription regulation \\
\hline DENR & $\begin{array}{l}\text { HUMAN Density-regulated } \\
\text { protein (DRP) (Protein DRP1) }\end{array}$ & Unknown & Unknown \\
\hline TB182 & $\begin{array}{l}\text { HUMAN } 182 \mathrm{kDa} \text { tankyrase } 1- \\
\text { binding protein }\end{array}$ & $\begin{array}{l}\text { Nucleus, } \\
\text { cytoplasm }\end{array}$ & $\begin{array}{l}\text { Binds to the ANK repeat domain of } \\
\text { TNKS1 and TNKS2 }\end{array}$ \\
\hline NHERF & $\begin{array}{l}\text { HUMAN Ezrin-radixin-moesin- } \\
\text { binding phosphoprotein } 50 \\
\text { (EBP50) }\end{array}$ & $\begin{array}{l}\text { Intracytopla } \\
\text { smic } \\
\text { membrane; } \\
\text { peripheral }\end{array}$ & Scaffold protein \\
\hline HD & $\begin{array}{l}\text { HUMAN Huntingtin (Huntington } \\
\text { disease protein) (HD protein) }\end{array}$ & $\begin{array}{l}\text { Cytoplasm, } \\
\text { nucleus }\end{array}$ & $\begin{array}{l}\text { May play a role in microtubule- } \\
\text { mediated transport or vesicle function }\end{array}$ \\
\hline NUDC & $\begin{array}{l}\text { HUMAN Nuclear migration } \\
\text { protein nudC }\end{array}$ & $\begin{array}{l}\text { Cytoplasm. } \\
\text { Nucleus }\end{array}$ & $\begin{array}{l}\text { Plays a role in neurogenesis and } \\
\text { neuronal migration }\end{array}$ \\
\hline PGRC1 & $\begin{array}{l}\text { HUMAN Membrane-associated } \\
\text { progesterone receptor component } \\
1 \text { (mPR) }\end{array}$ & $\begin{array}{l}\text { Microsome; } \\
\text { microsomal } \\
\text { membrane; } \\
\text { single-pass } \\
\text { membrane } \\
\text { protein }\end{array}$ & Receptor for progesterone \\
\hline CX026 & $\begin{array}{l}\text { HUMAN UPF0368 protein } \\
\text { Cxorf26 }\end{array}$ & Unknown & Unknown \\
\hline CLN3 & $\begin{array}{l}\text { HUMAN Battenin (Protein } \\
\text { CLN3) (Batten disease protein) }\end{array}$ & $\begin{array}{l}\text { Lysosome; } \\
\text { lysosomal } \\
\text { membrane }\end{array}$ & Unknown \\
\hline TOIP1 & $\begin{array}{l}\text { HUMAN Torsin-1A-interacting } \\
\text { protein } 1\end{array}$ & Nucleus & Unknown \\
\hline SQSTM & HUMAN Sequestosome-1 & $\begin{array}{l}\text { Cytoplasm. } \\
\text { Endosome; } \\
\text { late } \\
\text { endosome. } \\
\text { Nucleus }\end{array}$ & Signal trasduction \\
\hline LMNB1 & HUMAN Lamin-B1 & Nucleus & Unknown \\
\hline
\end{tabular}


Table A-3 (continued).

\begin{tabular}{|c|c|c|c|}
\hline $\begin{array}{l}\text { Swissprot } \\
\text { code }\end{array}$ & Protein name & $\begin{array}{l}\text { Subcellular } \\
\text { localization }\end{array}$ & Functional category \\
\hline RB12B & $\begin{array}{l}\text { HUMAN RNA-binding protein } \\
\text { 12B (RNA-binding motif protein } \\
\text { 12B) }\end{array}$ & Unknown & Unknown \\
\hline CTRO & $\begin{array}{l}\text { HUMAN Citron Rho-interacting } \\
\text { kinase (CRIK) }\end{array}$ & cytoplasm & Unknown \\
\hline ATX2L & HUMAN Ataxin-2-like protein & Membrane & Unknown \\
\hline TRA2B & $\begin{array}{l}\text { HUMAN Arginine/serine-rich- } \\
\text { splicing factor } 10 \text { (Transformer-2- } \\
\text { beta) }\end{array}$ & Nucleus & Translation \\
\hline UBP2L & $\begin{array}{l}\text { HUMAN Ubiquitin-associated } \\
\text { protein 2-like (Protein NICE-4) }\end{array}$ & Unknown & Unknown \\
\hline AKT1 & $\begin{array}{l}\text { HUMAN RAC-alpha } \\
\text { serine/threonine-protein kinase }\end{array}$ & $\begin{array}{l}\text { Cytoplasm. } \\
\text { Nucleus }\end{array}$ & Signal transduction \\
\hline CHD1 & $\begin{array}{l}\text { HUMAN Chromodomain- } \\
\text { helicase-DNA-binding protein } 1\end{array}$ & Nucleus & Gene Regulation \\
\hline CBX4 & $\begin{array}{l}\text { HUMAN E3 SUMO-protein } \\
\text { ligase CBX4 }\end{array}$ & Nucleus. & Transcription regulation \\
\hline BCKD & $\begin{array}{l}\text { HUMAN [3-methyl-2- } \\
\text { oxobutanoate dehydrogenase } \\
\text { [lipoamide]] kinase }\end{array}$ & $\begin{array}{l}\text { Mitochondr } \\
\text { ion; } \\
\text { mitochondri } \\
\text { al matrix. }\end{array}$ & Metabolic pathways \\
\hline IF4H & $\begin{array}{l}\text { HUMAN Eukaryotic translation } \\
\text { initiation factor } 4 \mathrm{H} \text { (eIF-4H) }\end{array}$ & Cytoplasm & Translation regulation \\
\hline PAIRB & $\begin{array}{l}\text { HUMAN Plasminogen activator } \\
\text { inhibitor } 1 \text { RNA-binding protein }\end{array}$ & $\begin{array}{l}\text { Cytoplasm. } \\
\text { Nucleus }\end{array}$ & $\begin{array}{l}\text { RNA processing and transcription } \\
\text { regulation }\end{array}$ \\
\hline AAKB1 & $\begin{array}{l}\text { HUMAN 5'-AMP-activated } \\
\text { protein kinase subunit beta-1 }\end{array}$ & Cytoplasm & Metabolic pathways \\
\hline MATR3 & HUMAN Matrin-3 & Nucleus & $\begin{array}{l}\text { RNA processing and transcription } \\
\text { regulation }\end{array}$ \\
\hline PGAM1 & $\begin{array}{l}\text { HUMAN Phosphoglycerate } \\
\text { mutase } 1\end{array}$ & Unknown & Unknown \\
\hline K1143 & $\begin{array}{l}\text { HUMAN Uncharacterized protein } \\
\text { KIAA1143 }\end{array}$ & Unknown & Unknown \\
\hline RGP2 & $\begin{array}{l}\text { HUMAN Rap1 GTPase-activating } \\
\text { protein } 1 \text { (Rap1GAP) }\end{array}$ & $\begin{array}{l}\text { Golgi } \\
\text { apparatus; } \\
\text { Golgi } \\
\text { apparatus } \\
\text { membrane }\end{array}$ & Signaling transduction \\
\hline
\end{tabular}


Table A-3 (continued).

\begin{tabular}{llll}
\hline $\begin{array}{l}\text { Swissprot } \\
\text { code }\end{array}$ & Protein name & $\begin{array}{l}\text { Subcellular } \\
\text { localization }\end{array}$ & Functional category \\
\hline TRAF4 & $\begin{array}{l}\text { HUMAN TNF receptor-associated } \\
\text { factor 4 }\end{array}$ & $\begin{array}{l}\text { Cytoplasm. } \\
\text { Nucleus }\end{array}$ & Signal transduction \\
MINT & $\begin{array}{l}\text { HUMAN Msx2-interacting } \\
\text { protein (SPEN homolog) }\end{array}$ & Nucleus & Signal transduction \\
FA76B & HUMAN Protein FAM76B & Unknown & Unknown \\
PPIG & $\begin{array}{l}\text { HUMAN Peptidyl-prolyl cis-trans } \\
\text { isomerase G }\end{array}$ & Nucleus & $\begin{array}{l}\text { RNA processing and transcription } \\
\text { regulation }\end{array}$ \\
ZO2 & $\begin{array}{l}\text { HUMAN Tight junction protein } \\
\text { ZO-2 (Zonula occludens 2 } \\
\text { protein) }\end{array}$ & $\begin{array}{l}\text { Cell } \\
\text { junction }\end{array}$ & Cell structure \\
& $\begin{array}{l}\text { HUMAN Chromobox protein } \\
\text { homolog 8 (Polycomb 3 homolog) }\end{array}$ & Nucleus & $\begin{array}{l}\text { RNA processing and transcription } \\
\text { regulation }\end{array}$ \\
\hline
\end{tabular}

The information was compiled from Swiss-Prot annotations. 
Table A-4 Details of characterized phosphoproteins.

\begin{tabular}{|c|c|c|c|c|c|c|c|c|c|}
\hline $\begin{array}{l}\text { Accession } \\
\text { number }\end{array}$ & Protein & Peptide & Site & $\begin{array}{l}\text { Phosphory- } \\
\text { lation site }\end{array}$ & $\begin{array}{l}99- \\
029\end{array}$ & $\begin{array}{l}98- \\
065\end{array}$ & $\begin{array}{l}99- \\
045\end{array}$ & $\begin{array}{l}99- \\
067\end{array}$ & $\begin{array}{l}99- \\
010\end{array}$ \\
\hline \multicolumn{10}{|c|}{$\begin{array}{l}\text { Phosphoproteins found in all } 5 \text { specimens } \\
\end{array}$} \\
\hline \multirow[t]{2}{*}{ Q05682 } & Caldesmon & K.RGS*IGENQVEVMVEEK.T & S202 (b6) & $\mathrm{Y}$ & $\mathrm{Y}$ & $\mathrm{Y}$ & $\mathrm{Y}$ & Y & $\mathrm{Y}$ \\
\hline & & K.RGS*IGENQVEVM\#VEEK.T & S202 & $\mathrm{Y}$ & $\mathrm{N}$ & Y & $\mathrm{N}$ & $\mathrm{N}$ & $\mathrm{N}$ \\
\hline \multirow[t]{3}{*}{ P17661 } & Desmin & R.TFGGAPGFPLGSPLSS*PVFPR.A & $\mathrm{S} 32(\mathrm{y} 5, \mathrm{y} 6)$ & $\mathrm{Y}$ & $\mathrm{N}$ & $\mathrm{Y}$ & $\mathrm{Y}$ & $\mathrm{N}$ & $\mathrm{N}$ \\
\hline & & R.TFGGAPGFPLGS*PLSSPVFPR.A & $\mathrm{S} 28(\mathrm{y} 9, \mathrm{y} 10)$ & $\mathrm{N}$ & $\mathrm{Y}$ & $\mathrm{Y}$ & $\mathrm{Y}$ & Y & $\mathrm{Y}$ \\
\hline & & R.TFGGAPGFPLGS*PLSS*PVFPR.A & $\mathrm{S} 32(\mathrm{y} 5, \mathrm{y} 6)$ & $\mathrm{Y}$ & $\mathrm{Y}$ & $\mathrm{N}$ & $\mathrm{Y}$ & $\mathrm{Y}$ & $\mathrm{N}$ \\
\hline \multirow[t]{3}{*}{ P04792 } & $\begin{array}{l}\text { Heat-shock protein } \\
\text { beta-1 (Heat shock } 27 \\
\text { kDa protein) }\end{array}$ & R.QLS*SGVSEIR.H & S82 (y7, y8) & $\mathrm{Y}$ & $\mathrm{Y}$ & $\mathrm{Y}$ & $\mathrm{Y}$ & $\mathrm{Y}$ & $\mathrm{Y}$ \\
\hline & & R.GPS*WDPFR.D & $\mathrm{S} 15(\mathrm{~b} 2, \mathrm{~b} 3)$ & $\mathrm{Y}$ & $\mathrm{Y}$ & $\mathrm{Y}$ & $\mathrm{Y}$ & $\mathrm{N}$ & $\mathrm{N}$ \\
\hline & & R.GPS*WDPFRDWYPHSR.L & S15 & $\mathrm{Y}$ & $\mathrm{N}$ & $\mathrm{N}$ & $\mathrm{N}$ & $\mathrm{Y}$ & $\mathrm{N}$ \\
\hline \multirow[t]{6}{*}{ Q9UMS6 } & Synaptopodin-2 & R.AQS*PTPSLPASWK.Y & S902 (y10, y11) & $\mathrm{Y}$ & $\mathrm{Y}$ & $\mathrm{Y}$ & $\mathrm{Y}$ & $\mathrm{Y}$ & $\mathrm{Y}$ \\
\hline & & R.AQS*PTPS*LPASWK.Y & S906 (y5, y7) & $\mathrm{Y}$ & $\mathrm{Y}$ & $\mathrm{N}$ & $\mathrm{Y}$ & $\mathrm{N}$ & $\mathrm{N}$ \\
\hline & & R.TAKPFPGSVNQPATPFS*PTR.N & $\mathrm{S} 604(\mathrm{y} 3, \mathrm{y} 6)$ & $\mathrm{Y}$ & $\mathrm{N}$ & $\mathrm{Y}$ & $\mathrm{N}$ & $\mathrm{Y}$ & $\mathrm{N}$ \\
\hline & & K.PFPGSVNQPATPFS*PTR.N & S604 & $\mathrm{Y}$ & $\mathrm{N}$ & $\mathrm{Y}$ & $\mathrm{N}$ & $\mathrm{Y}$ & $\mathrm{N}$ \\
\hline & & $\begin{array}{l}\text { R.GVSS*PIAGPAQPPPWPQPAPWSQPAFYD } \\
\text { SSER.I }\end{array}$ & S638 (not) & $\mathrm{Y}$ & $\mathrm{N}$ & $\mathrm{Y}$ & $\mathrm{N}$ & $\mathrm{N}$ & $\mathrm{N}$ \\
\hline & & K.SKS*PDPDPNLSHDR.I & S226 (not) & $\mathrm{N}$ & $\mathrm{N}$ & $\mathrm{N}$ & $\mathrm{N}$ & $\mathrm{Y}$ & $\mathrm{N}$ \\
\hline \multicolumn{10}{|c|}{ Phosphoproteins found in 4 out of 5 specimens } \\
\hline O14558 & $\begin{array}{l}\text { Heat-shock protein } \\
\text { beta-6 (Heat-shock } 20 \\
\text { kDa-like protein) }\end{array}$ & R.RAS*APLPGLSAPGR.L & S16 (y11, y12) & $\mathrm{N}$ & Y & Y & $\mathrm{N}$ & $\mathrm{Y}$ & Y \\
\hline Q14315 & Filamin-C & R.LGS*FGSITR.Q & $\mathrm{S} 2233$ (b2, b3) & $\mathrm{N}$ & $\mathrm{Y}$ & $\mathrm{N}$ & $\mathrm{Y}$ & $\mathrm{Y}$ & $\mathrm{Y}$ \\
\hline
\end{tabular}


Table A-4 (continued).

\begin{tabular}{|c|c|c|c|c|c|c|c|c|c|}
\hline $\begin{array}{l}\text { Accession } \\
\text { number }\end{array}$ & Protein & Peptide & Site & $\begin{array}{l}\text { Phosphory- } \\
\text { lation site }\end{array}$ & $\begin{array}{l}99- \\
029\end{array}$ & $\begin{array}{l}98- \\
065\end{array}$ & $\begin{array}{l}99- \\
045\end{array}$ & $\begin{array}{l}99- \\
067\end{array}$ & $\begin{array}{l}99- \\
010\end{array}$ \\
\hline \multirow[t]{2}{*}{ Q9H3Z4 } & $\begin{array}{l}\text { DnaJ homolog } \\
\text { subfamily C member } 5\end{array}$ & R.S*LSTSGESLYHVLGLDK.N & S8 (not) & $\mathrm{Y}$ & $\mathrm{Y}$ & $\mathrm{N}$ & $\mathrm{Y}$ & $\mathrm{Y}$ & $\mathrm{N}$ \\
\hline & & R.SLSTS*GESLYHVLGLDK.N & S12 (not) & Y & $\mathrm{N}$ & $\mathrm{Y}$ & $\mathrm{N}$ & $\mathrm{N}$ & $\mathrm{N}$ \\
\hline \multirow[t]{6}{*}{ Q9HBL0 } & Tensin-1 & R.RMS*VGDR.A & $\mathrm{S} 1393(\mathrm{y} 5, \mathrm{y} 6)$ & $\mathrm{N}$ & $\mathrm{Y}$ & $\mathrm{N}$ & $\mathrm{N}$ & $\mathrm{Y}$ & $\mathrm{N}$ \\
\hline & & R.WDS*YDNFSGHR.D & S338 (y8, y9) & $\mathrm{Y}$ & $\mathrm{Y}$ & $\mathrm{N}$ & $\mathrm{N}$ & $\mathrm{N}$ & $\mathrm{N}$ \\
\hline & & K.EAT*SDPSRTPEEEPLNLEGLVAHR.V & T854 (not) & $\mathrm{N}$ & $\mathrm{N}$ & Y & $\mathrm{N}$ & $\mathrm{N}$ & $\mathrm{N}$ \\
\hline & & R.T*PTQPLLESGFR.S & T1105 (not) & $\mathrm{N}$ & $\mathrm{N}$ & $\mathrm{N}$ & $\mathrm{Y}$ & $\mathrm{N}$ & $\mathrm{N}$ \\
\hline & & K.EATSDPSRT*PEEEPLNLEGLVAHR.V & T860 (not) & $\mathrm{N}$ & $\mathrm{N}$ & $\mathrm{N}$ & $\mathrm{Y}$ & Y & $\mathrm{N}$ \\
\hline & & R.SQS*FSEAEPQLPPAPVR.G & S621 (not) & $\mathrm{N}$ & $\mathrm{N}$ & $\mathrm{N}$ & $\mathrm{Y}$ & $\mathrm{N}$ & $\mathrm{N}$ \\
\hline \multicolumn{10}{|c|}{ Phosphoproteins found in 2 out of 5 specimens } \\
\hline Q15019 & Septin-2 & K.IYHLPDAES*DEDEDFKEQTR.L & $\mathrm{S} 218(\mathrm{y} 11, \mathrm{y} 12)$ & Y & $\mathrm{Y}$ & $\mathrm{N}$ & $\mathrm{Y}$ & $\mathrm{N}$ & $\mathrm{N}$ \\
\hline Q08554 & $\begin{array}{l}\text { Desmocollin-1 } \\
\text { precursor }\end{array}$ & R.MKVQDQDLPNT*PHSK.A & $\mathrm{T} 385$ (not) & $\mathrm{N}$ & $\mathrm{Y}$ & $\mathrm{N}$ & $\mathrm{N}$ & $\mathrm{N}$ & $\mathrm{Y}$ \\
\hline P21291 & $\begin{array}{l}\text { Cysteine and glycine- } \\
\text { rich protein } 1\end{array}$ & K.GFGFGQGAGALVHS*E & S192 (y2, y3) & $\mathrm{Y}$ & $\mathrm{Y}$ & $\mathrm{N}$ & $\mathrm{Y}$ & $\mathrm{N}$ & $\mathrm{N}$ \\
\hline Q8N283 & $\begin{array}{l}\text { Ankyrin repeat domain- } \\
\text { containing protein } 35\end{array}$ & R.QS*VGLLT*NELAM\#EK.E & $\begin{array}{l}\text { S672; T677 } \\
\text { (b3, b4/ not) }\end{array}$ & $\mathrm{N}, \mathrm{N}$ & $\mathrm{Y}$ & $\mathrm{N}$ & $\mathrm{Y}$ & $\mathrm{N}$ & $\mathrm{N}$ \\
\hline Q01995 & Transgelin & K.HVIGLQMGS*NR.G & $\mathrm{S} 181(\mathrm{y} 2, \mathrm{y} 6)$ & $\mathrm{Y}$ & $\mathrm{Y}$ & $\mathrm{Y}$ & $\mathrm{N}$ & $\mathrm{N}$ & $\mathrm{N}$ \\
\hline P08670 & Vimentin & R.ISLPLPNFS*SLNLR.E & S419 (y5, y6) & $\mathrm{Y}$ & $\mathrm{N}$ & $\mathrm{N}$ & $\mathrm{Y}$ & $\mathrm{Y}$ & $\mathrm{N}$ \\
\hline Q6P597 & Kinesin light chain 3 & K.APRTLSAST*QDLS*PH & $\begin{array}{l}\text { T493; S497 } \\
\text { (not/y2,y3) }\end{array}$ & $\mathrm{N}, \mathrm{N}$ & $\mathrm{N}$ & $\mathrm{N}$ & $\mathrm{Y}$ & $\mathrm{N}$ & $\mathrm{Y}$ \\
\hline O95425 & Supervillin & R.S*LSDFTGPPQLQALK.Y & S547 (not) & $\mathrm{N}$ & $\mathrm{N}$ & $\mathrm{N}$ & $\mathrm{Y}$ & $\mathrm{Y}$ & $\mathrm{N}$ \\
\hline Q15746 & $\begin{array}{l}\text { Myosin light chain } \\
\text { kinase }\end{array}$ & R.KSS*TGSPTSPLNAEK.L & S1773 (y12, y13) & $\mathrm{Y}$ & $\mathrm{N}$ & $\mathrm{N}$ & $\mathrm{Y}$ & $\mathrm{Y}$ & $\mathrm{N}$ \\
\hline \multirow[t]{2}{*}{ Q63ZY3 } & $\begin{array}{l}\text { Ankyrin repeat domain- } \\
\text { containing protein } 25\end{array}$ & R.ALAMPGRPES*PPVFR.S & S375 (y5,y6) & Y & $\mathrm{N}$ & $\mathrm{N}$ & $\mathrm{Y}$ & $\mathrm{Y}$ & $\mathrm{N}$ \\
\hline & & K.KIS*ITER.S & S406 (not) & $\mathrm{N}$ & $\mathrm{N}$ & $\mathrm{N}$ & $\mathrm{Y}$ & $\mathrm{N}$ & $\mathrm{N}$ \\
\hline
\end{tabular}


Table A-4 (continued).

\begin{tabular}{|c|c|c|c|c|c|c|c|c|c|}
\hline $\begin{array}{l}\text { Accession } \\
\text { number }\end{array}$ & Protein & Peptide & Site & $\begin{array}{l}\text { Phosphory- } \\
\text { lation site }\end{array}$ & $\begin{array}{l}99- \\
029\end{array}$ & $\begin{array}{l}98- \\
065\end{array}$ & $\begin{array}{l}99- \\
045\end{array}$ & $\begin{array}{l}99- \\
067\end{array}$ & $\begin{array}{l}99- \\
010\end{array}$ \\
\hline \multicolumn{10}{|c|}{ Phosphoproteins found in 1 out of 5 specimens } \\
\hline Q92736 & Ryanodine receptor 2 & R.SSSENAKVTS*LDS*SSHR.I & $\begin{array}{l}\text { S4546; S4549 } \\
\text { (not/not) }\end{array}$ & $\mathrm{N}, \mathrm{N}$ & $\mathrm{Y}$ & $\mathrm{N}$ & $\mathrm{N}$ & $\mathrm{N}$ & $\mathrm{N}$ \\
\hline P13796 & Plastin-2 & R.GS*VSDEEMMELR.E & S5 (y10, y11) & $\mathrm{Y}$ & $\mathrm{Y}$ & $\mathrm{N}$ & $\mathrm{N}$ & $\mathrm{N}$ & $\mathrm{N}$ \\
\hline Q15283 & $\begin{array}{l}\text { Ras GTPase-activating } \\
\text { protein } 2\end{array}$ & K.S*SFKETFMCEFFK.M & S559 (not) & $\mathrm{N}$ & $\mathrm{Y}$ & $\mathrm{N}$ & $\mathrm{N}$ & $\mathrm{N}$ & $\mathrm{N}$ \\
\hline P43353 & $\begin{array}{l}\text { Aldehyde dehydrogenase } \\
\text { 3B1 }\end{array}$ & R.FDY*IFFTGS*PR.V & $\begin{array}{l}\text { Y183; S189 } \\
\text { (b3/y2, y4) }\end{array}$ & $\mathrm{N}, \mathrm{N}$ & $\mathrm{Y}$ & $\mathrm{N}$ & $\mathrm{N}$ & $\mathrm{N}$ & $\mathrm{N}$ \\
\hline P17252 & $\begin{array}{l}\text { Protein kinase } \mathrm{C} \text { alpha } \\
\text { type }\end{array}$ & R.STLNPQWNES*FTFK.L & $\mathrm{S} 226(\mathrm{y} 4, \mathrm{y} 5)$ & Y & $\mathrm{N}$ & $\mathrm{Y}$ & $\mathrm{N}$ & $\mathrm{N}$ & $\mathrm{N}$ \\
\hline Q8IYX7 & Protein FAM154A & R.EY*QKGPIPMEGLTTSR.R & Y59 (not) & $\mathrm{N}$ & $\mathrm{N}$ & $\mathrm{Y}$ & $\mathrm{N}$ & $\mathrm{N}$ & $\mathrm{N}$ \\
\hline O75069 & $\begin{array}{l}\text { Transmembrane and } \\
\text { coiled-coil domains } \\
\text { protein } 2\end{array}$ & R.GAS*LHS*SSGGGSS*GSSSRRTK.S & $\begin{array}{l}\text { S166; S169 } \\
\text { (not/not) }\end{array}$ & $\mathrm{N}, \mathrm{N}$ & $\mathrm{N}$ & $\mathrm{Y}$ & $\mathrm{N}$ & $\mathrm{N}$ & $\mathrm{N}$ \\
\hline P27216 & Annexin A13 & K.YQKS*LSDMVRSDTSGDFR.K & S294 (not) & $\mathrm{N}$ & $\mathrm{N}$ & $\mathrm{Y}$ & $\mathrm{N}$ & $\mathrm{N}$ & $\mathrm{N}$ \\
\hline Q6PCE3 & $\begin{array}{l}\text { Phosphoglucomutase-2- } \\
\text { like } 1\end{array}$ & K.AVAGVMITAS*HNR.K & $\mathrm{S} 175(\mathrm{y} 3, \mathrm{y} 4)$ & $\mathrm{Y}$ & $\mathrm{N}$ & $\mathrm{Y}$ & $\mathrm{N}$ & $\mathrm{N}$ & $\mathrm{N}$ \\
\hline P01854 & Ig epsilon chain $\mathrm{C}$ region & R.VAHTPS*STDWVDNK.T & S92 (y8, y9) & $\mathrm{N}$ & $\mathrm{N}$ & $\mathrm{Y}$ & $\mathrm{N}$ & $\mathrm{N}$ & $\mathrm{N}$ \\
\hline Q8TCU4 & $\begin{array}{l}\text { Alstrom syndrome protein } \\
1\end{array}$ & R.SPLQEAES*KVSMALEETLR.Q & $\begin{array}{l}\text { S2367 (y11, } \\
\text { y14) }\end{array}$ & $\mathrm{N}$ & $\mathrm{N}$ & $\mathrm{Y}$ & $\mathrm{N}$ & $\mathrm{N}$ & $\mathrm{N}$ \\
\hline P80421 & $\begin{array}{l}\text { Ig heavy chain V-I region } \\
\text { DOT }\end{array}$ & R.DRLVMSSDTSANTVS*MQLR.N & $\mathrm{S} 79(\mathrm{~b} 14, \mathrm{~b} 15)$ & $\mathrm{N}$ & $\mathrm{N}$ & $\mathrm{Y}$ & $\mathrm{N}$ & $\mathrm{N}$ & $\mathrm{N}$ \\
\hline Q8TAP8 & $\begin{array}{l}\text { Putative uncharacterized } \\
\text { protein } \mathrm{C} 7 \text { orf } 47\end{array}$ & K.S*SLALGLELR.A & S108 (not) & $\mathrm{N}$ & $\mathrm{N}$ & $\mathrm{Y}$ & $\mathrm{N}$ & $\mathrm{N}$ & $\mathrm{N}$ \\
\hline P36871 & Phosphoglucomutase-1 & K.AIGGIILTAS*HNPGGPNGDFGIK.F & $\mathrm{S} 117(\mathrm{~b} 9, \mathrm{~b} 12)$ & Y & $\mathrm{N}$ & $\mathrm{Y}$ & $\mathrm{N}$ & $\mathrm{N}$ & $\mathrm{N}$ \\
\hline P60174 & $\begin{array}{l}\text { Triosephosphate } \\
\text { isomerase }\end{array}$ & R.KQS*LGELIGTLNAAK.V & $\mathrm{S} 21(\mathrm{y} 12, \mathrm{y} 13)$ & $\mathrm{Y}$ & $\mathrm{N}$ & $\mathrm{N}$ & $\mathrm{Y}$ & $\mathrm{N}$ & $\mathrm{N}$ \\
\hline Q9NZN4 & $\begin{array}{l}\text { EH domain-containing } \\
\text { protein } 2\end{array}$ & R.GPDEAMEDGEEGS*DDEAEWVVTK.D & S438 (not) & Y & $\mathrm{N}$ & $\mathrm{N}$ & Y & $\mathrm{N}$ & $\mathrm{N}$ \\
\hline P22059 & $\begin{array}{l}\text { Oxysterol-binding protein } \\
1\end{array}$ & K.GDMS*DEDDENEFFDAPEIITMPENLGHK.R & S351 (b4) & $\mathrm{Y}$ & $\mathrm{N}$ & $\mathrm{N}$ & $\mathrm{Y}$ & $\mathrm{N}$ & $\mathrm{N}$ \\
\hline
\end{tabular}


Table A-4 (continued).

\begin{tabular}{|c|c|c|c|c|c|c|c|c|c|}
\hline $\begin{array}{l}\text { Accession } \\
\text { number }\end{array}$ & Protein & Peptide & Site & $\begin{array}{l}\text { Phosphory- } \\
\text { lation site }\end{array}$ & $\begin{array}{l}99- \\
029\end{array}$ & $\begin{array}{l}98- \\
065\end{array}$ & $\begin{array}{l}99- \\
045\end{array}$ & $\begin{array}{l}99- \\
067\end{array}$ & $\begin{array}{l}99- \\
010\end{array}$ \\
\hline Q32MZ4 & $\begin{array}{l}\text { Leucine-rich repeat flightless- } \\
\text { interacting protein } 1\end{array}$ & R.RGS*GDTSISIDTEASIR.E & S120 (b3) & $\mathrm{N}$ & $\mathrm{N}$ & $\mathrm{N}$ & $\mathrm{Y}$ & $\mathrm{N}$ & $\mathrm{N}$ \\
\hline Q9UBT6 & DNA polymerase kappa & K.CDS*YKDDLLLR.M & S10 (b3) & $\mathrm{N}$ & $\mathrm{N}$ & $\mathrm{N}$ & Y & $\mathrm{N}$ & $\mathrm{N}$ \\
\hline Q86SQ6 & $\begin{array}{l}\text { Probable G-protein coupled } \\
\text { receptor } 123\end{array}$ & K.QVT*KKAPLCLDTDQPPYPR.Q & T833 (b4,b5) & $\mathrm{N}$ & $\mathrm{N}$ & $\mathrm{N}$ & $\mathrm{Y}$ & $\mathrm{N}$ & $\mathrm{N}$ \\
\hline Q9Y618 & Nuclear receptor corepressor 2 & K.REGTPPPPPPS*R.D & $\mathrm{S} 1390(\mathrm{y} 2, \mathrm{y} 3)$ & $\mathrm{N}$ & $\mathrm{N}$ & $\mathrm{N}$ & $\mathrm{Y}$ & $\mathrm{N}$ & $\mathrm{N}$ \\
\hline Q9H2J4 & Phosducin-like protein 3 & K.LS*ES*GAIMTDLEENPK.K & $\begin{array}{l}\text { S199; S201 } \\
\text { (not/y12, y14) }\end{array}$ & $\mathrm{N}, \mathrm{N}$ & $\mathrm{N}$ & $\mathrm{N}$ & Y & $\mathrm{N}$ & $\mathrm{N}$ \\
\hline P09496 & Clathrin light chain A & R.LQS*EPESIR.K & $\mathrm{S} 105(\mathrm{~b} 3, \mathrm{~b} 4)$ & $\mathrm{N}$ & $\mathrm{N}$ & $\mathrm{N}$ & $\mathrm{Y}$ & $\mathrm{N}$ & $\mathrm{N}$ \\
\hline O43237 & $\begin{array}{l}\text { Cytoplasmic dynein } 1 \text { light } \\
\text { intermediate chain } 2\end{array}$ & K.SGQKT*VLSNVQEELDR.M & $\mathrm{T} 463(\mathrm{y} 10, \mathrm{y} 12)$ & $\mathrm{N}$ & $\mathrm{N}$ & $\mathrm{N}$ & $\mathrm{Y}$ & $\mathrm{N}$ & $\mathrm{N}$ \\
\hline P35558 & $\begin{array}{l}\text { Phosphoenolpyruvate } \\
\text { carboxykinase }\end{array}$ & R.WM\#S*EEDFEK.A & S118 (y6, y7) & Y & $\mathrm{N}$ & $\mathrm{N}$ & $\mathrm{Y}$ & $\mathrm{N}$ & $\mathrm{N}$ \\
\hline P05386 & $60 \mathrm{~S}$ acidic ribosomal protein $\mathrm{P} 1$ & K.KEES*EES*DDDMGFGLFD & $\begin{array}{l}\text { S101; S104 } \\
\text { (b5, b6/y10, } \\
\text { y11) }\end{array}$ & $\mathrm{Y}, \mathrm{Y}$ & $\mathrm{N}$ & $\mathrm{N}$ & $\mathrm{Y}$ & $\mathrm{N}$ & $\mathrm{N}$ \\
\hline P13861 & $\begin{array}{l}\text { cAMP-dependent protein kinase } \\
\text { type II-alpha regulatory subunit }\end{array}$ & K.GDS*ES*EEDEDLEVPVPSR.F & $\begin{array}{l}\text { S77; S79 } \\
\text { (not/not) }\end{array}$ & $\mathrm{Y}, \mathrm{Y}$ & $\mathrm{N}$ & $\mathrm{N}$ & $\mathrm{Y}$ & $\mathrm{N}$ & $\mathrm{N}$ \\
\hline Q6P9B9 & Integrator complex subunit 5 & R.FQAPSPS*TLLR.Q & S1012 (not) & $\mathrm{N}$ & $\mathrm{N}$ & $\mathrm{N}$ & Y & $\mathrm{N}$ & $\mathrm{N}$ \\
\hline Q14195 & $\begin{array}{l}\text { Dihydropyrimidinase-related } \\
\text { protein } 3\end{array}$ & R.GSPT*RPNPPVR.N & T524 (y6, y9) & $\mathrm{N}$ & $\mathrm{N}$ & $\mathrm{N}$ & Y & $\mathrm{N}$ & $\mathrm{N}$ \\
\hline Q13618 & Cullin-3 & R.FLLES*FNNDRLFK.Q & S359 (not) & $\mathrm{N}$ & $\mathrm{N}$ & $\mathrm{N}$ & Y & $\mathrm{N}$ & $\mathrm{N}$ \\
\hline P48681 & Nestin & R.S*LGEQDQMTLRPPEK.V & S767 (not) & $\mathrm{Y}$ & $\mathrm{N}$ & $\mathrm{N}$ & $\mathrm{N}$ & Y & $\mathrm{N}$ \\
\hline Q9NSE4 & Isoleucyl-tRNA synthetase & K.GLVYRS*Y*KPVFWSPSSR.T & $\begin{array}{l}\text { S239; Y240 } \\
(\mathrm{b} 5, \mathrm{~b} 6 / \mathrm{b} 6, \mathrm{~b} 7)\end{array}$ & $\mathrm{N}, \mathrm{N}$ & $\mathrm{N}$ & $\mathrm{N}$ & $\mathrm{N}$ & $\mathrm{Y}$ & $\mathrm{N}$ \\
\hline
\end{tabular}


Table A-4 (continued).

\begin{tabular}{|c|c|c|c|c|c|c|c|c|c|}
\hline $\begin{array}{l}\text { Accession } \\
\text { number }\end{array}$ & Protein & Peptide & Site & $\begin{array}{l}\text { Phosphory- } \\
\text { lation site }\end{array}$ & $\begin{array}{l}99- \\
029\end{array}$ & $\begin{array}{l}98- \\
065\end{array}$ & $\begin{array}{l}99- \\
045\end{array}$ & $\begin{array}{l}99- \\
067\end{array}$ & $\begin{array}{l}99- \\
010\end{array}$ \\
\hline O43432 & $\begin{array}{l}\text { Eukaryotic translation } \\
\text { initiation factor } 4 \text { gamma } 3\end{array}$ & R.SS*ASSLNR.F & S1194 (not) & $\mathrm{N}$ & $\mathrm{N}$ & $\mathrm{N}$ & $\mathrm{N}$ & $\mathrm{Y}$ & $\mathrm{N}$ \\
\hline P27824 & Calnexin precursor & K.AEEDEILNRS*PR.N & S583 (y2, y3) & $\mathrm{Y}$ & $\mathrm{N}$ & $\mathrm{N}$ & $\mathrm{N}$ & Y & $\mathrm{N}$ \\
\hline P20711 & $\begin{array}{l}\text { Aromatic-L-amino-acid } \\
\text { decarboxylase }\end{array}$ & K.GLQAY*IR.K & Y377 (y2, y3) & $\mathrm{N}$ & $\mathrm{N}$ & $\mathrm{N}$ & $\mathrm{N}$ & Y & $\mathrm{N}$ \\
\hline O76041 & Nebulette & R.SMQHS*PNLR.T & S953 (y4, y5) & $\mathrm{N}$ & $\mathrm{N}$ & $\mathrm{N}$ & $\mathrm{N}$ & Y & $\mathrm{N}$ \\
\hline P08238 & $\begin{array}{l}\text { Heat shock protein HSP } \\
\text { 90-beta (HSP 90) }\end{array}$ & K.IEDVGS*DEEDDSGKDKK.K & S254 (b5, b8) & $\mathrm{Y}$ & $\mathrm{N}$ & $\mathrm{N}$ & $\mathrm{N}$ & Y & $\mathrm{N}$ \\
\hline O75385 & $\begin{array}{l}\text { Serine/threonine-protein } \\
\text { kinase ULK1 }\end{array}$ & R.KM\#S*LGGGR.P & S495 (y4, y6) & $\mathrm{N}$ & $\mathrm{N}$ & $\mathrm{N}$ & $\mathrm{N}$ & Y & $\mathrm{N}$ \\
\hline Q9Y5C1 & $\begin{array}{l}\text { Angiopoietin-related } \\
\text { protein } 3 \text { precursor }\end{array}$ & K.NMSLELNS*K.L & $\mathrm{S} 122(\mathrm{y} 2, \mathrm{y} 3)$ & $\mathrm{N}$ & $\mathrm{N}$ & $\mathrm{N}$ & $\mathrm{N}$ & Y & $\mathrm{N}$ \\
\hline Q6KC79 & Nipped-B-like protein & K.QNEST*IVEPK.Q & T667 (y3, y6) & $\mathrm{N}$ & $\mathrm{N}$ & $\mathrm{N}$ & $\mathrm{N}$ & Y & $\mathrm{N}$ \\
\hline P58107 & Epiplakin & R.QPLQAT*FRGLRKQVS*AR.D & $\begin{array}{l}\text { T1495, S1504 } \\
(\mathrm{b} 5, \mathrm{~b} 6 / \mathrm{b} 14, \mathrm{~b} 15)\end{array}$ & $\mathrm{N}, \mathrm{N}$ & $\mathrm{N}$ & $\mathrm{N}$ & $\mathrm{N}$ & $\mathrm{N}$ & Y \\
\hline P02545 & Lamin A/C & R.LRLS*PSPTSQR.S & $\mathrm{S} 390(\mathrm{y} 7, \mathrm{y} 8)$ & $\mathrm{Y}$ & $\mathrm{N}$ & $\mathrm{N}$ & $\mathrm{N}$ & $\mathrm{N}$ & Y \\
\hline Q13242 & $\begin{array}{l}\text { Splicing factor, } \\
\text { arginine/serine-rich } 9\end{array}$ & R.GS*PHYFSPFRPY & S211 (b2) & $\mathrm{Y}$ & $\mathrm{N}$ & $\mathrm{N}$ & $\mathrm{N}$ & $\mathrm{N}$ & Y \\
\hline Q13263 & $\begin{array}{l}\text { Transcription intermediary } \\
\text { factor 1-beta }\end{array}$ & R.SRS*GEGEVSGLMR.K & S473 (b2, b3) & $\mathrm{Y}$ & $\mathrm{N}$ & $\mathrm{N}$ & $\mathrm{N}$ & $\mathrm{N}$ & Y \\
\hline P07197 & $\begin{array}{l}\text { Neurofilament triplet M } \\
\text { protein }\end{array}$ & K.GKSPVPKS*PVEEK.G & S617 (y5, y6) & $\mathrm{Y}$ & $\mathrm{N}$ & $\mathrm{N}$ & $\mathrm{N}$ & $\mathrm{N}$ & Y \\
\hline P15924 & Desmoplakin & K.GLPSPYNMSSAPGS*R.S & S2825 (y2) & $\mathrm{Y}$ & $\mathrm{N}$ & $\mathrm{N}$ & $\mathrm{N}$ & $\mathrm{N}$ & Y \\
\hline O00264 & $\begin{array}{l}\text { Membrane associated } \\
\text { progesterone receptor } \\
\text { component } 1\end{array}$ & K.LLKEGEEPTVYS*DEEEPKDESAR.K & $\mathrm{S} 180$ (b11, b12) & Y & $\mathrm{N}$ & $\mathrm{N}$ & $\mathrm{N}$ & $\mathrm{N}$ & Y \\
\hline
\end{tabular}


Table A-5 Scansite results for the characterized phosphorylation sites.

\begin{tabular}{|c|c|c|c|c|}
\hline $\begin{array}{l}\text { Accession } \\
\text { number }\end{array}$ & Protein & Site & $\begin{array}{l}\text { Scansite result } \\
\text { Kinase/binding }\end{array}$ & Type \\
\hline O14558 & $\begin{array}{l}\text { Heat-shock protein beta- } 6 \\
\text { (Heat-shock } 20 \mathrm{kDa}-\text { like } \\
\text { protein) }\end{array}$ & S16 & 14-3-3 Mode 1 & $\begin{array}{l}\mathrm{pS} / \mathrm{T} \text { binding } \\
\text { group }\end{array}$ \\
\hline Q14315 & Filamin-C & S2233 & Akt Kinase & $\begin{array}{l}\text { Basophilic } \mathrm{S} / \mathrm{T} \\
\text { kinase group }\end{array}$ \\
\hline Q9H3Z4 & $\begin{array}{l}\text { DnaJ homolog subfamily } \mathrm{C} \\
\text { member } 5\end{array}$ & S8 & $\begin{array}{l}\text { Calmodulin dependent } \\
\text { Kinase } 2\end{array}$ & $\begin{array}{l}\text { Basophilic } \mathrm{S} / \mathrm{T} \\
\text { kinase group }\end{array}$ \\
\hline \multirow[t]{3}{*}{ Q9HBL0 } & Tensin-1 & S1393 & Protein Kinase A & $\begin{array}{l}\text { Basophilic } \mathrm{S} / \mathrm{T} \\
\text { kinase group }\end{array}$ \\
\hline & & $\mathrm{T} 1105$ & Erk1 Kinase & $\begin{array}{l}\text { Kinase binding } \\
\text { site group }\end{array}$ \\
\hline & & S621 & $\begin{array}{l}\text { Calmodulin dependent } \\
\text { Kinase } 2\end{array}$ & $\begin{array}{l}\text { Basophilic } \mathrm{S} / \mathrm{T} \\
\text { kinase group }\end{array}$ \\
\hline Q15019 & Septin-2 & S218 & Casein Kinase 2 & $\begin{array}{l}\text { Acidophilic } \mathrm{S} / \mathrm{T} \\
\text { kinase group }\end{array}$ \\
\hline Q9NZN4 & $\begin{array}{l}\text { EH domain-containing protein } \\
2\end{array}$ & S438 & Casein Kinase 2 & $\begin{array}{l}\text { Acidophilic } \mathrm{S} / \mathrm{T} \\
\text { kinase group }\end{array}$ \\
\hline Q9Y618 & Nuclear receptor corepressor 2 & S1390 & GSK3 Kinase & $\begin{array}{l}\text { Acidophilic } \mathrm{S} / \mathrm{T} \\
\text { kinase group }\end{array}$ \\
\hline P09496 & Clathrin light chain A & S105 & 14-3-3 Mode 1 & $\begin{array}{l}\mathrm{pS} / \mathrm{T} \text { binding } \\
\text { group }\end{array}$ \\
\hline P05386 & $\begin{array}{l}\text { 60S acidic ribosomal protein } \\
\text { P1 }\end{array}$ & S104 & Casein Kinase 2 & $\begin{array}{l}\text { Acidophilic } \mathrm{S} / \mathrm{T} \\
\text { kinase group }\end{array}$ \\
\hline \multirow[t]{2}{*}{ P13861 } & $\begin{array}{l}\text { cAMP-dependent protein } \\
\text { kinase type II-alpha regulatory } \\
\text { subunit }\end{array}$ & S77 & Casein Kinase 2 & $\begin{array}{l}\text { Acidophilic } \mathrm{S} / \mathrm{T} \\
\text { kinase group }\end{array}$ \\
\hline & & S79 & Casein Kinase 2 & $\begin{array}{l}\text { Acidophilic } \mathrm{S} / \mathrm{T} \\
\text { kinase group }\end{array}$ \\
\hline P48681 & Nestin & S767 & $\begin{array}{l}\text { 14-3-3 Mode 1; Protein } \\
\text { Kinase A }\end{array}$ & $\begin{array}{l}\mathrm{pS} / \mathrm{T} \text { binding } \\
\text { group; Basophilic } \\
\mathrm{S} / \mathrm{T} \text { kinase group }\end{array}$ \\
\hline P08238 & $\begin{array}{l}\text { Heat shock protein HSP 90- } \\
\text { beta (HSP 90) }\end{array}$ & S254 & Casein Kinase 2 & $\begin{array}{l}\text { Acidophilic } \mathrm{S} / \mathrm{T} \\
\text { kinase group }\end{array}$ \\
\hline P58107 & Epiplakin & S1504 & PKC zeta & $\begin{array}{l}\text { Basophilic } \mathrm{S} / \mathrm{T} \\
\text { kinase group }\end{array}$ \\
\hline
\end{tabular}




\section{VITA}

Li Chen was born on May 17, 1971, in Xinjiang, China. She graduated with a B.S. in chemistry in 1992 and a M.S. in pharmaceutical analysis in 1998. She joined in the Pharmaceutical Sciences program in the University of Tennessee Health Science Center in Memphis for her Ph.D. studies in 2005. She completed her doctorate degree in December 2009. 